\title{
Ruska K.Simeonova
}

\section{Die Segmentsysteme des Deutschen und des Bulgarischen}

\author{
Eine kontrastive \\ phonetisch-phonologische Studie
}

Verlag Otto Sagner München · Berlin - Washington D.C.

Digitalisiert im Rahmen der Kooperation mit dem DFG-Projekt „Digi20“

der Bayerischen Staatsbibliothek, München. OCR-Bearbeitung und Erstellung des eBooks durch den Verlag Otto Sagner:

http://verlag.kubon-sagner.de

( $)$ bei Verlag Otto Sagner. Eine Verwertung oder Weitergabe der Texte und Abbildungen, insbesondere durch Vervielfältigung, ist ohne vorherige schriftliche Genehmigung des Verlages unzulässig. 


\title{
SLAVISTISCHE BEITRÄGE
}

\author{
BEGRÜNDET VON \\ ALOIS SCHMAUS \\ HERAUSGEGEBEN VON \\ HEINRICH KUNSTMANN \\ PETER REHDER - JOSEF SCHRENK \\ REDAKTION \\ PETER REHDER
}

Band 244

\section{VERLAG OTTO SAGNER MÜNCHEN}


RUSKA SIMEONOVA

\section{DIE SEGMENTSYSTEME}

DES DEUTSCHEN UND DES BULGARISCHEN

Eine kontrastive phonetisch-phonologische Studie

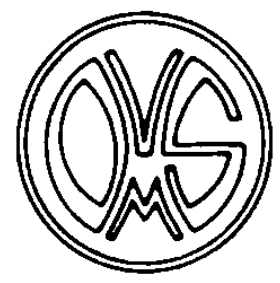

VERLAG OTTO SAGNER - MÜNCHEN 1989 
Bayerische Staatsbibliothok München

ISBN 3.87690.445.5

(c) Verlag Otto Sagner, München 1989 Abteilung der Firma Kubon \& Sagner, München 


\section{EINLEITUNG}

Aus den theoretischen und praktisch-angewandten Aufgaben des Fremdsprachenunterrichts in den letzten Jahrzehnten unseres Jahrhunderts hat sich der kontrastive Sprachvergleich ergeben, der sich auch in kurzer Zeit zu einer wesentlichen Richtung der linguistischen Forschung entwickelte. Kontrastive Untersuchungen verlaufen synchron, haben zum Forschungsobjekt ein Sprachenpaar, in den meisten Fällen eine Fremdsprache und die Muttersprache, und zum Gegenstand - die Aufdeckung der Unterschiede und der Übereinstimmungen der verglichenen Sprachen. Ihr Ziel kann auch ein rein linguistisches sein; dann tragen sie zur vergleichenden Charakteristik der untersuchten Sprachen bei und sind somit auch sprachtypologisch ausgerichtet. Kontrastive Untersuchungen verfolgen jedoch viel öfter Aufgaben, die sich aus dem Fremdsprachenunterricht ergeben. In solchen Fällen sind ihre Ergebnisse und Feststellungen als theoretische Grundlage zur anschlicßenden methodischen Aufbereitung anzusehen, damit sie den konkreten Aufgaben zur Verbesserung der Sprachausbilldung entsprechen.

Die vorliegende Schrift stellt sich zum Ziel, die Segmentsysteme des Gegenwartsdeutschen und des Gegenwartsbulgarischen in kontrastiver Sicht $\mathrm{zu}$ besschreiben und aus dieser Beschreibung Konsequenzen auf Unterschiede und Übereinstimmungen sowohl im Tätigkeitsprozeß ihrer Realisierung als auch in ihrem phonologischen Status, der Distribution und Kombinatorik zu ziehen.

Die beschreibende Darstellung wird nach den Grundaspekten des lautsprachlichen Kommunikationsvorgangs vorgenommen : nach dem physiologisch-genetischen, nach dem akustisch-perzeptiven und dem phonologischen.

Grundlage der vorgenommenen Konfrontation bildet die Standardform der Aussprache des Deutschen und des Bulgarischen in ihrer gemäßigten, jedoch neutralen Realisierungsvariante.

Die physiologisch-genetische Beschreibung beider Segmentsysteme erfolgt nach bereits vorhandenen Untersuchungen dieses Forschungsaspektes fur das Deutsche und für das Bulgarische.

Bei der kontrastiven akustischen Analyse werden in starkem Maße experimentalphonetische Untersuchungen des Verfassers benutzt, die im Experimentalphonetischen Laboratorium der Bulgarischen Akademie der Wissenschaften und der Sofioter Universität durchgeführt worden sind. 
Bei der phonologischen Gegenüberstellung beider Phonemsysteme wurdc auch ein möglichst vollständiges Bild der Distribution und Kombinatorik der Segmente angestrebt.

Neben den Zielen einer kontrastiven Untersuchung Deutsch-Bulgarisch, sollen die in der Schrift vorhandenen akustischen Daten sowie die Ergebnisse der distributionell-kombinatorischen Analyse beider Phonemsysteme als Grundlage für Sprachsynthese dienen, die in unserem Land immer noch in den Ansätzen steckt. 


\section{KONTRASTIVE BEOBACHTUNGS- UND EXPERIMENTALANALYSE DER ARTIKULATORISCHEN BESONDERHEITEN IM PROZESS DER VOKALBILDUNG DES DEUTSCHEN UND DES BULGARISCHEN}

\subsection{SEGMENTIERUNGSMÖGLICHKEITEN DER SPRACHLAUTE IM REDEFLUSS}

Ein jeder Versuch zur experimentellen Bestimmung der Basiskomponenten der Artikulationseinstellungen und der relevanten Sprechbewegungsabläufe, die in einer gegebenen Sprache für die Verwirklichung ihrer lautlichen Form benutzt werden, ein jeder Versuch zur experimentellen Festlegung der akustischen und funktionellen Charakteristika der Sprachlaute, wären eine bloße Fiktion, wenn man keine experimentell gesicherten Beweise für die tatsächliche Möglichkeit, die einzelnen Lautsegmente im Redefluß, d. h. in ihrem zeitlichen und räumlichen Bewegungsablauf eindeutig zu segmentieren, auf der Hand hätte.

Sind aber die einzelnen Sprachlaute im kontinuierlichen ProzeB des Sprechens voneinander abzugrenzen? Könnte man sie genetisch, gennematisch und perzeptiv segmentieren und auf welche Weise?

Es ist durchaus möglich, wie G. Lindner $(1975,121)$ behauptet, die Bewegungen der Sprechorgane während der Artikulation eines einzelnen Sprachlautes zu verfolgen. Die Phonetik (vor allem die Lautphysiologie) hat diese Tatsache bewiesen, indem sie mehr oder weniger genau sowohl die Einstellung wie auch die Bewegungen der Artikulationsorgane bei der Bildung der einzelnen Sprachlaute beschrieben hat. Es ist auch gar nicht verwunderlich, zumal der Mensch imstande ist, einzelne Sprachlaute hervorzubringen. Die Sache wird aber recht kompliziert, sobald man bedenkt, daß sich der sprachliche Kommunikationsproze $B$ durchaus nicht aus wechselseittigen Einzellautrepliken zwischen den Gespächspartnern zusammensetzt, sondern in einem kontinuierlichen, situationsbedingten Redefluß-Kontext verläuft. Dieser kontinuierlich verlaufende Kontext ist einer Reihe von phonetischen Gesetzen und verschiedenen Faktoren untergeordnet, die die experimentelle Untersuchung der tatsächlichen, ineinandergreifenden und sich überlagernden Bewegungen der Sprechorgane im ProzeB der Nacheinanderfol- 
ge und Gleichzeitigkeit der segmentalen und suprasegmentalen Einheiten im Redefluß äußerst erschweren.

Die Schwierigkeiten, die durch die Genese der Lautbildung bedingt sind, faßt G. Lind ne r $(1975,120)$ folgendermaßen zusammen: „Den Sprechbewegungsablauf als Gesamtgeschehen zu beschreiben und darzustellen ist deshalb so schwierig, weil es sich 1. um ein Geschehen handelt, bei dem eine Vielzahl von Organen gleichzeitig beteiligt ist, weil 2. deren Bewegungen miteinanderverflochten sind und weil 3.... nicht ohne Hilfsmittel möglich ist, alle Organe gleichzeitig in ihrem Bewegungsablauf zu beobachten."

Der Weg, den $G$. Lindner vorschlägt und auch selbst geht, um seinen Ansatz zu einer Hypothese der Sprechbewegungsabläufe des Deutschen aufzubauen, ist das absichtliche Auseinanderhalten, die Abstraktion während und zum Zwecke des Experiments, d. h. entweder die Untersuchung aller Bewegungen eines einzelnen Sprechorgans während der Artikulation, oder aber die absichtliche Lenkung der Aufmerksamkeit auf die Einstellungen bzw. Bewegungen aller an der Artikulation eines einzelnen Sprachlautes beteiligten Organe.

Der zweite Schritt auf diesem Wege wäre dann „die Ergebnisse durch gedankliche Analyse miteinander zu verbinden" (L in d ner, 1975, 120) und der dritte, „das oberste Kriterium", ihre Überprüfung in der Wirklichkeit, in der Sprachpraxis.

Lindner vertritt weiter in seiner „Phonetischen Studie“ die Auffassung, da B man für die Segmentierung der Sprechbewegungsabläufe naturgemā $B$ von den zweigliedrigen Lautfolgen ausgehen müßte, ,weil in ihnen die Bewegungen enthalten sind, die mit zwingender Notwendigkeit beim Übergang von der Einstellung eines Lautes in die eines anderen vollzogen werden müssen" (Lind ner, 1975, 122).

Doch weder die Abgrenzung der zweigliedrigen Lautfolgen voneinander noch die Segmentierung der Elemente, aus denen sie bestehen, ist eine natürliche, sprechphysiologische Gegebenheit. Die Abgrenzungsmöglichkeiten müßte man folglich auf dem Wege des Experiments suchen, festlegen und systematisieren, wobei man auch stets darauf bedacht sein muß, daß die Abgrenzungsmöglichkeiten je nach dem Aspekt der Untersuchung verschieden ausfallen.

Die experimentalphonetischen Versuche von Menzerath, de Lacerda u.a., die Grenzen der Laute im Redefluß zu bestimmen, haben bereits in den 30er Jahren die Grundlage für die Koartikulationstheorie geschaffen, nach welcher die lautliche Form der gesprochenen Rede eine kontinuierliche, dynamisch verlaufende Aufeinanderfolge von ständig variierenden, in- und übereinandergreifenden Signalen darsellt. „Sprechen ist Dauerbewegung. Das Dogma von Anglitt, Stellung und Abglitt muß fallen. ... Diese synchron verlaufende Bewegungsmehrheit (Gleitlaute gibt es nicht!) habe ich als 'Synkinese' oder 'Koartikulation' bezeichnet und damit sagen wollen, da $B$ die Wortartikulation im Sinne der Gesamtstruktur verläuft, so also, da $B$ sich die Teilbewegungen zur Ganzheit zusammenfügen, sich 'verflechten', nicht aber wie Glieder einer 'Kette' aufeinanderfolgen. Der Aufbau, die Ganzheit geht dem Teil voraus : sie weist den Weg" (Menzerath, 1935, 252). 
Seit Menzeraths Zeit sind ,alle Versuche, mit Hilfe von Registrierungen die Grenzen im Sprechbewegungsablauf zu finden, gescheitert" (Lind ne r, 1975, 123, 124).

Auch auf dem akustischen Niveau ist die Segmentierungsfrage noch nicht gelöst.

So scheint die durch die immer wieder scheiternden Segmentierungsversuche physiologischer und spektroakustischer Art aufgestellte These von der nicht segmentierbaren Kontinuität des Artikulationsprozesses die These der traditionellen Phonetik von dem diskreten Charakter der menschlichen Rede $\mathrm{zu}$ widerlegen.

Bei dem hohen Entwicklungsstand der für phonetische Untersuchungszwecke anwendbaren und speziell dafür entwickelten und konstruierten Untersuchungsgeräte heute könnte man kaum der Meinung zustimmen, daß der Grund für die Hilflosigkeit gegenüber der Abgrenzung eines Lautsegments vom „Nachbargeschehen genetisch und am Klangendprodukt... an der Unzulänglichkeit unserer experimentellen Mittel zu suchen wäre" (Wä n gle r, $1964,18)$.

Gegenwärtige Beobachtungen und Experimente haben bereits gezeigt, da $\mathbb{B}$ man ihn woanders suchen sollte. Es hat sich nämlich erwiesen, daß im Artikulationsmechanismus der Rede eine Vielzahl von "Schlüsselprozessen“ eine gute Abhilfe für die recht eindeutige Bestimmung der Lautgrenzen im Redefluß leisten können. Es ist logisch anzunehmen, daß diese Schlüsselprozesse als perzeptiv und artikulatorisch-aktiv-relevante Markierungsmuster der Lautgrenzen im sprachgeschichtlich und genetisch bedingten GedächtnisCode-System eines jeden Sprachträgers gespeichert sind.

An das Vorhandensein dieser perzeptiv-psycholinguistischen Signale knüpft auch $G$. Lindner an, indem er versucht, ihre Existenz experimentell nachzuweisen und die Brücke zu ihren Korrelaten auf dem physiologischartikulatorischen Niveau zu schlagen.

Gleichzeitig ist die experimentelle Aufbereitung und Auswertung eines umfangreichen sprachlichen Materials in der noch nicht veröffentlichten Studie von Tilkov, Mischeva und Peeva "Akustische Mikrostruktur der bulgarischen Sprachlaute" ein nicht zu bestreitender Beweis dafür, da $B$ man die Auffassung von dem diskreten Charakter der Lautsegmente auch auf dem akustischen Niveau der Rede bestätigen kann (Т и лков, М ише ва, П е ва, 1977, 8).

So entsprechen z. B. dem Ein- bzw. Ausschalten der Stimme, der Lösung des Verschlusses bei den Explosiven und Nasalen klar ausgeprägte Veränderungen in der Struktur der Schallwelle. Bei der Bildung der Frikative sind die Spektralgrenzmarken wegen der relativ langsamen Erweiterung der frikativen Enge nicht so eindeutig. Dagegen sind jedoch die für das Vokalsegment wie auch die für das Segment des Konsonanten typischen Spektralgrenzmarken auf dem spektralen Lautbild klar zu beobachten, wobei zwischen ihnen ein Übergangssegment umrissen bleibt. Wenn auch mit etwas Vorbehalt und Unsicherheit, so könnte man doch als Spektralgrenze zwischen den beiden Lautsegmenten die Mitte dieses Übergangsintervalls markieren. Die Messungen haben gezeigt, daß diese Segmentübergänge von einer geringen Dauer sind (etwa 0,002 Sek.), so daß der maximale Fehler, den man 
durch diese Prozedur einführt, nur noch 0,001 Sek. dauern würde, ein Wert, der sich in den Grenzen der Ausmessungsgenauigkeit bewegt und deshalb nicht von Belang ist. Den Ergebnissen der Spektraluntersuchungen entsprechend werden in der oben zitierten Studie auch Regeln fur die spektrale Abgrenzung der Lautsegmente im Redefluß aufgestellt. Die Segmentierungsmethodik und -regeln von Tilkov und Mischeva haben wir bei der Errechnung und Auswertung der Ergebnisse unserer Spektraluntersuchungen zur Intensität, Grundfrequenz und Dauer der deutschen und der bulgarischen Sprachlaute im Redekontinuum angewendet. Die daraus gewonnene Erfahrung läßt auf den universellen Charakter der spektralen Segmentierungsmöglichkeiten schließen (Siehe die vergleichenden Intonogramme in der Beilage).

\subsection{VOKALARTIKULATION DES DEUTSCHEN UND DES BULGARISCHEN}

\subsubsection{Relevante Einstellungen und Sprechbewegungsabläufe für} die Bildung der Kardinalvokale in den meisten Weltsprachen

Die Gesamtheit der fur die Artikulation der Vokallaute notwendigen physiologischen Faktoren ist eine relative Größe. Je nach den spezifischen Besonderheiten des Vokalsystems einer bestimmten Sprache variieren sie, obwohl in einem begrenzten Umfang.

Im Hinblick auf die für die Bildung der Kardinalvokale notwendigen Faktoren sind Meinungsunterschiede bei den verschiedenen Autoren anzutreffen.

So berücksichtigt das Hellwag'sche Vokaldreieck allein die Zungenbewegung in waagerechter und senkrechter Richtung. Danach werden die Vokallaute einerseits in Vorder-, Mittel- und Hinterzungenvokale, andererseits in hohe, mittlere und tiefe Vokale eingeteilt.

E. Sievers $(1901,79)$ hebt zwei wichtige Faktoren für die Vokalartikulation hervor: die orale Öffnung und die Tätigkeit des Zungenrückens.

J. Forchhammer (1921) versucht, die typische Einstellung des Ansatzrohres bei der Vokalartikulation originell durch seinen 'Vokalklotz' darzustellen. Er geht von der Auffassung aus, da $B$ die Resonatoren des Mundraumes dabei eine wesentliche Rolle spielen, und nennt in diesem Zusammenhang folgende wesentliche Faktoren fur die Bildung der Vokale : a) die Bewegungen der Lippen, b) die Bewegung der Zunge waagerecht. wodurch sich die Form und das Volumen des Mund- und Rachenraumresonators verändern, c) die Bewegung der Zunge (zusammen mit der des Unterkiefers) in senkrechter Richtung, was von entscheidender Bedeutung für die Breite des Ansatzrohres ist.

Das Neue. was WI. Georgiev und I. Duridanov zu den wesentlichen Faktoren für die Vokalbildung hinzufügen, ist „der Grad der Spannung der Sprechorgane und der Wandungen der Mundhöhle" (Георгиев, 
Дуриданов, 1965, 55). Die Faktoren, welche im zitierten Buch aufgezählt werden, und die, wenn man den Charakter des Buches mitberücksichtigt, auch fur universell gahalten werden können, sind:

a) Bewegung der Zunge auf der waagerechten und senkrechten Fläche,

b) Lippeneinstellung,

c) Tätigkeit des Gaumensegels,

d) Spannungsgrad der aktiven Sprechorgane und der Wandungen der Mundhöhle.

Aus dem kurzen Überblick der phonetischen Literatur in bezug auf die für die Vokalartikulation wesentlichen Faktoren und aus selbstgewonnener Beobachtungserfahrung können wir schließen : Universell für die Bildung der Kardinalvokale in den meisten Weltsprachen (ausgenommen den Tonsprachen) sind folgende physiologische Faktoren:

a) Tätigkeit der Lippen (einschließlich des Unterkiefers),

b) Bewegung des Zungenrückens in waagerechter und senkrechter Richtung,

c) Tâtigkeit des weichen Gaumens (Gaumensegeltätigkeit),

d) Spannungsgrad der unter a), b) und c) aufgezählten Organe, sowie der Wandungen der Mundhöhle.

\subsubsection{Relevante Einstellungen und Sprechbewegungsabläufe}

für die Vokalartikulation des Deutschen und des Bulgarischen

J. Laziscius $(1961,70,72)$ konkretisiert die für die Vokalartikulation des Deutschen wesentlichen Faktoren und Bewegungsabläufe der Sprechorgane wie folgt: Die orale Öffnung und die Artikulationstätigkeit des Zungenrückens bei aktiver, bzw. passiver Lippentätigkeit. Zwei Seiten weiter in seiner "Phonetik" hebt er auch die Tätigkeit des Unterkiefers als einen wesentlichen Faktor für die Bildung der deutschen Vokale hervor.

Für Otto von Essen $(1962,73)$ sind die entscheidenden Faktoren für die Bildung der deutschen Vokale drei : „Die Unterscheidbarkeit der verschiedenen Vokale beruht auf ihrer unterschiedlichen Klangwirkung, diese auf der verschiedenen Bildungsweise, an der der Mundöffnungsgrad, die Lippenform und die Zungenhebung maßgebend beteiligt sind."

H. Wängler $(1964,23,24)$ zählt vier Faktoren auf und zwar: die Tätigkeit des Unterkiefers, die Lippentätigkeit, die Zungenbewegung und die Gaumensegeltätigkeit.

T. Su ga re va unterstreicht in ihrer "Deutschen Phonetik“ $(1965,55)$ drei Faktoren: die Lippeneinstellung, die Zungenhebung und die Gaumensegeltätigkeit.

G. Lindner spricht von der relevanten Beteiligung vier aktiver Sprechorgane an der deutschen Vokalmodifizierung. Das sind: der Unterkiefer, die Lippen, die Zunge und das Gaumensegel (L ind ne r, 1975, 53). Dabei schlieBt die wechselseitige Bedingtheit der aktiv beterligten Sprechorgane weder psychologisch noch physiologisch die Tätigkeit eines von ihnen aus : „Die Bewegung jedes einzelnen am Sprechen beteiligten Organs ist in das Bewegungsgesamt der übrigen eingebettet. Deshalb muß der 
Bewegungsablauf eines jeden Organs immer in Relation zu den übrigen betrachtet werden" (Li nd ne r, 1975, 35, 36). Der Unterkiefer beteiligt sich zwar nicht unmittelbar an der Lautmodifikation. „Aber er ist Basisorgan für die Lippen und die Zunge“, die „bei Kieferbewegungen passiv mitbewegt werden" (Lindner, 1975, 53). Die Kieferbewegungen verlaufen relativ langsam, da aber Lippe und Zunge ein „erhebliches Bewegungstempo“ (1975, 53) entwickeln, sind die langsamen Kieferbewegungen nicht von Belang.

Bei der Bestimmung der für die Vokalartikulation des Bulgarischen wesentlichen Faktoren geht D. Tilk ov (Тилков, Бояджиев, 1977, 46) von drei Faktoren aus, deren Tátigkeit die Form und das Volumen des Ansatzrohres je nach der Modifizierung eines bestimmten Vokals anders gestaltet. Das sind:

a) Die Stelle der größten horizontalen Einengung des Ansatzrohres, bestimmt durch die Zungeneinstellung.

b) Der Grad dieser Einengung, der ebenfalls durch die Zungeneinstellung bedingt wird,

c) Die Lippeneinstellung.

Wir berücksichtigen bei unserer kontrastiven Analyse der Vokalartikulation des Deutschen und des Bulgarischen folgende für beide Sprachen wesentliche Faktoren:

1. Den Spannungsgrad der aktiven Sprechorgane und der Wandungen der Mundhöhle.

2. Die aktive Tätigkeit : a) des Unterkiefers, b) der Lippen, c) der Zungc, d) des Gaumensegels und e) der Glottis.

\subsubsection{Spannungsgrad der aktiven Sprechorgane und der Wandungen der Mundhöhle}

Die Sprechspannung der aktiven Organe, sowie der von der Phonationsluft auf die Wandungen des Mundraumes ausgeübte Spannungsdruck, ließen sich nur sehr umständlich und nicht vollkommen exakt durch experimentellphonetische Untersuchungen ergründen. Deshalb sind wir in unserer Arbeit einen anderen Weg gegangen, der nach unserer Auffassung doch einen relativ guten Einblick in den konfrontativen Vergleich zwischen dem Deutschen und dem Bulgarischen ermöglicht :

Wir haben dafür die erfahrungs- und untersuchungsgemä $B$ gewonnenen typischen Besonderheiten des Vokalismus im Deutschen und im Bulgarischen zu Hilfe gezogen und sie durch gedankliche Analyse im Hinblick auf den physiologischen Spannungsgrad verglichen.

Die deutsche Artikulationsbasis wird nach $\mathrm{H}$. Krech durch eine mittlere Sprechspannung und elastische, klar ausgeprägte Lautbildung charakterisiert (Die deutsche Sprache, 1970, 2. Bd., 761, 762).

Der kontrastive Vergleich in bezug auf die Sprechspannung während der Vokalartikulation in den beiden Sprachen zeigt einen verhältnismäßig höheren Spannungsgrad für das Deutsche.

Die Ursachen für die stärker ausgeprägte Sprechspannung bei der Vokalbildung des Deutschen im Gegensatz zu dem fast spannungslosen 
Verhalten der aktiven Sprechorgane bei der artikulatorischen Gestaltung der bulgarischen Vokallaute sind in den spezifisch-typischen Besonderheiten der beiden Vokalsysteme zu suchen:

So ist für die Gestaltung der deutschen Vokale an der Morphemgrenze der feste Vokaleinsatz charakteristisch. Er ist eine physiologisch-artikulatorische Erscheinung, bei deren Realisierung die Stimmlippen einen hohen Muskelspannungsgrad erreichen : Um den vor ihrer aktiven Vibrationstätigkeit nötigen Glottisverschluß zu schaffen, müssen sie mit ihrer ganzen Masse für Sekundenbruchteile in einer hoch gespannten Einstellung verharren. Bezogen auf die gesprochene Rede, ist der relative Anteil dieser Spannung von einem recht hohen Betrag, wenn man die hohe Gebrauchsfrequenz des festen Vokaleinsatzes respektiert.

Eine sprechwissenschaftlich-phonetische Untersuchung des festen Vokaleinsatzes im Deutschen hat nachgewiesen, daß $41,2 \%$ der abgehörten Vokaleinsätze mit festem Einsatz gesprochen wurden (K re ch, 1968,26).

Der bulgarische Vokalismus kennt den festen Vokaleinsatz nicht. Das ist vielleicht eine der Ursachen, warum die bulgarische Sprache „legato" klingt, im Gegensatz zu dem ausgeprägten, vor allem durch den festen Vokaleinsatz bedingten „Stakkato-Klangcharakter" des Deutschen. Gleichzeitig ist das Fehlen dieser vokalischen Erscheinung im Bulgarischen zweifellos auch eine Ursache dafür, daß sie im phonetischen Fremdsprachenunterricht an Bulgaren sehr oft von ihnen unmarkiert bleibt, was selbstverständlich zu Perzeptionsstörungen führt. Im Falle aber, daß ein Deutsch lernender Bulgare darauf bedacht ist, dieses eigenartige vokalische Grenzsignal nicht zu übersehen und korrekt „neu einzusetzen“, so kommt es nicht selten zu einer hyperkorrekten, d. h. pathologischen Bildung. Das führt auf die Dauer zu unangenehmen Schmerzverkrampfungen im Kehlkopf, weshalb man auch den Gebrauch des festen Vokaleinsatzes gern aufgibt (Vergleiche diesbezüglich die Glottisaufnahmen von vokalischen Morphemgrenzen im Deutschen und im Bulgarischen in der Beilage, Abb. 1, 2, 3).

Die lange geschlossene und die kurze offene Vokalreihe ist ebenfalls als spannungsschaffende Quelle im Bereich des deutschen Vokalismus zu betrachten.

Die Spannung der an der Vokalartikulation beteiligten Sprechorgane sowie der von der Phonationsluft gegen die Wandungen des Ansatzrohres ausgeủbte Spannungsdruck werden bedingt :

a) durch die Artikulationsdauer der beiden Vokalreihen,

b) durch die mit ihr verbundenen Veränderungen im Volumen der Ansatzrohrresonatoren und

c) durch den ständigen Wechsel zwischen Länge und Kürze, der ständige Einengungen und Ausweitungen der Resonatoren mit sich führt.

Die bulgarischen betonten Vokallaute nehmen eine Mittelstellung zwischen der Reihe der langen geschlossenen und der der kurzen offenen Vokale des Deutschen ein (Siehe die vergleichende Formantenkarte in der Beilage. Abb. 1). Ihre artikulatorische Modifizierung setzt dementsprechend eine für die aktiven Sprechorgane begünstigende .mittlere“ Sprechspannung voraus. Was den betonten Vokalismus der beiden Sprachen betrifft, so ist auch hier die für die Artikulation der deutschen unbetonten Vokale notwendige 
Spannung stärker. Die Reduktion ist eine so stark ausgeprägte Assimilationserscheinung im Bulgarischen, da $\beta$ der bulgarische Vokalismus in unbetonter Stellung gerade auf die Hälfte, $d$. h. bis auf drei unbetonte Vokale zusammenschrumpft (Trubetzk oу, 1939; Стойков, 1967; Тилков, Бояджиев, 1977). Diese drei unbetonten Vokallaute werden naturgemäß auch mit geringerer Intensität realisiert, was im Grunde genommen sparsamere Bewegungen und geringere Muskelspannung verlangt.

Im Deutschen werden die zwei betonten vokalischen Phonemreihen in unbetonter Stellung ebenfalls von der Reduktion betroffen. Die unbetonten Vokallaute, wie unsere Spektraluntersuchungen des unbetonten deutschen Vokalismus zeigen (Си ме онова, 1976), nehmen sowohl physiologisch als auch akustisch-perzeptiv eine Mittelstellung zwischen der Reihe der geschlossenen und der der offenen Vokale ein, mit einem mehr ausgeprägten, spektral registrierbaren und auditiv-wahrnehmbaren geschlossenen, d. h. dunkelklanglichen Charakter (Siehe Formantenkarte in der Beilage, Abb. 2). Doch ist die Gebrauchsfrequenz geschlossener unbetonter Vokale in der deutschen Gegenwartssprache relativ hoch: unbetonte und dabei recht geschlossene Vokale werden in der gesprochenen Rede in pro- und enklitischen Positionen realisiert; ebenfalls in unbetonter offener Silbe von mehrsilbigen Wörtern lateinischer, griechischer und neuerer fremdsprachiger Herkunft.

So ist das Verhältnis zwischen der Anzahl der deutschen und der bulgarischen unbetonten Vokallaute $8: 3$, was trotz der annähernd gleichen Intensität, mit der sie hervorgebracht werden, doch eine gute Voraussetzung fur die etwas mehr gespannte Artikulation des deutschen unbetonten Vokalismus ist.

-.. Die Reihe der labialisierten Vorderzungenvokale des Deutschen umfaßt vier Phoneme. Die bulgarische Sprache kennt sie nicht. Die Bildung der deutschen labialisierten Vorderzungenvokale ist mit einer recht aktiven Tätigkeit der Lippen verbunden, die je nach dem Phonem einen verschiedenen Rundungs- und Vorstülpungsgrad erreichen müssen. Dabei sind ihre energischen Bewegungen mit entsprechenden und gleichzeitig verlaufenden Hebungsbewegungen des Zungenrückens zum harten Gaumen gekoppelt, wobei auch die Zungenhebung graduell durch das entsprechende Phonem bedingt ist. Diese aktive und komplizierte Tatigkeit vollzieht sich natürlich auf Grund stärkerer Muskelspannung.

Für die Realisierung der bulgarischen Vokale werden die Lippen allein bei den zwei Hinterzungenvokalen [o] und [u] gerundet, bzw. vorgestülpt. Da die bulgarischen Vokale jedoch relativ kurz und offen ausgesprochen werden, ist die fur deren Bildung nötige Sprechspannung geringer.

\subsubsection{Sprechbewegungen der Lippen und des Unterkiefers}

Wie im Deutschen, so führt auch im Bulgarischen der Unterkiefer während der Vokalartikulation vertikale Bewegungen aus, ohne vorgeschoben zu werden. Im Gegensatz zu der bulgarischen Sprache sind jedoch seine Bewegungen fur die Gestaltung der deutschen Vokale energischer. Diese recht 
energischen Bewegungen werden in hohem Maße auch durch den Druck bedingt, den der Phonationsstrom nach der jähen Lösung des Glottisversichlusses beim festen Vokaleinsatz auf die Mundraumwandungen bzw. auch auf die Muskeln, die den Unterkiefer bewegen, ausübt.

„Die Lippen (im Deutschen) streben zur ausgeprägten Ausformung des Lautes und neigen mehr oder weniger zur hoch-ovakn Einstellung." Gleichzeitig ,ist die Öffnungsweite im Deutschen relativ groß und bertifft in erster Linie die Vokale. Als Anhaltspunkte seien die Ôffnungsweiten bei $(\mathrm{d})=15$ bis $25 \mathrm{~mm}$ und bei (i) = $5 \mathrm{~mm}$ angegeben“ (Die deutsche Sprache, 1970, 2. Bd., 761).

Die energischen Sprechbewegungen der Lippen bei der deutschen V'okalartikulation werden ebenfalls durch die Besonderheiten des vokalischen Phonemsystems des Deutschen bestimmt, wo das Verhältnis zwischen labialisierten und illabialisierten Vokalen sich auf 8:6 zugunsten der labialisierten Vokallaute beläuft.

Wenn man des weiteren bedenkt, da $B$ die Aussprache der deutschen Vokale klarer ausgeprägt ist, daß sowohl die labialisierten wie auch die illabialisierten Vokallaute je in geschlossener und in offener Form auftreten, so kann man schließen, daß der Wechsel zwischen energischer Lippenrundung und -vorstülpung (für $[\mathrm{u}:, \mathrm{U}, \mathrm{Y}:, \mathrm{Y}, \mathrm{O}:, 0, \sigma: \infty]$ ) und deren energischer Spreizung (für $[i:, 1, e:, \varepsilon]$ ) recht häufig geschieht.

Schon E. Sievers warnte vor einer lässigen Mundlippenartikulation zugunsten der Zunge und des Kehlkopfs, da sich so ein Verhalten der Lippen auf die Vokalbildung des Deutschen schlimm auswirkt (Sievers, 1901).

Und F. Geratewohl $(1937,15)$ nennt die Trägheit des Unterkiefers und der Mundlippen beim Deutschsprechen „den Todfeind der deutschen Artikulation".

Im Gegensatz zu der energischen labialen Tätigkeit des Deutschen verhalten sich die Lippen bei der Artikulation der bulgarischen Vokallaute eher passiv. Sogar die zwei bulgarischen Hinterzungenvokale [0] und [0] werden mit viel geringerer Lippenrundung und -vorstülpung als die entsprechenden deutschen hervorgebracht.

Daß diese Behauptung vollkommen der sprachlichen Wirklichkeit entspricht, zeigen der Vergleich ihrer Linguagramme und die kontrastiv gestaltete Tabelle ihrer Formantenwerte (Siehe Beilage, Abb. 3, 4; Abb. 5, 6; Tab. 1, 2). So charakterisieren die Formantendaten die bulgarischen [0] und [0] als Vokale, bei deren Bildung die Sprechenergie kompakter auf der spektralen Formantenskala verteilt ist als bei den entsprechenden deutschen. Parallel damit zeigen die bulgarischen [0] und $[0]$ auch eine höhere Tonalität. was für Gestaltungsresonatoren mit größerer Öffnung und kleinerem Volumen spricht, welches als Ergebnis einer nicht gerade energischen Lippentätigkeit anzusehen ist.

Die bulgarischen illabialisierten Vorderzungenvokale $[\mathrm{I}]$ und $[\varepsilon]$ verlangen ebenfalls keine so energische Lippenspreizung wie die entsprechenden deutschen; die Sprechbewegungen der Lippen sind bei ihrer Bildung recht sparsam und etwas träge. Diese Tatsache wird gleichfalls durch den Vergleich der Linguagramme und der akustischen Spektren der illabialisierten Vokale der heiden Sprachen bestätigt (Siehe Beilage. Abb. 7, 8; Abb. 9, 10). Von 
schwacher Mundlippentätigkeit der bulgarischen Artikulation schlechthin sprechen auch die bulgarischen Phonetiker St. St oik ov $(1967,46,47)$ und D. Tilk ov $(1977,46)$.

\subsubsection{Sprechbewegungen der Zunge}

Betrachtet man kontrastiv-vergleichend die Grundbewegungen der Zunge zur Vokalartikulation in den beiden Sprachen, so stellt man keine wesentlichen Unterschiede zwischen ihnen fest :

Die Zungenmasse führt sowohl bei den deutschen als auch bei den bulgarischen Vokalen in erster Linie horizontale Bewegungen aus, die nicht in allen Fällen auch mit vertikalen Zungenbewegungen gekoppelt sind. Eine spezifische Besonderheit der deutschen Vokalartikulation in bezug auf die Zungentätigkeit ist die apikale „Kontaktstellung“ der Zunge (L in d ne r, 1975, 198), die keine notwendige Voraussetzung für die Gestaltung der Vokallaute des Bulgarischen ist.

Aber wenn auch die Zungentätigkeit während der Vokalartikulation unserer zwei Sprachen ohne wesentliche Unterschiede verläuft, so stößt man doch aus Gründen der schon oben erwähnten Besonderheiten des Vokalsystems des Deutschen auf Unterschiede, was den Spannungsgrad der Zungenbewegungen anbelangt. Daß die Zungenspannung viel geringer bei der Gestaltung der bulgarischen Vokallaute ist, beweisen eine Reihe von Fällen aus der korrektiven deutschen Phonetik für Bulgaren : So stört z. B. die für einen Bulgaren ungewohnte Zungenspannung, gekoppelt mit einer ebenfalls ungewohnten Aussprachedauer, die korrekte Bildung der langen geschlossenen Vokale des Deutschen (Sime o n ova, 1982).

\subsubsection{Gaumensegeltätigkeit}

Wie die deutschen, so gelten auch die bulgarischen Vokallaute als „oral“. Das heißt, daß das Velum durch seine Bewegungen nach oben-hinten zum Passavantischen Wulst einen Verschluß mit der hinteren Rachenwand schafft, der kein nasales Ausweichen der Phonationsluft erlaubt.

Die Frage, ob das Gaumensegel völlig den Nasenweg verlegt oder ob nicht zwischen ihm und der hinteren Rachenwand eine geringe Öffnung von 2-3 mm Durchmesser frei bleibt, die den Vokalen einen ..gesunden nasalen Beiklang" verleiht (Wä ngle r, 1960, 51), oder aber keine höhere Nasenresonanz bewirken kann (Wängler, 1972), ist weder für das Deutsche noch für das Bulgarische endgültig geklärt. Wir teilen in dieser Hinsicht die Auffassung $G$. Lindners, daß der Eindruck einer leichten Nasalität durch die nasalkonsonantische Umgebung hervorgerufen wird.

Es sei zum Schluß noch einmal hervorgehoben, daß sich in bezug auf die Gaumensegeltätigkeit bei der Vokalartikulation beide Sprachen gleich verhalten. 


\section{KONTRASTIVE ANALYSE DER AKUSTISCHEN STRUKTUR DER DEUTSCHEN UND DER BULGARISCHEN VOKALLAUTE}

oib

\subsection{PRIMÄRE AKUSTISCHE MERKMALE DER VOKALLAUTE DES DEUTSCHEN UND DES BULGARISCHEN}

\subsubsection{Untersuchungsverfahren}

Für die vergleichende Analyse der primären akustischen Parameter der Vokallaute der beiden Sprachen, die deren kontrasten und analogen Artikulationsbasiskomponenten festzulegen und darzustellen versucht, berufen wir uns auf die Ergebnisse der Spektraluntersuchungen des deutschen und des bulgarischen betonten und unbetonten Vokalismus durch den Verfasser (С имеонова, 1975, 1976, 1978). Gleichzeitig erwies sich eine von uns durchgeführte akustische Analyse der muttersprachlichen Interferenz auf die Aussprache Deutsch sprechender Bulgaren als sehr interessant, und die Basiskomponenten der akustischen Grundstruktur der beiden Sprachen indirekt bestätigend. Deshalb halten wir es für angebracht, die Methodik und die Auswertung dieser Analyse in unsere Ausführungen einzubeziehen.

Sprecher für diese Untersuchungen sind vier bulgarische Germanistikstudenten und ein DDR-Bürger Lektor für deutsche Sprache am KIZ der DDR in Sofia, der eine korrekte Aussprache hat.

Die bulgarischen Studenten kommen entsprechend aus Sofia, Petritsch, Lovetsch und Tolbuchin, d. h. ihre ..deutsche Aussprache“ weist nicht allein die typischen Besonderheiten der bulgarischen Artikulationsbasis schlechthin, sondern auch charakteristische Merkmale ihrer muttersprachlichen Mundart auf. Sie haben alle ihr Deutschstudium nach beendetem dreizehnten Lebensjahr begonnen und die polytechnischen Oberschulen mit Deutsch als Unterrichtssprache absolviert.

Mit Hilfe eines Kay-Sonagraphen wurden 56 kurze Sätze (grundsätzlich Sprichwörter vgl. dic liste in der Beilage) mit terminalem und interrogativem Melodieverliuuf analysiert. Die Beispiele wurden so ausgewählt, daB sie alle deutschen Vokal- und Konsonantenlaute in ihrer typischen Kombinatorik und Distribution enthalten. Außerdem haben wir darauf geachtet. daß die Positionen, in denen die Vokale der beiden Sprachen in unseren Beispielen erscheinen, statistisch signifikant sind.

Durch die Spektraluntersuchungen dieser Beispiele versuchen wir die Spektralcharakteristika der typischen, durch die muttersprachliche phonetische Interferenz bedingten Schwierigkeiten eines Deutsch sprechenden Bulgaren im Bereich des betonten und unbetonten Vokalismus darzustellen. 
Sie dienen außerdem, wie schon oben erwähnt, zur Bestätigung der phonetischen Grundlagenkomponenten der beiden Sprachen auf dem akustischen Niveau.

Mit Hilfe des Sonagraphen wurde auch die Intensitätskurve der gesprochenen Beispiele aufgezeichnet. Die Intensitätsgipfel bei diesem Experiment wurden in $\mathrm{mm}$ und nicht in $\mathrm{dB}$ errechnet. Ein solches Verfahren sagt uns zwar nichts über die absoluten Intensitätswerte der einzelnen Vokallaute aus. Uns ging es jedoch um die Relationen und den kontrastiven Vergleich, die sich auch so präzise durchführen ließen.

Die Lautdauer der Vokallaute des Deutschen und des Bulgarischen haben wir zum Zweck des kontrastiven Vergleichs ebenfalls aus dem Sonagramm ermittelt.

Die auf Tonband aufgezeichneten Beispiele wurden des weiteren auch einem auditiv-statistischen Test unterzogen.

\subsubsection{Vergleichende Charakteristik der primären Spektralmerkmale der deutschen und der bulgarischen Vokallaute}

\subsubsection{Spektrale Besonderheiten der betonten Vokale des Deutschen}

Die lange geschlossene Vokalreihe des Deutschen tritt in phonologische Opposition zu der Reihe der kurzen offenen Vokale, so daß das vokalische Phonemsystem der deutschen Sprache 15 Vokalphoneme umfaßt : /a :, o :, u :, i :, e :, $\varepsilon:$ : $:$, Y:; a, D, U, I, $\varepsilon, \propto, y /$ (Ungeheuer, 1969).

Die sprachliche Quantität und Qualität der deutschen Vokallaute, die dank bestimmter Sprechbewegungsabläufe realisiert werden, finden ihren entsprechend unterschiedlich nuancierten Niederschlag auch im akustischen Spektrum des einzelnen Vokallautes; sie gehören zu seinen akustischen Parametern. Unterschiede zwischen lang und kurz sind tatsächlich in der Formantenstruktur der Vokallaute vorhanden, allerdings im Rahmen der fur einen Vokallaut spezifischen Relationsverhältnisse zwischen den Formantenbereichen. Neben der Quantität leisten auch die qualitativen Klangfarbenunterschiede Perzeptionshilfen, vor allem in Fällen, wo der Kontext keine Hilfe bietet (Lindner, 1969,192). Hier möchten wir der Annahme G. Lindners beipflichten, der schreibt : .Aus dieser Sicht kann man sämtliche Kurzvokale als ein in sich geschlossenes System auffassen, das, als Ganzes gesehen, zu dem System der Langvokale durch seine Dauer und in zweiter Linie durch Klangfarbenunterschiede insgesamt, somit auch durch die Lage der Formanten unterschiedlich ist" (L in d ner, 1969, 192).

Die Auswertung der Spektraluntersuchungen der deutschen betonten Vokallaute durch den Verfasser (Симеонова, 1975) ergibt folgende zusammenfassende Schlußfolgerung in bezug auf deren Klangfarbe (Verglichen werden die Frequenzwerte):

Die kurzen offenen Vokale des Deutschen sind kompakter als die langen geschlossenen, was sich akustisch in einer Erhöhung des $F_{\text {, und }}$ entsprechender Senkung des $F_{2}, d$. $h$. in einem Aneinanderrücken der beiden 
Formantenbereiche, äußert. Der Relationskoeffizient $\mathrm{R}_{2}^{*}$, der das Verhältnis $F_{2}: F_{3}$ zahlenmäßig ausdrückt, bleibt konstant. Die Frequenzcharakteristik des $F_{3}$ bleibt bei allen kurzen offenen Vokalen entweder konstant, oder aber sinkt gelegentlich herab, so da $B$ die Streuung der Gesamtenergie über die spektrale Frequenzskala eingeengt wird (Siehe Tab. 1 und die Formantenkarte: Abb. 4,5). - Während bei den zwei Hinterzungenvokalreihen [o:] - [0], [u :] - [0] die Unterschiede in den Teiltonfrequenzen nicht so stark ausgeprägt sind, kommen sie bei den Vorderzungenvokalreihen, insbesondere bei $[i:]-[i]$ und $[e:]-[\varepsilon]$ spürbar zum Vorschein, $d$. h., die kurzen illabialisierten Vorderzungenvokale sind in einem recht hohen Maße kompakter als die entsprechenden langen geschlossenen.

Physiologisch und akustisch läßt sich diese Tatsache wohl durch den niedrigeren Hebungsgrad des vorderen Zungenrückens zum harten Gaumen bei der kurzen offenen Vokalreihe erklären, was zur Volumenvergrößerung des präoralen Resonators und respektiv zur Senkung der Frequenzwerte von $F_{2}$ führt. Gleichzeitig wird durch diese niedrigere Zungenrückeneinstellung zum harten Gaumen die Ö́nnung des postoralen Resonators bei konstantem Volumen vergrößert; daraus resultiert akustisch eine Erhöhung der Freguenzwerte des $F_{1}$ (Siehe Tab. 1 und Formantenkarte, Abb. 1,2), die die Verteilung der Gesamtenergie bei den deutschen betonten Vokalreihen veranschaulichen).

\subsubsection{Spektrale Besonderheiten des bulgarischen betonten Vokalismus}

Am kompaktesten ist die spektrale Gesamtenergie bei den bulgarischen betonten Vokallauten [a] und [o] verteilt.

Die Kompaktheit der betonten Vokallaute des Bulgarischen sinkt von [a] zu [I] wie folgt :

[a] $\left(R_{3}=4,5\right) \rightarrow[0]\left(R_{3}=5\right) \rightarrow[\varepsilon]\left(R_{3}=6,6\right) \rightarrow\left[\right.$ o] $\left(R_{3}=6,7\right) \rightarrow[0]\left(R_{3}=7,7\right) \rightarrow[1]$ $\left(R_{3}=12,3\right)$.

Die zwei Hinterzungenvokale [0] und [0] zeigen bei unseren Spektralanalysen einen sehr schwach markierten, oft ganz fehlenden $F_{3}$. Bei allen übrigen betonten Vokalen des Bulgarischen sind die drei Formantenbereiche klar ausgeprägt.

Der Frequenzunterschied zwischen $[\varepsilon]$ und $[1]$ in bezug auf $F_{2}$ ist recht hoch (etwa $400 \mathrm{~Hz}$ ), was darauf schließen läßt, daß sich die zwei illabialisierten Vokallaute kontrast in ihrer Klangfarbe voneinander unterscheiden. Die deutschen illabialisierten Vorderzungenvokale [i:] und [e:] haben relativ naheliegende Frequenzwerte für $F_{2}$ (Siehe Tab. 2). Diese Tatsache könnte ohne die Perzeptionshilfen des Kontextes zu ihrer perzeptiven Vermischung führen. Fast gleich klingend hören sich die beiden Vokale auch von Deutsch lernenden Bulgaren an.

- $R_{1}, R_{2}, R_{3}=$ Relationskoeffizienten. die die Energiestreuungsverhälınisse, $d$. h. die Verhāltnisse der formantenbereiche zueinander zahlenmäBig ausdrücken ( $J_{\text {a }}$ obs on $U$. a., 1952). 
Die Frequenzwerte des für das bulgarische Vokalsystem charakteristischen und für das System der betonten Vokallaute des Deutschen fremden Vokals [0] beziehen ihn nach seinem $F_{1}$ auf die Spektralzonen des $[\varepsilon]$ und [0], während sein zweiter Formant ihn in die [a]-Spektralzone einordnet.

\subsubsection{Vergleichende Charakteristik der primären Spektralmerkmale des deutschen und des bulgarischen betonten Vokalismus}

Die betonten bulgarischen Vokallaute (6 Vokalphoneme: /a, I, $\varepsilon, \partial, 0, \partial /)$ nehmen nach ihrer Formantenstruktur eine Mittelstellung zwischen der Reihe der langen geschlossenen und der der kurzen offenen deutschen Vokale ein.

Die spektrale Mittelstellung der bulgarischen betonten Vokallaute ist auf den nach den Frequenzwerten und Relationskoeffizienten aufgestellten Tabellen in der Beilage zu sehen (Tab. 1,2; Abb. 1,2). Bulgarisches [a] unterscheidet sich in seiner Klangfarbe kaum von den deutschen [a:] und [a].

Die ermittelten Frequenzwerte (Siehe Tab. 2) sprechen jedoch von einer etwas näheren akustischen Verwandtschaft des [a] zu [a:]. Die Frequenzunterschiede zwischen $\left[\mathrm{a}^{\mathrm{b}}\right]$ und $\left[\mathrm{a}^{\mathrm{d}}\right]$ sind jedoch ebenfalls gering. so da $B$ man die drei betonten A-Laute als akustisch gleich bestimmen könnte.

Die Spektralanalyse weist eine fast volle Übereinstimmung der Teiltonfrequenzen des $\left[0^{b}\right]$ und $\left[0^{d}\right]$ auf. $F_{1}$ und $F_{2}$ des langen geschlossenen deutschen [o:] liegen dagegen etwa um $100 \mathrm{~Hz}$ niedriger, weshalb man es perzeptiv wie einen Vokallaut mit dunklerer Klangfarbe wahrnimmt.

Bulgarisches betontes [1] nimmt spektral eine Mittelstellung zwischen den deutschen [i:] und [1] ein. Die Kompaktheit nimmt bei diesen Vokalen, zahlenmäßig ausgedrückt, wie folgt, $a b$ :

$\left[{ }^{\mathrm{d}}\right] \quad\left(\mathrm{R}_{3}=11,4\right) \rightarrow[1 \mathrm{~b}] \quad\left(\mathrm{R}_{3}=12,5\right) \rightarrow[\mathrm{i}:] \quad\left(R_{3}=13,6\right)$.

Bulgarisches betontes $[\varepsilon]$ liegt nach seinen primären Spektralparametern dem deutschen $[\varepsilon]$ näher. Dafür sprechen die relativ gleichen Relationskoeffizienten, die wir für die beiden Laute ermittelt haben (Siehe Tab. 2). Die zwei Vokale sind demnach auch gleich kompakt, während die Spektralenergie des [e:] eine diffusere Streuung zeigt (Siehe Tab. 2).

\subsubsection{Spezifische spektrale Besonderheiten der deutschen betonten Vokale, realisiert von Deutsch sprechenden Bulgaren}

Die Auswertung der Spektraluntersuchungen für die einzelnen Vokallaute ist auf Tabellen 3,4 in der Beilage zu sehen.

$[a:]-[a]$

Bei dem deutschen Sprecher (Sprecher 1) ist kein wesentlicher Unterschied zwischen den Spektralcharakteristika der beiden Vokale festzustellen; beide Laute sind gleich kompakt, mit hohem $F_{1}(800-750 \mathrm{~Hz})$ und niedrigem $F_{2}$ $(1230-1200 \mathrm{~Hz})$. Der Unterschied von $50 \mathrm{~Hz}$ bei $F_{1}$ und $30 \mathrm{~Hz}$ bei $F_{2}$ kann perzeptiv nicht von Belang sein. Der quantitative Unterschied zwischen den beiden $\mathrm{A}$-Arten ist dagegen scharf ausgeprägt. Das Verhältnis dazwischen ist 
$2: 1$ zugunsten der [a:]-Laute. Die relativen Intensitätsverhältnisse zwischen den beiden A-Lauten sprechen für eine etwas höhere Intensität des [a :]. Da jedoch der Unterschied minimal ist $([a:]:[a]=22,5 \mathrm{~mm}: 18 \mathrm{~mm})$ und weil gegen Ende des terminal verlaufenden Ausspruchs mit einer relativ gleichen Intensitätsverminderung bei allen betonten Vokalen $\mathrm{zu}$ rechnen ist $([a:]:[a]=15: 13)$, so schätzen wir die Intensität der beiden Laute als gleich ein.

[a:] und [a] weisen in der Aussprache der vier Bulgaren keine Frequenzunterschiede auf. Deshalb haben wir zum Zwecke des Vergleiches ihre Mittelwerte ausgerechnet. So liegt $F_{1}$ des von den bulgarischen Versuchspersonen einheitlich realisierten A-Lautes etwa um $100 \mathrm{~Hz}$ tiefer als $F_{1}$ vom [a:] des deutschen Sprechers und um $50 \mathrm{~Hz}$ tiefer als $F_{1}$ des [a]. $F_{2}$ zeigt degegen etwa um $100 \mathrm{~Hz}$ höhere Werte als $F_{2}$ der A-Laute des deutschen Sprechers. Die Relationskoeffizienten der beiden Sprecherpaare beziehen sich aufeinander wie folgt: $[\mathrm{a}: \mathrm{d}]:[\mathrm{a}: \mathrm{b}]=1,5: 2$ und $\left[\mathrm{a}^{\mathrm{d}}\right]:\left[\mathrm{a}^{\mathrm{b}}\right]=1,6: 1,9$.

Die zahlenmäßig ausgedrückten Formantenverhältnisse der A-Laute, gesprochen von einem Deutschen und von vier Bulgaren, offenbaren eine etwas diffusere Verteilung der Sprechenergie bei der bulgarischen Realisierung, was eine dunklere Klangfarbe des vorderen [a] bedeutet.

Die Dauer des langen [a :] schwankt bei den bulgarischen Sprechern (mit Ausnahme des Sprechers IV) viel mehr als bei dem deutschen und zwar zwischen ziemlichen Extremen.

Das kurze [a] ist in seinen Dauerwerten recht stabil; hier sind nur unwesentliche Abweichnungen zwischen den einzelnen Sprechern zu beobachten. gleich.

Die relativen Intensitätswerte sind bei allen Sprechern verhältnismäßig

[i:]

$[$ l]

Der deutsche Sprecher bildet das kurze offene [1] kompakter und fast doppelt so kurz als das lange geschlossene [i :].

Intensitätsbezogen unterscheiden sich die beiden I-Laute bei ihm kaum voneinander.

Das spektrale Bild der deutschen 1-Laute sieht in der bulgarischen Realisierung folgendermaßen aus :

Die absoluten Formantenwerte sowie die Relationskoeffizienten sind für beide I-Laute gleich.

Mit Ausnahme des Sprechers II ist die spektrale Gesamtenergie für die I-Laute bei den übrigen Bulgaren im Gegensatz zum deutschen Sprecher und Sprecher II viel diffuser verteilt. Spektral liegen folglich die I-Laute zwischen der Klangfarbe des gespannten [i:] und der bulgarischen betonten [ $]$ ] (Vgl. Tab. 2).

Das lange [i :] ist bei allen bulgarischen Versuchspersonen von längerer Dauer als bei dem deutschen Sprecher, doch das Dauerverhältnis zwischen [i :] und [ 1$]$ ist etwa das gleiche.

Die Intensitätsunterschiede zwischen [i:] und [ $\mathrm{i}$ ] sind unwesentlich und bei allen Sprechern fast gleich.

Zusammenfassend kann man sagen, da $B$ die Unterschiede zwischen den deutschen I-Lauten, realisiert von Bulgaren, sich lediglich in einer etwas 
dunkleren Klangfarbe für beide Laute äußern. Perzeptiv sind jedoch diese Unterschiede nicht von Belang (Siehe die Auswertung des auditiven Testes, Tab. 5 und 6).

\section{$[e:]-[\varepsilon]$}

Die spektrale Gesamtenergie fur das [e:] ist beim Sprecher I recht diffus verteilt im Vergleich $z u[\varepsilon]\left(R_{3}[e:]: R_{3}[\varepsilon]=7,8: 5,6\right)$, was vollkommen der herkömmlichen Klangfarbenbestimmung vom [e :] als „dunkel“ und $[\varepsilon]$ als ..hell" entspricht.

Das spektrale Bild der deutschen betonten E-Laute, gesprochen von Bulgaren, ist ziemlich bunt :

- Bei drei bulgarischen Sprechern (II, IV, V) ist das [e :] kompakter, d. h. "heller". Sprecher III bewegt sich im Rahmen der korrekten deutschen Realisierung des [e:].

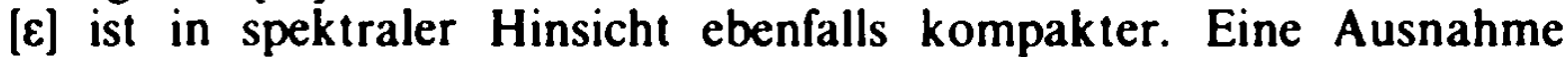
hierzu macht ebenfalls Sprecher III, dessen $[\varepsilon]$-Aussprache sich sehr der des deutschen Sprechers nähert (Vgl. Tab. 6).

Der Klangfarbenunterschied zwischen [e:] und $[\varepsilon]$ bewegt sich bei Sprechern II und III im Rahmen der für diese Laute zugelassenen normgerechten Abweichungen, bei Sprecher IV treten gar keine Klangfarbenunterschiede zwischen den beiden E-Lauten auf. Sprecher III realisiert das lange [e :] nur ein wenig länger als das kurze $[\varepsilon]$, Sprecher IV macht keinen quantitativen Unterschied zwischen [e :] und [E].

Dynamisch sind beide E-Laute bei allen Sprechern etwa gleich stark.

Zusammenfassend soll gesagt werden, daß das lange geschlossene [e:], gesprochen von Bulgaren, kompakter, $d$. h. heller hervorgebracht wird, was auch perzeptiv auffallt ( Vgl. die Auswertung des auditiven Testes, Tab. 5). Die normgerechte Bildung dieses Vokals durch Sprecher III ist regionalgebunden zu erklären : Der Student kommt aus einer Gegend Bulgariens (Lovetsch), fur die neben einer stärker ausgeprägten Konsonantenpalatalisierung auch eine gespanntere Vokalartikulation charakteristisch ist (Стойков, 1980).

\section{[u:] [o]}

Während die beiden Formen dieses Hinterzungenvokals sich beim deutschen Sprecher deutlich voneinander unterscheiden, was spektral ausgedrückt, sich in einer diffuseren Streuung der Gesamtenergie für das lange geschlossene [u :] im Gegensatz zum kurzen offenen [o] äußert, werden sie von Bulgaren qualitativ gleich realisiert. Die gewonnenen Frequenzwerte sowie die Relationskoeffizienten ordnen die von Bulgaren gesprochenen U-Laute zwischen der gespannten und der ungespannten Form der deutschen U-Laute ein. Perzeptiv klingt also dieser einheitliche U-Laut „offener“ als das deutsche gespannte [u:] und .,geschlossener" als das ungespannte [u] :

$$
\begin{aligned}
& R_{1}[u: d]: R_{1}[u: b]=2,4: 2,2 \\
& R_{1}[u d]: R_{1}\left[u^{b}\right]=1,9: 2,1 .
\end{aligned}
$$

Die qualitative Fehlleistung bei der Bildung der deutschen U-Laute von Bulgaren hätte perzeptiv sicher nicht auffallen können, wäre deren sprachliche Dauer normgerecht gewesen. Hier halten sich jedoch, genauso wie auch bei den anderen, bisher besprochenen Vokalen, Normgerecht und Normwidrig die Waage: Bei zwei der Sprecher entspricht das Dauerverhältnis dem des deutschen Sprechers, bei den anderen zwei sind [u:] und [u] gleich lang. 
Die Intensitătskurven zeigen keine abweichenden Besonderheiten auf.

$[o:]$ - [o]

Die Bulgaren sprechen qualitativ einen einheitlichen O-Laut aus. Seme Klangfarbe bewegt sich zwischen den Spektralwerten des geschlossenen und offenen deutschen Vokals, d. h., die von den Bulgaren realisierte O-Art klingt etwas heller als der gespannte deutsche O-Laut und dunkler als das ungespannte [o]:

$\mathbf{R}_{1}[\mathbf{o} \cdot \mathbf{d}]: \mathbf{R}_{1}[\mathrm{o}: \mathrm{b}]=2,5: 2,1$
$\mathbf{R}_{1}\left[\mathrm{o}^{\mathrm{d}}\right]: \mathbf{R}_{1}\left[\mathrm{~b}^{\mathrm{b}}\right]=1,8: 2$.

Quantitativ unterscheiden sich die beiden O-Laute auch in der bulgarischen Realisierung, obgleich die sprachliche Dauer des langen [o:] nicht so stabil ist, wie sie der deutsche Sprecher realisiert.

$[y:]-[r]$

Eine normgerechte Realisierung der beiden U-Laute ist bei keinem der bulgarischen Sprecher zu beobachten. Im Rahmen der standardisierten Korrektheit bewegt sich allein die Aussprache des langen geschlossenen [y :] bei Sprecher II. Einzelbeispiele qualitativer Korrektbildung sind auch bei den übrigen bulgarischen Versuchspersonen zu beobachten, allerdings bei der langen geschlossenen Form.

Im allgemeinen wird das lange geschlossene [y :] von Bulgaren (Vgl. Tab. 6) ,gemischt" realisiert, mit einem etwa um $100 \mathrm{~Hz}$ höheren $F_{1}$ und um $200-400 \mathrm{~Hz}$ niedrigeren $F_{2}$. Ein dritter Formant ist dabei auf der spektralen Frequenzskala nicht zu sehen.

Die Frequenzwerte fur das [y:] bewegen sich in der Frequenzzone eines offenen $[\mathbf{Y}]$ oder eines Schwa-Lautes; es fehlt jedoch, wie schon erwähnt, der fur das $[\mathrm{Y}]$ und $[0]$ charakteristische, klar ausgeprägte $\mathrm{F}_{3}$.

Die spektrale Gesamtenergie ist bei dieser „Mischbildung“ recht kompakt verteilt : $\mathbf{R}_{1}[\mathbf{y}: \mathrm{d}]: \mathbf{R}_{\mathbf{1}}[\mathbf{y}: \mathrm{b}]=6: 3,8$

$$
R_{1}[y: d]: R_{1}\left[y^{b}\right]=4,4: 3,8, \text { d. h., }
$$

der von Bulgaren gesprochene Vokal hat einen helleren, fast [1]-ähnlichen Charakter.

Die kurze offene Form ist in den meisten Beispielen der bulgarischen Lautverbindung [IU] ähnlich, oder aber wird sie wie ein [i] + einen [u] realisiert. Das bestätigen die ermittelten relativen Formantenwerte, die sich in der Frequenzzone der $U$-Laute bewegen, aber auch das Fehlen eines $F_{3}$. .Mischbildungen" wie etwa bei der langen U-Form sind jedoch auch in der Region des kurzen $[Y]$ anzutreffen. Normgerechte Dauerunterschiede zwischen $[y:]$ und $[\mathrm{y}]$ sind allein beim Sprecher II deutlich ausgeprägt, beim Sprecher III sind sie noch zu bemerken, bei den übrigen zwei Versuchspersonen - kaum zu spüren.

[o] [oe]

Hier herrschen unter den Deutsch sprechenden Bulgaren ähnliche Verhältnisse wie auch bei den U-Lauten, mit dem alleinigen Unterschied, da $B$ das lange geschlossene [ $[0$ :] sich bei allen Sprechern dessen korrekter Bildung nähert. Allerdings ist die für die Realisierung eines [ø:] nötige Energie etwas diffuser verteilt, d. h., es hat einen dunkleren, |o]-ähnlichen Charakter: 
$\mathrm{R}_{1}[\boldsymbol{b}: \mathrm{d}]: \mathrm{R}_{1}[\boldsymbol{\sigma}: \mathrm{b}]=4,4: 5,2$

$R_{3}[\sigma: d]: R_{3}[\sigma: b]=6,2: 7$.

Die Dauerverhältnisse sind bei drei der bulgarischen Sprecher verschwommen die beiden Formen sind quantitativ fast ausgeglichen, und allein bei Sprecher III ist die sprachliche Dauer der O-Laute etwa normgerecht.

\section{Primäre spektrale Besonderheiten der Diphthonge}

Das übliche spektrale Bild der deutschen Diphthonge ist gut auf der Formantenskala zu beobachten und zwar bei allen Sprechern. Diesem spektralen Bild sind folgende akustische Beweise für das Wesen der deutschen Diphthonge zu entnehmen:

a) Die spezifische „gleitende" Sprechbewegungsart bei der Bildung der Diphthonge :

b) Den "fallenden" Charakter der deutschen Diphthonge;

c) Die Abgrenzungsmöglichkeit der Bestandteile eines Diphthongs, sowie die phonetisch-akustische Interferenz unter ihnen.

Wie beim deutschen Sprecher, so konnte man auch bei den vier Bulgaren die Frequenzwerte der beiden Elemente eines jeden Diphthongs mit relativer Genauigkeit bestimmen. Hiermit seien folgende Spezifika hervorgehoben:

a) Bei den "Vorderzungendiphthongen" [äe] und [jö] sind auf dem Spektralbild sehr deutlich der zweite und der dritte Formantstreifen zu sehen, allerdings in der Form eines schräg nach oben verlaufenden Formantenstreifens. Die Frequenzwerte der beiden oberen Formanten haben wir ebenfalls ermittelt ;

b) Die sprachliche Dauer aller drei Diphthonge ist bei den 5 Sprechern relativ stabil und gleich. Sie bewegt sich, wie es die traditionelle Phonetik schon bestätigt hat (Esse n, 1962, 79), in den Quantitätsgrenzen eines langen Vokals :

c) Befindet sich der Diphthong im Wortauslaut und das Wort auch am Ende des terminal verlaufenden Ausspruchs unserer Beispiele, so sinkt dessen Intensität nicht so jäh herab, wie es der Fall bei den übrigen Vokalen in solcher Position ist (Vgl. Tab. 9, 11, 12).

In der Amplitudenkurve der Sonagramme sind bei den Diphthongen stets zwei deutliche Intensitätsgipfel zu beobachten, die jedoch dynamisch relativ ausgeglichen sind.

\section{Der Diphthong $[\bar{a} e]$}

Während das zweite Element dieses Diphthongs in der „bulgarischen“ Aussprache fast vollkommen mit dessen spektralem Bild beim deutschen Sprecher übereinstimmt, wird sein erster Bestandteil diffuser, etwa wie das bulgarische betonte [a] gebildet.

Der Unterschied ist jedoch gering, so da $B$ dieser Diphthong von deutschen Perzipienten als korrekt klingend empfunden wird.

\section{Der Diphthong [.0. ]}

Dieser Diphthong wird von Bulgaren im Rahmen seiner normgerechten deutschen Aussprache realisiert. 


\section{Der Diphthong $[\hat{a} o]$}

Auch bei diesem Diphthong sind weder spektral noch perzeptiv normwidrige Abweichungen in dessen Aussprache von Bulgaren festzustellen.

\subsubsection{Vergleichende Charakteristik der primären Spektralparameter des deutschen und des bulgarischen unbetonten Vokalismus}

Die primären spektralen Merkmale der deutschen und der bulgarischen Vokale in unbetonter Stellung sind auf Grund von umfangreichem Material vom Verfasser nach dem Visible Speech-Verfahren untersucht und auditiv getestet worden (Sime onova, 1975, 1976).

Zusammenfassend lassen sich die Untersuchungsergebnisse folgendermaßen formulieren :

Akustisch betrachtet sind die unbetonten deutschen Vokale im Mittel kompakter als die Vokale der betonten gespannten Reihe und diffuser als die der betonten ungespannten. In ihrem akustischen Spektrum überwiegen jedoch spektrale Merkmale der gespannten Vokalreihe. Perzeptiv läßt sich daher ihr Charakter eher als ..geschlossen" bestimmen.

Eine Sonderstellung unter den deutschen unbetonten Vokalen nimmt der Murmelvokal [a] ein. Spektral weist dieser Laut relativ enge Berührungspunkte zu drei unbetonten Vokalen auf: einem [e], [ø] und einem [a] (Sime on ova, 1976, 24).

Der unbetonte Murmelvokal [0] hat also in spektraler Hinsicht keinen einheitlichen Charakter. Seine Spektralcharakteristika sind stets durch dessen Position und Lautumgebung bedingt. Im System des deutschen unbetonten Vokalismus unterscheidet man demnach zwei spektrale Grundvarianten des [a] :

1. Eine [a]-Variante, deren akustische Parameter in der Spektralzone eines [e] oder eines [ø] liegen. Diese Variante, mit spezifischen Spektralunterteilungen, ist für folgende [ə]-Positionen charakteristisch:

[ə] in den Präfixen [bə-, ga-],

[a] im absoluten Auslaut,

[a] im Auslaut vor Nasal-, nach Explosiv-, Frikativ- und Sonorlauten.

[a] im Auslaut for [1].

[a] im Auslaut nach [r];

2. Eine [e]-Variante (Reduktionsvokal [e] - Ulbrich, 1972; Meinhold, 1973) bzw. [-or], deren Spektralcharakteristika in der Frequenzzone eines unbetonten [a]-Vokals liegen (S i m e o n o va, 1975).

Im bulgarischen unbetonten Vokalismus ist ein hoher Grad an assimilatorischer Reduktion zu beobachten. Im allgemeinen macht sich hier die Annäherungstendenz der sog. „breiten“ Vokale des Bulgarischen auf ihre .engen“ Korrelationsentsprechungen zu. bemerkbar: $[a \rightarrow a] .[0 \rightarrow 0],[e \rightarrow 1]$ (Тилков, Бояджиев, 1977, 69).

Am stärksten ist die Reduktion beim Korrelationspaar $[a \rightarrow$ a] vertreten, was sich in einer fast vollständigen Neutralisierung der die beiden Vokale in betonter Stellung interscheidenden Spektralmerkmale .kompakt-diffus“ ausdrückt. 
Die spektrale Bewegung [o $\rightarrow u$ ] und insbesondere [e $\rightarrow 1]$ ist in der gegenwärtigen bulgarischen Standardaussprache im allgemeinen viel schwächer ausgeprägt und trifft außerdem nicht für alle Positionen, in denen diese Vokale auftreten können, zu.

Die Ergebnisse der Spektraluntersuchungen (S i m e o n o va, 1975) zeigen, da $B$ mit Ausnahme des [a] die akustischen Spektren der übrigen unbetonten bulgarischen Vokale keine wesentlichen Abweichungen von denen der entsprechenden betonten Vokallaute aufweisen.

Konstant in betonter und unbetonter Stellung bleibt das akustische Spektrum des bulgarischen [ə]-Vokals.

Der spektrale Vergleich zwischen dem deutschen und dem bulgarischen unbetonten Vokalismus zeigt bei den bulgarischen unbetonten Vokallauten im allgemeinen eine diffusere Verteilung der Gesamtenergie (Си меонова, 1976, 28). Anders gesagt, die bulgarischen unbetonten Vokale haben eine dunklere Klangfarbe als die entsprechenden deutschen.

Dem deutschen Murmelvokal [o] entsprechen im System des bulgarischen unbetonten Vokalismus, je nach der Position und Lautumgebung des [ə], drei Vokallaute: [e], [a] und [e]. Die Distribution dieser bulgarischen Vokale entspricht der des Murmelvokals wie folgt:

1. Ein bulgarisches unbetontes [e] in den Positionen:

- Anlaut nach den stimmhaften Explosiven [b, g],

- Absoluter Auslaut nach Explosiven, Frikativen, meistenfalls auch nach Sonoren;

2. Ein bulgarisches unbetontes [e] oder ein [e] im Auslaut vor [t];

3. Ein bulgarisches $[e]$ im Auslaut vor $[r]-$ im Falle, da $B$ die entsprechende deusche Position [-วr] als [-әr] oder [-ว२] realisiert wird;

4. Ein reduziertes bulgarisches $[\varepsilon]$ im Auslaut vor $[r]-$ im Falle, daß [-ar] der deutschen Sprache monophthongisch wie [e] realisiert wird (S i me on ova, 1975).

2.1.7.1. Spezifische spektrale Besonderheiten der unbetonten deutschen Vokale, realisiert von Deutsch sprechenden Bulgaren

Die Auswertung der Spektraluntersuchungen für die einzelnen unbetonten Vokallaute ist auf Tabelle 4 in der Beilage zu sehen.

\section{Unbetontes [a]}

Wie man es auch erwarten konnte, wird das unbetonte [a] vom deutschen Sprecher etwas diffuser als die beiden betonten A-Arten gesprochen: $\mathbf{R}_{1}[\mathbf{a}:]: \mathbf{R}_{1}[\mathbf{a}]: \mathbf{R}_{1}[\mathbf{a}]=1,5: 1,4: 1,9$. Seine Klangfarbe hätte man folglich ab etwas dunkler im Vergleich zu den betonten A-Lauten bestimmen können. Die bulgarischen Versuchspersonen realisieren das deutsche $[a]$ ebenfalls diffuser. Bei ihnen lassen sich jedoch einige spezifische Besonderheiten beobachten:

a) Das unbetonte [a] hat einen merklich dunkleren Charakter als das [c] des deutschen Sprechers: $\mathbf{R}_{1}\left[\mathbf{a}^{\mathrm{d}}\right]: \mathbf{R}_{1}\left[\mathbf{a}^{\mathrm{b}}\right]=1,9: 2,2$,

b) Das unbetonte [a] wird in proklitisch gebrauchten Einsilbern (das, als) noch diffuser, d.h. noch dunkler gebildet: 


$$
\begin{aligned}
& \mathbf{R}_{1}: \mathbf{R}_{2}: \mathbf{R}_{3}\left(\text { das }^{d}\right)=2,3: 1,6: 3,6, \\
& \mathbf{R}_{1}: \mathbf{R}_{2}: \mathbf{R}_{3}\left(\text { das }{ }^{2}=4,2: 1,7: 7,2 .\right.
\end{aligned}
$$

Die Klangfarbe dieses von Bulgaren realisierten [a] nähert sich allzusehr dder Klangfarbe eines bulgarischen unbetonten [a] oder [e]. Selbst verständlich isist eine solche [a]-Bildung durch die im bulgarischen unbetonten Vokalismus sssehr stark ausgeprägte Reduktion bedingt, unter derem Einfluß die spektralen Merkmale „kompakt-diffus“ beim Korrelationspaar [a] - [ə] völlig neutralisisiert werden (Си меонова, 1975; Т и лков, Бояджи ев, 1977, 67-69).

\section{Unbetontes [i]}

Beim unbetonten [i] fallt das spektrale Ergebnis auf, daß der deutsche SSprecher einen Unterschied zwischen einem gespannten und einem uungespannten [I] auch in unbetonter Stellung macht: das ursprünglich uungespannte [1] wird im Gegensatz zu dem ursprünglich gespannten langen [i :] irin unbetonter Position kompakter gebildet:

$\mathbf{R}_{1}: \mathbf{R}_{\mathbf{2}}: \mathbf{R}_{\mathbf{3}}$ (urspr. [i :]) $=9,8: 1,7: 11,3$

$\mathbf{R}_{1}: \mathbf{R}_{2}: \mathbf{R}_{3}$ (urspr. [I]) $=7,3: 1,2: 8,7$.

Die Bulgaren bilden dagegen einen einheitlichen unbetonten I-Laut, dessen SSpektralmerkmale eine Mittelstellung zwischen dem gespannt und dem uungespannt realisierten deutschen [i] einnehmen und somit dem bulgarischen uunbetonten [i] sehr nahe kommen:

$$
\begin{gathered}
\mathbf{R}_{1}: \mathbf{R}_{2}: \mathbf{R}_{3}\left[{ }^{\mathrm{i}}\right]=9,8: 1,7: 11,3 \\
\mathbf{R}_{1}: \mathbf{R}_{2}: \mathbf{R}_{3}\left[{ }^{\mathrm{d}}\right]=7,3: 1,2: 8,7 \\
\mathbf{R}_{1}: \mathbf{R}_{2}: \mathbf{R}_{3}\left[{ }^{\mathrm{b}}\right]=8,2: 1,3: 10,3 \\
\left.\mathbf{R}_{1}: \mathbf{R}_{2}: \mathbf{R}_{3}\left[{ }^{\mathrm{b}}\right]=8: 1,2: 10 \text { (S i m e o n o v } \mathrm{a}, 1976,21\right) . \\
\text { Unbetonte }[e] \text {-Varianten }
\end{gathered}
$$

Bei der Auswertung der Sonagramme sind wir im Zusammenhang mit der RRealisierung des unbetonten [e] auf Besonderheiten gestoßen, die uns vweranlaßt haben, die spektral auffallenden [e]-Varianten gesondert $z u$ boehandeln.

\section{a) Deutscher Sprecher}

$[\mathfrak{e}]$ in unbetonten Präfixen vor $[r]$ und in proklitisch gebrauchten Einsilbern vor $[r]$

Das unbetonte Präfix 〈ver-〉, das in unseren Beispielen vorkommt, wird vvom deutschen Sprecher etwa wie [fe-] realisiert, ja sogar mit einer noch djunkleren Klangfarbe:

$\mathbf{R}_{1}: \mathbf{R}_{2}: \mathbf{R}_{3}\langle$ ver- $=2,6: 2: 5$,
$\mathbf{R}_{1}: \mathbf{R}_{2}: \mathbf{R}_{3}[f e-]=2,1: 1,9: 4$.

Der Spektralcharakter des proklitisch gebrauchten bestimmten Artikels 〈〈der 〉 ist fast diphthongartig. Eine Ausnahme bilden lediglich zwei Beispiele, djie einen [a]-gleichen $\mathrm{F}_{2}$ und sonst ein monophthongisches Frequenzbild aaufweisen. Der diphthongische Charakter der übrigen Beispiele ist zwar nicht scehr scharf ausgeprägt, aber es lassen sich immerhin zwei Elemente deutlich ecrkennen: ein [e]-artiges Segment am Anfang und ein zweites, [a]-artiges, wwelches in das darauffolgende vokalisch gelöste [r] einfließt. 


\section{$[e]$ in anderen proklitischen Einsilhern, die nicht auf ein $[r]$ ausgehen}

Unbetontes $[e]$ in solchen Proklitika hat den spektralen Charakter eines gespannten, doch gekürzten [e:]:

$R_{1}: R_{2}: R_{3}[e:]=4,8: 1,4: 6,4$,

$R_{1}: R_{2}: R_{3}$ [es], [den] $=4,4: 1,4: 6,3$.

b) Bulgarische Sprecher

Das unbetonte Präfix [fєr-] ergibt bei zwei der bulgarischen Sprecher (Sprecher II und IV) etwa das spektrale Bild des deutschen Sprechers, beim Sprecher II allerdings etwas diffuser:

$$
\begin{aligned}
& \mathbf{R}_{1}: \mathbf{R}_{2}: \mathbf{R}_{3}\langle\text { ver-d }\rangle=2,6: 2: 5, \\
& \mathbf{R}_{1}: \mathbf{R}_{2}: \mathbf{R}_{3}\langle\text { ver-IV }\rangle=2,9: 1,7: 4,9, \\
& \mathbf{R}_{1}: \mathbf{R}_{2}: \mathbf{R}_{3}\langle\text { ver-II }\rangle=3,3: 2: 6,4 .
\end{aligned}
$$

Die anderen zwei Bulgaren realisieren dieses unbetonte [e] mehr [e]-ähnlich. Das ließe sich offenbar dadurch erklären, daß sie das [ $r$ ] in dieser Position wie einen Vibranten realisieren. Das proklitisch gebrauchte $\langle$ der $\rangle$ hat bei drei der bulgarischen Sprecher (die das [r] vokalisch auflösen) ebenfalls einen diphthongischen Spektralcharakter. Dabei ist der Frequenzunterschied zwischen den beiden Elementen größer. Perzeptiv gesehen, sollte das heißen, da $B$ so eine Bildung im Munde eines Bulgaren dunkler klingen würde. Der auditive Test bestätigt allerdings diese Annahme nicht (Vgl. die Tabelle der Frequenzwerte und die des auditiven Testes Tab. 4; Tab. 7, 8).

Die Aussprache des unbetonten [e] in Proklitika wie beispielsweise [Es], [des]. [den], nähert sich bei den bulgarischen Sprechern einerseits dem gespannten langen [e :] in ihrer eigenen Realisierung, andererseits und zwar in einem höheren Maße weist sie den diffuseren Charakter des normgerechten deutschen [e :] auf:

$$
\begin{aligned}
& \mathrm{R}_{1}: \mathrm{R}_{2}: \mathrm{R}_{3}\left[\mathrm{e}^{\mathrm{b}}\right]=5: 1,3: 6,4 \\
& \mathrm{R}_{1}: \mathbf{R}_{2}: \mathbf{R}_{3}\left[\varepsilon \mathrm{s}^{\mathrm{b}}\right],\left[\mathrm{den}^{\mathrm{b}}\right]=5,7: 1,4: 8,2 \\
& \mathrm{R}_{1}: \mathrm{R}_{2}: \mathrm{R}_{3}[\mathrm{e}: \mathrm{d}]=6,2: 1,3: 7,8 .
\end{aligned}
$$

Ein Blick in die Tabelle mit den Frequenzwerten der bulgarischen unbetonten Vokale (Sime on ova, 1976, 26) hätte uns außerdem die sehr nahe spektrale Verwandtschaft dieser $[\mathrm{e}]$-Bildung zu einem bulgarischen unbetonten [e] oder [e] gezeigt.

Realisierungen des Murmeliokals in den Positionen [-ə], $[-\mathrm{o}+K]$, [bə-,gə-]

Die Bildung des Murmelvokals in den angeführten Positionen unterscheidet sich beim deutschen Sprecher nur unwesentlich von dessen [e]-Bildung in manchen Beispielen mit dem proklitisch gebrauchten 〈der〉:

$R_{1}: R_{2}: R_{3}\langle$ der $\rangle=3,2: 1,5: 4,7$

$\mathbf{R}_{1}: \mathbf{R}_{2}: \mathbf{R}_{3}$ [bo-, ga] $=3,5: 1,4: 4,8$,

$R_{1}: R_{2}: R_{3}[-0]=3,4: 1,5: 5,2$.

$\uparrow_{\text {diffus }}^{\text {kompakt }}$

Dabei ist dem Vergleich der Relationskoeffizienten zu entnehmen, daß die spektrale Gesamtenergie von $[e] \mathrm{zu}[-\mathrm{a}+\mathrm{K}]$ diffuser verteilt wird.

Bei den bulgarischen Versuchspersonen sind die Spektralunterschiede zvischen den verschiedenen Positionsvarianten des [0] ebenfalls relativ gering: 
$R_{1}: R_{2}: R_{3}[-2+K]=4,5: 1,5: 6,6$

$\mathbf{R}_{1}: \mathbf{R}_{2}: \mathbf{R}_{3}[-2]=5,1: 1,4: 7,3$

$\mathbf{R}_{1}: \mathbf{R}_{2}: \mathbf{R}_{3}$ [bo-, gə-] $=5,2: 1,4: 7,4$.

$\uparrow_{\text {diffus }}^{\text {kompakt }}$

Die spektrale Gesamtenergie ist jedoch bei den bulgarischen Sprechern im allgemeinen diffuser verteilt als bei dem deutschen Sprecher.

\section{Reduzierter Vokal [e]}

Das spektrale Bild des vom deutschen Sprecher realisierten [e] ließe sich einerseits mit dem eines unbetonten, ursprünglich vorderen [a] (z. B. in [das] - prokl. gobraucht) vergleichen, andererseits mit der Realisierung des unbetonten [e] im Präfix 〈ver- $\rangle$ :

$R_{1}: R_{2}: R_{3}[a]=2,3: 1,6: 3,6$

$R_{1}: R_{2}: R_{3}\left[e^{\mathrm{d}}\right]=2.1: 1,9: 4$

$R_{1}: R_{2}: R_{3}\langle$ ver- $=2,6: 2: 5$.

$\uparrow_{\text {diffus }}^{\text {kompakt }}$

Das deutsche $[\mathrm{e}]$. gebildet von den bulgarischen Sprechern, ist diffuser, $d . h$. dunkler:

$R_{1}: R_{2}: R_{3}\left[r^{d}\right]=2,1: 1,9: 4$

$R_{1}: R_{2}: R_{3}\left[e^{b}\right]=3,4: 1,8: 6$.

kompakt

Merkwürdigerweise decken sich die Relationskoeffizienten der bulgarischen Realisation vollkommen mit denen eines unbetonten bulgarischen [a] und liegen sehr nahe einem unbetonten bulgarischen [a] (S i m e o n o va, 1976, $26-27)$.

Wie schon erwähnt, klingen in unbetonter Stellung bulgarisches [a] und [a] gleich, so daB man hieraus schließen kann. Bulgaren realisieren den deutschen Reduktionsvokal [r] sowohl in Anlehnung an bulgarisches [a] als auch an bulgarisches [a].

\section{Unhetontes [u]}

$$
\begin{aligned}
& \mathrm{R}_{1}\left[\mathrm{u}^{\mathrm{d}}\right]: \mathrm{R}_{1}\left[\mathrm{u}^{\mathrm{d}}\right]: \mathrm{R}_{1}\left[\mathrm{u}^{\mathrm{d}}\right]=2,4: 1,8: 2,5, \\
& \mathrm{R}_{1}\left[\mathrm{u}:{ }^{\mathrm{b}}\right]: \mathrm{R}_{1}\left[\mathrm{u}^{\mathrm{b}}\right]: \mathrm{R}_{1}\left[\mathrm{u}^{\mathrm{b}}\right]=2,2: 2,2: 2,3 .
\end{aligned}
$$

Aus dem Vergleich der Relationskoeffizienten der drei U-Laute ist folgender Schluß zu ziehen:

Charakter.

- Das unbetonte [u] behält beim deutschen Sprecher seinen gespannten

- Die Bulgaren realisieren sowohl die betonte als auch die unbetonte Form der U-Laute gleich. Dabei bewegt sich die Klangfarbe dieses einheitlich gebildeten U-Lautes zwischen der des gespannten und der des ungespannten deutschen Vokals; man könnte aber den Klangcharakter dieses unbetonten U.Vokals wegen des geringen Unterschiedes in den Frequenzwerten mit dem Klangcharakter eines langen geschlossenen deutschen [ $u$ :] identifizieren. 
$\mathbf{R}_{1}\left[0^{d}\right]: \mathbf{R}_{1}\left[0^{d}\right]: \mathbf{R}_{1}\left[o^{d}\right]=2,5: 1,8: 2,2$.

$\mathbf{R}_{1}[0: b]: \mathbf{R}_{1}\left[\mathbf{o}^{\mathrm{b}}\right]: \mathbf{R}_{1}\left[\mathbf{o}^{\mathrm{b}}\right]=2,1: 2: 2,2$.

Der Vergleich der Relationskoeffizienten zeigt, daß es zwischen der deutschen und der bulgarischen Realisierung des unbetonten [o] keinen Unterschied gibt. Beim deutschen Sprecher liegen jedenfalls dessen Spektralwerte zwischen denen des gespannten und denen des ungespannten deutschen [0:] - [0], während die Bulgaren, sowohl in betonter als auch in unbetonter Position, alle O-Laute klanglich verhältnismäßig gleich realisieren.

\section{Die deutschen Diphthonge in unbetonter Stellung}

In unbetonter Stellung kommen in unseren Ausspruchbeispielen allein die Diphthonge [âe] und [âo] vor. Da wir bei der Auswertung der Spektraldaten fur die drei deutschen Diphthonge in betonter Stellung keine merklichen Verstöße gegen ihre normgerechte Realisierung seitens der bulgarischen Sprecher festgestellt haben, so können wir annehmen, da $\beta$ die Bulgaren den Diphthong [0̈] in unbetonter Position (wenn er überhaupt in so eine Position hătte auftreten können), nicht anders als die übrigen zwei Diphthonge in unbetonter Stellung realisieren würden. Die spektralen Unterschiede zwischen betont und unbetont sind bei dem deutschen Sprecher gering. Die diphthongische Formantenstruktur ist aber auch in unbetonter Stellung relativ deutlich umrissen. So konnte man auch hier noch einen dritten Formanten fixieren und auswerten.

Was die betonte und unbetonte Form des Diphthongs [äo] betrifft, so zeigen die bulgarischen Realisierungen das gleiche spektrale Bild. Der Diphthong [äe] weist in unbetonter Position eine diffusere Streuung der spektralen Gesamtenergie auf. Mit Ausnahme von Sprecher II sind jedoch bei den anderen Bulgaren keine Spuren von einem $F_{3}$ zu beobachten, die Konturen der unbetonten Diphthonge sind leicht verschwommen, und das gesamte Spektralbild ist temporal etwas zusammengeschrumpft.

Der Vergleich der Relationskoeffizienten, die das Verhăltnis zwischen $F_{2}$ und $F_{1}$ fur beide Elemente in betonter und unbetonter Position ausdrücken. sieht bei der deutschen und bulgarischen Realisierung wie folgt aus:

\begin{tabular}{|c|c|}
\hline 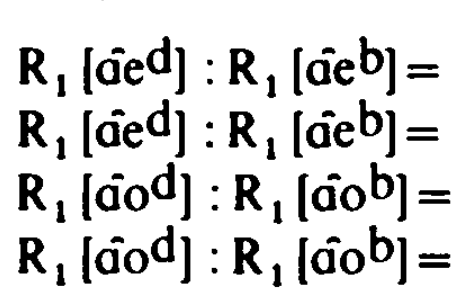 & $\begin{array}{l}\text { I. Element } \\
1,8: 2,4 \\
2,2: 2,7 \\
1,8: 1,7 \\
1,8: 1,9\end{array}$ \\
\hline
\end{tabular}

Beim Vergleich der für die unbetonten Vokale gewonnenen relativen Dauer- und Intensitătsverhăltnisse (gemessen an den Sonagrammen in $\mathrm{mm}$ ) sind nur geringe Abweichungen in der Realisierung der einzelnen Sprecher zu bemerken. Während die Vokaldauer der langen geschlossenen Vokale in der bulgarischen Realisierung recht stark schwankt (vgl. Seite 26-30), so kann man sie bei den unbetonten Vokalen als stabil bezeichnen. Sie bewegt sich hier um die relative Dauer der kurzen offenen Vokale herum. Dies ist übrigens auch beim deutschen Sprecher der Fall. 
Sämtliche unbetonte Vokale sind intensitătsschwächer als die entsprechenden betonten. Dabei sind die Intensitätsgrade zwischen betont und unbetont beim deutschen Sprecher stärker ausgepragt. Das relative Intensitätsverhältnis zwischen den betonten Vokallauten (lange geschlossene und kurze offene Form) und den unbetonten sieht nach unseren Errechnungen für den deutschen und für die bulgarischen Sprecher so aus:

lang betont : unbetont $=$

kurz betont : unbetont $=$ oder

lang betont : unbetont $=$ kurz betont : unbetont $=$

\section{Dt. Sprecher}

$20: 17$

$20: 17$

$1,2: 1$

$1,2: 1$
Bulg. Sprecher

$18: 17$

$19: 17$

$1: 1$

$1,1: 1$

\subsubsection{Zusammenfassung der Untersuchungsergebnisse}

Die Untersuchungsergebnisse der von Bulgaren realisierten Vokale des Deutschen erlauben folgende Zusammenfassung:

a) Betonte Vokale

Mit Ausnahme der labialisierten Vorderzungenvokale tritt bei der bulgarischen Realisierung der gespannten und ungespannten Vokalreihe des Deutschen qualitativ ein gewisser Ausgleich auf, d.h., beide Vokalreihen klingen etwa gleich.

Die Relationskoeffizienten und selbst die gewonnenen Frequenzwerte (Vgl. Tab. 3) zeigen dabei, daB sich die spektralen Merkmale der qualitativ einheitlichen Vokallaute, die die Bulgaren realisieren, wie folgt zusammenfassen lassen:

- A-Laute: Hier wird ein etwas diffuserer A-Vokal gebildet. Die qualitative Abweichung von den deutschen A-Lauten ist jedoch perzeptiv irrelevant.

- Die vordere labialisierte Vokalreihe wird im Durchschnitt kompakter, d. h. heller realisiert.

- Die hintere Vokalreihe [u:] - [0], [o:] - [0] wird ebenfalls kompakter gestaltet. Hierzu könnte man sagen, daß die von den Bulgaren realisierten gespannten Formen eher wie die deutschen ungespannten klingen.

Da sich im bulgarischen Vokalsystem historisch keine labialisierten Vorderzungenvokale herausgebildet haben. so sind bei deren Realisierung durch Bulgaren die meisten Besonderheiten anzutreffen:

- Sowohl bei den Ü-Lauten als auch bei den Ö-Lauten sind interessante „Mischbildungen“" zu beobachten. So bewegen sich die Frequenzwerte für das $[\gamma$ :] bei allen bulgarischen Sprechern in der Frequenzzone eines offenen $[y]$ oder des Murmelvokals. Dabei fehlt der für das $[Y]$ und [a] charakteristische $F_{3}$ auf der Frequenzskala. Im allgemeinen ist die Frequenzenergie für die von den bulgarischen Sprechern gebildete U-Art kompakter, so da $B$ deren Klangbild auch ,helle“, i-ähnliche Komponenten enthält.

- Recht interessant ist die Bildung des kurzen offenen [y]: Sie hat im Mittel den Charakter der bulgarischen Lautverbindung [I 0 ] oder wird gar in manchen Beispielen als $[i]+\mid \mathrm{u}]$ realisiert. 
- Die (O-Laute weisen im allgemeinen einen diffuseren Spektralcharakter auf. Etwa die Hälfte unserer Beispiele zeigt auch hier eine gemischt gebildete Form. die sich allerdings allein beim offenen [o] bemerkbar macht. Sie trägt einen Schwa-ähnlichen Charakter, wobei auf dem Spektralbild ebenfalls kein $F_{3}$ zu sehen ist.

- Das Bild der deutschen Diphthonge in betonter Stellung zeigt weder spektral noch perzeptiv wahrnehmbare Abweichungen von ihrer normgerechten Realisierung.

- Die Dauerverhältnisse zwischen der langen und der kurzen Vokalreihe des Deutschen, gebildet von Bulgaren, zeigen relativ grobe Verzerrungen, die jedoch keinen konstanten Charakter tragen: Unter Umständen (wenn der Sprechende darauf achtet) werden die langen Vokale auch mit der normgerechten Dauer realisiert. Das heißt aber, daß die Quantität neben der Qualität der Vokale ein sehr labiles Kettenglied, wenn auch nicht labiler als die Qualität, in der Phonetik des Deutschunterrichts an Bulgaren ist, und daß ihr Einverleiben in den phonetischen Funktionscode der Fremdsprache und von dort aus in die mötorische Tätigkeit der Sprechorgane einen hohen Zeitaufwand oder aber neue effektivere Unterrichtsverfahren benötigt.

b) Unhetonte Vokale

Die deutschen Vokallaute in unbetonter Position ergeben bei den bulgarischen Sprechern ein einheitliches spektrales Bild, unabhängig davon, ob der entsprechende Vokal in betonter Stellung gespannt oder ungespannt ist. während der deutsche Sprecher auch in unbetonter Position einen Unterschied dazwischen macht.

Im allgemeinen werden die deutschen unbetonten Vokale von Bulgaren diffuser, d.h. den gespannten Vokalen ähnlich realisiert.

- Augenfallig ist die recht dunkle Klangfarbe des unbetonten [a], besonders in den Proklitika [das]. [als]. Hier macht sich die starke Reduktion des bulgarischen Korrelationspaares $[a]-[a]$ in unbetonter Stellung besonders deutlich bemerkbar.

Die Realisierung des unbetonten $[e]$ in proklitisehen Einsilbern und in unbetonten Präfixen auf [r] hängt bei den Bulgaren von der Bildungsart des [r]-Lautes ab: In Fällen, in denen das [r] vokalisch gelöst wird, stimmen die gewonnenen Frequenzwerte mit denen des deutschen Sprechers fast überein, der hierzu den Reduktionsvokal [r] realisiert. Spricht man jedoch das [r] gerollt oder frikativ aus, so behält der Vokal seinen gespannten [c]-Charakter. Die spektrale Gesamtenergie für den Murmelvokal [o] in den Positionen [-a]. $[-a+K]$. [ba-, go-] ist bei den Bulgaren nur etwas diffuser verteilt als bei dem deutschen Sprecher. [e] für die Silbe [-ar] wird ebenfalls dunkler, mehr [o]-artig realisiert.

- Auch bei den Hinterzungenvokalen [u] und [o] machen die Bulgaren keinen Unterschied zwischen ursprünglich gespannt und ungespannt. Der von ihnen gebildete Vokallaut entspricht spektral eher der gespannten Form.

- Die deutschen Diphthonge behalten bei allen Sprechern auch in unbetonter Stellung ihren spezifischen Spektralcharakter. In ihrer Realisierungsart von Bulgaren sind keine normwidrigen Abweichungen zu beobachten. 
- Die Dauer der unbetonten Vokale ist bei allen Sprechern eine konstante Größe. Sie bewegt sich um die relative Dauer der kurzen offenen Vokale herum.

- Die unbetonten Vokale sind bei allen Sprechern im Durchschnitt intensitätsschwächer als die entsprechenden betonten. Dabei ist der Unterschied zwischen betont und unbetont in bezug auf die Intensität bei dem deutschen Sprecher stärker ausgeprägt als bei den bulgarischen Sprechern.

\subsubsection{Spezifische Besonderheiten des deutschen Vokalismus, realisiert von Deutsch sprechenden Bulgaren (Auditiver Test)}

Die 56 sonagraphisch untersuchten Sätze (Siche die Beispielsliste in der Beilage und die Erläuterungen zum Untersuchungsmaterial und den Versuchspersonen auf. S. 17), wurden auch einem auditiven Test unterzogen. Die Tonbandaufnahmen wurden von 60 Abhörern (Germanistik- und Slawistikstudenten der Martin-Luther-Universität in Halle/S, DDR) und von 5 ausgebildeten deutschen Phonetikern und Sprechpädagogen nach dem vorgeschriebenen Verfahren bearbeitet. Die Abhörer hatten die Aufgabe:

- die von der jeweiligen $V_{p}$ in den Textbeispielen lang ausgesprochenen Vokale zu unterstreichen:

- die unbetonten Vokale zu vermerken:

- die Qualität eines jeden Vokals zu bestimmen, indem sie die in phonetischer Transkription angegebene Realisierungsmöglichkeit ankreuzen. die sie wahrnehmen.

Die Ergebnisse dieses Testes erlauben folgende zusammenfassende Bemerkungen:

A. Isinge geschlossene Vokale

Eine Abweichung von der korrekten Aussprache der langen betonten Vokale durch dic vier bulgarischen Germanistikstudenten vermerken die Abhörer an erster Stelle im Hinblick auf ihre sprachliche Dauer-die Quantität ..sitzt“" nicht fest bei den Deutsch sprechenden Bulgaren. So wird die normgerechte Quantität der langen Vokale in $12.1 \%$ der Beispiele nicht eingehalten.

Normwidrige Abweichungen in bezug auf die Qualität wurden mit Ausnahme des Vokals $\mid \mathrm{a}:]$ bei allen langen Vokallauten und bei allen Versuchspersonen festgestellt. Im Mittel werden $11 \%$ der geschlossenen Vokale offen realisiert.

Am auffallendsten sind die Abweichungen bei den labialisierten langen [ $\gamma:]$ und [ $0:]$. in deren Aussprache sich in $25 \%$ der Fälle fremdartige Lautelemente cinmischen.

B. Kurze offene Vokule

Die normgerechte Realisierung der kurzen offenen Vokale beträgt nach den Ergebnissen der auditiven Untersuchung $74.9^{\circ}{ }^{\prime}$. Verstöße gegen die Quantität wurden bei dieser Vokalreihe nicht vermerkt - kein kurzer Vokal wird lang ausgesprochen. Dagegen werden $21^{\circ}$, aller in den Textbeispielen vorkommenden offenen Vokale geschlossen realisiert. Was die offenen labialisierten $[\mathrm{l}]$ und $\left[\alpha^{2}\right]$ betrifft, so fällt bei ihnen der hohe Prozentsatz einer 
wahrgenommenen fremdartigen Realisierung auf $134,1 \%$ der getesteten Beispiele).

C. Unhetonte Vokale

Da die Untersuchung der deutschen Aussprache der vier Bulgaren am Material von natürlichen Sätzen, vorwiegend Sprichwörtern, vorgenommen wurde, kommen in unseren Beispielen verständlicherweise keine Fremdwörter vor. Es wurde folglich allein die Realisierung der in deutschen Wörtern auftretenden unbetonten Vokale akustisch und auditiv untersucht, vor allem in pro- und enklitisch bedingten unbetonten Kürzen und speziell die Realisierung des Murmelvokals in seiner Distribution.

Die in unseren Textbeispielen vorkommenden unbetonten [i], [u], [o], [a] (33 Bsp.) werden von den Abhörern als korrekt empfunden; für das unbetonte [a] wird allerdings vermekt, daß es sich .dunkler“ als unbetontes deutsches [a] anhört.

Das unbetonte [e] wird im Präfix 〈ver-> und im proklitisch gebrauchten Einsilber $\langle$ der $\rangle$. d. h. in Position vor [r], in $63,8 \%$ der Fäle diphthongisch als [-วp] gelöst, in $36,1 \%-$ als [-วk].

In den proklitischen Einsilbern [des, den] hört man zu 50\% kurzes geschlossenes [e] und $\mathrm{zu} 50 \%$ kurzes offenes $[\varepsilon]$.

Der Murmelvokal [ə] wird in den Positionen [-ə], [bə-, gə-], [-ə+K] zu $100 \%$ korrekt wahrgenommen.

Die Auslautsilbe <-er> hört man in $44,2 \%$ der Fälle als [e], in $27 \%$ als [e r], in $15.4 \%$ als $\left[e^{\mathrm{h}} \mathrm{k}\right]$ und in $15.2 \%$ als [әк].

Zusammenfassend kann man sagen, da $B$ die auditiv getestete Realisierung der deutschen Vokale im allgemeinen den spektralen Untersuchungsergebnissen desselben Materials entspricht.

Sowohl die akustische Untersuchung der primären und sekundären Spektralmerkmale der von den Bulgaren realisierten deutschen Vokallaute als auch der auditiv durchgefuhrte Test offenbaren unbestreitbar die typischen Schwierigkeiten auf dem Gebiet des deutschen betonten und unbetonten Vokalismus, die sich für einen Deutsch lernenden Bulgaren durch den phonetischen Einfluß der Muttersprache ergeben, und stellen der korrektiven deutschen Phonetik für Bulgaren die verantwortliche Aufgabe, die Erarbeitung effektiver Methoden für die Überwindung der Schwierigkeiten anzustreben.

\subsection{SEKUNDÄRE AKUSTISCHE MERKMALE DES DEUTSCHEN UND DES BULGARISCHEN VOKALISMUS}

\subsubsection{Untersuchungsverfahren}

Für den kontrastiven Vergleich der sekundären akustischen Parameter der deutschen und der bulgarischen Vokale haben wir spezielle Spektraluntersuchungen im Laboratorium für experimentelle Phonetik der Bulgarischen Akademie der Wissenschaften durchgeführt. Dabei haben wir die schon bei 
den Spektraluntersuchungen bulgarischer Laute bewährte Methodik von Tilkov, Mischeva, Peeva benutzt. Was die konkreten Untersuchungsbeispiele betrifft, so haben wir zwei und mehrsilbige Wörter aus der gesprochenen Sprache und keine Logatome analysiert. Die Auswahl der Beispiele wurde nach folgenden Kriterien getroffen:

- Die Wörter aus den beiden Sprachen enthalten alle.Vokale in betonter und unbetonter Stellung;

- Die betonten und unbetonten Vokallaute treten in ihrer für die jeweilige Sprache natürlichen Distribution und Kombinatorik auf;

- Die Anzahl der Beispiele für die entsprechenden Positionen ist statistisch signifikant;

- In Fällen, wo aus phonemkombinatorischen und Distributionsgründen die eine Sprache über keine Beispiele aus dem nationalen Wortschatzgut verfügt, haben wir nach gebräuchlichen Lehn- und Fremdwörtern gegriffen;

- Alle Wortbeispiele wurden in den gleichen Ausspruchsrahmen mit terminalem Melodieverlauf gefaßt. Z. B. sag: bitte. sag : bekennen. usw. oder: nak : б̆umor. nak : бékon usw. (Siehe die Liste mit den analysierten Beispielen in der Beilage). Sprecher bei diesem Experiment sind vier Frauen: zwei DDR-Staatsangehörige und zwei Bulgarinnen, alle mit korrekter Aussprache. Drei von ihnen haben eine philologische Hochschulbildung, zwei unterrichten deutsche Phonetik. Eine bulgarische Sprecherin ist Diplomphysikerin am experimentalphonetischen Laboratorium der Bulgarischen Akademie der Wissenschaften und hat Erfahrung auf dem Gebiet der experimentalanalytischen Untersuchungen an bulgarischem Sprachmaterial.

Die Sprecher wurden angewiesen, die Beispiele mit neutraler Sprechmelodie, gleichmäßigem Tempo und gleichgehaltener Lautstärke vorzusprechen. Die Beispiele wurden von jedem Sprecher je zweimal gesprochen.

Die Zahl der analysierten Beispiele beträgt 698, darunter 366 bulgarische und 332 deutsche Ausspruchbeispiele.

Die Tonbandaufnahmen sind im Tonstudio der Sofioter Universität auf $19 \mathrm{~cm} / \mathrm{sek}$. Bandgeschwindigkeit und unter Berücksichtigung aller Bedingungen, die eine hohe Aufzeichnungsqualität gewährleisten, gemacht.

Zur Aufzeichnung der Grundfrequenz, des Intensitätsverlaufs, der Lautdauer und des gesamten Oszillogramms ist im experimentalphonetischen Laboratorium ein Registriergerät entwickelt und konstruiert. Durch diesen Intonographen wurden die sekundären akustischen Merkmale unserer Beispiele aufgegezeichnet.

\subsubsection{Vergleichende Charakteristik der sekundären Spektralmerkmale der deutschen und der bulgarischen Vokallaute}

Wie schon hervorgehoben, fassen wir die Spektralmerkmale der Sprachlaute unter dem Bergiff akustische Grundstruktur zusammen. Während jedoch die Formantenstruktur der Sprachlaute, vorwiegend der Vokale, eine recht lange systematisch und komplex angelegte Untersuchungsgeschichte hinter sich hat (Simeonova, 1975), sind die sekundären akustischen Merkmale der Laute in ihrer Komplexität. in ihrer Bezogenheit aufeinander 
und auf die Formantenstruktur noch keiner systematischen Untersuchung innerhalb einer Sprache unterzogen worden. Die Arbeit von Tilkov und Mischeva (Т и лков, М и ше в а, 1978) kann man als den ersten Versuch, die ..Mikrostruktur" der bulgarischen Sprachlaute einer systematischen Analyse zu unterziehen, betrachten. Spezifische Einzelfragen der Grundfrequenz. Intensität und Lautdauer versuchen allerdings viele Experimentalphonetiker aufzuklären. Zu den $u$. E. wichtigsten darunter nehmen wir im entsprechenden Abschnitt der vorliegenden Arbeit Bezug.

\subsubsection{Grundfrequenz des deutschen und des bulgarischen Vokalismus. Untersuchungsverfahren}

Die Untersuchungs- und Ausrechnungsverfahren der Grundfrequenz haben in der phonetisch-akustischen Literatur keine lange Tradition. Sie sind heute noch sowohl theoretisch als auch praktisch umstritten und problematisch.

So rechnen House und $\mathrm{F}$ a irbanks $(1953,25,1)$ die Grundfrequenz aus, indem sie die Anzahl der Perioden des am Oszillogramm fixierten Lautsegments durch dessen Dauer dividieren.

Lehiste und Peterson (1961, v. 33) beachten bei der Errechnung des $F_{0}$ das Maximum in der Kontur des Lautsegments.

M o h r (1971, H 23) schlägt ein graphisches Verfahren zur Bestimmung der Grundfrequenz vor, indem er alle für das Lautsegment wesentlichen Punkte (Grenzmarken, Maximal- und Minimalwerte) in Betracht zieht.

Tilkov und Mischeva (Т и л ко в, М ише в а, 1977, 22) setzen sich mit den Schwächen und Inkonsequenzen der oben angeführten Ausrechnungsverfahren auseinander und schlagen eine neue Methodik vor:

Es wird angenommen. da $\beta$ der integrale Mittelwert der Grundfrequenz vollkommen und genau das Lautsegment charakterisiert. Der Mittelwert des $F_{0}$ wird nach folgender Formel gewonnen:

$$
F_{0}=\frac{f_{1} t_{1}+f_{2} t_{2}+\ldots f_{n} t_{n}}{t_{1}+t_{2}+\ldots t_{n}},
$$

wo $f_{1}, f_{2} \ldots f_{n}$ die mittleren Frequenzwerte der einzelnen Punkte in der linearen $F_{1,}$-Kontur darstellen, und $t_{1}, t_{2} \ldots t_{n}$ - der Dauer eines jeden dieser Punkte entsprechen. Die Summe: $t_{1}+t_{2}+\ldots t_{n}$ gibt die Gesamtdauer des Lautsegments an.

Der durch die Einbeziehung der temporalen Relationen zwischen den einzelnen Punkten der $\mathrm{F}_{0}$-Kontur des jeweiligen Lautsegments gewonnene Mittelwert für die Grundfrequenz entspricht dann schon dem realen Integralwert des Grundtones des analysierten Lautsegments. Dieses Verfahren wurde in der vorliegenden Arbeit benutzt. 


\subsubsection{Grundtonfrequenz der betonten Vokale des Deutschen und des Bulgarischen}

Die absoluten Werte, die wir für den $F_{0}$ der betonten Vokale beider Sprachen ermittelt haben, lassen bestimmte Gesetzmäßigkeiten erkennen, nach denen die Grundfrequenzdaten der Vokale innerhalb der uns bekannten Vokalgruppen der beiden Sprachen streuen. So kann man aus den vergleichenden Tabellen (Tab. 13, 14) ersehen, daß die Grundfrequenz der deutschen und der bulgarischen Vokale nicht für alle Vokale die gleiche ist, da $B$ sie mit der Höhe der Zungenlage während der Artikulation folgerichtig korrespondiert. Dabei folgt sie einer recht stabilen GesetzmäBigkeit : Je höher die Zungenlage, desto höher der $F_{0}$. Am höchsten sind die Grundfrequenzwerte des Vorderzungenvokals [i] und des hohen Hinterzungenvokals [u], am niedrigsten sind sie bei den A-Lauten.

Diese Gesetzmäßigkeit beobachtet man in den zwei betonten deutschen Vokalreihen, sie gilt in gleichem Maße auch für den bulgarischen betonten Vokalismus. Da sie durch die Spektralanalysen von Vokallauten anderer Sprachen ebenfalls festgestellt ist, kann man sie für universell halten (Ho u se, Fairbanks.1953; Lehiste, Peterson, 1961; Boel, 1972; Mohr, 1971).

Die angeführten Autoren suchen verschiedene Erklärungen für diese Gesetzmäbigkeit: House - Fairbanks, Lehiste - Peterson, Mohr - Kim auf physiologischer Grundlage, Boel - auf elektro-akustischem Boden. House und Fairbanks (1953) versuchen, die höhere Grundfrequenz der Vokale mit hoher Zungenlage folgendermaßen zu erklären: Die Hebung der Zunge von den tiefen zu den hohen Vokalen vergrößert die Spannung der Zungenmuskeln; sie wird dann weiter auf die laryngiale Muskulatur und die Stimmlippen übertragen, wodurch auch die Frequenz des erzeugten Stimmtones ansteigt.

Lehiste und Peterson (1961) erklären die Ursache dieser auch von ihnen beobachteten GesetzmäBigkeit ebenfalls physiologisch. Nach ihnen liegt sie in der etwas mehr ausgeprägten zusätzlichen vertikalen Bewegung des Kehlkopfes während der Artikulation der hohen Vokale. Diese im Gegensatz zu den tiefen Vokalen mehr ausgeprägte vertikale Larynxbewegung erfolgt mechanisch, da die Zunge unmittelbar mit dem oberen Teil des hioidalen Knochens, während andere laryngiale Muskeln mit dessen unterem Teil verbunden sind. Diese mechanisch bewirkte Bewegung des Kehlkopfes vergrößert die Spannung der Stimmlippen, woraus eine Erhöhung der Grundfrequenz resultiert.

Mohr (1971) äußert die Meinung, daß die gegenwärtigen Kenntnisse in der Physiologie des Sprechmechanismus nicht ausreichen, um diese Erscheinung zufriedenstellend aufzuklären. Er berichtet, daß die letzten Untersuchungen über die Genese der Sprachlaute keine Korrelation zwieschen dem Hebungsgrad der Zunge und der zusätzlichen vertikalen Kehlkopfbewegung bei den hohen Vokalen bestätigen. Ebenso fehlen jedoch auch für die Bestätigung der House - Fairbanks' Hypothese experimentell fundierte Daten. Mohrs Hypothese baut auf der myoelastischen Theorie für das Zustandekommen der Stimme. Er berücksichtigt dabei die bestehende 
Korrelation zwischen der Ausweitung bzw. Einengung der Stimmritze für die verschiedenen Vokalgruppen, dem Grad des Luftdruckes und der Geschwindigkeit der Expirationsluft. So nimmt er an, daß die pharyngiale Einengung. welche bei den tiefen Vokalen [a] und [o] ihr Maximum erreicht. zu entsprechender Erhöhung des Luftdruckes oberhalb der Glottis führt, infolgedessen sich die Geschwindigkeit der ausströmenden Luftsāule, entsprechend auch die Schwingungsgeschwindigkeit der Stimmbānder verringern. Eine analoge Einengung allerdings zwischen dem Zungenrücken und dem Gaumen, wie man sie bei den hohen Vokalen beobachtet, kann nicht den gleichen Effekt hervorrufen, da der superglottale Raum ein recht großes Volumen bei ihrer Artikulation hat.

Indem Mohr in seiner Hypothese allein mit den wechselseitigen Beziehungen und der gegenseitigen Bedingtheit zwischen Pharynx und Larynx auszukommen versucht, was zu ẹner einseitigen Lösung des Problems führt, bezieht Boel (1972) in seinen Überlegungen die Einwirkung des gesamten superglottalen Ansatzraumes auf die Tätigkeit der Stimmlippen in die Lösung der Frage ein. Er beruft sich dabei auf die Gesetzmäßigkeiten der elektroakustischen Analogie. So bestimmt er theoretisch die Eingangsimpedanz des Ansatzrohres und rechnet ihre Werte fur die typischen, mit Hilfe eines Syntesators gewonnenen Vokalgebilde der französischen Sprache aus. Seine Ergebnisse zeigen, daß die Streuung der Werte der Eingangsimpedanz des Ansatzrohres mit der der Stimmlippen korreliert, besonders auffallig bei einem niedrigen $F_{1}$, wie es der Fall mit den hohen Vokalen [i] und [u] ist. Bei ihrer Bildung äußert sich die Impedanzeinwirkung in einer Steigerung der Schwingungsfrequenz der Stimmbänder.

Die zufriedenstellende Lösung dieser immer wieder ins Auge fallenden Gesetzmäßigkeit wird mehr Licht über das Wesen der Grundfrequenz werfen, und es ist an der Zeit, bei dem heutigen hohen Entwicklungsstand der experimentellen Phonetik, sie endgültig und eindeutig zu lösen. $\mathrm{Zu}$ diesem Zweck sollte man sowohl auf physiologischem als auch auf akustischem Niveau entsprechende Untersuchungen vornehmen. Bei unseren Spektralanalysen der Grundfrequenz der deutschen und bulgarischen Vokallaute sind wir auf eine Tatsache gestoßen, die zwar weiterer näherer Untersuchungen bedarf, jedoch ein gewichtiger Grund dafür ist, da $B$ man keine der angeführten Hypothesen zu ihrer Aufklärung heranziehen könnte:

Die zwei Reihen von betonten Vokalen im Deutschen folgen, eine jede fur sich, der Gesetzmäßigkeit, nach der die höhere Zungenhebung entsprechende Steigerung der Grundfrequenz mit sich zieht. Vergleicht man doch diesbezüglich die lange geschlossene mit der kurzen offenen Vokalreihe (Siehe Tab. 13), so weisen nicht, wie man hätte erwarten können, die geschlossenen, sondern die offenen Vokale etwas höhere Grundfrequenzwerte auf. Dabei charakterisiert sich ihre Artikulation im Gegensatz zu der der geschlossenen Vokale insgesamt durch eine etwas niedrigere Zungenlage.

Hierzu möchten wir die Meinung äußern, daß die Lösung dafür wahrscheinlich in der von uns beobachteten Korrelation zwischen Grundfrequenz und Lautdauer zu suchen wäre: so gestaltet sich die Grundfrequenz bei einem kurzen offenen Vokal wegen der kurzen Lautdauer nicht so variabel we bei cinem langen geschlossenen; hier verläuft sie recht unstabil, besonders im 
letzrten Segment der $F_{0}$-Kontur, wo sich das Schwingungstempo etwas verlangsamt und der Stimmton sinkt (Diese Erscheinung läßt sich am Oszillogramm beobachten: Vgl. Abb. 14).

\subsubsection{Grundfrequenz der unbetonten Vokale des Deutschen und des Bulgarischen}

Die unbetonten Vokallaute des Deutschen und des Bulgarischen charakterisieren sich durch niedrigere Frequenzwerte im Vergleich zu den betonten Vokalen. Diese Erscheinung korrespondiert gut mit der schon bei den betonten Vokalen beschriebenen Abhängigkeit der Grundfrequenz von der Höhe der Zungenlage: sämtliche unbetonte Vokale werden mit etwas niedrigerer Zungenhebung im Gegensatz zu den betonten realisiert - daher auch die niedrigeren Grundfrequenzwerte. Der Grundfrequenzunterschied $\mathrm{zW}$ ischen betont und unbetont offenbart sich bei allen Versuchspersonen, bewegt sich jedoch im Rahmen einer individuell bedingten Variabilität. So ist er z. B. bei H.P. (für die deutsche Sprache) stärker ausgeprägt als bei H.V. $(29,3 \mathrm{~Hz}$ gegen $11,9 \mathrm{~Hz})$; für das Bulgarische ist der Frequenzunterschied zwischen betont und unbetont bei M.P. größer als bei R.S. $(20,4 \mathrm{~Hz}$ gegen 14.2 Hz).

Sehr auffälig ist der Unterschied zwischen den Grundfrequenzwerten der betonten Vokale des Deutschen und denen des Murmelvokals [o] oder des Reduktionsvokals [e] (Siehe Tab. 13, 15). Dieser Umstand ist ein Beweis dafür, da $B$ die einzigen im spektralen Sinne des Wortes reduzierten Vokallaute in der gegenwärtigen deutschen Standardaussprache der [o]- und [e]-Vokal sind. Im Bulgarischen trift die Reduktion die drei sog. „breiten“"Vokale [a,o,e] in unbetonter Stellung (Стойков, 1966, 144; 1968, 136; Тилков, Бояджисв, 1977, 67-70). Das ist eine Tatsache, die schon Trubetzkoy beobachtet und beschrieben hat (Trubetzkoy, 1971, 131). Die Untersuchungen zur Formantenstruktur der bulgarischen betonten und unbetonten Vokallaute bestätigen unbestreitbar die Reduktion des unbetonten Vokallautes [a] der bulgarischen Standardaussprache. der, je nach der Position, stärker oder schwächer, seine Formantenstruktur in Richtung [ə] ändert.

Von einer Reduktion der sog. bulgarischen ..engen" Vokale wird in der Literatur über Fragen des bulgarischen Vokalismus nicht gesprochen. Die Ergebnisse unserer Spektraluntersuchungen lassen jedoch annehmen, da $B$ in mancher Position auch der „enge“ Vokal [o] einer assimilativen Reduktion fahig ist. und zwar bewegt sich seine Formantenstruktur dann in der Spektralzone des „breiten“ [a]. Da so eine Formantenbewegung als einen qualitativen Übergang von einem engen zu einem breiten Vokal betrachtet werden kann, haben wir hierfür die Bezeichnung „rückwärtige Reduktion“ vorgeschlagen, im Gegensatz zu der bekannten Reduktion der bulgarischen breiten Vokale, deren Formantenwerte sich stets in Richtung .enge“ Vokale bewegen (С и меонов ва, 1975, 94, 95).

Die Reduktion der bulgarischen Vokallaute in unbetonter Position läßt sich auch durch die Frequenzwerte des $F_{0}$ nachweisen: Die Grundfrequenzuerte fur die unbetonten $[a]$ und [a] zeigen den minimalen Unterschied von 
1.6 Hz (Sprecher R.S.) und $1,9 \mathrm{~Hz}$ (Sprecher M. P.), was für die Klangfarbenneutralisierung dieses Korrelationspaares spricht. Auf die Reduktion des [c] und [o] weisen lediglich die Grundfrequenzwerte, die wir für die eine Versuchsperson gewonnen haben, hin (Siehe Tab. 14, 16).

\subsubsection{Beeinflussung der Grundfrequenz der Vokallaute} des Deutschen und des Bulgarischen durch die konsonantische Lautnachbarschaft

Für jeden deutschen und bulgarischen Vokallaut wurden die Grundfrequenzwerte je nach der konsonantischen Lautumgebung ermittelt. Zunächst wurde der $F_{0}$ der Vokale separat in stimmhafter und stimmloser Lautnachbarschaft errechnet, danach in der Lautumgebung von Konsonanten, die sich nach der Art ihrer Bildung voneinander unterscheiden. Die gewonnenen Mittelwerte sind auf Tabellen 17-25 zu sehen.

Die Untersuchungsergebnisse für beide Sprachen zeigen keine wesentlichen Unterschiede im $F_{0}$ der betonten und unbetonten Vokale, die durch die stimmhafte bzw. stimmlose Lautumgebung bedingt wären. Nur bei manchen Vokallauten und bestimmten Versuchspersonen läßt sich eine gewisse Streuung der Frequenzwerte in Abhängigkeit von der stimmhaften oder stimmlosen konsonantischen Umgebung beobachten. Für das Deutsche macht sich dadurch die Tendenz zu einem leichten Ansteigen der Frequenzzahl des $F_{0}$ zugunsten der stimmlosen Lautumgebung bemerkbar (Siehe Tab. 17-21). Für das Bulgarische ist das jedoch nicht der Fall.

Die Untersuchungsergebnisse der bulgarischen Autoren Tilkov und Mischeva (Тилков. Миччева, Пеева, 1977) weisen ebenfalls keine folgerichtige Becinflussung der Grundfrequenz durch stimmhafte bzw. stimmlose konsonantische Umgebung auf.

Die Beeinflussung der Grundfrequenz der Vokale des amerikanischen Englisch durch die Art der sie umgebenden Konsonanten ist von $\mathrm{H}$ o u se und Fairbanks (1953) und von Lehiste und Peterson (1961) untersucht worden. Auf Grund von Spektralanalysen der Vokale [o] und [e] stellen House und Fairbanks fest, daB in der Nachbarschaft von Verschlußlauten die Grundfrequenz der Vokale am höchsten und in der Umgebung von Sonorlauten am niedrigsten ist.

Bei Lehiste und Peters on (1961) sind es die Engelaute, welche die Erhöhung der Grundfrequenz benachbarter Vokale bewirken, während sie in der Umgebung von Sonorlauten die niedrigsten Frequenzwerte aufweist.

Tilkov und Mischeva (Тилков, Милева, Пеева, 1977, 30) finden. da $\beta$ das Untersuchungsmaterial von House und Fairbanks für die statistische Auswertung nicht ausreicht. Ihre eigenen Untersuchungen, die sie am Material von zweisilbigen natürlichen bulgarischen Wörtern und Logatomen durchgeführt haben, bestätigen keine systematische Abhängigkeit zwischen der konsonantischen Umgebung und der Grundfrequenz der bulgarischen Vokale.

Die eventuelle Beeinflussung des $F_{0}$ durch die verschiedenen Konsonantengruppen haben wir in jeder der natürlichen sprachlichen Distribution der 
beiden Sprachen entsprechenden Position, vor und nach Explosiven, Frikativen und Sonoren, verfolgt. Die ermittelten Frequenzwerte sind auf Tab. $22-25$ zu sehen.

Die Auswertung unserer Ergebnisse erlaubt die Behauptung, daß die Grundfrequenz der betonten und unbetonten Vokallaute des Deutschen und Bulgarischen nicht durch die Art der sie umgebenden Konsonanten beeinflußt wird.

\subsubsection{Abhängigkeit der Grundfrequenz der Vokale des Deutschen und des Bulgarischen von der Akzentuierung im Wort}

Aus den Ergebnissen der intonographischen Analyse des Grundtones bei zwei-, drei- und viersilbigen Wörtern haben wir versucht, den Einfluß der Akzentuierung im Wort auf die Bewegung des Grundtones zu verfolgen, bzw. Schlüsse im Hinblick auf gewisse gesetzmäßige Abhängigkeiten zwischen der Akzentuierung und der Grundfrequenz der Vokale zu ziehen.

$\mathrm{Zu}$ diesem Zweck wurden 150 zweisilbige Wörter mit Betonung auf der ersten bzw. zweiten Silbe (Typ : bitte - бaтe, dafür - беглик), 249 dreisilbige Wörter mit betonter erster, zweiter bzw. dritter Silbe (Typ: Ameise - ядене, gewürzig - raтанка, populär - лековит), 299 vier- und mehrsilbige Wörter mit Betonung auf der zweiten, dritten oder letzten Silbe (Typ: berichtete мислителят, stimmulieren - ръкавица, Tonalität - необходим) aus beiden Sprachen analysiert.

Die gewonnenen Ergebnisse erlauben folgende zusammenfassende Schlußfolgerung (Siehe Tab. 26-29):

- Betonte erste Silbe, abgesehen von der Silbenzahl des Wortes, weist stets die höchste Grundfrequenz auf;

- Unbetonte letzte Silbe hat bei zwei-, drei- und viersilbigen Wörtern ausnahmslos die niedrigste Grundfrequenz:

- Wenn die zweite Silbe eines dreisilbigen Wortes oder die zweite bzw. dritte Silbe eines viersilbigen Wortes betont ist, so zeichnet sie sich in der Regel durch erhöhte Grundfrequenz aus, oder aber ist die Frequenz des Grundtones etwa der Grundfrequenz der vorangehenden unbetonten Silben gleich;

- Wenn die letzte Silbe eines zwei-, drei- oder viersilbigen Wortes unter Betonung liegt, so weist sic in manchen Fällen auch die höchste Grundfrequenz auf; meist ist sie aber annähernd gleich der Grundfrequenz der vorangehenden unbetonten Silbe; gelegentlich sinkt sie auch etwas darunter. Dieser Umstand läßt sich durch die terminale melodische Gestaltung unserer "Rahmenbeispiele“ (Vgl. S. 111 ก) erklären. Betont oder nicht betont, zeichnet sich die letzte Silbe eines terminal verlaufenden Ausspruchs durch die sog. ..spannungslose Tiefe" (Esse n, 1964, 37) aus, was sich akustisch in einer Spannungs-, Tempo- und Intensitätsabschwächung und in einer Verminderung der Grundfrequenz ausdrückt.

Bei der Vp. H. V. (für die deutsche Sprache) und M.P. (für das Bulgarische) beobachtet man ein mehr ausgeprägtes Herabsinken der vokalischen G-undfrequenz im betonten Wortauslaut. Eine Erklärung hierfür finden wir in 
der individuell stärker ausgeprägten terminalen Melodieführung der jeweiligen Versuchsperson.

Wir möchten die im betonten Wortauslaut beobachtete Erscheinung gleichzeitig als einen Beweis dafür betrachten, daß sich die terminale Melodieführung im Deutschen und im Bulgarischen akustisch gleich gestaltet.

\subsubsection{Spezifische Intensität der deutschen und der bulgarischen Vokallaute}

\subsubsection{Die wichtigsten Untersuchungen zur spezifischen Intensität der Vokale}

Der Frankfurter Ohrenarzt Oskar Wolf stellt 1871 die ersten systematischen auditiven Versuche zur spezifischen Intensität (Schallfülle) deutscher Sprachlaute an. Otto v. Esse n $(1962,108)$ berichtet über dessen Versuch und führt die sich daraus ergebende Schallfülleordnung der Sprachlaute (Entfernungsangaben in Schritt) an: So hat unter den deutschen Vokalen (ausgenommen die Vorderzungenvokale mit Lippenrundung) der Vokal [a] den höchsten Schallfüllewert, [u] - den niedrigsten.

Ausführliche Untersuchungen ähnlicher Art am Lautmaterial aus dem Französischen stellt R ou ss el ot $(1897,1924)$ an, ermittelt jedoch eine andere hierarchische Schallfülleordnung der Vokale. Hiernach haben die E-Laute die am weitesten reichende Schallkraft, es folgen dann die O-, A-, I- und U-Laute.

Jespersen $(1913,186)$ gibt folgende Ubersicht der Sprachlaute nach ansteigender spezifischer Schallfulle (wir führen nur die Vokale an):

1.a) p, t, k

6. $\ddot{\mathrm{u}}, \mathrm{u}, \mathrm{I}$

7. $\mathrm{o}, \mathrm{o}, \mathrm{e}$

8. $\supset, \mathfrak{x}, a$

Er benutzt die von ihm aufgestellte Schallfülleskala zur Charakterisierung seiner .Schallsilbe".

Die meßtechnischen Untersuchungen unserer Zeit bestätigen im großen und ganzen die einstmaligen auditiven Untersuchungen Wolfs. So folgt die von Fairbanks, House und Stevens (1959) gewonnene Schallfulleordnung der amerikanischen Vokale vollkommen der von Wolf.

Lehiste und Peters on (1959) untersuchen die spezifische Schallkraft der amerikanischen Vokale an von einer Versuchsperson isoliert gesprochenen Lauten und einsilbigen Wörtern. Bei ihnen bilden die $\mathrm{O}$ - und I-Laute die Extrempunkte : die O-Laute weisen die höchstregistrierte spezifische Intensität auf, die I-Laute dagegen die niedrigste.

Die Vokale der russischen Sprache sind von Ж и н ки н (1958) in bezug auf ihre spezifische Intensität analysiert worden. Nach ihm steht der Vokal [a] auf der höchsten Intensitätsstufe, [i] - auf der niedrigsten. Die Intensitătsreihen- 
ordnung der russischen Vokale ist die folgende: $a, o, e, u, i$. Eine Erklärung für diese Schallfülleordung der Vokale sucht Shinkin in der korrelativen Gesetzmäßigkeit zwischen dem Volumen des pharyngialen Raumes und der Schallkraft der Vokale: je kleiner das Volumen, desto größer die Schallfülle des jeweiligen Vokals.

Die Untersuchungsergebnisse O.v. Essens $(1953,81-88)$ verwerfen die von Jespersen und anderen angenommene "Schallfulleordung“ der Sprachlaute : .. a ist weit von seiner Vorrechtsstellung am Anfang der Skala fort und ins Innere der Reihe gerückt; o und ö haben sich um etwa das Eineinhalbfache (ca. $3 \mathrm{~dB}$ ) schallwirksamer erwiesen als a; die Nasale und / stehen im Range den Vokalen gleich und sogar oft voran" (Es se n, 1953, 87).

Tilk ov, M ischeva und Peeva (1977) haben die spezifische Intensität der bulgarischen betonten Vokale am Material von Logatomen Typ CVCV, durch den im phonetischen Laboratorium der Bulgarischen Akademie der Wissenschaften entwickelten und konstruierten Intonographen, gesprochen von 5 Versuchspersonen, registriert und ausgewertet (Ти лков, М и шева, П е в в , 1977, $73 \mathrm{~m}$.

Im Unterschied zu Untersuchungen über andere Sprachen kann man nach den von Tilkov und Mischeva ermittelten Untersuchungsergebnissen nicht von einer konstanten Schallfülleordnung der Vokale im Bulgarischen sprechen. Einen relevanten Wert haben lediglich die Intensitätsunterschiede zwischen der Gruppe der bulgarischen „,breiten“ Vokale $[a, \supset, \varepsilon]$ und der der .engen" Vokale [1. $U$, o].

Die Schallfülleordnung innerhalb der beiden Vokalgruppen verhält sich bei den 5 Versuchspersonen recht variabel. Die Ursache hierfür suchen die Autoren in der leichten mundartlichen Färbung der Aussprache mancher Versuchspersonen.

Der dB-Unterschied zwischen den beiden Gruppen von Vokalen des Bulgarischen beträgt $3 \mathrm{~dB}$; auch der Unterschied zwischen der höchsten und der niedrigsten Intensitätsstufe der Vokale ist im Mittel $3 \mathrm{~dB}$, was viel weniger ist als der Unterschied, den man für andere Sprachen ermittelt hat (Für das Ungarische beträgt er z. B. 12,3 dB (F o n a g , 1966), für das Englische $5,5 \mathrm{~dB}$ (Fairbanks, House, Stevens, 1950), für das Französische 4,5 dB (Rossi, 1971). Diesbezüglich heben Tilkov und Mischeva jedoch hervor, da $B$ die für die genannten Sprachen veröffentlichten Angaben allein für isoliert gesprochene Vokale gelten.

Die Verfasser stellen weiter fest (Т и л к в, М ише в а, Пе в а, 1977, 71), daß die spezifische Intensität der bulgarischen Vokale in einem stark ausgeprägten Abhängigkcitsverhältnis zu der Akzentuierung im Wort stehen, was die Untersuchung der spezifischen Schallfülle der unbetonten Vokallaute des Bulgarischen äuberst erschwert. Die konsonantische Lautnachbarschaft hat nach den bulgarischen Autoren keinen merklichen EinfluB auf die spezifische Intensität der bulgarischen Vokale. Es sei jedoch unsererseits im Zusammenhang mit der zitierten Untersuchung darauf hingewiesen, da $B$ das Untersuchungsmaterial von Tilkov und Mischeva (zweisilbige Logatome vom Typ (VCV) keinen vollständigen Einblick in das akustische Wesen der spezifischen Intensität der bulgarischen Vokale gewährleisten kann. 


\subsubsection{Vergleichende Intensitätscharakteristik der deutschen und bulgarischen Vokallaute}

Die physikalisch-akustische Größe .Intensität“, auf das Gennema der menschlichen Sprachlaute bezogen, hat in der phonetischen Literatur einen zweifachen Gebrauch: Einerseits beinhaltet dieser Begriff auf der linguistischfunktionellen Ebene die variable Schallkraftgröße, mit der je nach der Situation, den logischen Überlegungen, dem Gemütszustand des Sprechenden und Hörenden die Laute im kontextualen Zusammenhang verschieden ..druckstark" realisiert und perzeptiv aufgenommen werden; andererseits wird dadurch auf der physikalisch akustischen Ebene die fixe, einem jeden Sprachlaut innewohnende und allein für ihn charakteristische .spezifische Intensität“" (Schallfülle, Sonorität) bezeichnet. Im Redefluß läßt sich jedoch die spezifische Intensität meßtechnisch aus der linguistisch variablen Intensität nicht ohne weiteres extrahieren. Deshalb haben wir die einzelnen Beispiele unseres Versuchsmaterials, die keine Logatome, sondern Wörter aus dem natürlichen Wortschatz des Deutschen und des Bulgarischen darstellen, in einen quasi-natürlichen, terminal verlaufenden Ausspruchrahmen gefaßt. Die gleichzeitig gehaltene Lautstärke und das Sprechtempo, die wir von den Versuchspersonen beim Vorsprechen der Beispiele verlangt haben, wurde am fertigen Tonbandmaterial durch drei Diplomphonetiker vor der meßtechnischen Registrierung auditiv überprüft.

Die Intensitätskurven der deutschen und der bulgarischen Vokale wurden durch den Pegelschreiber - eine Vorrichtung des Intonographen, registriert. Die Intensität wurde auf ein willkürlich gewähltes Niveau von 40 Millivolt bezogen und in $\mathrm{dB}$ ausgerechnet. Da die spezifische Intensität im Sprechbewegungsablauf eines Lautes beträchtlich variiert, wurde für den jeweiligen Vokal der Mittelwert der Intensitätskurve als dessen typische Charakteristik genommen.

Die Mittelwerte von den meßtechnischen Angaben sind auf Tab. 30-37 zu sehen.

Die Konfrontation der gewonnenen Intensitätswerte läßt folgende Überlegungen zusammenfassen:

A. hetonte Vokale

Die Einordnung der betonten Vokale des Deutschen und des Bulgarischen nach ihrer spezifischen Intensität folgt im großen und ganzen der Sonoritätsskala von Jespersen. So zeigt unter den betonten Vokalen der Vokal [a] die höchsten Intensitätswerte, [u] - die niedrigsten.

Im einzelnen lassen sich folgende Schlußbemerkungen formulieren:

- Die deutschen kurzen offenen Vokale weisen, vor allem in der Aussprache der Vp.H.P., cine etwas höhere Intensität als die langen geschlossenen auf. Die Intensitätsunterschiede zwischen den beiden Vokalreihen sind aber gering $-29,1 \mathrm{~dB}$ für die offenen Vokale gegenüber $28,8 \mathrm{~dB}$ für die geschlossenen. Bei der $\mathrm{Vp}$. H. V. beobachtet man ebenfalls eine gewisse Intensitätssteigerung in der Reihe der offenen Vokale, sie trifft aber nicht fur alle offenen Vokale zu. 
Aus den angeführten Beobachtungen kann man schließen, daß die Quantität und Qualität der Vokale im Deutschen keinen wesentlichen Einfluß auf ihre spezifische Intensität ausüben.

- Innerhalb der offenen und der geschlossenen Vokalreihe des Deutschen und in der Reihe der betonten Vokale des Bulgarischen lassen sich zwischen den einzelnen Vokallauten ebenfalls keine wesentlichen Intensitätsunterschiede feststellen. Der höchste Intensitätsunterschied zwischen zwei Vokalen aus einer Vokalreihe beträgt $2.5 \mathrm{~dB}$ im Deutschen und $1,3 \mathrm{~dB}$ im Bulgarischen.

- Die Reihenfolge der deutschen und bulgarischen betonten Vokale in der auf Grund der gewonnen Intensitätswerte aufgestellten Skala (Siche Tab. 30, 31) gestaltet sich, bis auf die Initialstellung des [a]-Vokals, bei den einzelnen Versuchspersonen recht variabel. Relevante Unterschiede lassen allein für das Bulgarische zwei Gruppen von intensitätsunterscheidbaren Vokalen erkennen : die Gruppe der „breiten“ Vokale mit höherer Intensität und die Gruppe der ..engen" Vokale mit einer etwas niedrigeren Intensität.

- Sowohl im Deutschen als auch im Bulgarischen ist eine gesetzmäBige Abhängigkeit der Intensität von der Stellung der akzentuierten Silbe im Wort zu beobachten. So hat die Intensität einer ersten betonten Silbe in beiden Sprachen stets einen höheren Wert im Vergleich zu der Intensität einer zweiten, dritten oder letzten betonten Silbe.

- Statistisch relevante Intensitätsunterschiede, durch die Art der Silbe (offen oder geschlossen) bewirkt, sind weder im Deutschen noch im Bulgarischen festzustellen.

\section{B. unbetonte Vokale}

Die Intensität der unbetonten Vokale des Deutschen und des Bulgarischen wurde für jeden Vokallaut in erster, zweiter und dritter vorbetonter und in erster nachbetonter (Wortauslaut) Position, in offener und geschlossener Silbe crmittelt. Die Ergebnisse dieser Untersuchung sind auf Tab.34-37 veranschaulicht. Aus ihnen ergeben sich folgende zusammenfassende Schlubfolgerungen:

- Die spezifische Intensität der Vokallaute des Deutschen und des Bulgarischen ist stark akzentabhängig. So sind die deutschen unbetonten Vokale im Mittel um $2.7 \mathrm{~dB}$, die bulgarischen - um $2.3 \mathrm{~dB}$ druckschwächer als die betonten. Die angeführten I ifferenzen sprechen von etwa gleichen Intensitätsverhältnissen in beiden Sprachen: Der Unterschied zwischen betont und unbetont ist sowohl im Deutschen als auch im Bulgarischen recht grob.

- Die Rangordnung der unbetonten Vokale nach ihrer Intensität entspricht weder im Deutschen noch im Bulgarischen der der betonten Vokallautc. Der Vokal [a] behält zwar in beiden Sprachen seine Spitzenstellung. doch rücken der Vokal [e] und die labialisierten Vorderzungenvokale [ø] und $[y]$ des Deutschen, die Vokale [e] und [e] des Bulgarischen in unbetonter Stellung näher an ihn. Das ließe sich durch die sich in Richtung Schwavokal verändernde Konfiguration des Ansatzrohres in unbetonter Position erklären, was zur Abschwächung sämtlicher Vokale führt. Diese Abschwächung wird ihrerseits naturgemäB von assimilatorischen Klangfarbenneutralisierungen begleitet. 
Die ermittelten Intensitätswerte lassen deutlich ein Bild beobachten, was wir auch bei der Konfrontation der Formantenstruktur der betonten und unbetonten Vokale des Deutschen und in einem noch höheren Maße des Bulgarischen gewinnen könnten: die Rangordnung der unbetonten Vokale nach ihrer spezifischen Intensität verrät, wenn nicht ganz eindeutig, so doch in phonetisch relativ gut dekodierbaren Umrissen, die Reduktionstendenzen des unbetonten Vokalismus der beiden Sprachen.

- Die Differenzen in der spezifischen Intensität eines Vokallautes, durch sein Auftreten in erster, zweiter oder dritter vorbetonter Position bedingt, betragen fur das Deutsche und fur das Bulgarische im Mittel $1 \mathrm{~dB}$, der Intensitätsunterschied zwischen der ersten vorbetonten und der ersten nachbetonten (in unseren Versuchsbeispielen stets die letzte des Wortes) Silbe dagegen ist für beide Sprachen beträchtlich: 4,2 dB für das Bulgarische und $3.5 \mathrm{~dB}$ für das Deutsche. Da unsere Beispiele in einem terminal verlaufenden Rahmen eingefaßt sind, können wir aus den angeführten Intensitätsdifferenzen schließen, daB die unbetonte Silbe eines terminalen Auslautes in beiden Sprachen von etwa gleicher Druckstärke ist.

- Die spezifische Intensität des deutschen Murmelvokals nimmt zwar die unterste Stufe unserer Intensitätsskala ein (Tab.34), jedoch ist die Differenz zwischen dem [a] und beispielsweise einem [y], [u], [i] diesbezüglich gering: $0.2 \mathrm{~dB}([\mathrm{\partial}]-[\mathrm{y}]), 0,3 \mathrm{~dB}([\mathrm{\partial}]-[\mathrm{u}]), 0,5 \mathrm{~dB}([\mathrm{\partial}]-[i])$.

Unsere Versuchsergebnisse zur spezifischen Intensität der unbetonten Vokale sprechen folglich gegen die in der deutschen Phonetik vertretene Auffassung, da $B$ eines der wesentlichen akustischen Merkmale des Murmelvokals seine im Gegensatz zu den übrigen unbetonten Vokalen geringe Intensität sei (Lind ner, 1969, 194).

2.2.5. Spezifische Dauer der deutschen und der bulgarischen Vokallaute

\subsubsection{Die wichtigsten Untersuchungen zur spezifischen Dauer der Vokale}

Die spezifische (objektive) Dauer der Sprachlaute ist vielmals untersucht worden.

Die Ergebnisse einzelner Forscher lassen sich jedoch nicht unmittelbar miteinander vergleichen, weil sie durch unterschiedliche Meßverfahren und Segmentierungsmethodik gewonnen worden sind. Es lassen sich aber doch die Relationen innernalb einer Lautreihe festlegen, die man weiter als vergleichende Grundlage benutzen kann. Auf diese Weise können auch die Ergebnisse früherer Forscher und ihre dadurch gewonnenen Feststellungen über bestimmte Zusammenhänge zwischen den artikulatorischen und akustischen Eigenschaften der Laute und ihrer Dauer Aufschluß geben.

Der Phonetiker E. A. M e ye r $(1903,1904,1909)$ hat im ersten Jahrzehnt unseres Jahrhunderts eingehende Untersuchungen zur Lautdauer am Material 
aus dem Englischen, Deutschen, Ungarischen vorgenommen. Seine Feststellungen sind im großen und ganzen durch spätere Untersuchungen bestätigt worden. Er formuliert sie in der Monographie ..Englische Lautdauer“ (1903, 106, 109) wie folgt:

a) Ein breiter Vokal ist stets kürzer als seine enge Abart;

b) Je höher die Zungenhebung bei einem Vokal ist, um so kürzer ist er;

c) Ein vor Fortis-Konsonanten stehender Vokal ist stets kürzer als derselbe Vokal vor Lenes;

d) Der Vokal vor Verschlußlauten ist kürzer als derselbe vor Engelauten;

e) Die Konsonanten [l], [m], [n], [r] wirken auf den vorangehenden Vokal kürzend. usw.

Panconcelli Calzia stellt 1917 eine gewisse Abhängigkeit der Vokaldauer von der gesamten Wortlänge fest: ..Je mehr Laute dem akzentuierten Vokal folgen, desto kürzer ist er" (Pa nconcelli-Calzia, 1917, 127).

Auf die Einwirkung des Sprechtempos auf die objektive Dauer von Vokalen weist G. Lindner hin (Lindner, 1976, 407-414). E. Zwirner schlägt 1936 in „Phonometrischer Beitrag zur Frage der neuhochdeutschen Quantität“" (S.96-112) die Brücke von der objektiven, physikalischen Dauer der Sprachlaute zu ihrer Realisierungsdauer im sprachlichen Kommunikationsproze $B$. Er stellt durch seine phonometrische Experimente fest, da $B$ sich die sprachlich kurzen und die sprachlich langen Vokale um je einen mittleren Dauerwert gruppieren. Weiter versucht er die Schwellenwerte im Prozeß der Wahrnehmung der Dauerunterschiede analytisch nachzuweisen. Die Untersuchung ergab. daß ein Dauerunterschied von 2-3 Einheiten bei den kurzen Vokalen und von 5-6 bei den langen Vokalen dem deutschen Sprecher und Hörer unmerklich bleibt.

In .Objektive und subjektive Lautdauer deutscher Vokale“ $(1940,79)$ versucht E. Fischer-Jorgensen die These b) der Meyerschen Feststellungen zu bestätigen: ..Vielleicht kann man auch sagen, daß eine größere und kompliziertere Bewegung (Öffnung des Mundes, Rundung der Lippen) längere Zeit in Anspruch nehme" (1940, 79).

Ähnlich auch bei O. v. Esse n $(1962,119)$ : ,.Lautbildungen mit großem Atmungsaufwand beanspruchen im allgemeinen größere Dauer als solche mit geringerem."

In seinem Artikel ..Die spezifische Lautdauer deutscher Sonanten“ berichtet E. Maack über seine Untersuchungen zur Lautdauer deutscher Vokale, die er an Schallplattentexten verschiedener Sprecher vergleichsweise und unter Berücksichtigung des ..Gewichtes" der einzelnen Sonanten durchgeführt hat. Diese seine Untersuchungen bestätigen ..im großen ganzen den schon von E. Meyer aufgestellten Satz, daß die Lautdauer umgekehrt proportional ist zur Höhe der Zungenstellung" ( $\mathrm{M}$ a a ck, 1949, 232).

In einer weiteren Arbeit analysiert Maack die Beeinflussung der Lautdauer der Vokale durch die konsonantische Lautnachbarschaft, wobei er sich lediglich auf die Lautdauer der betonten Vokale konzentriert. Dabei faßt er die Ergebnisse, die er für die betonten Sonanten gewonnen hat, in folgenden Regeln zusammen: ..Ein Sonant ist im allgemeinen um so länger:

- je längere Zeit die Bildung des folgenden Konsonanten erfordert: 
- je größer die Expirationsstärke des vorhergehenden Konsonanten ist und je mehr sich diese auf die Stärke und Dauer des Sonanten auswirken kann:

- je weiter seine Artikulationsstelle von der des nachfolgenden Konsonanten entfernt ist;

- je näher sie an der des vorangehenden Konsonanten liegt" ( $\mathrm{M}$ a a ck, 1953, 128).

Lehiste und Peterson (1960) untersuchen ebenfalls den Einfluß der Lautnachbarschaft auf die Vokaldauer und stellen fest, daß die Dauer der Vokale kaum durch vorangehende Konsonanten beeinflußt wird, daß aber der Vokallaut im allgemeinen kürzer ist, wenn ein stimmloser Konsonant darauf folgt, länger - vor einem stimmhaften Konsonanten: Das Verhältnis ist im Durchschnitt $2: 3 \ldots$ plosives are preceded by the shortest syllable nuclei; nasals had approximately the same influence as voiced plosives. Syllable nuclei were longest before voiced fricatives" (1. Lehiste und Peterson, 1960. 702).

Die Lautdauer bulgarischer Sprachlaute ist zum ersten Mal von Stoik ov untersucht worden (С той ков, 1942).

Stoikov nennt in seiner Arbeit „Български книжовен изговор“ vier Arten von Vokaldauer:

- eine absolute Vokaldauer, die allein von den artikulatorischen Merkmalen eines gegebenen Lautes abhängt.

- eine positionshedingte. welche durch die phonetischen Bedingungen, unter denen ein Laut vorkommt, bestimmt wird,

- eine bedeutungsdifferenzierende (phonologische, funktionelle),

- eine expressive, wodurch Gemütsbewegungen zum Ausdruck gebracht werden.

Die Rangordnung der bulgarischen betonten Vokale nach ihrer objektiven Dauer sieht bei Stoikov. ZahlenmäBig ausgedrückt (in Millisekunden). folgendermaßen aus: 1 - 9.5, 1 - 9.6, a - 103, $\varepsilon-11,5$, a - 11,8, a - 12.5.

Die ..positionsbedingte" Lautdauer zeigt nach Stoikov Abhängigkeit von dem Akzent: die betonten Vokale sind im Mittel länger als die unbetonten; ebenso von der Art der Silbe: die Vokale in geschlossener Silbe sind kürzer als die in offener.

Die Beeinflussung der Lautdauer der bulgarischen Vokale durch die Position der Silhe im Wort und durch die konsonantische Lautnachbarschaft ist von Stoikov nicht analysiert worden.

Eine eingehende Untersuchung der objektiven Dauer der bulgarischen Vokale unter Berücksichtigung aller im Kontext wirkenden Faktoren nehmen Tilkov, Mischeva und Peeva (Тилков. Мишева, Пеева, 1977, 43-56) vor. Sie formulieren ihren Analysen entsprechend folgende Fes:stellungen:

- Die betonten Vokale sind im Mittel von längerer Dauer als de unbetonten:

- Sowohl bei den betonten als auch bei den unbetonten Vokalen macht sich die Abhängigkeitstendenz von der Artikulationscharakteristik der Vokace bemerkbar: die Dauer wächst progressiv von den engen zu den bretten Vokalen an: 
- In offener Silbe dauern sowohl die betonten als auch die unbetonten Vokale länger als in geschlossener;

- Die Beeinflussung der Lautdauer durch die konsonantische Umgebung hängt vor allen Dingen von der Bildungsart der den Vokal umgebenden Konsonanten ab und nicht so sehr von ihrer Artikulationsstelle.

\subsubsection{Vergleichende Charakteristik der spezifischen Lautdauer der deutschen und der bulgarischen Vokale}

Für unsere vergleichenden Untersuchungen der Lautdauer der deutschen und der bulgarischen Vokale haben wir dasselbe Versuchsmaterial benutzt. an dem auch ihre spezifische Intensität und die Grundtonbewegung untersucht wurden. Es sei hier noch einmal darauf hingewiesen, daß wir bei der Aufstellung der Versuchsmethodik ganz besonders auf die für die statistische Auswertung ausreichende Anzahl der Beispiele eines jeden uns interessierenden Faktors bedacht waren. Die Rahmenaussprüche mit terminaler Melodiegestaltung, die wir durch den Intonographen analysiert haben. enthalten zwei-. drei- und mehrsilbige Wörter aus dem natürlichen Wortschatz beider Sprachen. Aus den Versuchsergebnissen, die wir nach den entsprechenden Faktoren zusammengefaßt haben (Siehe Tab. 38-49). kann man folgende Schlüsse zichen:

A. Lautdauer der hetonten Vokale des Deutschen und des Bulgarischen

- Von einer klar ausgeprägten Rangordnung in bezug auf die Lautdauer der betonten Vokallaute beider Sprachen kann kaum die Rede sein. da die spezifische Dauer unter den einzelnen Vokalen recht ausgeglichen ist. Trotzdem markiert ein geringer Dauerunterschied die Grenze zwischen den ..breiten" und den ..engen" Vokallauten des Deutschen und des Bulgarischen, wobei sämtliche ...breite" Vokale etwas länger andauern als die ..engen" (Siehe Tab. 38, 40).

- Das Dauerverhälınis zwischen der langen geschlossenen und der kurzen offenen Vokalreihe des Deutschen beträgt im Mittel $1: 0.6$.

- Die objektive Lautdauer der betonten Vokale des Deutschen und des Bulgarischen wird kaum durch die Art der Bildung des vorangehenden Konsonanten beeinflußt (Siehe Tab. 44, 45).

- Die Bildungsweise des nachfolgenden Konsonanten wirkt sich dagegen etwas merklicher auf die Dauer des linksstehenden Vokals aus: die Untersuchungsergebnisse zeigen. daß die betonten Vokallaute beider Sprachen am kürzesten vor Explosiven ausfallen und daß nachfolgende Frikative, durch die Bildung der Enge, die Dauer des vorangehenden Vokals etwas verlängern (Siehe Tab. 44, 45).

- Für das Bulgarische, wo betonte Vokale auch in Auslautposition distribuiert sind. haben wir die spezifische Vokaldauer getrennt für den An-. In- und Auslaut in der entsprechenden konsonantischen Umgebung ermittelt. Die Ergebnisse sprechen von keiner merklichen Beeinflussung der Lautdauer der betonten Vokale durch die konsonantische Umgebung im An- und Inlaut. Der Unterschied in der Vokaldauer zwischen diesen Positionen und der Auslautposition fallt aber auf: der auslautende betonte Vokal wird nach 
unseren Angaben um 0,03 sek. länger realisiert. Dabei ist es nicht von Belang. auf was für einen Konsonanten eine geschlossene Auslautsilbe ausgeht.

Die längere Lautdauer der betonten bulgarischen Vokale im Wortauslaut ließe sich durch die abschließende Melodie des Auslautes erklären, was eine Abschwächung der Intensität der betonten Endsilbe und höchstwahrscheinlich eine dadurch bedingte Ausdehnung des Vokals mit sich zieht. Der betonte Auslaut untersteht wie bekannt keiner Reduktion, so daß die durch die melodische Gestaltung bedingte Längung des Vokals vollerhalten bleibt.

B. Lautdauer der unhetonten Vokale des Deutschen und des Bulgarischen

Die Lautdauer der unbetonten Vokale des Deutschen und des Bulgarischen wurde für jeden einzelnen Vokal ermittelt, wobei die Art der Silbe (offen. geschlossen), ihre Position im Wort (I., Il. vorbetonte und letzte unbetonte Silbe) und die konsonantische Umgebung berücksichtigt wurden (Siehe Tab. 39, 41).

Über die spezifische Lautdauer der unbetonten Vokale beider Sprachen läßt sich nach den von uns ermittelten Ergebnissen folgendes sagen:

- Die unbetonten Vokale sind in beiden Sprachen von kürzerer Dauer als die betonten.

- Die langen geschlossenen Vokallaute des Deutschen sind im Mittel um das doppelte länger als die unbetonten Vokale $(0,18: 0,009)$, während sich die spezifische Lautdauer der kurzen offenen zu der der unbetonten Vokale wie $0.11: 0,09$ verhält.

- Das Dauerverhältnis „.betont - unbetont“ für die bulgarischen Vokale ist im Mittel $0,11: 0,1$. Die etwas beträchtlichere Differenz zwischen betont und unbetont bei der Vp. M. P. $(0,1: 0,08)$ läßt sich u. E. durch ihre stärkere Neigung zur quantitativen Reduktion erklären.

- Die Art der Silbe bewirkt keine beträchtlichen Unterschiede des Bulgarischen. Es ist allerdings eine leichte Tendenz zur längeren Dauer des Vokals der geschlossenen Silbe im An- und Inlaut für beide Sprachen festzustellen (Siehe Tab. 48, 49).

- Der geschlossene unbetonte Auslaut ist in bezug auf die Lautdauer der bulgarischen unbetonten Vokale und des deutschen [D] stark einer quantitativen Reduktion ausgesetzt. Das ist aus den Angaben der Tab. 46, 47 ersichtlich, wo der quantitative Unterschied zwischen dem geschlossenen Inand Auslaut viel geringer ist als die Differenz zwischen dem offenen unbetonten In- und Auslaut.

- Die spezifische Lautdauer des einzigen vokalischen Vertreters des deutschen Auslautes [a] haben wir für die Positionen [-ən], [-əl] und die übrigen sprachlich möglichen Auslautpositionen getrennt ermittelt, da die Auslautsilben [-ən], [-al] einer starken quantitativen Reduktion unterliegen ( $\mathrm{Me}$ in h old, 1973). Die Ergebnisse für die Lautdaucr des [2] in den Endsilben [-2n], [-al] bestätigen zahlenmäßig die von unseren Versuchspersonen realisierte quantitative Reduktion: Die Lautdauer des [a] beträgt 0,08 sek. für die Auslautsilben [-an]. [-əl] gegenüber 0,13 sek. für seine übrigen Auslautpositionen. Im absoluten Auslaut dauert das deutsche [ə] allerdings länger seine Dauerwerte betragen hier im Mittel 0,18 sek., eine Lautdauer, die den bulgarischen unbetonten Vokalen in offener Auslautsilbe sehr nahe kommt. 


\section{VERGLEICHENDE CHARAKTERISTIK}

\section{DER DEUTSCHEN UND DER BULGARISCHEN VOKALPHONEME}

Bei unserem Versuch, die deutschen und die bulgarischen Vokalphoneme vergleichend zu charakterisieren, die Ähnlichkeiten und Unterschiede in den Vokalsystemen der beiden Sprachen hervorzuheben und auf diese Basis manche Interferenzerscheinungen aus dem Bereich des Vokalismus aufzuklären, richten wir uns nach den theoretischen Auffassungen zweier zeitgenössischer Forschungsgruppen: der Gruppe Jak obson, Fant und $\mathrm{H}$ alle (1952) und der Leningrader Forschungsgruppe Tschistowits $c h$, Kojewnikow, I wanow (Ч истович, 1961; Kozhevnikov, Chistovich, 1965).

Die Leningrader Wissenschaftler stellen die These auf, da $B$ man das Phonem als eine streng bestimmbare Einheit von distinktiven artikulatorischen und akustischen Merkmalen definieren sollte, und schlagen in diesem Zusammenhang entsprechende Verfahren zur gleichzeitigen Fixierung der akustischen und physiologischen Parameter der Rede vor.

Die Forschungsgruppe Jakobson. Fant. Halle vereinigt den akustischen, physiologischen und perzeptiven Gesichtspunkt bei der Interpretation der funktionellen Seite der Sprachlaute und formuliert auf dieser Grundlage die bekannten 12 distinktiven Merkmale.

Die Auffassung von der natürlichen Korrelation zwischen allen Realisierungsebenen der Lautsprache bildet die theoretische Grundlage der vorliegenden Arbeit. Die akustisch-physiologischen distinktiven Merkmale behaupten sich ..praktisch" bei der phonologischen Interpretation der Sprachlaute.

Für den konfrontativen Vergleich der Vokalphoneme des Deutschen und des Bulgarischen stellen wir die Merkmalmatrizen aus folgenden distinktiven Merkmalen zusammen :

1. gespannt - ungespannt (artikulatorische Charakteristik: geschlossen - offen)

2. kompakt - diffus (artikulatorische Charakteristik: breit - eng)

3. hoch - niedrig (artikulatorsiche Charakteristik: vorn - hinten)

4. hemol - nicht bemol (artikulatorische Charakteristik : labial - illabial).

Die Analyse der deutschen Vokalphoneme nach den oben angeführten distinktiven Merkmalen behauptet das Vorhandensein von zwei Reihen Vokalphonemen im Lautsystem des Deutschen, die als Merkmalpaare ..gespannt - ungespannt". ..kompakt - diffus" einander gegenübergestellt werden konnen:

\begin{tabular}{|c|c|c|c|c|c|c|c|c|}
\hline gespannt & $a:$ & $0:$ & $\mathbf{u}:$ & $\mathrm{i}:$ & e: & $\varepsilon:$ & $\boldsymbol{\theta}:$ & $y:$ \\
\hline ungespannt & $a$ & 0 & 0 & 1 & $\varepsilon$ & & $\overline{\mathbf{e}}$ & $y$ \\
\hline kompakt & $\mathbf{a}$ & 0 & 0 & 1 & $\varepsilon$ & & $\boldsymbol{\propto}$ & $Y$ \\
\hline
\end{tabular}


Eine jede Vokalphonemreihe kann weiter als ein selbständiges System dargestellt werden, indem die Vokalphoneme als Merkmalpaare „kompakt diffus". .,hoch-niedrig" in eine distinktive Opposition zueinander treten*:

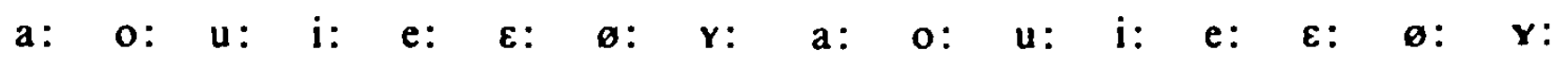
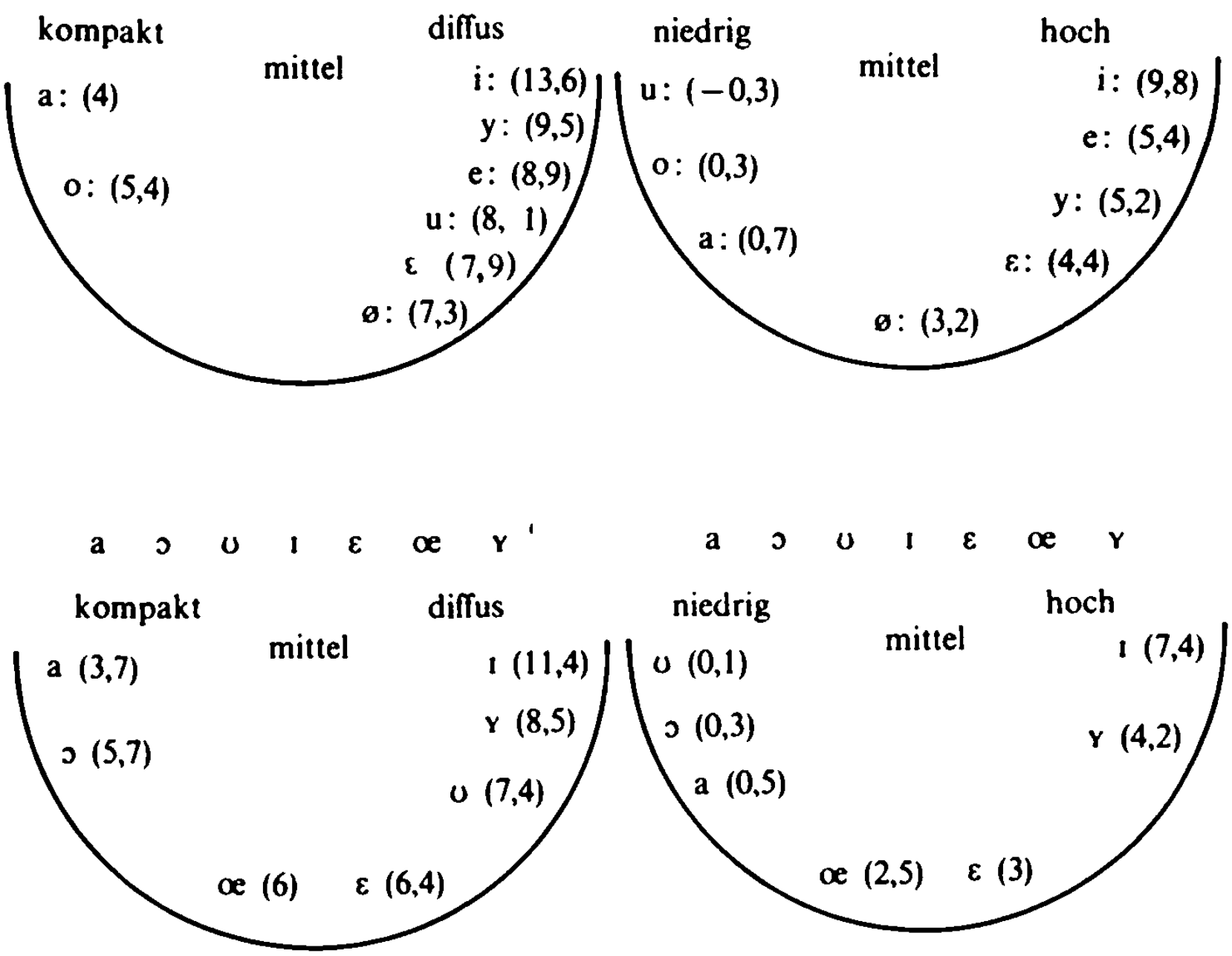

- Die Vokalphoneme sind nach den vom Verfasser gewonnenen spektralen RelationskoeffiIenten eingeordnet (Simeonova, 1976). Als Grenze bei der Bestimmung der Merkmalpaare ..kompak1-diffus" nehmen wir $R_{1}>7$ und $R_{3}<6$ an. was heiben soll, daB wir alle Vokalphoneme. die eine gröbere Energiestreuung als $R_{3}=7$ zwischen den formantenbereichen $F_{\text {, }}$ und $F_{3}$ zeigen. fur .diffus" halten. alle diejenigen dagegen. deren Streuungsrelationskoefizient kleiner als $R_{3}=6$ ausfallt - für ..kompakl“" hallen. Vokalphoneme mit einem Relationskoefízienten $R_{3} \approx 6$ halten wir fur ..merkmalarm" oder ..mittel" $\left(R_{3}=7\right.$ entspricht einem Frequen 2 unterschied $\angle$ wischen den Formantenzentren des $F_{\text {, }}$ und $F_{3}$ von ca. $2300 \mathrm{H}_{2}$. während der ..ideale“ Abstand $z$ wischen dem ersten und dem dritten formanten bei neutraler Lage des Ansat $2 r o h r e s=2000 \mathrm{H}_{2}$ beträgt).

Bei der Bestimmung der Merkmalpaare ..hoch-niedrig“" kann man sich sowohl nach dem $R_{1}$ Idem Relationskoeftizienten. der zahlenmäBig das Verhältnis $f, i$, ausdrückll) als auch nach dem $R_{2}$ (dem Relationskoefticienten für das Verhältnis $F_{3}: F_{1}$ ). die in einem indirekten Proportionälverhälınis zueinander stehen, richten: je gröber $R_{1}, b<w$. je kleiner $R_{2}$. desto höher die Tonalitäl des jeweiligen Vokals. Der Präzision und Bequemlichkeit wegen arbeiten wir mit der Differen /wischen $R_{1}$ und $R_{2}\left(R_{1}-R_{2}\right)$. Geltende Grenzen für uns dabei sind a) obere Grenze: $R_{1}-R_{2}=4$. d.h. $\left.F_{3}-F_{2}=1000 \mathrm{H}_{2}\left(\approx 1200 \mathrm{H}_{2}\right): F_{3}-F_{2} \approx 1000 \mathrm{H} / \quad(=900) \mathrm{H} /\right)$ und b) untere Grenze: $R_{1}-R_{2}=2.5$. d.h. $F_{2}-F_{1}=1\left(00 H_{2}\right.$ und $F_{3}-F_{2} \approx 1000 H_{2}$; so eine Energieverteilung nähert sich der ..neutralen". 
Die vergleichende Merkmalmatrix für die beiden Phonemreihen der deutschen Vokale ergibt folgende Gruppierung nach den vier distinktiven Merkmalem :

\begin{tabular}{|c|c|c|c|c|c|c|c|c|c|c|c|c|c|c|c|}
\hline \multirow{2}{*}{$\begin{array}{l}\text { Distunkuves } \\
\text { Merkmal }\end{array}$} & \multicolumn{15}{|c|}{ Vokalphoneme } \\
\hline & $\mathbf{a}:$ & a & o: & ? & $\mathbf{u}:$ & 0 & $\boldsymbol{\theta}:$ & $\boldsymbol{\alpha}$ & $y:$ & $r$ & e: & $(\varepsilon:)$ & $\varepsilon$ & i: & 1 \\
\hline gespannt & + & - & + & - & + & - & + & - & + & - & + & + & - & + & - \\
\hline ungespannt & - & + & - & + & - & + & - & + & - & + & - & - & + & - & + \\
\hline kompakt & + & + & + & + & - & - & - & \pm & - & - & - & - & - & - & - \\
\hline diffus & - & - & - & - & + & + & + & \pm & + & + & + & + & + & + & + \\
\hline hoch & - & - & - & - & - & - & \pm & \pm & + & + & + & + & \pm & - & - \\
\hline nicdrig & + & + & + & + & + & + & \pm & \pm & - & - & - & - & \pm & + & + \\
\hline hemol & - & - & + & + & + & + & + & + & + & + & - & - & - & - & - \\
\hline nicht bemol & 0 & 0 & - & - & - & - & - & - & - & - & + & + & + & + & + \\
\hline
\end{tabular}

Mit Ausnahme von /e:/ und / $\varepsilon: /$, die vollkommen gleiche Merkmalcharakteristika aufweisen. treten alle übrigen Vokalpaare in Opposition zueinander in bezug auf ein oder mehrere distinktive Merkmale.

Eine Reihe Minimalpaare in distinktiver Opposition aus dem Gegenwartsdeutschen bekräftigt den Phonemcharakter der durch die vier distinktiven Merkmale beschriebenen Vokalphoneme:

a) lang geschlossen/kurz offen:

Saat Sohn(e) Ruhm Miete Heer Höhle fühlen

satt Sonne Rum Mitte Herr Hölle füllen

b) lang geschlossen/lang geschlossen: iagen/logen/liegen/lügen/legen/lugen/lögen

c) kurz offen/kurz offen:

Racke'Rocke/Ricke/rücke/Recke/Röcke.

Die distinktive Opposition /e:/ $/ \varepsilon /$ (Beeren-Bären) hat nach unseren Spektraluntersuchungen, auditiven Beobachtungen und funktionellen Überlegungen keinen relevanten Charakter. Wie aus der Merkmalmatrix ersichtlich, haben beide Vokale vollkommen gleiche Charakteristika. Ihre absoluten Frequenzwerte liegen ebenfalls sehr nahe (Си меонова, 1976). Manche Verfasser, die ihre theoretisch-phonetische und pädagogische Tätigkeit der korrekten deutschen Hochlautung gewidmet haben (Wän gle r, 1964. 32: $\mathrm{M}$ a rıe $\mathrm{ns}, 1965,44)$, weisen ebenfalls auf die auditiv auffallende Verwandtschaft zwischen $e: /$ und $/ \varepsilon$ :/ hin. Wir möchten hier die Auffassung vertreten, daß die Opposition $/ \mathrm{e}: /-/ \varepsilon:$ funktionell erst durch den Sinnzusammenhang eines weiteren Kontextes gelöst werden kann. Deswegen wollen wir das $\varepsilon$ : als eine fakultative Variante des Vokalphonems /e:/ betrachten (Vgl. auch Trubetzk oy, 1939). 
Nach Trubetzk oy $(1971,131)$ läßt sich der bulgarische Vokalismus (6 Vokalphoneme) durch ein dreiklassiges dreieckiges phonologisches Schema darstellen, dessen mittlere Tonklasse /a/ einen neutralen Klangcharakter und einen höheren Öffnungsgrad aufweist. Für die Beschreibung der Vokalphoneme des Bulgarischen genügen drei der angeführten distinktiven Merkmale und zwar: kompakt/diffus, hoch/niedrig, bemol/ nicht bemol.

Eine konfrontativ-vergleichende Matrixtabelle der Vokalphoneme des Deutschen und des Bulgarischen, aufgestellt nach den vier distinktiven Merkmalen, würde die folgt aussehen: (Das Zeichen 0, das Fehlen des entsprechenden Merkmals bedeutet, haben wir für die A-Laute eingeführt, die ohne Lippenbeteiligung gebildet werden).

\begin{tabular}{|c|c|c|c|c|c|c|c|c|c|c|c|c|c|c|c|c|c|c|c|c|c|}
\hline \multirow{3}{*}{$\begin{array}{l}\text { Distinktives } \\
\text { Merkmal }\end{array}$} & \multicolumn{21}{|c|}{ Vokalphoneme } \\
\hline & \multicolumn{15}{|c|}{ Deutsch } & \multicolumn{6}{|c|}{ Buigarisch } \\
\hline & o: & $a$ & $0:$ &. & $\mathbf{u}:$ & 0 & a: & $x$ & $y:$ & $r$ & e: & (E:) & $\varepsilon$ & i: & 1 & a & s & i & 8 & 1 & 0 \\
\hline gespannt & + & - & + & - & $t$ & - & $t$ & - & + & - & + & + & - & + & - & - & - & - & - & - & - \\
\hline ungespannt & - & + & - & $t$ & - & + & - & + & - & + & - & - & + & - & + & - & - & - & - & - & - \\
\hline kompakt & + & + & + & $t$ & - & - & - & \pm & - & - & - & - & - & - & - & + & + & - & + & - & - \\
\hline diffus & - & - & - & - & $t$ & + & + & \pm & + & + & + & + & + & + & + & - & - & + & - & + & + \\
\hline hoch & - & - & - & - & - & - & \pm & \pm & + & + & + & + & \pm & + & + & - & - & - & $t$ & + & \pm \\
\hline nicdrig & + & + & + & + & + & + & $\overline{ \pm}$ & \pm & - & - & - & - & \pm & - & - & + & + & + & - & - & \pm \\
\hline bxemol & - & - & + & + & + & + & + & + & $t$ & $t$ & - & - & - & - & - & - & + & + & - & - & - \\
\hline nicht bemol & 0 & 0 & - & - & - & - & - & - & - & - & + & + & + & + & + & 0 & - & - & + & + & + \\
\hline
\end{tabular}

Aus der vergleichenden Merkmalmatrix kann man folgende Schlüsse ableiten :

- Für die bulgarischen Vokalphoneme ist das Merkmal ..gespannt ungespannt" irrelevant.

- Die bulgarische Sprache hat zwei spektral kompakte, artikulatorisch breite Vokalphoneme gegenüber vier der deutschen Sprache; zwei Vokalphoneme $(/ \alpha /$ und $/ \varepsilon /)$ des Deutschen und eins des Bulgarischen $(/ \varepsilon /)$ kann man hierzu als .merkmalarm“ oder „mittel” bezeichnen.

- Eine merkwürdige Erscheinung des bulgarischen betonten Vokalismus ist das diffuse (enge) Vokalphonem /a/, das die deutsche Sprache nicht kennt. Daraus ergeben sich Schwierigkeiten für Bulgarisch lernende Deutsche; dadurch lassen sich auch die Inkonsequenzen in der deutschen Transkription bulgarischer Namen: Bulgarien. Tirnovo, Kardshaly u. a. erklären. Unsere spektralen und auditiv-statistischen Untersuchungen haben gezeigt, da $B$ das bulgarische $:$; seinem Klangcharakter nach, den A-Lauten am nächsten steht. Man müßte es folglich in der deutschen Transkription bulgarischer Namen folgerichtig durch /a/ wiedergeben.

- Im Deutschen und im Bulgarischen halten sich die Merkmalpaare ..hoch-niedrig" (artikulatorisch ..vorn-hinten") relativ die Waage. Das 
Verhältnis ist $2: 3+1$ merkmalarmen Vokalphonem für das Bulgarische. $6: 6+3$ merkmalarmen Vokalphonemen für das Deutsche.

- „bemol - nicht bemol“" (artikulatorisch .labial - illabial“): Hierzu weist das deutsche Vokalsystem eine spezifische Besonderheit auf, die für das Bulgarische nicht zutrifft: im deutschen Vokalismus ist die Reihe der labialisierten vorderen Vokale (4 Vokalphoneme) vertreten; im bulgarischen Vokalismus fehlen sie. Das Verhältnis zwischen den Merkmalpaaren .labial - illabial“" im Deutschen ist $8: 4+2$ Vokalen ohne Lipenbeteiligung. im Bulgarischen ist es $2: 3$ +einem Vokal ohne Lippenbeteiligung.

\subsection{VERGLEICHENDE PHONOLOGISCHE INTERPRETATION DES UNBETONTEN VOKALISMUS DES DEUTSCHEN UND DES BULGARISCHEN}

\section{A. Deutsche unbetonte Vokale}

Vergleicht man die für die unbetonten Vokallaute des Deutschen gewonnenen Spektralmerkmale (gemeint sind in der nachfolgenden Konfrontation ihre Relationskoeffizienten - Vgl. S. 83, 84) mit denen der betonten Vokalphoneme in bezug auf die distinktiven Merkmale .gespannt ungespannt“. "kompakt - diffus", ,hoch - niedrig“, so bekommt man folgendes Bild:

- Im Hinblick auf das Merkmal ..gespannt - ungespannt“” lassen sich die unbetonten Vokallaute des Deutschen in die Reihe der ungespannten Vokalphoneme einordnen - schon allein aus dem Umstand, daß sie mit ihnen etwa die gleichen Temporalcharakteristika aufweisen.

- Das distinktive Merkmal ..kompakt - diffus“ orientiert sie wie folgt:

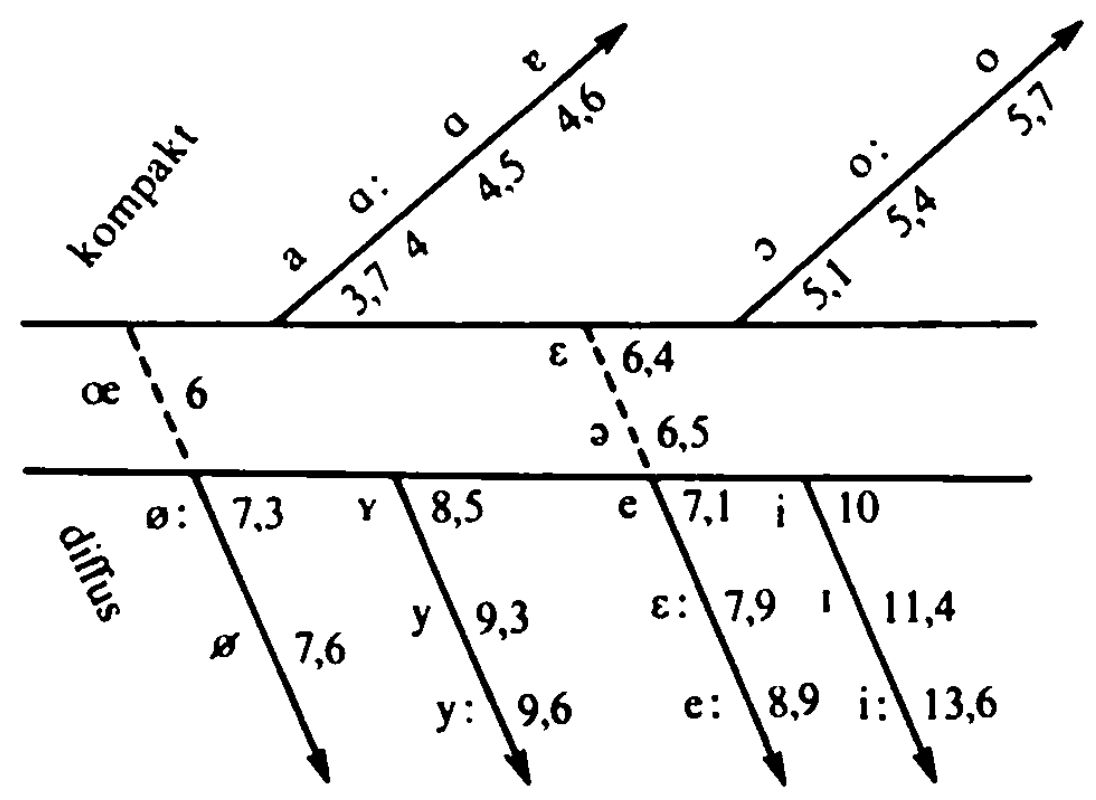

so aus: 


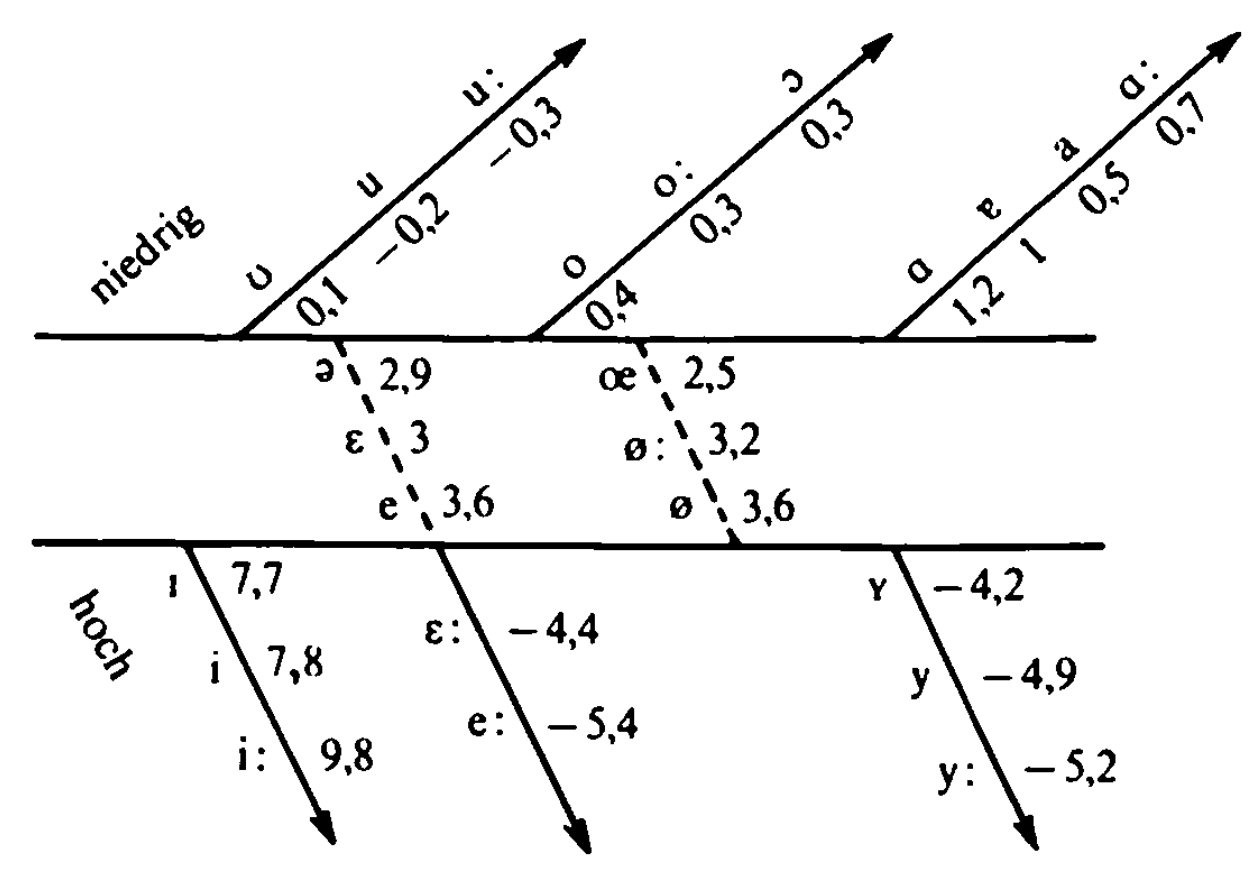

Die vergleichende Matrixtabelle nach den vier distinktiven Merkmalen trgibt folgendes Bild:

\begin{tabular}{|c|c|c|c|c|c|c|c|c|c|c|c|c|c|c|c|c|c|c|c|c|c|c|c|c|}
\hline Distinktives & \multicolumn{24}{|c|}{ Vokaltaute } \\
\hline & $\mathbf{0}:$ & a & o & p & o: & o & 0 & $\mathbf{u}:$ & 0 & u & o: & $\boldsymbol{x}$ & () & $y$. & $\mathbf{Y}$ & y & c: & $(\varepsilon:)$ & $\varepsilon$ & e & o & $i:$ & 1 & 1 \\
\hline gespannt & + & -1 & - & - & + & - & - & + & - & - & + & - & - & + & - & - & + & + & - & - & - & + & - & - \\
\hline ungespannt & - & + & + & + & - & + & + & - & + & + & - & + & + & - & + & + & - & - & + & + & + & - & + & + \\
\hline kompakt & + & + & + & + & + & + & + & - & - & - & - & \pm & - & - & - & - & - & - & \pm & - & \pm & - & - & - \\
\hline diffus & - & - & - & - & - & - & - & + & + & + & + & \pm & + & + & + & + & + & + & \pm & + & \pm & + & + & + \\
\hline hoch & - & - & - & - & - & - & - & - & - & 一 & \pm & \pm & \pm & + & + & + & + & + & \pm & \pm & \pm & + & + & + \\
\hline niedrig & + & + & + & + & + & + & + & + & + & + & \pm & \pm & \pm & - & - & - & - & - & \pm & \pm & \pm & - & - & - \\
\hline bemol & - & -1 & - & - & + & + & + & + & + & + & + & + & + & + & + & + & - & - & - & - & - & - & - & - \\
\hline nicht bemol & 0 & 0 & 0 & 0 & - & - & - & - & - & - & - & - & 一 & - & - & - & + & + & + & + & + & + & + & + \\
\hline
\end{tabular}

Aus der obigen Matrixtabelle läßt sich folgendes zusammenfassen:

a) Die unbetonten Vokale des Deutschen treten in distinktive Opposition zu den langen geschlossenen Vokalphonemen in bezug auf das Merkmal .gespannt - ungespannt", d. h., sie unterscheiden sich von ihnen quantitativ.

b) Vergleicht man die Charakteristika der unbetonten Vokale mit denen der kurzen offenen Vokalphoneme des Deutschen, so stellt man volle Gleichheit in bezug auf alle vier distinktiven Merkmale fest. Nach dem dichotomischen Prinzip würde diese Tatsache genügen, um die unbetonten Vokallaute des Deutschen als Phonemvarianten zu bestimmen. Funktionell gesehen kann diese These durch die äußerst niedrige Gebrauchsfrequenz der unbetonten Vokale (mit Ausnahme von [o]) im Deutschen, die natürliche 
distinktive Oppositionen auf semantischer oder gar morphologischer Grundlage ausschlie $B t^{*}$, bestätigt werden.

c) Die phonetischen Charakteristika der deutschen unbetonten Vokale räumen ihnen eine Mittelstellung zwischen der Reihe der gespannten und der der ungespannten Vokalphoneme ein.

Eine besondere Erscheinung im unbetonten Vokalismus des Deutschen ist der Murmelvokal [a]. Da dieser Vokal durch verschiedene Variantenschattierungen in den meisten Weltsprachen vertreten ist, ist auch das Interesse an dessen phonetischen Merkmalen und phonologischem Status berechtigt.

Die Interpretationen des phonologischen Status des [0] gehen in zwei Richtungen vor sich: eine, die die These von dem selbständigen Phonemcharakter des Vokals aufstellt, und eine zweite, die den Murmelvokal als Allophon eines bestimmten Phonems interpretiert.

Angefangen bei Trubetzk oy (1939), der den ..unbestimmten“ Vokal als ein selbständiges ...außerhalb der Eigentonklassen stehendes Vokalphonem“ behandelt (1939. 105), erstreckt sich diese Interpretation des [a] bis in den heutigen Tag hinein in den Überlegungen Ungeheuers $(1969,32)$ und M. Philipps $(1974,47)$.

Die Beweggründe, die Ungeheuer zugunsten des selbständigen Phonemcharakters des Murmelvokals heranzieht, können allerdings nicht überzeugen: Ungeheuer hebt richtig hervor, da 3 die deutsche Sprache keine Minimalpaare bereitstellen kann. wo das [a] in eine distinktive Opposition zu [e] oder [ $\varepsilon]$ hätte treten können: die komplementäre Distribution des [a] $z u / e$ und $/ \varepsilon /$ ist auch nicht völlig gewahrt (z. B. [ge'nı :I] gegen [go'näo]; ["i :tem] gegen ["a:təm]). Trotz alledem möchte er das" [0] als selbständiges Phonem ansehen aus folgendem Grund: Wenn man das [a] beispielsweise als Allophon zum Vokalphonem ' $\varepsilon$. einordnet. ..Dann müßte jedenfalls genau festgestellt werden, nach welchen kombinatorischen Regeln dieses Phonem in unbetonten Silben einmal als $\varepsilon$, das andere $M a l$ als [0] ausgesprochen wird. Da diese Regeln bis jetzt nur teilweise bekannt sind und außerdem das [0] im Vokalismus der unbetonten Silbe ein so hervorstechendes Merkmal ist, sei es als selbständiges Phonem angenommen" (Ungeheuer. 1969, 32).

Die ersten Einwände gegen die Auffassung Trubetzkoys in diesem Zusammenhang begegnen uns in Gerhardts Werk "Phonometrie und Phonologie" (1941). Gerhardt kehrt später noch einmal zu diesem Problem zurück $(1963,92)$ und schlägt vor, den Vokal der Indifferenzlage als ..kombinatorische Variante vom Sektor [e]" zu klassifizieren.

Diese ..allophone" Richtung in der phonologischen Betrachtung des [a]-Vokals vertreten ebenfalls $S$ inder und Strojewa ( 3 индер. C т poeBd, 1957, 36: 1965. 145). Hutterer (1965, 47). Wurzel (1970, 149). Tilk ov (1972).

Durch spektrale und auditiv-statistische Untersuchungen haben wir festgestellt, daß der Vokal der Indifferenzlage keinen einheitlichen spektralen

- (j. Lngeheuer versucht hierzu gewisse Oppositionen aufzustellen, aber lediglich im Rahmen des fremden Wortguts im Deutschen. Minımalpaare für den Vokal [o] hat er jedoch nicht finden können. 
Charakter aufweist, da er stets positionsbedingt im sprachlichen Kontext erscheint (Sim e o n o va, 1978). Trotz seines recht variablen Charakters kann er nur den Variantensektoren eines [a], [e] und [ø] zugeordnet werden. Spektral und auditiv haben wir zwei positionsbedingte Varianten des [ə] ermitteln können: den Reduktionsvokal [e] für die Auslautverbindung [-ər] und die Variante [D] mit einem mehr oder weniger ausgeprägten Charakter eines Eoder Ö-Lautes.

Aus dem kontrastiv-phonologischen Vergleich der zwei Varianten des Murmelvokals mit den beiden Vokalphonemreihen und den unbetonten Vokallauten des Deutschen ist folgendes ersichtlich:

- [a] bildet eine binäre Opposition zu / $\mathrm{a}: /$ in bezug auf das distinktive Merkmal .gespannt - ungespannt"; sonst fallen seine Charakteristika mit denen von /a/ und /a:/ zusammen.

Hätte man den Monophthong [e], den man anstelle der Auslautverbindung [-or| realisiert, für eine $100 \%$ sprachliche Realität halten können, so wären distinktive Oppositionen von Minimalpaaren auf morphologischer Grundlage vollkommen natürlich und hätten, obwohl nur bedingt (da sich eigentliche Minimalpaaroppositionen auf semantischer Basis nicht aufstellen lassen), für den selbständigen Phonemstatus des Monophthongs [e] plädieren können. Solche Oppositionen wären beispielsweise: Bauer - (ich) baue; Banner (im) Banne; Messer - Messe; Leser - Lese; Kater - Kate; höher - Höhe; leiser - leise; Kummer - Kumme; Wunder - Wunde; verlieren - verliehen; mildern - milden; verzieren - verziehen; hören - Höhen u.a.

Der Monophthong [e] ist jedoch trotz der standardisierten Realisierung der auslautenden [-ar] als [e] noch keine hundertprozentige sprachliche Wirklichkeit (M e in h old, 1973). Die Realisierung der [-ər] als [e] gehört noch zu den Entwicklungstendenzen der gegenwärtigen deutschen Hochlautung. Deswegen sehen wir das [p] als eine positionsbedingte Variante der A-Phoneme an.

- Das [ə] der Positionen [bə-], [gə-] des absoluten Auslauts und des Auslauts vor Nasal wird den O-Phonemen nach dem Merkmal „labial illabial" und dem /e:/ nach dem Merkmal .gespannt - ungespannt“ gegenübergestellt. Gleiche Charakteristika weist diese Variante zu / $\varepsilon /$ und $/ e /$ auf. Unsere funktionell-linguistischen Überlegungen würden auch bei dieser Variante zu Oppositionen führen, die jedoch lediglich die Flexion betreffen. Im absoluten Auslaut könnte die [a]-Variante in Oppositionen zu einer Null-Position in der Flexion treten: Bann(e) (Dat. Sing.) - Bann; Wille - will (1. P. Sing.) u. a.

Doch die spektrale und die auditiv wahrnehmbare Verwandtschaft des auslautenden [ə] zu den E-Lauten ist so hervorstechend, daß es kaum nötig wäre, allein auf Grund der oben angeführten Oppositionen den selbständigen phonologischen Status des Murmelvokals zu verteidigen. Wir möchten das deutsche Murmel-[a] in den Positionen [ba-, ga-], absolutem Auslaut und Auslaut vor Nasal als eine stellungsbedingte Variante des Phonems $/ \varepsilon /$ betrachten.

B. Bulgarische unbetonte Vokale

Für die phonologische Beschreibung der Vokallaute des Bulgarischen reichen, wie schon erwähnt, die distinktiven Merkmale „kompakt - diffus“. 
..moch - niedrig“, .,bemol - nicht bemol“ aus. Da wir uns der Auffassung amschließen, daß die Akzentuierung ein prosodisches und kein Lautmerkmal ist (Siehe Тилков, 1966, 107; Николов, 1972, 4, 17, u. a.), sehen wir die bulgarischen unbetonten Vokale als Phonemvarianten an.

Die phonologischen Relationen zwischen betonten und unbetonten Vokallauten haben wir nach den oben angeführten Merkmalen verfolgt, fur die wir über eigene akustische Daten verfügen.

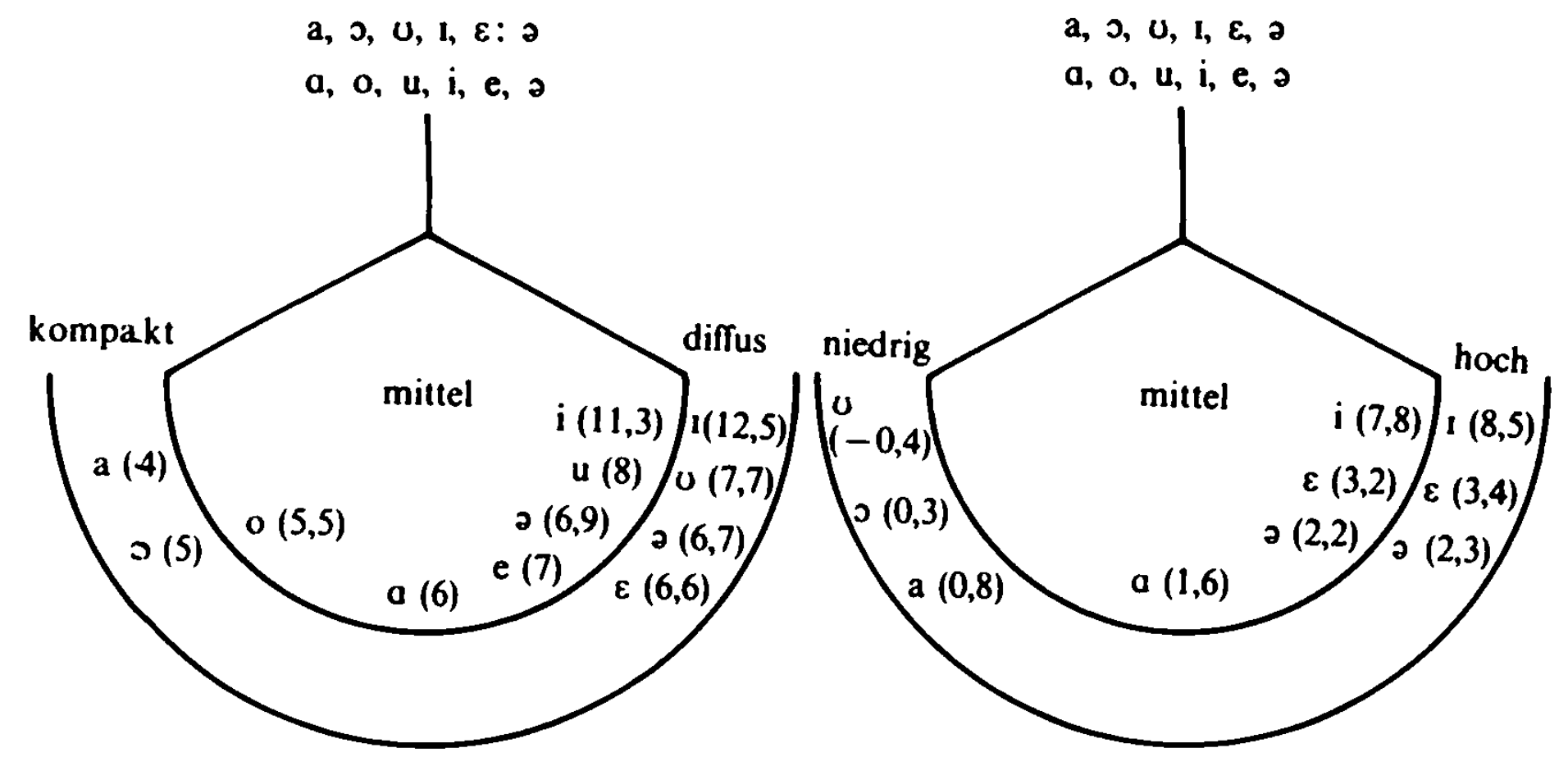

Wie aus dem obigen Vergleich der Relationskoeffizienten für die Merkmale ..kompakt - diffus". ".hoch - niedrig" ersichtlich, weisen die unbetonten Vokallaute des Bulgarischen ein etwas mehr diffuseres Spektrum als die betonten Vokale auf. Ihre Grundfrequenz zeigt ebenfalls etwas niedrigere Werte (Vgl. S. 136-137).

Die Spektraldifferenzen zwischen den beiden Vokalreihen sind jedoch gering und so sprachlich irrelevant. Auffallend sind allein die spektralen Veränderungen des Vokals [a], der in unbetonter Position als „merkmalarm“ in bezug auf die Merkmale "kompakt - diffus“, ,hoch - niedrig“" erscheint. Diese spektrale Bewegung des unbetonten [a] in Richtung [o] ist unserer Auffassung nach ein Beweis dafür, daß die Reduktion des [a] sich auch auf die standardisierte bulgarische Aussprache erstreckt. Hier wird in hohem Maße der Klangunterschied zwischen den unbetonten [a] und [ə] aufgehoben. Die Neutralisierung der anderen zwei Korrelationspaare $\varepsilon \leftrightarrow 1, \partial \leftrightarrow 0$ in unbetonter Position ist in den Ergebnissen unserer Spektraluntersuchungen nicht belegt. 


\subsection{DISTRIBUTION DER VOKALLAUTE IN DER DEUTSCHEN UND IN DER BULGARISCHEN SPRACHE}

\section{A. Betonte Vokalphoneme}

Die zwei Reihen Vokalphoneme im Deutschen (15 Vokalphoneme nach der herkömmlichen Klassifikation) haben eine komplementäre oder halb komplementäre Verteilung im Wort: ein langer geschlossener Vokal erscheint in offener Silbe, ein kurzer offener Vokal ist ein Zeichen für eine geschlossene Silbe. Lange geschlossene Vokale kommen an-, in- und auslautend vor, wobei ihre Auslautposition allein für einsilbige Wörter gilt. Kurze offene Vokale kommen niemals im Auslaut vor.

Ein langer geschlossener Vokal kann im Wortanlaut selbständig eine Silbe bilden (Typ V), ein kurzer offener Vokal ist als Silbenkern stets von einem oder mehreren Konsonantensatelliten begleitet.

Die häufigsten Silbentypen für die langen geschlossenen deutschen Vokallaute sind: $V$ : Abend, Igel. Ofen, Ufer, Öde, üben; $V K, K V K$ (lediglich für Einsilber): Ahn, ihn, er, Uhr, Ohr, Öhr; Bahn, Tag, Dor, Zug, der, für, vor, her-; $K V$ : so, zu, malen, lieben, Boden, lösen, lesen, lügen.

Für die kurzen offenen: $V K$ : Affe, essen, irren, üppig; $V K K:$ Amt, Ast, Ort, ist, elf, örtlich, östlich. Ülk; $K V K K$ : hart, bersten, hüpfen, Rost, bürsten. rösten; $K V K$ : Salbe, gerben, finden, murmeln, köstlich, wülstig.

Das bulgarische Vokalsystem kommt mit viel weniger Vokalphonemen aus als das deutsche. Den 6 bulgarischen Vokalphonemen stehen 15 deutsche gegenüber.

Das vokalisch-konsonantische Phonemverhältnis ist in der deutschen Gegenwartssprache gut ausgeglichen: den 15 Vokalphonemen und 3 Diphthongen entsprechen 19 Konsonantenphoneme. Im Bulgarischen ist dieses Verhältnis 6:39 zugunsten der Konsonantenphoneme. Trotz dieser auffallenden Differenz zeichnet sich die bulgarische Sprache ebenfalls durch ein gut ausgewogenes vokalisch-konsonantisches Gleichgewicht aus (Тилков, Бояджиев, 1977, 45). Das läßt sich durch die Tatsache erklären. daß die bulgarischen Vokale dank des freien Wortakzents syntagmatisch fast keine stellungsbedingten Einschränkungen kennen: sie könen sowohl im Anlaut als auch im In- und Auslaut, in offener und geschlossener Silbe von ein- und mehrsilbigen Wörtern auftreten; sie nehmen folglich einen recht breit gefächerten Anteil am phonetischen Bau des bulgarischen Wortes. Die häufigst vorkommenden Silbentypen für die betonten Vokallaute des Bulgarischen sind: $V$ : ехо, ьгъл, азот, удар, огън. ивица ; $V K$ : изба, ыли, ахвам, оглед, евтин, умник; $K V$ : риба, пари, дело, дете. водя, длето, баба, делба, буря, бижу, къпя; $K^{\prime} V$ : изява, беля, кюнец. ревю, кьорав ; $K V K$ : тип, мед, вар, куп, пьт, поп, титла, метил, вечност, петел. въздух, пъдпъдък, кукла, боклук, тиган, шапка, топка, потоп; $K^{\prime \prime} / K^{\prime}$ : бял, кюп, гьо, увяхвам, летят, тютюн, коняр, Гьончо.

B. Unhetonte Vokallaute

Kurze geschlossene Vokale in unbetonter Position sind im eigentlichen deutschen Wortschatz eine Seltenheit (lebendig, Heimat). Kurz und moivienfalls geschlossen werden die deutschen Vokale [e]. [i]. [y] in ..pro- unc 
iknklitischen betonungsunfähigen Morphemen" (T rubetzk oy, 1971) realisisiert. Kurz und geschlossen bildet man ebenfalls die Vokale [e], [i], [o], [u], [y] in ppro- oder enklitisch gebrauchten Einsilbern. Sonst kommen kurze geschlosscsene Vokale in unbetonter Position lediglich in Fremdwörtern vor. Ohne die FFremdwörter würden die kurzen geschlossenen Vokale in unbetonter Silbe bis auuf den Murmelvokal [ə] tatsächlich verschwinden. Das [ə] hat jedoch eine sesehr hohe Gebrauchsfrequenz in der deutschen Gegenwartssprache: $20,68 \%$ alaller Vokallaute (Lindner, 1969). Dieser Umstand, zusammen mit den uunbetonten geschlossenen Vokalen in pro- und enklitisch gebrauchten Morphemen und Einsilbern und den unbetonten geschlossenen Vokalen in FFremdwörtern, erhält im Deutschen das Gleichgewicht „,betont - unbetont“ aluufrecht.

Distributiv entsprechen dem deutschen Schwa-Vokal die unbetonten boulgarischen Vokallaute $[\mathrm{e}],[\mathrm{a}],[\mathrm{p}]^{*}$ :
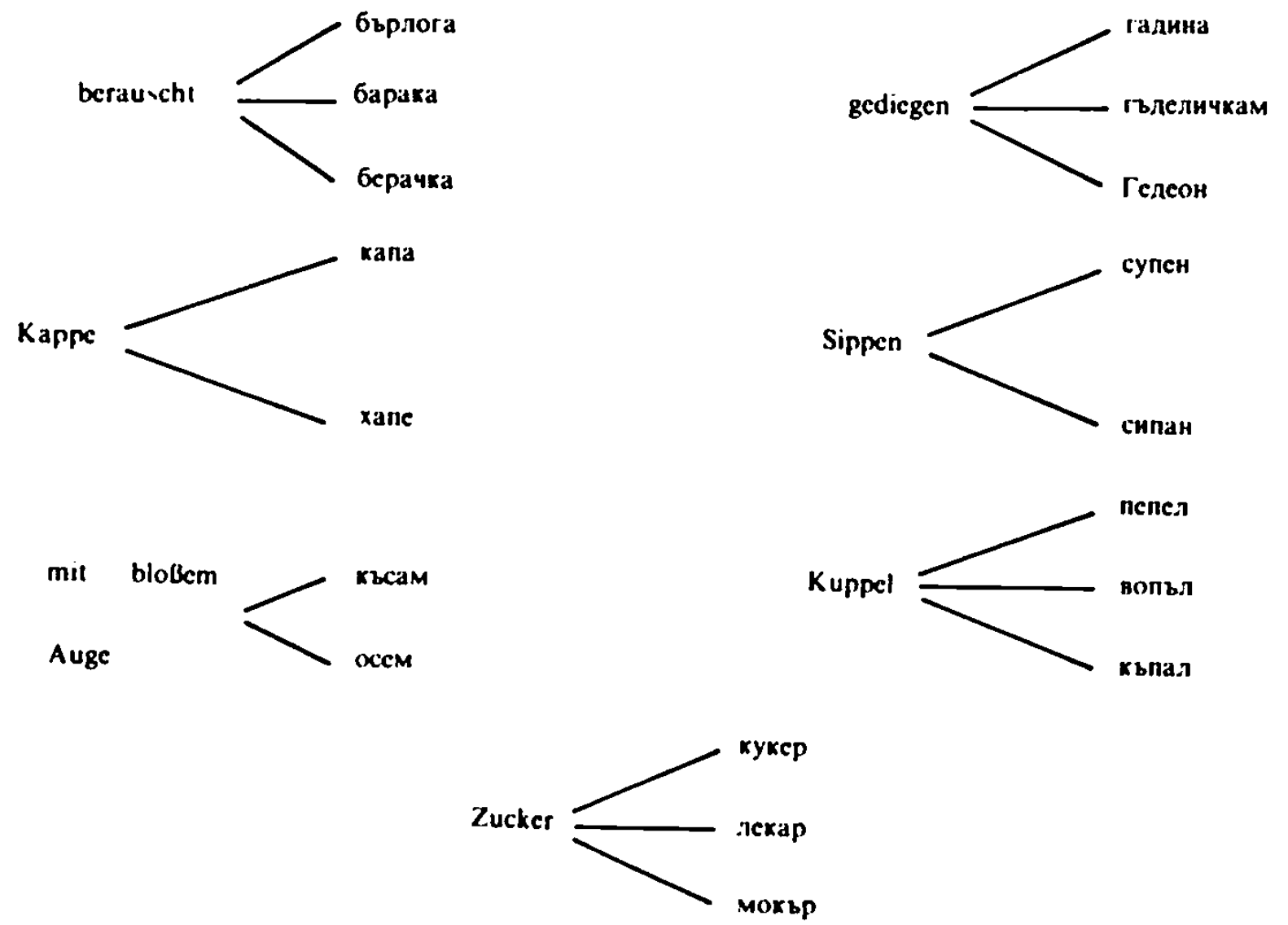

Die bulgarischen unbetonten Vokale, genauso wie die betonten. unnterstehen keiner strengen Gesetzmäßigkeit in bezug auf ihre Verteilung im WJort: sie treten an-, in- und auslautend, in offener und geschlossener Silbe voor. Die Silbentypen, die sie bilden, unterscheiden sich strukturell nicht von deenen der betonten Vokale.

- Näheres über die spektrale und auditiv wahrnehmbare Verwandtschafl des [a] mil einem von diexsen $\mathrm{hm}$ distributiv entsprechenden bulgarischen unbetonten Vokallaulen findet man auf $\mathrm{S} .48 .49$ der.r vorliegenden Arbeit. 


\section{ZUSAMMENFASSENDE SCHLUSSBEMERKUNGEN}

Auf der Suche nach effektiven Methoden zur Überwindung der schwierigen Schwellen in der Phonetik des Fremdsprachenunterrichts haben wir uns zum Ziel gesetzt, die für einen Deutsch lernenden Bulgaren typischen Ausspracheschwierigkeiten aufzudecken, zu systematisieren und analytisch $\mathrm{zu}$ untersuchen.

Dieses Vorhaben kann und muß allein auf konfrontativem Wege zwischen den beiden Sprachen verwirklicht werden.

Dabei gehen wir von der These aus, daß Basiskomponenten aller Ebenen der Lautsprache ihr einheitliches System durchdringen, daß sie ihren Niederschlag in jeder einzelnen lautlichen Erscheinung finden.

Assoziationen zu lautlichen Basiskomponenten löst der in der Phonetik der Vergangenheit und der Gegenwart gebräuchliche Begriff .,Artikulationsbasis" einer gegebenen Sprache aus.

Unseres Erachtens jedoch entspricht die traditionelle Formulierung dieses Begriffes nicht seinem eigentlichen Inhalt. Auf theoretischer Grundlage setzen wir uns mit den verschiedenen Formulierungen und Interpretationen dieses Begriffes auseinander und schlagen eine für die systematische Verwirklichung unseres Vorhabens brauchbare Arbeitsdefinition der Artikulationsbasis einer gegebenen Sprache vor (S i m e o n o va, 1979, 19). Dieser Definition folgen wir in der vorliegenden Arbeit, welche den ersten Teil einer breit angelegten Konfrontation der phonetischen Grundlagen des Deutschen und des Bulgarischen darstellen soll.

Die Vokalsysteme beider Sprachen werden auf physiologischer, physikalisch-akustischer und funktioneller Grundlage miteinander verglichen. Als theoretisches Postulat dient die Auffassung, daß es neben den psycholinguistischen auch experimentell gesicherte Mittel und Methoden für die Abgrenzung der einzelnen Lautsegmente im Redeflu $B, d . h$. in ihrem zeitlichen und räumlichen Bewegungsablauf, gibt. In der praktischen Arbeit bei der Auswertung der Untersuchungsergebnisse werden die Segmentierungsmethodik und die Regeln der bulgarischen Experimentalphonetiker Tilkov und Mischeva benutzt.

A. Vergleichende Charakteristik der Vokalartikulation im Deutschen und im Bulgarischen

Der Vergleich der physiologischen Basiskomponenten fur die Vokalartikulation im Deutschen und im Bulgarischen ergibt folgendes:

- Der Spannungsgrad der aktiven Sprechorgane und der Wandungen der Mundhöhle im Prozeß der Vokalgestaltung ist für das Deutsche verhältnismäßig höher als für das Bulgarische. Ursachen: a) Fester Vokaleinsatz im Deutschen - kein vokalisches Grenzsignal im Bulgarischen; b) Lange geschlossene und kurze offene Vokale im Deutschen - nur kurze und relativ offene Vokale im Bulgarischen.

- Energische Sprechbewegungen der Lippen und des Unterkiefers für das Deutsche gegenüber lässige Bewegungen der Lippen und des Unterkiefers für die Vokalartikulation des Bulgarischen. 
Eine der wesentlichen Ursachen: Das Verhältnis „labialisierte - illabialisierte" Vokale ist im Deutschen 8:7 zugunsten der labialisierten Vokale, im Bulgarischen - 2:4 zugunsten der illabialisierten Vokale.

- Die Gestaltung der Vokallaute des Deutschen ist mit einer höheren Zungenspannung im Gegensatz zum Bulgarischen verbunden. Ursache: Die Quantität und Qualität der deutschen Vokale.

Die für einen Deutsch lernenden Bulgaren ungewohnte höhere Spannung der Sprechorgane im Prozeß der Vokalartikulation des Deutschen wird durch eine Reihe Beispiele aus der korrektiven deutschen Phonetik für Bulgaren bestätigt.

B. Physikalisch-akustische kontrastive Untersuchung der Vokale des Deutschen und des Bulgarischen

Die spektrale Untersuchung der Vokallaute des Deutschen und des Bulgarischen umfaßt sonagraphische und intonographische Analysen aller Komponenten der akustischen Grundstruktur der Vokale $(698+55 \mathrm{Ver}-$ suchsbeispiele), die Auswerung der gewonnenen Ergebnisse, ihre Systematisierung und ihr Aufeinanderbeziehen mit dem Zweck, die kontrastiven und analogen Spektralerscheinungen aufzudecken. Die phonetische Interferenz zwischen der Mutter- und der Fremdsprache wird experimentell an der Aussprache von vier Deutsch sprechenden Bulgaren analysiert.

Aus der akustischen Untersuchung der primären Spektralmerkmale des deutschen und des bulgarischen Vokalismus lassen sich folgende Schlüsse ableiten :

- Die kurzen offenen Vokale des Deutschen sind im Mittel kompakter als die langen geschlossenen.

- Die betonten bulgarischen Vokallaute nehmen nach ihrer Formantenstruktur eine Mittelstellung zwischen der Reihe der langen geschlossenen und der der kurzen offenen deutschen Vokale ein.

- Akustisch betrachtet sind die unbetonten deutschen Vokale im Mittel kompakter als die Vokale der betonten gespannten Reihe und diffuser als die der betonten ungespannten. In ihrem akustischen Spektrum überwiegen jedoch spektrale Merkmale der gespannten Vokalreihe.

- Eine besondere Stellung unter den deutschen unbetonten Vokalen nimmt der Murmelvokal [a] ein. Spektral weist dieser Laut relativ enge Berührungspunkte zu drei unbetonten Vokalen auf: einem [e], [ø] und einem $[a]$.

- Im bulgarischen unbetonten Vokalismus ist ein hoher Grad an assimilatorischer Reduktion $\mathrm{zu}$ beobachten. Im allgemeinen macht sich spektral die Annäherungstendenz der sog. „breiten“ Vokale des Bulgarischen auf ihre .engen" Korrelationspaare zu, bemerkbar.

- Am stärksten ist die Reduktion beim Korrelationspaar /a-a/ vertreten, was sich in einer fast vollständigen Neutralisierung der die beiden Vokale in betonter Stellung unterscheidenden Spektralmerkmale ,kompakt-diffus“ ausdrückt.

- Der spektrale Vergleich zwischen dem deutschen und dem bulgarischen unbetonten Vokalismus zeigt im allgemeinen eine diffusere Verteilung der Gesamtenergie bei den bulgarischen unbetonten Vokallauten. 
- Dem deutschen Murmelvokal [ə] entsprechen im System des bulgarischen unbetonten Vokalismus je nach dessen Position und Lautumgebung drei Vokallaute: [e]. [a] und [a]. Die Distribution dieser bulgarischen Vokale entspricht der des Murmelvokals.

Die Untersuchungsergebnisse der von Bulgaren realisierten Vokale des Deutschen ergeben folgendes:

- Mit Ausnahme der labialisierten Vorderzungenvokale tritt bei der bulgarischen Realisierung der gespannten und ungespannten Vokalreihe qualitativ ein gewisser Ausgleich ein, d.h., beide Vokalreihen klingen etwa gleich.

- Da sich im bulgarischen Vokalsystem historisch keine labialisierten Vorderzungenvokale herausgebildet haben, sind bei deren Realisierung durch Bulgaren die meisten Besonderheiten anzutreffen: Sowohl bei den Ü-Lauten

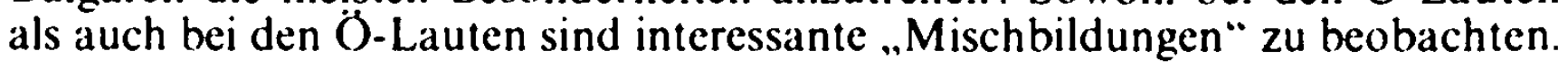
So bewegen sich die Frequenzwerte für das [y:] bei allen bulgarischen Versuchspersonen in der Frequenzzone eines offenen [Y] oder des Murmelvokals. Dabei fehlt der für das $[Y]$ und [a] charakteristische $F_{3}$ auf der Frequenzskala. Interessant ist die Bildung des kurzen offenen $[\gamma]$ : Sie hat im Mittel den Charakter der bulgarischen Lautverbindung [io]. Die Oे-Laute weisen im allgemeinen einen diffuseren Charakter auf. Etwa die Hälfte der Versuchsbeispiele zeigt auch hier eine gemischt gebildete Form, die sich allerdings allein beim offenen $[\propto]$ bemerkbar macht. Sie trägt einen Schwa-ähnlichen Charakter..

- Die Dauerverhältnisse zwischen der langen und der kurzen Vokalreihe des Deutschen zeigen relativ große Verzerrungen. die jedoch keinen konstanten Charakter tragen.

- Die deutschen Vokallaute in unbetonter Position ergeben bei den bulgarischen Versuchspersonen ein einheitliches spektrales Bild unabhängig davon, ob der entsprechende Vokal in betonter Stellung gespannt oder ungespannt ist, während der deutsche Sprecher auch in unbetonter Position einen Unterschied dazwischen macht.

- Augenfällig ist dic recht dunkle Klangfarbe des unbetonten [a]. besonders in den Proklitika [das]. [als]. Hier macht sich die starke Reduktion des bulgarischen Korrelationspaares $[\mathrm{a}-$ o] in unbetonter Stellung besonders deutlich bemerkbar.

- Die unbetonten Vokale sind bei allen Versuchspersonen im Durchschnitt intensitätsschwächer als die entsprechenden betonten.

Die spektral-analytische Untersuchung der sekundären akustischen Merkmale des deutschen und des bulgarischen Vokalismus läßt folgendes zusammenfassen:

1. Grundfrequenz der Vokallaute des Deutschen und des Bulgarischen

- Die Grundfrequenz der deutschen und der bulgarischen Vokale ist nicht für alle Vokale gleich. Sie korrespondiert folgerichtig mit der Höhe der Zungenlage während der Artikulation. Dabei folgt sie folgender recht stabilen Gesetzmäßigkeit: Je höher die Zungenlage, destc, höher der $F_{0}$.

Diese Gesetzmäßigkeit beobachtet man in den zwei betonten deutschen Vokalreihen, sie gilt in gleichem Maße auch für den bulgarischen betonten Vokalismus. 
Dic zwei Reihen von betonten Vokalen im Deutschen folgen, eine jede für sich, dieser Gesetzmäßigkeit. Vergleicht man jedoch diesbezüglich die lange geschlossene mit der kurzen offenen Vokalreihe, so weisen nicht, wie man hätte erwarten können, die geschlossenen, sondern die offenen Vokale etwas höhere Grundfrequenzwerte auf. Die Erklärung dafür wäre wahrscheinlich in der von uns beobachteten Korrelation zwischen Grundfrequenz und Lautdauer zu suchen: so gestaltet sich die Grundfrequenz bei einem kurzen offenen Vokal wegen der kurzen Dauer nicht so variabel wie bei einem langen geschlossenen: hier verläuft sie recht unstabil. besonders im letzten Segment der $F_{0}-$ Kontur, wo sich das Schwingungstempo etwas verlangsamt und der Stimmton sinkt.

- Die unbetonten Vokallaute des Deutschen und des Bulgarischen charakterisieren sich durch niedrigere Frequenzwerte im Vergleich zu den betonten Vokalen.

- Sehr auffallig ist der Unterschied zwischen den Grundfrequenzwerten der betonten Vokale des Deutschen und denen des Murmelvokals [D] oder des Reduktionsvokals [p]. Dieser Umstand ist ein Beweis dafür, daß die einzigen im spektralen Sinne des Wortes reduzierten Vokallaute in der gegenwärtigen deutschen Standardaussprache der [o]- und [e]-Vokal sind.

- Die Reduktion der bulgarischen Vokallaute in unbetonter Position läßt sich auch durch die Frequenzwerte des $F_{0}$ nachweisen: Die Grundfrequenzwerte für die unbetonten [a] und [e] zeigen den minimalen Unterschied von 1,6 (Sprecher R. S.) und $1,9 \mathrm{~Hz}$ (Sprecher M. P.), was für den Ausgleich der Klangfarbenunterschiede dieses Korrelationspaares spricht.

- Die Grundfrequenz der Vokallaute des Deutschen und des Bulgarischen wird nicht durch die Art der sie umgebenden Konsonanten beeinflußt.

- Die Grundfrequenz der Vokale der beiden Sprachen hängt von der Ahantuierung im Wort ab:

Betonte erste Silbe. abgesehen von der Silbenzahl des Wortes, weist stets die höchste (irundfrequenz auf:

Unbetonte letzte Silbe hat ohne Ausnahme die niedrigste Grundfrequenz;

Wenn die letzte Silbe eines zwei-, drei- oder viersilbigen Wortes unter Betonung liegt, weist sie in manchen Fällen auch die höchste Grundfrequenz auf: meist ist sie aber annähernd gleich der Grundfrequenz der vorangehenden unbetonten Silbe; gelegentlich sinkt sie auch etwas darunter. Dieser Umstand läßt sich durch die terminale melodische Gestaltung unserer „Rahmenbeispiele" erklären. Diese Erscheinung weist gleichzeitig darauf hin, daß sich der terminale Melodieverlauf im Deutschen und im Bulgarischen gleich gestaltet.

2. Spezifische Intensität der deutschen und der bulgarischen Vokallaute

- Die Einordnung der betonten Vokale des Deutschen und des Bulgarischen nach ihrer spezifischen Intensität folgt im großen und ganzen der Sonoritätsskala von Jespersen. So zeigt unter den betonten Vokalen der Vokal [a] die höchsten Intensitätswerte, [u] - die niedrigsten.

- Die Quantität und Qualität der deutschen Vokale üben keinen uesentlichen Einfluß auf ihre spezifische Intensität aus.

- Innerhalb der offenen und der geschlossenen Vokalreihe des Deutschen und in der Reihe der betonten Vokale des Bulgarischen lasen sich zwischen 
den einzelnen Vokallauten ebenfalls keine wesentlichen Intensitätsunterschiede feststellen.

- Relevante Intensitätsunterschiede lassen allein für das Bulgarische zwei Gruppen von intensitätsunterscheidbaren Vokalen erkennen : die Gruppe der .breiten“ Vokale mit höherer Intensität und die der „engen“ Vokale mit einer etwas niedrigeren Intensität.

- Sowohl im Deutschen als auch im Bulgarischen ist eine gesetzmäBige Abhängigkeit der Intensität von der Stellung der akzentuierten Silbe im Wort zu beobachten. So hat die Intensität einer ersten betonten Silbe in beiden Sprachen stets einen höheren Wert im Vergleich zu der Intensität einer zweiten, dritten oder letzten betonten Silbe.

Relevante Intensitätsunterschiede, durch die Art der Silbe (offen oder geschlossen) bewirkt, sind weder im Deutschen noch im Bulgarischen festzustellen.

Die spezifische Intensität der Vokallaute des Deutschen und des Bulgarischen ist stark akzentabhängig. So sind die deutschen unbetonten Vokale im Mittel um 2,7 dB, die bulgarischen - um 2,3 dB druckschwächer als die betonten. Die angeführten Differenzen sprechen von etwa gleichen Intensitätsverhältnissen in beiden Sprachen.

Die Rangordnung der unbetonten Vokale nach ihrer Intensität entspricht weder im Deutschen noch im Bulgarischen der der betonten Vokallaute. Sie verrät, genauso wie die Konfrontation der Formantenstruktur der Vokale der beiden Sprachen, die Reduktionstendenzen im Deutschen und im Bulgarischen.

Die spezifische Intensität des deutschen Murmelvokals nimmt zwar die unterste Stufe unserer Intensitätsskala ein, jedoch ist die Differenz zwischen dem [ə] und beispielsweise einem [y], [u], [i] diesbezüglich gering. Die Versuchsergebnisse zur spezifischen Intensität der unbetonten Vokale sprechen folglich gegen die in der deutschen Phonetik vertretene Auffassung. daß eines der wesentlichen Merkmale des Murmelvokals seine im Gegensatz zu den übrigen unbetonten Vokalen geringe Intensität sei.

3. Spezifische Lautdauer der Vokale des Deutschen und des Bulgarischen

Von einer klar ausgeprägten Rangordnung in bezug auf die Lautdauer der betonten Vokale beider Sprachen kann kaum die Rede sein, da die spezifische Dauer der einzelnen Vokale untereinander recht ausgeglichen ist. Trotzdem markiert ein geringer Dauerunterschied die Grenze zwischen den ..breiten“ und den „engen“ Vokallauten des Deutschen und des Bulgarischen, wobei sämtliche ..breite“ Vokale etwas länger andauern als die ,engen“.

Das Dauerverhältnis zwischen der langen geschlossenen und der kurzen offenen Vokalreihe des Deutschen beträgt im Mittel $1: 0.6$.

Die objektive Lautdauer der betonten Vokale des Deutschen und des Bulgarischen wird kaum durch die Art der Bildung des vorangehenden Konsonanten beeinflußt.Die Bildungsweise des nachfolgenden Konsonanten wirkt sich dagegen etwas merklicher auf die Dauer des linksstehenden Vokals aus: Am kürzesten fallen die betonten Vokale der beiden Sprachen vor Explosiven aus, nachfolgende Frikative verlängern durch die Bildung der Enge die Dauer des vorangehenden Vokals. 
Die unbetonten Vokale sind in beiden Sprachen von kürzerer Dauer als die betonten.

Die langen geschlossenen Vokallaute des Deutschen sind im Mittel um das doppelte länger als die unbetonten Vokale $(0,18: 0,09)$, während sich die spezifische Lautdauer der kurzen offenen zu der der unbetonten Vokale wie $0,11: 0,09$ verhält.

Das Dauerverhältnis „betont - unbetont“ ist für die bulgarischen Vokale im Mittel $0.11: 0,1$.

Die Art der Silbe bewirkt keine beträchtlichen Unterschiede in der spezifischen Dauer der unbetonten Vokale des Deutschen und des Bulgarischen.

Der geschlossene unbetonte Auslaut ist in bezug auf die Lautdauer der bulgarischen unbetonten Vokale und des deutschen [a] stark einer quantitativen Reduktion ausgesetzt.

Die Lautdauer des [ə] beträgt $0,08 \mathrm{sec}$. für die Auslautsilben [-an], [-əl] gegenüber $0,13 \mathrm{sec}$. für seine übrigen Auslautpositionen. Im absoluten Auslaut dauert allerdings das deutsche [ə] länger --- seine Dauerwerte betragen hier im Mittel 0,18 sec., eine Lautdauer, die den bulgarischen unbetonten Vokalen in offener Auslautsilbe sehr nahe kommt.

4. Die vokalischen Phonemsysteme des Deutschen und des Bulgarischen

- Für den konfrontativen Vergleich der Vokalphoneme des Deutschen und des Bulgarischen erweisen sich folgende distinktive Merkmale als relevant: 1. gespannt ungespannt; 2. kompakt - diffus; 3 . hoch - niedrig; 4. bemol - nicht bemol.

- Für die bulgarischen Vokalphoneme ist das Merkmal „gespannt - ungespannt“" irrelevant.

Die bulgarische Sprache hat zwei spektral kompakte, artikulatorisch breite Vokalphoneme gegenüber vier der deutschen Sprache. Zwei Vokalphoneme $(/ \propto) /$ und $/ \varepsilon /)$ des Deutschen und eins des Bulgarischen $(/ \varepsilon /)$ kann man hierzu als „merkmalarm” oder „mittel” bezeichnen.

Eine merkwürdige Erscheinung des bulgarischen betonten Vokalismus ist das diffuse (enge) Vokalphonem /a/, das die deutsche Sprache nicht kennt. Daraus ergeben sich Schwierigkeiten für Bulgarisch lernende Deutsche; dadurch lassen sich auch die Inkonsequenzen in der deutschen Transkription bulgarischer Namen erklären.

- Im Deutschen und im Bulgarischen halten sich die Merkmalpaare „hoch niedrig" relativ die Waage. Das Verhältnis ist $2: 3+1$ merkmalarmen Vokalphonem für das Bulgarische, 6:6+3 merkmalarmen Vokalphonemen für das Deutsche.

In bezug auf die distinktiven Merkmale „,bemol nicht bemol“" weist das deutsche Vokalsystem eine spezifische Besonderheit auf, die für das. Bulgarische nicht zutrifft: Im deutschen Vokalismus ist die Reihe der labialisierten Vorderzungenvokale (4 Vokalphoneme) vertreten.

Der phonologische Status des [3] wird auf Grund der spektral-analytisch und auditiv-statistisch ermittelten 2 stellungsbedingten Varianten dieses Vokals interpretiert : Den Monophthong [ $[\mathrm{e}]$ für die Auslautverbindung $[-\partial r]$ sehen wir als eine positionsbedingte Variante der A-Phoneme, das [o] in 
den Positionen [bə-], [gə-], absolutem Auslaut und Auslaut vor Nasal - als eine stellungsbedingte Variante des Phonems / $\varepsilon /$ an.

- Sämtliche bulgarische unbetonte Vokale betrachten wir als Phonemvarianten, da wir uns der Auffassung anschließen, da $B$ die Akzentuierung ein prosodisches und kein Lautmerkmal ist. Außerdem sind die Spektraldifferenzen zwischen den betonten und unbetonten bulgarischen Vokalen gering und so sprachlich irrelevant.

Die funktionelle Gegenüberstellung der beiden Vokalreihen des Bulgarischen bestätigt den hohen Grad qualitativer Reduktion des Korrelationspaares [a - - $]$ in unbetonter Position und die etwas schwächeren Klangfarbenveränderungen der Korrelationspaare $[\varepsilon-1]$, [0-o -0 . Spektral läßt sich der Klangfarbenausgleich zwischen den unbetonten [a] und [e] sehr deutlich beobachten, während die qualitative Reduktion der Korrelationspaare $[0-u],[\varepsilon-1]$ durch unsere Spektraluntersuchungen nicht belegt werden konnte.

Die Distribution der Vokallaute im Deutschen und im Bulgarischen läßt folgende Schlußbemerkungen zusammenfassen :

Das bulgarische Vokalsystem kommt mit viel weniger Vokalphonemen aus als das deutsche. Den 6 bulgarischen Vokalphonemen stehen 15 deutsche gegenüber.

Das vokalisch-konsonantische Verhältnis ist in der deutschen Gegenwartssprache gut ausgeglichen: den 15 Vokalphonemen und 3 Diphthongen entsprechen 19 Konsonantenphoneme. Im Bulgarischen ist dieses Verhältnis 6:39 zugunsten der Konsonantenphoneme. Trotz dieser auffallenden Differenz zeichnet sich die bulgarische Sprache ebenfalls durch ein gut ausgewogenes vokalisch-konsonantisches Gleichgewicht aus. Das läßt sich durch die Tatsache erklären, daß die bulgarischen Vokale dank des freien Wortakzents syntagmatisch fast keine stellungsbedingten Einschränkungen kennen.

- Das Gleichgewicht „betont - unbetont“ in bezug auf die vokalische Distribution wird im Deutschen durch die hohe Gebrauchsfrequenz des [a]. durch den Gebrauch der pro- und enklitisch abgekürzten Morpheme und Einsilber sowie durch die in Fremdwörtern vorkommenden kurzen geschlossenen Vokale aufrechterhalten.

Die bulgarischen unbetonten Vokale unterstehen wie die betonten keiner strengen GesetzmäBigkeit bei ihrer Verteilung im Wort. Die Silbentypen, die sie bilden, unterscheiden sich strukturell nicht von denen der betonten Vokale.

- Dem deutschen Schwa-Vokal entsprechen distributiv die unbetonten bulgarischen Vokale [e], [a], [a]. 


\section{DIE KONSONANTENSYSTEME DES DEUTSCHEN UND DES BULGARISCHEN}

\subsection{ALLGEMEINES ZUR KONSONANTENBILDUNG}

Das entscheidende Element bei der Bildung der Konsonanten ist die Ausbildung einer akustisch wirksamen Hemmstelle im Ansatzraum mit Hilfe des Luftstromes. Dagegen werden zur Erzeugung der Vokale die Hohlräume des Ansatzrohres durch das Zusammenwirken von Stimmgebung, Atmung und Artikulation zur Resonanz angeregt. und der im Kehlkopf erzeugte primäre Stimmklang wird darin modifiziert. Mit Ausnahme des Konsonanten [h] findet bei der Bildung der Konsonanten eine starke Verengung in der sagittalen Mittellinie des Ansatzraumes statt, die als Hemmung der Ansatzluft entgegentritt. Physikalisch betrachtet, sind Konsonanten Mischungen aus Klang und Geräusch. Nur bei der Bildung reiner Geräuschlaute (Zischlaute) wird an der jeweiligen Artikulationsstelle ein Geräusch erzeugt, das entwerder stimmhaft oder stimmlos ertönt. Die Geräuschlaute zeigen auch durch die Ausbildung einer schmalen Rinne bzw. durch das Hindurchpressen der Luft und deren starken Reibung sehr hohe Teilfrequenzen auf (bei s- und f-Lauten bis zu $10 \mathrm{kHz}$ ). Dagegen zeigen die Sonorlaute, einbezüglich das [L], bei dem nur unerhebliche Geräusche nachzuweisen sind, ein vokalähnliches Spektrum. ..An den Stellen zwischen $i-j$ und zwischen $u$ und bilabialem $v$ gehen Vokalund Konsonantensystem genetisch unmittelbar ineinandēr über. Die Einstellung der Artikulationsorgane im Ansatzraum ist nahe verwandt; nur die Art, welche Wirkung die Enge akustisch ausübt, entscheidet darüber, ob der erzeugte Laut als Vokal oder Konsonant gewertet wird. Wenn die Enge nur klangformenden Charakter hat (bei [i:, u:]), wird der Laut als Vokal empfunden...; wird aber durch die Enge eine sekundüre Schallquelle gebildet, dic ein Geräusch erzeugt, so werden die Laute zu Konsonanten“ (Lindner, 1981, 244).

Phonologisch gesehen, können Konsonanten in den meisten Sprachen keine Silbenkerne sein. In der Silbenstruktur bilden sie deren Rand. Ein Vokal kann allein eine betonte Silbe bilden ein Konsonant nicht. Was den Phonemstatus mancher Konsonanten und Konsonantenverbindungen in unseren zwei Sprachen betrifft, so gibt es umstrittene Probleme hinsichtlich einer monophonematischen Wertung. Auf diese Probleme wird unter konfrontativer Sicht bei der Darstellung der jeweiligen Konsonanten eingegangen.

\subsection{PHONETISCHE K LASSIFIZIERUNG DER KONSONANTEN}

Die traditionellen Einteilungsgesichtspunkte, die bei der physiologischen Klassifikation der Konsonanten im Vordergrund stehen, sind die Einteilung nach dem artikulierenden Organ und der Artikulationsstelle, nach dem Artikulations- und Überwindungsmodus. 
Hinsichtlich der Beteiligung des artikulierenden Organs an der Ausbildung der Hemmstelle gelten für die Bestimmung der Konsonanten folgende Bezeichnungen :

labial bzw. bilabial: artikulierendes Organ sind die Lippen; labiodental: artikulierende Organe sind die Unterlippe und die oberen Schneidezähne ;

coronal : artikulierendes Organ ist der vordere Zungensaum (von Eckzahn bis Eckzahn);

apical: artikulierendes Organ ist der vorderste Teil der Zungenspitze ; dorsal: artikulierendes Organ ist der Zungenrücken, und da er genauer bestimmt werden muß, so unterscheidet man folgende Bezeichungen für die dorsalen Konsonanten :

prädorsal-mit dem vorderen Zungenrücken als artikulierendes Organ ;

mediodorsal - mit dem mittleren Zungenrücken als artikulierendes Organ ;

postdorsal-mit dem hinteren Zungenrücken als artikulierendes Organ;

lateral bzw. bilateral : artikulierendes Organ ist der eine Zungenrand bzw. beide Zungenränder.

In bezug auf die Artikulationsstelle, mit deren Hilfe das artikulierende Organ die Hemmstelle ausbildet, gelten folgende Bezeichnungen:

labial bzw. hilabial: mit der Oberlippe bzw. beiden Lippen als Artikulationsstelle ;

dental: mit den oberen Schneidezähnen als Artikulationstelle;

alveolar: Artikulationsstelle sind die Zahntaschen der oberen Schneidezähne ; palatal: Artikulationsstelle ist der harte Gaumen, und da dieser genauer bestimmt werden muß, so ergeben sich daraus folgende Bezeichnungen für die palatalen Konsonanten:

präpalatal: Artikulationsstelle ist der vordere Teil des harten Gaumens ;

mediopalatal: Artikulationsstelle ist der mittlere Teil des harten Gaumens ;

postpalatal: Artikulationsstelle ist der hintere Teil des harten Gaumens ; velar: Artikulationsstelle ist der weiche Gaumen (das Gaumensegel); unular: Artikulationsstelle ist das Zäpfchen; pharyngal: Artikulationsstelle ist die Pharynxhinterwand.

Für die bulgarischen Konsonanten sollte außerdem bei jeder Lautklasse die Bezeichnung ..mediodorsal" hinsichtlich des artikulierenden Organs und .mediopalatal" in bezug auf die Artikulationsstelle eingeführt werden, da sie im Unterschied $\mathrm{zu}$ den deutschen Konsonantenphonemen je eine palatale, "weiche" Form mit Phonemwert aufweisen, bei deren Bildung eine zusätzliche Hebung der Mittelzunge zum Gaumen zustandekommt.

Die Art der Ausbildung der Hemmstellen wird als Artikulationsmodus bezeichnet. Nach dem Artikulationsmodus ergeben sich folgende Bezeichnungen für die deutschen und die bulgarischen Konsonanten: 
Verschlußlaute: die Hemmstelle wird als Verschluß des Ansatzraumes ausgebildet ;

Engelaute: die Hemmstelle ist eine im Ansatzraum ausgebildete Enge; Nasale (Nasenlaute): VerschluB des Ansatzraumes bei gleichzeitiger nasaler Öfnung ;

Intermittierende Verschlußlaute: Ausbildung eines ständig unterbrochenen Verschlusses im Ansatzraum.

Ein weiterer Gesichtspunkt zur Charakterisierung der Konsonanten ist der Üherwindungsmodus, der angibt, wie der Luftstrom die Hemmstelle des Ansatzraumes bzw. im Ansatzraum überwindet. Für den Überwindungsmodus der Konsonanten sind ebenfalls spezielle Bezeichnungen und Einteilungsprinzipien entwickelt worden. So überwindet die Ausatmungsluft die Hemmstelle durch :

- Sprengung (bei Ausbildung eines Verschlusses);

- Reibung (bei Ausbildung einer Enge);

- Flattern (bei Ausbildung eines intermittierenden Verschlusses);

-. Bildung eines Hauches.

Die Bezeichnungen lassen sich bei der Darstellung des Konsonantensystems einer gegebenen Sprache und besonders zweier Sprachen unter konfrontativem Aspekt kombinieren, da die angegebenen Größen: Artikulationsstelle, artikulierendes Organ, Artikulationsmodus und Überwindungsmodus nicht immer überschneidungsfrei sind. Außerdem ist es bei der Gegenüberstellung der Konsonanten von zwei Sprachen zweckmäßig, auch andere Einteilungskriterien bzw. Bezeichnungen dafür heranzuziehen, die die spezifischen Besonderheiten der jeweiligen Konsonantengruppe oder des einzelnen Konsonanten genau angeben (Siehe Einteilungstabelle der deutschen und der bulgarischen Konsonanten nach physiologischen Kriterien S. 73).

\subsection{PHONOLOGISCHE KLASSIFIKATION DER KONSONANTEN}

Die distinktiven Merkmale der Phoneme, die eine relevante linguistische Information tragen, basieren grundsätzlich auf phonetischen (physiologischakustischen) Gegebenheiten. So werden die meisten schon behandelten Bezeichnungen zur phonetischen Einteilung der Konsonanten auch zu deren phonologischen Klassifikation benutzt. Jedoch sind nicht alle Lautklassen, die auf der Tabelle (S. 73) 1 vorkommen, als Realisierungen eines Phonems zu werten. „Auch müssen nicht alle Artikulationsmerkmale, die in den Spalten und Zeilen spezifiziert werden, als distinktive Merkmale behandelt werden" (Meinhold, S to ck, 1982, 122).

Für die phonologische Darstellung der deutschen Konsonanten reichen nach Meinhold und Stock 10 distinktive Merkmale aus. Die von beiden Autoren aufgestellte Konsonantenmatrix sieht wie folgt aus (Siehe S. 74).

Dadurch. daß für das Bulgarische palatalisierte (weiche) Konsonanten charakteristisch sind, ist die Anzahl der bulgarischen Konsonantenphoneme fast um das Doppelte größer als die der deutschen (38 gegenüber 20). 
Distributionell betrachtet, erscheinen die bulgarischen Palatalkonsonantenphoneme nur vor Hinterzungenvokalen: [bał - b’ał], [kup -..k'up]. Leicht palatalisierte Allophone kommen auch vor $[\mathrm{r}]$ und $[\varepsilon]$ auf: [tak - lek …lık]. Durch die Einführung der distinktiven Merkmale „palatalisiert - nicht palatalisiert" bzw. "weich - hart" steigt auch die Anzahl der distinktiven Merkmale in der Matrix der bulgarischen Konsonantenphoneme an. Tilkov (Т и лков, Бояджие в, 1977, 136) baut die Merkmalmatrix der bulgarischen Konsonanten auf 15 distinktiven Merkmalen auf. Seine Matrixtabelle sieht wie folgt aus (Siehe S. 74-75).

Aus dem Vergleich der Merkmalmatrizen des Deutschen und des Bulgarischen ergeben sich folgende Unterschiede :

- Für die Darstellung der bulgarischen Konsonantenphoneme ist das distinktive Merkmal „palatalisiert“ (,weich“) notwendig. Dieses Markmal ist für das deutsche Konsonantensystem fremd.

- Dem Merkmal "fortis" in der Matrix des Deutschen entspricht das Merkmal "stimmhaft" in der Matrix des Bulgarischen, weil hier die Stimmlosigkeit bzw. Stimmhaftigkeit relevant ist.

- Für die Bestimmung der bulgarischen Konsonantenphoneme ist das distinktive Merkmal "laryngal-pharyngal“" nicht notwendig.

Die deutschen Konsonanten [c] und [x] werden von Meinhold und Stock als selbständige Phoneme bewertet und daher nicht in die Matrix aufgenommen.

Das bulgarische Konsonantenphonem [1] nimmt nach Tilkov eine Sonderstellung unter den bulgarischen Konsonantenphonemen ein. Als distinktives Merkmal für diesen Konsonanten führt Tilkov die Bezeichnung ..gleithaft" ein.

Die deutschen Affrikaten [pf], [ts] werden von Meinhold und Stock biphonematisch gewertet. 


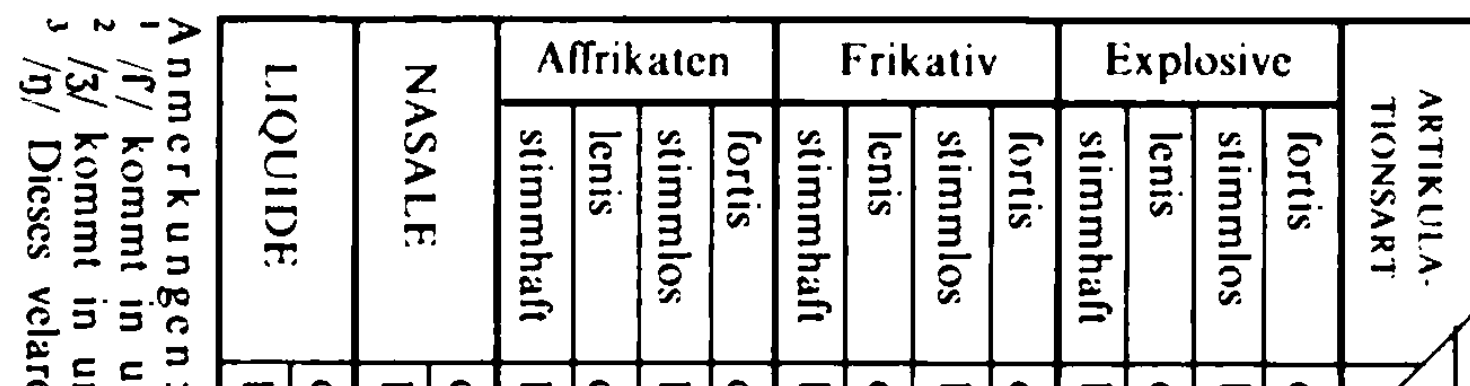

ว도? ?

\begin{tabular}{|l|l|l|l|l|l|l|l|l|l|l|l|l|l|l|l|}
\hline$\sigma$ & $a$ & $\sigma$ & $a$ & $\sigma$ & $a$ & $\sigma$ & $a$ & $\sigma$ & $a$ & $\sigma$ & $a$ & $\sigma$ & $a$ & $\sigma$ & $a$ \\
\hline
\end{tabular}

叉글.

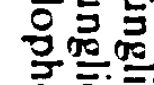

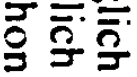

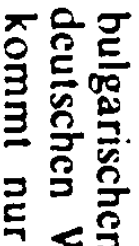

$\exists$

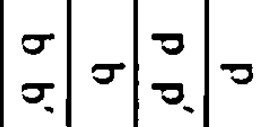

D $<<\quad \geq$

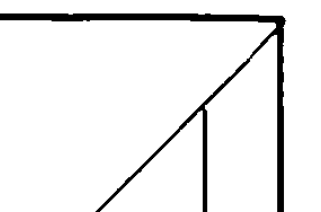

ว

코.글

乐高

(1)

结

ב.

$\vdots$

20

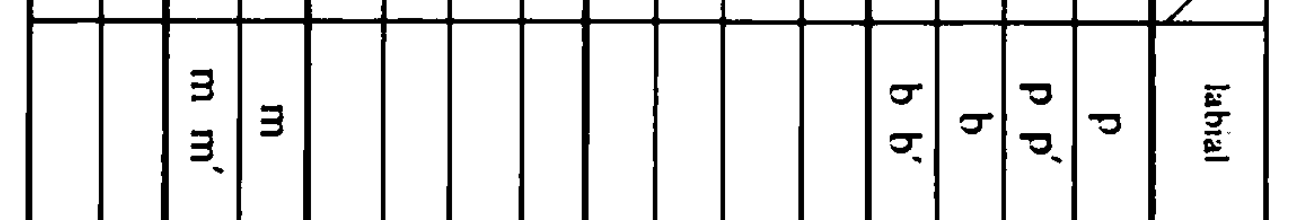

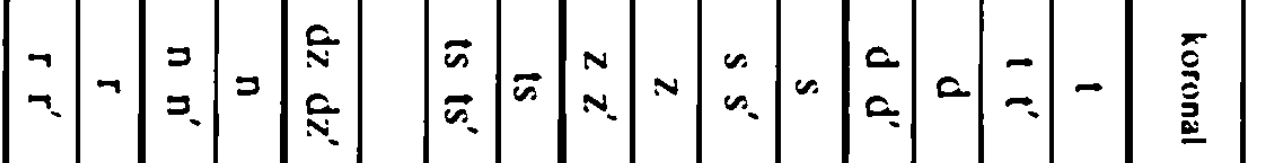

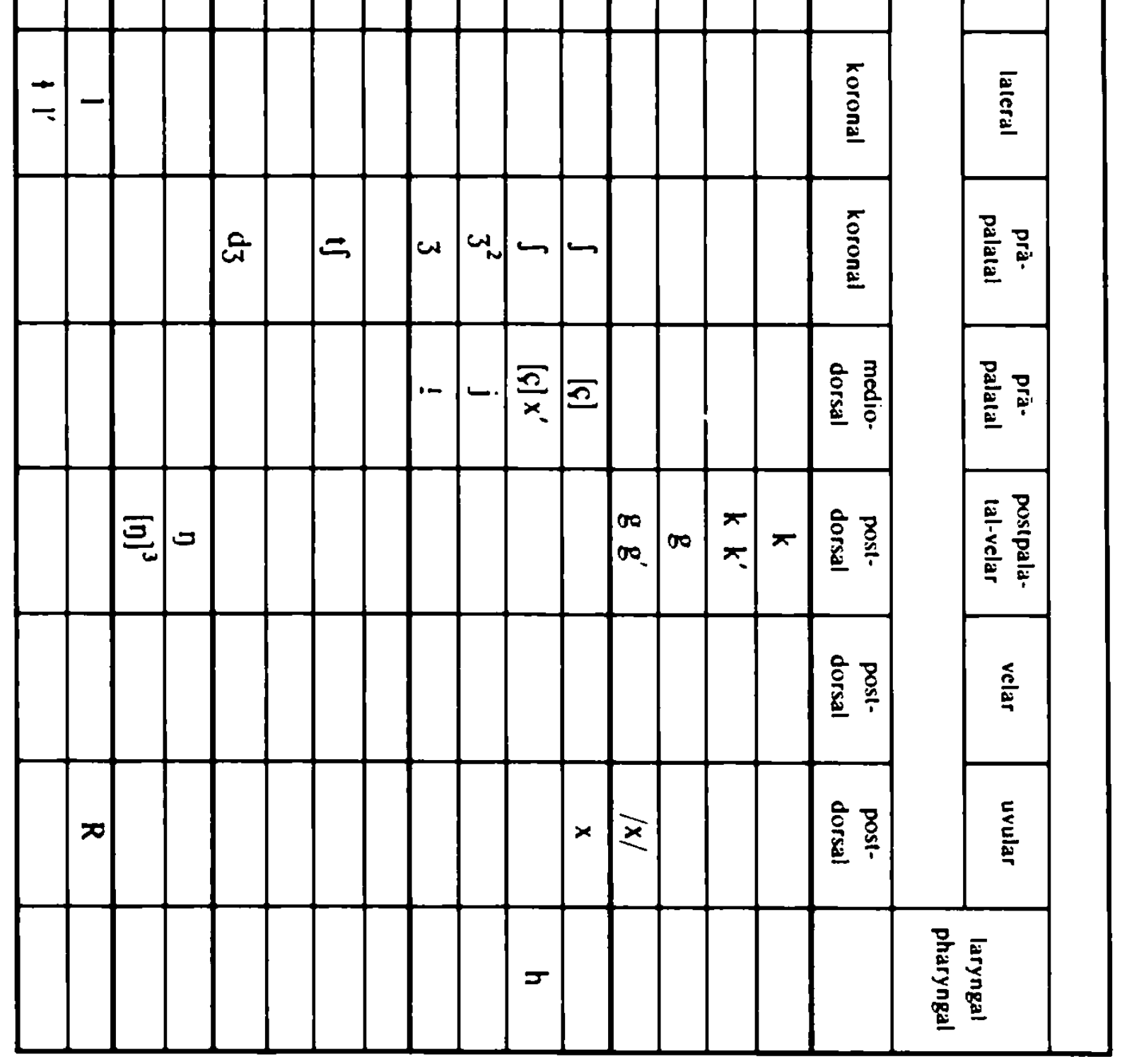


Matrixtabelle der deutschen Konsonanten nach Meinhold und Stock $(1982,124)$

\begin{tabular}{|l|c|c|c|c|c|c|c|c|c|c|c|c|c|c|c|c|c|c|c|}
\hline \multicolumn{1}{|c}{ Merkmal } & p & b & m & f & v & t & d & n & l & s & z & f & f $-x$ & j & k & g & g & r & h \\
\hline konsonantisch & + & + & + & + & + & + & + & + & + & + & + & + & + & + & + & + & + & + & + \\
obstruent & + & + & - & + & + & + & + & - & - & + & + & + & + & + & + & + & - & + & + \\
frikativ & - & - & 0 & + & + & - & - & 0 & 0 & + & + & + & + & + & - & - & 0 & + & + \\
fortis & + & - & 0 & + & - & + & - & 0 & 0 & + & - & + & + & - & + & - & 0 & - & - \\
laryng.-pharyng. & 0 & 0 & 0 & 0 & - & 0 & 0 & 0 & 0 & 0 & - & 0 & 0 & - & 0 & 0 & 0 & - & + \\
nasal & 0 & - & + & 0 & 0 & 0 & - & + & 0 & 0 & 0 & 0 & 0 & 0 & 0 & - & + & 0 & 0 \\
vorn & + & + & + & + & + & + & + & + & + & + & + & - & - & - & - & - & - & - & 0 \\
koronal & - & - & - & - & - & + & + & + & + & + & + & + & - & - & - & - & - & - & - \\
hinten & - & - & - & - & - & - & - & - & - & - & - & - & - & - & + & + & + & + & 0 \\
lateral & 0 & 0 & 0 & 0 & 0 & - & - & - & + & - & - & - & 0 & 0 & 0 & 0 & 0 & 0 & 0 \\
\hline
\end{tabular}

Anmerkung: - Sofern ein Merkmal fur die Unterscheidung eines Phonems nicht ausgenutzt wird, erscheint an der entsprechenden Stelle der Matrix das Zeichen 0.

- Es wurde ein in der Matrixtabelle von Meinhold und Stock z.ugelassener Druckfehler korrigiert : „laryngal - pharyngal“" ist im Deutschen allein das Phonem $/ \mathrm{h} / ; / \mathrm{v} /, / \mathrm{z} /, / \mathrm{j} /, / \mathrm{r} /$ müssen mit dem Zeichen $/-/$ markiert werden und nicht mit $/ 1 /$, wie es bei Meinhold und Stock $(1982,124)$ ist.

Distinktive Merkmale der hulgarischen Konsonantenphoneme

(nach Tилков/Бояджиев, 1977, 136)

\begin{tabular}{|c|c|c|c|c|c|c|c|c|c|c|c|c|c|c|c|}
\hline $\begin{array}{l}\text { Диф. } \\
\text { пр. }\end{array}$ & 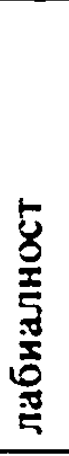 & 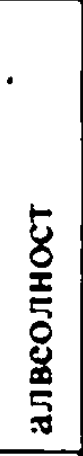 & 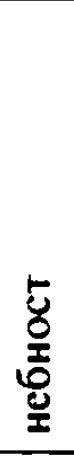 & 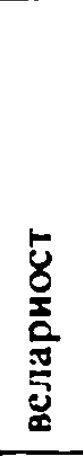 & 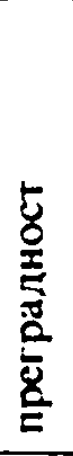 & $\begin{array}{l}5 \\
\bar{\delta} \\
\underline{2} \\
5 \\
0 \\
x \\
0 \\
= \\
\end{array}$ & 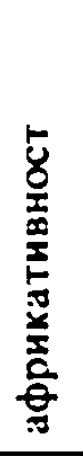 & 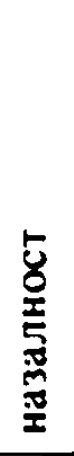 & 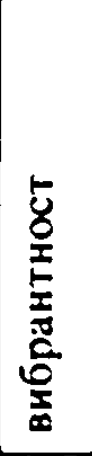 & 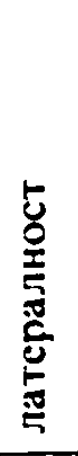 & 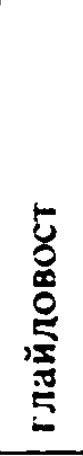 & 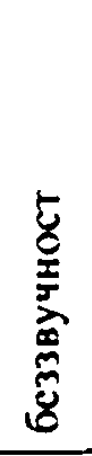 & 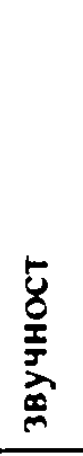 & 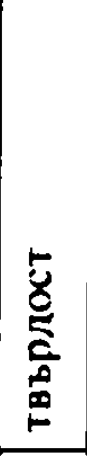 & $\begin{array}{l}5 \\
\bar{z} \\
z \\
\Sigma \\
\end{array}$ \\
\hline 1 & 2 & 3 & 4 & 5 & 6 & 7 & 8 & 9 & 10 & 11 & 12 & 13 & 14 & 15 & 16 \\
\hline $\mathbf{n}$ & + & & & & + & & & & & & & + & & + & \\
\hline$n^{\circ}$ & + & & & & + & & & & & & & + & & & + \\
\hline 6 & + & & & & + & & & & & & & & + & + & \\
\hline $6^{\circ}$ & + & & & & + & & & & & & & & + & & + \\
\hline$\phi$ & + & & & & & $t$ & & & & & & + & & + & \\
\hline$\phi^{\prime}$ & + & & & & & + & & & & & & + & & & + \\
\hline B & + & & & & & + & & & & & & & + & + & \\
\hline$B^{*}$ & + & & & & & + & & & & & & & + & & + \\
\hline M & + & & & & & & & + & & & & & & + & \\
\hline$M^{\circ}$ & + & & & & & & & + & & & & & & & + \\
\hline
\end{tabular}




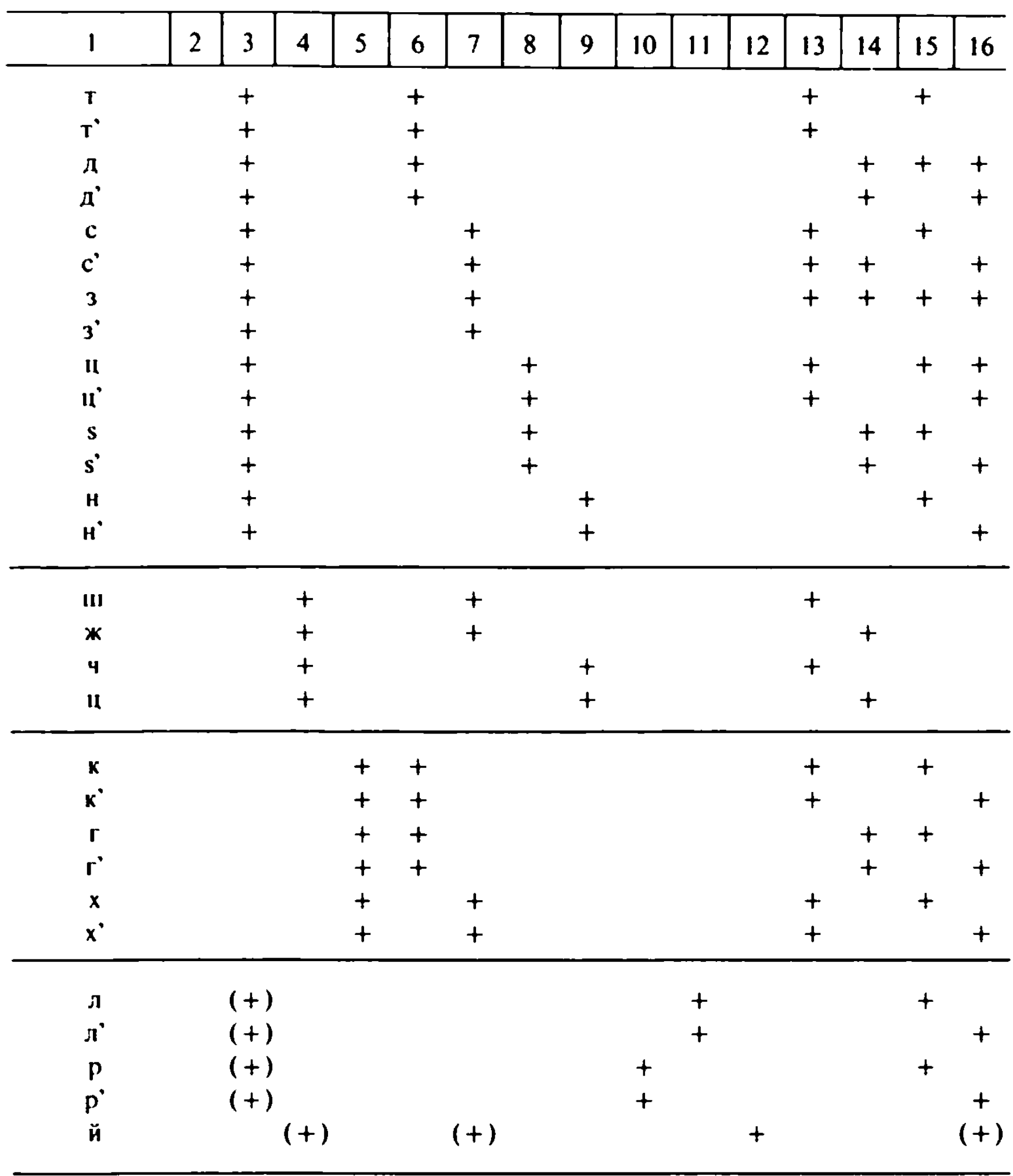


Matrixtabelle der hul

\begin{tabular}{|c|c|c|c|c|c|c|c|c|}
\hline & $p p^{\prime}$ & $b \quad b^{\prime}$ & $m m^{\prime}$ & r $r$ & $v \quad v$ & $11^{\prime}$ & d d & is is \\
\hline konsonantisch & ++ & ++ & ++ & ++ & ++ & ++ & ++ & ++ \\
\hline obstruent & ++ & ++ & -- & ++ & ++ & ++ & ++ & ++ \\
\hline frikativ & - & -- & o $\quad 0$ & ++ & ++ & -- & -- & o 0 \\
\hline stimmlos & ++ & -- & -- & ++ & -- & ++ & -- & ++ \\
\hline nasal & 00 & 00 & ++ & $0 \quad 0$ & o 0 & $0 \quad 0$ & 00 & 00 \\
\hline vorn & ++ & ++ & ++ & ++ & ++ & ++ & ++ & ++ \\
\hline koronal & -- & -- & -- & -- & -- & ++ & ++ & ++ \\
\hline hinten & -- & -- & -- & - & - & -- & - & -- \\
\hline lateral & $\begin{array}{ll}0 & 0\end{array}$ & $0 \quad 0$ & o $\quad 0$ & o 0 & $\begin{array}{ll}0 & 0\end{array}$ & -- & -- & -- \\
\hline palatal & -+ & -+ & -+ & -+ & -+ & $-t$ & -+ & -+ \\
\hline
\end{tabular}

- Tilkov führt in die Konsonantenmatrix des Bulgarischen das distinktive Merkmal ..affrikativ" ein, da er die bulgarischen Affrikaten $/ \mathrm{ts} /, / \mathrm{t} /, / \mathrm{dz} /, / \mathrm{d} 3 /$ als selbständige Phoneme betrachtet.

Damit die Unterschiede besser zum Vorschein kommen, benutzen wir die von Meinhold und Stock gebrauchten Zeichen $1+;-; 0)$ und vereinfachen die von Tilkov aufgestellte Merkmalmatrix. So sieht demnach die Konsonantenmatrix des Bulgarischen wie folgt aus (Siehe S. 76-77). 
garischen Konsonanten

\begin{tabular}{|c|c|c|c|c|c|c|c|c|c|c|}
\hline$d z d z^{\prime}$ & if $d z$ & $n n^{\prime}$ & 11 & $r \quad r$ & $s s^{\prime}$ & $z \quad z^{\prime}$ & S $3 !$ & $k \quad k^{\prime}$ & $88^{\prime}$ & $\times c$ \\
\hline++ & ++ & ++ & ++ & ++ & ++ & ++ & $++t$ & ++ & ++ & ++ \\
\hline$+t$ & ++ & -- & - & - & ++ & ++ & +++ & ++ & ++ & ++ \\
\hline o 0 & 00 & 00 & $\begin{array}{ll}0 & 0\end{array}$ & 00 & ++ & ++ & $++t$ & -- & -- & ++ \\
\hline-- & +- & -- & - & -- & ++ & -- & +-- & ++ & - & ++ \\
\hline $0 \quad 0$ & 00 & ++ & o 0 & 00 & o 0 & 00 & $\begin{array}{lll}0 & 0 & 0\end{array}$ & 00 & 00 & o 0 \\
\hline++ & ++ & ++ & ++ & ++ & ++ & $+t$ & --- & -- & - & - \\
\hline++ & ++ & ++ & $0 \quad 0$ & 00 & ++ & ++ & $+t+$ & - & - & - \\
\hline-- & - & -- & o 0 & o 0 & -- & -- & --- & ++ & ++ & ++ \\
\hline-- & -- & -- & ++ & o 0 & - & -- & --- & 00 & 00 & $0 \quad 0$ \\
\hline-+ & -- & -+ & -+ & -+ & -+ & -+ & --- & -+ & -+ & -+ \\
\hline
\end{tabular}

\section{DIE KONSONANTENPHONEME DES DEUTSCHEN UND DES BULGARISCHEN IM EINZELNEN}

\subsection{VERSCHLUSSLAUTE (EXPLOSIVE)}

Deutsch /b, p/: d, t/ $: / \mathrm{g}, \mathrm{k} /$

Bulgarisch $/ b, b^{\prime} ; p, p^{\prime} / d^{\prime}, d^{\prime} ; t, t^{\prime} / ; / g, g^{\prime} ; k, k^{\prime} /$

\subsection{PHONETISCHE CHARAKTERISTIK DER DEUTSCHEN UND DER BULGARISCHEN VERSCHLUSSLAUTGRUPPE}

b. $p-b, p / ; i d, t-d, t / ; / g, k-g, k /$

Dic deutschen Verschlußlaute und ihre bulgarischen harten Entsprechungen werden durch folgende gemeinsame artikulatorische Merkmale gekennzeichnet :

Bei ihrer Bildung wird der gesamte Ansatzraum vollkommen verschlossen, was eine kurzzeitige Unterbrechung des Luftstromes bewirkt. 
- Der Verschluß wird durch eine aktive Bewegung des artikulierenden Organs überwunden.

- Eine unerläBliche Voraussetzung für die Bildung der deutschen und der bulgarischen Verschlußlaute ist der feste Abschluß des Nasenraumes durch die Hebung des Gaumensegels.

Unterschiedliche artikulatorische und akustische Merkmale weist sowohl die stimmhafte als auch die stimmlose Reihe der deutschen und der bulgarischen Verschlußlaute auf.

Die phonetisch-phonologischen Unterschiede lassen sich wie folgt zusammenfassen :

- Das Unterscheidungskriterium „stimmhaft - stimmlos“ ist fur die bulgarischen Verschlußlaute von Bedeutung; im Deutschen ist es nicht in allen Fällen zusammenhängender Rede anzutreffen. Hier bleibt der Gegensatz „Lenis - Fortis“ („Gelindlaut-Strammlaut“ bei Trubetzk o y, 1939) erhalten.

- Bei der Bildung der deutschen [p, $t, k]$ entsteht durch die schnelle und aktive Verschluß-Lösungsbewegung des artikulierenden Organs und die daraus folgernde Entspannung der Luftsäule ein hörbarer Hauch. Deshalb wird diese Gruppe als die der behauchten Verschlußlaute (Fortes) bezeichnet. Die Verschluß-Lösungsphase der bulgarischen $[\mathrm{p}, \mathrm{t}, \mathrm{k}$ ] dauert etwas länger an, und die Muskelspannung des artikulierenden Organs ist geringer. Daher werden die bulgarischen Verschlußlaute $[p, t, k]$ nicht behaucht (Siehe S. 156

Sonagramme mit sichtbarem Unterschied* der Lösungsphase der deutschen $[p, t, k]$ und $\operatorname{der}$ bulgarischen $[p, t, k])$.

-- Die Lösung des Verschlusses bei den deutschen $[b, d, g]$ geschieht zwar bei geschlossener Glottis im Gegensatz zu [p, t, k], „doch wird der Luftstrom aus der Lunge zuerst an den Stimmlippen gebremst, die sofort mit dem Einsetzen des Luftstromes wieder zu schwingen beginnen. Deshalb ist zwar eine Sprengung des Verschlusses, die Plosion, hörbar, aber kein nachfolgender Hauch. Deshalb wird die Gruppe der mit geschlossener Glottis produzierten Verschlußlaute $[\mathrm{b}, \mathrm{d}, \mathrm{g}]$ auch als die der nichtbehauchten (Lenes) bezeichnet. Ihre Stimmhaftigkeit aber kann im Deutschen fehlen" (Lin d n e r, 1981, 250). Die bulgarischen Verschlußlaute $[b, d, g]$ sind immer stimmhaft, so daß das unterscheidende Merkmal zwischen den beiden bulgarischen Verschlußlautgruppen eindeutig das Vorhandensein bzw. Fehlen der Stimmhaftigkeit ist.

Akustisch sind die einzelnen Verschlußlaute kaum voneinander zu unterscheiden. Ihre Spektralstruktur wird vor allem durch die kurzzeitige Schallunterbrechung und die breitbandigen, kurzzeitigen Geräusche charakterisiert, die die lautsprachlichen Zeichen vertikal gliedern (L ind ne r, 1981, 260). Die gute auditive Differenzierung der Verschlußlaute voneinander wird durch die Art, wie sie sich mit den Vokalen verbinden, gewährleistet. „Die wesentliche Information, welcher Verschlußlaut gesprochen worden ist, liegt nicht in der VerschluBbildung, sondern in dem Ubergang zum folgenden Laut, der mit einer ganz bestimmten Art der Offnungsbewegung verbunden ist ; diese

- Sichtbar ist die hohe Frequenzzone des Geräusches bei den deutschen Explosiven. Beim bulgarischen [ $k$ ] isı die Geräuschbildung während der Lösungsphase etwas intensiver als bei [p] und $[1]$. jedoch liegt die Frequenzzone beträchtlich tiefer als die des deutschen [k]. 
erfolgt an einer für die Verschlußbildung typischen Stelle des Ansatzraumes. Daher sind beim Übergang von Verschlußlauten zu Vokalen auch für die einzelnen VerschluBlaute bestimmte akustische Ursprungsstellen (Loci) festgestellt worden, von denen die Formantenstrukturen der Vokale herzukommen scheinen. In dieser für jeden Verschlußlaut typischen Locus-Bildung, die mit einer Verbiegung der vokalischen Formantenstrukturen einhergeht, liegt die eigentliche akustische Information über den Verschlußlaut" (F a n t, 1960, 268f, zit. nach Lind ner, 1982, 260).

$/ b, p-b^{\prime}, p^{\prime} / ; / d, \mathrm{t}-d^{\prime}, t^{\prime} / ; / g, \mathrm{k}-g^{\prime}, \mathrm{k}^{\prime} /$

Das unterschiedliche Merkmal zwischen den deutschen Verschlußlauten und ihren bulgarischen weichen Entsprechungen läßt sich phonetisch folgendermaßen beschreiben :

Für die Bildung der bulgarischen weichen Verschlußlaute braucht man eine zusätzliche Zungenartikulation, die auf der Basis der harten Verschlußartikulation realisiert wird. Bei den labialen Konsonanten wird sie durch die leichte Hebung der Mittelzunge zum harten Gaumen realisiert; bei den dentalen ... durch eine leichte Verschiebung der Artikulationsstelle zu dem unteren Teil des Alveolargebietes nach oben und bei den velaren - durch die völlige Verlegung des Verschlusses in das Gebiet des harten Gaumens. Die zusätzliche Artikulation verändert die Konfiguration des Ansatzraumes in Richtung des Vokals [1], woher auch die spezifischen Spektralcharakteristika der palatalen Explosive resultieren. Der palatale Effekt ist am stärksten bei den velaren Explosiven wegen der größeren Veränderung der Ansatzraumkonfiguration.

Diese zusätzliche Artikulation führt akustisch zu einigen Unterschieden in der Spektralstruktur der palatalen Konsonanten im Vergleich zu den nicht palatalen und ihren deutschen Entsprechungen, und zwar : Der Geräuschanteil in der Anfangsbildungsphase wird stärker ; es verändern sich die Richtung und die Dauer des Übergangssegments zum nachfolgenden Vokal.

\subsection{PARADIGMATISCHE UND SYNTAGMATISCHE DARSTELLUNG DER DEUTSCHEN UND DER BULGARISCHEN EXPLOSIVE}

Die deutschen und die bulgarischen Verschlußlaute treten in allen drei möglichen Positionen auf : an-, in- und auslautend (initial, medial und final). In initialer und medialer Position lassen sich, mit wenigen Ausnahmen, die die weichen Verschlußlautphoneme des Bulgarischen betreffen, Minimalpaarbeispiele anfuhren, wodurch ihre distinktive Funktion bestätigt werden kann. In finaler Position kennen beide Sprachen die Auslautverhärtung, so da $B$ auslautend nur Fortes- (für das Deutsche) bzw. stimmlose VerschluBlaute (für das Bulgarische) paradigmatisch möglich sind. Im folgenden seien in konfrontativer Sicht Minimalpaarbeispiele in ein- und mehrdimensionaler Opposition angeführt. 
6.3.1. Oppositionen der Fortis-Korrelation (für das Deutsche) bzıw. der Korrelation stimmlos/stimmhaft (für das Bulgarische)

(eindimensional) :

d. $/ \mathrm{p} /-/ \mathrm{b} /$ : pellen - bellen ['pelan] - ['belan]

Staupe - Staube l'Stâopə] - ['Stâobə]

b. $/ \mathrm{p} /-/ \mathrm{b} /$ : пия - бия ['pna] - ['bıа]

cona - coбa ['s'spa] - ['ssba]

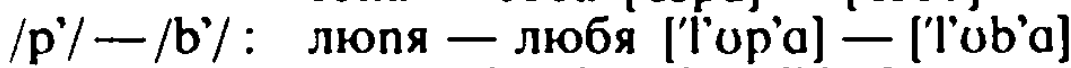

пяx - бяx [p’ax] - $\left[b^{\prime} a x\right]$

d. $/ \mathrm{t} /-/ \mathrm{d} /:$ Tank - Dank $[\operatorname{tank}]$ - [dank]

Seite - Seide ['záeto] — ['záedo]

b. $/ \mathfrak{t} /$-/d/: Танка - Данка ['tanka] - ['dayka] вата - вада ['vata] - ['vada]

$/ \mathrm{t} /-/ \mathrm{d} \%:$ тясно - дясно ['t'asno] - ['d'asno]

слетя - следя [sle't'a] - [sle'd'a]

d. $/ \mathrm{k} /-/ \mathrm{g} /$ : Kunst - Gunst [kunst] - [gunst]

Rocken - Roggen ['rokən] - ['rogən]

b. $/ \mathrm{k} /-/ \mathrm{g} /$ : кост - гост [kost] - [gost]

Лука - луга [tu'ka] - - [tu'ga]

$/ \mathbf{k}^{\prime} /$ - /g'/: кьорчо -- Гьорчо ['k'ərtfo] - ['g'ərtfo]

Oppositionen zu Nasalen (eindimensional):

d. $/ \mathrm{b} /-/ \mathrm{m} /:$ Biene - Miene ['bi :na] — ['mi : no]

b. $/ \mathrm{b} /-/ \mathrm{m} /$ : бухал мухал ['buxal]. ['muxal]

беся - меся ['bes'a] - ['mes'a]

рима риба ['rıma] ['rıba]

$/ b^{\circ} / / m^{\prime} /:$ бяx - мяx $\left[b^{\prime} a x\right]-\left[m^{\prime} a x\right]$

d. /d/ /n/: Dame - Name ['da :ma] ['na:ma]

baden - bahnen ['ba :dan] - ['ba : nən]

b. $/ \mathrm{d} /-/ \mathrm{n} /$ : дар - нap [dar] - [nar]

вада - вана ['vada] - ['vana]

$/ \mathrm{d}^{\prime} /-/ \mathrm{n}^{\prime} \%$ : видя - виня [vi'd'a] - [vi'n'a]

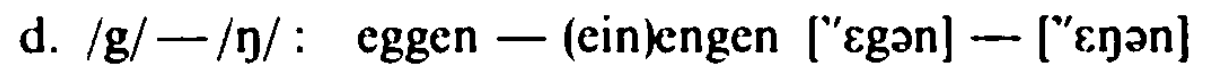

Oppositionen zu anderen Explosiven (eindimensional):

d. $/ \mathrm{p} /-/ \mathrm{t} /:$ Panne - Tanne ['pana] - ['tana]

Mappe - Matte ['mapo] ['mato]

b. $/ \mathrm{p} /-/ \mathrm{t} /$ : nop - тор [por] [tor]

пипам питам ['pipam] - ['pitam]

$/ p^{*} / \quad / t^{*}:$ лепя - летя [le'p'a] - [le't'a] 


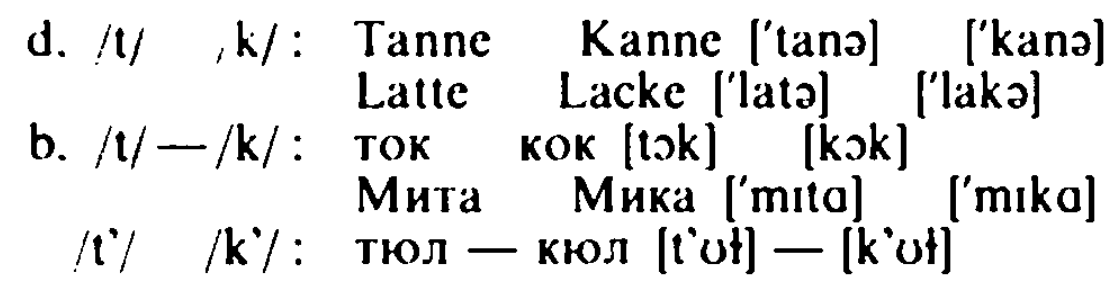

d. $/ \mathrm{p} /-/ \mathrm{k} /$ : Panne Kanne ['panə] - ['kanə]

Poppen - Pocken ['popon] - ['pokən]

b. $/ \mathrm{p} /-/ \mathrm{k} /$ : nak kan [pak] [kap] kana kaka ['kapa] ['kaka]

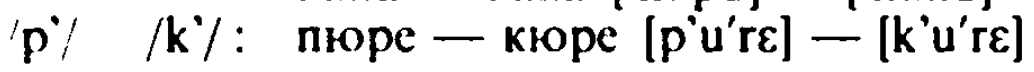

d. $/ \mathrm{b} /-/ \mathrm{d} /$ : Born - Dorn [born] - [dorn]

laben laden ['la :ban] - ['la : dan]

b. $/ b /-/ d /$ : бесен - десен ['besen] ['desen]

$/ b^{\prime} \%-/ \mathbf{d}^{\prime} \%$ : бясно - дясно ['b'asno] ['d'asno]

d. $/ \mathrm{d} /-/ \mathrm{g} /$ : Daumen Gaumen ['dôomən] - ['gôomən]

b. $/ \mathrm{d}^{\prime} \quad / \mathrm{g} /$ : дума - гума ['duma] ['guma] сядам - (по)сягам ['s'adom] - ['s'agam]

$/ \mathrm{d}^{\prime} /-/ \mathrm{g} /$ : дюля гюля (диал.) ['d'ul'a] - ['g'ul'a]

d. $/ \mathrm{b} /-/ \mathrm{g} /$ : Becken Gecken ['bekan] - ['gekon]

b. $/ \mathrm{b} /-/ \mathrm{g} /$ : бира rира ['bira] ['gira] laben - lagen ['la :bon] ['la :gon]

$\mathrm{b} \%-/ \mathrm{g} / \mathrm{:}$ бюpo Гюpo ['b'uro] - ['guro]

6.3.2. Distribution und Kombinatorik der Explosive im Deutschen und im Bulgarischen (Übersicht auf der Grundlage

von An- und Auslautstrukturen von konsonantischen Allophonen)

\subsubsection{Anlautstrukturen}

Sämtliche Explosive treten in beiden Sprachen vor Vokal auf. Einschränkungen sind allein die bulgarischen weichen Verschlußlaute unterworfen, die vor $/ \varepsilon /, / 1 /$ und $/ 2 /$ nicht vorkommen. Beispiele :

Deutsch: Panne, Bauer; Tinte, Donner; Köder, günstig;

Bulgarisch : пиле. болен; пял, бял ; том, дума ; тясно, десен, дюля ; кърт, гъба: кюп, гьол.

Gedoppelt kommen weder die deutschen noch die bulgarischen Explosive in Initialposition vor.

Wesentliche Unterschiede sind bei zwei-bzw. dreigliedrigen Verbindungen mit Konsonanten festzustellen. Sie lassen sich folgendermaßen systematisieren : 
Deutsch

1. Explosive schließen einander aus.

2. Fortes und Lenes lassen sich mit anderen Konsonanten kombinieren. Dabei sind folgende zweigliedrige Verbindungen möglich :

/br/ braun [brâon]

/bl/ blau [blâo]

/pr/ Prall [pral]

/pl/ Platz [plats]

/pn/ Pneuma

['pnjoma]

/dr/ Drang [dray]

$/ \operatorname{tr} /$ treu [tróø]

/ts/ zehn [tse : $\mathrm{n}]$

$/ \mathrm{t} \int /$ tschüs [t $\left.\int \mathrm{y}: s\right]$

/gr/ grau [gráo]

/gl/ Glanz [glants]

/gn/ Gnade ['gna :da]

/kr/ Kraut [kraoot]
Bulgarisch

1. Zwei Explosive treten - wenn auch selten - nebeneinander auf: птица, где, бдя, ктитор.

2. Die stimmlosen und die stimmhaften Explosive haben im Anlaut grōßere Kombinationsmōglichkeiten mit anderen Konsonanten als die deutschen Fortes und Lenes. Es sind folgende zweigliedrige Verbindungen möglich : /br/ 6par [brat] /br'/ бряг [br'ak] /bt/ благ [błak] /bl'/ блян [bl'an] /pr/ npar [prak] /pr'/ пряк [pr'ak] /pt/ плач [ptats] /pl'/ пляс [pl'as] /pn/ пневматика [pnev'matika]

/ps/ nce [pse]

/pt/ птица ['ptitsa]

/pts/ пчела [ptse'ta]

$/ \mathrm{p} \int /$ пшеница [pse'nitsa]

/dr/ apar [drak]

/dr'/ дрян [dr'an]

/dł/ длан [dłan]

/dn/ дневен ['dneven]

$/ d v /$ две [dve]

$/ \mathrm{tr} /$ три [trr]

/tr'/ трясък ['tr'asək]

/tt/ тласьк [tłasək]

/tv/ твой [tvoii]

/gr/ rpax [grax]

/gr\%/ rpgx [gr'ax]

/gl/ глеч [glet]]

/gn/ гнет [gnet]

/gn/ гняв [gn'an]

/gm/ rмyреu [gmu'rets]

/gv/ гвоздей ['gvozdei]

/gd/ rдe [gde]

$/ \mathrm{kr} /$ кpak [krak]

/kr'/ крясък ['kr'asək] 

/kl/ klauen ['klâoəon]
$/ \mathrm{kn} /$ knacken ['knakən]
/kv/ Quelle ['kvela]
/ks/ Xerox ['kse : roks]

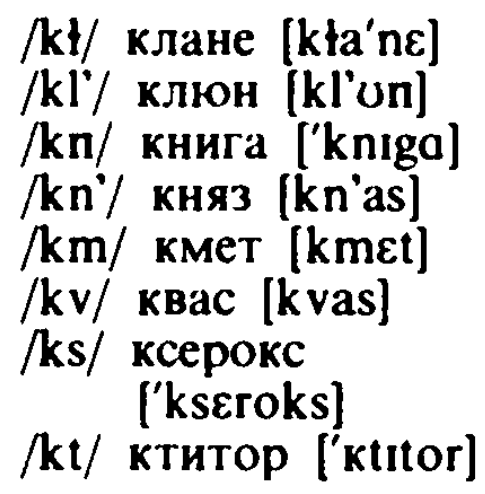

6.3.2.2. Kombinatorik der Explosive mit Konsonanten als allophonische Auslautstrukturen

Im absoluten Auslaut vor Pause sind sowohl im Deutschen als auch im Bulgarischen keine stimmhaften Allophone möglich (Auslautverhärtung); im Bulgarischen auch keine palatalisierten Allophone.

Im Deutschen und im Bulgarischen sind folgende zweigliedrige Auslautstrukturen mit Explosiv im absoluten Auslaut möglich : $(\sim=$ Vorkommen der Verbindung in Fremdwörtern)

Deutsch

/p/ Kalb [kalp]

Alp [alp]

/mp/ plump [plump]

/rp/ derb [derp] Gezirp [ga'tsırp]

$/ k t / J a g d$ [ja:kt] nackt [nakt]

/pt/ Abt [a:pt] Haupt [hoopt]

/ft/ Schrift [srift]

/It/ bald [balt] Welt [velt]

/mt/ Hemd [hemt] Amt [amt]

/nt/ Band [bant] bunt [bunt]

$/ \mathrm{t} /$ bedingt [bo'dint]

$/ r t /$ Pferd [pfe : $r t]$ Wort [vort]

/st/ Fest [fest]

/ct// Recht [reçt]

/xt/ Tracht [traxt]

/lk/ Volk [folk]

/jk/ Wink [vink]
Bulgarisch

/tp/ стьлб [stałp] пулn [potp]

/mp/ - ромб [romp]

/rp/ rep6 [gerp]

cъpn [sarp]

$/ k t / \sim$ nakt [pakt]

/pt/ копт [kopt]

/ft/ дзифт [dzift]

/tt/ жълт [3att]

/nt/ бинт [bint]

/rt/ смърт [smart]

/st/ noct [post]

/çt/ Bext [veçt]

/tk/ дълг [dətk]

вълк [vətk]

/nk/ гонг [gonk] 
/rk/ Berg [berk]

Werk [verk]

/sk/ 〜 brüsk [brysk]

$/ \mathrm{st} /$ Gischt [gIJt]

$$
\begin{aligned}
& \text { /rk/ корк [kərk] } \\
& \text { /sk/ риск [risk] } \\
& / \mathrm{st} \text { мош [most] } \\
& \text { вожд [vost] }
\end{aligned}
$$

Wie aus der Gegenüberstellung ersichtlich, ist eine einzige Auslautstruktur im Deutschen nicht in ursprünglich deutschen Wörtern möglich:/sk/. Im Bulgarischen dagegen sind 7 Auslautstrukturen nur in gebräuchlichen Fremdwörtern möglich. Es fehlen außerdem Entsprechungen der deutschen Auslautstrukturen $/ \mathrm{mt} /, / \mathrm{nt} /, / \mathrm{xt} /$.

Diachronisch gesehen, steigt die Anzahl der möglichen konsonantischen Auslautverbindungen bei der Entwicklung vom Mittelhochdeutschen zum Neuhochdeutschen steil an. Ursache dafür ist der Ausfall des Endsilben-[a] in den verschiedenen Konjugations- und Deklinationsformen im Neuhochdeutschen. So weist das Neuhochdeutsche etwa 50 neue dreigliedrige Konsonantenverbindungen im Auslaut gegenüber 12 des Mittelhochdeutschen. Viergliedrige konsonantische Auslautverbindungen gibt es im Mittelhochdeutschen überhaupt nicht. Bei der Entwicklung zum Neuhochdeutschen sind degegen etwa 30 viergliedrige Auslautverbindungen entstanden. Was die Explosive als Endglied der drei- bzw. viergliedrigen Auslautstruktur betrifft, so kommt hier allein das Allophon [t] in Frage, [p] und $[k]$ erscheinen nicht.

Konsonantische Anhäufungen von drei- und mehr Gliedern sind als Auslautstrukturen im Bulgarischen unmöglich. Man könnte nur auf sehr wenige Fremdwörter mit dreigliedriger konsonantischer Auslautstruktur verweisen : текст, карст.

Im folgenden sind die im Deutschen möglichen drei- und viergliedrigen Auslautstrukturen mit $[t]$ im absoluten Auslaut angeführt.

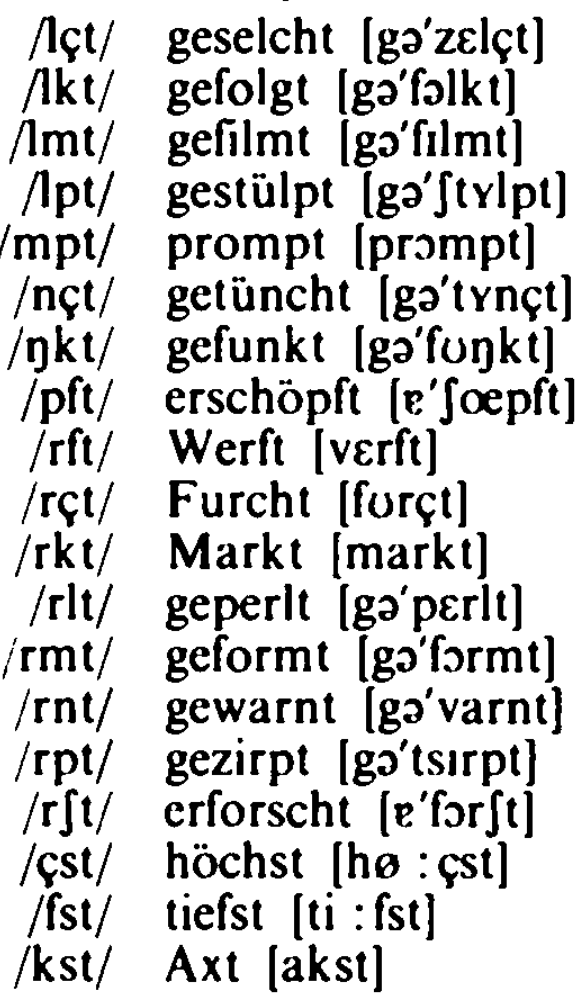


/st/ Schwulst [Svolst]

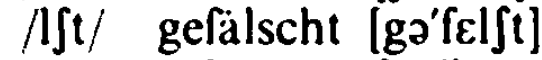

/mst/ gebremst [ga'bremst]

$/ \mathrm{m} \int \mathrm{t} /$ geramscht [ga'ramft]

inst/ Dienst [di : nst]

inft/ gewünscht [gə'vynft]

/nst/ Angst [anst]

/pst/ Propst [pro:pst]

/rst/ Durst [durst]

$/ \int s t /$ (du) duschst [du : $\left.\int s t\right]$

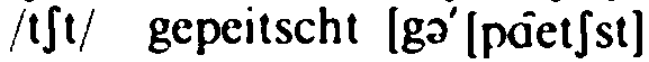

Viergliedrige Auslautstrukturen mit $/ \mathrm{t} / \mathrm{im}$ absoluten Auslaut:

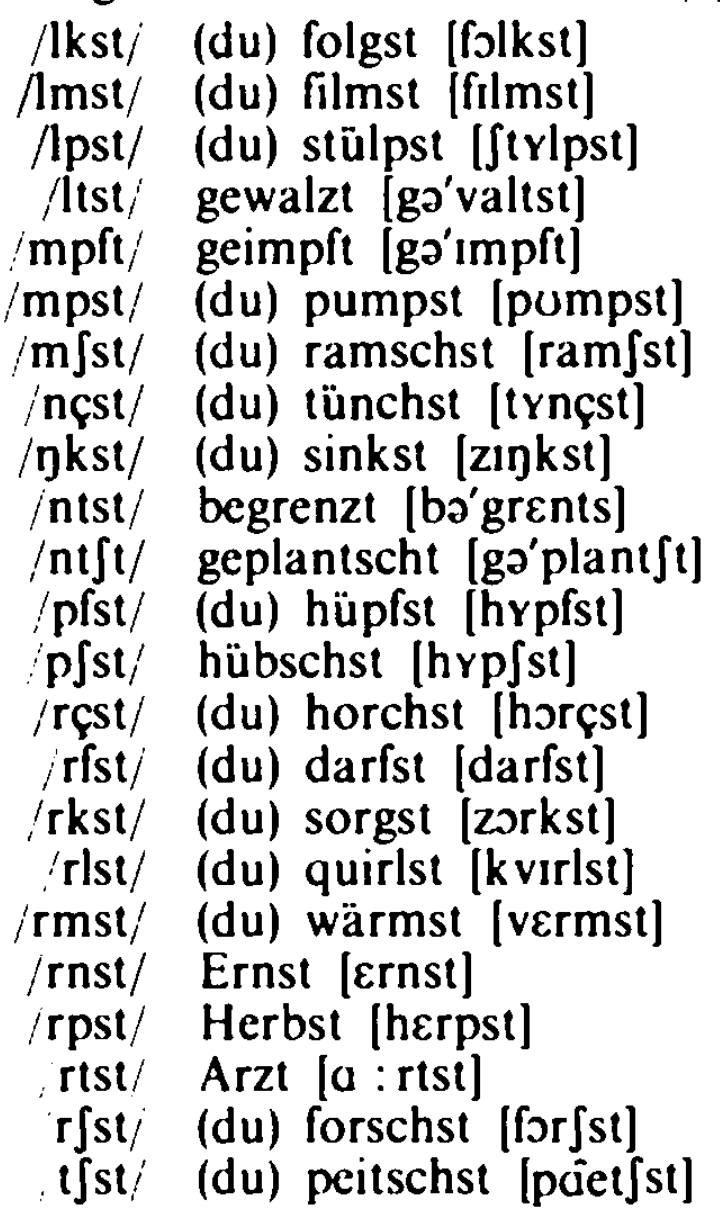

Verben, deren Infinitiv schon dreigliedrige Auslautstrukturen aufweist, ergeben in der 2. Person Singular vier- und füngliedrige Konsonantenverbindungen : rümpfen - du rümpfst [rympfst] ; impfen - du impfst [Impfst] ; plantschen - du plantschst [plant Jst]; röntgen du röntgst [rœentkst].

\section{ENGELAUTE (FRIKATIVE)}

Deutsch : /v, $/ / ; / z, s / ; / j /, / \mathfrak{c} / ; / \mathrm{J} / ; / \mathrm{x} / ; / \mathrm{h} / ;$

Bulgarisch: $/ v, v^{\prime} ; f, f \% / z, z ; s, s^{\prime} /: / 1 / ; / 3 / ; / \int / ; / x /$

Die Erzeugung der Engelaute ist ein komplizierter Prozeß. Die Ausbildung der jeweiligen Hemmstelle im Ansatzraum durch die Annäherung des 
artikulierenden Organs an die Artikulationsstelle erfolgt wie bei den Verschlußlauten unter Abschluß des Nasenweges. Bei allen Engelauten wird der Weg des Luftstromes durch den Ansatzraum so stark verengt, da $B$ er eine hohe Geschwindigkeit erreicht, um die als Enge ausgebildete Hemmstelle überwinden zu können. „Diese hohe Strömungsgeschwindigkeit führt dazu, daß eine turbulente Strömung entsteht ; dadurch wird ein Geräusch erzeugt, das durch die vor der Enge liegenden Hohlräume seinerseits überformt wird" (Lindner, 1981, 252). Alle Engelaute lassen sich im Gegensatz zu den Verschlußlauten als isolierte Laute produzieren.

Da trotz der allgemeinem Bildungszüge die Artikulation der einzelnen Engelaute kompliziert ist, weil es zwischen den deutschen und den bulgarischen Engelauten phonetische und phonologische Unterschiede gibt, erfolgt im nächsten ihre konfrontative Darstellung gruppenweise, so wie sie nach phonetischen Prinzipien eingeteilt worden sind (Siehe Tab. S. 73).

\subsection{LABIODENTALE FRIKATIVE}

/d. $v-$ b. $v /-/$ d. $f-$ b. $f / ; /$ d. $v-$ b. $v /-/$ d. $f-$ b. $f \%$

Man beobachtet artikulatorisch keinen Unterschied zwischen den deutschen labiodentalen Frikativen und ihren bulgarischen harten Entsprechungen. Die labiodentale Hemmstelle wird in beiden Sprachen durch leichte Berührung der Unterlippe mit den oberen Schneidezähnen, bzw. durch ihre Annäherung bis auf einem schmalen Spalt hergestellt. Die Phonationsluft entweicht - - tönend oder nicht tönend - in breitem Strahl, der perzeptiv als „weiches Geräusch“ zu klassifizieren ist. Die Zunge ist an der Bildung nicht beteiligt. Das Gaumensegel ist gehoben.

Akustisch festgelegt und perzeptiv beurteilt, ist das frikative Geräusch beim deutschen fortis [f] stärker als beim bulgarischen stimmlosen [i].

Das deutsche Lcnis-[v] und das bulgarische stimmhafte [v] unterscheiden sich akustisch wesentlich von [d.f]. [b.I] und zwar nicht allein durch den Stimmanteil, sondern auch durch ihre Formantenstruktur. Während [d. П] und [b. I] ein typisch frikatives Spektralbild ergeben, zeigen [d. v]. [b. v] eher eine sonorähnliche Struktur mit gut erkennbaren Formantenstreifen (Siehe Sonagramme S. 157*).

Akustisch lassen sich die deutschen [ $\mathrm{v}, \eta]$ und die bulgarischen $[v, \eta]$ nach ihrer Geräuschdauer als ununterbochen, nach der Schallenergiekonzentration als diffus und nach dem auditiven Effekt als tief und hart (bemol) bestimmen.

Die bulgarischen palatalen [ $\left.v^{\prime}, f^{\prime}\right]$ werden im Unterschied zu den nichtpalatalen $[v, f]$ und den deutschen $[v, f]$ durch eine zusätzliche Hebung der Mittelzunge zum harten Gaumen in Richtung i-Bildung artikuliert. Akustisch weisen sie das zusätzliche Merkmal „diés“ auf, auditiv lassen sie sich als .weich“ bestimmen.

- Am Sonagramm ist das intensive Gerāusch des [d. I] und [b. П] sichtbar; bei [d. v] und [b. v] sind $F_{1}$ und $F_{2}$ gut erkennbar. Weiter ist auch die lenisierte [b]-Bildung im deutschen ['bra:vo] und cie stimmhafte Bildung des bulgarischen [b] in ['bravo] zu beobachien. 


\title{
7.2. PRÄDORSAL-ALVEOLARE FRIKATIVE
}

\author{
/d.z - b.z/ - /d.s - b.s/; /d.z-b.z'/ - /d.s - b. s'/
}

Bei der prädorsal-alveolaren Engebildung strömt die Luft in eine Enge, die sich nach dem vorderen Teil der Zunge hin immer mehr verengt. Der an der Hemmstelle gebildete Kanal ist rinnenähnlich und sehr schmal, wodurch die Luft eine sehr hohe Strömungsgeschwindigkeit erhält. Dadurch ist die Ausbildung von Wirbeln sehr aktiv. An der oberen Kante der unteren Scheidezähne, worauf der feine Luftstrahl trifft, wird er gebrochen, so daß an dieser Schneide nochmals Wirbel entstehen. Durch die zweifache turbulente Geräuschbildung entsteht ein scharfes, hoch abgestimmtes Geräusch, das auditiv als „Zischlaut" charakterisiert wird. Die Schallstruktur dieses scharfen Geräusches erreicht ihr Maximum bei 6000 bis $8000 \mathrm{~Hz}$.

Sowohl die deutschen S-Laute als auch die bulgarischen harten $[z, s]$ lassen Bildungsvarianten zu, die obwohl mit anderen artikulatorischen Mitteln (dorsale, apikale S-Bildung) doch einen gleichwertigen Effekt erzeugen. Bei der Bildung der S-Laute können in beiden Sprachen verschiedene Fehlbildungen auftreten, die als Sigmatismen bezeichnet werden.

Akustisch lassen sich die deutschen $[z, s]$ und ihre bulgarischen harten Entsprechungen $[z, s]$ nach ihrer Geräuschdauer als ununterbrochen, nach der Schallenergiekonzentration als diffus und nach dem auditiven Effekt als hoch bestimmen.

Die palatale Hemmstellenbildung für die bulgarischen [ $\left.z^{\prime}, s^{\prime}\right]$ wird durch eine leichte Verschiebung der Artikulationsstelle vom unteren zum oberen Teil des Alveolargebietes und durch einen engeren Kontakt des äußeren Randes der Mittelzunge mit dem harten Gaumen erzielt. Die dadurch bewirkte Veränderung in der Konfiguration des Ansatzraumes ist eine geringe, so da $B$ die "palatale" Information eher in den Formantenübergängen zum nachfolgenden Vokal liegt.

Akustisch weisen sie das Merkmal „diès“ auf, auditiv sind sie als „weich“ einzuschätzen.

\subsection{PRÄDORSAL-PALATALE FRIKATIVE}

/d.j - b. $1 /$; /d. c - b.c $/$;

d. $/ \mathrm{j} / \mathrm{i}-\mathrm{b} . / \mathrm{l} /$

Je nachdem, ob ein Geräusch an der Hemmstelle entsteht, wird der erzeugte Lautklang zu der Klasse der Vokale oder der Konsonanten gerechnet.

Artikulatorische Ausgangsbasis fur die Bildung des bulgarischen [!] ist die Bildung des Vokals [1]: Die Zunge wird nach vorn verlegt und wölbt sich mit dem vorderen und mittleren Zungenrücken gegen den vorderen Teil des harten Gaumens. Die Zungenränder liegen seitlich am Hartgaumen, die Zungenspitze hat Kontaktstellung. Das Gaumensegel ist gehoben, die Stimmlippen schwingen. Das längere Einhalten der anfänglichen Bewegungen wird durch ein Hinübergleiten zum angrenzenden Vokal unterbrochen. Deshalb bezeichnet D. Tilkov das bulgarische [I] als .glide“, Gleitlaut 
(Тилков, 1975, 35). Ein Reibegeräusch kann bei dieser gleitenden Artikulation als zweitrangiges Merkmal auftreten, ist jedoch fur die Identifizierung des Lautes nicht notwendig. In der Position zwischen zwei Vokalen konnte z. B. Tilkov keine Reibung beobachten.

Im Vergleich zum bulgarischen [I] wird deutsches [j] mit etwas kleinerer und zeitlich längerer Engebildung, größerer Artikulationsspannung und stärkerem Expirationsdruck realisiert. Das Reibegeräusch ist obligatorisch und stärker als beim bulgarischen [I] (Siehe Sonagramme zum deutschen [j] und bulgarischen $[1]-S .158-160$ ).

Die phonematische Wertung des deutschen [j] ist umstritten. Der Streit besteht darin, ob man es als Konsonantenphonem oder aber als Halbvokalallophon interpretieren sollte. Trube tzk o y $(1971,64)$ wertet [j] als $i$-Allophon wegen seiner mit [i:, l] supplementären Distribution. Ebenfalls als Allophon des Phonems /i/ behandelt es auch Morciniec $(1958,64)$. Für das Phonem /i/ gibt er folgende drei Varianten an:

1. In akzentuierter Stellung: kurzes offenes [1], z. B. ist, Biß.

2. Nach akzentuiertem Vokal: [I], d.h. Laut mit zwischen [1] und [e] variierendem Öffnungsgrad, z. B. frei, läuten.

3. Vor akzentuiertem Vokal: enges geräuschhaftes [j], z. B. jung, ja.

Gegen eine allophonische Zuordnung des /j/ zum Vokalphonem /i/ wendet sich Werner $(1972,47)$ mit Beispielen wie jiddisch, injizieren, plehejisch. $\mathrm{Zu}$ beachten ist auch seine systembezogene Begründung. daß die Bewertung des /j/ als i-Variante die im Deutschen sonst durchgehende Zweiteilung in silbentragende Vokale - marginale Konsonanten durchkreuzt.

Von O.v. Essen (1979, 109). Meinhold und Stock (1982, 139). Lindner $(1981,255-256)$ und Wurzel $(1984,975)$ wird das /j/ als konsonantisches Phonem bestimmt.

Im phonologischen System der bulgarischen Konsonanten nimmt das [1] eine Sonderstellung ein. Es hat weder eine stimmlose Entsprechung noch eine palatalisierte Form. Das Gesamt seiner Charakteristika ordnet das [I] in die Gruppe der Konsonantenphoneme, seine Distribution ist aber selten eine konsonantische. Distinktive Oppositionen wie твой - твои, порой - порои beweisen jedoch seinen selbständigen phonologischen Status und sprechen gegen seine Bewertung als Allophon des i-Vokals.

Das bulgarische Phonem / $/$ erscheint immer an einen Vokal gebunden, indem er mit ihm zusammen eine Art Diphthong bildet. Insgesamt sind 11 1-Verbindungen mit Vokal möglich. In fünf davon erscheint es als erstes

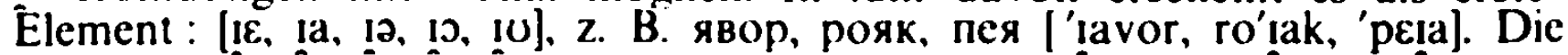
Lautverbindung [ï] ist im Bulgarischen unmöglich. In sechs Lautverbindun-

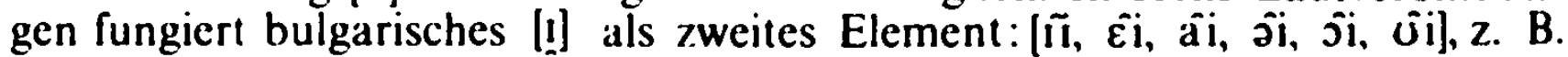
айран, майка, край : тъй ; пейка, шшибой, пийна : кройка; пуйка. Zwischen zwei Vokalen (die sog. Jotation): тоягa [to'yaga]. отчаяи [ot'tfa!an]. Sehr

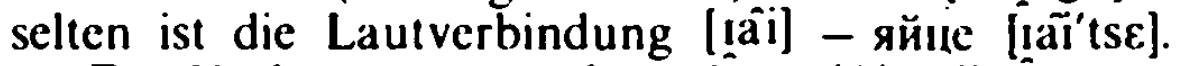

Das Vorkommen von deutschem / $\mathrm{j}$ / ist distrıbutionell stark beschränkt. Es kann nur in Silbenanlaut vor Vokalen (außer vor i) auftreten: Jahr, jedoch, jene, Jugend: Objekt, Maja. Mayonnaise. In Positionen nach Vokal, wo das bulgarische/!/ auch vorkommt, tritt im Deutschen kein Reibelaut, sondern ein Vokal in Erscheinung: Heide, heute. 


\section{d. $[\varsigma]-h \cdot[\zeta]$}

Die Bildungsweise des deutschen stellungsbedingten Allophons [c] ist die des [i]. nur daß es stimmlos erzeugt wird.

Das bulgarische palatalisierte [c], das vor und nach den Vokalen [1] und [E] erscheint, ähnelt dem deutschen [c], ist ihm aber sowohl artikulatorisch als auch akustisch und distributionell nicht gleichzusetzen. Im Unterschied zum deutschen [s] wölbt sich die Mittelzunge zum hinteren Teil des harten Gaumens, und ihre seitlichen Ränder werden an den harten und weichen Gaumen gelegt. Dabei ist die Berührungsfläche größer als beim bulgarischen $[x]$ in [xo'ro]. Das bulgarische Allophon [c] ist stärker palatalisiert als andere

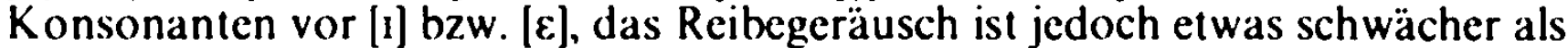
beim deutschen [c] (Siehe Sonagramme des deutschen [c] und bulgarischen [c] - S. 167).

Akustisch lassen sich das deutsche [c] und das bulgarische [c] als Geräuschkonsonanten mit einem ununterbrochenen diésen Spektrum und kompakter Energieverteilung bestimmen.

Die I Distribution der beiden Allophone stimmt nur in einigen wenigen Positionen und Lautumgebungen überein:

Deutsches [s]:

- in Initialposition vor $/ \mathrm{i} / \mathrm{e} / \mathrm{e} / \mathrm{in}$ wenigen Fremdwörtern: Chirurg. Chemie:

- in- und auslautend nach $/ \mathrm{i} / \mathrm{,} / \mathrm{e} / \mathrm{,} / \mathrm{y} /$, oo, o $n /, / 1 /, / r /$ : Licht, Hecht, Küche, leuchten. Fenchel, Lerche. mich, Blech, euch, manch, welch, durch (Die Inlautposition weist eine hohe Gebrauchsfrequenz. auf).
Bulgarisches [s]:

- in Initialposition vor $/ 1 /, / \varepsilon /:$ хитър, Хемyс (Diese Position weist eine hohe Gebrauchsfrequenz auf); - in- und auslautend in einigen wenigen Wörtern nach $/ \mathrm{l} /, / \varepsilon_{0} /$ : Ихтиман, стихия, ехиден, техен, вехт, стих; Folgt dem [c] jedoch ein Hinterzungenvokal, so wird das velare $/ x /$ realisiert: exo, pexaв, мexyp, uxy!

d. $[x]-b . / x /$

Sowohl das deutsche Allophon $[x]$ als auch das bulgarische „harte“ Phonem $x$ werden velar gebildet. Der organgenetische Unterschied zwischen beiden besteht darin, da $B$ im Gegensatz zum bulgarischen $/ x /$ der hintere Zungenrücken bei der Bildung des deutschen $[\mathrm{x}]$ viel stärker zum hinteren Teil des Gaumens, etwa an der Grenze zwischen hartem und weichem Gaumen, aufgewölbt wird. Es entsteht dabei entweder eine etwas engere Spalte im Vergleich zum bulgarischen $/ \mathbf{x} /$, oder es kommt zu einer leichten Berührung zwischen Zungenrücken und Velum. Daher kann das deutsche velare Allophon [x] klanglich verschiedene Färbung annehmen. „Es kann den Klang eines dumpfen Geräusches haben, das durch den davor liegenden großen Hohlraum resonatorisch tief abgestimmt wird, kann aber auch, wenn das Gaumensegel ins Schwingen gerät, ein unregelmäßiges Geräusch erzeugen“ (Lind ne r, 1982. 256-257) (Siehe vergleichende Sonagramme des deutschen $[x]$ und des bulgarischen $/ x /-S .165)$.

Akustisch lassen sich das deutsche velare $[x]$ und das bulgarische velare, „harte“ Phonem / $/$ als Geräuschkonsonanten bestimmen, deren Geräuschin- 
tensität mit ansteigender Frequenz abschwächt und etwa in der Zone um $4 \mathrm{KHz}$ am schwächsten ist. Was die Geräuschdauer betrifft, so weist das bulgarische $/ \mathrm{x}$ / ein ununterbrochenes, kompaktes Spektrum auf, während für das deutsche $[x]$ zwei Spektralbilder möglich sind - ein ununterbrochenes kompaktes und ein intermittierendes - beim Mitschwingen des Gaumensegels während der Bildung. Beide Laute weisen akustisch das zusätzliche Merkmal „bemol“ auf, auditiv sind sie als "tief" zu bestimmen.

Das deutsche $[x]$ ist distributionell beschränkt; es erscheint in deutschen Wörtern nur nach hinteren Vokalen und zwar niemals in Initialposition: Buch, Bach, Bauch; Rache, Kuchen, fauchen.

Das bulgarische ,harte“ Phonem / $x /$ hat dagegen eine viel breiter gefächerte Distribution. Im Gegensatz zum deutschen $[x]$ erscheint es auch in Initialposition vor Hinterzungenvokalen, Sonoren und dem Frikativ /v/: хала, хубав, хоро; храна, хляб, хмел; хвалба. Außerdem kommt es vor:

- anlautend nach Hinterzungenvokalen: ax! ox! ух! ухо, охлюв, охрана, охтика; ахна, ахвам;

- anlautend nach $/ \mathrm{v} /, / \mathrm{s} /:$ вход, схватка, сходен;

- inlautend zwischen zwei Hinterzungenvokalen: буха, доход, прахан, слухов ;

- inlautend nach Vorderzungenvokal, vor Hinterzungenvokal: exo, pexaB, тихо, мexyp, бaxyp;

- inlautend nach Hinterzungenvokal, vor Konsonant: тухла, смахнат, рохкав, пухче ; b. $/ \mathrm{c}^{\circ} /-d$. -

- in finaler Position nach Hinterzungenvokal: грах, дух, въздух, метох.

Das bulgarische palatalisierte Phonem /ç/ (.,weiches" [ç]) erscheint nur in Fremdwörtern und zwar allein vor [0] und hat daher eine sehr begrenzte Gebrauchsfrequenz: Хюстън. Хюйгенс. Aus diesem Grunde ist es als peripheres Element des bulgarischen Phonemsystems zu betrachten. Eine allophonische Entsprechung im Deutschen existiert nicht.

\subsubsection{Laryngal-pharyngaler Hauchlaut}

d. $/ \mathrm{h} /-$ b. -

Das deutsche Phonem /h/ hat keine Entsprechung im bulgarischen Konsonantensystem. Distrubutionell und lautkombinatorisch gesehen, entsprechen ihm im Bulgarischen entweder das palatalisierte bulgarische Allophon [c] oder das harte bulgarische /x/ (Siehe S. 165).

Unter den deutschen Konsonanten nimmt das [h] eine Sonderstellung ein. Artikulatorisch ist es als .OÖnnungsk onsonant“ zu bestimmen (E s s e n, 1979. 116). Die Enge wird bei ihm im Kehlkopf durch die Hauchstellung der Stimmlippen gebildet. An den in Hauchstellung getretenen, jedoch nicht schwingenden Stimmlippen reibt sich dann die Ausatmungsluft; dabei nimmt das Ansatzrohr bereits die Einstellung des nachfolgenden Vokals ein. Ein interessantes Bild ergeben Sonagramme des deutschen [h], wenn es zwischen Vokalen realisiert wird (Siehe Sonagramm S. 168).

Auf dem Sonagramm ist Stimmton auch beim h-Segment ersichtlich. Das vermutlich stimmhaft realisierte [h] wird dann ...als stark gehauchter Laut 
gesprochen, bei dem die Stimmlippen Randschwingungen ausfüren" (Lind ner, 1982, 258).

Bei der Realisierung des deutschen [h] durch Bulgaren ist stets ein stärkeres, palatal bzw. velar erzeugtes Geräusch herauszuhören. Das im Vergleich zur Bildung des [h] von Muttersprachlern stärkere Reibegeräusch bei dessen Artikulation durch Bulgaren ist auch aus dem vergleichenden Sonagramm ersichtlich (Siehe Sonagramm des deutschen [h] im Ausspruch: Hinter Heinrichs Hühnerhaus hängen hundert Hemden 'raus - S. 161-162).

Nach der Meinung bestimmter Phonetiker (Vgl. Гв льбо в, 1956) wird im Ausrufen axa! exe! für $\langle x\rangle$ ein dem deutschen [h] ähnlicher Laut realisiert (Siehe Sonagramm S. 169).

Verglichen mit dem deutschen [h], in intervokalischer Position(Siehe S. 168) gesprochen, zeigt bulgarisches $[\mathrm{x}$ ] in Interjektionen zwischen Vokalen ein ähnliches Spektralbild. Der Unterschied besteht lediglich darin, daß man beim deutschen [h] auch Streifen von höheren Geräuschfrequenzen beobachtet. Man sollte jedoch vielmehr Spektraluntersuchungen und zwar im natürlichen Redekontext anstellen, denn nach auditiven Beobachtungen des Vefassers realisieren Bulgaren gerade bei Interjektionen mit intervokalischem $[x]$ eine phonetische Erscheinung, die eher dem deutschen festen Vokaleinsatz nahe kommt.

\subsection{MEDIODORSAL-MEDIOPALATALE FRIKATIVE}

\section{d. $/ \int /, / 3 /^{\sim}-$ b. $/ \int /, / 3 /$}

Artikulatorisch-akustisch und perzeptiv sind sich die deutschen [S ], [3] und die bulgarischen [ $]$, [3] gleich. Die Hemmstelle für die Engebildung wird mediopalatal und mediodorsal gebildet. Dabei werden die Lippen leicht vorgestülpt, wobei der Resonanzraum für ihre Bildung vergrößert und die tieffrequenten Anteile des Geräusches dadurch verstärkt werden. Deutsche [S], [3] und bulgarische [S], [3] sind die einzigen Frikative, die mit Lippenbeteiligung und die einzigen Konsonanten, die mit Lippenrundung produziert werden. Diese phonetische Besonderheit ist für die Koartikulation bedeutsam, da sich die Lippenrundung auf voraufgehende Vokale übertragen kann. Eine solche normwidrige Erscheinung läßt sich unter Umständen vor allem bei deutschem [J] beobachten: [tIf] wie [trf].

Das akustische Spektrum von $\left[\int\right],[3]^{\sim},\left[\int\right],[3]$ ist ein ununterbrochenes und kompaktes und weist die stärkste Geräuschintensität unter den frikativen Konsonanten auf. Auditiv werden die mediodorsal-mediopalatalen Frikative als .tier" cingeschätzt.

Das Besondere am phonologischen Status der bulgarischen $/ \mathrm{S} /$ und $/ 3 /$ besteht darin, da $B$ sie keine palatalen Korrelate haben. Kontrastivphonologisch gesehen, sind deutsches / $/$ und bulgarisches / $/$ Phoneme mit recht hoher funktioneller Belastung. Hoch ist auch die Häufigkeitsfrequenz des bulgarischen [3], während die des deutschen $/ 3 /{ }^{\sim}$. .das nur in Fremdwörtern vorkommt, von untergeordneter Bedeutung ist. Nach Lind ner $(1981,256)$ liegt die Haüfigkeit seines Auftretens unter einem Promille. 


\subsection{PARADIGMATISCHE UND SYNTAGMATISCHE DARSTELLUNG DER FRIKATIVE IM DEUTSCHEN UND IM BULGARISCHEN}

7.5.1. Oppositionen der Fortis-Korrelation (für das Deutsche) bzw. de:r Korrelation stimmlos/stimmhaft (für das Bulgarische) (eindimensionall)

d. $/ \mathfrak{f} /-/ v_{/} /$: Fink - Wink [fink] - [vınk]

Frack - Wrack [frak] - [vrak]

b. $/ f /-/ v /:$ фa 3a - вa 3a ['faza] - ['vaza]

coфa - сова ['sofa] - ['sova]

флаг - влак [frak] - [vtak]

фрак - вpar [frak] - [vrak]

d. $/ \mathbf{s} /-/ z /:$ Mußc - Muse ['mu:sə] - ['mu:za]

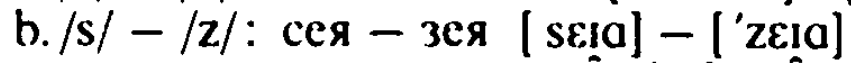

коса - коза [ko'sa] - [ko'za]

сладко-Златко ['słatko]-['złatko]

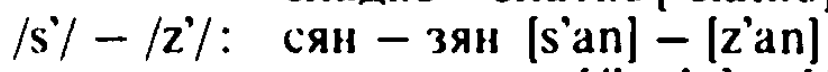

кося - козя ['kos'a] - ['ksza ]

Deutsches $/ \mathrm{h} /,[\mathrm{c}],[\mathrm{x}], / \mathrm{J} /$ und bulgarisches $/ \mathrm{x} /, / \mathrm{g} /$ treten in keinie eindimensionale Fortis/Lenis-Korrelation ein.

b. $/ \int /-/ 3 /:$ шал - жал [Sał] - [заł]

เилеп - жлеб [Slєp] - [z|єp]

срена - срежа ['sre $\left.\int \mathrm{a}\right]-[$ 'sreza]

Ein- und mehrdimensionale Oppositionen der Frikative

d. $/ \mathfrak{f} /$ - /s/: Kaffee - Kasse ['kafo] - ['kasə]

Graf - Gras [gra:f] - [gra:s]

$/ \mathfrak{f} /-/ z /$ : Fang - sang [fan] - [zan]

saufen - sausen ['zōofon] - ['zäozon]

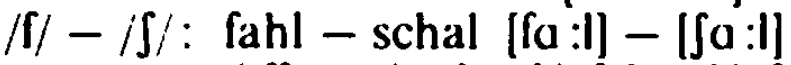

Affe - Asche ['afo] - ['a $a$ o]

raufen - rauschen ['rôofon]-

['rôosan]

$/ \mathfrak{f} /$ - /j/: faulen - jaulen ['fóolon] - ['jôolon]

$/ \mathrm{f} / \mathrm{-} / \mathrm{h} /$ : fauchen - hauchen ['fóoxəon] ['hoioxon]

$/ \mathrm{v} /-/ \mathrm{z} /:$ Wand - Sand [vant] - [zant]

$/ v /-/ \delta /:$ wohnen - schonen ['vo:nan] ['Jo:nən]

$/ \mathrm{v} / \mathrm{-} / \mathrm{j} /:$ wagen - jagen ['va:gən] - ['ja :gən]

$/ \mathrm{v} / \mathrm{-} / \mathrm{h} /:$ Weide - Heide ['vöeda] - ['höedo]

b. $|\mathbf{f} /-| \mathbf{s}|-| \mathbf{z}|-| \mathbf{s}|-| \mathbf{z}|-| \mathbf{x} /$ :

фауна - cayнa ['fauna] - ['sauna]

фap - zap - wap [far] - [zar] - [3ar] 


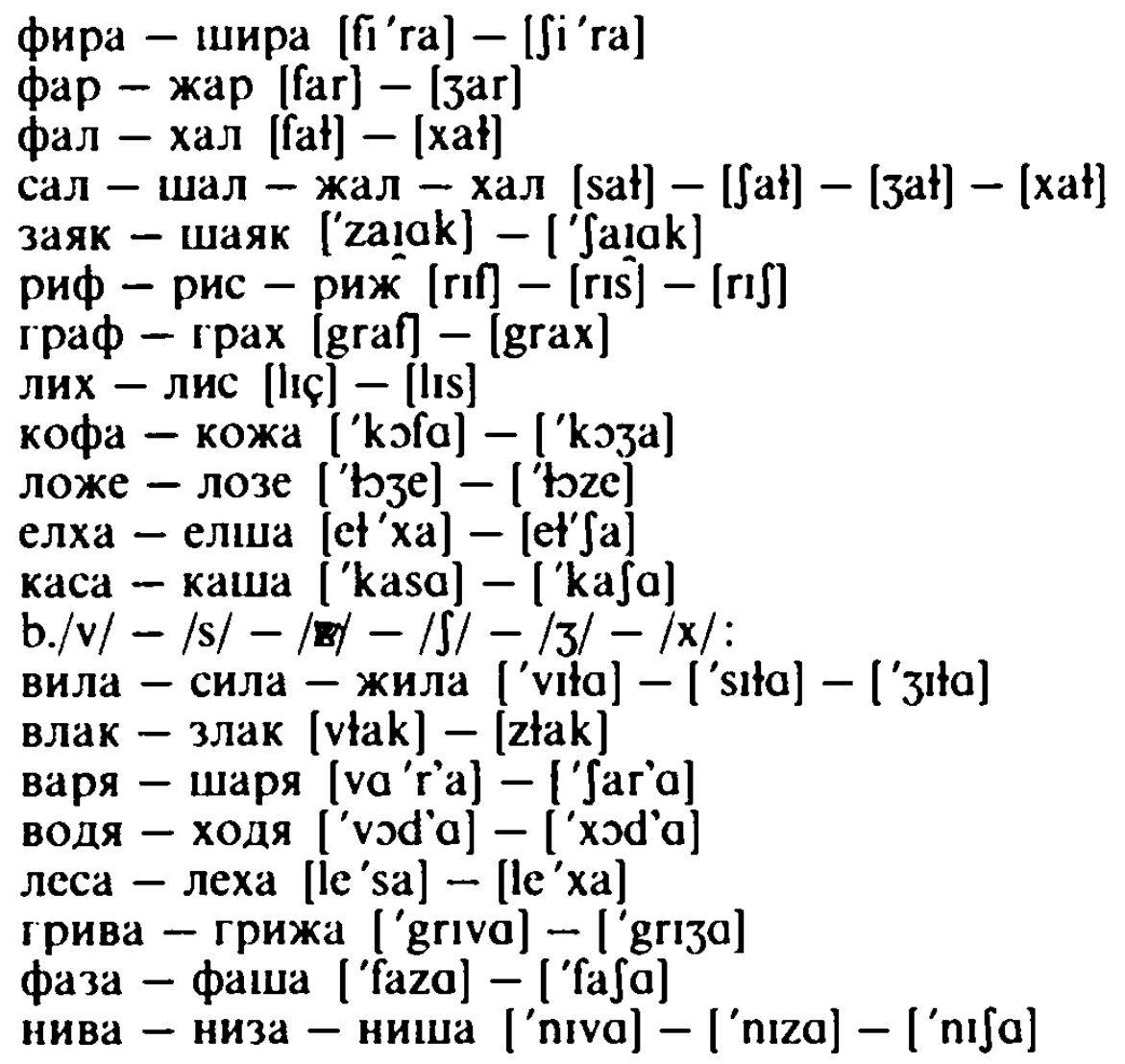

7.5.2. Distribution und Kombinatorik der Frikative im Deutschen und im Bulgarischen (Übersicht auf der Grundlage von An- und Auslautstrukturen von konsonantischen Allophonen)

\subsubsection{Anlautstrukturen}

Im Wortanlaut bestehen für das Deutsche und das Bulgarische folgende Beschränkungen für die Frikativkonsonanten:

- In beiden Sprachen kommen anlautend keine Doppelfrikativkonsonanten auf.

- Es sind ausgeschlossen vor Vokal:

\section{Deutsch}

$\mathrm{m} / \mathrm{h} / \mathrm{s} /$; in nativen

Wörtern auch [c $-\mathbf{x}$ ]

- Es verbinden sich nicht mit anderen Konsonanten zu zweigliedrigen Anlautstrukturen:

Deutsch

I $/ . / \mathrm{j} /, / \mathrm{h} /$

Wesentliche Unterschiede sind bei zwei- bzw. dreigliedrigen Verbindungen der Frikative mit Frikativen und anderen Konsonanten festzustellen. Sie lassen sich folgendermaßen auflisten:

\section{Bulgarisch}

palatalisierte Frikative vor

$[\varepsilon, I, \partial /$ 
Deutsch

Unter den Frikativen ist allein die Kombination

/S/ + /v/ möglich:

Schwung [Svun]
Deutsch

Als frikative Konsonanten vor Sonor treten in deutschen Wörtern allein $/ \mathrm{f} / \mathrm{h} / \mathrm{v} /$ (sehr begrenzt!) und / $\mathrm{f} /$ auf: Flamme ['flamə], Frieden ['fri:dən], wringen ['vrinan], Schmalz [Jmalts], Schnecke ['fneka], Schlinge ['Jlına], Schrank [frank]

\section{Deutsch}

Frikativ +

Frikative sind unvereinbar mit $/ n$, $d$, g, $\mathbf{k} /$; mit /p, $\mathrm{t}$ bildet Anlautstrukturen allein $/ \delta /$ : Spalt [Spalt], Staub [stooop]

\section{Zweigliedrige Anlautstrukturen \\ Frikativ + Frikativ}

\section{Bulgarisch}

Im Bulgarischen ist die Kombinatorik unter Frikativen im Anlaut umfangreicher, wobei sie meistens sowohl als erstes als auch als zweites Glied vorkommen: взимам ['vzımam], всеки ['fseki], всявам ['fs'avam], звук [zvuk]; свирка ['sfirka], свят [sv'at], сват [sfat]; вживявам [vfi'v'avam], вшивам ['ffivam]; жвакам ['zvakam]; вход [fxot], хвош [xvoft]

\section{Frikativ + Sonor}

\section{Bulgarisch}

Die Verbindung Frikativ + Sonor tritt hāufig auf: вместо ['vmesto], внос [vnos], влак [vłak], враг [vrak], влюбен ['vl'uben], вмятам

['vm'atam], врял [vr'at]; флота ['fłta], фльонга ['fl'onga], фрак [frak]; змия [zmi'ıa], знак [znak], злак [złak], зритёл ['zritef], (при)злява ['zl'ava], зрял [zr'ał], шмугвам (ce)

['Smugvam], шнола ['Snsta], шлеп

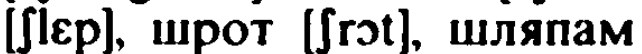

['Jl'apam]; смок [smok], сноп [snop], слива ['sliva], срам [sram], смяна ['sm'ana], сняг [sn'ak], сляп [sl'ap], сряда ['sr'ada]; хмел [хmєł], хлад [xłat], храм [xram], хляб [xl’ap], хрян [xr'an]

\section{Explosiv}

\section{Bulgarisch}

Die Verbindung Frikativ + Explosiv tritt hāufig auf: вбивам ['vbıvom], впивам ['fpivam]; вдовец [vdo'vets], втор [ftor]; вдявам ['vd'avam]; вграждам ['vgrazdom], вкарвам ['fkarvam], сбор [zbor], спор [spor], сбия ['sbna], сдъвквам ['sdəfk vam], стон [ston], стяг [st’ak], сгур [zgor], сговор ['zgovor], скала ['skala], шпора ['Spora], щит [stit], школа ['jksla] 
Deutsch

Dreigliedrige Anlautstrukturen mit

Frikativ als erstes Glied sind im

Deutschen wesentlich selten. Es sind nur folgende Verbindungen möglich:

$/$ pr/ Sprache ['Spra:xə]

$/ \int \mathrm{pl} /$ Splitter ['Splite],

/str/ Strähne ['Stre:na]

/skr/ Skrupel ['skru:pal]

\section{Dreigliedrige Anlautstrukturen}

Bulgarisch

Dreigliedrige Anlautstrukturen mit

Frikativ als erstes Glied sind im

Bulgarischen viel umfangreicher. Es

können sogar Anlautstrukturen von zwei, sogar drei Frikativen vorkommen. Die viel größere Anzahl von dreigliedrigen Kompositionen im Vergleich zum Deutschen entsteht durch die Präfixe $\langle b-\rangle$ und $\langle c-\rangle$. Beispiele:

/vzl/ взлом [vzłom]

/fsl/ вследствие ['vsletstvie]

/vzr/ взрив [vzrif]

/fsr/ всрастнал ['fsrastnał]

/fst/ встъпвам ['fstopvam]

/fpl/ вплитам ['fplitam]

/fpr/ впряг [fpr'ak]

/vgr/ вграден [vgra'den]

/vgl/ вглеждам [vglezdam]

/fkl/ вклинявам [fkli'n'avam]

/vdl/ вдлъбнат ['vdłəbnat]

/ftr/ втривам ['ftrivam]

/zbl/ сбльсък ['zbłasek]

/spl/ сплав [spłal]

/zbr/ сбръчкам ['zbratjkam]

/spr/ сприя ['sprịa]

/zdr/ здраве ['zdrave]

сдружавам [zdru'favam]

/str/ cтpax [strax]

/zgl/ сгледа ['zgleda]

/skl/ склад [skłat]

/zgr/ страда ['zgrada]

/skr/ скреж [skre]]

/skv/ скверен ['skveren]

/sxv/ cxвatka ['sxvatka]

/sxl/ схлупен ['sxłupen]

/sxr/ схрускан ['sxruskan]

/svr/ сврака ['svraka]

/smr/ смрадлика ['smradlika]

/fdr/ ждрело [3dre'b]

/xvr/ хвръквам ['xvrokvam]

Durch das Hinzufügen des Präfixes

$\langle b-\rangle$ sind im Bulgarischen auch vier-

gliedrige Anlautstrukturen möglich:

/fstr/ встрани [fstra'nI]

/fsmr/ всмръквам ['fsmrəkvam] 


\subsubsection{Auslautstrukturen (vor Pause)}

Deutsch

ilf, $\mathrm{nf}, \mathrm{rf} /$ :

Wolf [volf]

Senf [zenf]

Dorf [dorf]

/ps, ps, ks, pfs/:

Krebs [kre:ps]

hübsch [hypf]

Keks [ke :ks]

Fuchs [foks]

tags [ta :ks]

Kopf(s) [kopfs]

/fs, çs, Xs, fs/:

betreffs [bo'trefs]

Blechs [bleçs]

$\operatorname{Dach}(s)$ [daxs]

Tausch(s) [tâofs]

/ls, rs, ms, ns, ns/:

Fels [f $\varepsilon$ ls]

Vers [fers]

Sims [zıms]

Gans [gans]

längs [lens]

/lf, $\mathrm{r} \int, \mathrm{m} \int, \mathrm{nf} / \mathrm{:}$

falsch [falf]

Marsch [marf]

Ramsch [rams]

Mensch [menf]
Zweigliedrige Auslautstrukturen

mit Frikativ in finaler Position

\section{Bulgarisch}

Af, $\mathrm{mf}, \mathrm{rf} /$ :

голф [gotf]

триумф [tri'omf]

нерв [nerf]

торф [torf]

/ps, ks/:

гипс [gıps]

лукс [łoks]

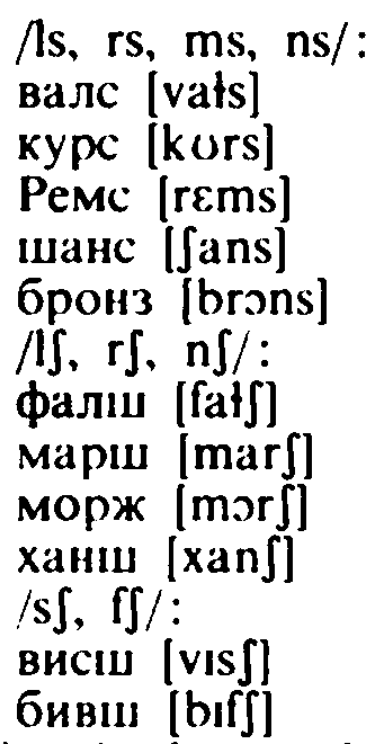

- Im Deutschen sind 19 zweigliedrige Auslautstrukturen mit Frikativ in Finalstellung. im Bulgarischen - 14 möglich.

- Unmöglich für das Bulgarische sind die Auslautstrukturen: $/ \mathrm{nf} /, / \mathrm{pf} /$, $/ \mathrm{xs} / \mathrm{.} / \mathrm{fs} / \mathrm{.} / \mathrm{s} \mathrm{s} / \mathrm{.} / \mathrm{gs} /$.

- Unmöglich für das Deutsche sind die bulgarischen Auslautstrukturen: $/ \mathrm{s} \int /, / \mathrm{fs} !$

- Ausgenommen is $\iiint$. kommen die zweigliedrigen Auslautstrukturen mit Frikativ in Finalposition ausschlieblich in gebräuchlichen Fremdwörtern auf. 


\section{Drei- und mehrgliedrige Auslautstrukturen}

Deutsch

/pts, kts/:

Haupt(s) [hoopts]

Effekt(s) [ ['fekts]

Its, mts, nts, rts/:

Inhalt(s) ['inhalts]

Amt(s) [amts]

Element(s) [ele'ments]

Wort(s) [vorts]

/fts, çts, sts/:

Heft(s) [hefts]

Gerücht(s) [go'ryçts]

Test(s) [tests]

mps, Ips, rps/:

Mups [mops]

Rülps [rylps]

Knirps [knırps]

/lks, rks, jks/:

Volk(s) [volks]

Murks [morks]

links [links]

Ifs, nfs, rfs/:

Wolf(s) [volfs]

Hanf(s) [hanfs]

Wurf(s) [vorfs]

/lçs, nçs, rçs/:

Kelch(s) [kยlçs]

Mönch(s) [monçs]

durchs [durçs]

$\mathrm{m} \int \mathrm{s}, \mathrm{n} \int \mathrm{s}, \mathrm{r} \int \mathrm{s} / \mathrm{:}$

Ramsch(s) [ramss]

Wunsch(s) [vonfs]

Hirsch(s) [hırfs]

mpifs, $\mathrm{nt}$ /:

Krampf(s) [krampifs]

Mantsch [mant]]

Ims, rms, rns, rls/:

Schelm(s) [ [ $\varepsilon l m s]$

Schwarm(s) [Svarms]

Kern(s) [kerns]

Kerl(s) [kerls]

- Mehr als dreigliedrige Auslautstrukturen mit Frikativ in Initialposition sind nicht allein für das Bulgarische, sondern auch für das Deutsche ungewöhlich. Auch die dreigliedrigen ergeben sich im Deutschen durch das Hinzufügen des Genitivs -s bzw. Plurals -s.
Den recht vielen dreigliedrigen Auslautstrukturen mit Frikativkonsonanten in Finalposition im Deutschen entspricht im Bulgarischen eine einzige und zwar im Fremdwort / $\mathrm{gks} /$ : сфинкс [sfinks]. 


\section{VERSCHLUSS-ENGELAUTE (AFFRIKATEN)}

Deutsch: $/ \hat{\mathrm{p} f} /, / \hat{\mathrm{ts}} /$

Bulgarisch: $/ \mathrm{ts} /-/ \mathrm{dz} /, / \mathrm{ts}^{*} /-/ \mathrm{dz} / ; / \mathrm{t} \mathrm{f} /-/ \mathrm{d} z /$

Die Affrikaten stellen artikulatorisch eine spezifische Konsonantenbildung dar. Sie können als homorgane Lautbildungen aufgefaßt werden, da ihr plosiver und frikativer Bestandteil etwa an der gleichen Artikulationsstelle gebildet wird (Vgl. Essen, 1969, 104; Zacher, 1969, 106; Тилков, Бояджи ев, 1977, 109; Heike, 1972, 44; M a ngold, 1974, 64; М осков, 1982, $72 \mathrm{u}$. andere). Der gebildete Verschluß wird dabei nicht durch kurze, plötzliche Öffnung gelöst, sondern durch langsame Loslösung des artikulierenden Organs von der Artikulationsstelle und gleichzeitigen Ubergang in die Enge eines sehr kurz andauernden Frikativs mit ungefär gleicher Artikulationsstelle. Verschluß und Enge stellen also zwei Phasen eines einheitlichen Artikulationsaktes dar, wodurch ein Konsonnant neuer Qualität entsteht (Тилков, Бояджиев, 1977, 109).

Akustisch zeigen die Bestandteile der Affrikaten die gleichen Spektralcharakteristika wie die Explosiv- und Frikativkonsonanten, denen sie entsprechen. Der frikative Teil einer Affrikate ist jedoch kürzer als der entsprechende Frikativkonsonant, so daß ihre Gesamtdauer nicht die spezifische Dauer eines frikativen Konsonanten überschreitet (Neppert, Pétursson, 1984, 142; Тилков, Бояджиев, 1977, 109).

Die bulgarischen Affrikaten werden von der Mehrheit der bulgarischen und ausländlischen Autoren monophonematisch aufgefaßt (С т о й к о в, 1967; Леков, 1980; Стоянов, 1977; Тилков, Бояджиев, 1977, 1982; Младенов, 1970; Янакиев, 1960; Scatton, 1983; А ронсон, 1974 u. a.). Umstritten ist der phonologische Status der deutschen Affrikaten.

Verfolgt man die Auffassungen, die hinsichtlich des phonematischen Status der deutschen Affrikaten in der phonetischen Literatur vertreten sind, so stellt man fest, daß sie sich geradezu teilen: monophonematisch werden die deutschen Affrikaten beispielsweise von Trube $\mathrm{zk}$ o y (1971), Es s en (1979), Zacher (1969), Mangold (1974), Philipp (1974), Wurzel $(1970,1980)$ u. a. aufgefaßt, biphonematisch dagegen von Moreiniec (1958, 1968), Große (1967), Heike (1972), Werner (1972). Meinhold und Stock (1982) u.a.

Argumente für das Für und Wider in diesem phonematischen Streit suchen die Forscher im Funktionieren der Affrikaten im Sprachsystem des Deutschen.

Bei seiner monophonematischen Wertung der deutschen Affrikaten stützt sich Trubetzk oy auf phonetischen Angaben, nach denen auch bei den Affrikaten, wie bei allen ..typischen Fällen der monophonematischen Wertung", wo es sich .immer um den allmählichen Abbau eines Artikulationsgebildes handelt", ein .Verschluß" zuerst bis zu einer „Engebildung“ erweitert, dann ganz aufgehoben wird $(1958,52)$, was heißen soll, daß die Affrikaten phonetisch in sich geschlossene, nicht segmentierbare Einheiten sind. Ihm wird von Meinhold und Stock $(1980,127)$ in Anlehnung an Morcinicc (1958,56m) vorgeworfen, daß er gegen seine eigenen Regeln für die monophonematische Wertung von Lautverbindungen 
verstöße. Scine vierte Regel besagt nämlich folgendes: „Eine potentiell monophonematische (d.h. den Forderungen der Regeln I-III entsprechende) Lautverbindung muß als Realisation eines einzigen Phonems gewertet werden, wenn sie als Einzelphonem behandelt wird, d.h., wenn die in solchen Lautstellungen vorkommt, wo in der betreffenden Sprache Phonemverbindungen nicht zugelassen werden" (T r ube tzk oy, 1958, 53).

Morciniec sucht und findet (u. E. nicht immer phonematisch einwandfrei) gegenübergestelte Phonemverbindungen, die der Distribution von /pf/ und /ts/ entsprechen:

phlanzen - splittern - Sklave /pfl/ - / $p \mathrm{pl}, \mathrm{skl} /$

pfropfen - sprießen - streiten - Skrupel $/ \mathrm{pfr} /-/ \mathrm{ppr}$, Jtr, skr

Sumpf - Hamster /mpf/ - /mst/

Salz - Geschwulst /lts/ - /lst/

Herz - ernst. Herbst /rts/ - /rnst, rpst/

Tanz-Gunst, sanft /nts/ - /nst, nft/ (M orciniec, 1958, $56 \mathrm{fr}$, zitiert nach Meinhold, Stock, 1980, 128).

Auch die weiter angeführten Überlegungen von $\mathrm{Me}$ inhold und $\mathrm{Stock}$ (1980, 128f) scheinen phonologisch und zwar im Lichte von Trubetzkoy gut begründet $\mathrm{zu}$ sein: während Trubetzkoy z. B. die monophonematische Wertung des /pf/mit Wortpaaren wie Pfeil-feil; Pfad-fad; Pfand-fand belegt, führen sie Beispiele an wie etwa treiben - reiben; Schubs - Schuß .zwei Segment verbindungen, dic den Affrikaten phonetisch sehr ähnlich sind“ und die trotzdem nicht als einheitliche Phoneme aufgefaßt werden. Weiter wird von beiden Autoren auch eine Kommutationsprobe versucht, um zu beweisen, daß sowohl das erste als auch das zweite Element von /pf/ und /ts/ .austauschbar ist und distinktiv wirkt" $(1980,128)$ : Pfahle-prahle, hïpfe - hühsche u. a.; Zahn - Tran, Witz - Wichs u.a.

Meinhold und Stock haben jedoch manches aus den Uberlegungen Trubetzkoys nicht beachtet und zwar gerade zu Regel IV: „Das Deutsche duldet wohl im Anlaute Verbindungen eines Konsonanten mit I (klar, glatt, plump, Blei, fliegen, schlau) oder mit w (Qual, schwimmen); von Verbindungen zwei Konsonanten +1 , w' werden aber im Anlaut nur spl (Splitter), pn (Pflaume, Pflicht, Pflanze) und tsw (zwei, zwar, Zwerg, Zwinger usw.) geduldet, und da dreigliedrige Konsonantenverbindungen im Anlaut deutscher Wörter sonst nicht geduldet werden (außer str, spl und spr), so ist es schon aus diesem Grunde notwendig, die deutschen pf und ts (wenigstens in der Schriftsprache!) als einheitliche Phoneme zu betrachten" (Trubetzkoy, 1958, 53).

Die Ausführung Trubetzkoys könnte u. E. etwa in folgendem Sinne weiter. kommentiert werden: Die phonetische Unähnlichkeit erlaubt nicht, wenigstens nach den Normen der deutschen Standardaussprache, anlautendes /pl/, $/ \mathrm{kl} / \mathrm{t} / \mathrm{r} / \mathrm{,} / \mathrm{pr} / \mathrm{monophonematisch} \mathrm{zu}$ werten, da die stimmlosen Explosive gerade in der Position vor $\Lambda_{\prime}^{\prime}, / r /$ artikulatorisch drei Phasen beinhalten (Okklusion, Plosion und Aspiration), gegenüber einer einzigen in den Afrrikaten $/ \mathrm{pf} / \mathrm{s} / \mathrm{ts} /$. Auch die Sonagramme, die wir als Anschauungsmaterial dazu gemacht haben, weisen deutlich darauf hin (Siehe S. 170-188).

Die von Morciniec und Meinhold und Stock angeführten Beispiele enthalten im Anlaut den Explosiv immer nur als zweites Element; anlautende Lautverbindungen im Explosiv als erstes Element (ausgenommen /pf/, /ts/) 
sind nämlich in nativen deutschen Wörtern unmöglich, worauf übrigens auch Trubetzkoy verweist: .Außerdem werden im Anlaut echt deutscher Wörter Verbindungen des Typus 'Verschlußlaut + Engelaut' nicht zugelassen (Wörter wie Psalm, Xanthype tragen das deutliche Gepräge der Fremdartigkeit), was auch auf die monophonematische Wertung von pf und ts $/ z /$ einwirkt" (Trubetzk oy, 1971, 53, 54).

Wir akzeptieren weiter auch die Argumente von Philipp $(1974,38)$, die mit Recht bemerkt, da 3 die Kommutation von $/ p /$ und $/ t /$ nicht vor akzentuiertem Vokal möglich ist, und noch mehr die Erwägungen von Wurzel $(1982,937-940)$. daß durch die monophonematische Wertung der deutschen Affrikaten sich sowohl die Morpheme im Lexikon als auch die Morphemstrukturbedingungen einfacher und eindeutiger beschreiben lassen können.

Ein letzter, für diese Arbeit nicht unwesentlicher Beweggrund, die deutschen Affrikaten als einheitliche Phoneme anzusehen, ist durch die Notwendigkeit einer kontrastiven Betrachtung nach einheitlichen Beschreibunsprinzipien der miteinander zu konfrontierenden Sprachsysteme bedingt.

Am Rande sei auch die diachronische Bemerkung Otto von Essens angeführt: „Die Lautgeschichte des Deutschen bezeugt, da $3 / \mathrm{pf} /, / \mathrm{ts}$ / durch - natürlich unbewußte und ungewollte - Verschiebungen aus phonetisch einheitlichen Lauten $p$ unt $t$ entstanden sind und auch in der Folgezeit die resultierenden Afrikaten als einheitliche Lautbildungen begriffen wurden" (v. Essen, 1979, 105).

\subsection{PHYSIKALISCHE DAUER DER DEUTSCHEN UND DER BULGARISCHEN AFFRIKATEN}

Die physikalische Dauer der deutschen und der bulgarischen Affrikaten wurde ermittelt im Vergleich zu einfachen Konsonanten, die als Bestandteile einer Affrikate betrachtet werden können, bzw. zu Konsonantenverbindungen, die in der gleichen und in der umgekehrten Aufeinanderfolge in der Position der jeweiligen Affrikate auftreten; außerdem wurden die letzteren auch mit Verbindungen aus Affrikate + Konsonant temporal verglichen. Versuchspersonen waren je zwei Muttersprachler (Männer) für beide Sprachen. Für die bulgarische Sprache wurde auch eine Sprecherin für einige zusätzliche Sonagramme wegen der statistisch signifikanten Zahl der Beispiele gewonnen. Die Affrikaten und die mit ihnen zu konfrontierenden Konsonanten und Konsonantenverbindungen erscheinen in folgenden Positionen: Anlaut, Inlaut in intervokalischer Position. Auslaut. Es sei auf einige Besonderheiten der Auswertung hingewiesen: a) Stimmlose Explosive erscheinen anlautend immer kürzer, da die Okklusionsphase auf dem Sonagramm unsichtbar bleibt (Es wurde also allein die Plosionsphase anlautend ausgemessen); inlautend und auslautend dagegen ist die Okklusionsphase als der Abstand zwischen Ende des vorangehenden Lautes und Anfang der Plosionsphase immer gut zeitlich auszumessen; b) Sämtliche Konsonanten (wie übrigens auch sämtliche Vokale - siehe S. 142 - 143) dauern am terminalen Ausspruchsende länger als in allen übrigen Positionen. Die Wortbeispiele wurden in einem terminalen 
Ausspruchsrahmen eingeschlossen. Die gewonnenen Mittelwerte der physikalischen Dauer der deutschen und der bulgarischen Affrikaten im Vergleich zu einfachen Konsonanten und Konsonantenverbindungen sind nach Konsonantenart und Position in die Tab. auf $\mathrm{S} .106$ eingeordnet. In der Beilage sind sonagraphische Beispiele. die die physikalische Dauer der Affrikaten in der jeweiligen Position im Vergleich zu anderen Konsonantenverbindungen veranschaulichen (Siehe S 170-188).

\subsubsection{Zusammenfassung der Ergebnisse}

aus der kontrastiven sonagraphischen Analyse der deutschen und der bulgarischen Affrikaten

Die Auswertung der physikalischen Dauer der deutschen und der bulgarischen Affrikaten in konfrontativer Sicht zu einfachen Konsonante 1, die als Bestandelemente einer Affrikate fungieren, und zu Konsonantenvirbindungen, die in der gleichen bzw. in der umgekehrten Aufeinanderfolge in der Position der jeweiligen Affrikate auftreten, ergab folgendes:

- In Anlautposition: Hier dauert im Deutschen und im Bulgarischen die Affrikate $i t i_{i}$ am kürzesten - im Mittel um $110 \mathrm{~ms}$ für das Deutsche und $100 \mathrm{~ms}$ für das Bulgarische. Diese Dauer gleicht etwa der Dauer des Frikativs $/ \int$ : und ist geringer als die physikalische Dauer der für das Deutsche und für das Bulgarische möglichen Verbindungen des / $/$ / mit anderen Konsonanten. Die Affrikaten d. $/ \mathrm{pf} /$, d. $/ \mathrm{ts} /$ und b./ts/ dauern anlautend um etwa $30-40 \mathrm{~ms}$ länger als $\mathrm{d} . / \mathrm{t} / \mathrm{s}$.

- In Inlautposition: Wegen der Messung der Okklusionsphase des [p] bzw. [ $\mathrm{t}]$ ist die Dauer der miteinander zu konfrontierenden Konsonanten und Konsonantenverbindungen inlautend länger. In dieser Position haben nämlich die stimmlosen Explosive um das Doppelte höhere Dauerwerte als in Inlautposition. Der perzeptive Eindruck ändert sich jedoch nicht, da die Okklusionsphase unhörbar ist. Das läßt die Verhältnisse mit denen des Anlauts übereinstimmen.

- In Auslautposition: Hier muß man akustisch ebenfalls mit der sichtbaren (Okklusionsphase rechnen und akustisch/perzeptiv auch mit der physiologischen Ausdehnung des konsonantischen Auslauts. So bleiben die Verhälınisse relativ die gleichen wie an- und inlautend.

- Zu vermerken ist auch die ..simultane" Bildung der Affrikaten in beiden Sprachen. Sic läßt sich an unseren sonagraphischen Beispielen in konfrontativer Sicht zur komplizierten Bildung von Konsonantenverbindungen aus stimmlosem Explosiv + Frikativ klar beobachten, d.h., die Verringerung der Explosiv-Bildungsphasen der Affrikaten ist am Sonagramm gut zu sehen. Gerade diese komprimierte, simultane Bildung der Affrikaten bestimmt sie als einheitliche Konsonanten von neuer Qualität. Deswegen dürfte man sie bei der phonologischen Analyse nicht in einfache Konsonanten zerlegen und so distinktive Oppositionen $\mathrm{zu}$ anderen Konsonantenphonemen aufbauen, sondern man sollte sie als Einheit, also als Monophonem akzeptieren und so ihre Distinktion im Sprachsystem prüfen. 


\subsection{DIE AFFRIKATEN DES DEUTSCHEN UND DES BULGARISCHEN IM EINZELNEN}

\subsubsection{Deutsches /pf/}

Dic labiale Affrikate/pf/ des Deutschen hat keine Entsprechung im bulgarischen Konsonantensystem.

Phonetische Charakteristik : Es wird ein labialer VerschluB gebildet, dessen verlangsamte Lösung eine frikative Übergangslänge zwischen Unterlippe und oberen Schneidezähnen als zusätzliche Hemmstelle schafft. Akustisch ist die frikative Komponente von kürzerer Dauer als der frikative Konsonant [ $]$ ], weist jedoch die gleichen akustischen Eigenschaften auf.

\subsubsection{Deutsches /ts/, Bulgarisches /ts/, /ts'/; $/ \mathrm{dz} /, / \mathrm{dz} /$}

Die apikal- bzw. koronal-alveolare (dentale) Affrikate [ts] und ihre bulgarische Entsprechung [ts] haben phonetisch gleiche Merkmale. Die langsame Lösung des Verschlusses für ein [t] schafft die frikative Übergangsenge eines [s], die von kürzerer Dauer ist als die Enge des frikativen Konsonanten [s]. Die Gesamtdauer der Affrikaten d. [ts] und b. [ts] bewegt sich im Rahmen der Dauer eines Frikativkonsonanten. Bulgarisches /ts/ hat auch eine stimmhafte Entsprechung/dz/ mit begrenzter Gebrauchsfrequenz.

Für das bulgarische palatale [ts'] wird die Artikulationsstelle vom hinteren Teil der Alveolen zu ihrem oberen Teil verschoben. Der VerschluB wird mediodorsal gebildet. Die palatale Information wird perzeptiv, genauso wie bei [c]. von der Transition zum nachfolgenden Vokal gewährleistet.

\subsubsection{Bulgarisches $/ \mathrm{t} \int /-/ \mathrm{d} 3 /$, Deutsches [t $\left.\int\right]$}

Während das bulgarische $/ \mathrm{t} \mathrm{J} /$ eindeutig als einheitliches affrikatives Monophonem aufgefaßt wird, wertet man das deutsche [t $f]$ vorwiegend als eine biphonematische Konsonantenverbindung. Diachronisch gesehen, handelt es sich dabei um eine recht späte Bildung des Mittelhochdeutschen (ahd. diutisc $>$ deutsch). Neue Bildungen sind onomathopoetische Wörter wie Quatsch, lutschen (Vgl. Es se n, 1979, 105). Das begrenzte Auftreten des [t $]$ im heutigen Deutsch, dabei distributiv begrenzt, entscheidet über ihre biphonematische Wertung. Ihre physikalische Dauer fallt aber sogar etwas kleiner aus als die der anderen Affrikaten (Siehe S.115).

Bulgarisches $/ \mathrm{t} /-/ \mathrm{d}_{3} /$ weisen wie die entsprechenden Frikative $/ \mathrm{J} /$ und $/ 3 /$ keine palatalen Entsprechungen auf. Ihr Artikulationsmechanismus unterscheidet sich nicht von dem der anderen Affrikaten. Unter dem Einfluß der frikativen Komponente ist der [t]-VerschluB palatal-dorsal etwas nach hinten verschoben. $[\mathrm{t} f]$ und $\left[\mathrm{d}_{3}\right]$ zeichnen sich wie die anderen Affrikaten durch eine langsame Lösung des gebildeten Verschlusses und eine recht kurze Dauer des frikativen Geräusches aus im Vergleich zu der jähen Verschlußlösung des [J] und der längeren Dauer von [S] bzw. [3]. 


\subsection{PARADIGMATISCHE UND SYNTAGMATISCHE DARSTELLUNG DER DEUTSCHEN UND DER BULGARISCHEN AFFRIKATEN}

\subsubsection{Minimalpaaroppositionen der deutschen und der bulgarischen Affrikaten}

d. $/ \mathrm{pf} /-/ \mathrm{ts} /$ :

b. $/ \mathbf{t s} /-/ \mathrm{t} s /$ :

$/ \mathrm{ts} /-/ \mathrm{d} 3 /:$

d. $/ \mathbf{p} f /-/ p /-/ b /:$

$/ \mathrm{pf} /-/ \mathrm{t} /-/ \mathrm{d} /:$

$$
/ \mathrm{pf} /-/ \mathrm{k} /-/ \mathrm{g} /:
$$

d. $/ \mathrm{ts} /-/ \mathrm{t} /-/ \mathrm{d} /$ :

b. $/ \mathrm{ts} /-/ \mathrm{t} /-/ \mathrm{d} /$ :

$$
/ \mathbf{t s} /-/ \mathrm{p} /-/ \mathrm{b} /:
$$$$
/ \mathrm{ts} /-/ \mathrm{k} /-/ \mathrm{g} /:
$$

\section{Innerhalb der Affrikatengruppe}

Pfahl - Zahl [pfo:l] - [tsa:l]

Tropf - Trotz [tropf] - [trots]

Kipfel - Kitzel ['kipfal] - ['kitsal]

Lap - чap [tsar] - [t]ar]

плаи - плач [płats] - [płats]

дръвие - дръвче [dreftse] - [dreft $f \varepsilon]$

чам - джам [t $\mathrm{am}]$ - [dzam]

Oppositionen zu Explosiven

Pfanne - Panne ['pfano] - ['pana]

Topf - Topp [topi] - [top]

stopfen - stoppen ['Stopfon] - ['Stopan]

Pfeil - Beil [pfáel] - [bäel]

Pfeil - Teil [pfáel] - [tôel]

Tropf - Trott [tropf] - [trot]

Opfer - Otter ['apfe] - ['ote]

pfuschen - duschen ['pfufan] - ['dofon]

Pfeil - Keil [pfóel] - [káel]

Stopf - Stock [ [ top $f]-\left[\int t o k\right]$

stopfen - stocken ['Stopfon] - ['Stokon]

Pfeiler - Geiler ['pfriele] - ['gáelé]

Zahl - Tal [tsa:l] - [ta:l]

Schutz - Schutt [sots] - [sot]

wetzen - wetten ['vetson] - ['veton]

Zank - Dank [tsank] - [dank]

цел - тел [tset] - [tet]

плац - плат [płats] - [płat]

пица - пита ['pitsa] - ['pita]

цар - дар [tsar] - [dar]

Цена - Пена ['tsena] - ['pena]

каца - kana ['katsa] - ['kapa]

бон - 606 [bots] - [bop]

Lap - 6ap [tsar] - [bar]

цел - кел [tset] - [ket]

каца - кака ['katsa] - ['kaka]

виц - вик [vits] - [vik]

цол - гол [tsot] - [got] 


$$
\begin{aligned}
& \text { b. } / \mathbf{t s}^{\prime} /-/ \mathrm{t}^{\prime} /-/ \mathrm{d}^{\prime} /: \text { цяло - тяло ['ts'ało] - ['t'ało] } \\
& / \mathbf{t s}^{\prime} /-/ \mathbf{p}^{\prime} /-\mathrm{b}^{\prime} /: \\
& / \mathbf{t s}^{\prime} /-/ \mathbf{k}^{\prime} /: \\
& \text { b. } / \mathrm{t} \mathrm{j} /-/ \mathrm{t} /-/ \mathrm{d} / \text { : } \\
& / \mathrm{t} s /-/ \mathrm{p} /-\mathrm{b} /: \\
& / \mathrm{t} s /-/ \mathrm{k} /-\mathrm{g} /: \\
& \text { цял - дял [ts'at] - [d'at] } \\
& \text { цял - пял - бял [t'’at] - [p'at] - [b'at] } \\
& \text { цяр - кяр [ts'ar] - [k’ar] } \\
& \text { час - Tac [t fas] - [tas] } \\
& \text { плач - плат [płat }] \text { - [płat] }
\end{aligned}
$$

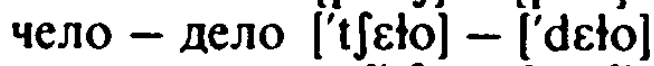

$$
\begin{aligned}
& \text { чанта - панта ['t Janta] - ['panta] }
\end{aligned}
$$

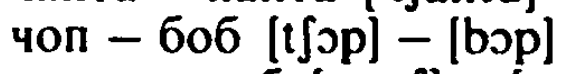

$$
\begin{aligned}
& \text { грьч - гръб [grat } \left.\int\right]- \text { [grəp] } \\
& \text { чаша - каша ['t } \left.a \int a\right]-\left[' k a \int a\right] \\
& \text { коч - кок [kotf] - [kok] } \\
& \text { чума - гума ['t Juma] - ['guma] }
\end{aligned}
$$$$
\text { d. }[p]-[]-[v]:
$$$$
/ \mathrm{p} f /-/ \mathbf{z} /:
$$$$
/ \mathrm{pf} /-/ \mathrm{s} /:
$$$$
/ \mathrm{pf} /-/ \mathrm{j} /:
$$$$
/ \mathrm{pf} /-/ \mathrm{s} /:
$$$$
/ \mathrm{pf} /-/ \mathrm{h} /:
$$$$
\text { d. } / \mathrm{ts} /-/ \mathrm{f} /-/ \mathrm{v} / \text { : }
$$$$
|\mathrm{ts} /-| \mathbf{z}|-| \mathrm{s} /:
$$

Pflug - Flug [pflu :k] - [nu :k]

Tupf - Tuff [topf] - [tuf]

Pfanne - Wanne ['pfana] - ['vanə]

Pfahl - Saal [pfa:l] - [za:l]

Schlupf - SchluB [slopf] - [slus]

Pfad(e) - Jade ['pfa :də] - ['ja :də]

Pfote - Schote ['pfo:to] - ['So:to]

tupfen - tuschen ['tupfan] - ['tufan]

Pfeil - Heil [pfáel] - [háel]

Zeile - Feile - Weile ['tsáelə] - ['fâelə] - ['vâeləi]

Stolz - Stoff [ [stolts] - [stof]

putzen - puffen ['putsan] - ['pufon]

Zahl - Saal [tsa:l] - [za:l]

Schutz - SchuB [ $\left.\int u t s\right]-\left[\int u s\right]$

$$
\begin{aligned}
& \text { beizen - beißen ['bóetzan] - ['boiesən] } \\
& \text { Weizen - Waisen ['váetsan] - ['váezan] }
\end{aligned}
$$

$/ \mathrm{ts} /-/ \mathrm{s} /-/ \mathrm{j} /:$

Zunge - Junge ['tsuna] - ['junə]

$/ \mathrm{ts} /-/ \mathrm{h} /:$

Zahl - Schal [tsa: :l] - [ [ a : :l]

Fritz - frisch [frits] - [frif]

Zahn - Hahn [tsa:n] - [ha: n]

b. $/$ ts $/-/ \mathbf{f} /-/ \mathrm{v} /$ :

uap - фap - Bap [tsar] - [far] - [var]

$/ \mathrm{ts}^{\circ} /-/ \mathrm{v}^{\circ} /$ :

$|\mathrm{ts} /-| \mathrm{s} /-\mid \mathbf{z} /$ :

цял - вял [ts’at] - [v'ał]

цол - сол [tsot] - [sot]

цар - зар [tsar] - [zar]

$/ \mathbf{t s}^{\circ} /-/ \mathbf{s}^{\circ} /:$

$/ \mathrm{ts} /-/ \mathrm{s} /-/ 3 /:$

цял - сял [t'ał] - [s’ał]

цол - шол [tsot] - [โวt]

цар - жap [tsar] - [3ar]

каца - каша - кажа ['katsa] - ['ka $a$ a] - ['kaza]

градеи - градеж [gra'dets] - [gra'def]

$$
/ \mathrm{ts} /-/ \mathbf{l}:
$$

Цонка - Ионка ['tsonka] - ['ingka]

$/ \mathbf{t s} /-/ \mathbf{x} /$ :

цял - ял [ts'al] - [!af]

uon - xon [tsop] - [xop]

kyu - kyx [kots] $-[k(s x]$ 


$$
\begin{aligned}
& \text { b. } / \mathrm{t} f /-/ \mathrm{f} /-/ \mathrm{v} /: \text { 4ap - фap - Bap }\left[\mathrm{t} \int \mathrm{ar}\right]-[\mathrm{far}]-[\mathrm{var}] \\
& 1 \int \Omega /-/ s /-/ 2 /: \\
& \text { чан - сан [t } \left.\int a n\right]-[\operatorname{san}] \\
& \text { 4ap - 3ap [t far] - [zar] }
\end{aligned}
$$

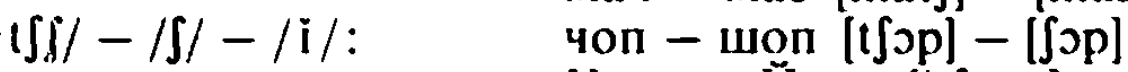

$$
\begin{aligned}
& \operatorname{ts} / /-/ x /: \\
& \text { Чона - Йона ['t } \\
& \text { чума - хума ['tfoma] - ['xuma] } \\
& \text { Oppositionen zu Sonoren }
\end{aligned}
$$

d. $/ \mathrm{p} f /-/ \mathrm{m} /-/ \mathrm{n} /-/ \mathrm{l} /-/ \mathrm{r} /:$

Pfad - Rad [pfa :t] - [ra :t]

Pfeile - Meile ['pfáela] - ['môela]

Pfote - Note - Rote ['pfo : ta] - ['no : to] - ['ro : to]

Pfand - Land [pfant] - [lant]

Zopf - Zoll [tsopf $]-[$ tsol]

Napf - Narr [napf] - [nar]

Wipfel - Wimmel ['vipfol] - ['vimal]

köpfen - können ['kœpfon] - ['kœnan]

Zöpfe - Zölle ['tsoepfa] - ['tsola]

d. $/ \mathrm{ts} /-/ \mathrm{m} /-/ \mathrm{n} /-/ \mathrm{g} /-/ \mathrm{r} /-/ \mathrm{l} /$ :

a) Zahl - Mal [tsa:l] - [ma:l]

Harz - Harm [harts] - [harm]

schwitzen - schwimmen ['Svitsan] - ['Sviman]

b) Zote - Note ['tso :ta] - ['no : ta]

Sitz - Sinn [zits] - [zın]

Spitze - Spinne ['Spitso] - l'Spına]

c) schwitzen - schwingen ['Svitsan] - ['Jvijan]

d) Ziegel - Riegel ['tsi : gol] - ['ri :gal]

Hetz - Herr [hets] - [her]

schwitzen - schwirren ['Svitson] - ['Sviron]

c) Ziege - Liege ['tsi : go] - ['li :gə]

Schatz - Schall [Sats] - [Sal]

petzen - pellen ['petson] - ['pelon]

b. $/ \mathbf{t s} /-/ \mathrm{m} /-/ \mathrm{n} /-/ \mathrm{r} /-/ \mathbf{l} /$ :
а) пеля - меля ['tsel’a] - ['mel’a]
цол - мол [tsot] - [mot]
куц - кум [kots] - [kom]
ryıa - ryma ['gotsa] - ['gomo]
b) llap - нap [tsar] - [nar]
плац - план [płats] - [płan]
буна - буна ['botsa] - ['bona]
c) цапвам - paпвам ['tsapvam] - ['rapvam]
цев - рев [tsef] - [ref]
вици - вир [vits] - [vir]
Мица - Мира ['mitsa] - ['mira]
d) цепя - лепя ['tsep'a] - ['lep'a]

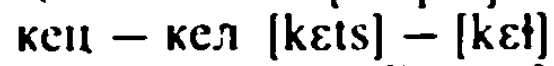
Мица - Мила ['mitsa] - ['mıla] 
b. $/ \mathrm{ts}^{\prime} /-/ \mathrm{m}^{\prime} /-\mid \mathrm{r}^{\prime} /-/ \mathbf{l}^{\prime} /$ :

каца - кала ['katsa] - ['kała]

цяр(а) - мяра ['ts'ara] - ['m’ara]

цял - рял - лял [t'ał] - [r'at] - [l'ał]

b. $/ \mathrm{t} \int /-/ \mathrm{m} /-/ \mathrm{n} /-/ \mathrm{r} /-/ \mathrm{l} /$ :

чайка - майка ['t

чин - лин [t $\left.\int \mathrm{In}\right]-[\mathrm{lin}]$

чар - нар [t far] - [nar]

чук - лук [t fuk] - [tuk]

rрач - грам [grat f] - [gram]

коч - кон [kJt $]-[$ kon]

кич - кир - кил [kit $]-[k i r]-[k i t]$

брачен - бранен ['brat fen] - ['branen]

жичен - жилен ['3it fen] - ['zilen]

точен - торен ['tot $\left.\int e n\right]-[$ toren]

лучен - лунен ['tut Sen] - ['łonen]

Dauer der deutschen und der bulgarischen Affrikaten (in ms)

\begin{tabular}{|c|c|c|c|c|c|c|c|}
\hline \multicolumn{4}{|c|}{ DEUTSCH } & \multicolumn{4}{|c|}{ BULGARISCH } \\
\hline \multirow{2}{*}{ Laut } & \multicolumn{3}{|c|}{ POSITION } & \multirow{2}{*}{ Laut } & \multicolumn{3}{|c|}{ POSITION } \\
\hline & Anlaut & Inlaut & Auslaut & & Anlaut & Inlaut & Auslautt \\
\hline $\begin{array}{l}\text { fs } \\
\mathbf{t} \\
\text { s } \\
\mathbf{t}+\text { Son. } \\
K .+ \text { fs }\end{array}$ & $\begin{array}{r}152 \\
58 \\
143 \\
148 \\
238\end{array}$ & $\begin{array}{l}232 \\
148 \\
183 \\
253 \\
-\end{array}$ & $\begin{array}{l}383 \\
171 \\
307 \\
242 \\
447\end{array}$ & $\begin{array}{l}\text { ts } t s^{\prime} \\
\mathbf{t} \\
\text { s } \\
\mathrm{t}+\text { Son. } \\
\text { s+ Expl. } \\
\text { Expl.+s } \\
\text { ts + Fr. }\end{array}$ & $\begin{array}{r}114 \\
75 \\
110 \\
96 \\
203 \\
228\end{array}$ & $\begin{array}{r}155 \\
98 \\
154 \\
210 \\
218 \\
-\end{array}$ & $\begin{array}{c}285 \\
217 \\
248 \\
- \\
332 \\
353\end{array}$ \\
\hline $\begin{array}{l}\text { is } \\
\int_{t} \\
\int\end{array}$ & $\begin{array}{r}113 \\
58 \\
137 \\
212\end{array}$ & $\begin{array}{l}246 \\
148 \\
213 \\
307\end{array}$ & $\begin{array}{r}435 \\
171 \\
35 \\
413\end{array}$ & $\begin{array}{l}\text { ts } \\
t \\
\int \\
\text { Expl. }+ \text { Expl. } \\
\text { Son. }+ \text { tf }\end{array}$ & $\begin{array}{r}97 \\
75 \\
129 \\
214 \\
146\end{array}$ & $\begin{array}{l}143 \\
133 \\
152 \\
233 \\
234\end{array}$ & $\begin{array}{c}280 \\
217 \\
258 \\
- \\
335\end{array}$ \\
\hline $\begin{array}{l}p f \\
p \\
f \\
p+\text { Son. } \\
p+F r . \\
f+\text { Son. } \\
\text { pf + Son. }\end{array}$ & $\begin{array}{r}150 \\
70 \\
133 \\
130 \\
\overline{181} \\
213\end{array}$ & $\begin{array}{c}200 \\
151 \\
163 \\
- \\
238 \\
250 \\
-\end{array}$ & $\begin{array}{l}361 \\
202 \\
275 \\
= \\
= \\
=\end{array}$ & & & & \\
\hline
\end{tabular}




\subsubsection{Distribution und Kombinatorik der Affrikaten im Deutschen und im Bulgarischen*}

\subsubsection{Anlautstrukturen}

Im Anlaut sind sowohl im Deutschen als auch im Bulgarischen einige wenige zweigliedrige Konsonantenstrukturen möglich.

Die deutsche labiale Affrikate /pf/kombiniert sich anlautend allein mit den Sonorlauten /// und / $\mathrm{r} /$ : Pflanze, Pfropfen.

Die Affrikate /ts/ tritt im Deutschen in eine einzige Anlautverbindung mit /v/ ein: Zwist. Im Bulgarischen ist außer mit /b/noch die Kombination mit / $p /$ möglich: цвете ['tsvete], цръквам ['tsrakvam].

Eine Besonderheit des bulgarischen Anlauts ergibt sich durch das Hinzufügen der Präfixe $\langle b-\rangle,\langle c-\rangle$, wobei die Affrikate /ts/ als zweites Glied der Anlautverbindung erscheint : вцепенявам [ftsepe'n'avam], сцена ['stsena]. Die palatale Affrikate /ts'/ verbindet sich im Anlaut mit keinem Konsonanten.

Die deutsche Konsonantenverbindung / $\mathrm{t} /$ ist anlautend ausgeschlossen.

Die bulgarische Affrikate / $\mathrm{t} / \mathrm{j} / \mathrm{a} ß \mathrm{t}$ sich im Anlaut mit den Konsonanten $/ \mathbf{v} !$,

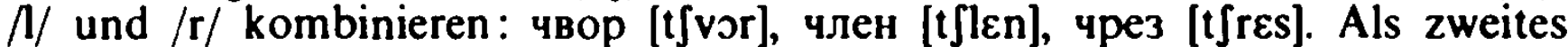
Glied im Anlaut erscheint bulgarisches $/ \mathrm{t} / \mathrm{f}$ nach / $\mathrm{v} /$ und /s/: Buepa ['ft $\left.\int \varepsilon r a\right]$, счукан ['st Jukan], als auch nach /n/: пчела [ptse'la].

\subsubsection{Auslautstrukturen}

Der Auslaut ist ebenfalls nicht reich an Konsonantenverbindungen mit Affrikaten in Finalstellung.

Deutsches /pf/ bildet zweigliedrige Kombinationen nur mit $/ \mathrm{m} /$ und $/ \mathrm{r} /$ : Kampf [kampi], Karpf [karpf].

Die Affrikate /ts/ kombiniert sich im Deutschen auslautend mit $/ 1 /, / n /, / r /$ und mit /द̧/: Pilz [pilts], Kranz [krants], Herz [herts], Geächz [ga'Eçts]. Bulgarisches /ts/ bildet zweigliedrige Auslautstrukturen mit $/ \mathrm{n} /, 1 /, / \mathrm{r} /$ und zwar in gebräuchlichen Fremdwörtern: танц [tants], филц [filts], кварц [kvarts].

Die deutsche Konsonantenverbindung / $\mathrm{t} /$ bildet im Auslaut keine weiteren Kombinationen mit anderen Konsonanten. Bulgarisches / $\mathrm{t} / \mathrm{s}$ verbindet sich mit den Sonoren $/ \mathrm{n} /, / \mathrm{r} /$ im Auslaut: хленч [xlent $]$ ], смърч [smort]].

- Trotz der biphonematischen Wertung des deutschen [t]] wird auch diese Konsonantenverbindung in die konfrontative Ubersicht der Distribution und Kombinatorik aufgenommen. 


\section{SONORLAUTE}

Deutsch: Nasale $/ \mathrm{m} /, / \mathrm{n} /$

Lateralengelaut $/ 1 /$

Vibrantes $/ \mathrm{r} /$

Bulgarisch: Nasale $/ \mathrm{m} /, / \mathrm{m}^{\prime} / ; / \mathrm{n} /, / \mathrm{n}^{\prime} /$

Laterale $/ \mathrm{l} / \mathrm{/l} /$

Vibrantes $/ \mathbf{r} /, / \mathbf{r}^{\prime} /$

\subsection{ALLGEMEINES ZU DEN SONOREN}

Sowohl phonetisch als auch phonologisch bilden die Sonorlaute eine Gruppe für sich. Artikulatorisch weisen sie gleichzeitig konsonantische und vokalische Merkmale auf: Es wird entweder ein oraler VerschluB bei gleichzeitiger Nasalöfnung gebildet (Nasale) oder ein oraler VerschluB mit gleichzeitiger Lateralenge (Lateralengelaute), oder aber ein intermittierender VerschluB (Vibrantes). Ihr Spektrum zeigt eine vokalähnliche, klar ausgeprägte Formantenstruktur, mit schwächerer Intensität der Formantenstreifen als bei den Vokalen. Ein weiteres gemeinsames phonetisches Charakteristikum der Sonorlaute, das sich am Sonagramm leicht beobachten läßt, ist der stark ausgeprägte koartikulatorische Einfluß der Lautnachbarschaft auf ihre Bildung.

In den meisten Weltsprachen weisen die Sonorlaute keine stimmlosen Korrelate auf. Deswegen verhalten sie sich im Redekontext assimilatorisch neutral.

\subsubsection{Nasale}

Die Nasale sind eng verwandt mit den VerschluBlauten - sie alle weisen orale Verschlußbildung an Lippen, Alveolen, Gaumen auf, d.h. dort, wo die entsprechenden Verschlußlautgruppen artikuliert werden. Eine artikulatorische Konfrontation mit den entsprechenden oralen Verschlußkonsonanten ergibt, daß die Nasale einen größeren Kieferwinkel haben, also offener sind (Ne pe r t, Pét urs s o n, 1984, 143). Allgemeines Keinnzeichen der Nasale und gleichzeitiger Unterschied zu den Verschlußlauten ist die nasale Öfnnung für die ausströmende Luft, gewährleistet durch die orale Senkung des Gaumensegels. Der Nasenhohlraum ist in seiner Form unveränderlich; daher ist die Resonanz der Luftsäule im Nasaltrakt für alle Nasalkonsonanten die gleiche. Die Spektralunterschiede, wenn auch sehr gering, sind in Abhängigkeit von der Lage der oralen Verschlußstelle, die die Nebenluftsäule unterschiedlich lang gestaltet: beim bilabialen Verschluß am längsten, beim postpalatalen bzw. velaren am kürzesten. Die perzeptive Identifizierung der einzelnen Nasalkonsonanten erfolgt im Sprechverlauf aufgrund der Vokalformantenübergänge. Sie sind nämlich für jeden Nasalkonsonanten spezifisch und charakteristisch, da sie von der Artikulationsstelle abhängen ( $\mathrm{Neppert}$, Pétursson, 1984, 144; Тилков, Бояджиев, 1977, 116). 
Nasalkonsonanten sind in den meisten Weltsprachen stimmhaft.

Da die Nasale sowohl das Merkmal ,konsonantisch" als auch das Merkmal „vokalisch“ besitzen, kann als differenzierendes Merkmal zwischen ihnen und den homorganen stimmhaften Verschlußlautkonsonanten das Vorhandensein bzw. das Fehlen von Nasalität fungieren. So ergibt sich für das Deutsche und für das Bulgarische folgende Korrelation:

Deutsch: / + nasal/ $m, n, \eta$.

$$
\text { / - nasal/ b, d, g }
$$

Bulgarisch: / + nasal/ $m, m$, $n, n^{\prime}$

Deutsches $/ \mathrm{m} /$

$$
\text { /-nasal/ v, v, d, d' }
$$

Bulgarische $/ \mathrm{m} / \mathrm{.} / \mathrm{m}^{\prime} /$

Die Artikulation ist die gleiche wie bei d. [p, b] bzw. b. [p, b; p', b']. Der palatale Effekt des bulgarischen [ $\left.\mathrm{m}^{\prime}\right]$ im Vergleich zu deutschem [m] und bulgarischem [m] wird durch die zusätzliche Artikulation des mittleren Zungenrückens erreicht. Ihr akustisches Spektrum, ein kompaktes, weist eine klar ausgeprägte Formantenstruktur auf, deren Intensität zu den höheren Frequenzen hin verhältnismäßig stark abfällt. Nach perzeptivem Eindruck lassen sich die bilabialen Nasale als „tief" bestimmen.

Deutsches $/ n /$

Bulgarische $/ n /, \quad n^{\circ} /$

Die Artikulation unterscheidet sich nicht von der der deutschen $[t, d]$ und der bulgarischen [t, $t^{\prime} ; d, d^{\prime}$ ]. Der palatale Effekt des bulgarischen [ $\left.n^{\prime}\right]$ wird durch die Verlegung des Verschlusses von den Alveolen auf den harten Gaumen erzielt.

Das akustische Spektrum von d. [n], b. [n, n'] ist ein diffuses, perzeptiv werden sie als "hoch" charakterisiert.

Deutsches $/ \mathfrak{y} /$

Bulgarisches velares Allophon / $\mathrm{j} /$ vor $/ \mathrm{g} . \mathrm{k} /$

Bei den Velarnasalen: deutsches [ $\eta]$, bulgarisches $[\eta]$ vor $[\mathrm{g}, \mathrm{k}]$, erfolgt die Gestaltung des Mundraumes wie bei [g. $k]$. Ihr Spektrum zeigt ein diffuses Bild, perzeptiv sind sie ebenfalls als "hoch" zu bestimmen.

Deutsches $/ \mathfrak{g} /$ ist distributionell eingeschränkt: Es kommt nur in- und auslautend und zwar stets nach kurzem Vokal, bzw. auslautend vor [0]; außerdem noch vor $/ \mathrm{k} /, / \mathrm{s} /$ in nativen Wörtern und vor $/ \mathrm{g} /$ in Fremdwörtern: Junge, Übung; Enkel, Zank, hangst; Mangan. Vorwiegend wegen der starken distributionellen Einschränkung wird deutsches / $\mathfrak{g} /$ von Is a č enk o (1974, $77 \mathrm{~m})$ und W u rzel $(1984,959 \mathrm{~m})$ als Phonemverbindung von $[\mathrm{n}+\mathrm{g}]$ gewertet.

Meinhold und Stock akzeptieren in Anlehnung an Morciniec (1968, 64m) die phonetische Einheitlichkeit des / $\mathfrak{n}$ / und die Unmöglichkeit, es durch Kommutation weiter zu segmentieren, und werten es monophonematisch. Sie heben außerdem noch hervor, da $\beta$ sich $/ \mathfrak{\eta} /$ in der Kommutation als distinktiv erweist (Meinhold, Stock, 1982, 131).

Bulgarisches [ $\mathbf{\eta}]$ tritt als Allophon von $/ \mathrm{n} /$ nur vor $/ \mathbf{g} /$ und $/ \mathbf{k} /$ auf: Ангел, црьнкам. Am Wortende und zwischen zwei Konsonanten wird im Gegensatz zum Deutschen nie ein [n] realisiert.

Bulgaren realisieren deutsches $/ \mathfrak{y} /$ meist als Lautverbindung. Die Ursachen fur die Fehlbildung sind in den distributionellen Unterschieden zwischen 
deutschem velarem $/ \mathfrak{\eta} /$ und bulgarischem [ $\eta]$, aber auch in der graphischen Darstellung des $/ \mathfrak{y} /-\langle n g\rangle$ zu suchen (Siehe Sonagramme - S. 194-196).

\subsubsection{Lateralengelaute}

Deutsches /1/

Bulgarische $\Lambda /, / 1$

Die Lateralengelaute sind in den verschiedenen Sprachen durch eine ganze Reihe verschiedener Bildungsarten vertreten. Das artikulatorisch Gemeinsame aller dieser Bildungsarten ist die Ausbildung einer Lateralenge durch die Berührung verschiedener Teile des Vordersaums oder der Oberfläche der Zunge mit dem Gaumen irgendwo in seiner Mittellinie und durch die gleichzeitige Senkung der Zungenränder im Bereich der Backenzähne auf einer oder zu beiden Seiten. Der erzeugte Schall wird auf diesem seitlichen Umweg übertragen, wodurch die mitschwingende Luftsäule eine spezifische Hohlraumform bekommt. Die Lateralengelaute sind einem starken koartikulatorischen EinfluB ausgesetzt.

Im Hochdeutschen gibt es nur einen phonetischen Haupttypus von $/ 1 /$, der mehreren koartikulatorischen Modifikationen unterliegt, wobei aber seine lautbestimmenden Artikulationsmerkmale gleichartig bleiben (Wängler, 1960, 87). Deutsches (l] ist ein apikal-alveolarer Lateralengelaut. Die Zungenspitze berührt bei leicht geöffneten Lippen das Alveolargebiet. Lateral bilden die Zungenränder mit den Backenzähnen einen schmalen Spalt, der die hinausströmende Luftsäule klangbestimmend modifiziert.

Bulgarisches [l] wird ebenfalls apikal-alveolar bis dental gebildet. Die Lautnachbarschaft übt auf seine Bildung einen sehr starken koartikulatorischen Einflu $B$ aus; je danach ob es vor einem hinteren bzw. einem vorderen Vokal zu stehen kommt, wandert der perzeptive Eindruck von einer "harten“ zu einer „weichen" Klangfarbe. Dieser starke koartikulatorische EinfluB der vokalischen Lautnachbarschaft auf das [l] läßt sich auch in vielen anderen Sprachen beobachten, nur nicht bei den deutschen [I]-Allophonen. O. v. Essen hat der Klangverwandtschaft zwischen [I] und [i] und [I] und [u] eine experimentalphonetische Studie gewidmet. Er nennt die beiden l-Spektren „ein dunkles, u-artiges“ und „ein helles, i-artiges“ (E s s e n, 1963, 53).

Die phonetischen Charakteristika des weichen bulgarischen l-Allophons fallen vollkommen mit denen des deutschen [1] zusammen (Siehe Sonagramme - S.197). Es weichen allerdings stark auseinander bulgarisches hartes [t] und deutsches [I], was sich im Deutschunterricht für Bulgaren interferierend auswirkt (Siehe Sonagramme - S. 198).

Bulgarisches palatales [I'] wird gebildet, indem der Verschluß von dem unteren auf das obere Alveolargebiet verlegt, und die Berührungsfläche zwischen Zunge und Palatum vergrößert wird, da auch die vorderen Zungenränder während der Artikulation am harten Gaumen kleben.

Spektral zeigen die Formantengebiete des [l'] eine Tendenz zur schwachen Erhöhung im Vergleich zu [l].

Die artikulatorisch-akustischen Unterschiede zwischen dem deutschen [l] und dem bulgarischen [ $\left.I^{\prime}\right]$ sind stark ausgeprägt. Die Interferenz im Deutschunterricht für Bulgaren ist aus diesem Grunde auffallend (Siehe Sonagramme - S. 200). 


\subsubsection{Vibrantes}

Das Charakteristische der Vibrantes besteht in der kurzzeitigen intermittierenden Berührung zwischen einem Teil der Zunge und dem gegenüberliegenden Gaumengebiet. Dabei kann ein- bzw. mehrmaliges flatterndes Anschlagen des artikulierenden Organs an die Berührungsfläche zustandekommen. Die Vibration entsteht dadurch, „daß, analog zu den Verhältnissen bei der Phonation, ein relativ weich gehaltenes Organ verschlußbildend leicht gegen eine Oberfläche gedrückt wird" (N e p pert, Pé t u r s s o n, 1984, 148) und den kurzzeitigen Verschluß gleich wieder freigibt. Der perzeptive Eindruck davon ist ein „dem Rollen eines Gegenstandes ähnliches Geräusch“" (Ess e n, 1979, 111).

Der Stimmklanganteil der Vibrantes wird als Vokalspektrum gegliedert, denn in den Öfnungsphasen liegt eine spezifisch geformte Luftsäule als Resonator vor. In der Öfnungsphase wird die bereits durch die Stimmlippen modiflzierte Luftsäule ein zweites $\mathrm{Mal}$ in einer anderen Frequenz moduliert (L in d n e r, 1981, 259). Artikulationsstelle und artikulierendes Organ schlagen sich aber akustisch nieder, was in den Spektren an der unterschiedlichen Lage der Formanten erkennbar ist und perzeptiv unterschiedliche r-Qualitäten erkennen läßt (Siehe Sonagramme - S. 201).

\subsubsection{Deutsches $/ r /$ und seine Allophone}

Deutsches $/ \mathrm{r} /$ wird unterschiedlich realisiert:

- Durch drei fakultative Allophone:

Zäpfchen-R[R], Zungenspitzen-R[r] und frikatives $R[\mathbf{k}]$ im Morphemanlaut: Rat [ra:t], [Ra:t], [ka:t]; Im Morphemauslaut nach Kurzvokal: Narr [naR], [пав]; Wort [voRt], [vost].

- Durch das vokalische $R[\mathrm{e}]$ :

Auslautend fur -er: Vater ['fa:te]; in den Affixen er-, her-, ver-, zer-: erleben [e'le:ban]; nach langem Vokal in einsilbigen Wörtern: Tor [to: $\left.:^{\mathbb{E}}\right]$, der [de $\left.:^{\mathbb{E}}\right]$; als Ersatzdehnung des vorangehenden Vokals: Wort [ $\left.v^{\mathbb{E}} t\right]$, wirklich [vi ${ }^{\mathbb{E}}$ kliç].

- Durch vollkommene Auslassung nach [a:]: war [va:], Gefahr [gə'fa:] (Vgl. Ulbrich, 1972).

Die drei fakultativen Allophone [r], [R] und [s] sind gleichberechtigt. Obwohl Zungenspitzen- $R$ historisch ursprünglich ist, ist Zäpfchen- $R$ viel mehr verbreitet; Ulbrich stellt 1972 durch statistisch-experimentelle Untersuchungen an etwa 10600 R-Realisierungen von Rundfunksprechern und Schauspielern ein sehr häufiges Auftreten von Reibe- $R$ [k] in der Position des $[r]$ und $[R]$ fest. Das Reibe- $R$ ist kommunikativ in keiner Weise hinderlich, so da $B$ man heute von drei und nicht von zwei gleichberechtigten fakultativen Allophonen des konsonantischen $R$ im Deutschen sprechen kann.

Für Zäpfchen-R und Zungenspitzen- $R$ ist die Bezeichnung ,gerollte R-Laute“ üblich. Ungeachtet dessen, ob die entspannte Zungenspitze oder das entspannte Zäpfchen Flatterbewegungen ausführen, beträgt die Anzahl der flatterartigen Schläge 1-2. In ihrem Merkmal "Stimmhaftigkeit“ enthalten beide $R$-Laute akustische Unterschiede und weisen perzeptiv unterschiedliche Vokalqualitäten auf. 
Das frikative $R[\mathbf{k}]$ stellt artikulatorisch eine Andeutung der postdorsalen Rinnenbildung dar, aber das Zäpfchen wird nicht zum Schwingen gebracht, sondern bildet nur den Kanal unter Geräuschproduktion (L i nd ner, 1981, 259). Das akustische Spektrum des [6] weist keinen Stimmklanganteil auf.

Das vokalische $R[e]$ ist, phonetisch gesehen, ohne Zweifel ein Vokal. Mit dem Murmelvokal [a] hat es die Positionen gemeinsam, in denen es aufritt (nur unbetont!). Der Klangunterschied zwischen den beiden unbetonten Vokalen wird artikulatorisch durch eine beim $[\mathfrak{e}]$ angedeutete postdorsale Zungenhebung bewirkt, mit einer größeren pharyngalen Annäherung verbunden (Lindner, 1981, 239). Sein akustisches Spektrum zeigt a-artige Elemente (Siehe Sonagramme - S.191).

Sprachentwicklungsgemä $B$ und normativ hat sich eine feste Lautform, das Zungenspitzen- $R$ in einer verhältnismäßig kurzen Zeitspanne in mehreren Allophonen aufgelöst. Ob der Prozeß der Auflösung sich gerade im Rahmen eines Jahrhunderts abgespielt hat ( $\mathrm{i}$ ind $\mathrm{n}$ e $\mathrm{r}, 1981,259)$ (als Ansatz wird der von Siebs 1898 vorgeschriebene Gebrauch des Zungespitzen-R angegeben), ist fraglich, denn Lautveränderungen erstrecken sich gewöhnlich auf längere geschichtliche Perioden. Die vollzogenen Veränderungen, ja sogar die Dynamik eines sich weiter vollziehenden Wandels, sind aber experimentell belegt und belegbar, und das macht die phonologische Wertung des deutschen [r] schon problematisch.

Bis etwa vor einem Jahrzehnt wurde das Phonem / $r /$ auf Grund der Allophone [r] und [R], die nur an isolierten Oppositionen beteiligt waren, als isoliertes Phonem ausgewiesen. $\mathrm{Me}$ inhold und Stock (1982, 134) versuchen auch das frikative $[\boldsymbol{\sigma}] \mathrm{mit}$ in die phonologischen Überlegungen einzubeziehen und bieten eine Modifizierung der Fortis-Korrelation im Deutschen an, in der das [k] mit dem potentiellen Korrelationspartner [ $\mathrm{x}]$ in Opposition tritt. Diese Fortis-Korrelation sieht wie folgt aus:

$/+$ fortis/ p f $t$ s $\int \mathrm{c} k \mathrm{x}$
$/-$ fortis/ b $v \mathrm{~d} z \mathrm{j} \mathrm{j} \mathrm{r}$

Damit wäre diese Korrelation für das System der deutschen Konsonantenphoneme außerordentlich produktiv. Außerhalb von Korrelationen ständen nur noch $/ /$ und $/ \mathrm{h} /{ }^{\circ}$ (M e in hold, S tock, 1982, 134).

Die vokalisierten Allophone wären dann in die Gruppe der zentralisierten Vokale zu integrieren. Interessant verhält sich der Reduktionsvokal [e], gesprochen im Auslaut für die graphische Verbindung -er. Er bildet nämlich Minimalpaaroppositionen zum schwachtonigen [a]: Spinne - Spinner ['Jprno - 'spme], doch ist sowohl die Position eine alleinstehende als auch die funktionale Belastung eine geringe.

\subsubsection{Bulgarisches $/ \mathrm{r} /$}

Zur Bildung von $/ r /$ wird die Zunge gehoben, wobei sie mit ihren seitlichen Rändern den oberen Zahndamm berührt. Die locker gehaltene Zungenspitze wird durch den Phonationsstrom in Schwingung versetzt und bildet vibrationsartig einen intermittierenden VerschluB an das Zahn-Alveolargebiet. Dabei weist der bulgarische R-Laut in Positionen vor betontem Vokal 2-3 
verschlußbildende Schläge auf, in Positionen vor unbetontem Vokal verringern sie sich. Das akustische Spektrum des [r] unterscheidet sich nicht von dem des deutschen Zungenspitzen-R.

Das bulgarische $/ r /$ hat eine breit gefächerte Distribution und reiche Kombinationsmöglichkeiten.

\subsubsection{Bulgarisches $/ \mathrm{r}^{\prime} /$}

Im Vergleich zum [r] wird die Artikulationsstelle von [r'] weiter nach oben verlagert, so daß die Zungenspitze an den harten Gaumen schlägt. Der Verschluß mit dem Gaumen, mit den mittleren Zungenrändern gebildet, ist großnächiger und intensiver.

Vergleicht man die akustischen Bilder von [r] und [r'], so stellt man eine Gleitbewegung der Formantenfrequenz in Richtung Transition (Übergang) zum nachfolgenden Vokal fest, wie bei jeder palatalen Artikulation.

Interferenzerscheinungen sind bei der Realisierung aller deutschen R-Allophone durch Bulgaren zu erwarten.

\subsection{PARADIGMATISCHE UND SYNTAGMATISCHE DARSTELLUNG DER DEUTSCHEN UND DER BULGARISCHEN SONORLAUTE}

9.2.1. Minimalpaaroppositionen der deutschen und der bulgarischen Sonorlaute

\section{Innerhalb der Sonorgruppe}

d. $/ \mathrm{m} /-/ \mathrm{n} /:$ Mull - Null $/ \mathrm{mol} /$ - /nol/

Same - Sahne ['za:mə] - ['za:nə]

Kimm - Kinn [kım] - [kın]

$/ \mathrm{m} /-/ \mathrm{g} /:$ Klamm - Klang [klam] - [klan]

Imme - Inge ['ima] - ['ino]

schwimmen - schwingen ['Sviman] - ['Svinan]

/m/ - /1/: Möwe - Löwe ['mø:va] - ['lø:va]

Stimme - Stille ['stıma] - ['Stıla]

Grimm - Grill [grım] - [gril]

$/ \mathrm{m} /-/ \mathrm{r} /:$ Motte - Rotte ['mota] - ['rota]

schwimmen - schwirren ['Sviman] - ['Sviran]

Mumm - Murr [mom] - [mor]

$/ \mathrm{m} /-/ \mathrm{e} /:$ Ohm - Ohr $[0: \mathrm{m}]-[0: \mathrm{e}]$

Lehm - leer [le:m] - [le:e ]

Ruhm - Ruhr [ru:m] - [ru: $\left.:^{\text {e }}\right]$

$\mathrm{m} /$ - /o/: Rahm - rar [ra:m] - [ra:]

b. $/ \mathrm{m} /-/ \mathrm{m}^{\prime} /$ : Mapa - мяpa ['mara] - ['m'ara]

$/ \mathrm{m} /-/ \mathrm{n} /$ : мош - нош $[\mathrm{mos} \mathrm{t}]-[\mathrm{n} \rho \mathrm{st}]$

чим - чин [t $\left.\int \mathrm{Im}\right]-\left[\mathrm{t} \int \mathrm{In}\right]$

Камен - канен ['kamen] - ['kanen] 
$/ \mathbf{m}^{\prime} /$ - /g'/: Мянка - нянка ['m'anka] - ['n'anka]

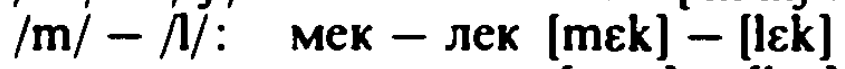

мъX - льх [məx] - [tax]

сом - сол [som] - [sot]

рамо - рало ['ramo] - ['rało]

$/ \mathrm{m}^{\prime} /-\Lambda^{\prime} /$ : тамян - талян [ta'm'an] - [ta'l'an]

$/ \mathrm{m} /-/ \mathbf{r} /:$ мaca - paca ['masa] - ['rasa]

мед - ред [met] - [ret]

гама - rapa ['gama] - ['gara]

чам - чар [t $\left.\int \mathrm{am}\right]$ - [t $\left.\int \mathrm{ar}\right]$

$/ \mathbf{m}^{\prime} /-/ \mathbf{r}^{\prime} /:$ мамя - Mapя ['mam'a] - ['mar'a]

d. $/ \mathbf{n} /-/ \mathbf{n} /:$ Tann - Tang $[\tan ]-[\tan ]$

sinnen - singen ['zınən] - ['zınən]

$/ \mathrm{n} /$ - / $/$ : Neid - Leid [noiet] - [löet]

Wonne - Wolle ['vona] - ['vola]

Bann - Ball [ban] - [bal]

$/ \mathbf{n} /-/ \mathbf{r} /: \quad$ Nest - Rest [nest] - [rest]

Dünne - Dürre ['dynə] - ['dyra]

Gewinn - Gewirr [ga'vin] - [ga'vir]

$/ \mathbf{n} /-/ \dot{e} /: \quad$ Föhn - Föhr [fø:n] $-[$ [ø :e $]$

$k u ̈ h n-k u ̈ r ~[k y: n]-[k y: \mathbb{e}]$

/n/ - /o]: Clan - klar [kla:n] - [kla:]

b. $/ \mathrm{n} /-\Lambda /:$ ниже - лиже ['nife] - ['life]

кон - кол [kon] - [kot]

кана - кала ['kana] - ['kała]

канен - кален ['kanen] - ['kalen]

$/ n^{\prime} /$ - /l': : сменя - смеля ['smen'a] - ['smel'a]

$/ \mathbf{n} /-/ \mathbf{n}^{\prime} /:$ нам - ням [nam] - [n'am]

$/ \mathbf{n} /-/ \mathbf{r} /:$ низа - риза ['niza] - ['rıza]

тон - тор [ton] - [tor]

тонен - торен ['tonen] - ['toren]

$/ \mathbf{n}^{\prime} /-/ \mathbf{r}^{\prime} /:$ пеня - перя ['pen'a] - ['per'a]

d. $/ /-/ \mathbf{r} /:$ Luder - Ruder ['lu:de] - ['ru :de]

hell - Herr [hel] - [her]

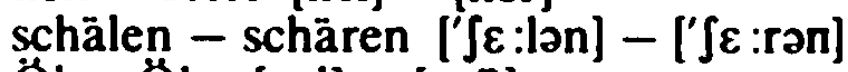

$/ 1-/ e /: \quad$ Öl - Öhr [ø:l] - [ø:e $]$

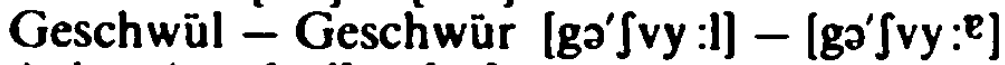

/1/- $0 /: \quad$ Aal - Aar [a:l] - [a:]

b. $\Lambda /-/ \mathrm{r} /:$ лов - ров [bI] - [rof]

вал - вар [vat] - [var]

бала - бара ['bata] - ['bara]

$\Lambda^{\prime} /-/ \mathbf{r}^{\prime} /:$ меля - меря ['mel'a] - ['mer'a]

b. $/ \mathbf{r} /-/ \mathbf{r}^{\prime} /:$ pana - pяna ['rapa] - ['r'apa] 


\section{Oppositionen zu Explosiven \\ (Siehe S. 81) \\ Oppositionen zu Frikativen}

d. $/ \mathbf{m} /-/ \mathbf{v} /-/ \mathbf{f} /$ :

Mal - Wahl - fahl [ma:l] - [va:l] - [fa:l]

reimen - reifen ['räemən] - ['ráefən]

Reim - Reif [râem] - [râef]

b. $/ \mathbf{m} /-/ \mathbf{v} /-/ \mathbf{f} /$ :

Mac - Bac - фac [mas] - [vas] - [fas]

слама - слава ['słama] - ['słava]

Алма - алфа ['ałma] - ['atfa]

$/ \mathrm{m}^{\prime} /$ - /v' : мяра - вяра ['m'ara] - ['v'ara]

d. $/ \mathrm{m} /-\mid \mathrm{z} /-/ \mathrm{s} /$ :

Mal - Saal [ma:l] - [za:l]

Name - Nase ['na:mə] - ['na :za]

Ramme - Rasse ['ramə] - ['rasa]

Ruhm - RuB [ru:m] - [ru:s]

b. $/ \mathrm{m} /-\mid \mathrm{z} /-/ \mathrm{s} /$ :

мор - 3оp [mor] - [zor]

Мила - сила ['miła] - ['sıła]

рима - риза ['rıma] - ['rıza]

мама - мaca ['mama] - ['masa]

вам - вac [vam] - [vas]

$\left|\mathbf{m}^{\prime}\right|-\left|\mathbf{z}^{\prime}\right|-\mid \mathbf{s}^{\prime} /:$

срамя - сразя [sra'm’a] - [sra'z'a]

мяра - cяpa ['m'ara] - ['s'ara]

d. $/ \mathbf{m} /-\mid \mathbf{j} /-/ \mathbf{c} /-/ \mathrm{s} /$ :

maulen - jaulen ['mâolən] - ['jôolən]

Reim - Reich [raem] - [raeç]

leimen - leichen ['läemən] - ['läeçan]

Mal - Schal [mo:l] - [ $a: l]$

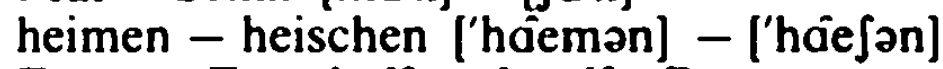

Farm - Farsch [farm] - [farf]

b. $/ \mathrm{m} /-/ \mathrm{y} /-/ \mathrm{f} /-/ \mathrm{s} /$ :

мода - йод[a] ['moda] - ['ixda]

Мица - жица ['mitsa] - ['fitsa]

кома - кожа ['koma] - ['ksfa]

мирен - ширен ['mıren] - ['Siren]

шумна - шушна ['Sumna] - ['Jufna]

вам - ваш [vam] - [vaf]

d. $/ \mathrm{m} /-/ \mathbf{x} /-/ \mathrm{h} /$ :

kommen - kochen ['kəmən] - ['kəxən]

Baum - Bauch [boiom] - [boiox]

Mehl - Hehl [me:l] - [he:l]

b. $/ \mathrm{m} /-/ \mathbf{x} /$ : майка - хайка ['mäika] - ['xaiika]

сумa - cyxa ['suma] - ['suxa]

грам - rpax [gram] - [grax] 
d. $/ \mathbf{n} /-/ \mathbf{v} /-/ \mathbf{f} /$ :

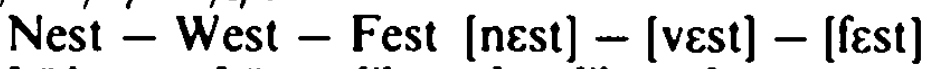

Löhne - Löwe ['lø:na] - ['lø:və]

Zone - Zofe ['tso:na] - ['tso:fa]

rein - reif [râen] - [ráef]

b. $/ \mathbf{n} /-/ \mathbf{v} /-/ \mathbf{f} /$ :

Hac - Bac - фac [nas] - [vas] - [fas]

брана - брава ['brana] - ['brava]

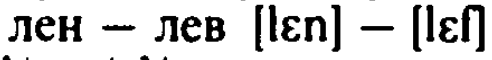

$/ \mathbf{n}^{\prime} /-/ \mathbf{v}^{\prime} /:$ кърня - кървя [ker'n’a] - [ker'v’a]

d. $/ \mathrm{n} /-|\mathbf{z}|-\mid \mathrm{s} /$ :

Nonne - Sonne ['nona] - ['zona]

löhnen - lösen ['lø:nən] - ['lø:zən]

Tanne - Tasse ['tanə] - ['tasə]

Mohn - Moos [mo:n] - [mo:s]

b. $/ \mathbf{n} /-|\mathbf{z}|-\mid \mathbf{s} /$ :

нар - 3ap [nar] - [zar]

нос - coc [nos] - [sos]

вана - ваза ['vana] - ['vaza]

кана - каса ['kana] - ['kasa]

КOH - KOC [kon] - [kos]

$\left|\mathbf{n}^{\prime} /-\right| \mathbf{z}^{*}|-| \mathbf{s}^{\prime} \mid:$

нянка - сянка ['n’anka] - ['s'ajka]

коня - козя ['kon'a] - ['k⿰z'a]

d. $/ \mathbf{n} /-/ \mathbf{j} /-/ \mathrm{c} /-/ \mathrm{s} /$ :

nagen - jagen ['na :gən] - ['ja :gən]

Leine - Leiche ['läenə] - ['láeça]

rein - reich [röen] - [râeç]

NuB - SchuB [nos] - [jus]

Tanne - Tasche ['tana] - ['tafo]

Kinn - Kisch [kın] - [kı $]$

b. $/ \mathrm{n} /-/ \mathrm{L} / \mathrm{L} / \mathrm{L} / \mathrm{J} /$ :

Нона - Иона ['nona] - ['ına]

нар - жар [nar] - [3ar]

налче - шалче ['nalt $\left.\int e\right]$ - ['Salt $\left.\int e\right]$

мрена - мрежа ['mrєna] - ['mrєza]

кана - каша ['kana] - ['kaja]

d. $/ \mathrm{n} /-/ \mathrm{x} /-/ \mathrm{h} /$ :

Bann - Bach [ban] - [bax]

Wonne - Woche ['vona] - ['voxa]

Nebel - Hebel ['ne :bal] - ['he :bal]

b. $/ \mathbf{n} /-/ \mathrm{c} /:$ нижа - хижа ['nıza] - ['cıza]

d. $/ \mathfrak{y} /$ - $/ \mathbf{f} /$ : Hang - Haff [han] - [haf]

bangen - baffen ['bayən] - ['bafən]

$/ \mathfrak{y} /-/ \mathbf{s} /:$ Fang - FaB [fay] - [fas] mengen - messen ['menən] - ['mesən]

$/ \mathfrak{y} /$ - / $/:$ Rang - rasch [ran] - [raf]

Finger - Fischer ['fine $]-\left[{ }^{\prime} f\left(\int e\right]\right.$ 


$$
\begin{aligned}
& / \mathrm{y} /-/ \mathrm{c} /: \text { Ding - dich [din] - [dic] } \\
& \text { sprengen - sprechen ['Sprenən] - ['Spreçən] } \\
& / \mathfrak{y} /-/ \mathrm{x} /: \text { bang - Bach [bay] - [bax] } \\
& \text { Wange - Wache ['vanə] - ['vaxə] }
\end{aligned}
$$

d. $/ 1 /-/ z /$ : Land - Sand [lant] - [zant]

b. $|\mathbf{l}|-|\mathbf{z}|-\mid \mathrm{s} /$ :

$$
\begin{aligned}
& \text { лапад - запад ['łapat] - ['zapat] } \\
& \text { кола - коза [ko'ła] - [ko'za] } \\
& \text { лито - сито ['lito] - ['sito] } \\
& \text { кол - кос [kot] - [kos] } \\
& / \mathrm{l} \%-/ \mathrm{s} \% \text { : лягам - cяraм ['l'agam] - ['s'agam] }
\end{aligned}
$$

d. $/ \mathrm{l} /-/ \mathrm{j} /-/ \mathrm{q} /-/ \mathrm{J} /$ :

Lade - Jade ['la:də] - ['ja:də]

Teil - Teich [táel] - [tâeç]

Eile - Eiche ['âelo] - ['áeça]

lohnen - schonen ['lo:nən] - ['Jo:nən]

heilen - heischen ['hoielan] - ['haiesan]

b. $/ 1 /-/ 1 /-/ f /-/ \mathrm{s} /$ :

$$
\begin{aligned}
& \text { лов - Иов [њf] - [10f] } \\
& \text { лито - жито ['lito] - ['fito] } \\
& \text { кола - кожа ['ksta] - ['ksfa] } \\
& \text { лайка - шайка ['läika] - ['Säika] } \\
& \text { кила - киша ['kiła] - ['kı } \left.\int a\right] \\
& \text { кол - кош [kot] - [kof] }
\end{aligned}
$$

d. $/ \mathbf{l} /-/ \mathrm{x} /-/ \mathrm{h} /$ :

Ball - Bach [bal] - [bax]

wallen - wachen ['valon] - ['vaxən]

leben - heben ['le:bən] - ['he:bən]

b. $/ 1 /-/ x /$ : лайка - хайка ['läika] - ['xaika]

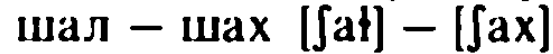

кула - куха ['koła] - ['kuxa]

9.2.2. Distribution und Kombinatorik der Sonorlaute im Deuschen und im Bulgarischen (Übersicht auf der Grundlage von An- und Auslautstrukturen)

\subsubsection{Anlautstrukturen}

Deutsch

- Sonorlaute lassen sich nicht im Anlaut miteinander kombinieren.

- Ein Sonorlaut kann nur als zweites Glied einer konsonantischen Anlautverbindung auftreten.

\section{Bulgarisch}

- Sonorlaute (ausgenommen $\Lambda, r /$ kommen als erstes Glied einer konsonantischen Verbindung nur vor andere Sonore vor: млад [młat], мляко ['ml’ako], мраз [mras], мряна ['mr'ana], много ['mnogo], нрав [nraf]. 
$-/ \mathrm{f} /, / \mathrm{v} /$ (sehr begrenzt) sind die einzigen Frikative, die in nativen Wörtern vor Sonor auftreten. In Fremdwörtern können auch $/ \mathrm{v} /, / \mathrm{s} /$ als erstes Glied vor Sonor erscheinen (Beispiele - Siehe S.94).

- Von der Explosivgruppe können nur $/ g /, / k$ / vor Sonor in deutschen Wörtern vorkommen; in Fremdwörtern kommt noch /p/ hinzu (Beispiele - Siehe $\mathrm{S} .81$ - 84).

- Die Affrikate /pf/ kombiniert sich anlautend allein mit $\Lambda, r /$ : Pflicht [pfliçt], Pfropfen ['pfropfan].

$-/ \mathrm{t} J /$ ist anlautend ausgeschlossen.

- Der velare Nasal $/ \mathfrak{\eta} /$ ist im Anlaut ausgeschlossen.

- In dreigliedrigen Anlautstrukturen kommen im Deutschen und im Bulgarischen $/ /, / r /$ auf und zwar immer als drittes Glied:

Deutsch:

/Spr/ Sprache

$/ \int \mathrm{pl} /$

['Spra:xə]

$/ \int t r /$

Splitter ['Splite]

Straße ['Jtra:sə]
- Frikativ und Sonor bilden häufig zweigliedrige Anlautstrukturen (Beispiele - Siehe S. 81-84).

- Alle nicht palatalisierten Explosive treten vor Nasal auf (Beispiele Siehe S. 82-84).

- Bulgarisches /ts/ kombiniert sich anlautend allein mit $/ \mathrm{r} /$ : цръквам ['tsrak vam]

- Die bulgarische Affrikate /t $\mathrm{f} / \mathrm{er}$ scheint als erstes Glied im Anlaut vor h, r/: член [t $\left.\int l \varepsilon n\right]$, чрез [t $\left.\mathrm{r} r \mathrm{~s}\right]$.

- Palatalisierte Konsonanten sind in Initialstellung vor Sonor ausgeschlossen.

Bulgarisch:

/Spr/ шпроти ['Sproti]

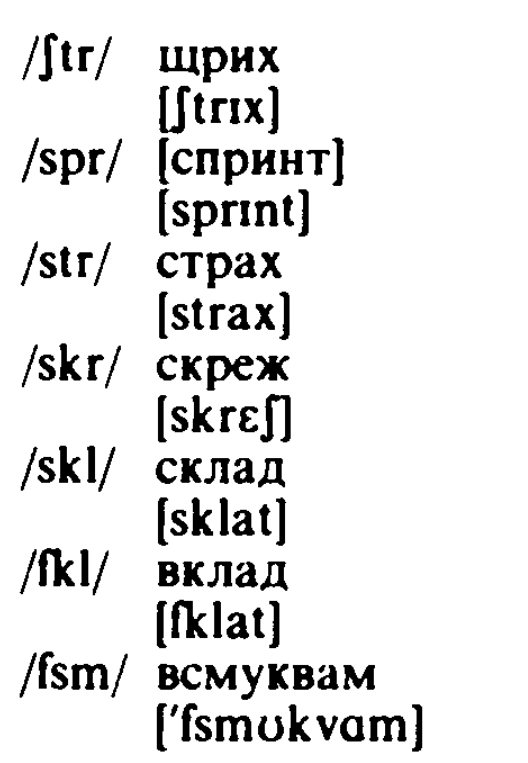

und andere (Siehe S. 81-84).

\subsubsection{Auslautstrukturen mit Sonor in Finalstellung}

Sowohl im Deutschen als auch im Bulgarischen sind zweigliedrige Auslautstrukturen mit Sonor in Finalstellung möglich. Im Bulgarischen ist ihr 
Auftreten jedoch sehr begrenzt. Deutsches $/ \mathfrak{n} /$ in Finalstellung nach Konsonant ist ausgeschlossen. Beispiele für Auslautstrukturen:

Deutsch

/rl/ Kerl [kerl]

/rn/ Dorn [dorn]

/lm/ Halm [halm]

$/$ ln/ rütteln ['rytaln]
Bulgarisch

/rl/ върл [varł]

$/ \mathrm{lm} /$ хълм [xәtm]

/n/ кълн [kałn]

\section{ZUSAMMENFASSENDE SCHLUSSBEMERKUNGEN}

Als Ergebnis der kontrastiven Analyse des deutschen und des bulgarischen Konsonantismus lassen sich folgende phonetische und phonologische Ubereinstimmungen, Unterschiede und sprachspezifische Besonderheiten zusammenfassend explizieren:

\section{Auf der Ebene der phonetischen Repräsentation Verschlußlaute (Explosive)}

Ubereinstimmungen:

- In beiden Sprachen werden die Verschlußlaute durch kurzzeitige Unterbrechung des Luftstromes realisiert, wobei der gesamte Ansatzraum während der Okklusionsphase vollkommen verschlossen wird.

- Deutsche und bulgarische Verschlußlaute werden nicht nasaliert - der Nasenraum wird im Bildungsproze $B$ durch die Hebung des Gaumensegels gegen den Passavantischen Wulst abgeschlossen; der gebildete orale VerschluB erfolgt durch eine aktive Bewegung des artikulierenden Organs, die Luft strömt oral aus.

Unterschiede :

- Die Stimmhaftigkeit der deutschen Explosive [b, d, g] kann fehlen. Dies ist sehr oft im Redekontext an Morphemgrenzen der Fall, wenn dort keine reale Pause verwirklicht wird. Die bulgarischen $[b, d, g]$ sind dagegen immer stimmhaft.

- Im Gegensatz zur Bildung der bulgarischen stimmlosen [p, $t, k]$ dauert die Lösungsbewegung des artikulierenden Organs bei der Artikulation der deutschen $[\mathrm{p}, \mathrm{t}, \mathrm{k}]$ kürzer an, und da sie außerdem noch dynamischer ist, bewirkt die folgernde Entspannung der Luftsäule einen hörbaren Hauch. Die bulgarischen $[\mathrm{p}, \mathrm{t}, \mathrm{k}$ ], bei denen die Lösungsphase von längerer Dauer und die Muskelspannung des artikulierenden Organs geringer ist, werden nicht aspiriert.

Sprachspezifische Besonderheiten:

Im Bulgarischen ist die Reihe der palatalisierten Verschlußlautphoneme vertreten. Bildungsmäßig werden sie durch eine zusātzliche Mittelzungenartikulation in Richtung harter Gaumen auf der Basis der "harten“ Verschlußlautkonsonanten realisiert. Akustisch schlägt sich diese zusātzliche 
Artikulation in Veränderung von Richtung und Dauer der Übergangssegmente (Transitionen) zum nachfolgenden Vokal nieder.

Engelaute (Frikative)

Übereinstimmungen:

- Artikulatorische Gleichheit beobachtet man zwischen den deutschen Frikativkonsonanten aus der labiodentalen und prädorsalalveolaren Gruppe und ihren .,harten“ bulgarischen Entsprechungen.

Unterschiede schälen sich heraus wie folgt:

a) Bei den prädorsal-palatalen Frikativen:

Im Vergleich zum bulgarischen / $1 /$ wird deutsches [j] mit kleinerer und zeitlich längerer Engebildung, größerer Artikulationsspannung und stärkerem Expirationsdruck realisiert (Vgl. S.87-88).

b) Den deutschen positionsbedingten Allophonen [c, $x$ ] entsprechen artikulatorisch und klanglich bulgarisches [c] und $[x]$ : хитър ['çter], хоро ['xo'rs]; der phonetische Unterschied besteht in dem geringeren Geräuschanteil bei den bulgarischen [ç], [x] im Gegensatz zu den deutschen [ç], [x]. Sprachspezifische Besonderheiten:

- Bis auf $/ \mathrm{y} /$ und $/ \mathrm{J} /$ weisen die bulgarischen Frikativkonsonanten entsprechende palatalisierte Frikativphoneme auf, deren artikulatorische Besonderheit durch eine zusätzliche Hebung der Mittelzunge in Richtung ibzw. e-Bildung gekennzeichnet wird.

- Deutsches $/ \mathrm{J} /$ hat in nativen Wörtern keine stimmhafte Entsprechung, dem bulgarischen $/ \mathrm{S} /$ entspricht das stimmhafte $/ 3 /$.

- Deutsches /h/ hat keine Entsprechung im Bulgarischen.

\section{Verschluß-Engelaute (Affrikaten)}

Üereinstimmungen:

Es besteht kein artikulatorischer Unterschied zwischen dem deutschen Affrikatenkonsonanten [ts] und dem bulgarischen "harten" [ts]; keine phonetischen Unterschiede sind auch zwischen bulgarischem [t $]$ ] und deutschem [t $\mathrm{f}]$ festzustellen.

Sprachspezifische Besonderheiten :

- Die deutsche Affrikate/pf/ ist im Bulgarischen nicht vorhanden.

- Die bulgarischen Affrikaten $/ \mathbf{t s} /-/ \mathbf{s s}^{\prime} /$ haben auch stimmhafte Entsprechungen: $/ \mathrm{dz} /-/ \mathrm{dz} /$, die jedoch eine sehr begrenzte Gebrauchsfrequenz aufweisen.

- Bulgarisches $/ \mathrm{t} \mathrm{j} /$ hat eine stimmhafte Entsprechung - /dz/, aber keine palatalisierte.

- Deutsche /ts/, /t $\mathrm{f} /$ weisen keine stimmhaften Entsprechungen auf.

\section{Sonorlaute}

Übereinstimmungen:

- Gleiche Artikulationsmerkmale weisen die deutschen und bulgarischen Nasale d. [m] - b. [m], d. [n] - b. [n] auf.

- Deutsches [I] und das bulgarische Allophon [I] sind sich artikulatorisch und dem Klang nach sehr ähnlich. 
- Dem deutschen Velarnasal [ $\eta]$ entspricht phonetisch und distributionell bulgarisches $[\mathrm{g}]$ vor $[\mathrm{g}],[\mathrm{k}]$.

Sprachspezifische Besonderheiten:

- $\mathrm{Zu}$ allen Sonorlauten des Bulgarischen gibt es palatalisierte Phonementsprechungen, für deren Bildung ebenfalls eine zusätzliche Mittelzungenartikulation notwendig ist.

- Der deutsche Vibrant / $r$ / weist eine interessante, orthoepisch normierte, positionsbedingte Palette von konsonantischen und vokalischen Varianten auf (Siehe S.111-112).

- Die bulgarischen $/ \mathbf{r} /, / \mathbf{r} /$ sind artikulatorisch stabil als ZungenspitzenFlatterlaute zu realisieren.

\section{Auf der Ehene der phonologischen Repräsentation}

- Durch das Vorhandensein der palatalisierten Konsonantenphoneme des Bulgarischen ist die Zahl der Konsonanten im Bulgarischen um das Doppelte größer als im Deutschen.

- Paradigmatisch-kontrastiv lassen sich die deutschen und die bulgarischen Konsonantenphoneme durch 10 distinktive Merkmale beschreiben; dabei wird in der bulgarischen Matrixtabelle die Leerstelle des Merkmals .laryngal - pharyngal" - relevant für das deutsche Konsonantensystem und irrelevant für das bulgarische - durch das allein für die bulgarischen Konsonantenphoneme relevante Merkmal „palatalisiert“ ersetzt (Siehe S. $74-77)$.

- Syntagmatisch lassen sich die Gemeinsamkeiten. Unterschiede und Sprachspezifika in bezug auf Distribution und Kombinatorik der Konsonantenphoneme in beiden Sprachen wie folgt zusammenfassen:

\section{A. Anlautstrukturen}

- Es fällt die an Konsonantenkombinatorik reichere Vielfalt im bulgarischen Wortanlaut im Gegensatz zum deutschen Wortanlaut auf. Im Deutschen überwiegen die zweigliedrigen konsonantischen Anlautstrukturen. und nur in sehr begrenztem Maße sind dreigliedrige Konsonantenverbindungen möglich: Sprache ['Spra:xə], Splitter ['Splıte], Strähne ['Jtre:nə], Skrupel ['skru:pal]. Im Bulgarischen bietet das Hinzufügen der Präfixe $\langle c\rangle$, 〈b〉 eine gute Möglichkeit zur Bildung von drei-, ja auch von viergliedrigen konsonantischen Anlautstrukturen (Siehe S. 101).

- Affrikaten bilden sowohl im Deutschen als auch im Bulgarischen einige wenige Konsonantenstrukturen im Anlaut. Deutsches [t $]]$ ist in nativen Wörtern anlautend ausgeschlossen.

- Im Deutschen lasser. sich Sonorlaute im Wortanlaut nicht miteinander kombinieren, während Sonor + Sonor im bulgarischen Wortanlaut öfter vorkommt.

- Dreigliedrige Anlautstrukturen sind in beiden Sprachen nur mit den Sonoren $/ \mathrm{l} /$ und $/ \mathrm{r} / \mathrm{möglich}$, wobei sie stets als drittes Glied erscheinen.

\section{B. Auslautstrukturen}

Hier sind die Gegebenheiten zu denen im Wortanlaut gerade umgekehrt: Der deutsche Auslaut ist viel reicher an mehrgleidrigen Konsonanten- 
verbindungen. So sind zum Beispiel in der 2. P. Sing. des Verbparadigmas bis auf funfgliedrige konsonantische Auslautstrukturen möglich. Im Bulgarischen treten weniger zweigliedrige Konsonantenverbindungen als im Anlaut auf, drei- und mehrgliedrige sind in nativen Wörten schon ausgeschlossen.

Im absoluten Auslaut kommen im Deutschen kein $/ \mathrm{h} /$ und kein $/ \mathrm{j} /$ vor. Bulgarisches /1/ verbindet sich auslautend ebenfalls mit keinem Konsonanten.

Die konsonantischen Kombinationsmöglichkeiten des Deutschen und des Bulgarischen sind im folgenden auf Tabellen veranschaulicht worden.

\section{Zweigliedrige Anlautstrukturen im Deutschen}
$\begin{array}{ll}\text { br } & \text { pr } \\ \text { bl } & \text { pl }\end{array}$
$\begin{array}{ll}\text { gr } & \mathrm{kr} \\ \mathrm{gl} & \mathrm{kl} \\ \mathrm{gn} & \mathrm{kn}\end{array}$
vr fr
gn $k n$
kv
$\begin{array}{ll}\text { (sn) } & \int \mathrm{r} \\ \text { (sm) } & \int \mathrm{n} \\ & \int \mathrm{v}\end{array}$
$\begin{array}{ll}\text { pfr } & \\ \text { pfl } & \text { tsv } \\ \text { (ps) } & t 5\end{array}$
(ks)

Zweigliedrige Anlautstrukturen im Bulgarischen

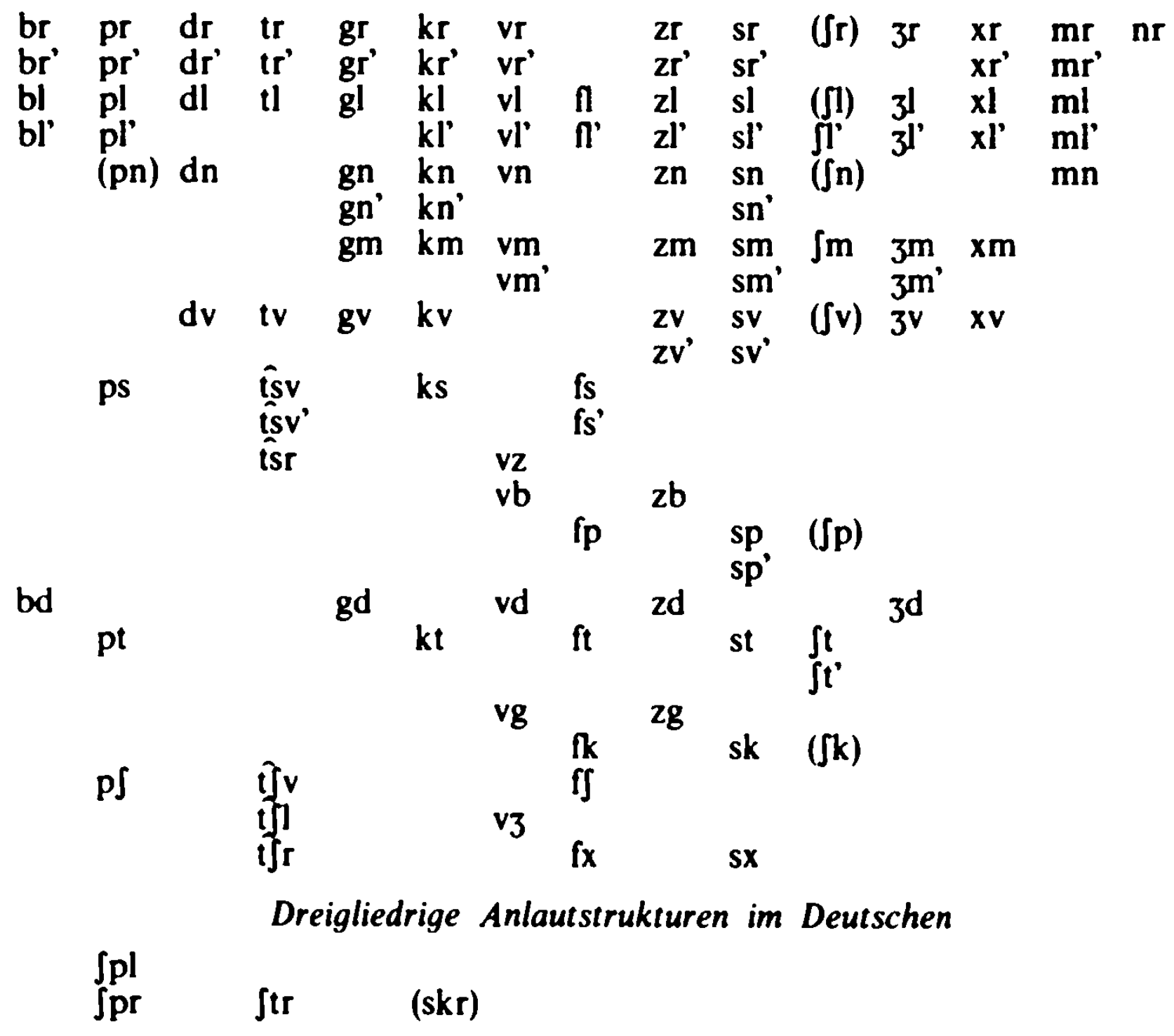


Dreigliedrige Anlautstrukturen im Bulgarischen

vgl

vdl

vdr zgl dzv

vgr

vgn

$\mathrm{zdr}$ $3 \mathrm{dr}$

zgr

$2 \mathrm{br}$

tsvr

XVr

fpl spl

fkl skl

fpr spr

fpr' spr'

$\mathrm{fkr} \quad \mathrm{skr}$

ftr str ftr

vzr

fsl

fsr

fsm

fst

skv

sxv

sxl

smr

Auslautstrukturen

Infolge der Auslautverhärtung sind im Deutschen und im bulgarischen Wortauslaut nur stimmlose Konsonantenverbindungen möglich.

Zweigliedrige Auslautstrukturen im Deutschen

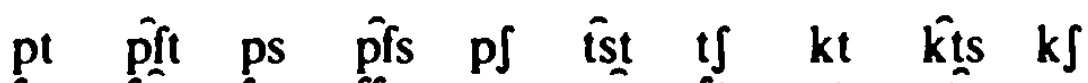

ft fts fs ff st sts $f t$ çt çts çs $x t \quad x \hat{t s} \quad x s$

mp mpf mt mts ms mf nt nts nf ns nf nç nt nk ns

Ip it its lk if ls if lf $\mathrm{lm}$ in

rp rt rts rk $\mathrm{rf}$ rs $\mathrm{rf}$ rç $\mathrm{rm}$ rn $\mathrm{rl}$

Zweigliedrige Auslautstrukturen im Bulgarischen

$\begin{array}{llllllll}\mathrm{pt} & \mathrm{ps} & \mathrm{pf} & \mathrm{kt} & & & & \\ \mathrm{ft} & \mathrm{f} & \mathrm{st} & \mathrm{sk} & \mathrm{s} f & \int \mathrm{t} & \mathrm{fk} & \mathrm{xt}\end{array}$

mp ms mn nt $\mathrm{nk}$ ns $\mathrm{nj} \mathrm{nm}$

Ip it ik if is if $\mathrm{lm}$ in

rp rt rk rt rs rX rm rn $\mathrm{rl}$

Mehrgliedrige Auslautstrukturen im Deutschen

pfst pst pft $t \int t \quad k s t$

fst fst çst xst

mpt mpft mps mpfs mst $\mathrm{m} f \mathrm{t}$

ntst nft nfts nfs nst nft nçt nçs $n t \int$ nkt nkts nks

lpt lps ltst lkt lks Ift lfs lst ift lçt lçs lmt Ims 
rpt rps rtst rft rfs rst rft rçt rçs rmt rms rnt rns rlt rls

pjst t tsst

mpst $\quad \mathrm{m} \int \mathrm{st}$

nt $5 s t \quad n f s t \quad n \int s t \quad n c ̧ s t \quad$ jkst

lpst lkst lfst lfst lçst Imst

$\begin{array}{llllll}\text { rpst rkst rfst } & \text { r } \int s t & \text { rçst } & \text { rmst } & \text { rnst } & \text { rlst }\end{array}$

Im Bulgarischen sind nur drei dreigleidrige Auslautverbindungen möglich : kst,

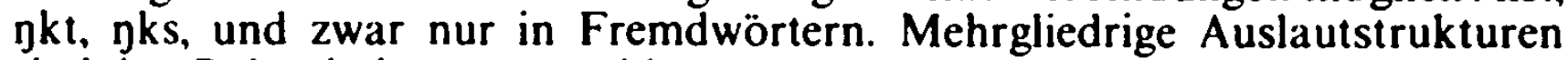
sind im Bulgarischen ausgeschlossen.

\section{BEILAGE}

\subsection{BENUTZTE TRANSKRIPTIONSZEICHEN}

Alle deutschen und bulgarischen Beispiele in der Monographie sind nach der von der Verfasserin im Buch „Grundzüge einer kontrastiven Phonetik Deutsch/Bulgarisch" (1988) vorgeschlagenen einheitlichen phonetischen Umschrift transkribiert worden. Als Grundlage dafür dienen die Transkriptionszeichen der API. Für die spezilischen Besonderheiten des bulgarischen Segmentsystems werden zum Teil auch diakritische Zeichen aus den in „Българска фонетика“ von Tilkov und Bojadshiev (Т и лков, Бояджи ев, 1977, 234-235) enthaltenen, benutzt.

Im folgenden werden die Zeichen (mit Beispielen) für die normgerechte Transkription des Deutschen und des Bulgarischen angeführt.

\section{Vokale}

\section{A. Phonetische Transkription der deutschen Standardaussprache}

[i:]= geschlossenes, langes: Liebe, ihn, Igel ['li:bə], ['i:n], ['i:gal]

[1] = offenes, kurzes: lispeln, Mitte [lispaln], [mita]

$[1]=$ kurzes, unsilbisches: Linie, Million ['li :nia],[mı'lıo:n]

$[\mathrm{e}:]=$ geschlossenes, langes: gehen, geben, Beet ['ge:àn], ['ge:ban], [be:t]

$[\varepsilon:]=$ offenes, langes: ähnlich, Bär [" $\varepsilon: n l i c]$, [be:e]

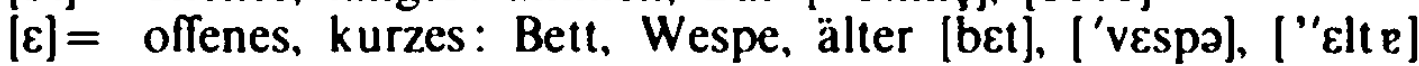

$[\partial]=$ schwachtoniges, gemurmeltes: Sage, beleben, gebieten ['za :gə], [bə'le :bən], [ga'bi:tan]

$[a:]=$ hinteres, langes: Abend, Tag, ahnen, Aal ["a:bənt], [ta:k], ["a:nən]. ['a:l]

$[a]=$ vorderes, kurzes: hatte, Hast ['hato], [hast]

$[\mathrm{u}:]=$ geschlossenes, langes: Rute, gut, Fuhre ['ru:to], [gu:t], [fu:ra]

$[u]=$ offenes, kurzes: Mutter, dulden ['mute], ['duldan]

$[0:]=$ geschlossenes, langes: Bohne, loben, Boot ["bo:na], ["lo:ban], [bo:t]

$[0]=$ offenes, kurzes: hoffen, kosten ['hofon], ['kaston]

$[y:]=$ geschlossenes, langes: lügen, Bühne ['ly:gen], ['by:nə]

$[y]=$ offenes, kurzes: üppig, lüften ["ypıç], ['Irften] 
[ø:]= geschlossenes, langes: lösen, Löhne ['lø:zən], ['lø:nə]

$[\propto]=$ offenes, kurzes: öffnen, löschen ["œfnan], ['lœ\}an]

\section{Diphthonge}

[âe]: Eiche, beide ["äeça], [bôeda]

[áo]: auch, sausen ['áox],['zóozan]

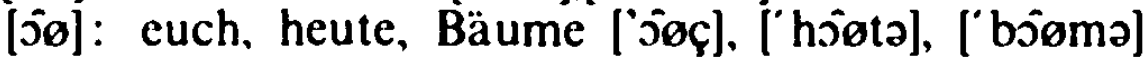

\section{Konsonanten}

[p] = stimmloser (bilabialer) Verschlußlaut: Puppe ['popa]

[b] = stimmhafter (bilabialer) VerchluBlaut: Bitte ['bita]

$[\mathrm{m}]=$ stimmhafter (bilabialer) Nasal: Mutter ['mote]

$[t]=$ stimmloser (alveolarer) Verschlußlaut: Tat $[t a: t]$

[d] = stimmhafter (alveolarer) Verschlußlaut: Daumen ['deomən]

[n] = stimmhafter (alveolarer) Nasal: Name ['na:ma]

$[k]=$ stimmloser (velarer) Verschlußlaut: Kanne, Sack, quer ['kanə], [zak], [kve:r]

$[\mathrm{g}]=$ stimmhafter (velarer) Verschlußlaut: gut, legen [gu:t], ["le:gen]

$[\eta]=$ stimmhafter (velarer) Nasal: Angel, Ubung, sinken ["anal], ["y:bun], ["zınkan]

$[\Pi=$ stimmloser (labiodentaler) Engelaut: Finger, Vater ['fine], ['fa:te]

$[\mathrm{v}]=$ stimmhafter (labiodentaler) Engelaut: Wagen, Vase ['va:gən], ['va:zə]

$[\mathbf{s}]=$ stimmloser (dentialveolarer) Engelaut: essen, Haß, ['Esan], [haß]

$[z]=$ stimmhafter (dentialveolarer) Engelaut: sausen ['zôozan]

[j] = stimmhafter (palataler) Engelaut: Jahr, jeder [ja:]. ['je:de]

$[c]=$ stimmloser (palataler) Engelaut: ich, möchte, China ["ıç]. ["moeçta], ['çi :na]

$[x]=$ stimmloser (velarer) Engelaut: Bach, Buch, auch [bax] [bu :x], ['aox]

[I] = stimmhafter (alveolarer) Lateralengelaut: List, alle [IIst], ["alə]

$[r]=$ stimmhafter (uvularer bzw. alveolarer) Flatterlaut: rot, Beere [ro:t], ['be:ro]

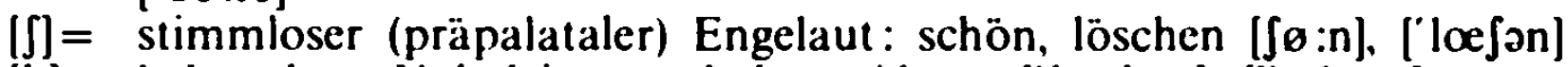

[h] = behauchter Vokaleinsatz: haben, Ahorn ['ha:bon], ["a:horn]

$[\mathrm{p}]=$ Reduktionsvokal: Vater, bitter ['fa:te]. ['bit $\varepsilon$ ]

Kurze geschlossene Vokale kommen in Fremdwörtern in offenen unbetonten Silben vor: Delegation [delega'tsio:n], physikalisch [fyzi'ka :lif]. Abgekürzt werden lange Vokale in klitisch gebrauchten Einsilbern: der Vater [de'e 'fa:te], sagt er [za:kt 'e·r].

\section{Diakritische Zeichen}

Länge: durch Doppelpunkt [ :]

Abgekürzte Länge: durch einen Punkt [·]

Hauptakzent: durch hochgesetzten, von rechts nach links verlaufenden Strich, vor der hauptbetonten Silbe: geben ['ge :ban]

Nebenakzent: durch hochgesetzten, von links nach rechts verlaufenden Strich, vor der nebenbetonten Silbe: langsam ['lay'sa:m]

Fester Vokaleinsatz: durch hochgesetzten Beistrich vor der vokalisch anlautenden Silbe: Arm in Arm ['arm in 'arm] 


\section{B. Phonetische Transkription der bulgarischen Standardaussprache}

\section{Vokale}

[ı] = betontes, kurzes: лист [list], изпит ['ispit], уши [u'jı]

[i] = unbetontes, schwach reduziertes: измама [iz'mama], Витиня [vi'tın'a], мини ['mini]

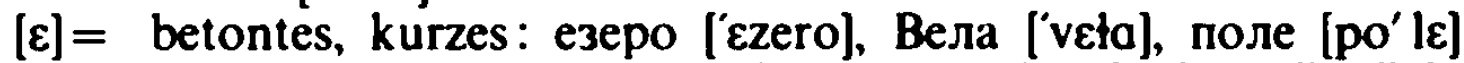

$[\mathrm{e}]=$ unbetontes, schwach reduziertes: ела [e' ła], беля [be'l'a]

$[a]=[\mathbf{b}]$, betont: ьгъл ['agel], бързо ['borzo]

[a] = betontes, kurzes: ад [at], бasa ['bała], rexa [le'xa]

$[a]=$ unbetontes, schwach reduziertes: araka [a' taka], билет [bi' let]

$[\mathrm{e}]=$ unbetontes, stark reduziertes [a] bzw. [ə] (rückwärtige Reduktion): мокър ['moker], лекар, бърлога ['leker], [ber' bga]

[э] = betontes, kurzes: остров, болен, хоро ['sstrof], ['bolen], [хо'ro]

$[0]=$ unbetontes, schwach reduziertes: болнав, кокиче [bof'naf], [ko'kit $\left.\int e\right]$

[u] = betontes, kurzes: умен, бутам, Перу ['umen], ['butam], [pe'ru]

$[u]=$ unbetontes, schwach reduziertes: мухи, перуника [mu'xı], [peru'nıka]

\section{Konsonanten}

[p] = stimmloser (bilabialer) Verschlußlaut: пипам, поп, 306 ['pıpam], [pop], [zop]

[p'] = stimmloser (bilabialer) palatalisierter Verschlußlaut : пяна, сипя ['p'ana], ['sip'a]

[b] = stimmhafter (bilabialer) Verschußlaut: 6a6a ['baba]

$\left[b^{\prime}\right]=$ stimmhafter (bilabialer) palatalisierter Verschlußlaut: бял, любя [b'al], ['l'u'ba]

$[t]=$ stimmloser (alveodentaler) Verschlußlaut: танк, вата, пот, под [tank], ['vata], [pot], [pot]

$\left[t^{\prime}\right]=$ stimmloser (alveodentaler) palatalisierter Verschlußlaut: тясно, клатя, Тотю ['t’asno], ['kłat'a], ['tot'u]

[d] = stimmhafter (alveodentaler) VerschluBlaut: ден, падам [den], ['padam]

[d'] = stimmhafter (alveodentaler) palatalisierter Verschlußlaut : дясно, следя, дюля ['d'asno], [sle 'd'a], ['d'ul'a]

[n] = stimmhafter (alveodentaler) Nasal: нос, кана, звън [nos], ['kana], [zvon]

$[k]=$ stimmloser (velarer) Verschlußlaut : кука, кок, глог [ kuka], [kok], [głok]

$\left[k^{\prime}\right]=$ stimmloser (velarer) palatalisierter Verschlußlaut: кьорав, кюп, кяр ['k'oraf], ['k'up], [k'ar]

$[g]=$ stimmhafter (velarer) Verschlußlaut : гост, тora [gost], ['toga]

$\left[\mathbf{g}^{\prime}\right]=$ stimmhafter (velarer) palatalisierter Verschlußlaut : rbон [ ['on]

[n] = stimmhafter (velarer) Nasal: звънко, мангал ['zvəђko], [man'gał]

$[i]=$ stimmloser (labiodentaler) Engelaut : фаза, кофа, лов ['faza], ['kofa], [ło]

$[v]=$ stimmhafter (labiodentaler) Engelaut : вa3a, совa ['vaza], ['sova]

[v'] = stimmhafter (labiodentaler) palatalisierter Engelaut : вял, свят [v'ał]. [sv'at]

$[s]=$ stimmloser (dentialveolarer) Engelaut : сам, коса, бос, без [sam], [ko'sa]. [bos], [bes]

$\left[\mathbf{s}^{\prime}\right]=$ stimmloser (dentialveolarer) palatalisierter Engelaut : сяно, кося ['s'ano], [ko's'a] 
[z] = stimmhafter (dentialveolarer) Engelaut: зима, коза ['zıma], [ko'za]

$\left[z^{\prime}\right]=$ stimmhafter (dentialveolarer) palatalisierter Engelaut : зяnaм ['z'apam]

[I] = stimmhafter Glide-Laut : Йовка, байно, ратай ['ıfka], ['baino], [ra'tai]

$[\hat{[c]}=$ stimmloser (palataler) Engelaut : хитър, Хемус, пихтия, ехиден ['çıter], ['çEmus], [piç'tıla], [e'çıden]

$[x]=$ stimmloser (velärer) Engelaut: хаван, хоро, хубав, ехо, мехур, рехав ['xa'van], [xo'ro], ['xubaf], ['Exo], [me'xur], ['rexaf]

$[\nmid]=$ stimmhafter (dentialveolarer) Lateralengelaut: лук, лош, лак, ела [łok], [łof], [tak], [e'ła]

[I] = stimmhafter (alveolarer) Lateralengelaut : лист, лесен [list], ['Iesen]

$[i]=$ stimmhafter (dentipalataler) palatalisierter Lateralengelaut: лято, люляк, поля ['l'ato], ['l'ul'ak], [po'l'a]

$[r]=$ stimmhafter (apikaler) Flatterlaut : риза, кораб, мокър ['riza], ['korap], ['moker]

$\left[r^{\prime}\right]=$ stimmhafter (apikaler) palatalisierter Flatterlaut: pяпа, коря ['r’apa], [ko'r'a]

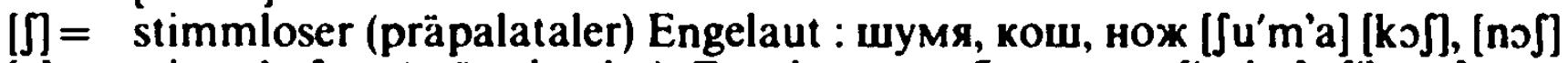

$[3]=$ stimmhafter (präpalataler) Engelaut : жаба, кожа ['3aba], ['kəza]

Diakritische Zeichen

[']= nach dem jeweiligen Konsonanten bezeichnet seine palatalisierte Aussprache : ['r’apa]

$[:]=$ eventuelle Länge des linksstehenden Vokals in Redezusammenhang

$\left[^{\prime}\right]=$ Hauptakzent - durch hochgesetzten, von rechts nach links verlaufenden Strich, vor der hauptbetonten Silbe : ['pita], [de't $\varepsilon$ ]

[]$=$ über Vokalen bedeutet diphthongische Aussprache: ['koika] 


\subsection{ABBILDUNGEN UNI) TABELLEN}

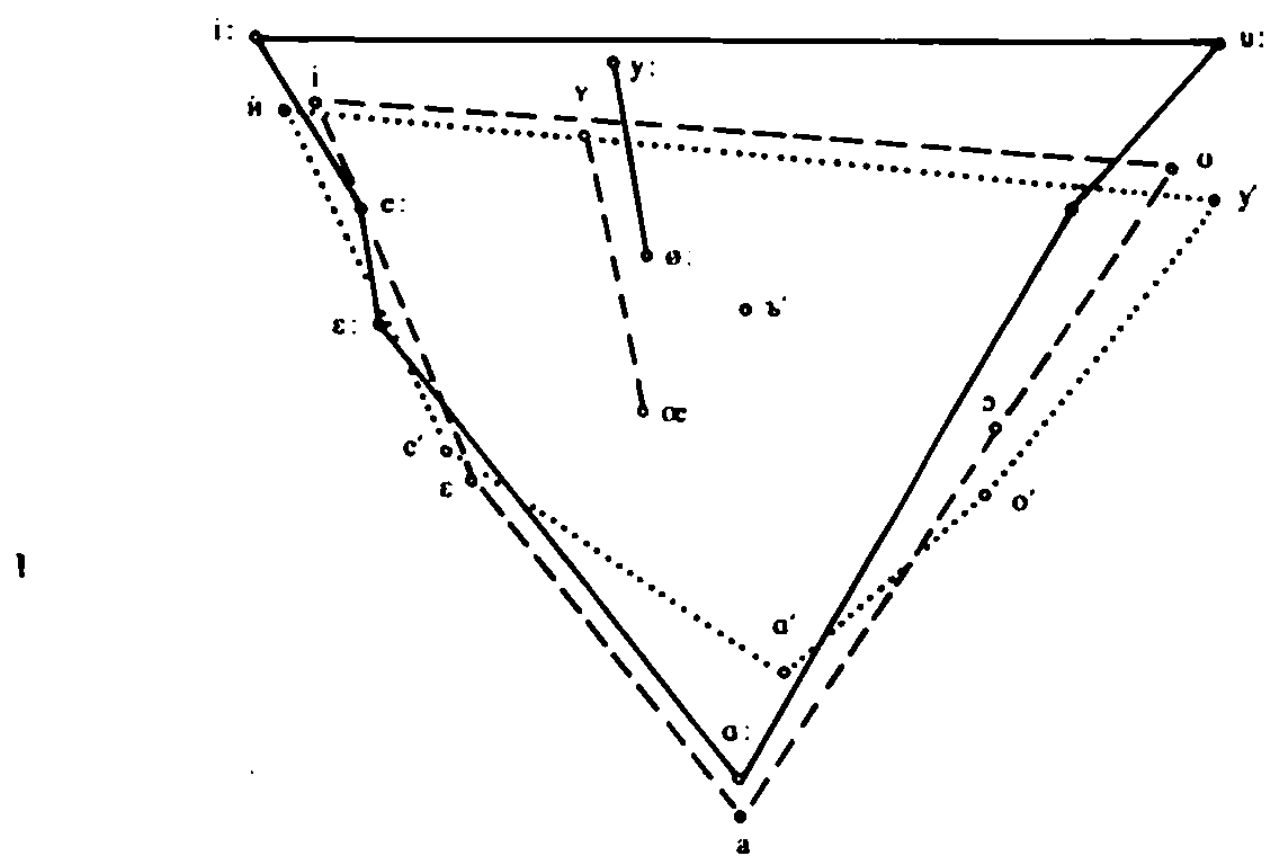

Abb. I. Vergleichende Formantenkarte der betonten Vokale des Deutschen und des Bulgarischen: lange geschlossene Vokale; kurze offene Vokale; bulgarische betonte Vokale

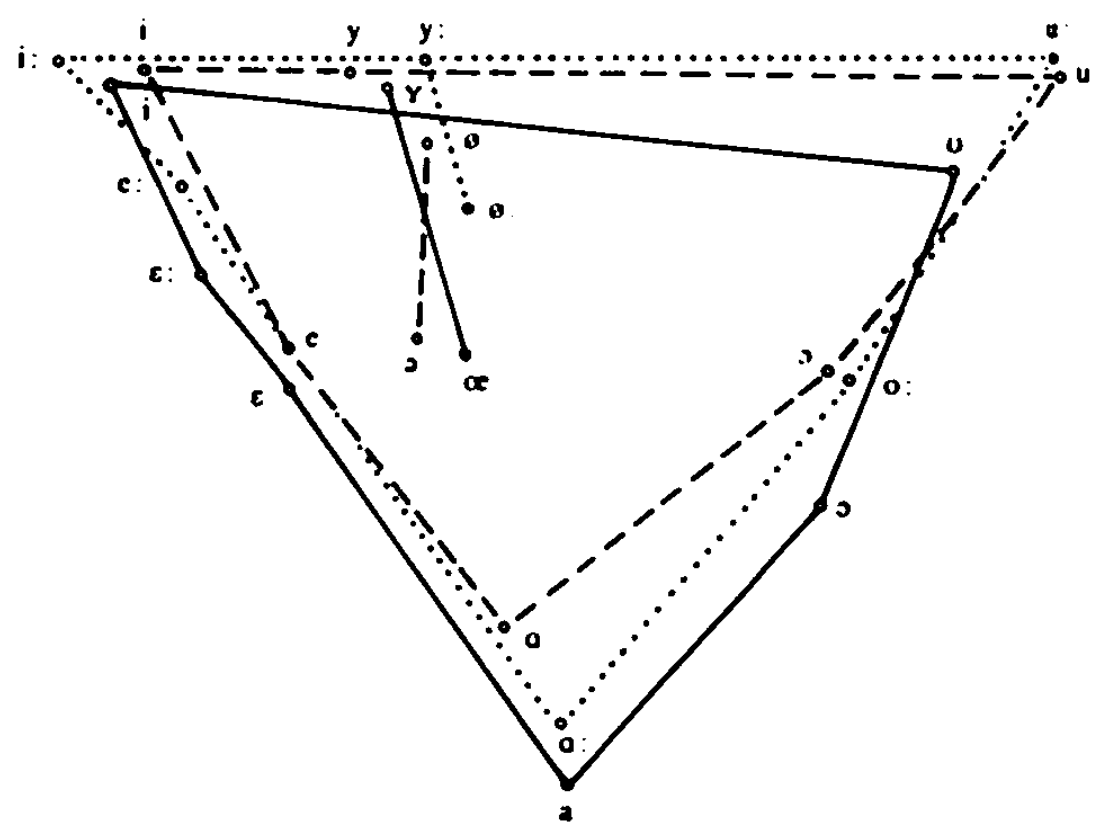

Abb. 2. Vergleichende Formantenkarte der betonten und der unhetonten Vokallaute des Deutschen: lange geschlossene Vokale; kurze offene Vokale. unbetonte Vokale 


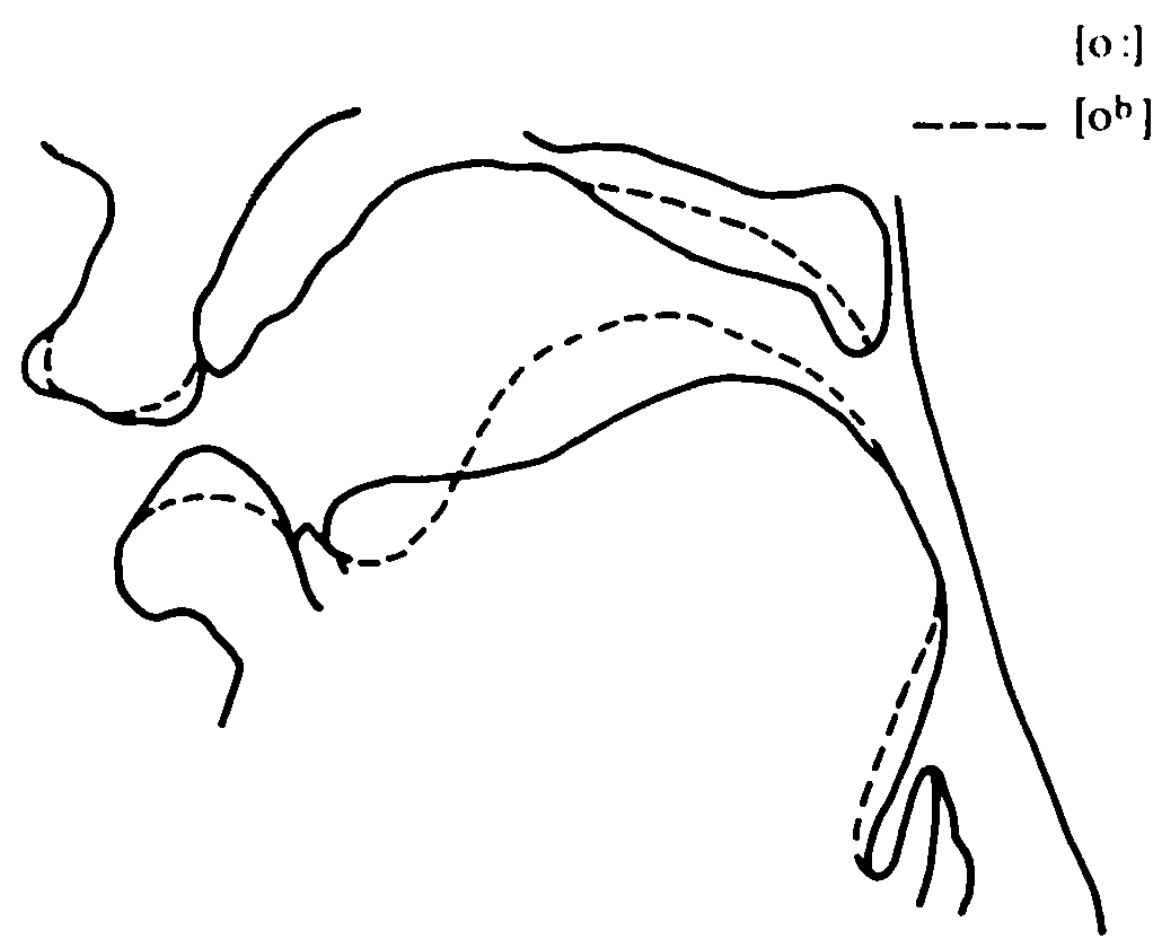

Abb. 3

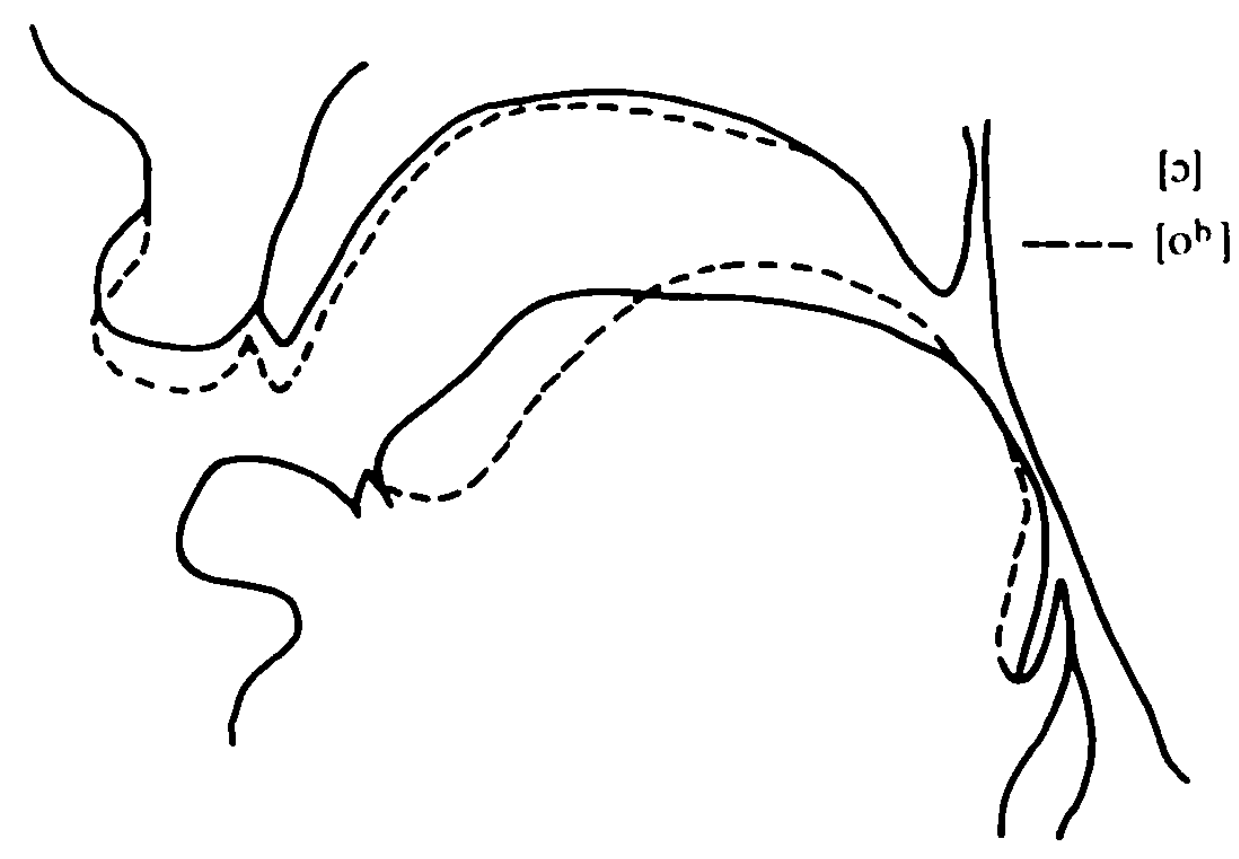

Abb. 4 
00050386

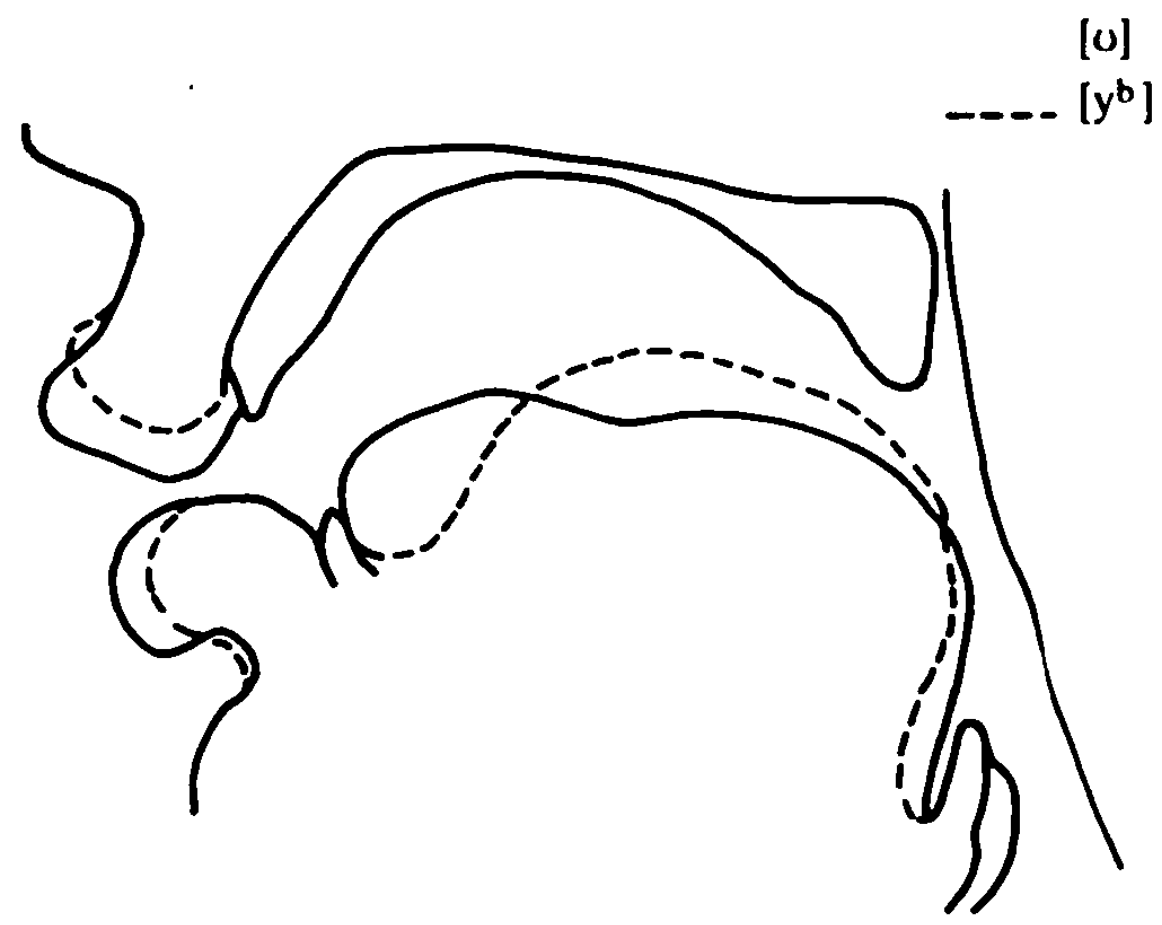

Abb. 5

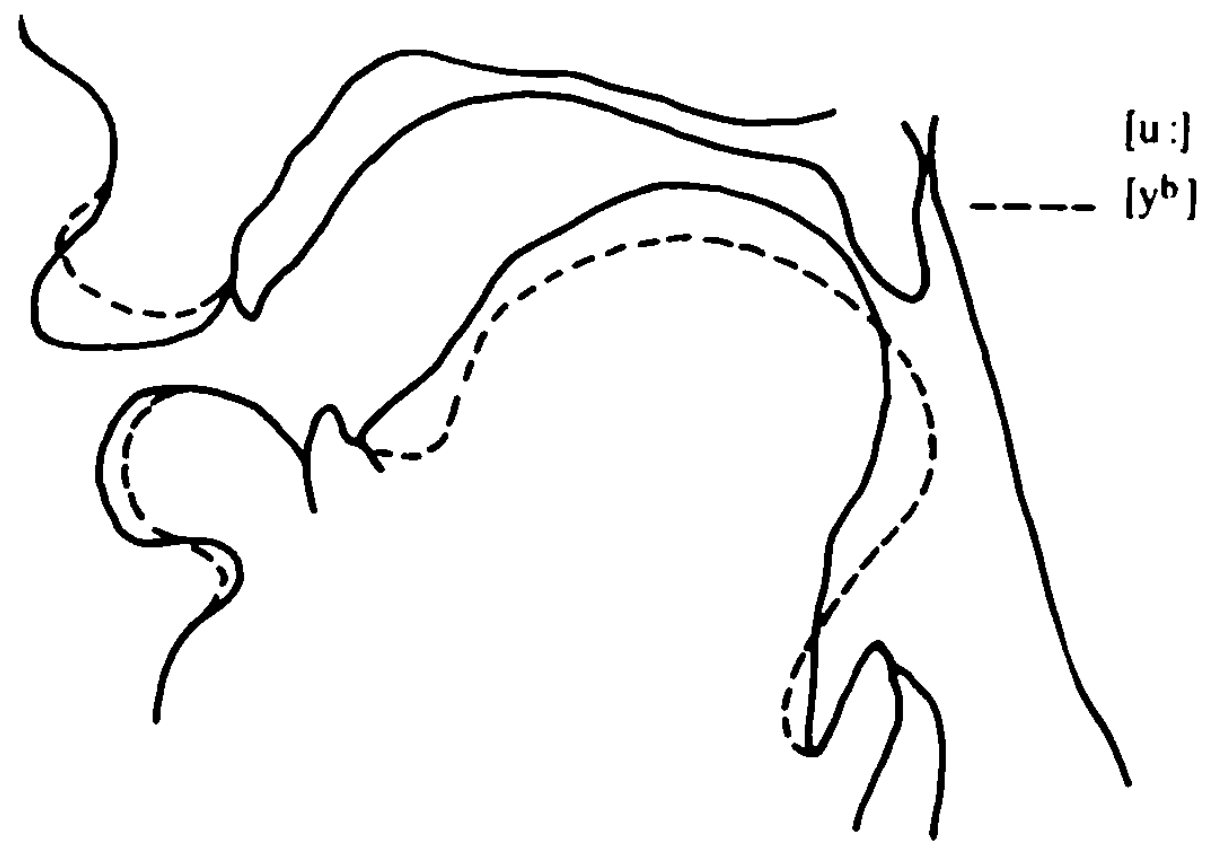

Abb. 6

130

Rusk K. Simeonova - 9783954791989

Downloaded from PubFactory at 01/10/2019 03:47:15AM

via free access 
[i:]

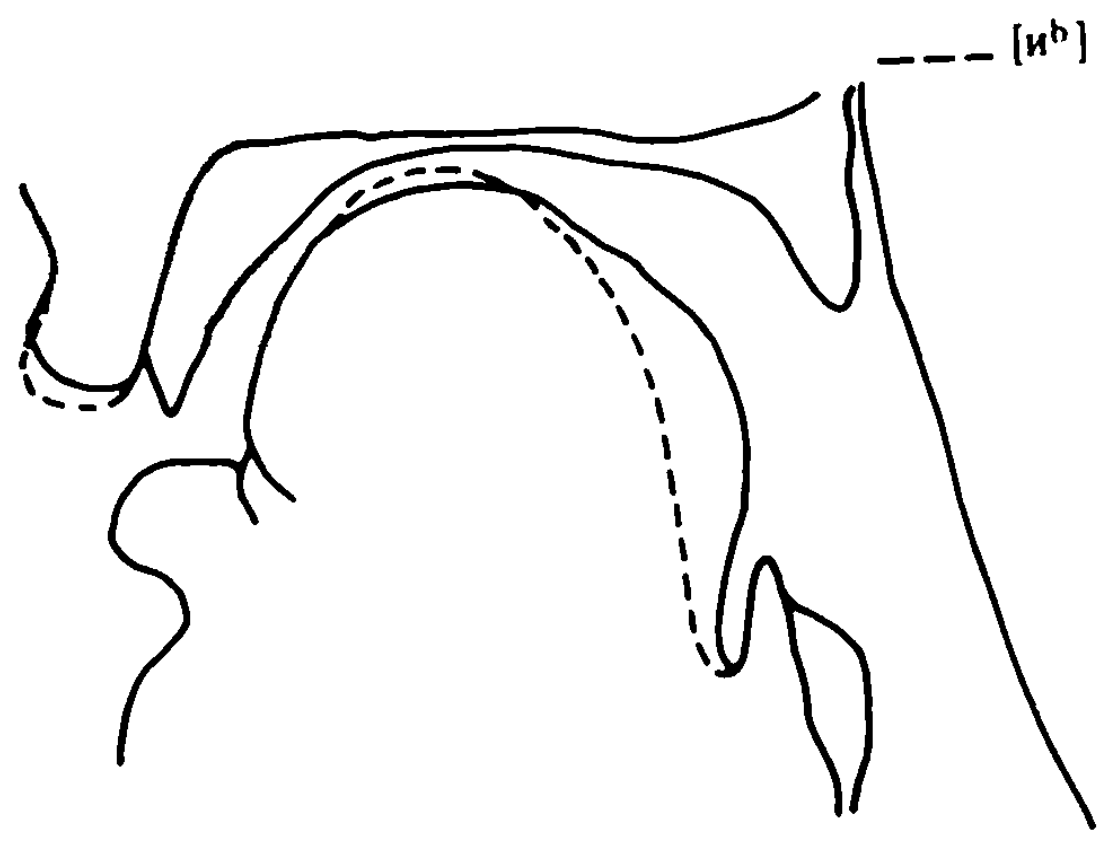

Abb. 7

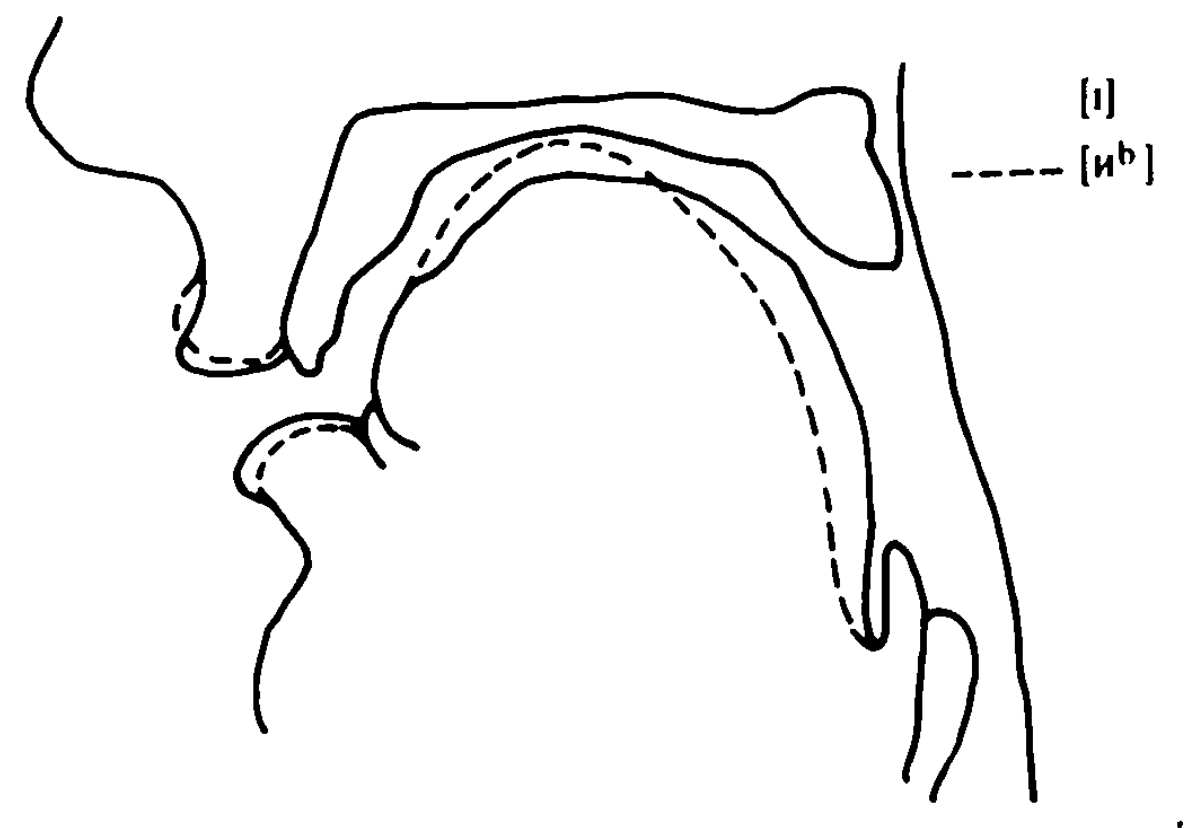

Abb. 8 


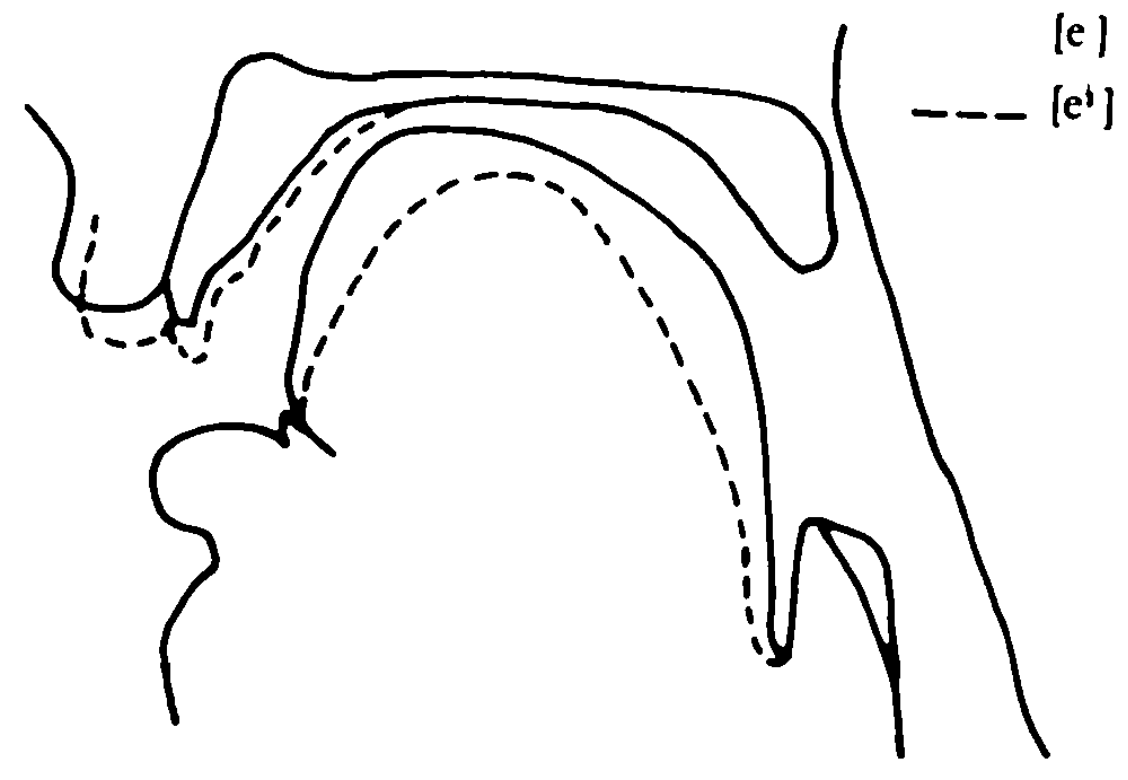

Abb. 9

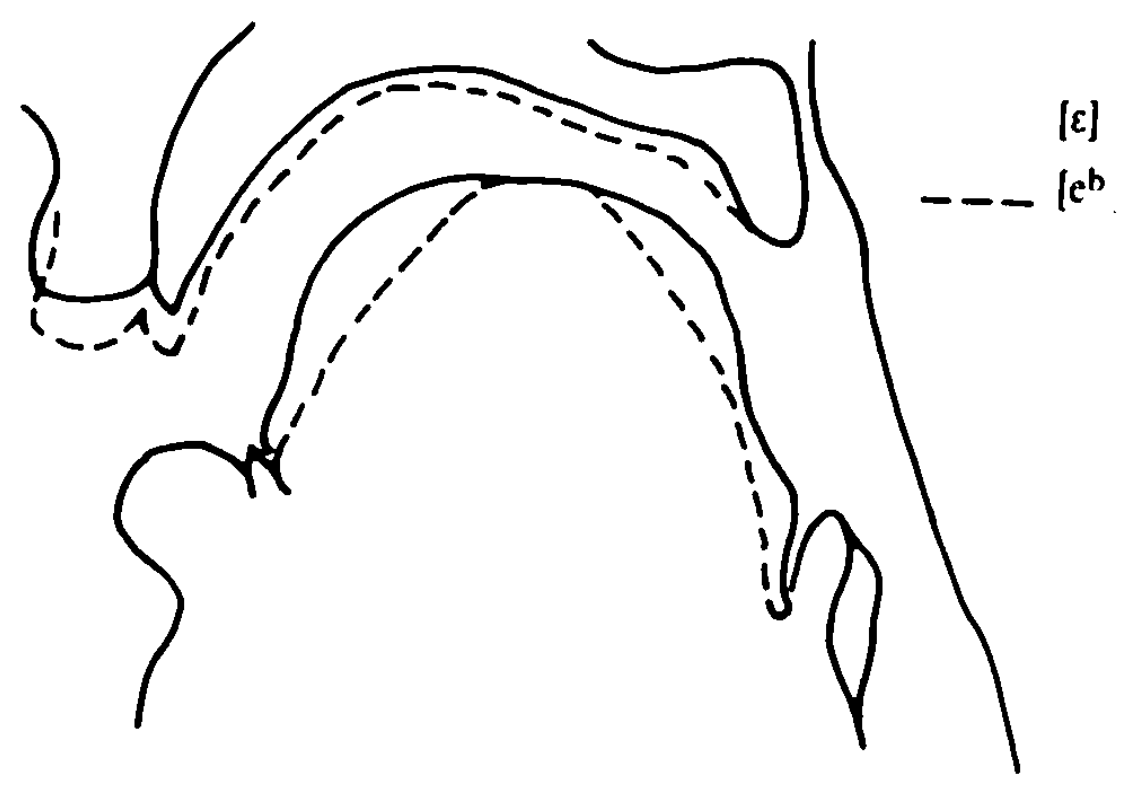

Abb. 10 
Tah. I. Deutsche hetonte Vokale. Vergleich ihrer Formantenwerte [in $\mathrm{Hz}$ ]

\begin{tabular}{|c|c|c|c|c|c|c|c|c|c|c|c|c|c|}
\hline Vuokale & $r_{1}$ & $r_{2}$ & $r_{3}$ & $R_{1}$ & $R_{2}$ & $\mathbf{R}_{3}$ & Vokale & $F_{1}$ & $r_{2}$ & Fs & $R_{1}$ & $R_{2}$ & $R_{3}$ \\
\hline $\mathrm{i}$ : & 221 & 2434 & 3011 & 11 & 1.2 & 13.6 & 1 & 257 & 2304 & 2924 & 9 & 1,3 & 11,4 \\
\hline$y:$ & 244 & 1615 & 2320 & 6.6 & 1,4 & 9.5 & $\mathbf{Y}$ & 293 & 1684 & 2492 & 5,7 & 1,5 & 8,5 \\
\hline e : & 323 & 2153 & 2877 & 6.7 & 1.3 & 8.9 & $\varepsilon$ & 433 & 1921 & 2758 & 4.4 & 1.4 & 6.4 \\
\hline $\mathbf{u}:$ & 294 & 796 & 2394 & 2.7 & 3 & 8.1 & 0 & 329 & 909 & 2423 & 2,8 & 2.7 & 7,4 \\
\hline$\varepsilon$ : & 365 & 2106 & 2871 & 5,8 & 1.4 & 7,9 & $\varepsilon$ & 433 & 1921 & 2758 & 4.4 & 1.4 & 6.4 \\
\hline o: & 332 & 1566 & 2416 & 4.7 & 1.5 & 7,3 & $\boldsymbol{x}$ & 402 & 1588 & 2397 & 4 & 1.5 & 6 \\
\hline $0:$ & 420 & 1038 & 2288 & 2.5 & 2.2 & 5.4 & o & 476 & 1145 & 2409 & 2,4 & 2.1 & 5.1 \\
\hline $\mathbf{a}:$ & 613 & 1442 & 2459 & 2.4 & 1.7 & 4 & $\mathbf{a}$ & 648 & 1442 & 2392 & 2,2 & 1.7 & 3,7 \\
\hline
\end{tabular}

Tah. 2. Bulgarische hetonte Vokale. Vergleich ihrer Formantenwerte [in $\mathrm{Hz}$ ]

\begin{tabular}{|c|c|c|c|c|c|c|}
\hline Vokale & $F_{1}$ & $F_{2}$ & $F_{3}$ & $R_{1}$ & $R_{2}$ & $R_{3}$ \\
\hline n & 237 & 2325 & 2963 & 9.8 & 1.3 & 12.5 \\
\hline$y$ & 319 & 835 & 2450 & 2.6 & 3 & 7.7 \\
\hline b & 362 & 1403 & 2416 & 3.9 & 1.7 & 6.7 \\
\hline$c$ & 396 & 1884 & 2620 & 4.8 & 1.4 & 6.6 \\
\hline 0 & 450 & 1089 & 2267 & 2.4 & 2.1 & 5 \\
\hline 0 & 543 & 1397 & 2455 & 2.6 & 1.8 & 4.5 \\
\hline
\end{tabular}


Tab. 3 Formantenwerte der deutschen betonten Vokale und der drei

\begin{tabular}{|c|c|c|c|c|c|c|c|c|c|c|c|c|c|c|}
\hline \multirow{2}{*}{$\frac{\frac{0}{3}}{\frac{\vec{b}}{c}}$} & \multicolumn{6}{|c|}{ Deutscher Sprecher } & \multicolumn{6}{|c|}{ II. Vp. } & \multirow[b]{2}{*}{$F_{1}$} & \multirow[b]{2}{*}{$\mathbf{F}_{2}$} \\
\hline & $F_{1}$ & $F_{2}$ & $F_{3}$ & $R_{1}$ & $R_{2}$ & $R_{3}$ & $F_{1}$ & $F_{2}$ & $F_{3}$ & $\mathbf{R}_{\mathbf{1}}$ & $\mathbf{R}_{2}$ & $\mathbf{R}_{3}$ & & \\
\hline $\mathrm{i}:$ & 240 & 2450 & 2980 & 10,2 & 1,2 & 12,4 & 300 & 2180 & 2760 & 7,3 & 1,4 & 9,2 & 230 & 2380 \\
\hline $\mathbf{I}$ & 320 & 2250 & 2820 & 7.5 & 1.2 & 9,4 & 280 & 2050 & 2580 & 7,4 & 1,3 & 9.2 & 230 & 2390 \\
\hline \multirow[t]{2}{*}{$y:$} & \multirow[t]{2}{*}{300} & \multirow[t]{2}{*}{1800} & \multirow{2}{*}{2300} & \multirow[t]{2}{*}{6} & \multirow{2}{*}{1,3} & \multirow[t]{2}{*}{7,3} & \multirow[t]{2}{*}{250} & \multirow{2}{*}{1750} & \multirow{2}{*}{2250} & \multirow{2}{*}{7} & \multirow{2}{*}{1,3} & \multirow{2}{*}{9} & 280 & 1800 \\
\hline & & & & & & & & & & & & & 250 & 700 \\
\hline \multirow{2}{*}{$\mathbf{Y}$} & \multirow{2}{*}{350} & \multirow{2}{*}{1440} & \multirow{2}{*}{2250} & \multirow{2}{*}{4,1} & \multirow{2}{*}{1.5} & \multirow{2}{*}{6.4} & 350 & 1650 & 2550 & 4.4 & \multirow[t]{2}{*}{1,5} & \multirow[t]{2}{*}{7,3} & & \multirow[b]{2}{*}{720} \\
\hline & & & & & & & 350 & 880 & & 2.5 & & & 280 & \\
\hline$e:$ & 360 & 2230 & 2810 & 6.2 & 1.3 & 7,8 & 400 & 1910 & 2650 & 4.8 & 1,4 & 6.4 & 340 & 2180 \\
\hline$\varepsilon$ & 470 & 1870 & 2650 & 4 & 1,5 & 5.6 & 630 & 1620 & 2680 & 2.5 & 1.7 & 4.3 & 450 & 2000 \\
\hline$u$ : & 300 & 720 & & 2.4 & & & 380 & 900 & & 2.3 & & & 300 & 700 \\
\hline 0 & 380 & 800 & & 1,9 & & & 400 & 880 & & 2.2 & & & 290 & 650 \\
\hline $\boldsymbol{\theta}$ & 390 & 1710 & 2430 & 4.4 & 1,4 & 6.2 & 330 & 1610 & 2500 & 4,9 & 1.6 & 7,6 & 330 & 1830 \\
\hline \multirow[t]{2}{*}{$\boldsymbol{\infty}$} & 370 & 1480 & 2300 & 4 & 1.6 & 6 & 320 & 1580 & 2410 & 5 & & & 310 & 1780 \\
\hline & & & & & & & 450 & 1450 & & 3,3 & 1.6 & 7.5 & 480 & 1620 \\
\hline 0 & 370 & 920 & & 2.5 & & & 440 & 910 & & 2 & & & 470 & 930 \\
\hline $\boldsymbol{J}$ & 450 & 800 & & 1.8 & & & 580 & 1100 & & 2 & & & 560 & 1100 \\
\hline$a:$ & 800 & 1230 & 2650 & 1.5 & 2.2 & 3,3 & 720 & 1420 & 3020 & 2 & 2.1 & 4,2 & 650 & 1230 \\
\hline $\mathbf{a}$ & 850 & 1200 & 2450 & 1.6 & 2 & 3 & 690 & 1380 & 3120 & 2 & 2,3 & 4.5 & 720 & 1270 \\
\hline ie & 730 & 1320 & & 1.8 & & & 610 & 1650 & & 2.7 & & & 680 & 1430 \\
\hline & 470 & 1970 & 2550 & 4.2 & 1.3 & 5.4 & 410 & 1850 & 2750 & 4.5 & 1,5 & 6.7 & 480 & 2000 \\
\hline do & 650 & 1150 & 2400 & 1.8 & 2.1 & 3,7 & 700 & 1170 & 2700 & 1.7 & 2.3 & 3.9 & 680 & 1220 \\
\hline & 450 & 1050 & & 2.3 & & & 420 & 870 & & 2.1 & & & 530 & 980 \\
\hline jo & 650 & 1300 & & 2 & & & 600 & 1200 & & 2 & & & 550 & 1150 \\
\hline & 400 & 2100 & 2850 & 5.3 & 1.4 & 7,1 & 450 & 1650 & 2650 & 3.7 & 1.6 & 5.9 & 350 & 1450 \\
\hline
\end{tabular}


Diphthonge, realisiert von einem Deutschen und vier Bulgaren [in $\mathrm{Hz}$ ]

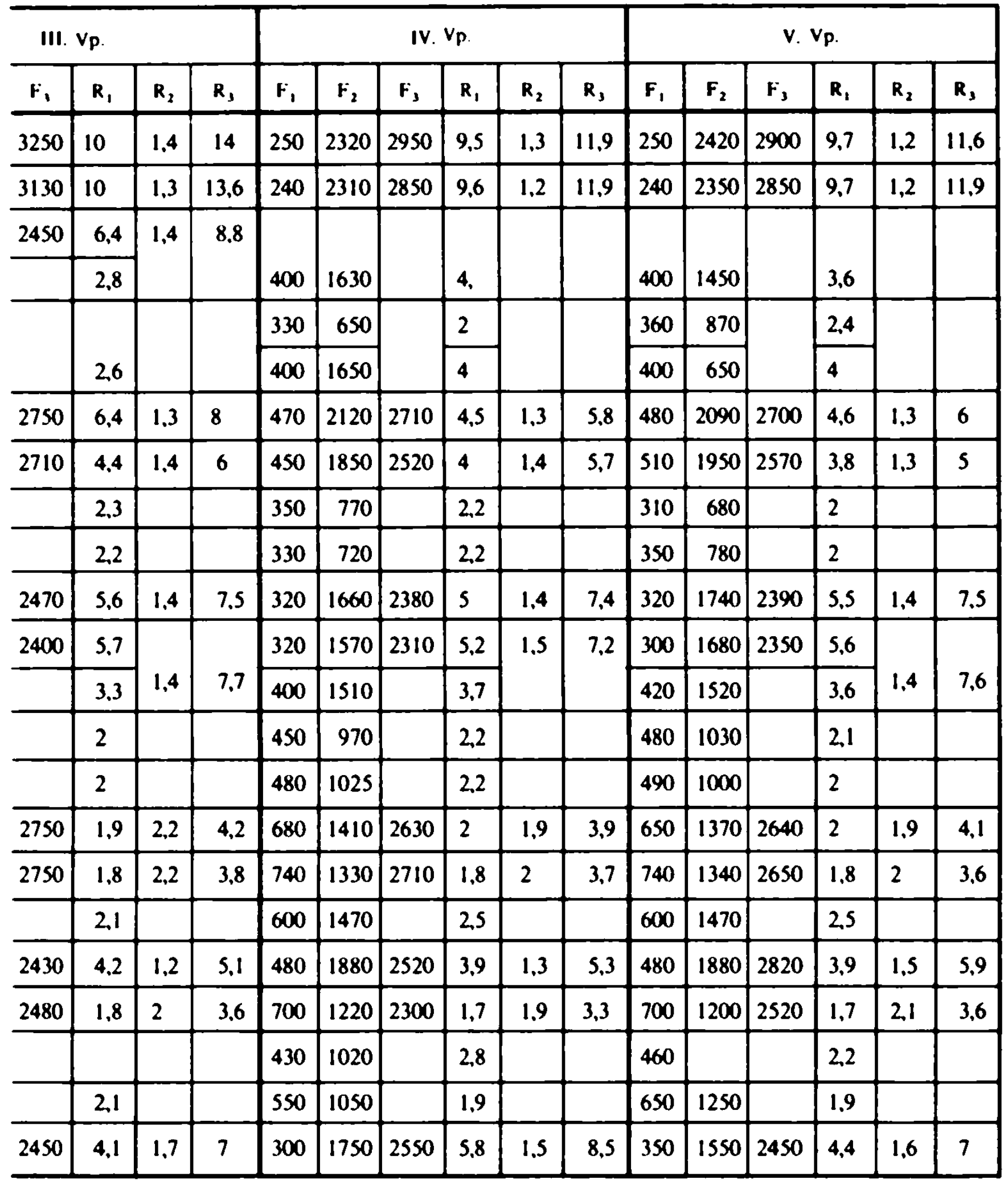


Tah. 4. Formantenwerte deutscher Vokale in unbetonter Stellung.

\begin{tabular}{|c|c|c|c|c|c|c|c|c|c|c|c|c|c|c|c|c|c|c|}
\hline \multirow{2}{*}{ Vokale } & \multicolumn{6}{|c|}{ Deutscher Sprecher } & \multicolumn{6}{|c|}{ II. Vp. } & \multicolumn{6}{|c|}{ III. $v_{p}$. } \\
\hline & $r_{1}$ & $\mathrm{r}_{2}$ & $r_{3}$ & $\mathbf{R}_{\mathbf{l}}$ & $\mathbf{R}_{2}$ & R, & $r_{1}$ & $r_{2}$ & $r_{3}$ & $R_{1}$ & $R_{2}$ & $\mathbf{R}_{\mathbf{3}}$ & $r_{1}$ & $\mathrm{r}_{2}$ & F, & $R_{1}$ & $R_{2}$ & $\mathbf{R}_{3}$ \\
\hline $\mathbf{o}^{*}$ & 700 & 1310 & & 1.9 & & & 580 & 1330 & 2500 & 2.3 & 1,9 & 4,3 & 650 & 1320 & 2500 & 2.1 & 1.7 & 3,6 \\
\hline $\mathbf{a}$ & 650 & 1500 & 2350 & 2.3 & 1.6 & 3.6 & 330 & 1450 & 2730 & 4.4 & 1,9 & 8.3 & 400 & 1630 & 2800 & 4.1 & 1.7 & 7.0 \\
\hline $\mathbf{i}$ & 250 & 2440 & 2820 & 9.8 & 1,7 & 11.3 & 270 & 2220 & 2870 & 8.2 & 1,3 & 10.6 & 250 & 2420 & 2990 & 9.7 & 1.2 & 12 \\
\hline $1^{* *}$ & 310 & 2250 & 2710 & 7.3 & 1.2 & 8.7 & & & & & & & & & & & & \\
\hline ver- & 450 & 1150 & 2250 & 2.6 & 2.0 & 5.0 & 400 & 1300 & 2550 & 3.3 & 2.0 & 6.4 & 350 & 1850 & 2450 & 5,3 & 1.3 & 7,0 \\
\hline $\begin{array}{l}\text { es des } \\
\text { den }\end{array}$ & 430 & $\mid 1900$ & 2720 & 4.4 & 1.4 & 6.3 & 290 & 1730 & 2690 & 6.0 & 1.6 & 9.3 & 350 & 2100 & 2850 & 6.0 & 1.4 & 8.1 \\
\hline der & $\begin{array}{l}500 \\
380 \\
500\end{array}$ & $\mid \begin{array}{l}1300 \\
1980 \\
1600\end{array}$ & $\left\{\begin{array}{l}2150 \\
2380 \\
2350\end{array}\right.$ & $\begin{array}{l}2.6 \\
5.2 \\
3.2\end{array}$ & $\begin{array}{l}1.7 \\
1.2 \\
1.5\end{array}$ & $\begin{array}{l}4.3 \\
6.2 \\
4.7\end{array}$ & 290 & 1730 & 2690 & 6.0 & 1.6 & 9.3 & 370 & $\begin{array}{l}2000 \\
1660\end{array}$ & 2770 & $\begin{array}{l}5,4 \\
3,3\end{array}$ & $\begin{array}{l}1,4 \\
1.6\end{array}$ & 7.5 \\
\hline-2 & 480 & 1640 & 2490 & 3.4 & 1.5 & 5.2 & 360 & 1690 & 2540 & 4.7 & 1.5 & 7,1 & 350 & 1940 & 2670 & 5.5 & 1.4 & 7.6 \\
\hline$-r$ & 580 & 1220 & 2300 & 2.1 & 1.9 & 4.0 & 400 & 1440 & 2700 & 3.6 & 1.9 & 6.8 & 400 & 1400 & 2600 & 3.5 & 1.9 & 6.5 \\
\hline$-o+k$ & 430 & 1680 & 2450 & 3.9 & 1.5 & 5.7 & 400 & 1760 & 2600 & 4.4 & 1.5 & 6.5 & 350 & 1860 & 2550 & 5,3 & 1.4 & 7.3 \\
\hline bo-go- & 460 & 16.30 & 2200 & 3.5 & 1.4 & 4.8 & 350 & 1810 & 2610 & 5.2 & 1.4 & 7.5 & 360 & 2080 & 2720 & 5.8 & 1.3 & 7,6 \\
\hline $\mathbf{u}$ & 360 & 900 & & 2.5 & & & 360 & $9 \times 0$ & & 2.5 & & & 350 & 790 & & 2,3 & & \\
\hline o & 450 & 1000 & & 2.2 & & & 440 & 1050 & & 2.4 & & & 450 & 1000 & & 2.2 & & \\
\hline
\end{tabular}

- Uu unbetonter, ungespannter a-Laut in proklitischen Einsilbern *[i] unbetonter, ungespannter i-Laut in proklitischen Einsilbern $* *-2+k] \quad[a]$ in Nebensilben und im Auslaut vor Konsonant

*.* Die zweite Reihe von Frequenzwerten bei 〈der〉 gibt die Frequenzwerte der diphthongisch realisierten Elemente an 
realisiert von einem Deutschen und vier Bulgarien [in $\mathrm{Hz}$ ]

\begin{tabular}{|c|c|c|c|c|c|c|c|c|c|c|c|c|c|c|c|c|c|}
\hline \multicolumn{6}{|c|}{ IV. Vp. } & \multicolumn{6}{|c|}{ V. $V p$} & \multicolumn{6}{|c|}{$\begin{array}{l}\text { Allgemeine Mitlelwerte } \\
\text { der Bulgaren }\end{array}$} \\
\hline$F_{1}$ & $r_{2}$ & $r_{3}$ & $R_{1}$ & $R_{2}$ & $\mathbf{R}_{3}$ & $F_{1}$ & $r_{2}$ & $r_{3}$ & $R_{1}$ & $\mathbf{R}_{2}$ & $R_{3}$ & $F_{1}$ & $F_{2}$ & $F_{3}$ & $R_{1}$ & $\mathbf{R}_{2}$ & $R_{3}$ \\
\hline 680 & 1430 & 2450 & 2.1 & 1.7 & 3.6 & 570 & 1300 & 2400 & 2.3 & 1,9 & 4,2 & 620 & 1350 & 2470 & 2,2 & 1.8 & 4.0 \\
\hline 380 & 1730 & 2730 & 4.6 & 1.6 & 7,2 & 410 & 1610 & 2700 & 4,0 & 1.7 & 6,6 & 380 & 1610 & 2700 & 4.0 & 1.7 & 7.2 \\
\hline 300 & 2420 & 3000 & 8,0 & 1.2 & 10 & 320 & 2400 & 3130 & 7.5 & 1.3 & 9,8 & 290 & 2370 & 3000 & 8.2 & 1.3 & 10.3 \\
\hline 300 & 1850 & 2350 & 6,2 & 1.3 & 7,8 & 500 & 1450 & 2450 & 2,9 & 1,7 & 4.9 & & & & & & \\
\hline 380 & 2000 & 2750 & 5.3 & 1,4 & 7.2 & 350 & 1980 & 2830 & 5.7 & 1,4 & 8.1 & 340 & 1950 & 2780 & 5.7 & 1.4 & 8,2 \\
\hline 480 & 2050 & 2750 & 4.3 & 1.3 & 5.7 & 390 & 1970 & 2900 & 5,1 & 1.5 & 7.4 & & & & & & \\
\hline 520 & 1500 & 2550 & 2,9 & 1.7 & 4.9 & 580 & 1610 & 2850 & 2,8 & 1.8 & 4,9 & & & & & & \\
\hline 360 & 1800 & 2490 & 5.0 & 1.4 & 6.9 & 380 & 1950 & 2750 & 5.1 & 1.4 & 7.2 & 360 & 1850 & 2610 & 5.1 & 1,4 & 7,3 \\
\hline 430 & 1570 & 2650 & 3,7 & 1.7 & 6.2 & 530 & 1500 & 2650 & 2.8 & 1.8 & 5.0 & 440 & 1480 & 2650 & 3,4 & 1,8 & 6.0 \\
\hline 400 & 1760 & 2600 & 4.4 & 1.5 & 6.5 & 460 & 1850 & 2710 & 4.0 & 1.5 & 5.9 & 400 & 1810 & 2620 & 4.5 & 1.5 & 6.6 \\
\hline 350 & 1810 & 2620 & 5,2 & 1,5 & 7,5 & 380 & 1830 & 2670 & 4,8 & 1.5 & 7,0 & 360 & 1880 & 2660 & 5.2 & 1,4 & 7,4 \\
\hline 380 & 820 & & 2.2 & & & 360 & 800 & & 2,2 & & & 360 & 830 & & 2,3 & & \\
\hline 420 & 900 & & 2.1 & & & 460 & 980 & & 2.1 & & & 440 & 980 & & 2.2 & & \\
\hline
\end{tabular}


Tah. 5. Lange geschlossene Vokale des Deutschen, realisiert von Bulgaren (Auditiver Test)

\begin{tabular}{|c|c|c|c|c|c|c|c|}
\hline 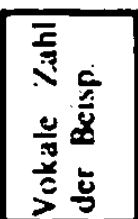 & \multicolumn{3}{|c|}{$\begin{array}{l}\text { Wahrgenommene } \\
\text { Realisierung }\end{array}$} & 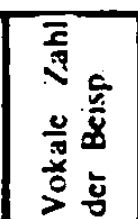 & \multicolumn{3}{|c|}{$\begin{array}{l}\text { Wahrgenommene } \\
\text { Realisierung }\end{array}$} \\
\hline \multirow[b]{3}{*}{$\begin{array}{c}10: 1 \\
14 \\
\text { Bsp. }\end{array}$} & $\%$ & $\%$ & $\%$ & & $\%$ & $\%$ & $\%$ \\
\hline & $|a:| \rightarrow[a:]$ & $|a:| \rightarrow\left[a^{\circ}\right]$ & 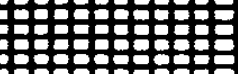 & 进 & $|i:| \rightarrow[i:]$ & $|i:| \rightarrow[i]$ & $|i:| \rightarrow[i]$ \\
\hline & $\begin{array}{l}\text { II. Vp. } 100 \\
\text { III. Vp. } 64.3 \\
\text { IV. Vp. } 57,1 \\
\text { V.Vp. } 76,8\end{array}$ & $\begin{array}{l}\text { II. Vp. } \\
\text { III. Vp. } 35,7 \\
\text { IV. Vp. } 42,9 \\
\text { V. Vp. } 21,4\end{array}$ & 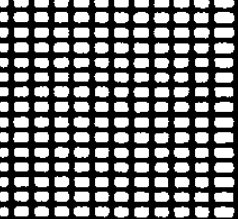 & $\begin{array}{c}\mid i: 1 \\
14 \\
\text { Bsp. }\end{array}$ & $\begin{array}{l}\text { II. Vp. } 85,7 \\
\text { III. Vp. } 78,6 \\
\text { IV. Vp. } 100 \\
\text { V. Vp. } 71,4\end{array}$ & $\begin{array}{l}\text { II. Vp. } 14,3 \\
\text { III. Vp. } 21,4 \\
\text { IV. Vp. } \\
\text { V. Vp. } 14,3\end{array}$ & $\begin{array}{l}\text { II. Vp. }- \\
\text { III. Vp } \\
\text { IV. Vp. }- \\
\text { V. Vp. } 14,3\end{array}$ \\
\hline \multirow[t]{2}{*}{$\begin{array}{l}\text { Mittel- } \\
\text { werte }\end{array}$} & $75 \%$ & $25 \%$ & 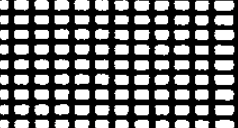 & $\begin{array}{l}\text { Mittel- } \\
\text { werte }\end{array}$ & $83.9 \%$ & $12,5 \%$ & $3,6 \%$ \\
\hline & $|u:| \rightarrow[u:]$ & $|u:| \rightarrow\left[u^{\circ}\right]$ & $|u:| \rightarrow|u|$ & & $|0:| \rightarrow[0:]$ & $10: 1 \rightarrow[0:]$ & $|0:| \rightarrow[0]$ \\
\hline \begin{tabular}{|c}
$\mid u: 1$ \\
12 \\
Bsp.
\end{tabular} & $\begin{array}{l}\text { II. Vp. } 100 \\
\text { III. V. } 50 \\
\text { IV. Vp. } 83,3 \\
\text { V. Vp. } 41,7\end{array}$ & $\begin{array}{l}\text { II. Vp. }- \\
\text { III. V. } 25 \\
\text { IV. Vp. } \\
\text { V. Vp. } 16,7\end{array}$ & $\begin{array}{l}\text { II. Vp. }- \\
\text { III. V. } 2.5 \\
\text { IV. Vp. } 16,7 \\
\text { V. Vp. } 41.7\end{array}$ & $\begin{array}{c}10: 1 \\
12 \\
\text { Bsp. }\end{array}$ & $\begin{array}{l}\text { II. Vp. } 100 \\
\text { III. V. } 75 \\
\text { IV. Vp. } 58.3 \\
\text { V. Vp. } 41,7\end{array}$ & $\begin{array}{l}\text { II. Vp. }- \\
\text { III. V. } 25 \\
\text { IV. Vp. 41,7 } \\
\text { V. Vp. } 50\end{array}$ & $\begin{array}{l}\text { II. Vp. }- \\
\text { III. V. }- \\
\text { IV. Vp. } \\
\text { V. Vp. } 8,3\end{array}$ \\
\hline \multirow[t]{2}{*}{$\begin{array}{l}\text { Mittel- } \\
\text { werte }\end{array}$} & $68.8 \%$ & $10,4 \%$ & $20.9 \%$ & $\begin{array}{c}\text { Mittel- } \\
\text { werte }\end{array}$ & $68,8 \%$ & $29.2 \%$ & $2.1 \%$ \\
\hline & $|y:| \rightarrow|y:|$ & $|y:| \rightarrow \begin{array}{l}\text { fremd- } \\
\text { artig }\end{array}$ & 曲 & 进 & $|\theta:| \rightarrow[\theta:]$ & $\mid \omega: 1 \rightarrow \underset{\text { artig }}{\text { fremd- }}$ & \\
\hline $\begin{array}{c}|y:| \\
10 \\
\text { Bsp. }\end{array}$ & $\begin{array}{l}\text { II. Vp. } 100 \\
\text { III. Vp. } 60 \\
\text { IV. Vp. } 70 \\
\text { V. Vp. } 50\end{array}$ & $\begin{array}{l}\text { II. Vp. }- \\
\text { III. Vp. } 40 \\
\text { IV. Vp. } 30 \\
\text { V. Vp. } 50\end{array}$ & 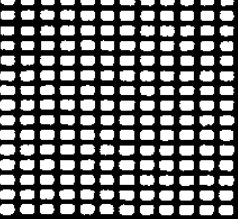 & $\begin{array}{c}10: 1 \\
10 \\
\text { Bsp. }\end{array}$ & $\begin{array}{l}\text { II. Vp. } 100 \\
\text { III. Vp. } 80 \\
\text { IV. Vp. } 80 \\
\text { V. Vp. } 60\end{array}$ & \begin{tabular}{|l|} 
II. Vp. \\
III. Vp. 20 \\
IV. Vp. 20 \\
V. Vp. 40
\end{tabular} & 弗 \\
\hline \multirow[t]{2}{*}{$\begin{array}{l}\text { Mittel- } \\
\text { werte }\end{array}$} & $70 \%$ & $30 \%$ & 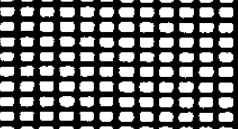 & $\begin{array}{l}\text { Mittel- } \\
\text { werte }\end{array}$ & $80 \%$ & $20 \%$ & \\
\hline & $|e:| \rightarrow|e:|$ & $|e:| \rightarrow\left|e^{\prime}\right|$ & $|e:| \rightarrow[\varepsilon:]$ & & $\mid e: t \rightarrow[\varepsilon]$ & & \\
\hline \begin{tabular}{|c}
$|e:|$ \\
16 \\
Bsp.
\end{tabular} & $\begin{array}{l}\text { II. Vp. } 87.5 \\
\text { III. Vp. } 92.2 \\
\text { IV. Vp. } 75 \\
\text { V. Vp. } 75\end{array}$ & $\begin{array}{l}\text { II. Vp. } \\
\text { III. Vp. } 18,8 \\
\text { IV. Vp. } \\
\text { V. Vp. }\end{array}$ & $\begin{array}{l}\text { II. Vp. } 12,5 \\
\text { III. Vp. } \frac{}{\text { IV. }} \\
\text { IV. Vp. } 18,8 \\
\text { V. Vp. }\end{array}$ & 进 & $\begin{array}{l}\text { II. Vp. } \\
\text { III. Vp. } \\
\text { IV. Vp. } 8,3 \\
\text { V. Vp. } 25\end{array}$ & & 車 \\
\hline $\begin{array}{l}\text { Miltel- } \\
\text { wertc }\end{array}$ & $82.6 \%$ & $4.7 \%$ & $14,1 \%$ & $\begin{array}{l}\text { Mittel- } \\
\text { werte }\end{array}$ & $2.1 \%$ & & \\
\hline
\end{tabular}




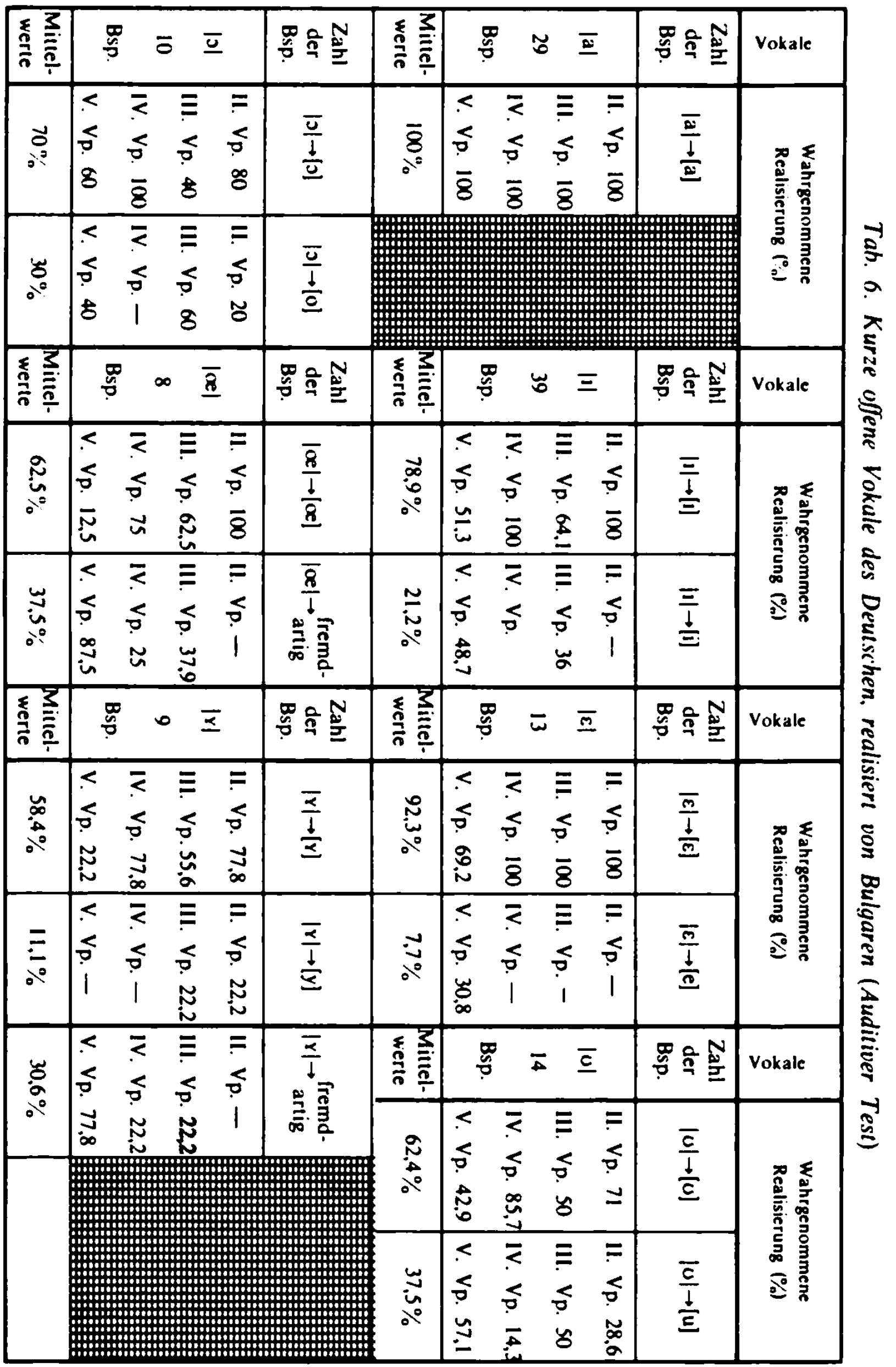


Tab. 7. Pro- und enklitisch abgekürzte E-Laute in der Aussprache von Bulgarem* (Auditiver Test)

\begin{tabular}{|c|c|c|c|c|c|}
\hline $\begin{array}{l}\text { Position } \\
\text { Ziahl der }\end{array}$ & \multicolumn{2}{|c|}{$\begin{array}{l}\text { Realisierte } \\
\text { Varianten }\end{array}$} & \multirow{2}{*}{$\begin{array}{c}\text { Position } \\
\text { Zahl der } \\
\text { Bsp. }\end{array}$} & \multicolumn{2}{|c|}{$\begin{array}{l}\text { Realisierte } \\
\text { Varianten }\end{array}$} \\
\hline$\frac{\pi}{4}$ & [əre] & [әк] & & [e] & {$[\varepsilon]$} \\
\hline $\begin{array}{c}\text { 〈ver-, der } \\
18 \\
\text { Bsp. }\end{array}$ & $\begin{array}{l}\text { II. Vp. } 100 \% \\
\text { III. Vp. } 22 \% \\
\text { IV. Vp. } 100 \% \\
\text { V. Vp. } 33,3\end{array}$ & $\begin{array}{c}\overline{-} \\
77,8 \% \\
\overline{6} \overline{7} \%\end{array}$ & $\begin{array}{c}\langle\text { des, den }\rangle \\
7 \\
\text { Bsp. }\end{array}$ & $\begin{array}{l}\text { II. Vp. } 100 \% \\
\text { III. Vp. }- \\
\text { IV. Vp. } 100 \% \\
\text { V. Vp. }-\end{array}$ & $\begin{array}{c}\overrightarrow{100 \%} \\
\overline{100 \%}\end{array}$ \\
\hline Mittelwerte & $63.8 \%$ & $36.1 \%$ & Mittelwerte & $50 \%$ & $50 \%$ \\
\hline
\end{tabular}

- Die Vokale [i], [u], [o] u. [a], die in pro- und enklitisch gebrauchten Einsilbern vorkommen, werdeen audutiv als korrekt klingend registriert; für das [a] wird jedoch vermerkt, daß es dunkler als deutsches [a] klingt

Tah. 8. Der deutsche Murmelvokal [a] in der Aussprache von Bulgaren

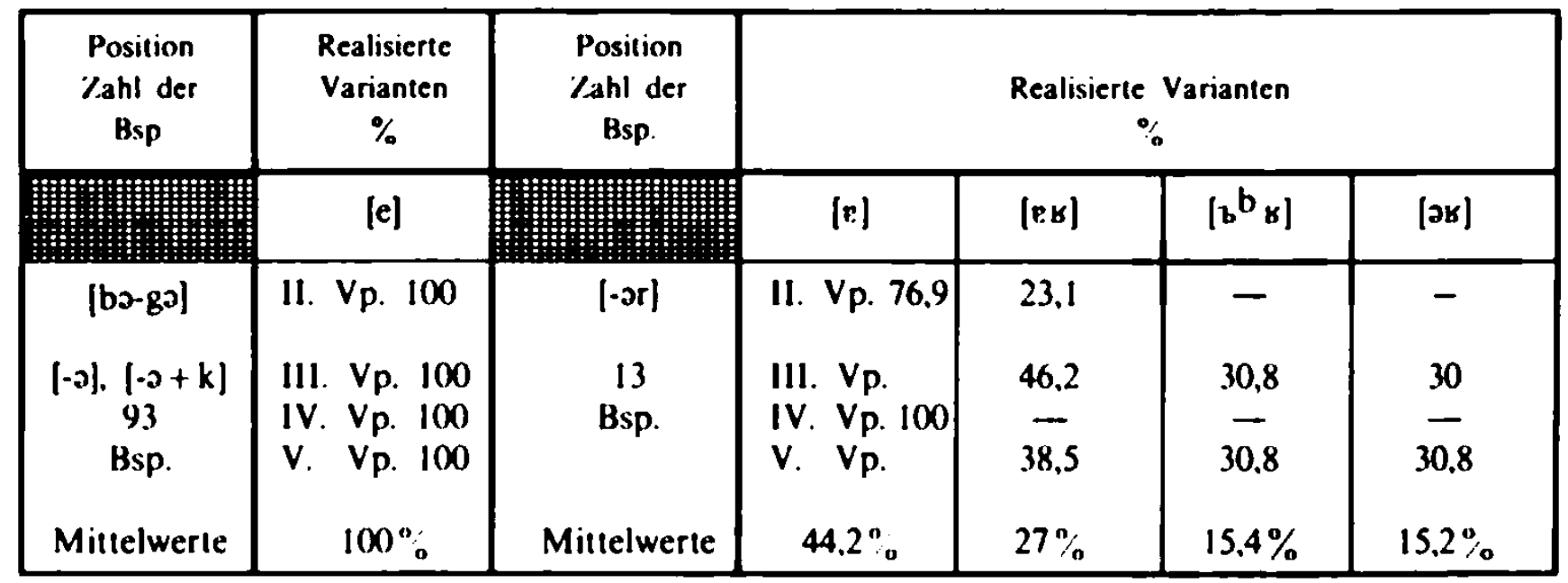

- Die Klangfarbe wird jedoch als ..dunkel” bestimmt 
Twab. 9. Intensität der deutschen Vokale, realisiert von einem Deutschen und vier Bulgaren

\begin{tabular}{|c|c|c|c|c|c|c|c|c|c|c|c|c|c|}
\hline \multirow{2}{*}{ 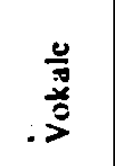 } & \multicolumn{6}{|c|}{ Intensitāt der Vokale in $\mathrm{mm}$} & \multirow{2}{*}{$\begin{array}{l}\frac{u}{\vec{n}} \\
\frac{\vec{b}}{c} \\
\end{array}$} & \multicolumn{6}{|c|}{ Intensität der Vokale in mm } \\
\hline & 1. $v_{p}$ & II. $V_{p}$ & III. $v_{p}$ & IV. $V_{p}$ & v. $v_{r}$ & $\mathbf{D}: \mathbf{B}$ & & I. $V_{p}$. & II. $v_{p}$ & III $v_{p}$ & IV Vp. & $v \cdot v_{p}$ & $\mathbf{D}: \mathbf{B}$ \\
\hline $\mathbf{a}:$ & 23 & 17 & 16 & 18 & 17 & $23: 17$ & o: & 22 & 18 & 17 & 16 & 20 & $22: 18$ \\
\hline a & 21 & 13 & 17 & 16 & 17 & $21: 16$ & 0 & 21 & 16 & 17 & 17 & 17 & $21: 17$ \\
\hline a & 20 & 16 & 16 & 18 & 16 & $20: 17$ & 0 & 23 & 20 & 17 & 17 & 18 & $23: 18$ \\
\hline ii: & 19 & 18 & 19 & 20 & 18 & $19: 19$ & $y$ & 20 & 18 & 21 & 21 & 22 & $20: 21$ \\
\hline i & 17 & 17 & 17 & 17 & 18 & $17: 17$ & $y$ & 18 & 17 & 18 & 20 & 19 & $18: 19$ \\
\hline I & 20 & 17 & 18 & 17 & 20 & $20: 18$ & $r$ & 22 & 17 & 20 & 23 & 24 & $22: 21$ \\
\hline e & 20 & 20 & 18 & 20 & 19 & $20: 19$ & o & 22 & 17 & 19 & 19 & 20 & $22: 19$ \\
\hline $\mathbf{e}$ & 18 & 19 & 17 & 17 & 20 & $18: 18$ & a & 20 & 17 & 17 & 18 & 19 & $20: 18$ \\
\hline E. & 17 & 17 & 18 & 17 & 18 & $17: 18$ & $\mathbf{x}$ & 20 & 17 & 19 & 19 & 19 & $20: 19$ \\
\hline-0 & 15 & 14 & 16 & 16 & 17 & $15: 16$ & $\ddot{a} \dot{e}$ & 19 & 17 & 16 & 17 & 19 & $19: 17$ \\
\hline$-0 \cdot+k$ & 15 & 15 & 16 & 17 & 16 & $15: 16$ & àe & 16 & 15 & 15 & 16 & 16 & $16: 16$ \\
\hline boa- & 14 & 19 & 17 & 16 & 24 & $14: 19$ & ōo & 20 & 19 & 17 & 16 & 21 & $20: 18$ \\
\hline$\cdots$ & 18 & 14 & 17 & 19 & 17 & $18: 17$ & ōo & 15 & 16 & 16 & 14 & 17 & $15: 16$ \\
\hline us : & 18 & 17 & 18 & 17 & 19 & $18: 18$ & $\overline{\boldsymbol{x}}$ & 19 & 18 & 17 & 17 & 20 & $19: 18$ \\
\hline iu & 19 & 14 & 18 & 17 & 20 & $19: 17$ & & & & & & & \\
\hline (1) & 18 & 15 & 17 & 17 & 20 & $18: 19$ & & & & & & & \\
\hline
\end{tabular}


Tab. 10. Lautdauer der deutschen Vokale, realisiert von einem Deutschen und vier Bulgareen

\begin{tabular}{|c|c|c|c|c|c|c|c|c|c|c|c|c|c|}
\hline \multirow{2}{*}{$\begin{array}{l}\frac{u}{v} \\
\frac{\partial}{\partial}\end{array}$} & \multicolumn{6}{|c|}{ Dauer der Vokale in $\mathrm{mm}$} & \multirow{2}{*}{$\begin{array}{l}\frac{u}{\pi} \\
\frac{0}{0} \\
>\end{array}$} & \multicolumn{6}{|c|}{ Dauer der Vokale in $\mathrm{mm}$} \\
\hline & I. $v_{p}$ & II. $v_{p}$ & II. $v_{p}$. & IV. $v p$ & v. $v$ & $\mathbf{D}: \mathbf{B}$ & & I. $V_{p}$ : & II. $v_{p}$ & III. $v p$ : & IV. $v_{p}$ & v. $v_{p}$ & $\mathbf{D}: \mathbf{B}$ \\
\hline $\mathbf{a}:$ & 24 & 18 & 16 & 20 & 24 & $24: 20$ & o: & 20 & 20 & 18 & 18 & 21 & $20: 20$ \\
\hline $\mathbf{a}$ & 14 & 10 & 12 & 10 & 12 & $14: 11$ & o & 12 & 12 & 18 & 20 & 18 & $12: 17$ \\
\hline $\mathbf{a}$ & 12 & 14 & 11 & 12 & 13 & $12: 13$ & o & 13 & 15 & 14 & 12 & 14 & $13: 14$ \\
\hline $\mathrm{i}:$ & 15 & 23 & 19 & 20 & 18 & $15: 12$ & $y:$ & 19 & 22 & 18 & 16 & 19 & $19: 19$ \\
\hline i & 13 & 10 & 11 & 10 & 8 & $13: 10$ & y & 12 & 13 & 15 & 12 & 17 & $12: 14$ \\
\hline I & 9 & 12 & 11 & 10 & 8 & $9: 10$ & $\gamma$ & 10 & 10 & 13 & 12 & 16 & $10: 13$ \\
\hline e: & 21 & 21 & 19 & 12 & 22 & $21: 19$ & o: & 18 & 22 & 19 & 17 & 19 & $18: 19$ \\
\hline e & 10 & 9 & 13 & 10 & 10 & $10: 11$ & ต : & 12 & 12 & 14 & 15 & 14 & $12: 14$ \\
\hline$\varepsilon$ & 12 & 13 & 15 & 12 & 15 & $12: 14$ & $\propto$ & 11 & 13 & 14 & 15 & 14 & $11: 14$ \\
\hline-2 & 11 & 10 & 11 & 11 & 11 & $11: 11$ & de & 23 & 23 & 21 & 18 & 20 & $23: 21$ \\
\hline $.2+k$ & 8 & 10 & 9 & 10 & 10 & $8: 10$ & $\dot{\mathrm{ce}}$ & 17 & 12 & 16 & 15 & 17 & $17: 15$ \\
\hline - bo- & 8 & 9 & 9 & 9 & 11 & $8: 9$ & ฮ̄o & 20 & 22 & 20 & 19 & 22 & $20: 21$ \\
\hline$-E$ & 17 & 11 & 15 & 10 & 14 & $17: 13$ & coo & 19 & 13 & 14 & 20 & 20 & $19: 17$ \\
\hline $\mathbf{u}:$ & 21 & 23 & 16 & 14 & 22 & $21: 19$ & 50 & 22 & 21 & 20 & 18 & 20 & $22: 20$ \\
\hline $\mathbf{u}$ & 10 & 9 & 11 & 9 & 12 & $10: 10$ & & & & & & & \\
\hline 0 & 9 & 10 & 11 & 12 & 10 & $9: 11$ & & & & & & & \\
\hline & & & & & & & & & & & & & \\
\hline
\end{tabular}


Tah. II. Lautdauer und Intensität der langen geschlossenen und kurzen offenen Vokale des Deutschen in der Aussprache eines Deutschen und vier Bulgaren (Mittelwerte, gem. in mm)

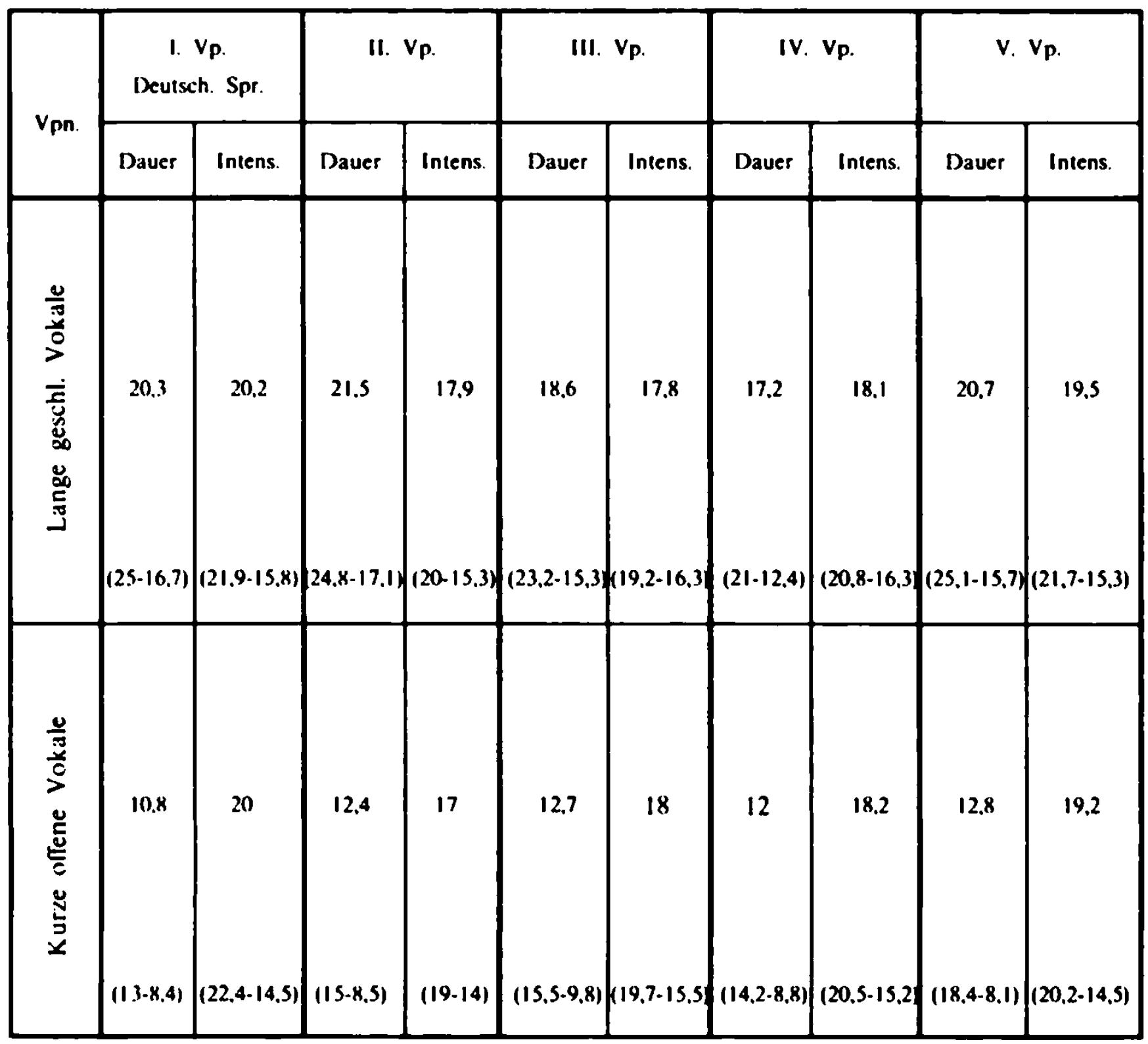

Tah. 12. Dauer- und Intensitätsverhälinisse zwischen den langen geschlossenen und kurzen offenen Vokalen des Deutschen, realisiert von einem Deutschen und vier Bulgaren

\begin{tabular}{|c|c|c|c|c|c|c|}
\hline Vpn. & I. Vp. & II. Vp. & III. Vp. & IV. Vp. & V. Vp. & D:B \\
\hline $\begin{array}{c}\text { Dauer } \\
\text { lang: }: \text { kurz }\end{array}$ & $2: 1$ & $1.7: 1$ & $1: 1$ & $1: 1$ & $1: 1$ & $1.6: 1$ \\
\hline $\begin{array}{c}\text { Intensitāt } \\
\text { lang:kurz }\end{array}$ & $1: 1$ & $1: 1$ & $1: 1$ & $1: 1$ & $1: 1$ & $1: 1$ \\
\hline
\end{tabular}


Grundfrequenz der betonten und unbetonten Vokale des Deutschen und des Bulgarischen (in $\mathrm{H}_{\mathrm{z}}$ )

Tab. 13

\begin{tabular}{|c|c|c|c|c|c|c|c|c|c|c|c|c|c|c|c|c|}
\hline \multirow{2}{*}{ Vsp. } & \multicolumn{15}{|c|}{ Grundfrequenz der betonien Vokale des Deutschen } & \multirow{2}{*}{ 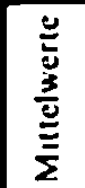 } \\
\hline & i : & 1 & $\mathbf{u}:$ & () & y: & $\mathbf{r}$ & e: & $\varepsilon:$ & $\varepsilon$ & o: & ? & $\boldsymbol{\theta}:$ & $\propto$ & a : & $\mathbf{a}$ & \\
\hline H.P. & 233 & 234.1 & 231.5 & 232,6 & 232.1 & 232,6 & 230,3 & 230.8 & 230.2 & 230.4 & 230.8 & 229 & 230,3 & 228,1 & 228,7 & \\
\hline H.V. & 178.6 & 181.2 & 180.1 & 181,2 & 180.1 & 180.9 & 174,4 & 174,5 & 174,6 & 174,6 & 174,9 & 179.3 & 181 & 170,8 & 171,6 & 17 \\
\hline
\end{tabular}

Tah. 14

\begin{tabular}{|c|c|c|c|c|c|c|c|}
\hline \multirow{2}{*}{ Vsp. } & \multicolumn{6}{|c|}{ Grundfrequenz der betonten Vokale des Bulgarischen } & \multirow{2}{*}{$\begin{array}{l}\text { Mittel- } \\
\text { werte }\end{array}$} \\
\hline & $u^{b}$ & $y^{b}$ & $c^{b}$ & $o^{b}$ & $b^{b}$ & $a^{b}$ & \\
\hline M. P. & 211,1 & 207.5 & 202,6 & 205.8 & 205.2 & 200.4 & 205.4 \\
\hline R. S. & 184.3 & 185.3 & 183 & 182.2 & 183,5 & 181.3 & 183,3 \\
\hline
\end{tabular}

Tab. 15

\begin{tabular}{|c|c|c|c|c|c|c|c|c|c|c|}
\hline \multirow{2}{*}{ Vsp. } & \multicolumn{9}{|c|}{ Girundfrequeny der unbetonten Vokale des Deutschen } & \multirow{2}{*}{$\begin{array}{l}\text { Mittel- } \\
\text { werte }\end{array}$} \\
\hline & $\mathbf{i}$ & $\mathbf{u}$ & y & c & $\rightarrow$ & $\mathbf{o}$ & a & 2 & $\mathbf{r}$ & \\
\hline H. P. & 209.6 & 210.8 & 209.6 & 209.2 & 208 & 205,4 & 202.8 & 160.9 & 170.2 & 201.7 \\
\hline H. V. & 174.5 & 175.7 & 175,6 & 174.1 & 171,9 & 175.3 & 169.6 & 145.5 & 139.9 & 165.4 \\
\hline
\end{tabular}

Tab. 16

\begin{tabular}{|c|c|c|c|c|c|c|c|}
\hline \multirow{2}{*}{ V.sp. } & \multicolumn{6}{|c|}{ Grundfrequenz der unbetonien Vokale des Bulgarischen } & \multirow{2}{*}{$\begin{array}{c}\text { Mittel- } \\
\text { werte }\end{array}$} \\
\cline { 2 - 8 } & $u^{b}$ & $y^{b}$ & $c^{b}$ & $o^{b}$ & $b^{b}$ & $a^{b}$ & \\
\hline M. P. & 198.1 & 201,9 & 190.5 & 196.5 & 179.8 & 181,4 & 191.4 \\
\hline R. S. & 162.2 & 168.9 & 161 & 163.6 & 159.9 & 161,8 & 162.9 \\
\hline
\end{tabular}




\begin{tabular}{|c|c|c|c|}
\hline $\begin{array}{l}\text { ס } \\
\text { ת }\end{array}$ & ס & \multicolumn{2}{|c|}{ 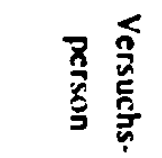 } \\
\hline$\overbrace{0}^{\infty}$ & $\underset{\dot{\alpha}}{\vec{\alpha}}$ & $\hat{\xi}$ & \multirow{4}{*}{ 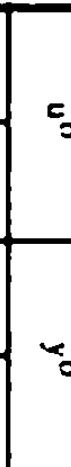 } \\
\hline$\cong$ & $\underset{\sim}{\stackrel{\infty}{\sim}}$ & $\cong$ & \\
\hline $\begin{array}{l}\underset{\delta}{x} \\
\dot{x}\end{array}$ & $\underset{\infty}{\infty}$ & $\stackrel{\mu}{\xi}$ & \\
\hline هـ & $\underset{\sim}{\vec{x}}$ & $\cong$ & \\
\hline هُ & $\underset{x}{\vec{x}}$ & $\stackrel{n}{F}$ & \\
\hline$\underset{\infty}{\infty}$ & $\underset{\sim}{\bar{x}}$ & $\dddot{\underline{ }}$ & 0 \\
\hline 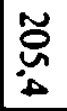 & 离 & $\stackrel{n}{\xi}$ & \\
\hline 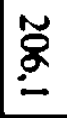 & $\underset{\infty}{\infty}$ & $\cong$ & 0 \\
\hline$\underset{\tilde{Q}}{\tilde{L}}$ & $\underset{w}{\vec{x}}$ & $\stackrel{n}{F}$ & $\sigma$ \\
\hline$\underset{\infty}{\stackrel{\sim}{\not}}$ & $\underbrace{\vec{x}}_{i 0}$ & $\stackrel{\varrho}{=}$ & 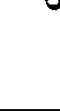 \\
\hline 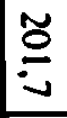 & $\begin{array}{l}\bar{\alpha} \\
\stackrel{0}{0}\end{array}$ & $\frac{\tilde{y}}{\hat{y}}$ & 0 \\
\hline$\overline{8}$ & $\underset{\dot{0}}{\stackrel{\infty}{\sigma}}$ & $\stackrel{\varrho}{=}$ & $=$ \\
\hline 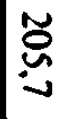 & $\underset{v}{\vec{x}}$ & $\stackrel{n}{\leftrightarrows}$ & 3 \\
\hline$\underset{\sim}{\tilde{O}}$ & $\underset{\underset{\infty}{\infty}}{\vec{x}}$ & $\stackrel{n}{=}$ & $\frac{n}{2}$ \\
\hline
\end{tabular}

\begin{tabular}{|c|c|c|c|c|}
\hline $\begin{array}{l}I \\
<\end{array}$ & $\begin{array}{l}I \\
\tau\end{array}$ & \multicolumn{3}{|c|}{ 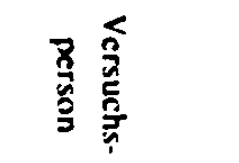 } \\
\hline 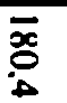 & $\underset{\sim}{\tilde{\alpha}}$ & $\stackrel{n}{F}$ & \multirow{2}{*}{-} & \\
\hline $\overrightarrow{\widetilde{N}}$ & $\underset{\sigma}{\stackrel{\leftrightarrow}{a}}$ & $\cong$ & & \\
\hline $\bar{x}$ & $\underset{\sim}{\tilde{N}}$ & $\frac{n}{F}$ & \multirow{2}{*}{$c$} & \\
\hline$\underset{\omega}{\widetilde{\infty}}$ & $\underset{\sim}{\sim}$ & $\stackrel{\varrho}{=}$ & & $\underset{c}{\pi}$ \\
\hline$\underset{\infty}{\infty}$ & 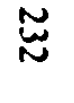 & 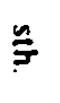 & \multirow{2}{*}{$<$} & $\begin{array}{l}\text { ते } \\
\stackrel{2}{9}\end{array}$ \\
\hline $\begin{array}{l}\bar{x} \\
\dot{0} \\
\dot{0}\end{array}$ & $\underset{\sim}{\sim}$ & $\cong$ & & $\begin{array}{l}\tilde{n} \\
\frac{\sigma}{2} \\
\frac{\hat{x}}{\alpha}\end{array}$ \\
\hline$\overline{\boldsymbol{\alpha}}$ & $\tilde{\tilde{\sigma}}$ & $\frac{n}{\xi}$ & \multirow{2}{*}{8} & 5 \\
\hline$\overline{\boldsymbol{x}}$ & $\underset{\tilde{O}}{\tilde{u}}$ & $\stackrel{n}{=}$ & & 哥 \\
\hline$\vec{\Delta}$ & $\underset{\infty}{\sim}$ & $\stackrel{n}{F}$ & \multirow{2}{*}{$m$} & 壳 \\
\hline$\vec{\Delta}$ & $\underset{\infty}{\sim}$ & $\stackrel{\varrho}{\varrho}$ & & $\frac{a}{3}$ \\
\hline $\overrightarrow{\tilde{u}}$ & $\tilde{W}_{\tilde{\omega}}^{\sim}$ & $\hat{\widehat{F}}$ & \multirow{2}{*}{$u$} & $\frac{\mathbf{x}}{\mathfrak{n}}$ \\
\hline $\overrightarrow{\vec{A}}$ & $\underset{\omega}{\omega}$ & $\stackrel{\varrho}{=}$ & & 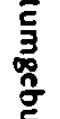 \\
\hline $\bar{\Xi}$ & $\underset{\sim}{\infty}$ & $\stackrel{n}{\xi}$ & \multirow{2}{*}{$\infty$} & $\bar{\Xi}$ \\
\hline$\overline{\check{\partial}}$ & $\underset{\sim}{\mathbb{N}}$ & $\cong$ & & \\
\hline$\underset{\infty}{\vec{J}}$ & $\underset{\mathscr{O}}{\sim}$ & $\stackrel{n}{\xi}$ & \multirow{2}{*}{$\begin{array}{l}3 \\
\frac{3}{\hat{n}} \\
\vdots \\
\vdots \\
\end{array}$} & \\
\hline$\underset{\text { ज゙ }}{\bar{\Xi}}$ & $\stackrel{N}{\sim}$ & $\cong$ & & \\
\hline
\end{tabular}

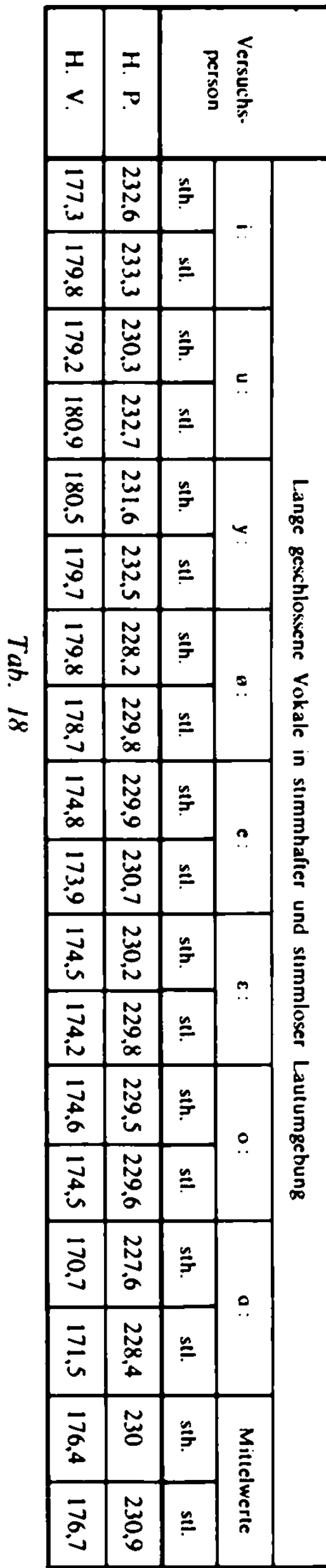

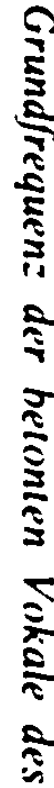

골ำ

2) 気 要余 N

 


\begin{tabular}{|c|c|c|c|c|}
\hline $\begin{array}{l}x \\
0 \\
y\end{array}$ & $\begin{array}{l}3 \\
0\end{array}$ & \multicolumn{3}{|c|}{ 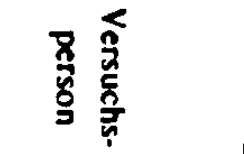 } \\
\hline$\underset{\sim}{8}$ & $\bar{a}$ & $\underline{\underline{F}}$ & \multirow{2}{*}{$\sigma^{\prime}$} & \\
\hline 莒 & $\overline{\breve{\Delta}}$ & $\cong$ & & 焉 \\
\hline శ్ర్ర & $\bar{\Xi}$ & $\dddot{a}$ & \multirow{2}{*}{$\xi$} & 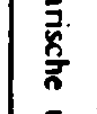 \\
\hline$\frac{\widetilde{\sigma}}{\omega}$ & 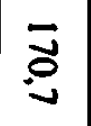 & $\cong$ & & 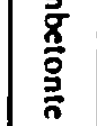 \\
\hline $\bar{D}$ & $\begin{array}{l}\overline{\mathscr{\infty}} \\
\substack{\infty \\
\infty}\end{array}$ & $\underline{\underline{\xi}}$ & \multirow{2}{*}{$\tilde{\sigma}$} & 㝘 \\
\hline$\stackrel{\bar{\infty}}{\stackrel{a}{\sigma}}$ & $\underset{\omega}{\bar{\omega}}$ & $\underline{\underline{\prime}}$ & & $\underline{\underline{u}}$ \\
\hline $\begin{array}{l}\mathbf{\Phi} \\
\mathbf{\infty} \\
0\end{array}$ & $\bar{\alpha}$ & $\underline{\underline{F}}$ & \multirow{2}{*}{$\circ$} & 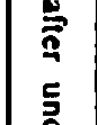 \\
\hline$\underset{\sim}{\tilde{N}}$ & $\overline{\bar{N}}$ & $\underline{\varrho}$ & & 毫 \\
\hline$\overline{8}$ & $\frac{\bar{a}}{6}$ & $\underline{\underline{\xi}}$ & \multirow{2}{*}{$\sigma_{\sigma}$} & 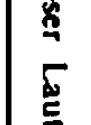 \\
\hline$\stackrel{\overline{\mathbf{s}}}{\dot{\omega}}$ & 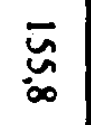 & $\cong$ & & 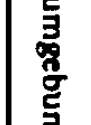 \\
\hline 离 & $\bar{\Phi}$ & $\stackrel{n}{\xi}$ & \multirow{2}{*}{ : } & ${ }_{0}^{\infty}$ \\
\hline$\vec{\Phi}$ & $\overline{\breve{o}}$ & $\cong$ & & \\
\hline 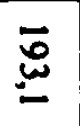 & $\underset{\sim}{\bar{Q}}$ & $\stackrel{\mu}{\vec{F}}$ & \multirow{2}{*}{\multicolumn{2}{|c|}{$\underline{\underline{\hat{\beta}}}$}} \\
\hline $\bar{w}$ & $\bar{a}$ & $\cong$ & & \\
\hline
\end{tabular}

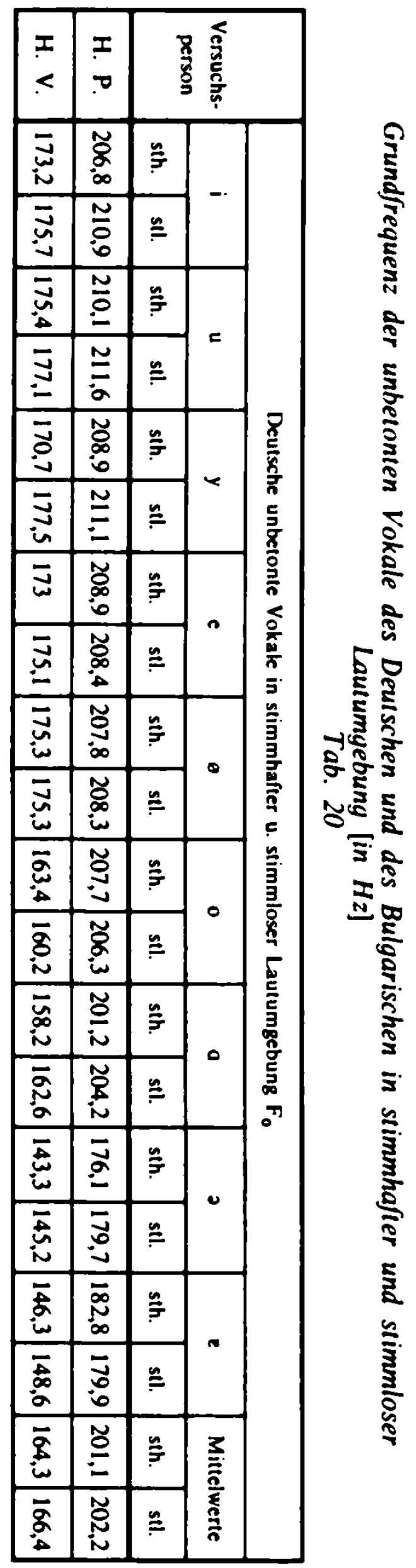


$F_{0}$ der betonten und unbetonten Vokale des Deutschen und des Bulgarischen in Abhängigkeit von der Art der konsonantischen Umgebung [in $\mathrm{Hz}$ ]

Tab. 22

\begin{tabular}{|c|c|c|c|c|c|c|c|c|c|}
\hline \multirow{2}{*}{$\begin{array}{c}\text { Versuchs- } \\
\text { person }\end{array}$} & $\begin{array}{c}\text { vor } \\
\text { Explosiv }\end{array}$ & $\begin{array}{c}\text { nach } \\
\text { Explosiv }\end{array}$ & $\begin{array}{c}\text { Mittel- } \\
\text { werte }\end{array}$ & $\begin{array}{c}\text { vor } \\
\text { Frikativ }\end{array}$ & $\begin{array}{c}\text { nach } \\
\text { Frikativ }\end{array}$ & $\begin{array}{c}\text { Mittel- } \\
\text { werte }\end{array}$ & $\begin{array}{c}\text { vor } \\
\text { Sonor }\end{array}$ & $\begin{array}{c}\text { nach } \\
\text { Sonor }\end{array}$ & $\begin{array}{c}\text { Mittel- } \\
\text { werte }\end{array}$ \\
\hline H. P. & 227,4 & 226,2 & 226,8 & 228,2 & 225,9 & 227,5 & 227,3 & 226.5 & 226,9 \\
\hline H. V. & 172 & 174,7 & 175,3 & 178.5 & 178,4 & 178,5 & 176,5 & 176,5 & 176 \\
\hline
\end{tabular}

Tab. 23

\begin{tabular}{|c|c|c|c|c|c|c|c|c|c|}
\hline \multirow{2}{*}{$\begin{array}{c}\text { Versuchs- } \\
\text { person }\end{array}$} & $\begin{array}{c}\text { Grundirequenz der unbetonten Vokale des Deutschen in Abhāngigkeit von der Art der } \\
\text { konsonantischen Lautumgebung } \\
\text { Explosiv }\end{array}$ & $\begin{array}{c}\text { nach } \\
\text { Explosiv }\end{array}$ & $\begin{array}{c}\text { Mittel- } \\
\text { werte }\end{array}$ & $\begin{array}{c}\text { vor } \\
\text { Frikativ }\end{array}$ & $\begin{array}{c}\text { nach } \\
\text { Frikativ }\end{array}$ & $\begin{array}{c}\text { Mittel- } \\
\text { werte }\end{array}$ & $\begin{array}{c}\text { vor } \\
\text { Sonor }\end{array}$ & $\begin{array}{c}\text { nach } \\
\text {.Sonor }\end{array}$ & $\begin{array}{c}\text { Mittel- } \\
\text { werte }\end{array}$ \\
\hline H. P. & 203,2 & 198,6 & 200,8 & 203,3 & 201,2 & 202,3 & 201,9 & 202,4 & 202,4 \\
\hline H. V. & 166,8 & 163,7 & 165,3 & 164,9 & 163 & 164 & 167,6 & 165,7 & 166,7 \\
\hline
\end{tabular}

Tab. 24

\begin{tabular}{|c|c|c|c|c|c|c|c|c|c|}
\hline \multirow{2}{*}{$\begin{array}{c}\text { Versuchs- } \\
\text { person }\end{array}$} & $\begin{array}{c}\text { Gor } \\
\text { Explosiv }\end{array}$ & $\begin{array}{c}\text { nach } \\
\text { Explosiv }\end{array}$ & $\begin{array}{c}\text { Mittel- } \\
\text { werte }\end{array}$ & $\begin{array}{c}\text { vor } \\
\text { Frikativ }\end{array}$ & $\begin{array}{c}\text { nach } \\
\text { Frikativ }\end{array}$ & $\begin{array}{c}\text { Mittel- } \\
\text { werte }\end{array}$ & $\begin{array}{c}\text { vor } \\
\text { Sonor }\end{array}$ & $\begin{array}{c}\text { nach } \\
\text { Sonor }\end{array}$ & $\begin{array}{c}\text { Mittel- } \\
\text { werte }\end{array}$ \\
\hline M. P. & 184.3 & 181,7 & 183 & 183.5 & 182.2 & 182,9 & 182,3 & 185,6 & 184 \\
\hline R. S. & 204.6 & 205,2 & 204.9 & 205,2 & 201,6 & 203,4 & 208.3 & 207,4 & 207.9 \\
\hline
\end{tabular}

Tab. 25

\begin{tabular}{|c|c|c|c|c|c|c|c|c|c|}
\hline \multirow{2}{*}{$\begin{array}{c}\text { Versuchs- } \\
\text { persion }\end{array}$} & $\begin{array}{c}\text { vor } \\
\text { Explosiv }\end{array}$ & $\begin{array}{c}\text { nach } \\
\text { Explosiv }\end{array}$ & $\begin{array}{c}\text { Mittel- } \\
\text { werte }\end{array}$ & $\begin{array}{c}\text { vor } \\
\text { Frikativ }\end{array}$ & $\begin{array}{c}\text { nach } \\
\text { Frikativ }\end{array}$ & $\begin{array}{c}\text { Mittel- } \\
\text { werte }\end{array}$ & $\begin{array}{c}\text { vor } \\
\text { Sonor }\end{array}$ & $\begin{array}{c}\text { nach } \\
\text { Sonor }\end{array}$ & $\begin{array}{c}\text { Mittel- } \\
\text { werte }\end{array}$ \\
\hline M. P. & 162.9 & 161.7 & 162.3 & 162.3 & 163.9 & 162,8 & 164.2 & 163.5 & 163,6 \\
\hline R. S. & 192.3 & 191.8 & 192.1 & 192.1 & 191.3 & 191.7 & 190,4 & 192.3 & 191.4 \\
\hline
\end{tabular}




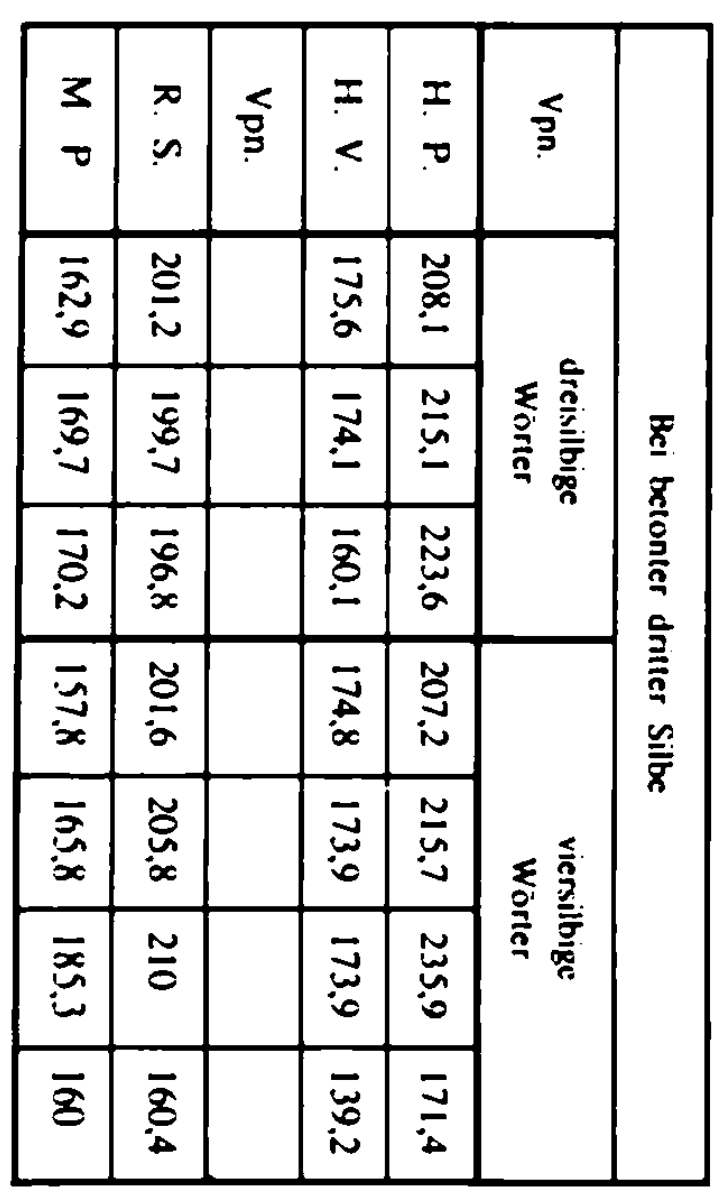

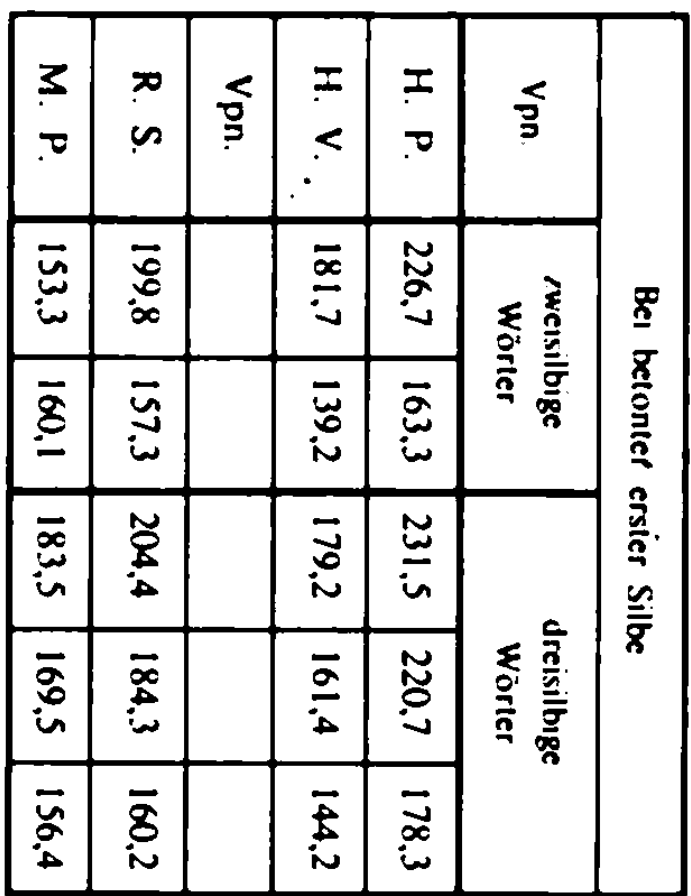



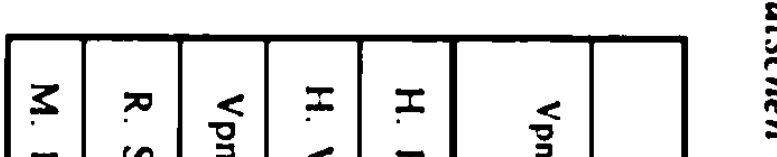

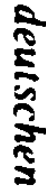

ริ

\begin{tabular}{|c|c|c|c|c|c|c|}
\hline$?$ & ర & $\stackrel{2}{\mathfrak{s}}$ & I & $=$ & ’ & \multirow{5}{*}{ 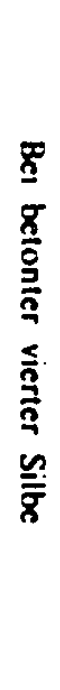 } \\
\hline$\underbrace{\bar{N}}_{i}$ & $\underset{\infty}{\tilde{\delta}}$ & & $\underset{\vec{x}}{\vec{\infty}}$ & ָٌ & \multirow{4}{*}{ 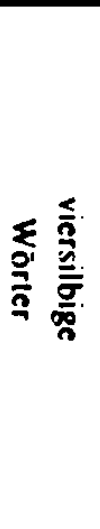 } & \\
\hline$\underset{i}{\bar{E}}$ & $\underset{\infty}{\overline{8}}$ & & تَ & $\stackrel{N}{\Delta}$ & & \\
\hline $\bar{i}$ & $\underset{a}{\overrightarrow{0}}$ & & $\underset{\Delta}{\vec{\sim}}$ & $\frac{N}{b}$ & & \\
\hline $\bar{i}$ & $\underset{\infty}{\vec{\infty}}$ & & 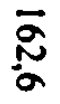 & 孞 & & \\
\hline
\end{tabular}

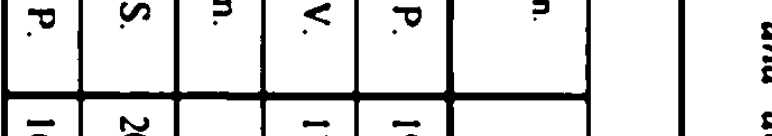

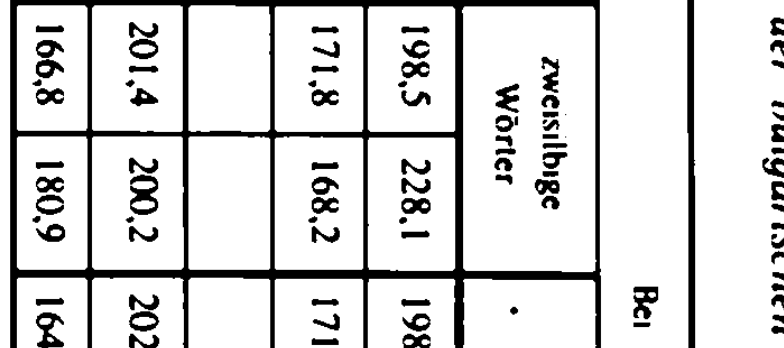

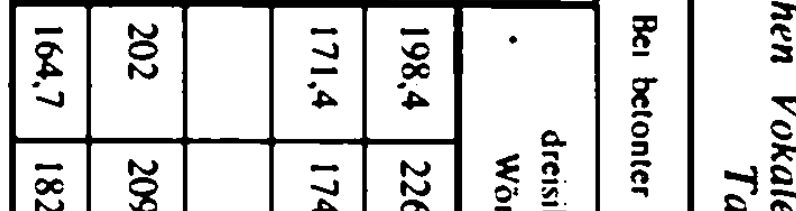

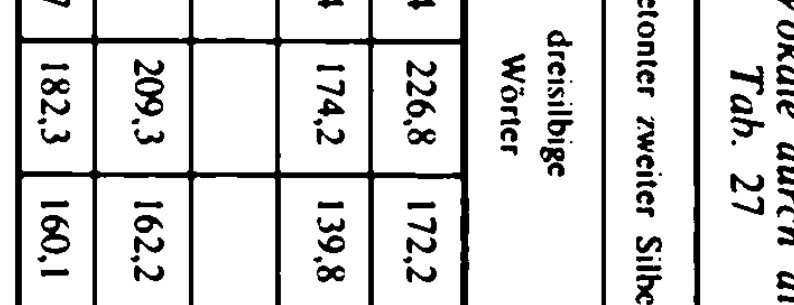

क.

\begin{tabular}{|c|c|c|c|c|}
\hline 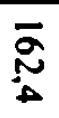 & $\stackrel{\sim}{\stackrel{N}{\mathcal{N}}}$ & $\overline{\vec{D}}_{\infty}$ & $\stackrel{N}{\equiv}$ & \\
\hline$\underset{\vec{x}}{\bar{\nu}}$ & $\underset{\mathscr{\infty}}{\stackrel{\mathscr{*}}{\sim}}$ & $\overrightarrow{\vec{b}}$ & $\underset{\sim}{\sim} \underset{\sim}{\sim}$ & - $\leq$ \\
\hline $\bar{\sim}$ & $\overrightarrow{8}$ & 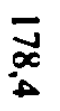 & $\underset{\sim}{\stackrel{n}{n}}$ & 言 $\overline{\bar{g}}$ \\
\hline
\end{tabular}

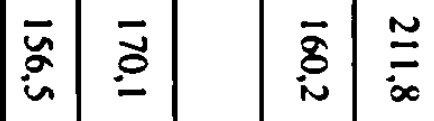




\begin{tabular}{|c|c|c|c|c|}
\hline $\begin{array}{l}T \\
<\end{array}$ & $\begin{array}{l}T \\
T\end{array}$ & \multicolumn{3}{|c|}{ 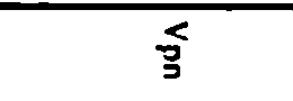 } \\
\hline$\overline{\boldsymbol{x}}$ & $\bar{n}$ & $\circ$ & & \\
\hline$\overline{\overrightarrow{0}}$ & $\underline{\underline{a}}$ & $\infty$ & $\overline{\bar{z}}$ & 䓂 \\
\hline $\bar{\pi}$ & $\overline{8}$ & : & $=$ & $\begin{array}{l}\frac{20}{2} \\
\frac{2}{2}\end{array}$ \\
\hline $\bar{\omega}$ & $\Phi$ & $\infty$ & $\overline{\overline{\bar{n}}}$ & $\frac{\pi}{0}$ \\
\hline $\bar{i}$ & $\Phi$ & $\circ$ & $\equiv$ & $\frac{\mathscr{\overline { n }}}{\overline{\mathrm{n}}}$ \\
\hline $\bar{\omega}$ & $\underline{\Xi}$ & $\infty$ & $\overline{\bar{z}}$ & 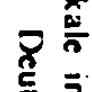 \\
\hline$\Xi$ & $\Xi$ & $\circ$ & $\bar{\Omega}$ & 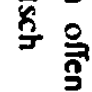 \\
\hline $\bar{a}$ & $\bar{A}$ & $\infty$ & is & $\vec{c}$ \\
\hline$\vec{\Delta}$ & $\stackrel{\Xi}{\sim}$ & $\circ$ & $\underline{3}$ & $\frac{2}{0}$ \\
\hline $\bar{i}$ & $\Phi$ & $\infty$ & กิ & \\
\hline
\end{tabular}

\begin{tabular}{|c|c|c|c|}
\hline $\begin{array}{l}\text { s. } \\
\text { in }\end{array}$ & 3 & \multicolumn{2}{|c|}{3} \\
\hline$i_{i}^{\sim}$ & $\stackrel{\widetilde{D}}{\sim}$ & ? & \\
\hline స & W. & $\infty$ & $\overline{\bar{x}}$ \\
\hline ज़ & $\begin{array}{l}\tilde{0} \\
\infty\end{array}$ & ० & \\
\hline$\stackrel{\tilde{x}}{-}$ & $\stackrel{0}{0}$ & $\infty$ & $\bar{~}$ \\
\hline$\ddot{\Xi}$ & $\begin{array}{l}\tilde{B} \\
\text { i }\end{array}$ & $\circ$ & $\equiv$ \\
\hline$\underset{\Delta}{\tilde{A}}$ & $\stackrel{\widetilde{\Xi}}{-}$ & $\infty$ & $\underline{\underline{x}}$ \\
\hline$\underset{a}{a}$ & : & $\circ$ & 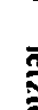 \\
\hline$\tilde{\omega}$ & $\underset{\sim}{\tilde{N}}$ & $\infty$ & \\
\hline$\stackrel{\infty}{-}$ & : & P & \\
\hline$\tilde{\infty}$ & $\underset{ه}{\widetilde{D}}$ & $\infty$ & $\bar{z}$ \\
\hline
\end{tabular}

\begin{tabular}{|c|c|c|c|}
\hline $\begin{array}{l}I \\
<\end{array}$ & $\begin{array}{l}\bar{T} \\
\end{array}$ & \multicolumn{2}{|c|}{ ड़ } \\
\hline$\tilde{\sigma}$ & & $\approx$ & \\
\hline$\stackrel{\tilde{x}}{\sim}$ & $\underset{\sim}{*}$ & c & \\
\hline$\underset{\sim}{\tilde{x}}$ & 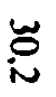 & v & \\
\hline$\tilde{x}$ & $\overbrace{i}$ & o & \\
\hline$\underset{a}{\sim}$ & : & $\hat{a}$ & \\
\hline$\tilde{x}$ & $\underset{\sim}{\widetilde{c}}$ & $=$ & \\
\hline$\stackrel{\sim}{\sim}$ & $\underset{\substack{\infty \\
\infty}}{\tilde{\infty}}$ & 0 & \\
\hline $\begin{array}{l}\tilde{a} \\
\dot{a}\end{array}$ & $\stackrel{\tilde{x}}{-}$ & E & \\
\hline$\underset{\sigma}{*}$ & $\stackrel{0}{0}$ & - & \\
\hline$\underset{i \sim}{\sim}$ & $\underset{\infty}{\infty}$ & $\because$ & \\
\hline 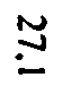 & $\tilde{\boldsymbol{x}}$ & . & \\
\hline 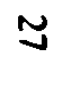 & $\underset{\infty}{\sim}$ & r & \\
\hline$\tilde{\omega}$ & $\underset{\substack{\tilde{x} \\
0}}{\tilde{n}}$ & $\sigma$ & \\
\hline
\end{tabular}

\begin{tabular}{|c|c|c|c|}
\hline $\begin{array}{l}\text { o } \\
\text { is }\end{array}$ & $\begin{array}{l}3 \\
0\end{array}$ & \multicolumn{2}{|c|}{ §ֵ } \\
\hline$\overline{\boldsymbol{x}}$ & $\bar{n}$ & : & 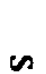 \\
\hline $\bar{\infty}$ & I & : & 䲶 \\
\hline $\bar{\infty}$ & 韹 & o & 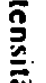 \\
\hline $\bar{b}$ & $\Xi$ & 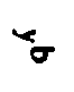 & $\begin{array}{l}\vec{a} \\
\bar{g}\end{array}$ \\
\hline $\bar{a}$ & $\bar{A}$ & $\approx$ & $\vec{n}$ \\
\hline $\bar{g}$ & $\stackrel{\bar{N}}{\mathrm{I}}$ & $\sigma_{\sigma}$ & a \\
\hline$\stackrel{\bar{a}}{\nu}$ & $\bar{\AA}$ & 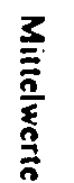 & 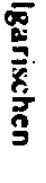 \\
\hline
\end{tabular}

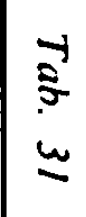

\begin{tabular}{|c|c|c|c|}
\hline$\approx$ & $\stackrel{\infty}{\infty}$ & & \\
\hline & $\stackrel{\infty}{\mathscr{\infty}}$ & & \\
\hline 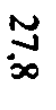 & 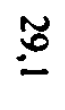 & $\circ$ & $\underline{z}$ \\
\hline & $\underset{\underset{x}{\tilde{x}}}{\stackrel{\sim}{x}}$ & $\infty$ & $\frac{\hbar}{\hat{n}}$ \\
\hline
\end{tabular}


Spezifische Intensität der unbetonten Vokale des Deutschen und des Bulgarischen [in $\mathrm{dB}$ ] Tab. 34

\begin{tabular}{|c|c|c|c|c|c|c|c|c|c|}
\hline \multirow{2}{*}{ Vpn. } & \multicolumn{8}{|c|}{ Spezifische Intensitat der unbetonten Vokale des Deutschen } \\
\cline { 2 - 11 } & o & e & o & 0 & y & u & i & 0 & . \\
\hline H. P. & 27,8 & 26,3 & 26,1 & 26 & 26 & 25,7 & 25,3 & 24,5 & 24 \\
\hline H. V. & 27,5 & 27,1 & 25,5 & 26,3 & 25,6 & 25,6 & 25,2 & 22,3 & 201,84 \\
\hline
\end{tabular}

Tab. 35

\begin{tabular}{|c|c|c|c|c|c|c|}
\hline \multirow{2}{*}{ Vpn. } & \multicolumn{5}{|c|}{ Spezifiscine Intensitä der unbetonten Vokale des Bulgarischen } \\
\cline { 2 - 7 } & $\mathrm{a}$ & $\mathrm{e}$ & $\mathrm{o}$ & $\mathrm{b}$ & $\mathrm{u}$ & $\mathrm{y}$ \\
\hline R. S. & 14.9 & 14.8 & 14,5 & 14.3 & 14,2 & 13,8 \\
\hline M. P. & 13.8 & 12.6 & 13,2 & 13,7 & 11.5 & 12 \\
\hline
\end{tabular}




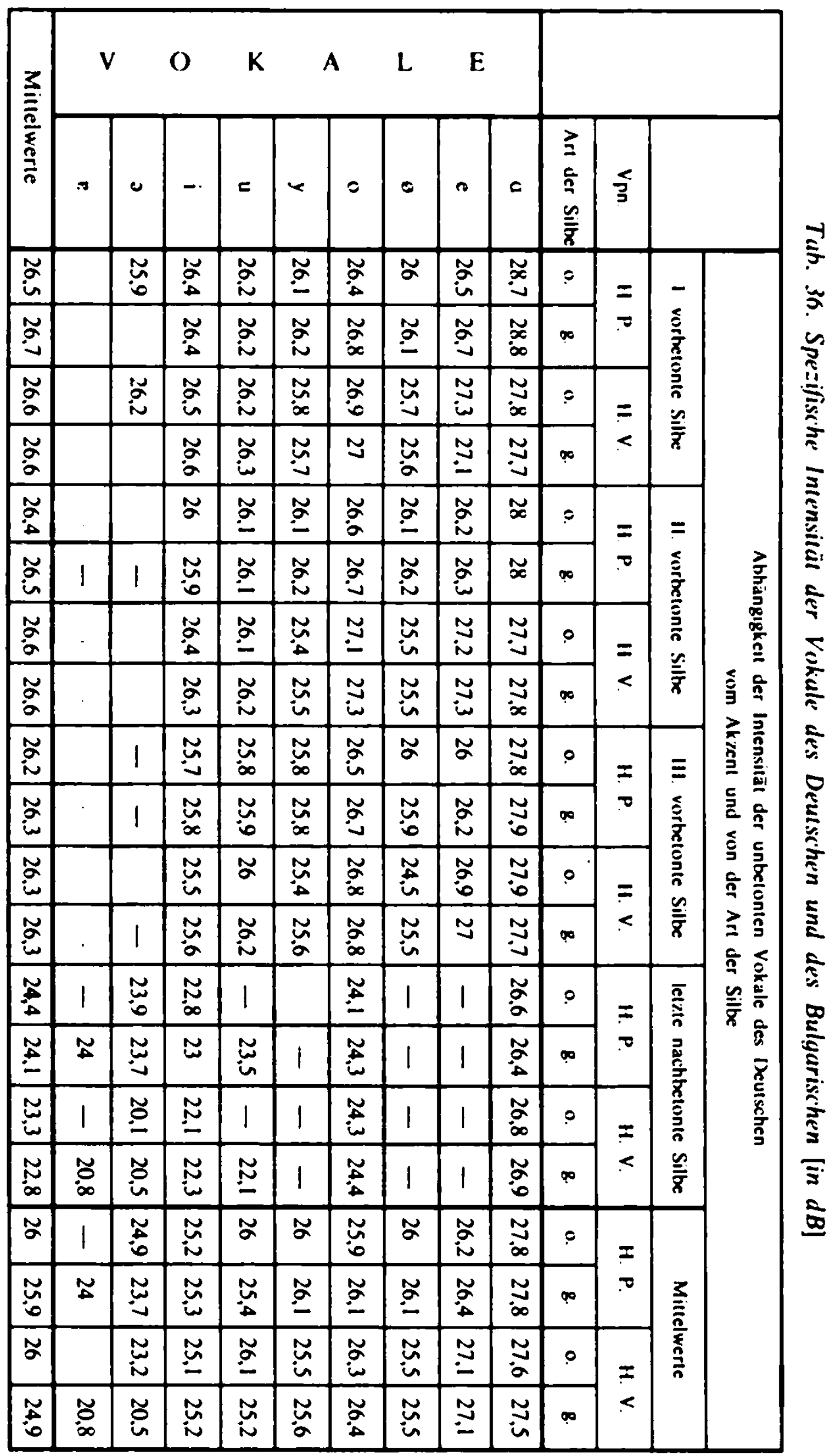




\begin{tabular}{|c|c|c|c|c|c|c|c|c|c|c|}
\hline \multirow{2}{*}{ 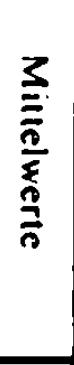 } & \multicolumn{6}{|c|}{$V \quad O \quad K A A L E$} & & & & \\
\hline & $k$ & $=$ & $\sigma$ & 0 & 0 & $=$ & $\begin{array}{l}\frac{a}{3} \\
\stackrel{0}{\bar{z}} \\
\end{array}$ & ș & & \\
\hline $\bar{n}$ & $\bar{n}$ & $\bar{n}$ & 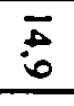 & $\overline{\tilde{n}}$ & $\bar{n}$ & $\overline{\ddot{c}}$ & 0 & \multirow{2}{*}{$\begin{array}{l}\text { o } \\
\text { is }\end{array}$} & \multirow{4}{*}{$\begin{array}{l}- \\
\grave{o} \\
\frac{\partial}{z}\end{array}$} & \multirow{20}{*}{ 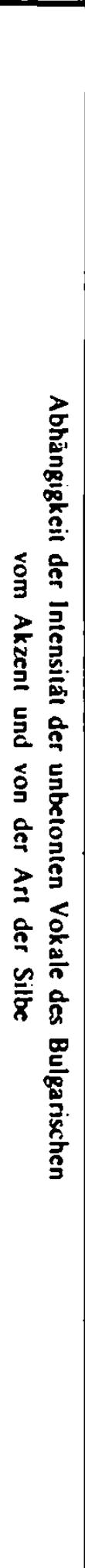 } \\
\hline$\overline{\tilde{n}}$ & $\bar{n}$ & $\bar{a}$ & $\bar{\vdots}$ & $\bar{\sim}$ & $\bar{\omega}$ & $\bar{g}$ & $\infty$ & & & \\
\hline$\vec{w}$ & $\bar{\Xi}$ & $\stackrel{\bar{n}}{i}$ & 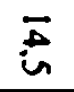 & $\overline{i n}$ & $\bar{A}$ & $\underset{\infty}{\bar{\infty}}$ & ○ & 3 & & \\
\hline$\underset{\infty}{\bar{\infty}}$ & 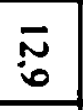 & $\bar{n}$ & $\bar{\omega}_{0}$ & $\bar{\infty}$ & 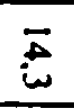 & $\bar{u}$ & $\infty$ & $\tau$ & & \\
\hline $\bar{n}$ & \pm & $\overline{\tilde{\omega}}$ & $\bar{n}$ & $\bar{n}$ & $\vec{a}$ & $\bar{n}$ & $\circ$ & \multirow{2}{*}{$\begin{array}{l}\infty \\
\infty\end{array}$} & $=$ & \\
\hline$\overline{\bar{w}}$ & i & $\bar{u}$ & \pm & $\bar{a}$ & $\bar{n}$ & $\bar{n}$ & $\infty$ & & $\frac{\delta}{8}$ & \\
\hline $\bar{\sigma}$ & $\underset{N}{\overline{0}}$ & $\bar{N}$ & $\Xi$ & $\bar{\omega}_{0}$ & $\stackrel{\Phi}{i}$ & \pm & ० & \multirow{2}{*}{$\begin{array}{l}3 \\
0\end{array}$} & 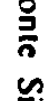 & \\
\hline 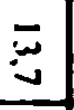 & $\bar{\omega}$ & 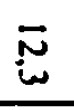 & $\bar{A}$ & $\bar{\omega}_{\infty}$ & $\stackrel{\vec{A}}{i}$ & : & $\infty$ & & $\overline{\bar{x}}$ & \\
\hline \pm & $\bar{A}$ & 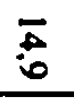 & 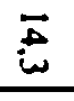 & $\bar{n}$ & $\bar{n}$ & $\overline{\tilde{\omega}}$ & ○ & \multirow{2}{*}{$\begin{array}{l}x \\
0\end{array}$} & \multirow{4}{*}{ 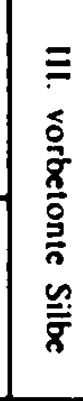 } & \\
\hline $\bar{n}$ & $\Phi$ & $\check{\Xi}$ & $\stackrel{E}{D}$ & $\bar{\Delta}$ & $\bar{a}$ & $\bar{\Delta}$ & $\infty$ & & & \\
\hline $\bar{i}$ & $\bar{N}$ & $\overline{0}$ & $\underset{\infty}{\bar{\infty}}$ & $\bar{\omega}$ & $\bar{\varpi}_{\infty}$ & $\vec{E}$ & $\circ$ & 3 & & \\
\hline $\bar{\Sigma}$ & $\bar{N}$ & $\bar{\omega}$ & $\vec{A}$ & $\bar{i}$ & $\vec{a}$ & \pm & $\infty$ & ס. & & \\
\hline $\bar{a}$ & $\bar{\Sigma}$ & : & 1 & $\bar{\omega}$ & $\bar{N}$ & $\underset{\omega}{\sim}$ & $\circ$ & \multirow{2}{*}{$\begin{array}{l}\text { هִ } \\
\text { סִ }\end{array}$} & \multirow{4}{*}{ 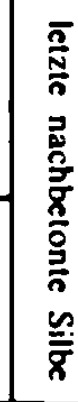 } & \\
\hline $\bar{F}$ & $\Xi$ & $\bar{\Sigma}$ & $\vec{\infty}$ & $\equiv$ & $\bar{N}$ & i & $\infty$ & & & \\
\hline$:$ & in & $\stackrel{\circ}{\sim}$ & 1 & $\stackrel{0}{\sim}$ & $\underset{i}{i}$ & $\begin{array}{l}\overrightarrow{0} \\
\infty \\
\end{array}$ & . & 3 & & \\
\hline i & $\therefore$ & $: 0$ & $\bar{a}$ & $\underset{i}{D}$ & : & $=$ & $\infty$ & ס & & \\
\hline$\Phi$ & $\underset{\infty}{\infty}$ & $\Phi$ & $\vec{a}$ & $\Phi$ & \pm & 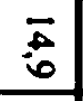 & ○ & \multirow{2}{*}{$\begin{array}{l}x \\
\text { is }\end{array}$} & \multirow{4}{*}{ 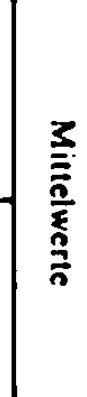 } & \\
\hline 志 & $\underset{\infty}{\vec{\infty}}$ & $\stackrel{I}{\mathbf{N}}$ & $\bar{D}$ & $\vec{a}$ & $\underset{\infty}{\Phi}$ & $\bar{n}$ & $\infty$ & & & \\
\hline $\bar{a}$ & $\bar{i}$ & $\bar{\Delta}$ & 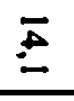 & i & $\overline{-}$ & \pm & ${ }^{\circ}$ & \multirow{2}{*}{$\begin{array}{l}3 \\
0\end{array}$} & & \\
\hline$\overline{0}$ & $\bar{\sigma}$ & $\Xi$ & $\bar{\Delta}$ & $\bar{N}_{\infty}$ & $\bar{\omega}$ & $\bar{\omega}$ & $\infty$ & & & \\
\hline
\end{tabular}


Spezifische Dauer der Vokallaute des Deutschen und des Bulgarischen Tab. 38

\begin{tabular}{|c|c|c|c|c|c|c|c|c|c|c|c|c|c|c|c|}
\hline \multirow{2}{*}{ Vpn. } & \multicolumn{15}{|c|}{ Spezifische Lautdauer der betonten Vokale des Deutschen } \\
\hline & $\mathbf{a}$ & a & o: & $\mathbf{3}$ & r: : & $\varepsilon$ & c: & i: & $\mathbf{I}$ & o : & $\mathbf{\alpha}$ & $y:$ & $\mathbf{r}$ & $\mathbf{u}:$ & 0 \\
\hline H. P. & 0.2 & 0.13 & 0,2 & 0.13 & 0.2 & 0.12 & 0,19 & 0,18 & 0.1 & 0.19 & 0,11 & 0.18 & 0.1 & 0,18 & 0,09 \\
\hline H. V. & 0.17 & 0.13 & 0.17 & 0.12 & 0,16 & 0.11 & 0,16 & 0.16 & 0.1 & 0.16 & 0.1 & 0.16 & 0.11 & 0.15 & 0.1 \\
\hline
\end{tabular}

Tah. 39

\begin{tabular}{|c|c|c|c|c|c|c|c|c|c|c|c|}
\hline \multirow{2}{*}{ Vpn. } & \multicolumn{11}{|c|}{ Sperifische Lautdauer der unbetonten Vokale des Deutschen } \\
\hline & o & o & c & i & a & y & u & b2. & د. & חו & : \\
\hline H. P. & 0.1 & 0.11 & 0.08 & 0.08 & 0.09 & 0.08 & 0,09 & 0,07 & 0.17 & 0.07 & 0.09 \\
\hline H. V. & 0.1 & 0,1 & 0,09 & 0.09 & 0.08 & 0.08 & 0,08 & 0.08 & 0.17 & 0,07 & 0.1 \\
\hline
\end{tabular}

Tah. 40

\begin{tabular}{|c|c|c|c|c|c|c|}
\hline \multirow{2}{*}{ Vpn. } & \multicolumn{5}{|c|}{$\begin{array}{c}\text { Spez. Lauldauer der betonten } \\
\text { Vokale des Bulgarischen }\end{array}$} \\
\cline { 2 - 7 } & 0 & 0 & y & u & c & b \\
\hline M. P. & 0.12 & 0.09 & 0.10 & 0.09 & 0.11 & 0.11 \\
\hline R. S. & 0.14 & 0.13 & 0.11 & 0.11 & 0.12 & 0.11 \\
\hline
\end{tabular}

Tab. 42

\begin{tabular}{|c|c|c|c|c|c|c|c|c|}
\hline \multirow{4}{*}{$V_{p n}}$. & \multicolumn{8}{|c|}{$\begin{array}{c}\text { l.auldauer der bulgarischen Vokale in Abhàngigkeit } \\
\text { von der Art der Silbe }\end{array}$} \\
\hline & \multicolumn{4}{|c|}{ betonte Vokale } & \multicolumn{4}{|c|}{ unbetonte Vokale } \\
\hline & \multicolumn{2}{|c|}{ often } & \multicolumn{2}{|c|}{ gexchl. } & \multicolumn{2}{|c|}{ often } & \multicolumn{2}{|c|}{ geschl. } \\
\hline & $\begin{array}{c}\text { An-. } \\
\text { Inlaut }\end{array}$ & $\begin{array}{l}\text { Aus- } \\
\text { laut }\end{array}$ & $\begin{array}{l}\text { An-. } \\
\text { Inlaut }\end{array}$ & $\begin{array}{l}\text { Aus- } \\
\text { laut }\end{array}$ & $\begin{array}{c}\text { An-. } \\
\text { Inlaut }\end{array}$ & $\begin{array}{l}\text { Aus- } \\
\text { laut }\end{array}$ & $\begin{array}{l}\text { An-. } \\
\text { Inlaut }\end{array}$ & $\begin{array}{l}\text { Aus- } \\
\text { laut }\end{array}$ \\
\hline M. P. & 0.09 & 0.13 & 0,08 & 0.11 & 0.6 & 0.12 & 0,07 & 0,08 \\
\hline R. S. & 0.1 & 0.17 & 0.11 & 0.12 & 0.09 & 0.15 & 0.1 & 0.13 \\
\hline
\end{tabular}

Tab. 41

\begin{tabular}{|c|c|c|c|c|c|c|}
\hline \multirow{3}{*}{ Vpn. } & \multicolumn{5}{|c|}{$\begin{array}{c}\text { Spez. Lautdauer der unbetonten } \\
\text { Vokale des Bulgarischen }\end{array}$} \\
\cline { 2 - 7 } & a & 0 & y & u & e & b \\
\hline M. P. & 0.09 & 0.08 & 0.10 & 0.07 & 0.07 & 0.07 \\
\hline R. S. & 0.12 & 0.10 & 0.10 & 0.10 & 0.11 & 0.11 \\
\hline
\end{tabular}

Tah. 43

\begin{tabular}{|c|c|c|c|}
\hline \multirow{2}{*}{$v_{p n}$} & \multicolumn{3}{|c|}{$\begin{array}{c}\text { Untet. Vokale } \\
\text { des Bulgarischen nach } \\
\text { der Posttion der Silbe } \\
\text { im Wort }\end{array}$} \\
\cline { 2 - 4 } & $\begin{array}{c}\text { I. } \\
\text { betonte } \\
\text { Silbe }\end{array}$ & $\begin{array}{c}\text { II. } \\
\text { betonte } \\
\text { Silbe }\end{array}$ & $\begin{array}{c}\text { letate } \\
\text { betonte } \\
\text { Silbe }\end{array}$ \\
\hline M. P. & 0.08 & 0.07 & 0.10 \\
\hline R. S. & 0.1 & 0.09 & 0.14 \\
\hline
\end{tabular}



Spezifische Lautdauer der Vokale des Deutschen und des Bulgarischen
Tab. 44

\begin{tabular}{|c|c|c|c|c|c|c|c|c|c|c|c|c|}
\hline \multirow{3}{*}{$V_{p n}}$. & \multicolumn{12}{|c|}{$\begin{array}{l}\text { Becinflussung der Lautdauer der betonten Vokale des Deutschen } \\
\text { durch die kons. Umgebung }\end{array}$} \\
\hline & \multicolumn{2}{|c|}{$\begin{array}{l}\text { vor } \\
\text { Explosiv }\end{array}$} & \multicolumn{2}{|c|}{$\begin{array}{l}\text { nach } \\
\text { Explosiv }\end{array}$} & \multicolumn{2}{|c|}{$\begin{array}{l}\text { vor } \\
\text { Frikativ }\end{array}$} & \multicolumn{2}{|c|}{$\begin{array}{l}\text { nach } \\
\text { Frikativ }\end{array}$} & \multicolumn{2}{|c|}{$\begin{array}{l}\text { vor } \\
\text { Sonor }\end{array}$} & \multicolumn{2}{|c|}{$\begin{array}{l}\text { nach } \\
\text { Sonor }\end{array}$} \\
\hline & 1. $8^{\circ}$ & k. o. & I. 8 & k. $o$. & I. 8 & k. o. & 1. 8 & k. o. & 1. 8 & k. o. & 1. 8 & k. co. \\
\hline H. P. & 0.19 & 0,09 & 0.19 & 0.12 & 0,22 & 0,17 & 0,20 & 0,12 & 0,18 & 0,14 & 0,18 & $0,1: 2$ \\
\hline H. V. & 0,18 & 0,11 & 0,18 & 0.12 & 0,19 & 0,16 & 0,17 & 0,12 & 0,18 & 0,13 & 0,18 & $0,1.2$ \\
\hline
\end{tabular}

- I. g. = lang geschlossen; k. o.=kurz offen

Tab. 45

\begin{tabular}{|c|c|c|c|c|c|c|c|c|c|c|c|c|}
\hline \multirow{3}{*}{ Vpn. } & \multicolumn{12}{|c|}{$\begin{array}{l}\text { Beeinflussung der Lautdauer der betonten Vokale des Bulgarischen } \\
\text { durch die kons. Umgebung }\end{array}$} \\
\hline & \multicolumn{2}{|c|}{$\begin{array}{c}\text { vor } \\
\text { Explosiv }\end{array}$} & \multicolumn{2}{|c|}{$\begin{array}{l}\text { nach } \\
\text { Explosiv }\end{array}$} & \multicolumn{2}{|c|}{$\begin{array}{c}\text { vor } \\
\text { Frikativ }\end{array}$} & \multicolumn{2}{|c|}{$\begin{array}{l}\text { nach } \\
\text { Frikativ }\end{array}$} & \multicolumn{2}{|c|}{$\begin{array}{l}\text { vor } \\
\text { Sonor }\end{array}$} & \multicolumn{2}{|c|}{$\begin{array}{l}\text { nach } \\
\text { Sonor }\end{array}$} \\
\hline & $\begin{array}{l}\text { An-. } \\
\text { Inlaut }\end{array}$ & $\begin{array}{l}\text { Aus- } \\
\text { laut }\end{array}$ & $\begin{array}{l}\text { An-, } \\
\text { Inlaut }\end{array}$ & $\begin{array}{l}\text { Aus- } \\
\text { laut }\end{array}$ & $\begin{array}{l}\text { An-, } \\
\text { Inlaut }\end{array}$ & $\begin{array}{l}\text { Aus- } \\
\text { laut }\end{array}$ & $\begin{array}{c}\text { An-. } \\
\text { Inlaut }\end{array}$ & $\begin{array}{l}\text { Aus: } \\
\text { laut }\end{array}$ & $\begin{array}{c}\text { An-. } \\
\text { Inlaut }\end{array}$ & $\begin{array}{l}\text { Aus- } \\
\text { laut }\end{array}$ & $\begin{array}{l}\text { An-, } \\
\text { Inlaut }\end{array}$ & $\begin{array}{l}\text { Auss- } \\
\text { lauit }\end{array}$ \\
\hline M. P. & 0.09 & 0,13 & 0.08 & 0.13 & 0.11 & 0.13 & 0.10 & 0.12 & 0.09 & 0.14 & 0,10 & 0.11 \\
\hline R. S. & 0.09 & 0.12 & 0.10 & 0.14 & 0.12 & 0.13 & 0.12 & 0.14 & 0,09 & 0.13 & 0,11 & $0.1: 2$ \\
\hline
\end{tabular}

Tab. 46

\begin{tabular}{|c|c|c|c|}
\hline & \multicolumn{3}{|c|}{$\begin{array}{c}\text { Lautdauer der unb. } \\
\text { Vokale des Deutschen } \\
\text { nach der Stellung der } \\
\text { unbet. Silbe zur betonten }\end{array}$} \\
\cline { 2 - 4 } & $\begin{array}{c}\text { I. } \\
\text { vorbet. } \\
\text { Silke }\end{array}$ & $\begin{array}{c}\text { II. } \\
\text { vorbet. } \\
\text { Silbe }\end{array}$ & $\begin{array}{c}\text { letzite } \\
\text { Silbe }\end{array}$ \\
\hline H. P. & 0.07 & 0.08 & 0.10 \\
\hline H. V. & 0.08 & 0.08 & 0.10 \\
\hline
\end{tabular}

Tab. 47

\begin{tabular}{|c|c|c|c|c|}
\hline \multirow{3}{*}{ Vpn. } & \multicolumn{4}{|c|}{$\begin{array}{l}\text { Lautdauer der unbet. } \\
\text { Vokale des Deutschen } \\
\text { nach der Position } \\
\text { der Silbe }\end{array}$} \\
\hline & \multicolumn{2}{|c|}{ olten } & \multicolumn{2}{|c|}{ geschlossen } \\
\hline & $\begin{array}{l}\text { I. II. } \\
\text { Silbe }\end{array}$ & $\begin{array}{l}\text { letzte } \\
\text { Silbe }\end{array}$ & $\begin{array}{l}\text { I. II. } \\
\text { Silbe }\end{array}$ & $\begin{array}{l}\text { letzle } \\
\text { Silbe }\end{array}$ \\
\hline H. P. & 0,08 & 0,02 & 0,08 & $0,08+0.1^{\bullet}$ \\
\hline H. V. & & & & $0.07+0.1$ \\
\hline
\end{tabular}

*- In geschl. letzter Silbe ist die Lautdauer des [0] separat fur die Positionen [-on] und [-20+ anderen Konsonanten] ermittelt. 
Beeinflussung der Lautdauer der unbetonten Vokale des Deutschen und des Bulgarischen durch die konsonantische Lautumgebung

Tab. 48

\begin{tabular}{|c|c|c|c|c|c|c|c|c|c|c|c|}
\hline \multicolumn{1}{|c|}{} & \multicolumn{8}{|c|}{ Becinflussung der Lautdauer der deutschen unbetonten Vokale } \\
durch die konsonantische Umgebung
\end{tabular}

Tab. 49

\begin{tabular}{|c|c|c|c|c|c|c|c|c|c|c|}
\hline \multirow{3}{*}{ Vpn. } & \multicolumn{10}{|c|}{$\begin{array}{l}\text { Beeinflussung der Lautdauer der unbetonten Vokale } \\
\text { des Bulgarischen durch die konsonantische Lautumgebung }\end{array}$} \\
\hline & \multirow{2}{*}{$\begin{array}{c}\begin{array}{c}\text { vor } \\
\text { Fxplosiv }\end{array} \\
\begin{array}{c}\text { An-. } \\
\text { Inlaut }\end{array}\end{array}$} & \multicolumn{2}{|c|}{$\begin{array}{l}\text { nach } \\
\text { Explosiv }\end{array}$} & \multirow{2}{*}{ 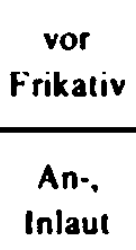 } & \multicolumn{2}{|c|}{$\begin{array}{l}\text { nach } \\
\text { Frikativ }\end{array}$} & \multicolumn{2}{|c|}{$\begin{array}{l}\text { vor } \\
\text { Sonor }\end{array}$} & \multicolumn{2}{|c|}{$\begin{array}{l}\text { nach } \\
\text { Sonor }\end{array}$} \\
\hline & & $\begin{array}{l}\text { An-, } \\
\text { Inlaut }\end{array}$ & $\begin{array}{l}\text { Aus- } \\
\text { laut }\end{array}$ & & $\begin{array}{l}\text { An-, } \\
\text { Inlaut }\end{array}$ & $\begin{array}{l}\text { Aus- } \\
\text { laut }\end{array}$ & $\begin{array}{l}\text { An-. } \\
\text { Inlaut }\end{array}$ & $\begin{array}{l}\text { Aus- } \\
\text { laut }\end{array}$ & $\begin{array}{l}\text { An-. } \\
\text { Inlaut }\end{array}$ & $\begin{array}{l}\text { Aus- } \\
\text { laut }\end{array}$ \\
\hline M. P. & 0,08 & 0.08 & 0.10 & 0,09 & 0,08 & 0.12 & 0,09 & 0,09 & 0,09 & 0.10 \\
\hline R. S. & 0,09 & 0,09 & 0.15 & 0,10 & 0,09 & 0.14 & 0,09 & 0.11 & 0,09 & 0,14 \\
\hline
\end{tabular}


Sonagramme der deutschen und der bulgarischen

Frikative [?], [v] [I], [v]
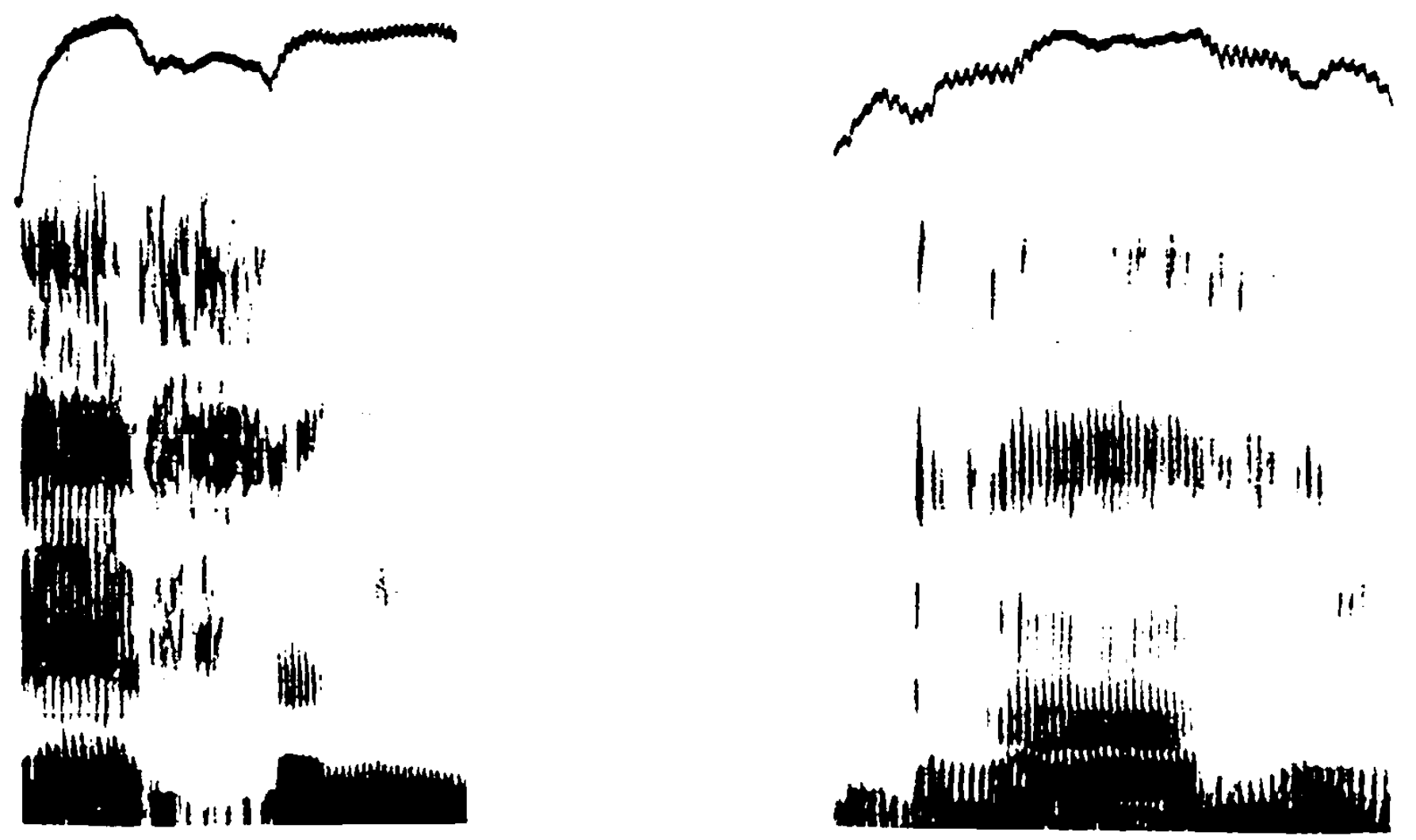

|'s:an]
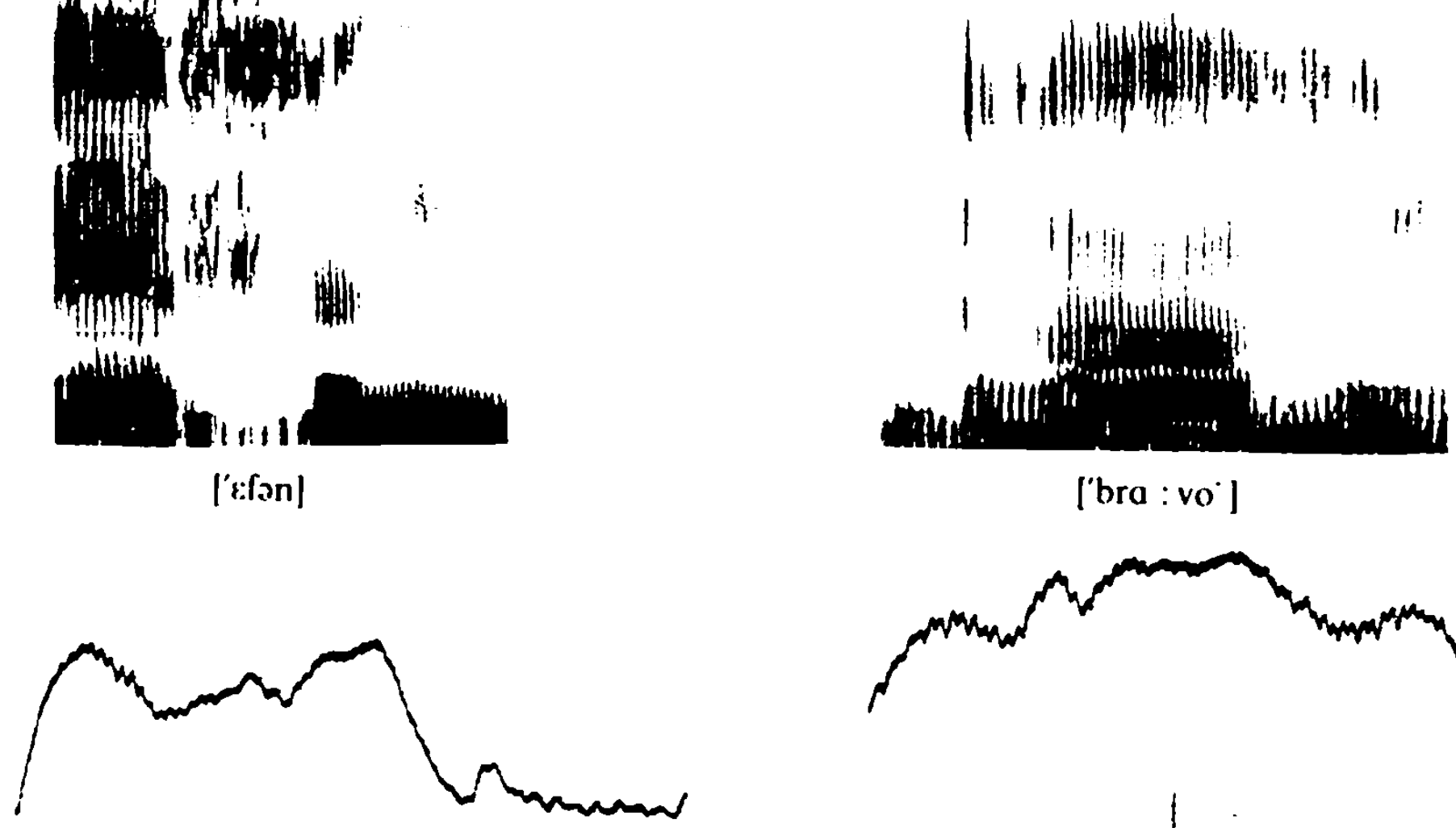

['bra : vo']

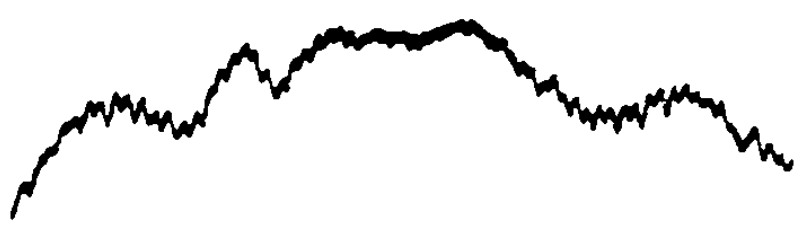

11

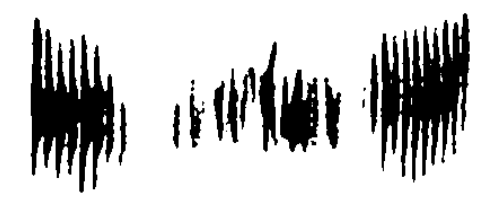

Ulll!!

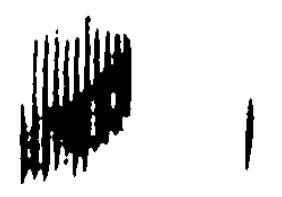

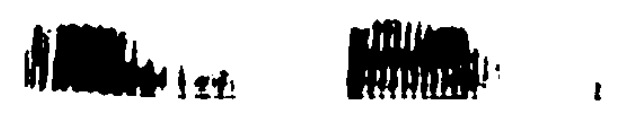

[c'frkt]

['bravo] 
Sonagramme des deutschen [j] und des bulgarischen [1]
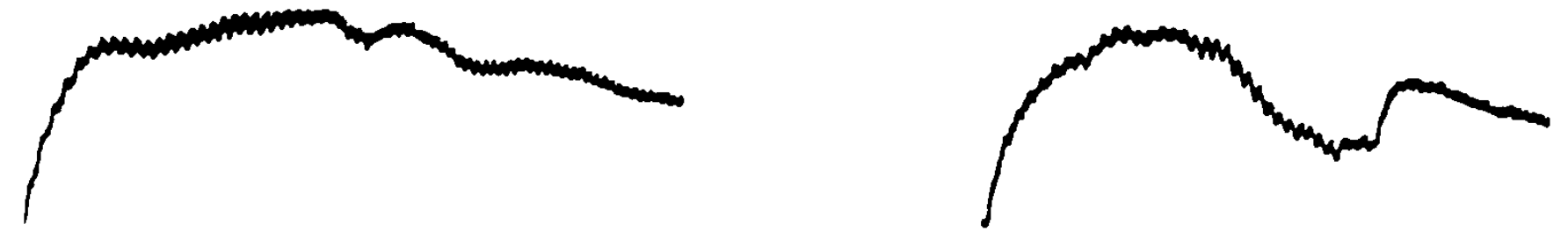

1119
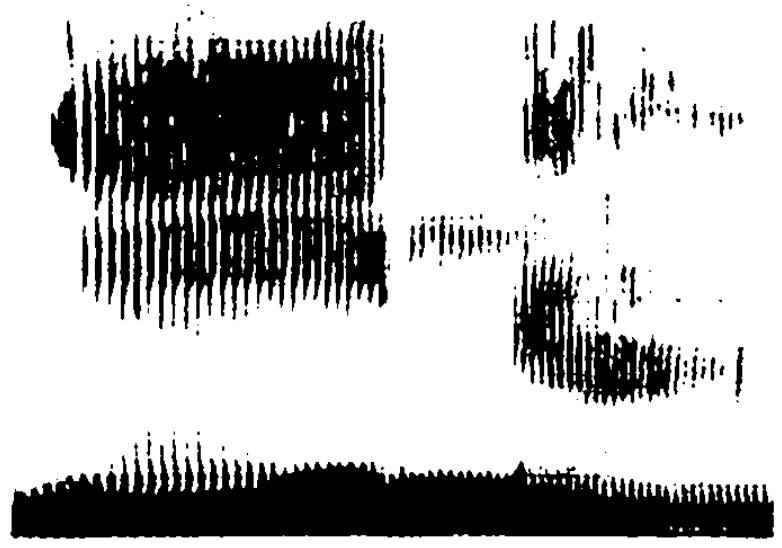

['jena]

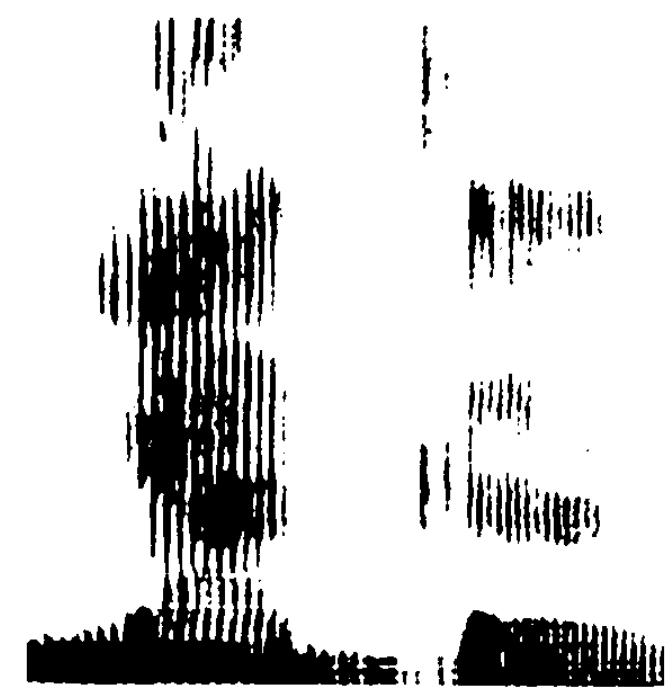

['jakə]
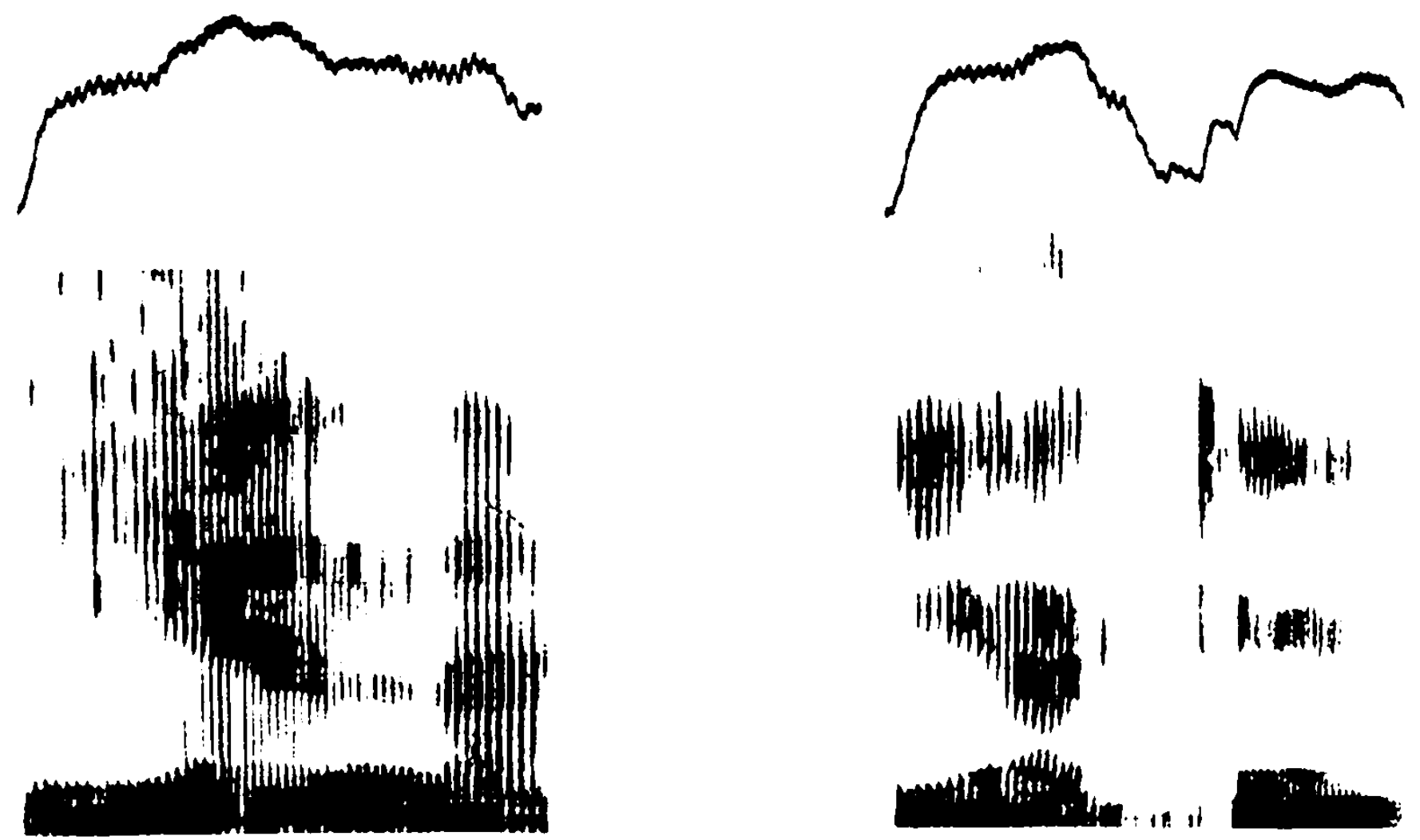

if

$$
\text { ['!Ena] }
$$
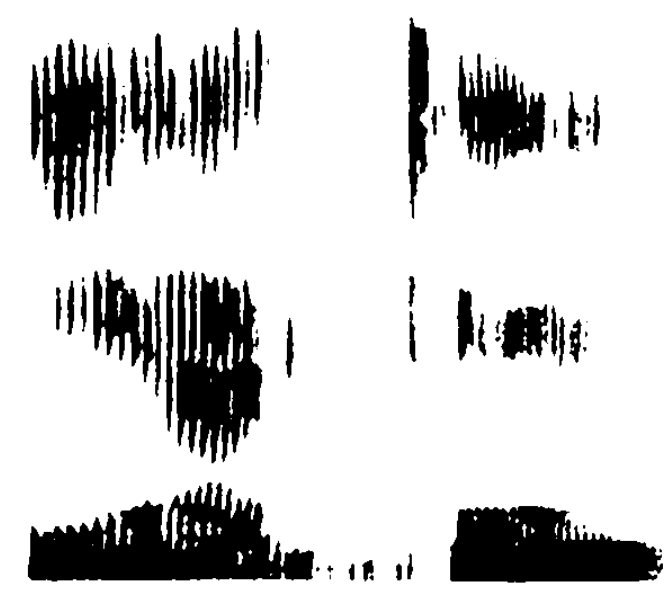

['lake] 

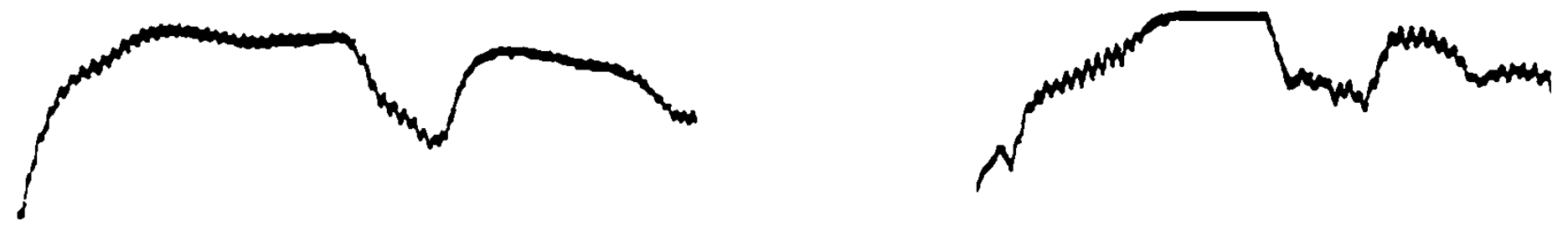

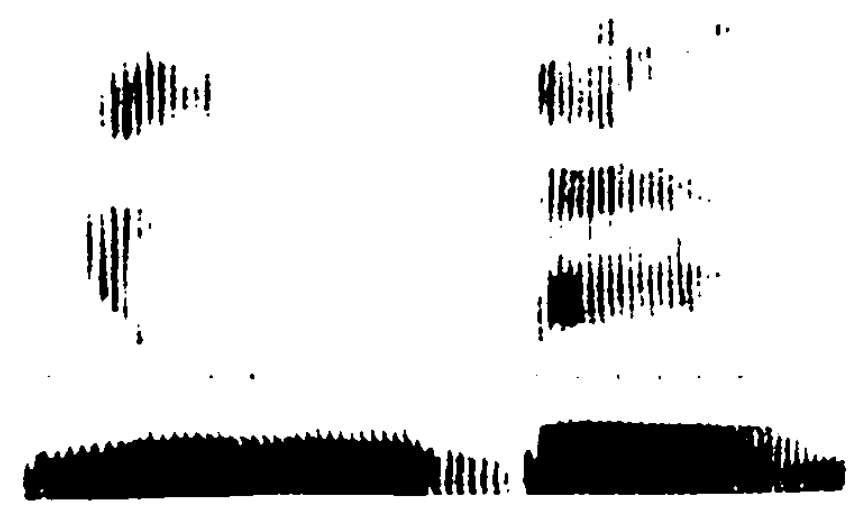

d. ['ju : bal]

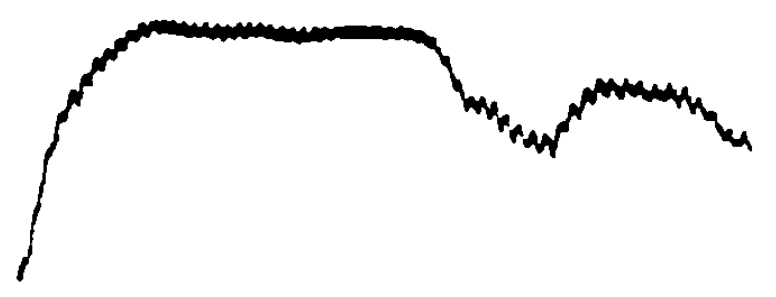

1

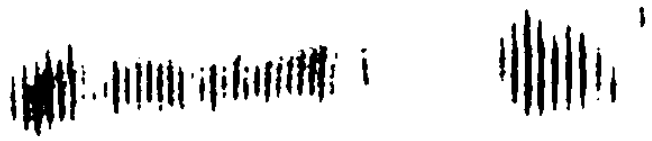
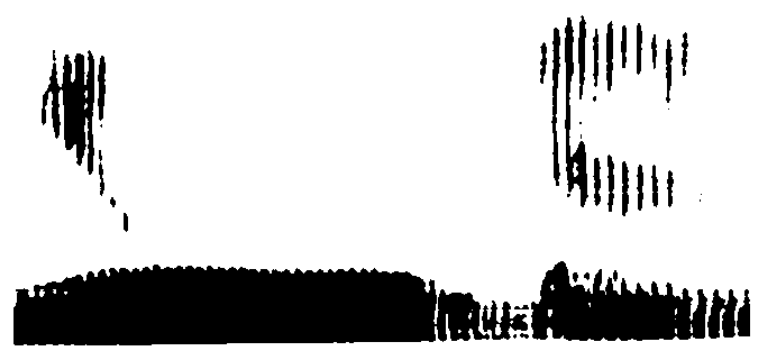

d. ['jo : ga']

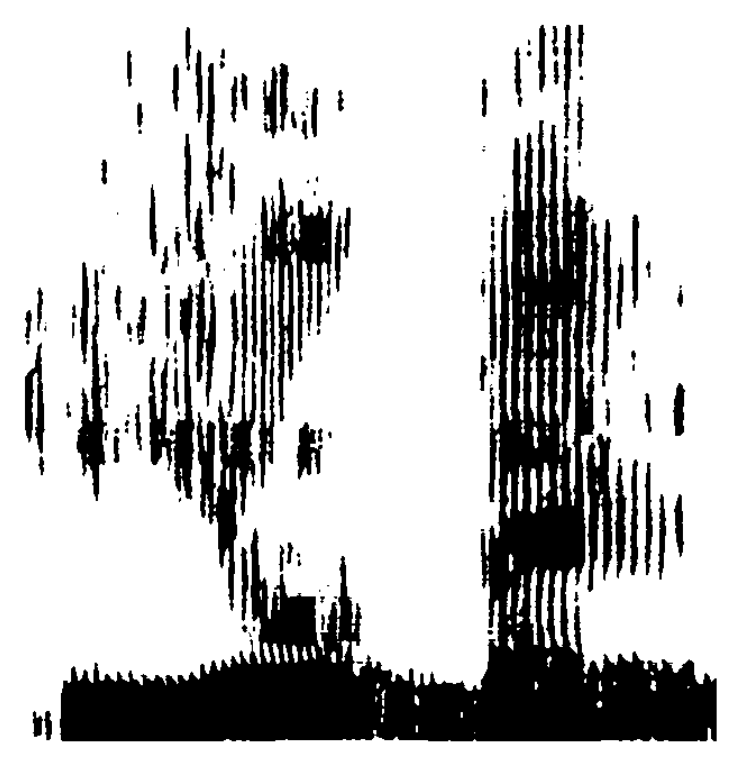

b. ['iv' bal]
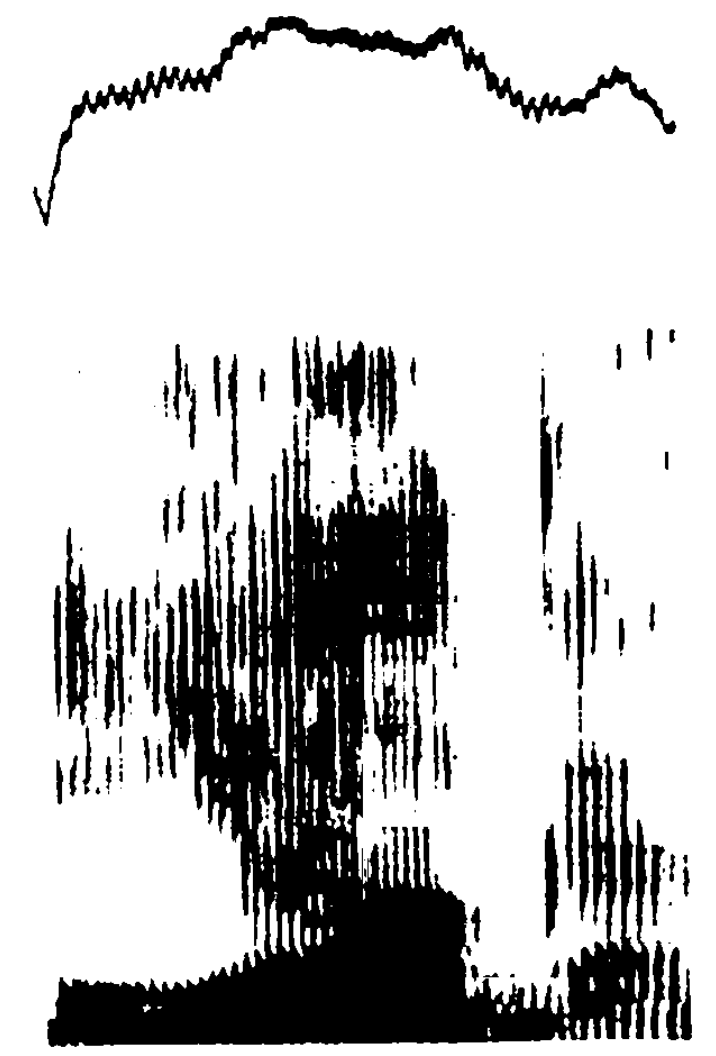

b. ['Ioga ] 


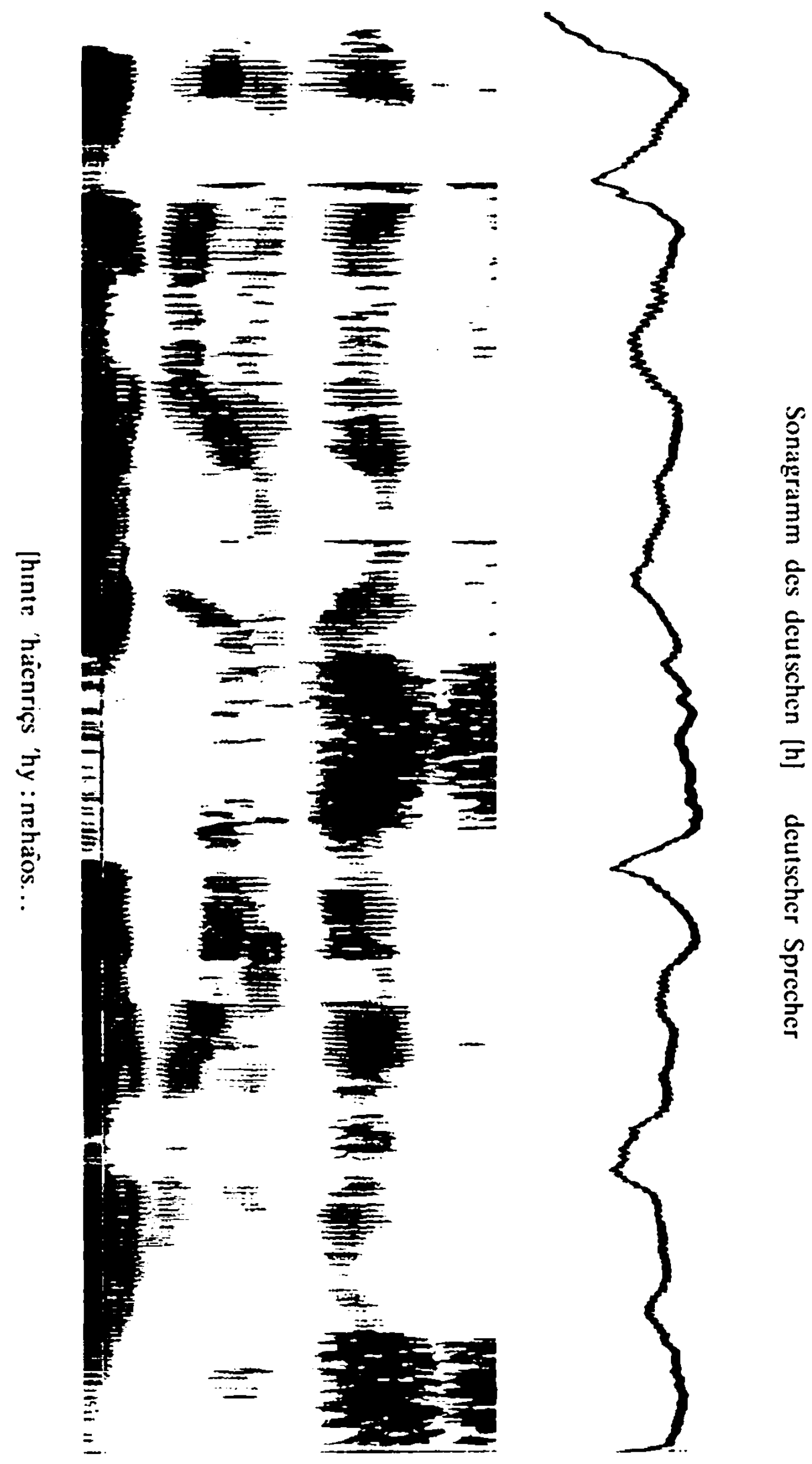




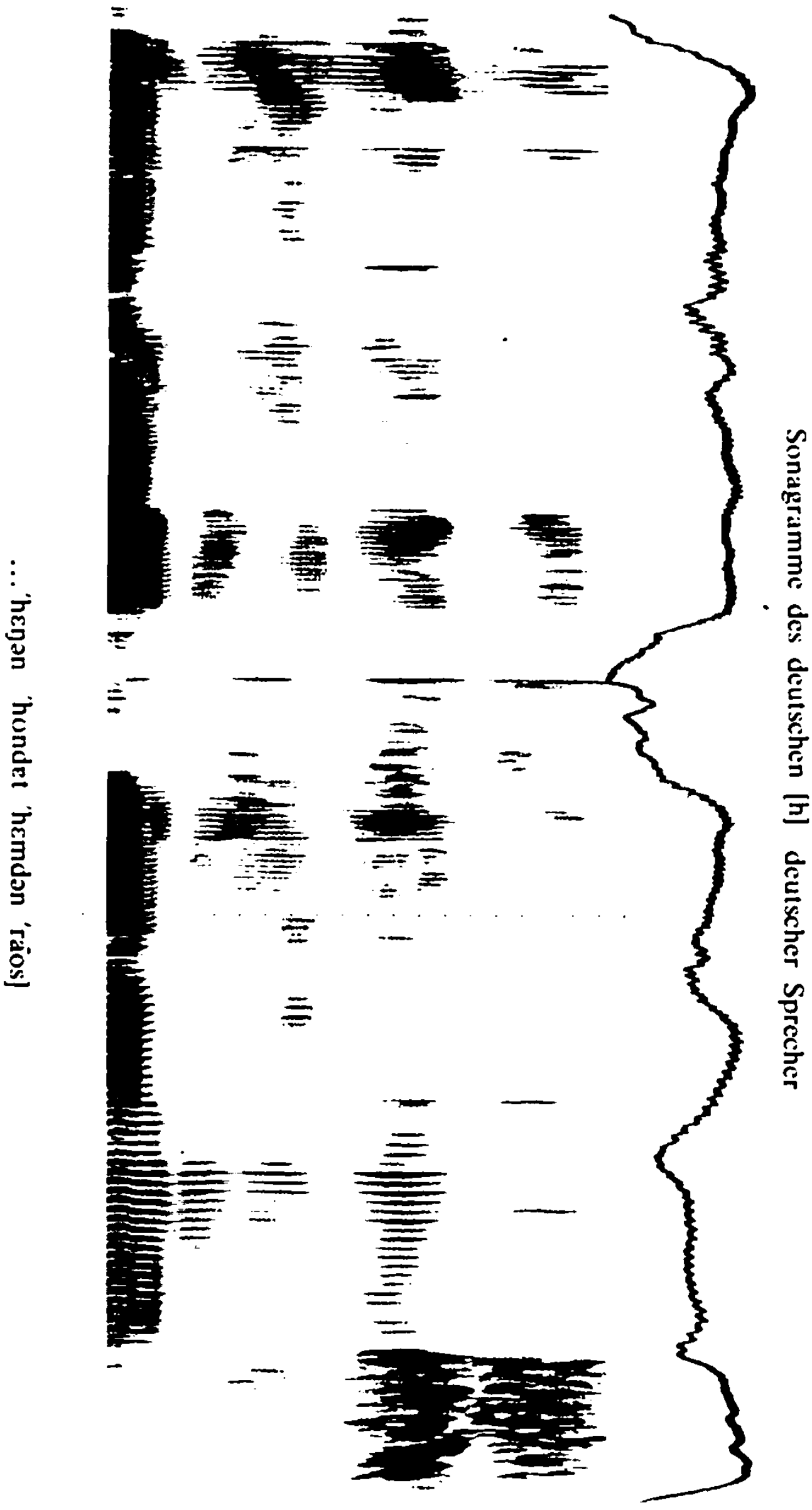



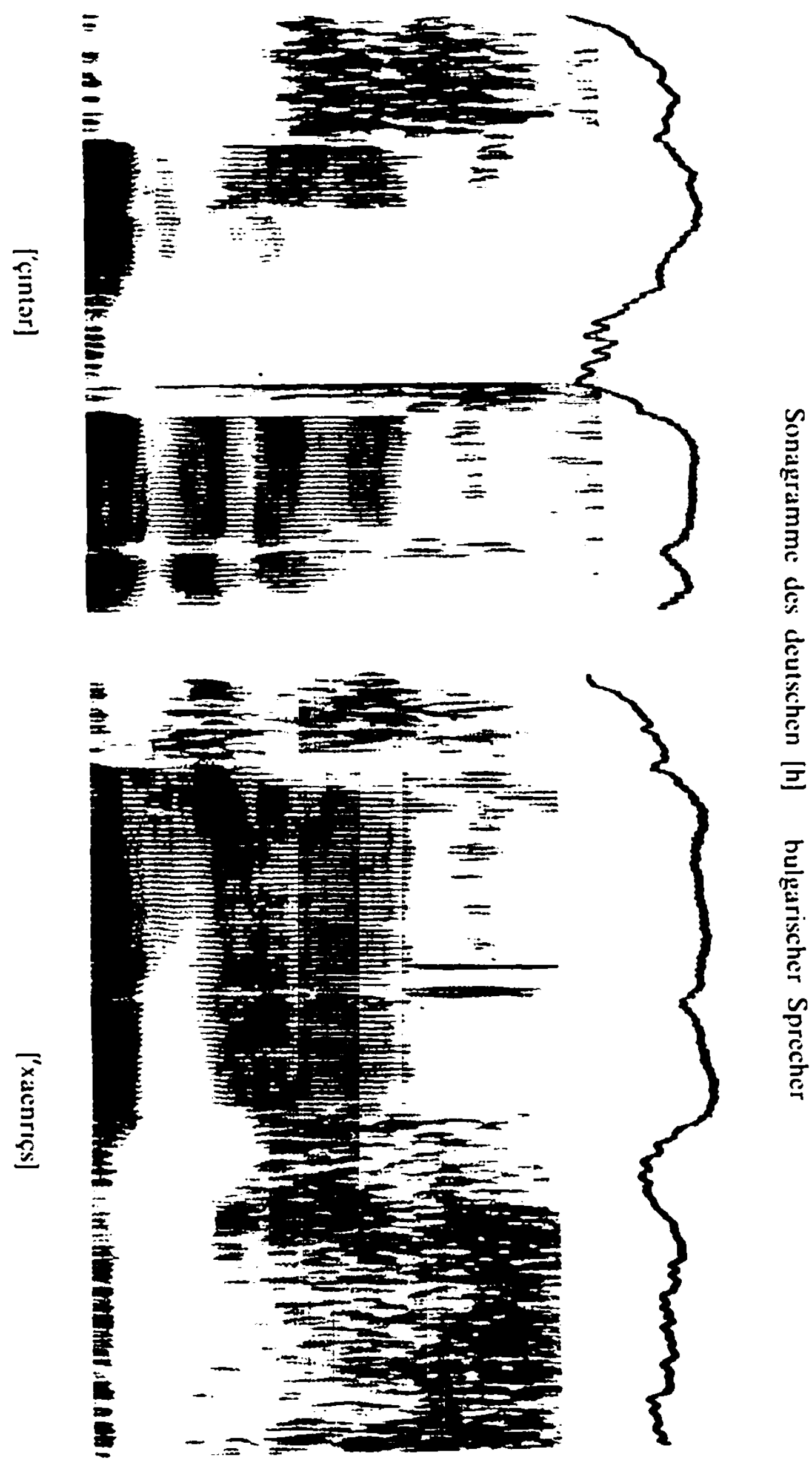

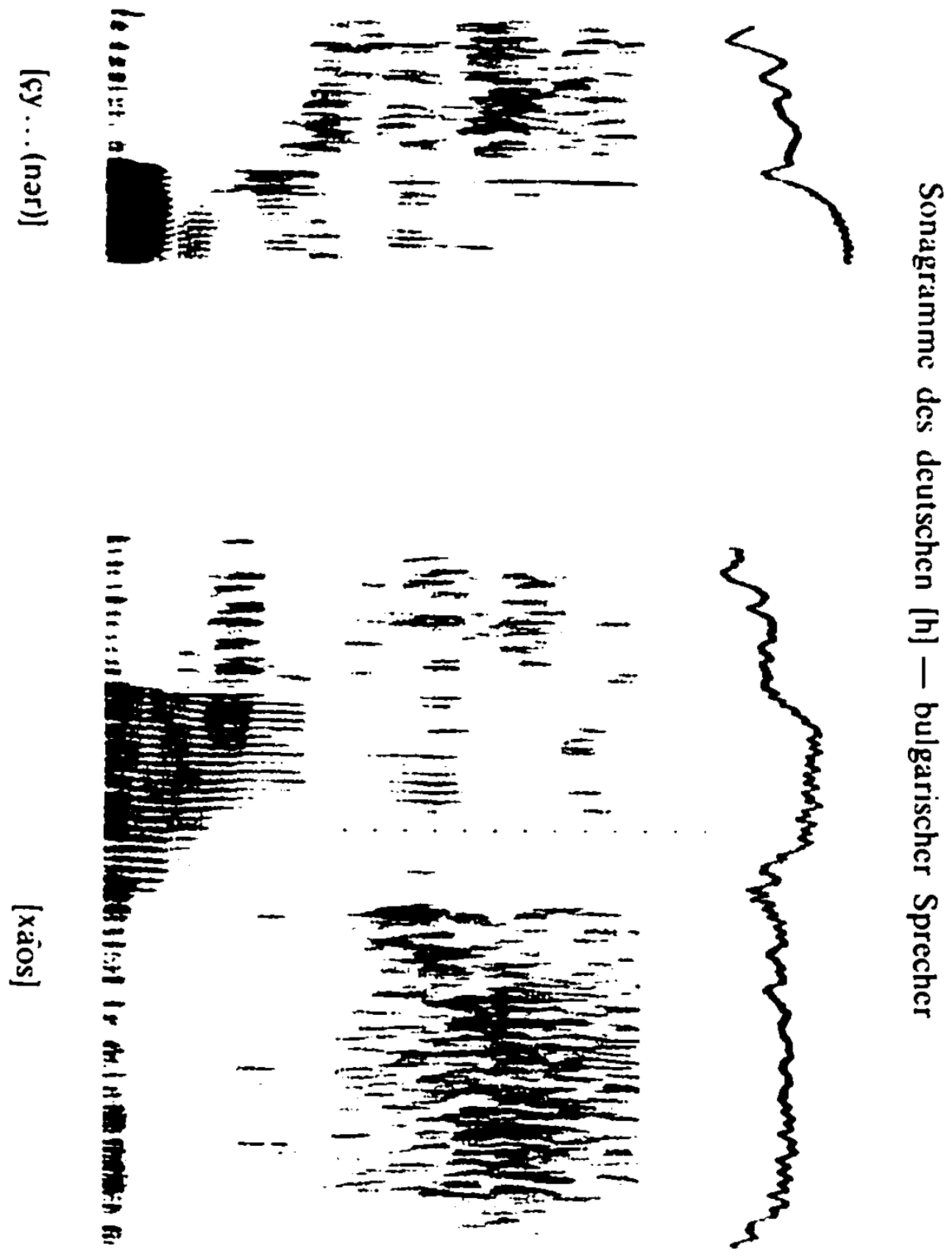

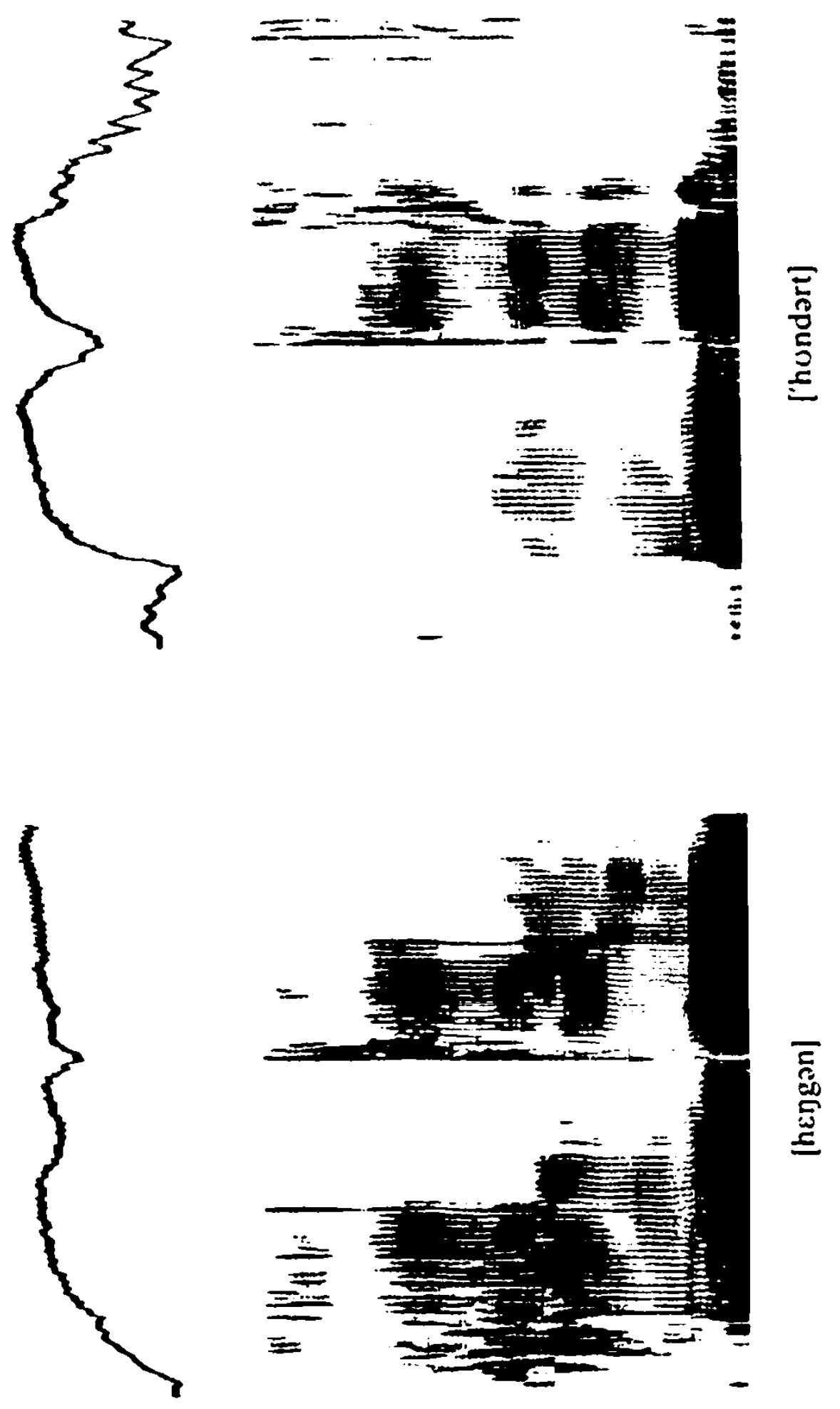


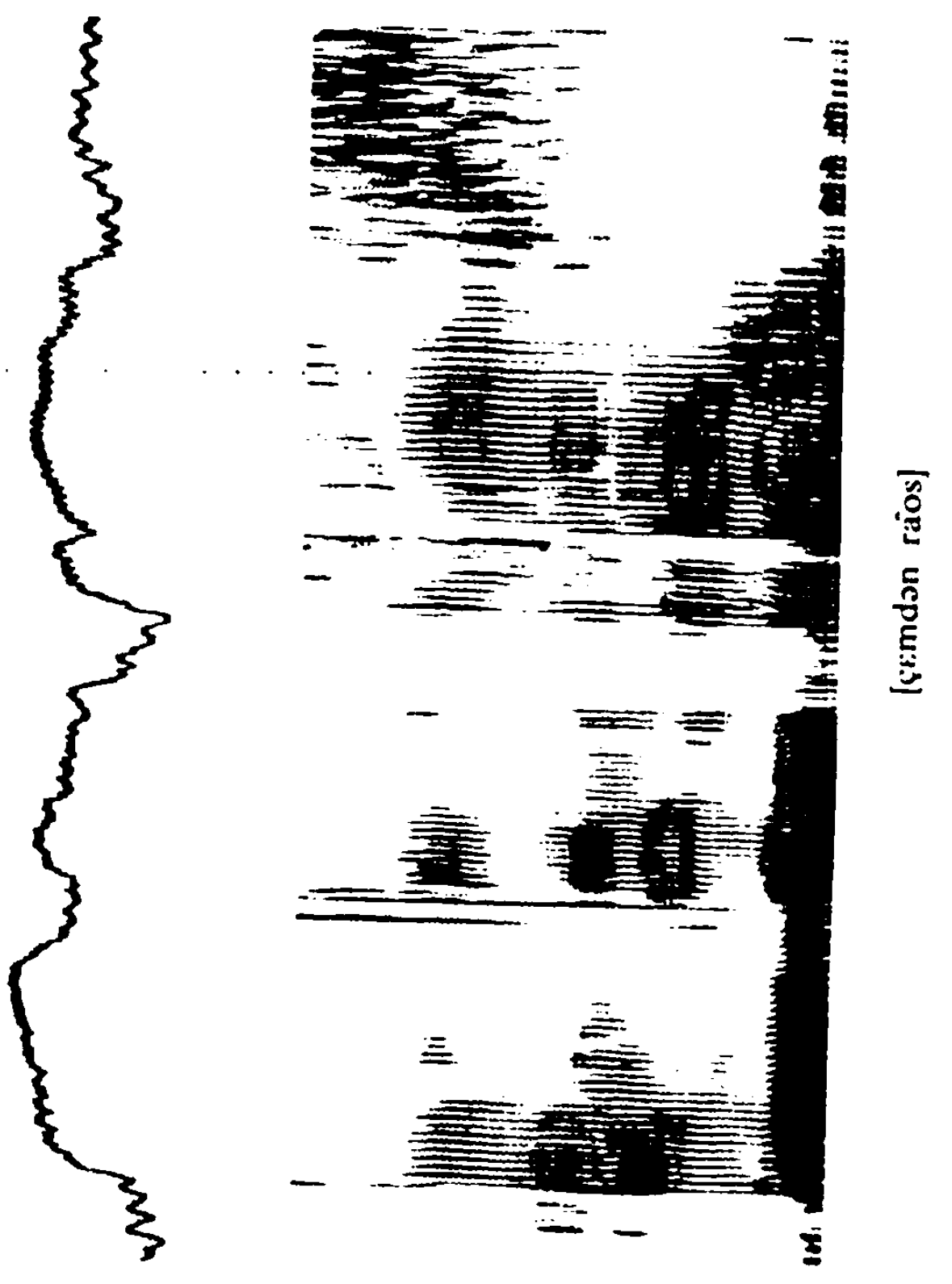


Sonagramme des deutschen [c] und des bulgarischen [c]

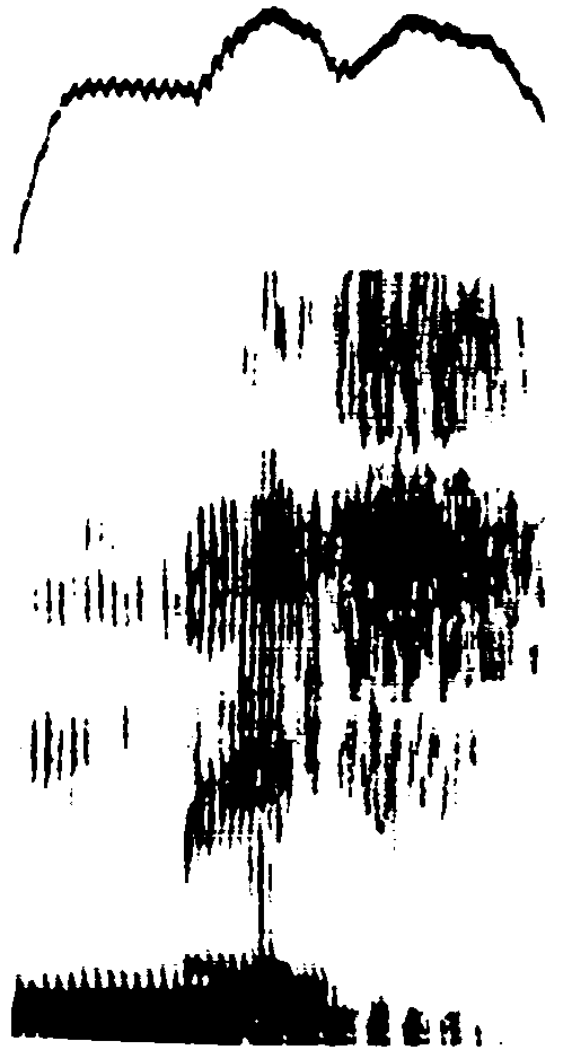

d. [mıç]

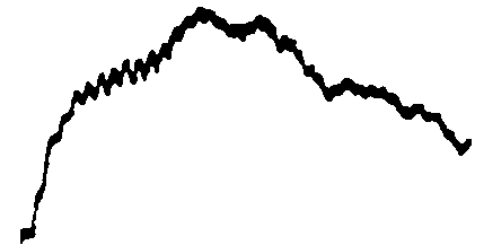

$1^{\prime}$

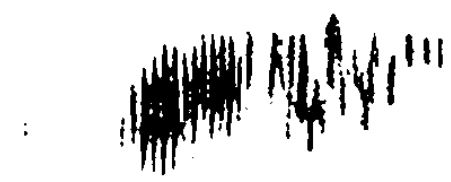

lippintion 1

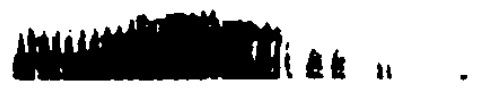

b. [mıç]

Sonagramme des deutschen $[x]$ und des bulgarischen $[x]$

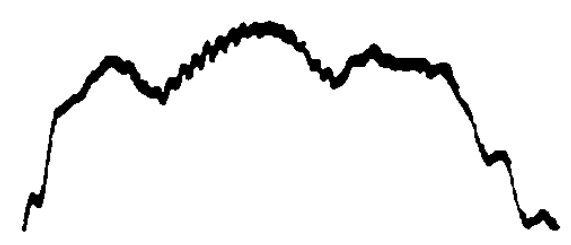

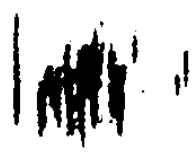
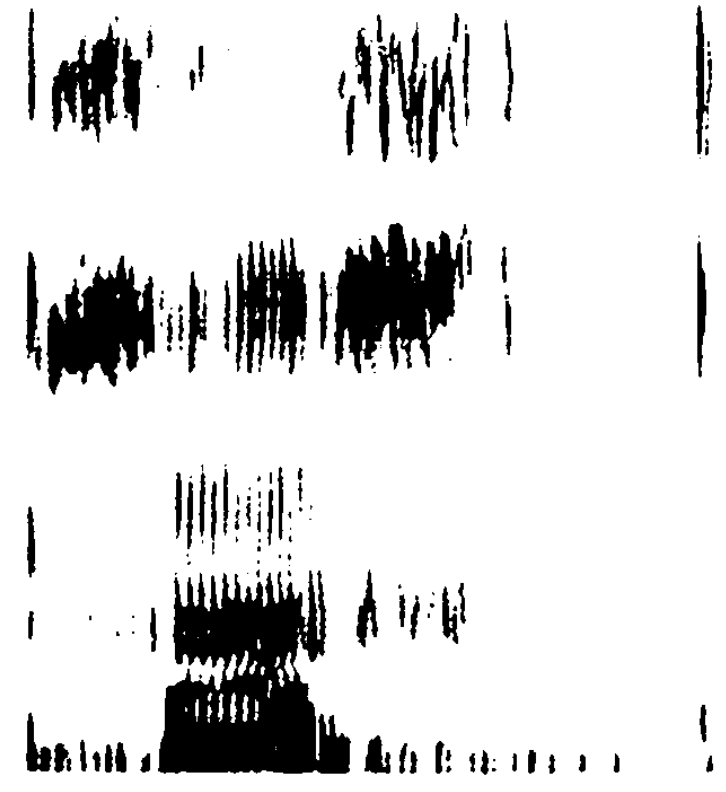

d. [praxt]
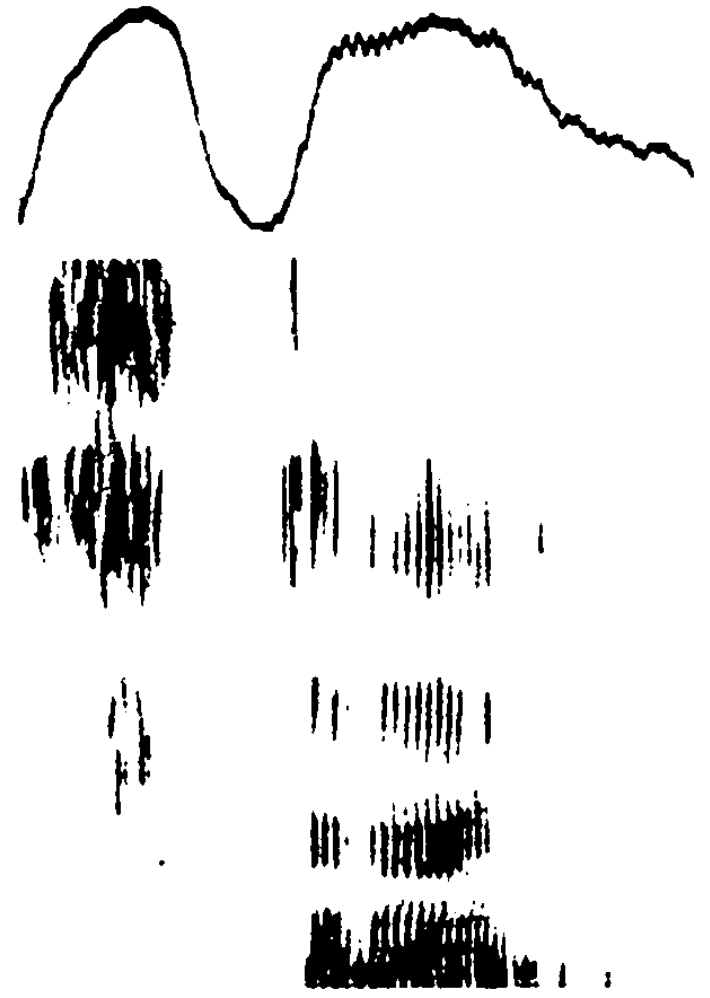

b. $[$ strax $]$ 
Das deutsche [h] in intervokalischer Position:

Sonagramm des Wortes ["a: horn] in dem Ausspruch:

Der Ahorn ist cine Baumart.

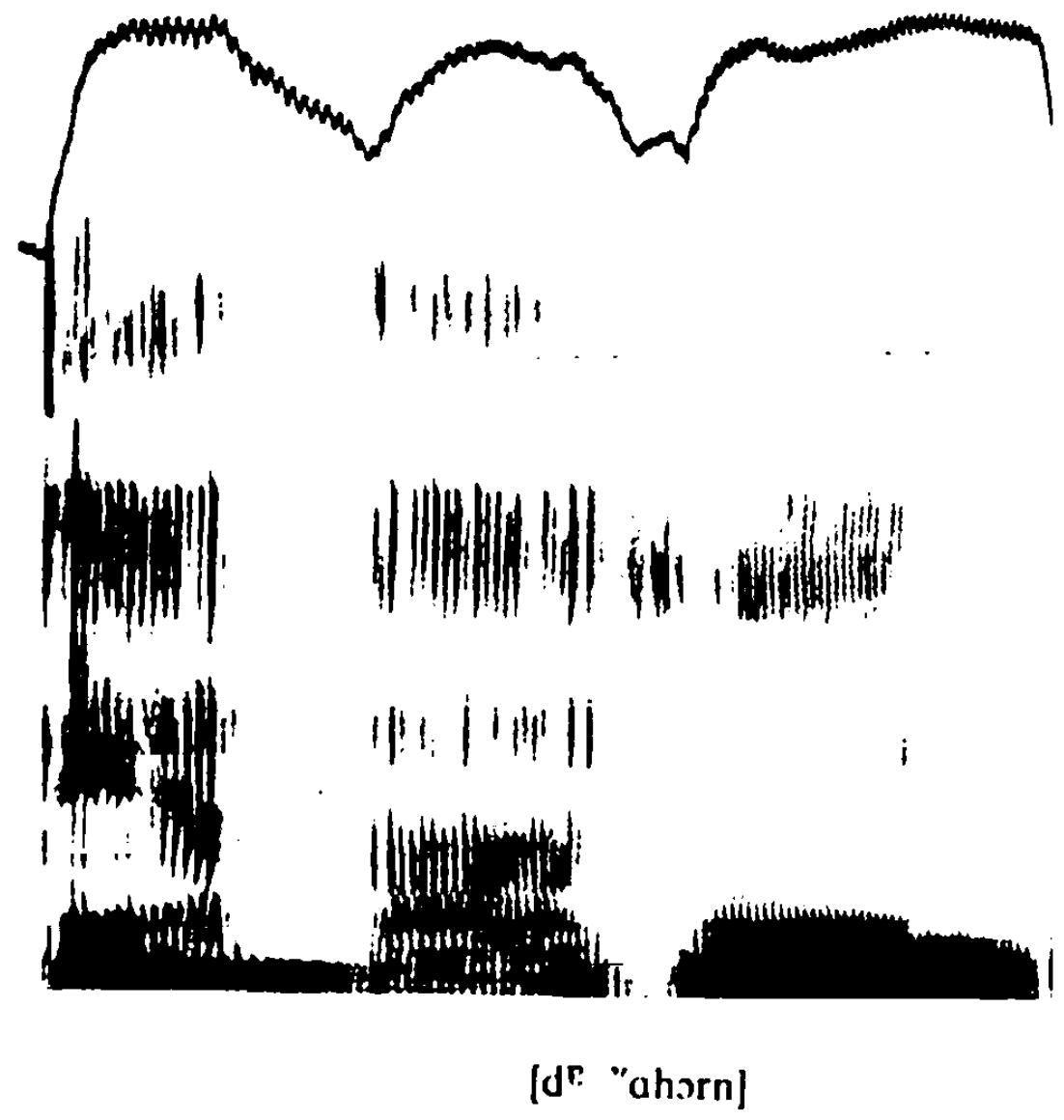


Phonostilistisch bedingte stimmlose und stimmhafte Realisierung des bulgarischen $/ x$ in Position zwischen Vokalen
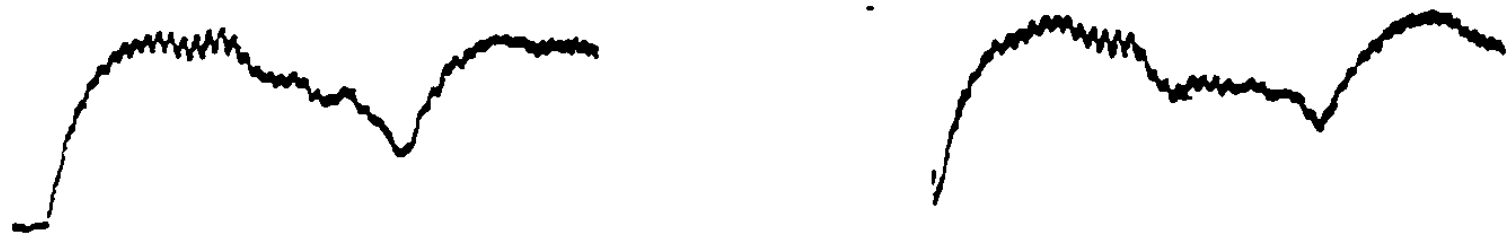

fi.

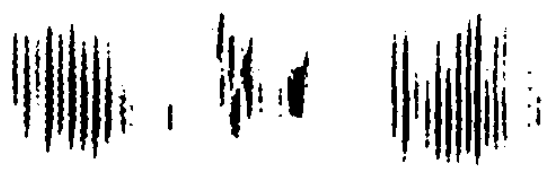

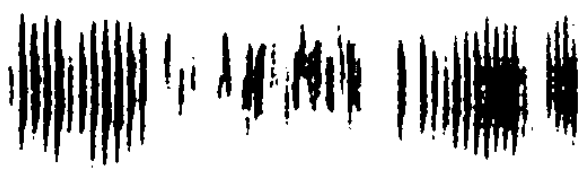

\|ning!

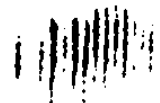

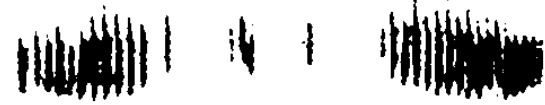

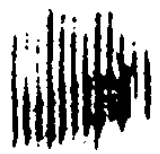

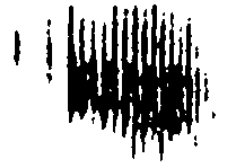

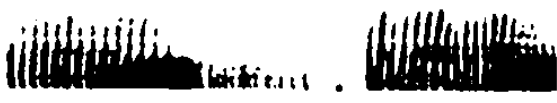

[a'xa]
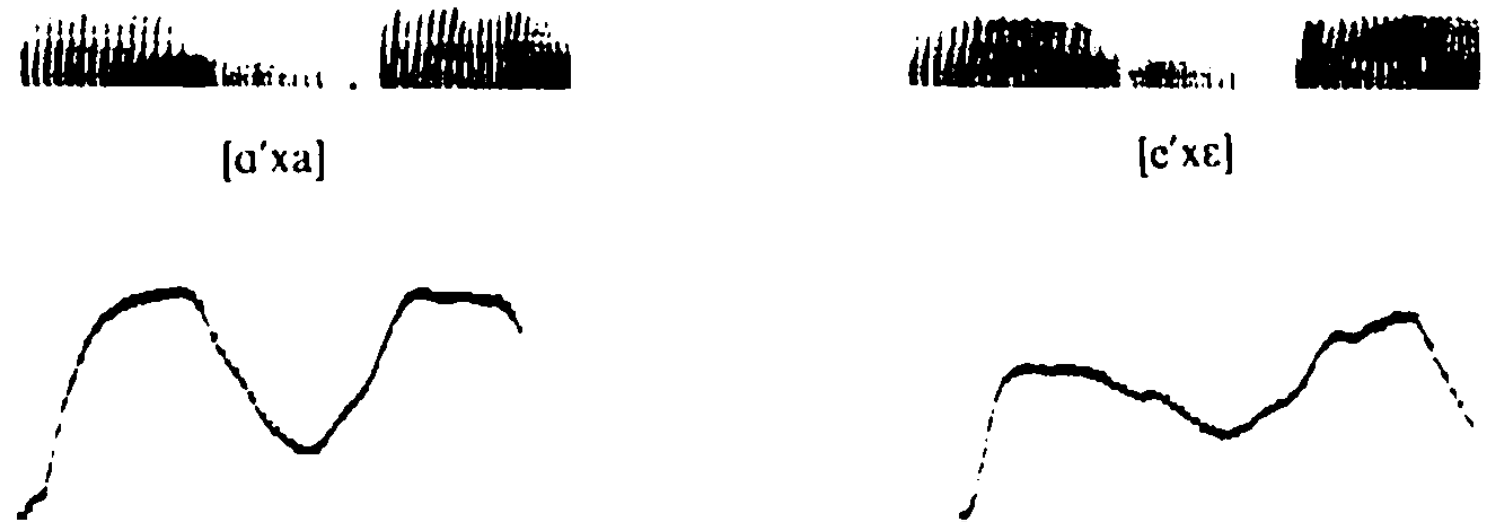

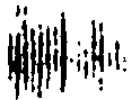

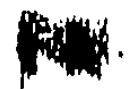

H⿻
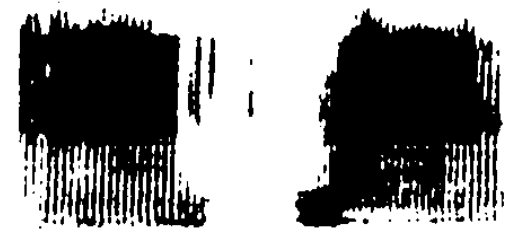

[u'xa]

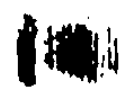

|

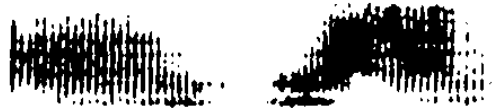

$\left[\mathrm{c}^{\prime} \times \varepsilon\right]$ 
d. $[\mathrm{p} f]-[\mathrm{pl}] ;[\mathrm{pr}]-[\mathrm{kr}]$ im Anlaut.

Die drei Bildungsphasen von [p, t. $k]$ in $[\mathrm{pl}]$, [pr], [kr] sind klar zu beobachten. Dagegen ist die Bildung der Affrikate [pf] einheitlich, mit nur einer Bildungsphase fur das [p].

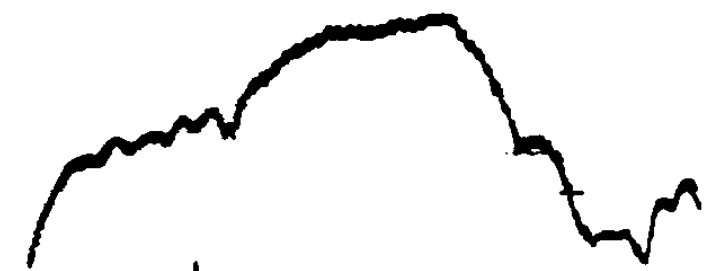

11

iffyy<smiles>[Al]=C(I)I</smiles>

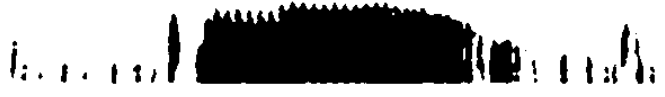

[phlu :k]
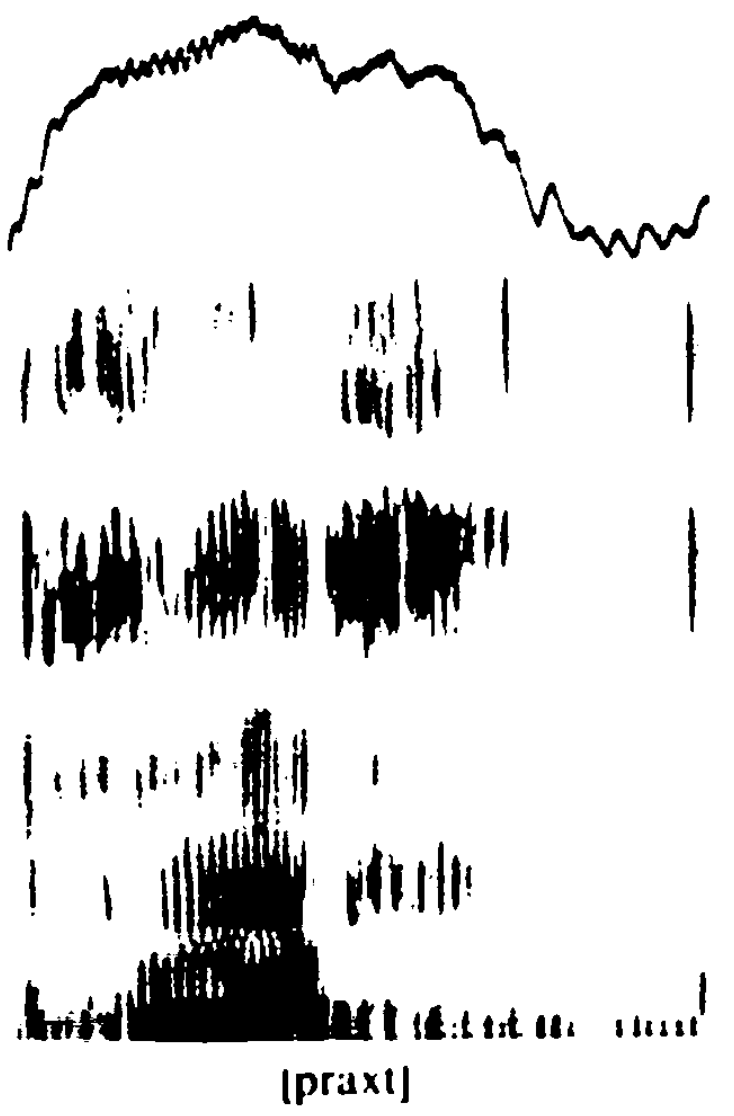

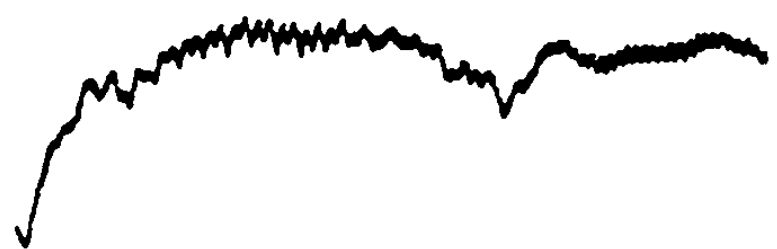

il 1111111
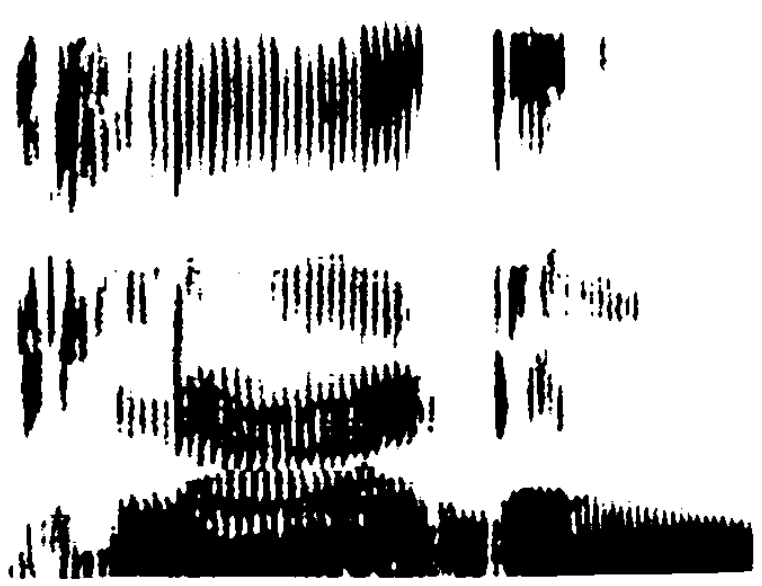

['klo : gən]
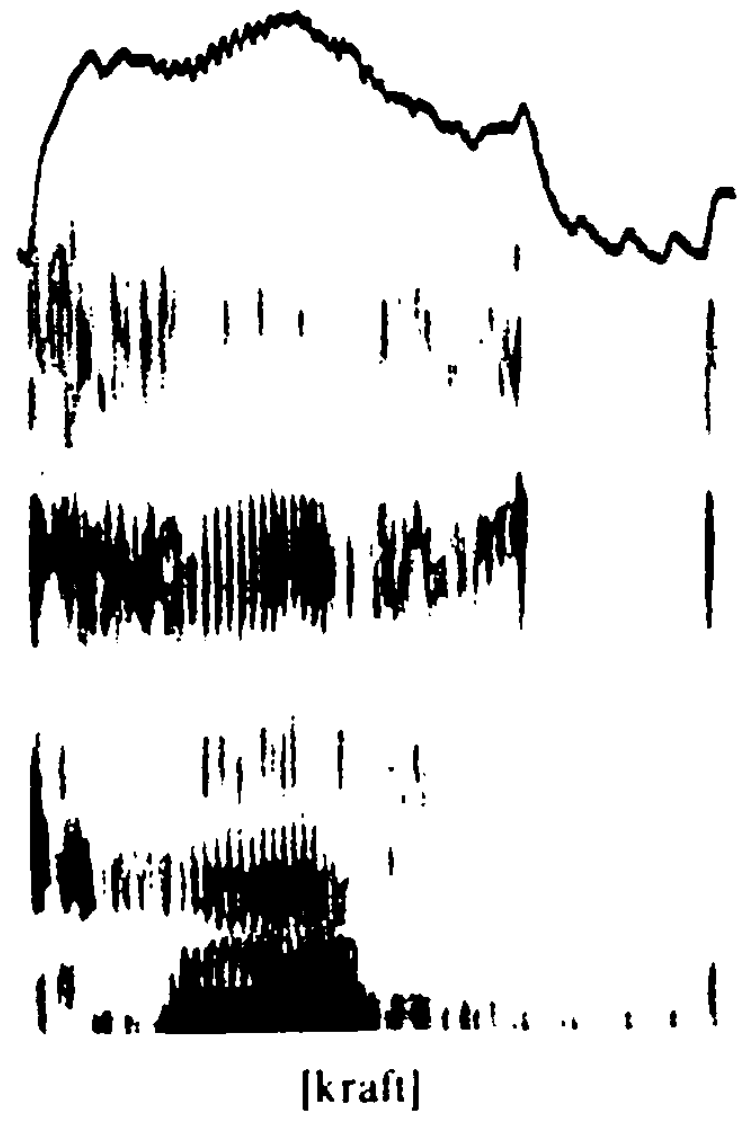
H. $[p]]-[f]-[p s]$ im Inlaut.
Hieer ist die cinheitliche Bildung der Afrikate [pf] gegenüber [ps] deutlicher als im Anlaut.

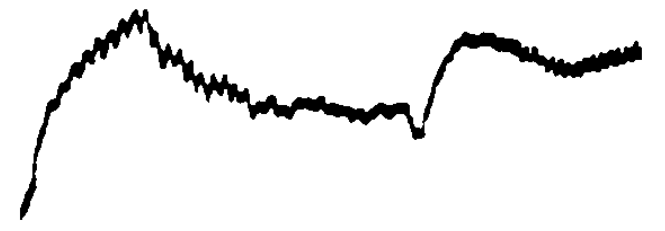

,

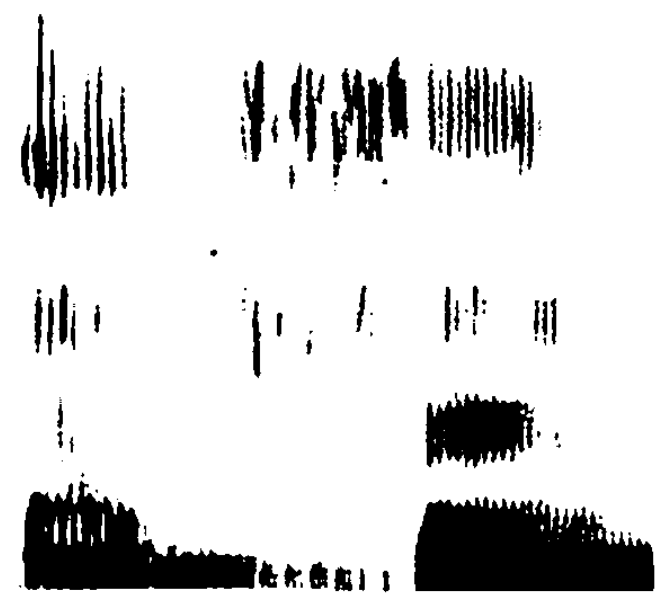

["opfr.]

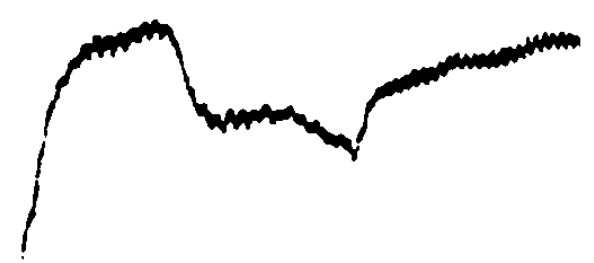

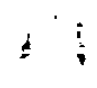

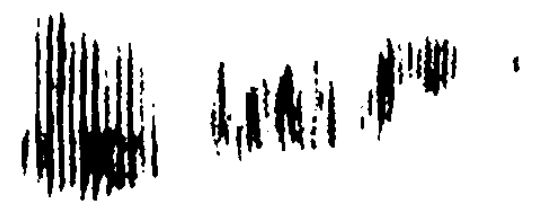

\|lillili!' : : :l

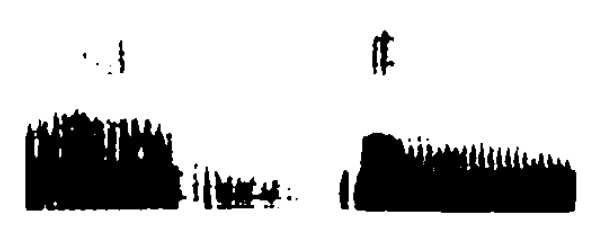

["sfan]

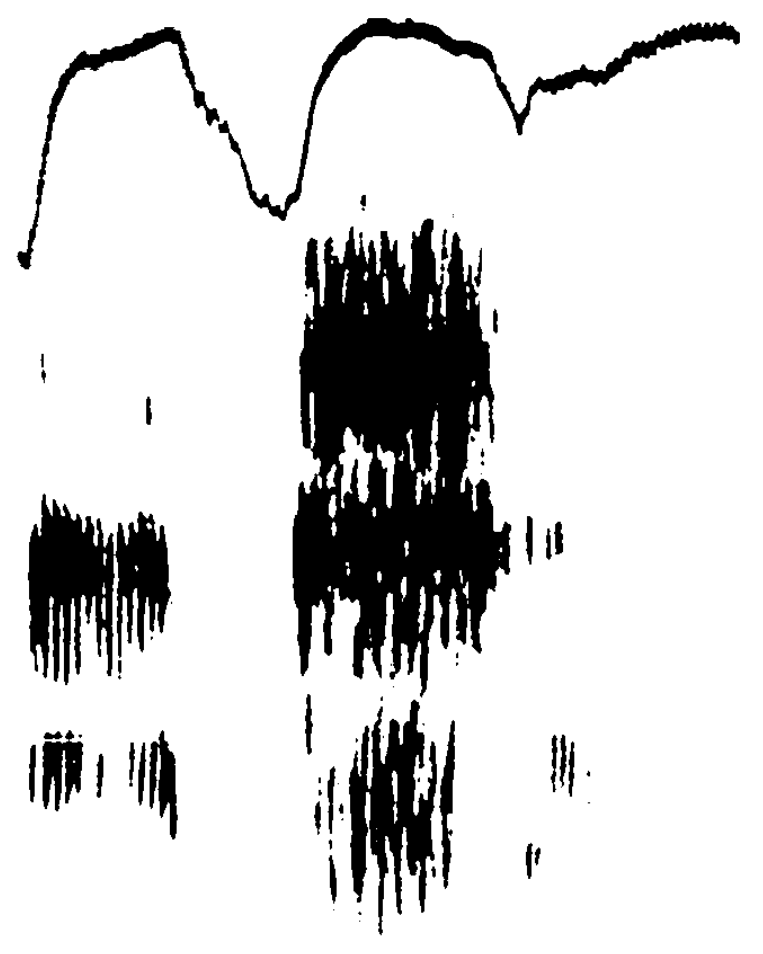

mumming ate of $\left.\right|_{10}$

["ıpson] 
d. $[t s]-[t r] ;[t]-[t r]$ im Anlaut mit sichtbarer cinheitlicher Bildung der Afrikatern
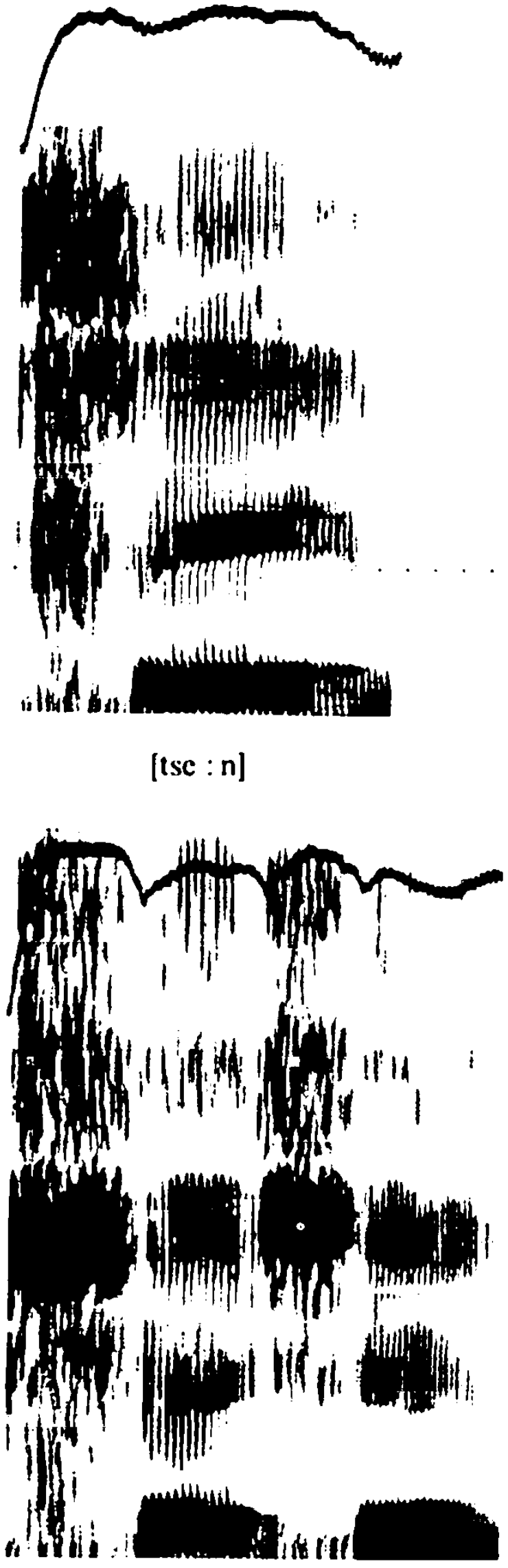

['ts\&çə]

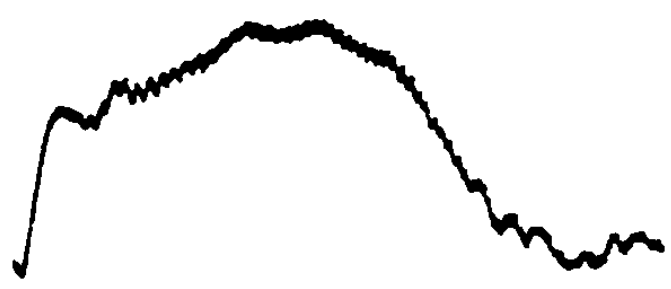

$5 \mid$ abl|
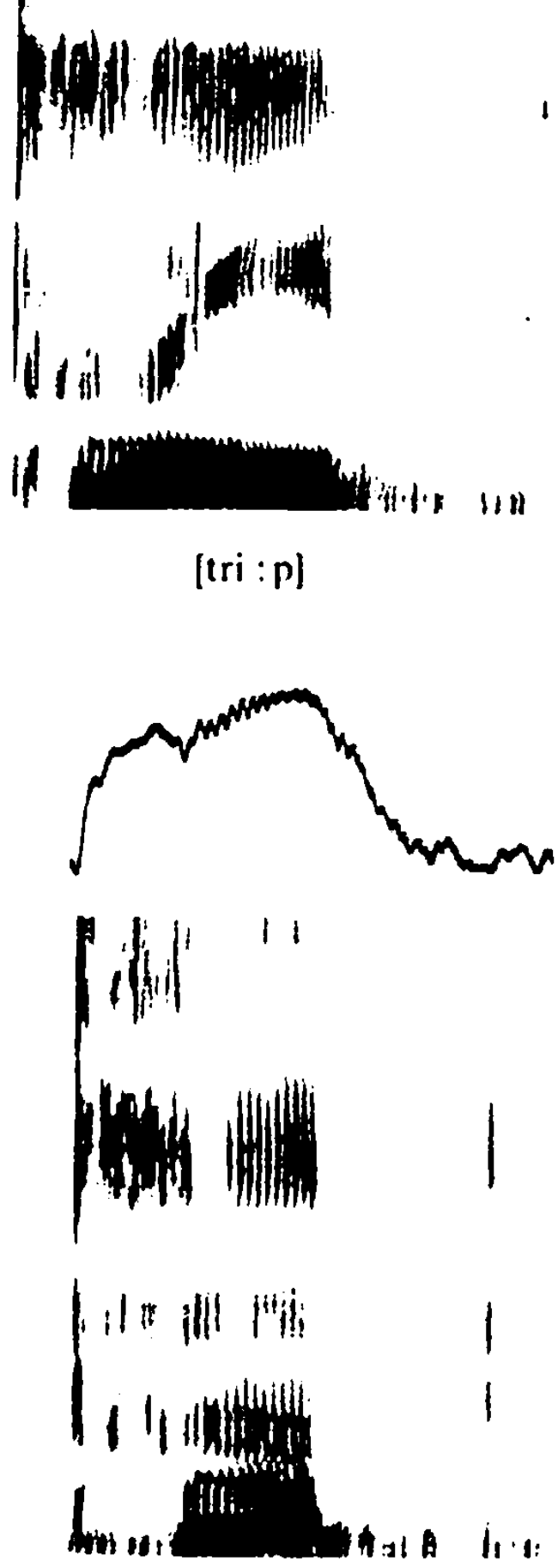

[tro:p] 
d. $|t s|-|k s|$ im Inlaul
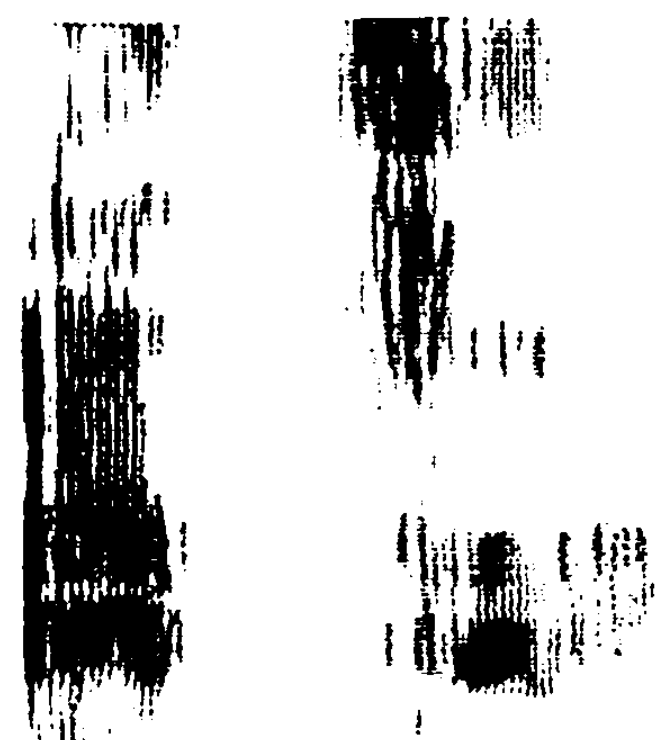

i) :

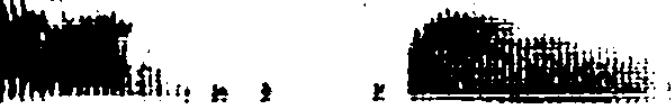

["s:tson]
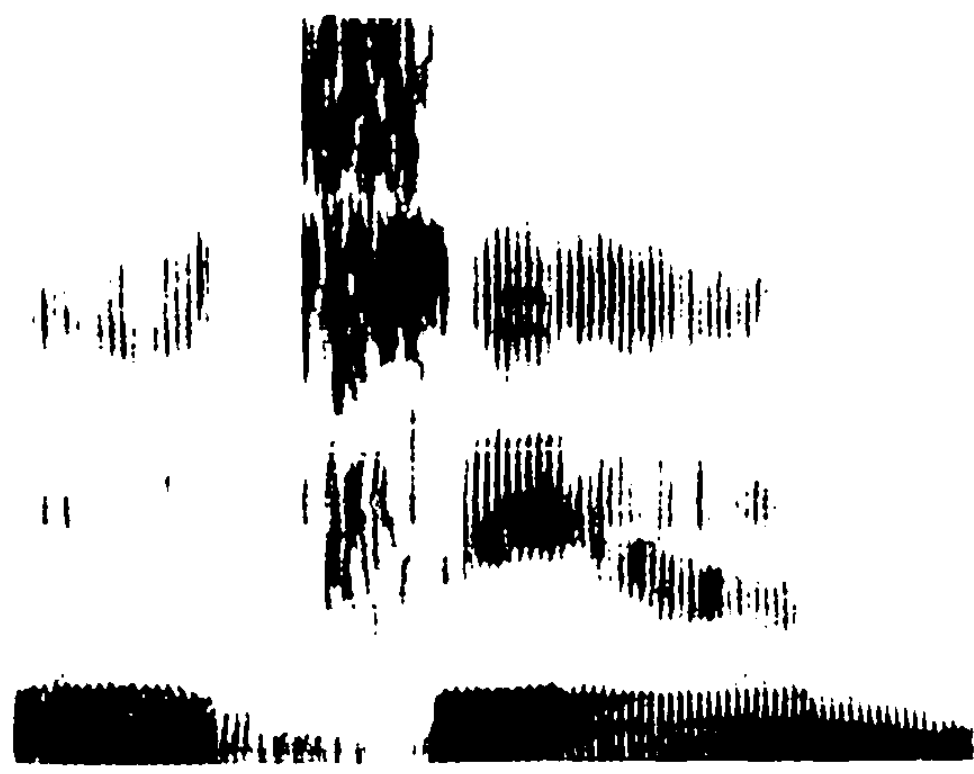

["o: tsea :n]
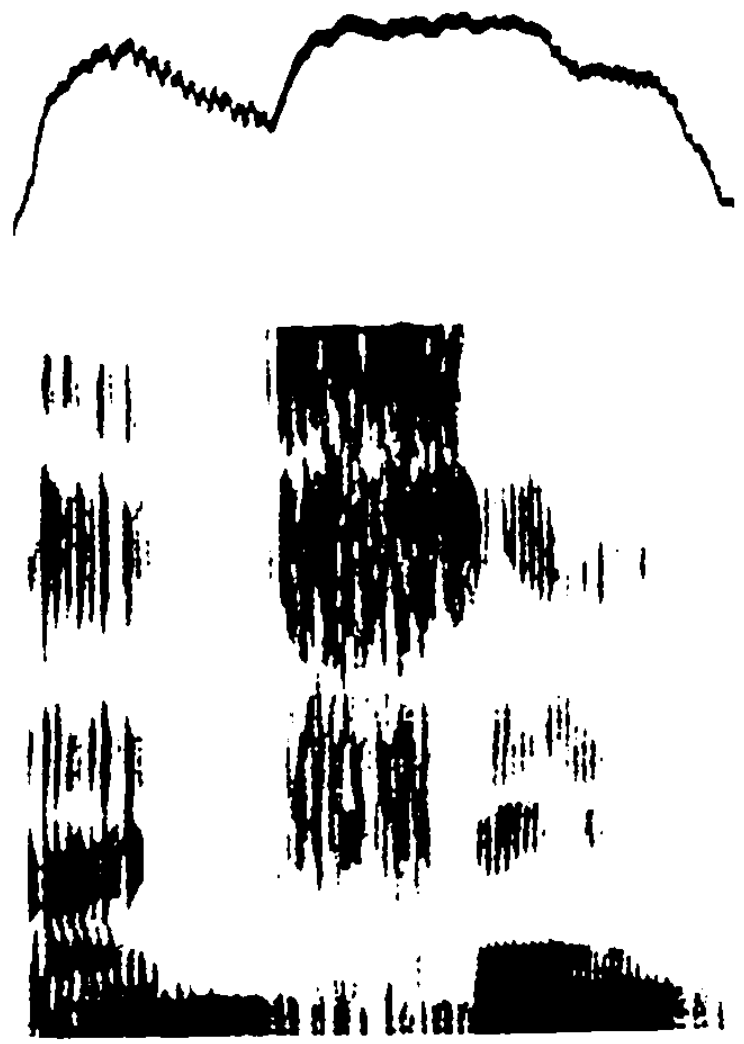

["aksa] 
d. $[p]]-[p s]$ im Anlaut
d. [pf]-[ps] im Inlaut
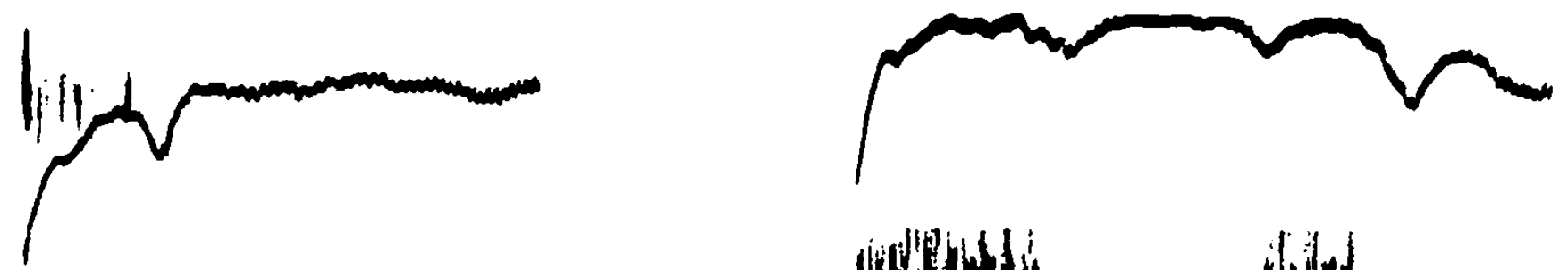

Hifl, 1,11

How

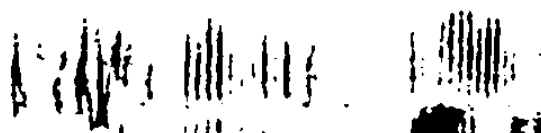

Whilling on

['prana]

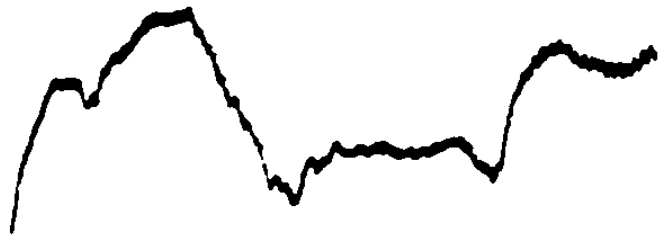

II lifh "II, "

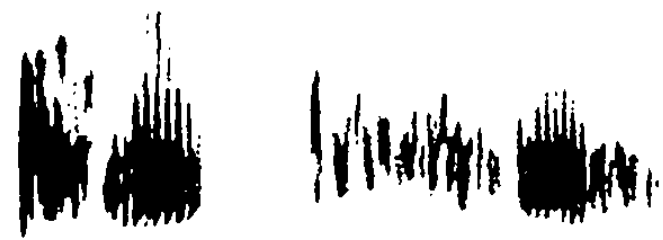

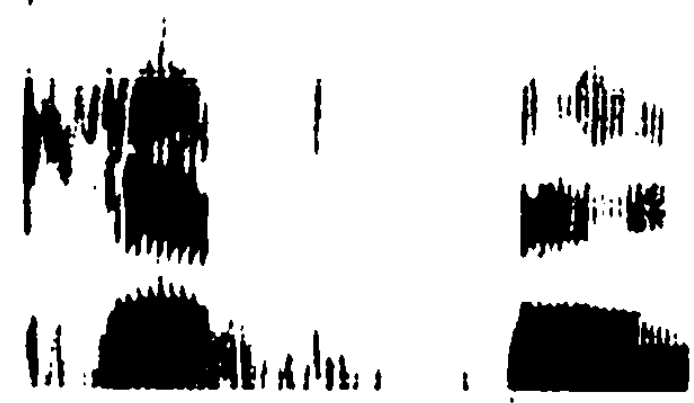

['kocplo]
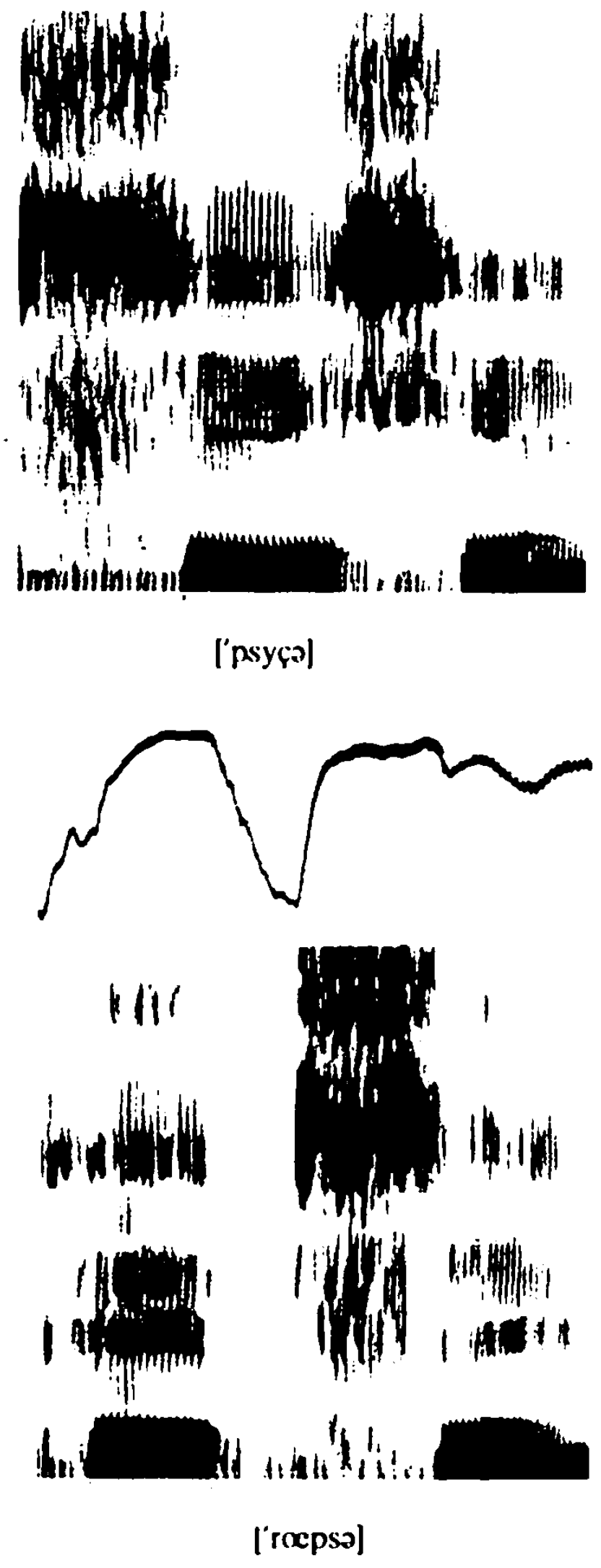
d. $[p[]-[p s]-[p s t]$ im Auslaut

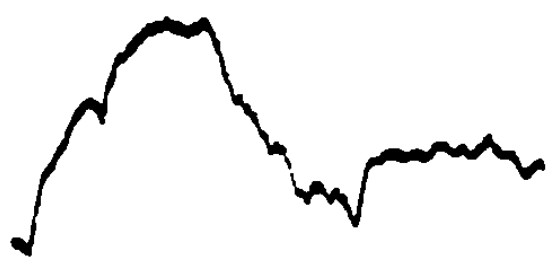

1

| inl 1
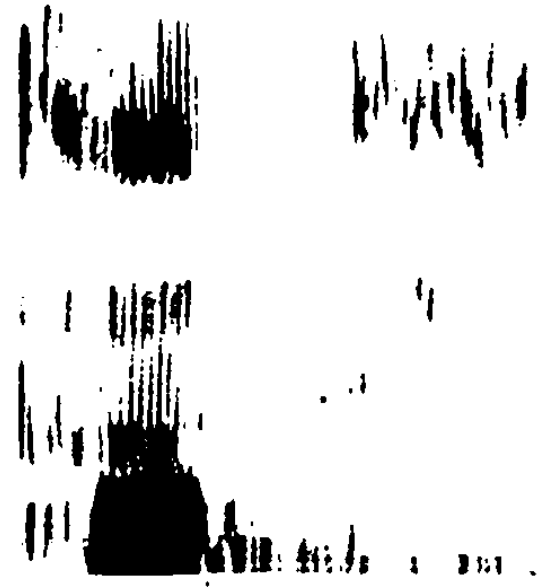

[k.oo]
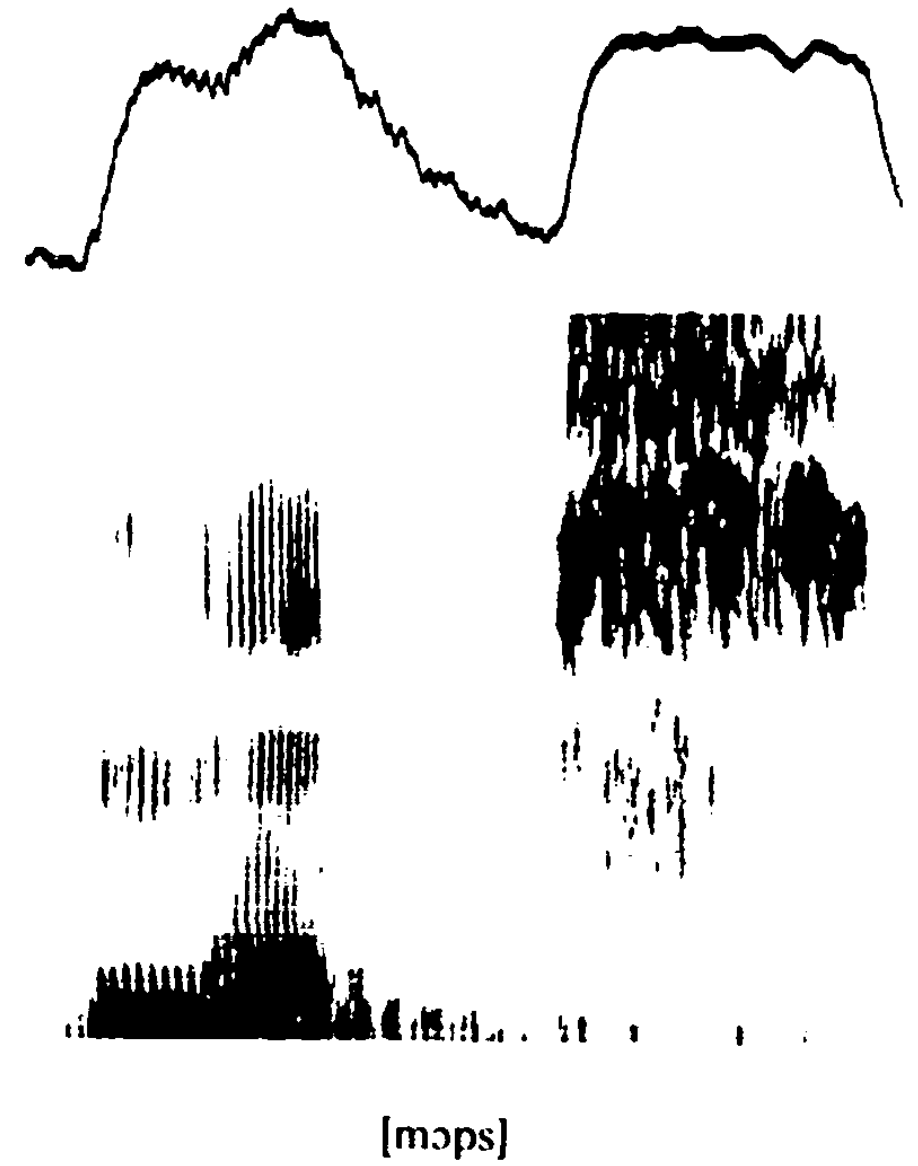
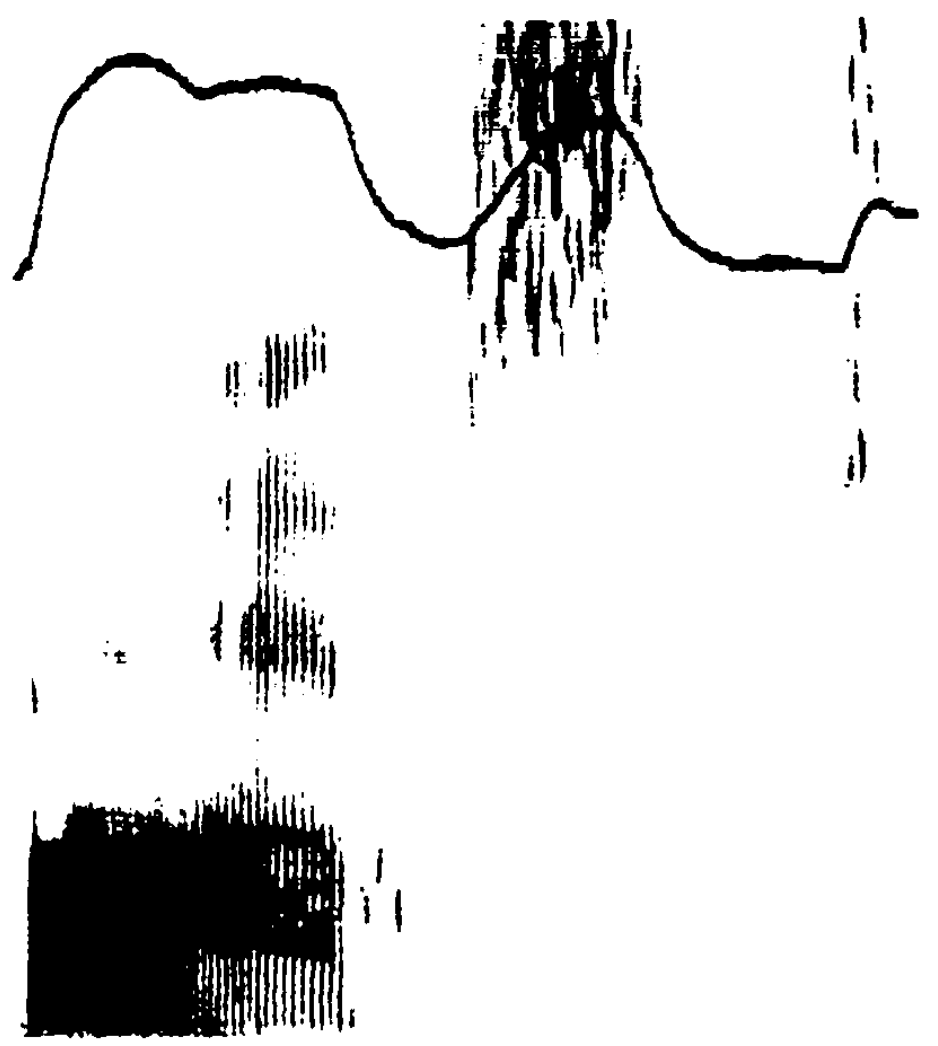

[po : pst] 
d. $[t s]-[t]]-[s]$ im Auslaut
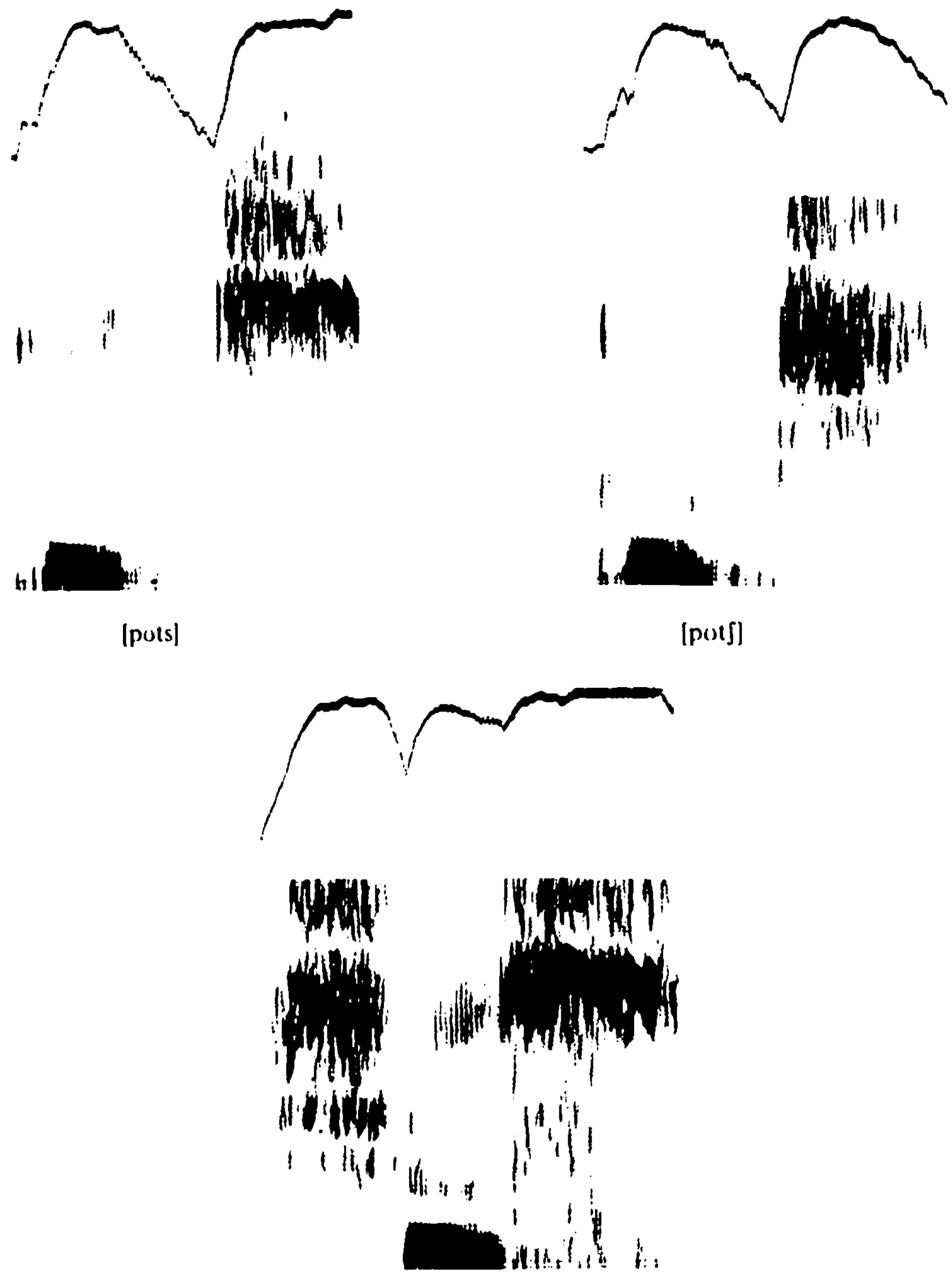

[sos] 
d. $\left[t \int\right]-\left[\int\right]-\left[k \int\right]$ im Inlaut
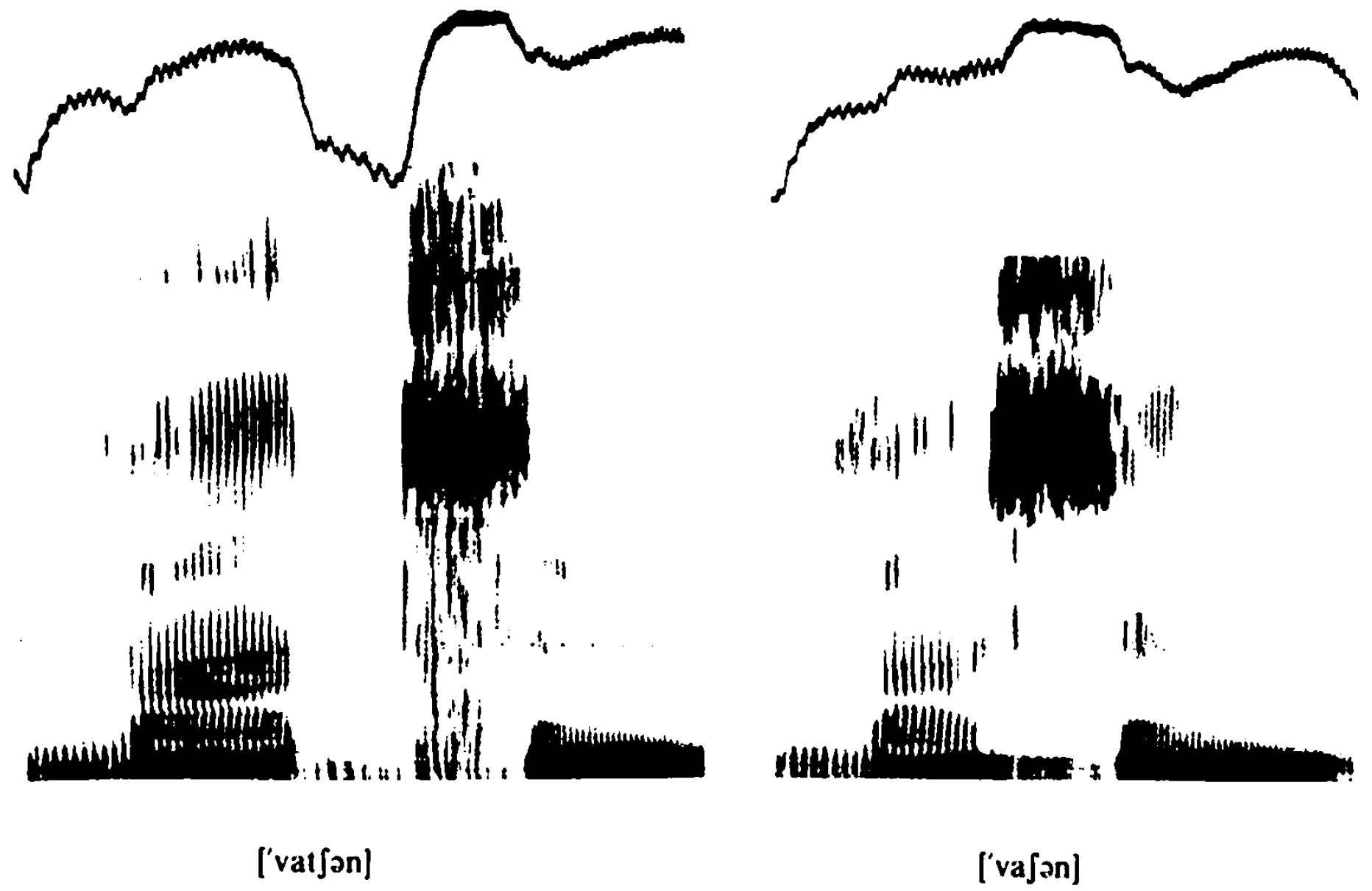

['vatjon]
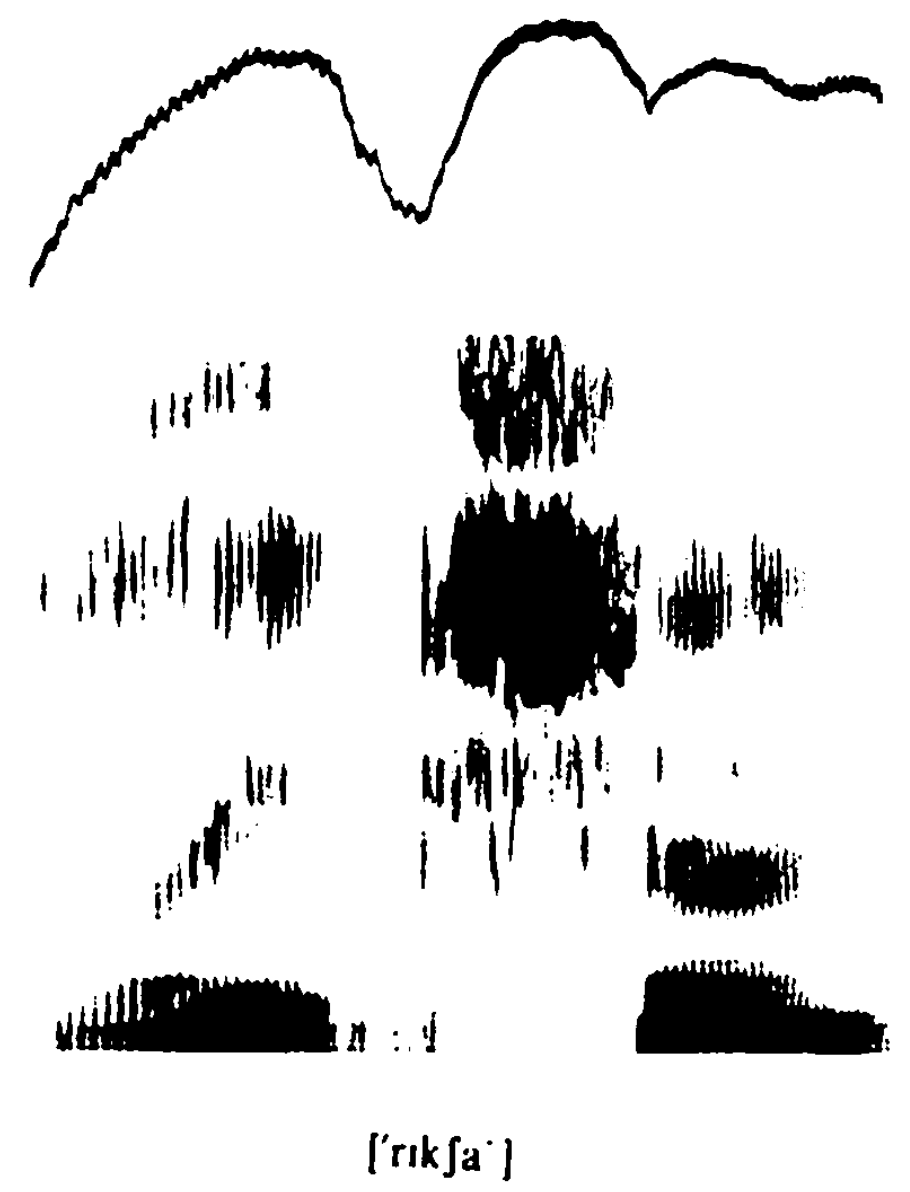
d. $[1]-\left[\int\right]$ im Auslau:
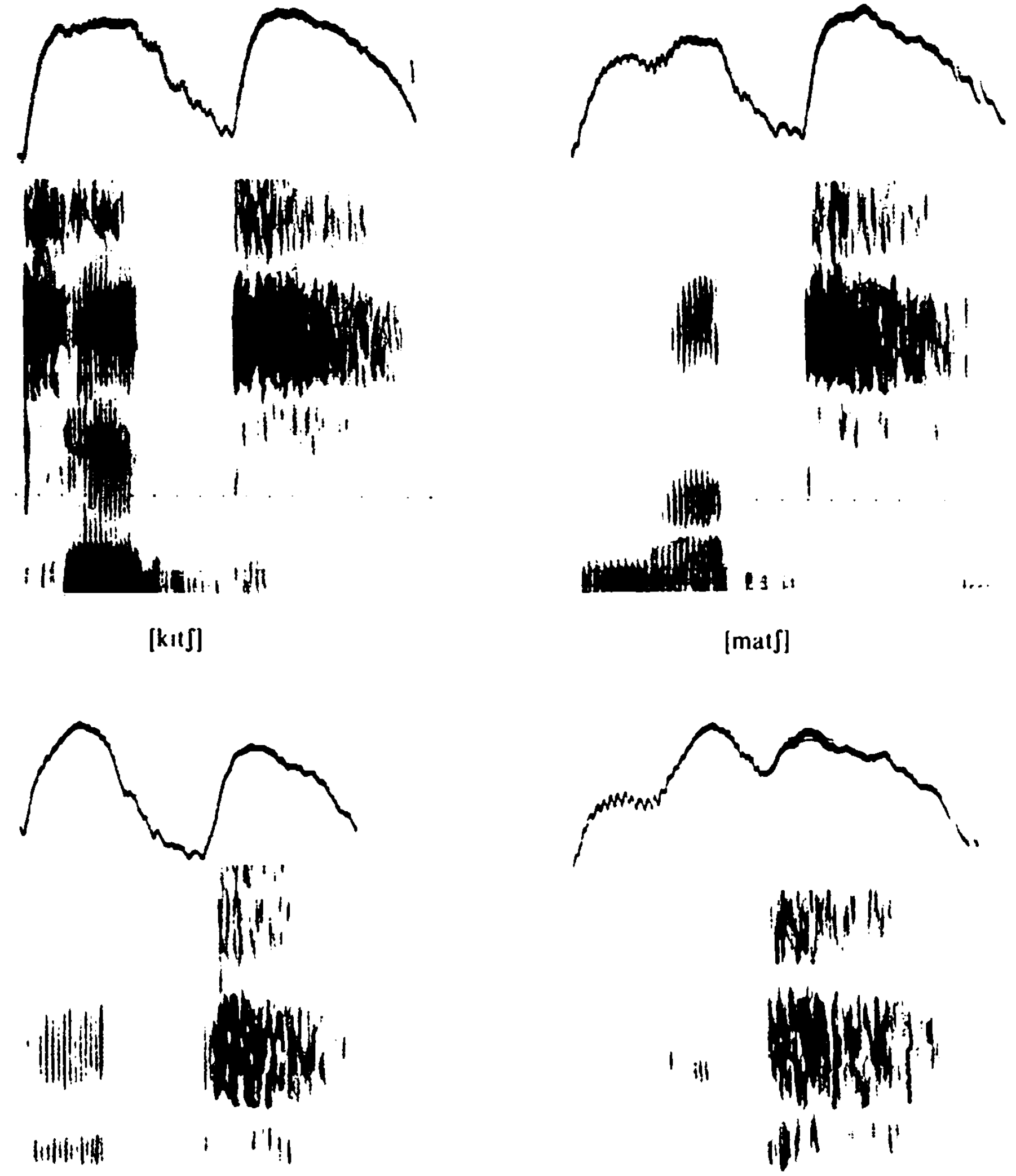

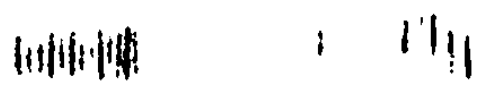
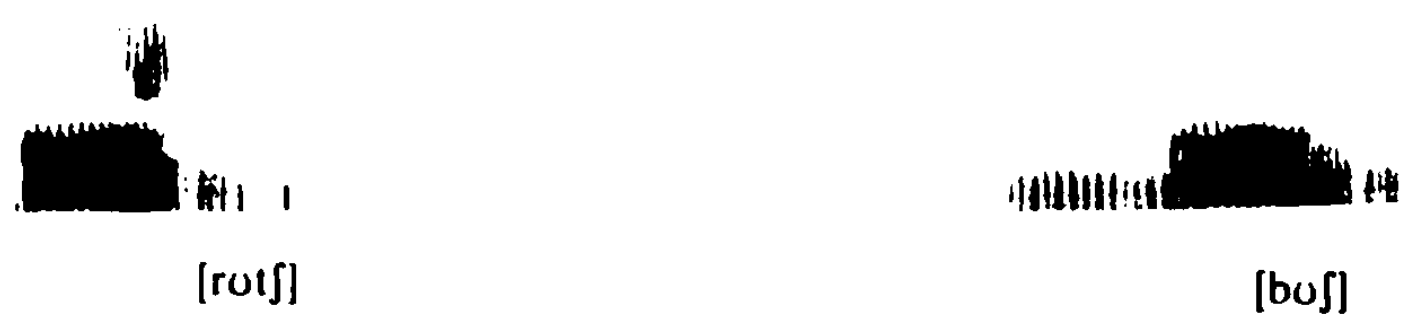
b. $[t s]-[s t]-[s t s]$ im Anlaut
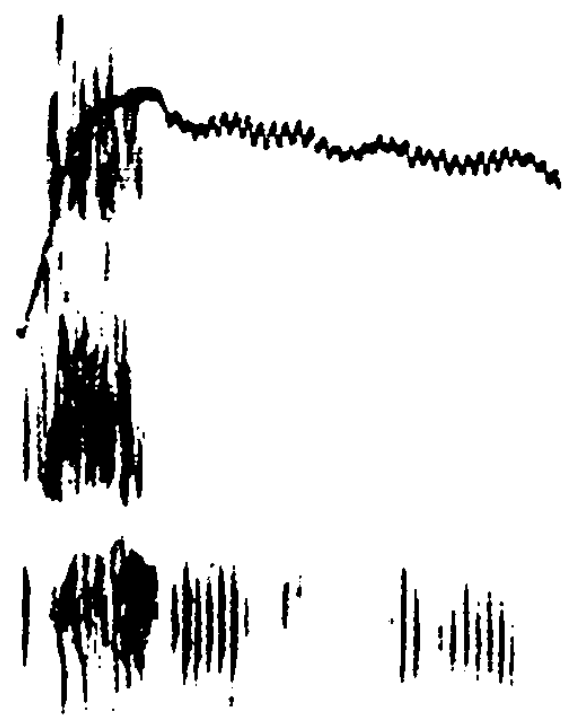

in

Iilinin!

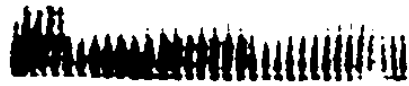

['tsena]
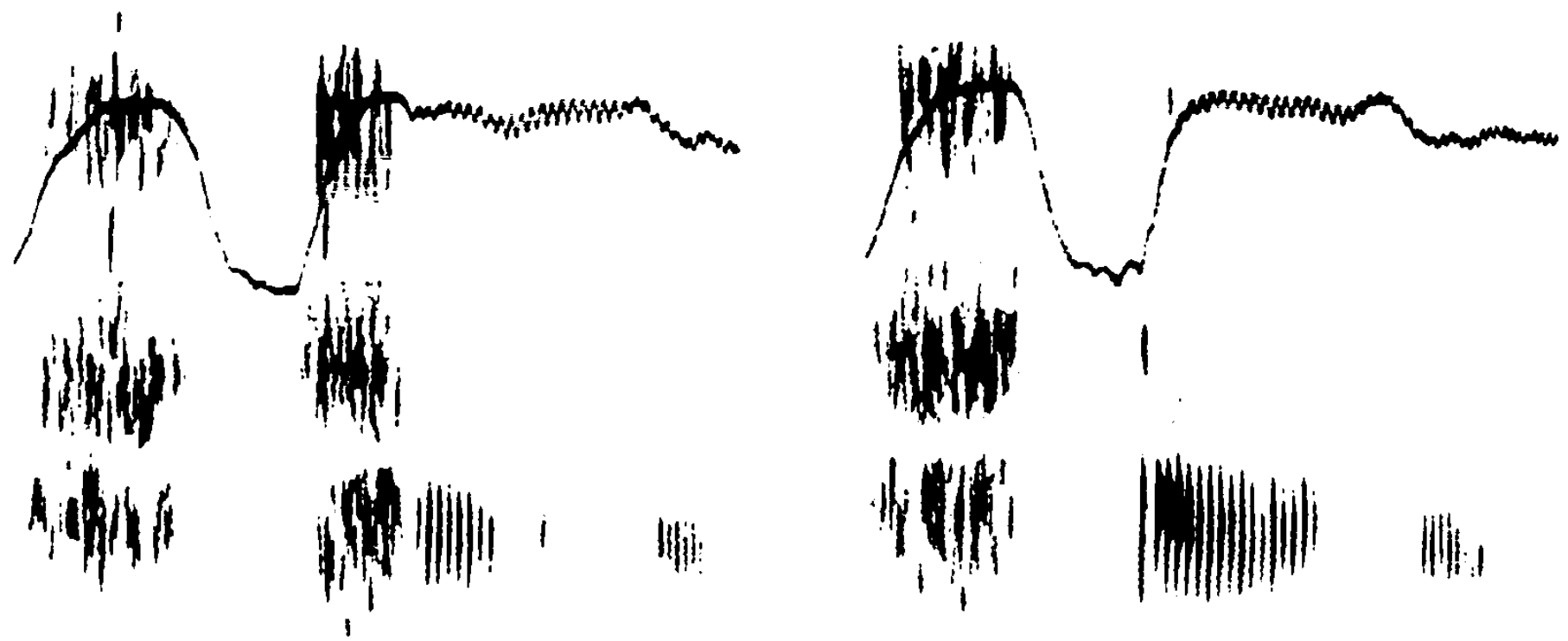

IIij:
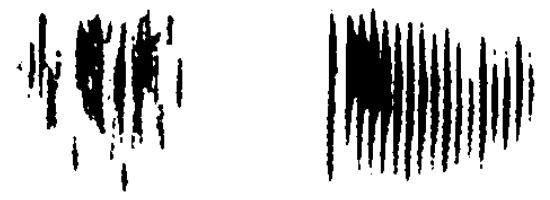

情:<smiles>[Si]#[W]#[W]</smiles>

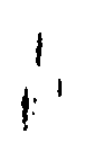

is.

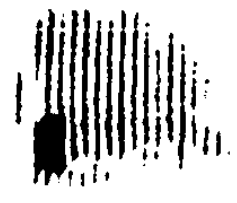

ith

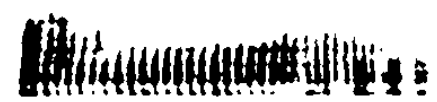

['stsena]

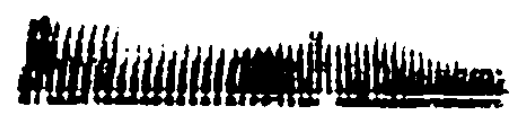

['stena] 
b. $[t s]-[s t]-[t]]$
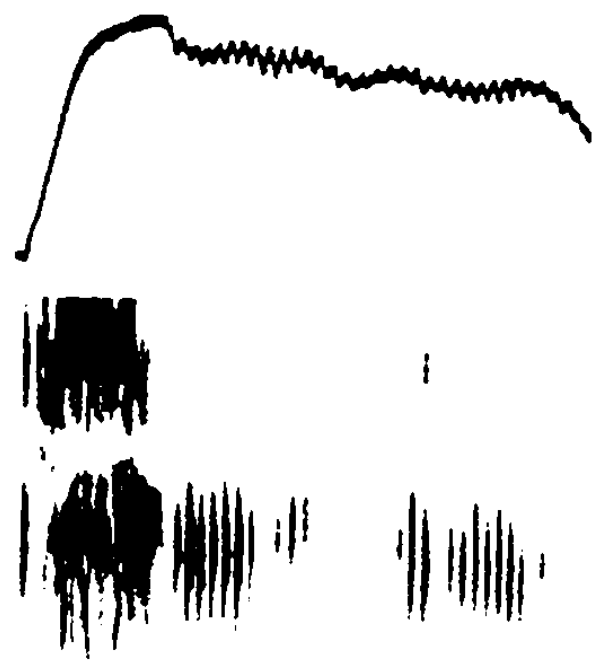

ifl| $\|$ fill|

INininiti
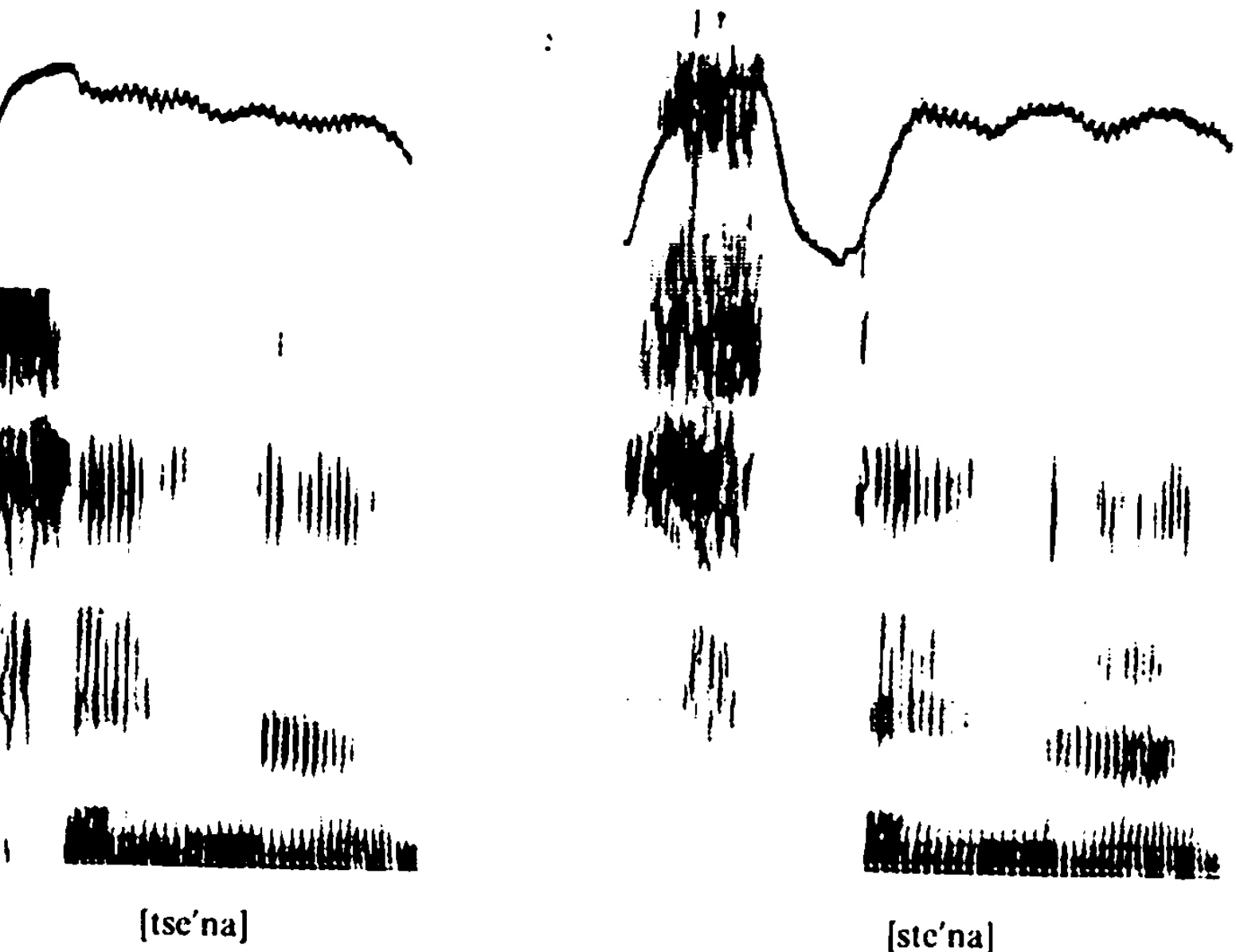

1)|l||l|l

$\|_{1}$

|ll|

il|j!

(II)

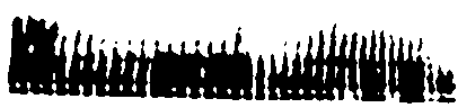

[stc'na]

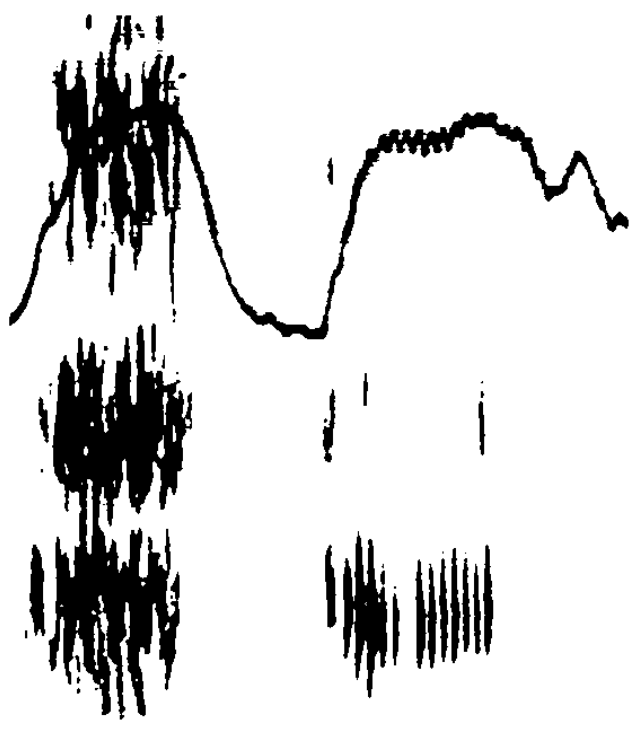

14

[star]
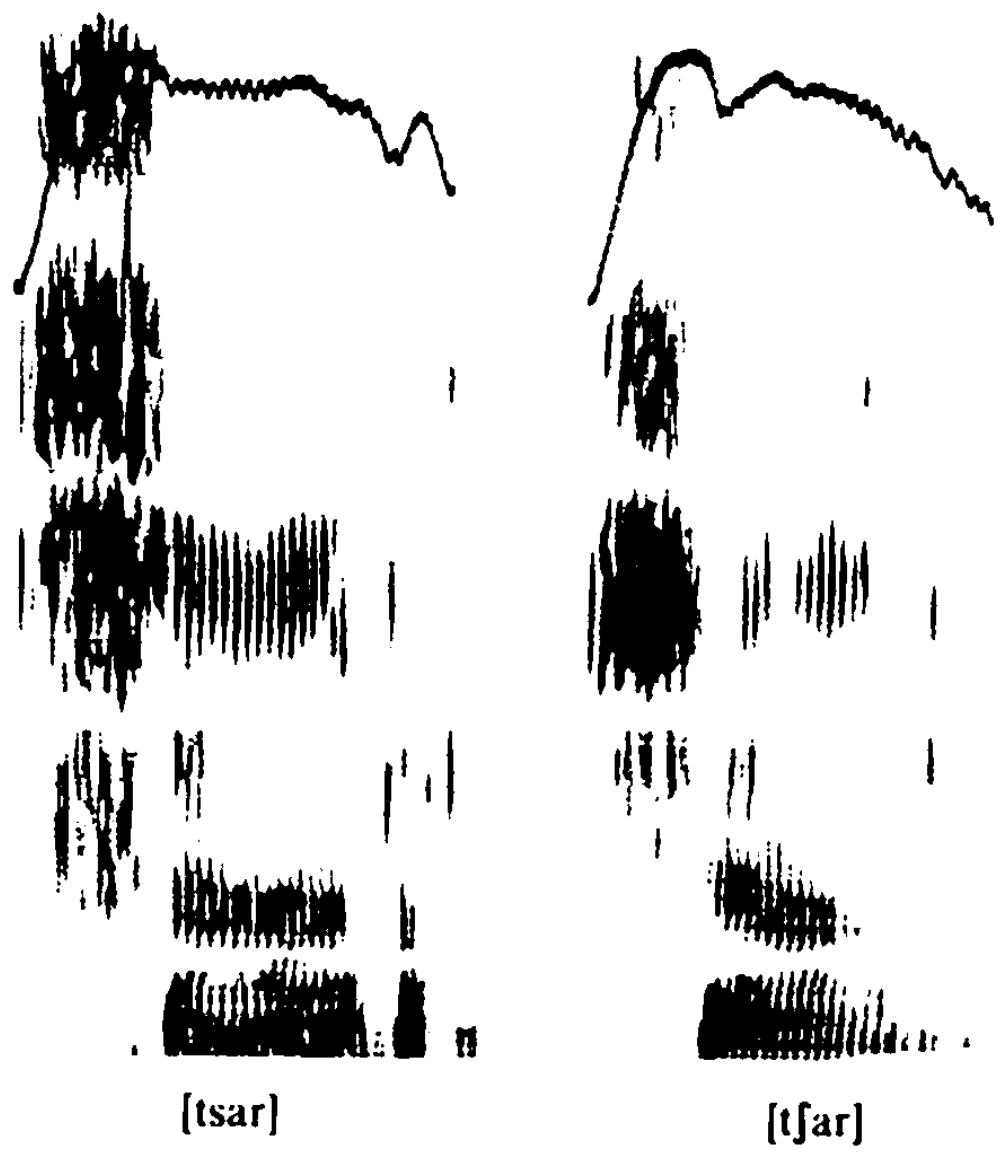
b. $[t s v]-[t s r]-[s t v]-[s t r]$ im Anlaut
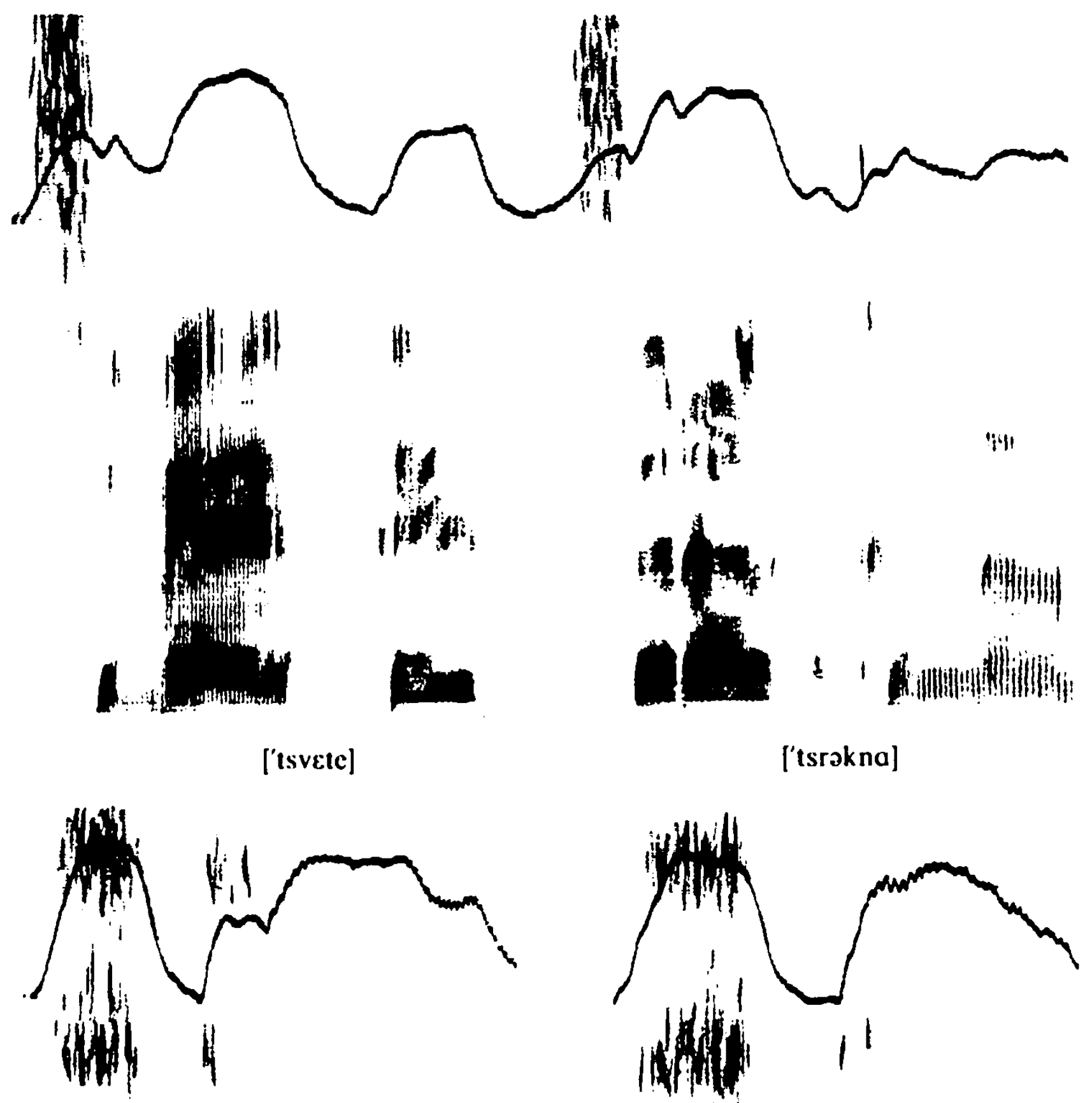

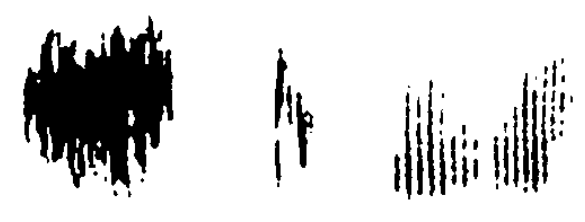

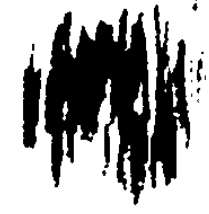

II

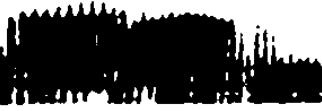

[stvst]

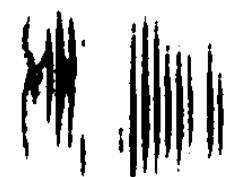

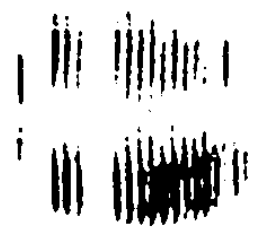

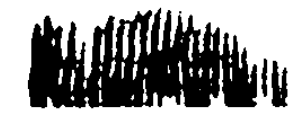

[strax] 
b. $[k s]-[p s]-[t s]-[p t s]$ im Inlaut
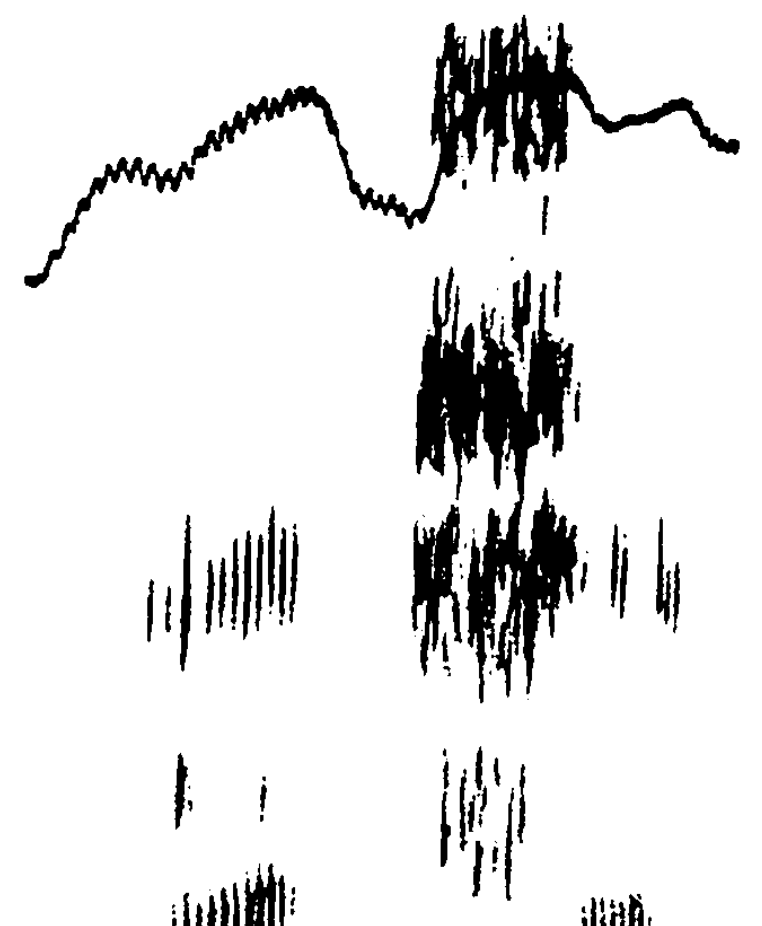

intllint:

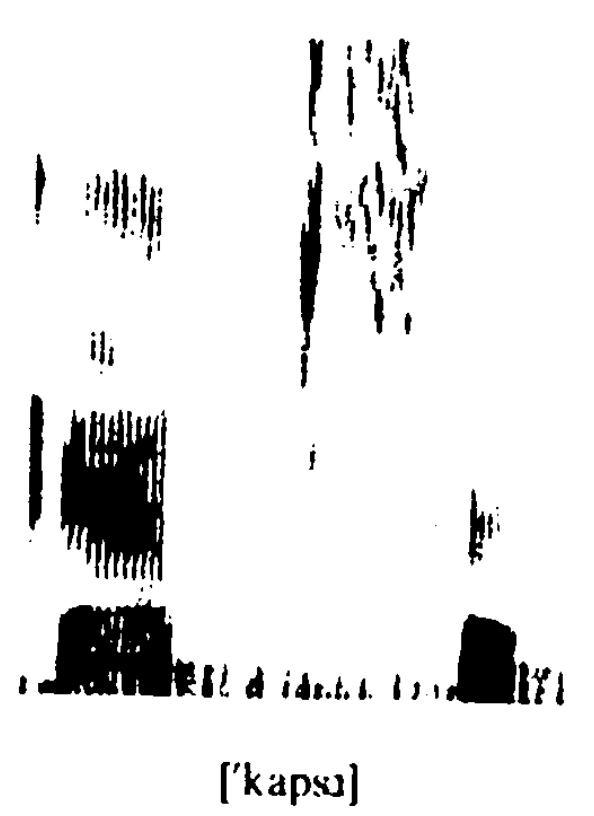

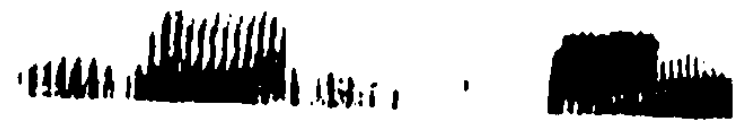

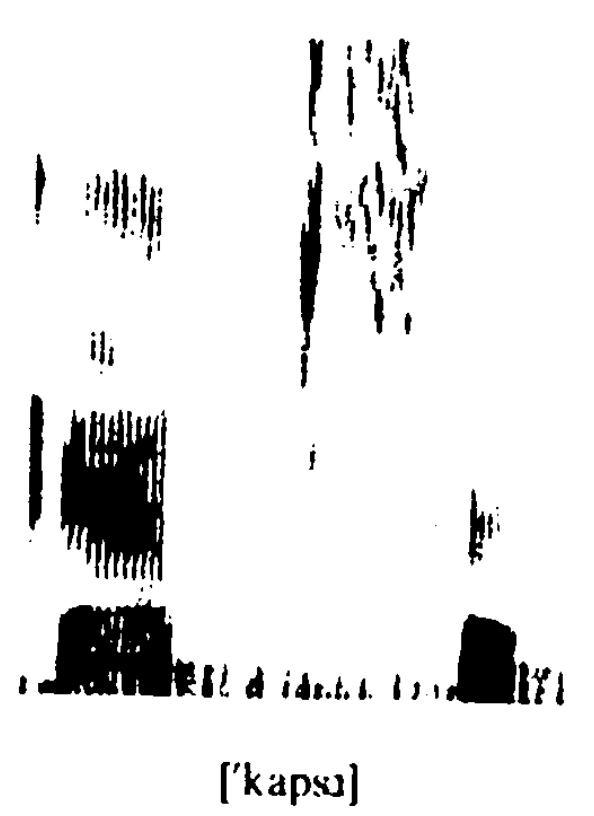

['vaksa]
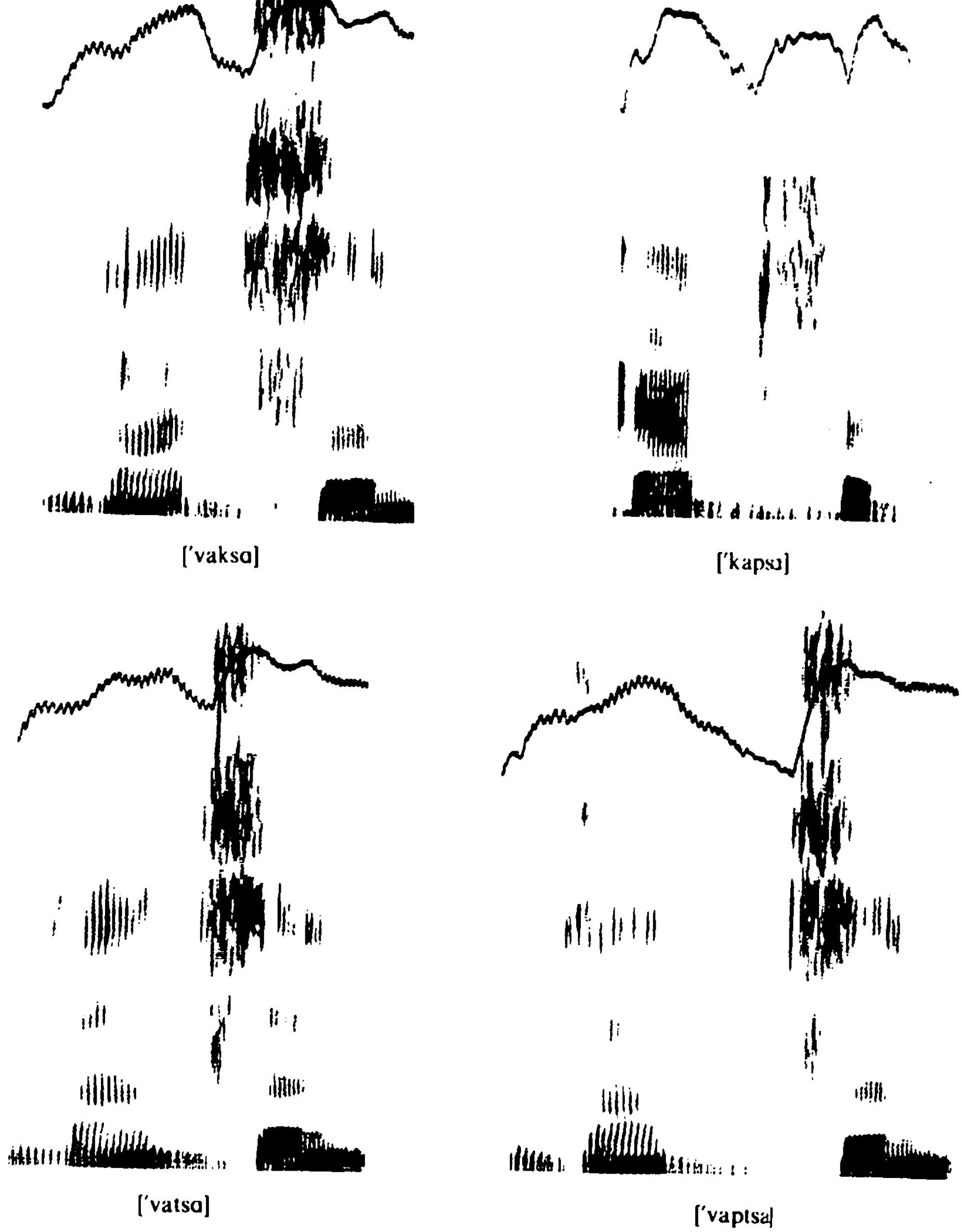
b. $[t s]-[s t]$ im Inlaut

b. $[\mathrm{ts}]-[\mathrm{st}]$ im Auslaut

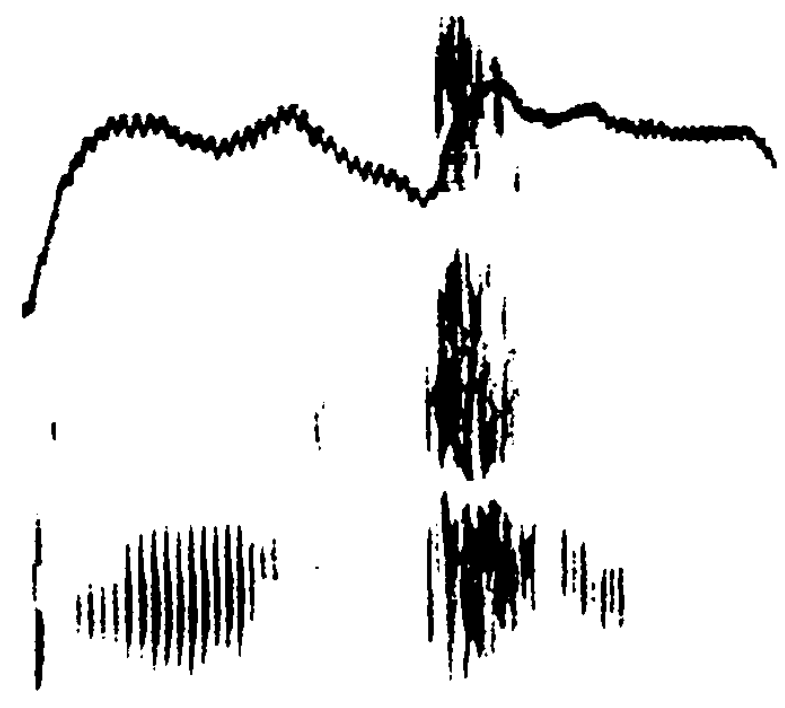

$\|$

iillili: 梢
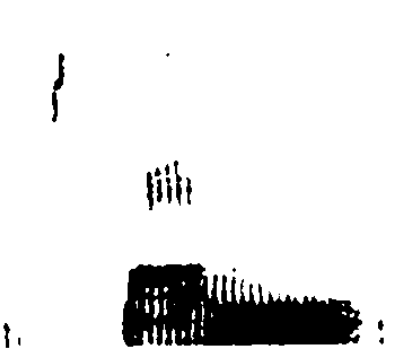

['płatsa]
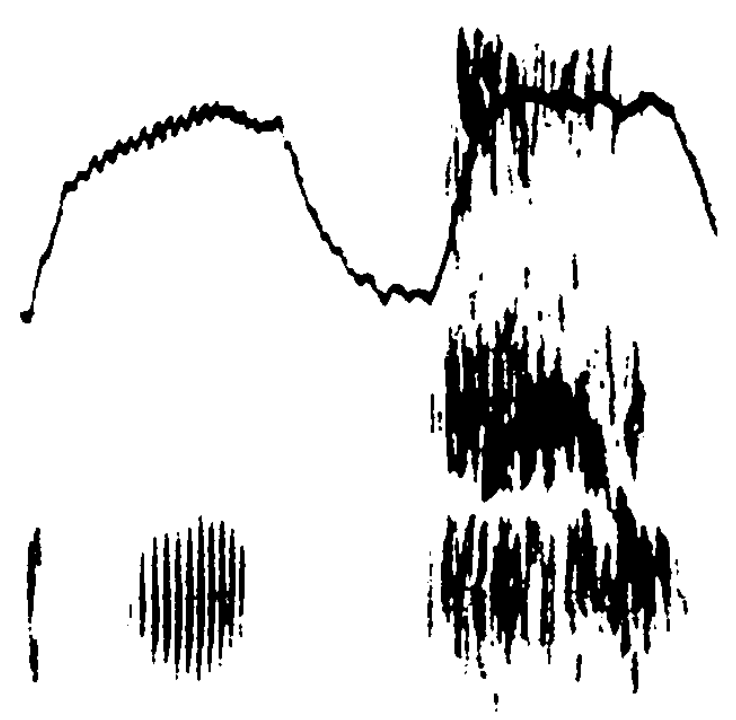

IIllill!
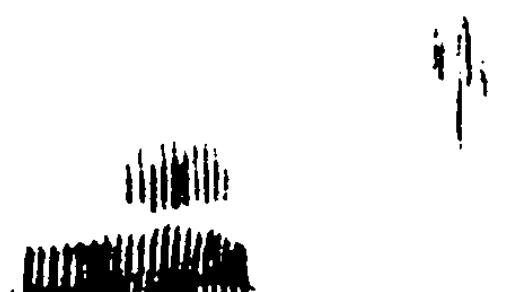

ient

[ptats]

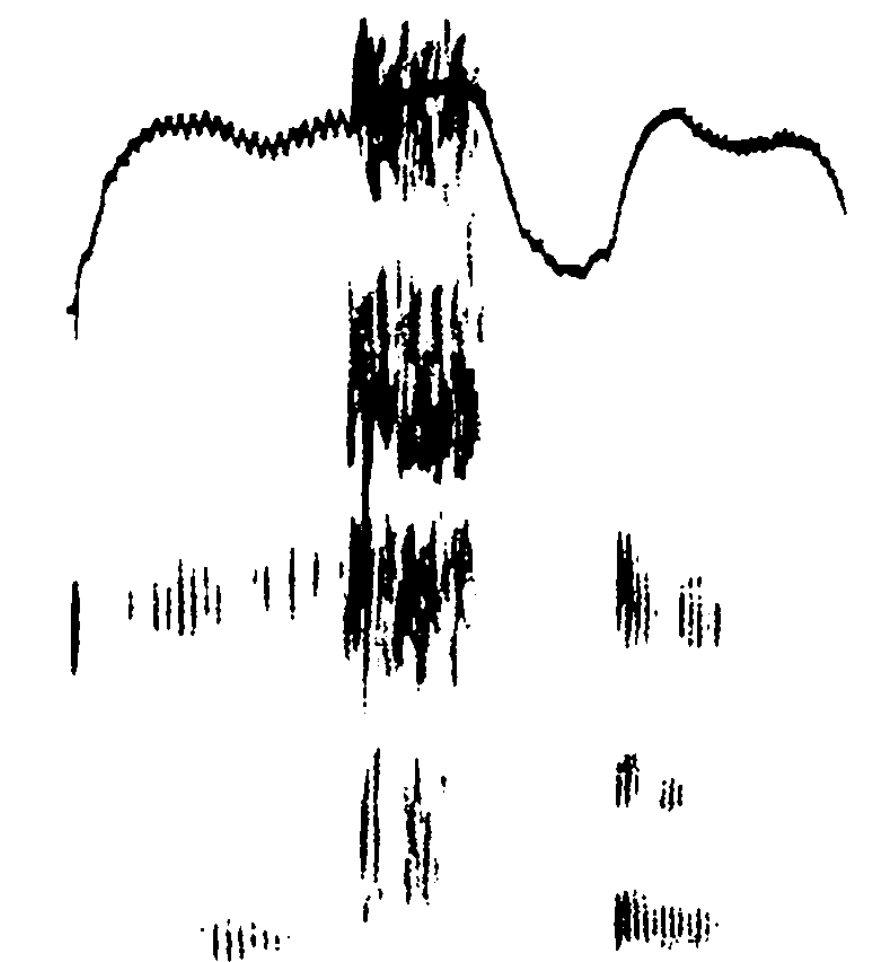

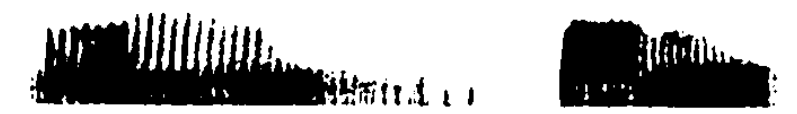

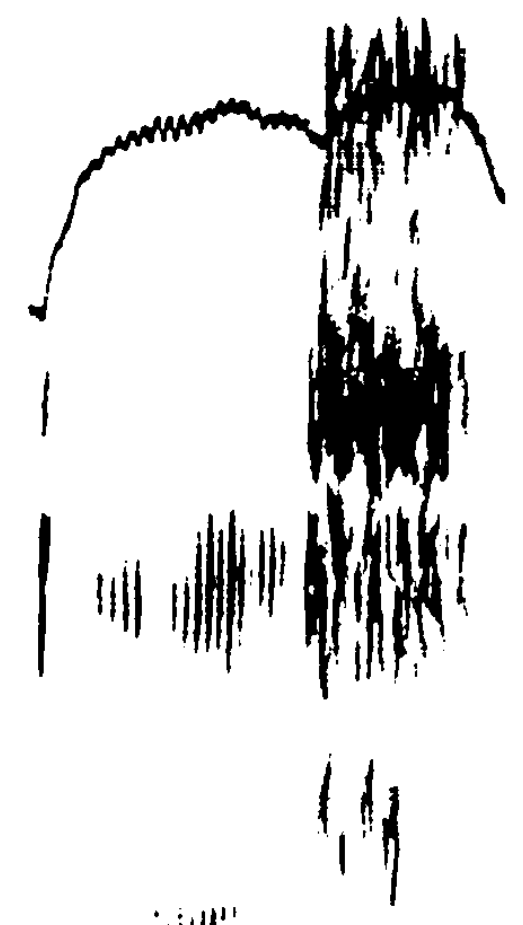

inilfill

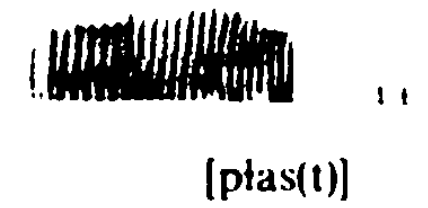


b. $[t s]-[s t]-[t]]-[s t]$ im Inlaut
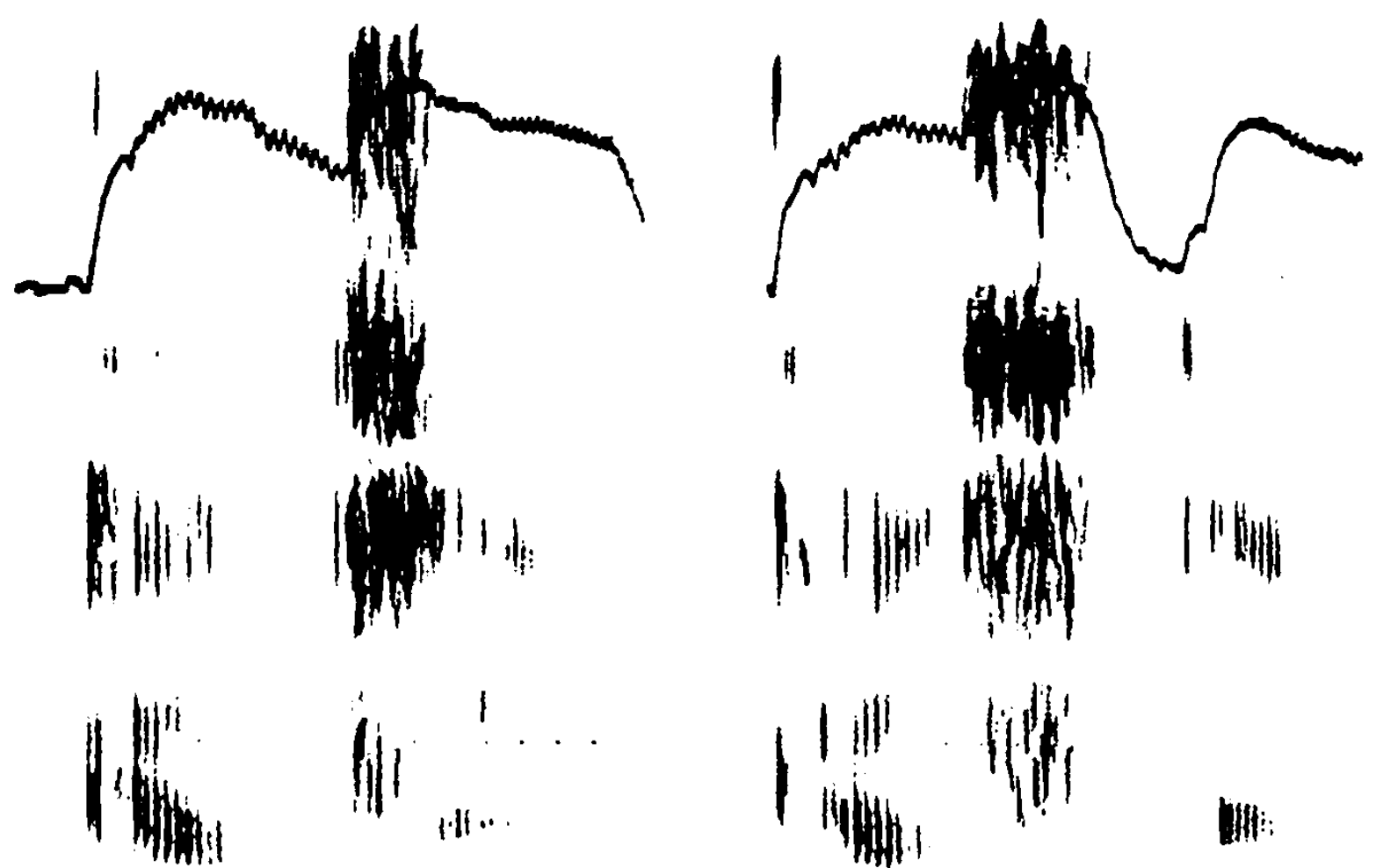

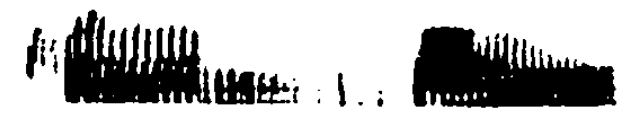

['katsa]

\$

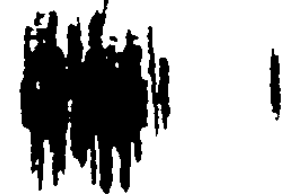

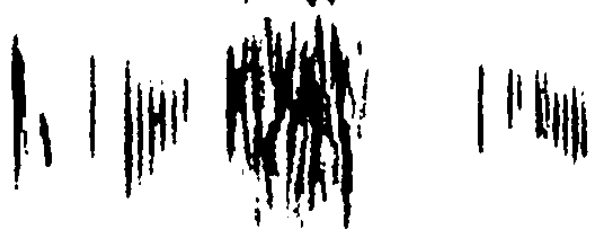

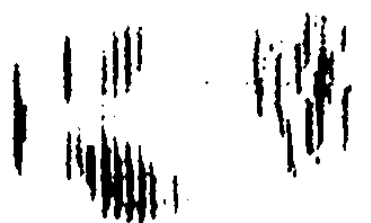

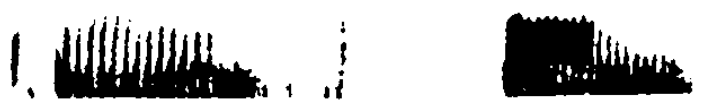
['kasta]
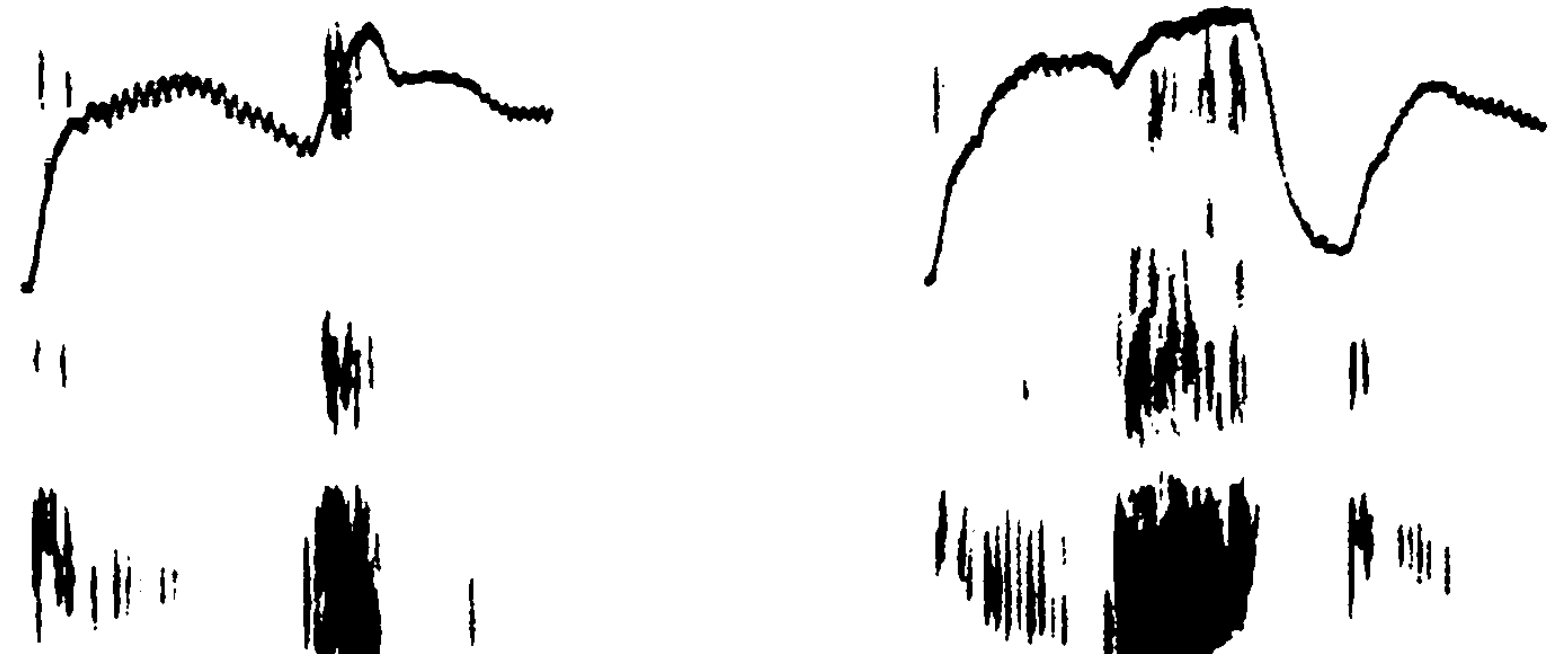

粗保

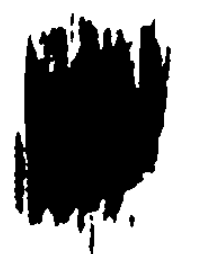

$\beta \|$

ni il

Lill|l|nis

II Hilin

Inilin

1 Hiwlill,
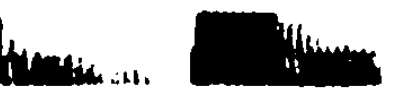

['katja]

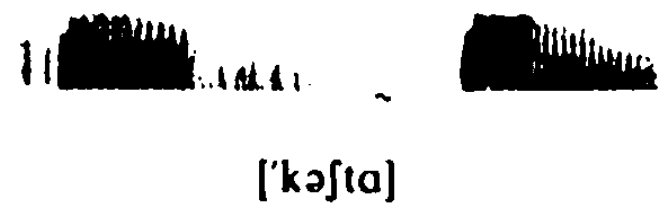


b. $[t]]-\left[\int t\right]$ im Inlaut
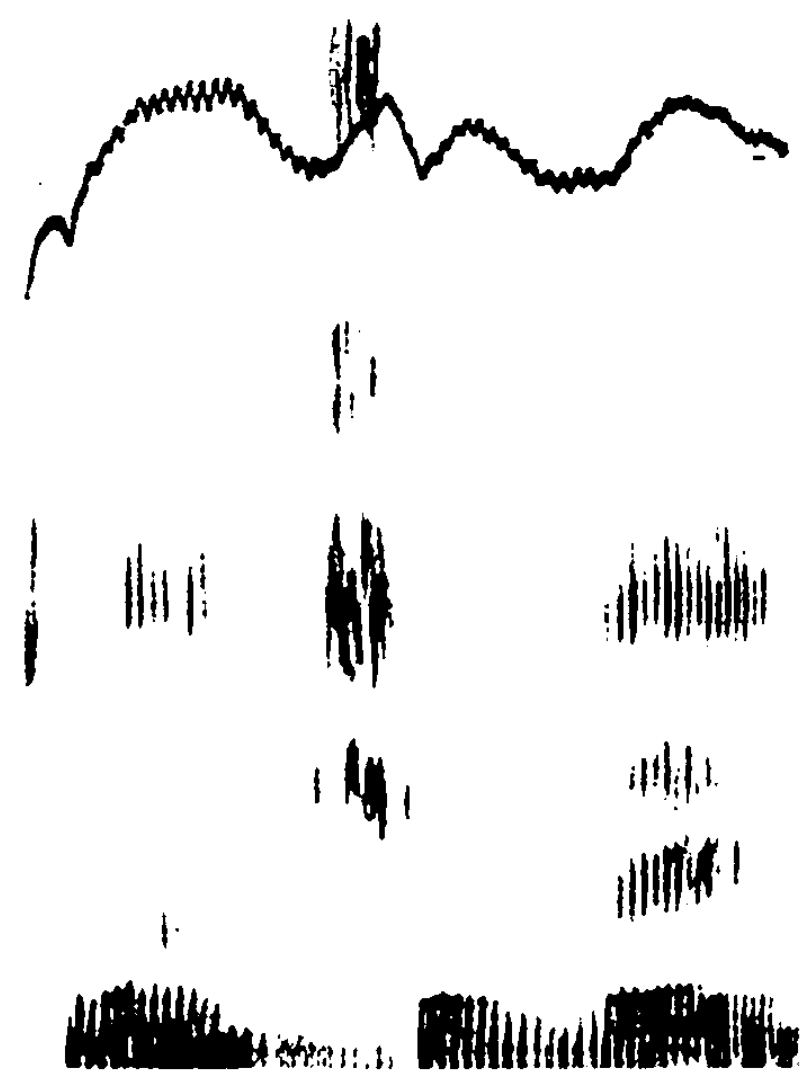

['phatfove]
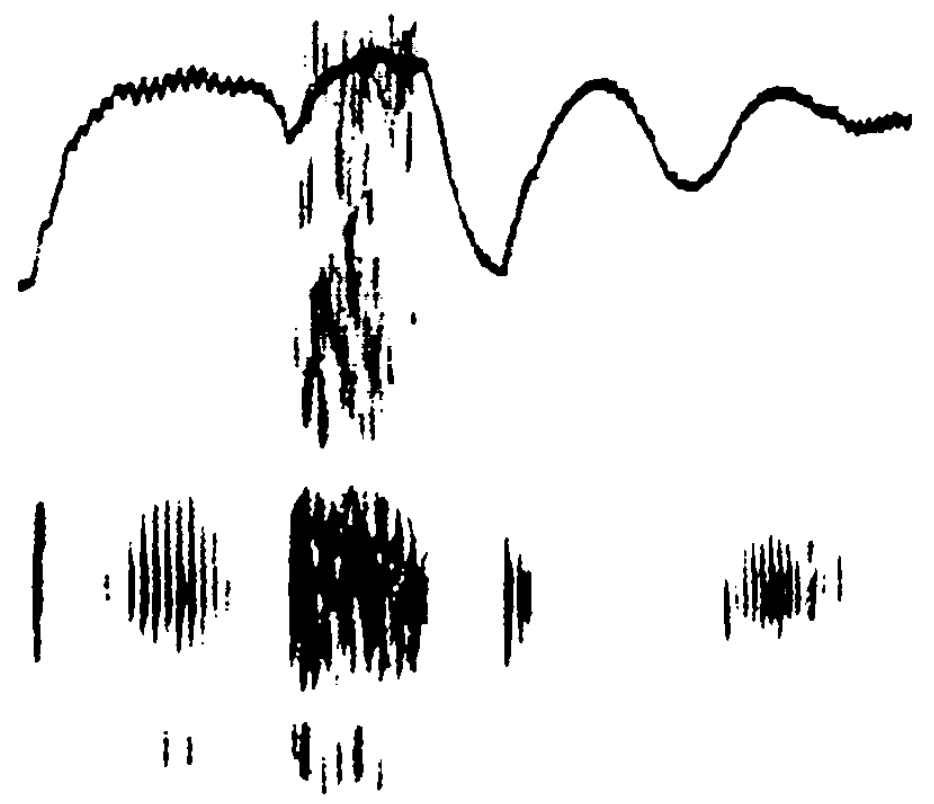

ilillili

里

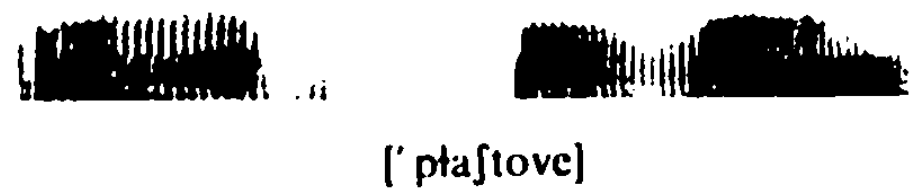


b. $[t c]-[s t]$ im An- und Inlaut
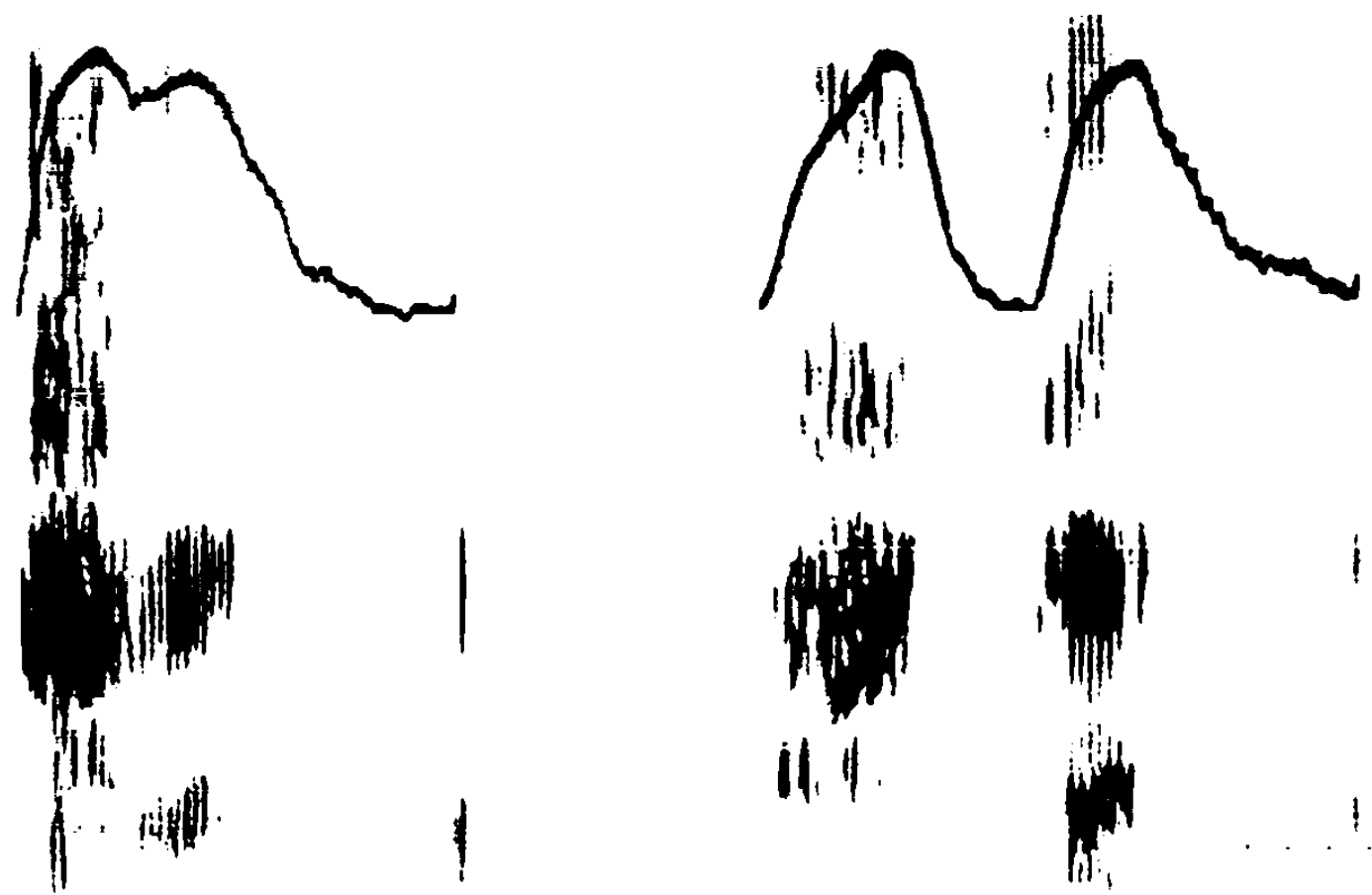

ili.

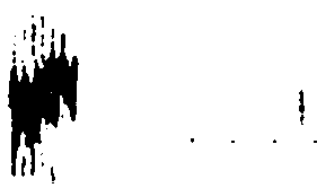

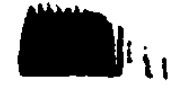

$\left[t \int 1 k\right]$
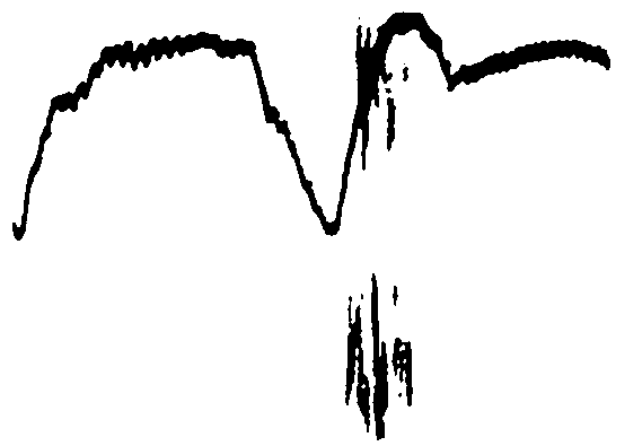

1 ||lifij|

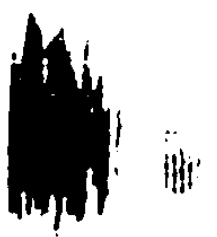

II

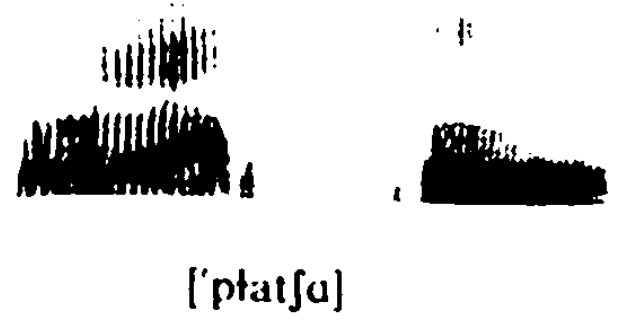

\section{Vili}

[stık]
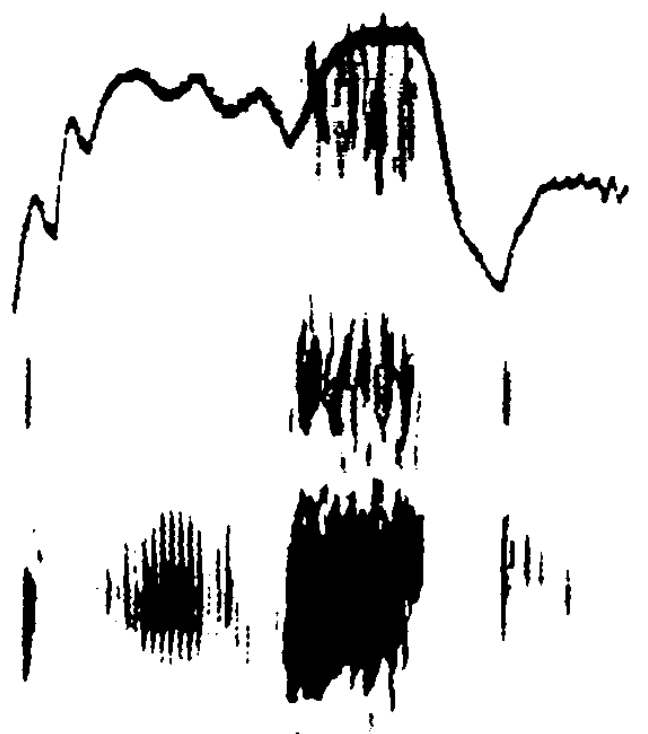

$\cdot i k$

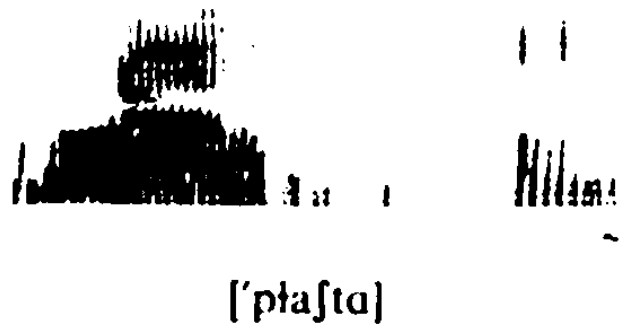


d. il] vor [ae], realisiert von einem Deutschen und einem Bulgaren

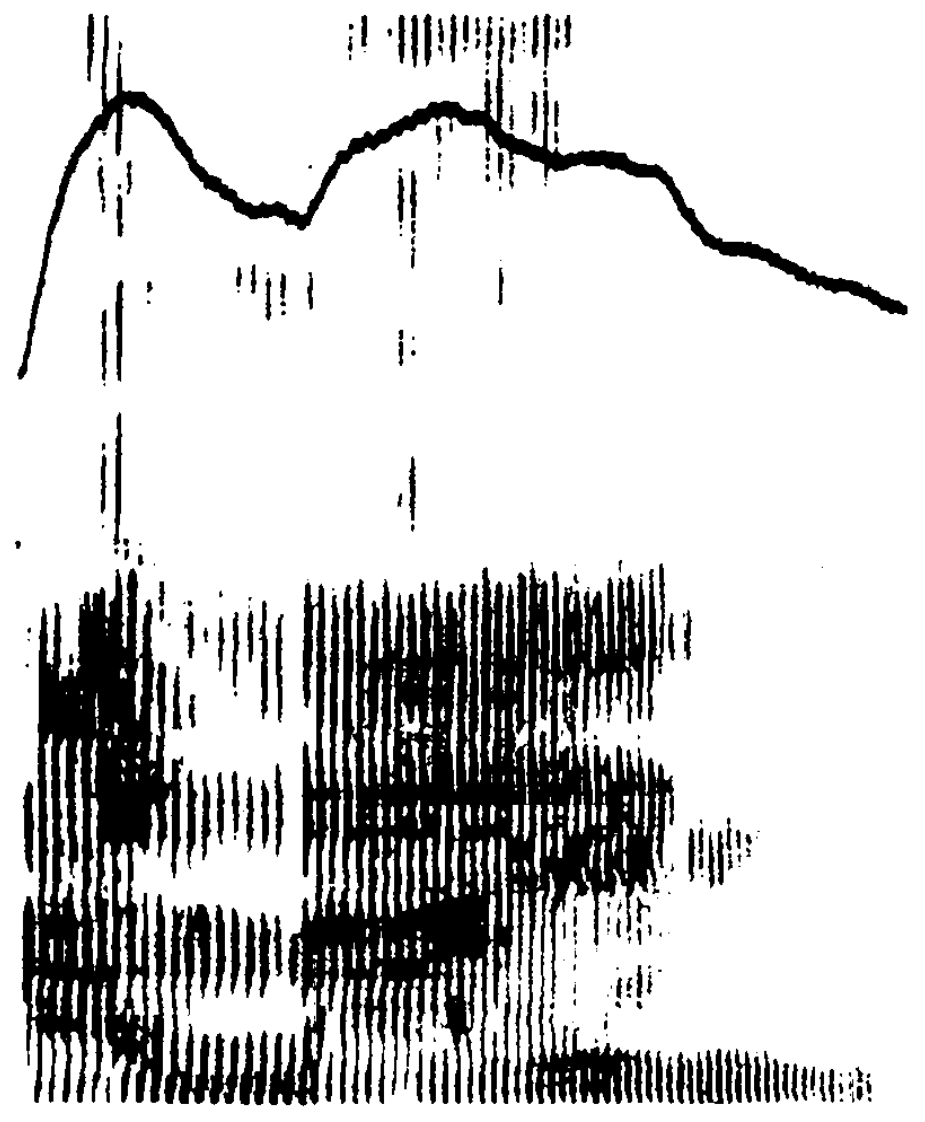

['al'àen]
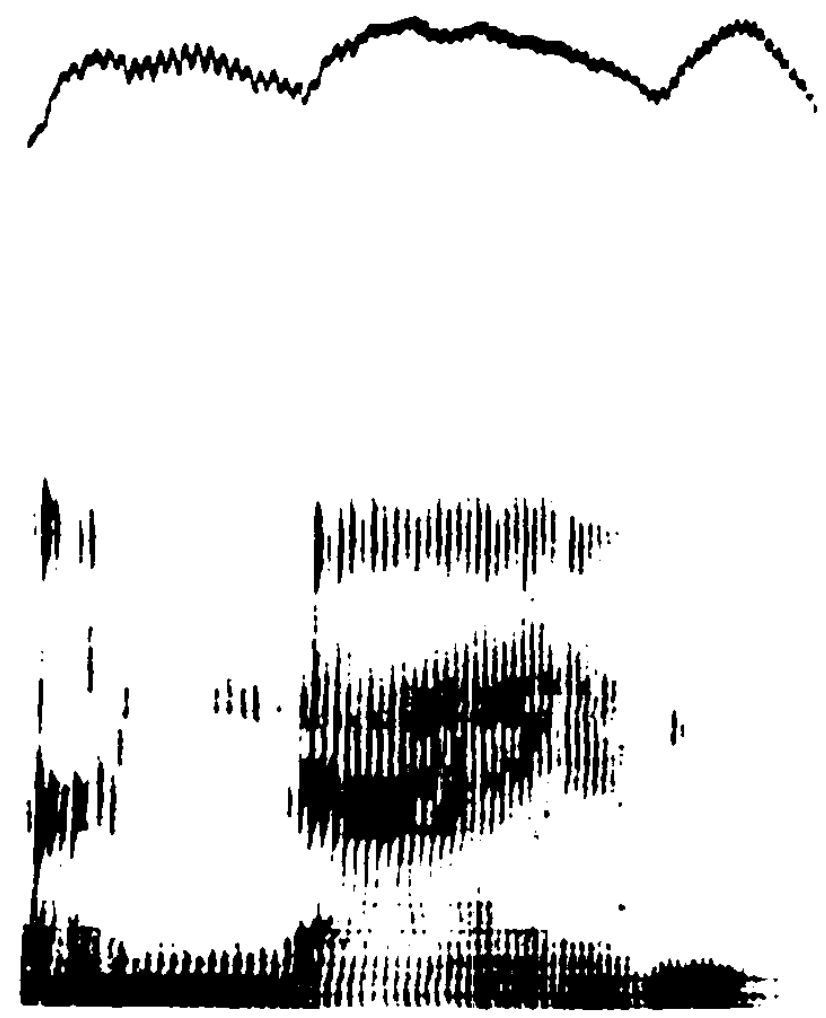

['al'àen] 
b. $\left[t \int\right]-\left[k \int\right]-[p s]-[k s]$ im Inlaut
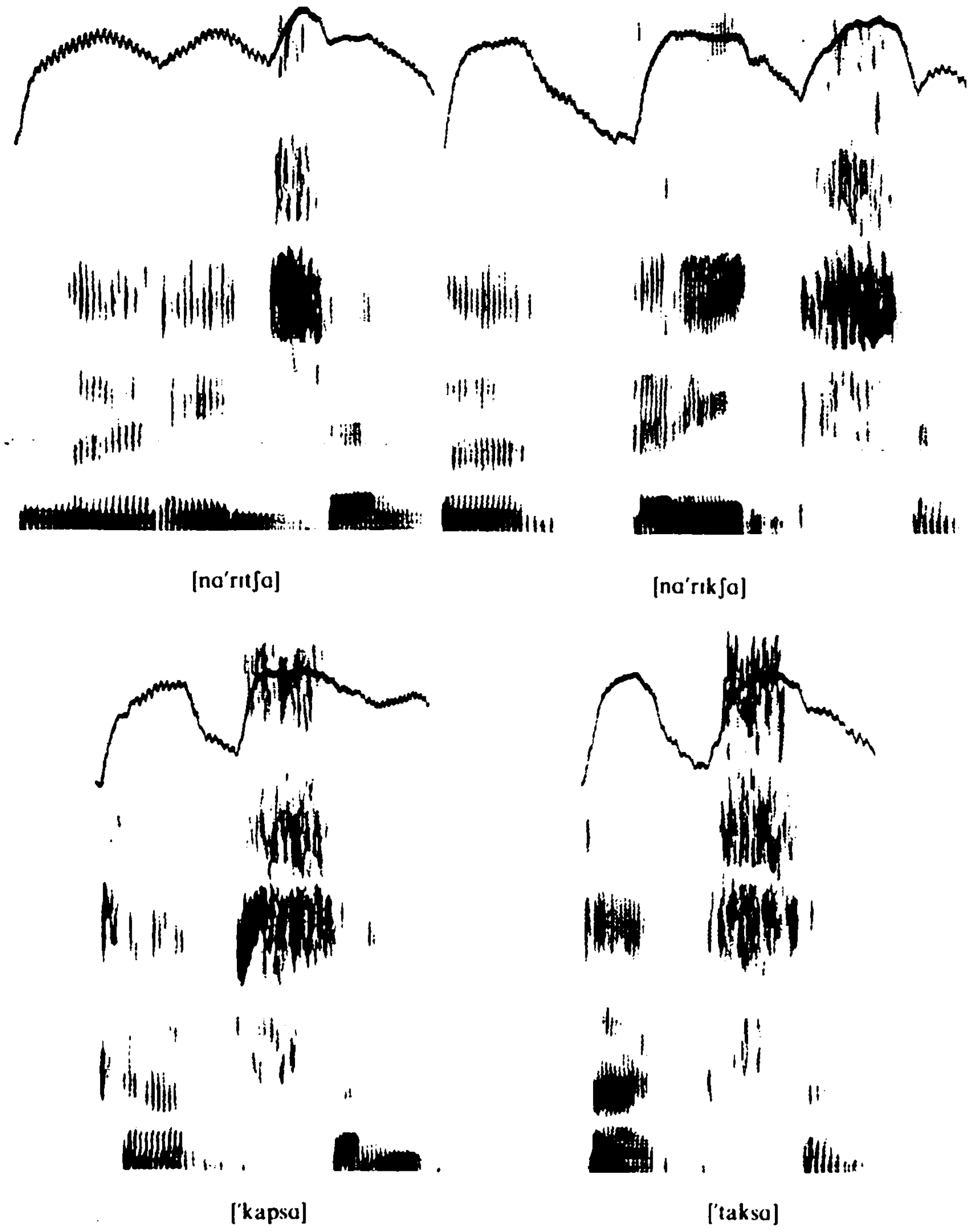
d. [r] im Auslaut für 〈-cr〉, gegenübergestellt dem [ə] im absoluten Auslaut b. $[-r, r]$ im Auslaut fur $\langle-a p\rangle,\langle-b p\rangle$
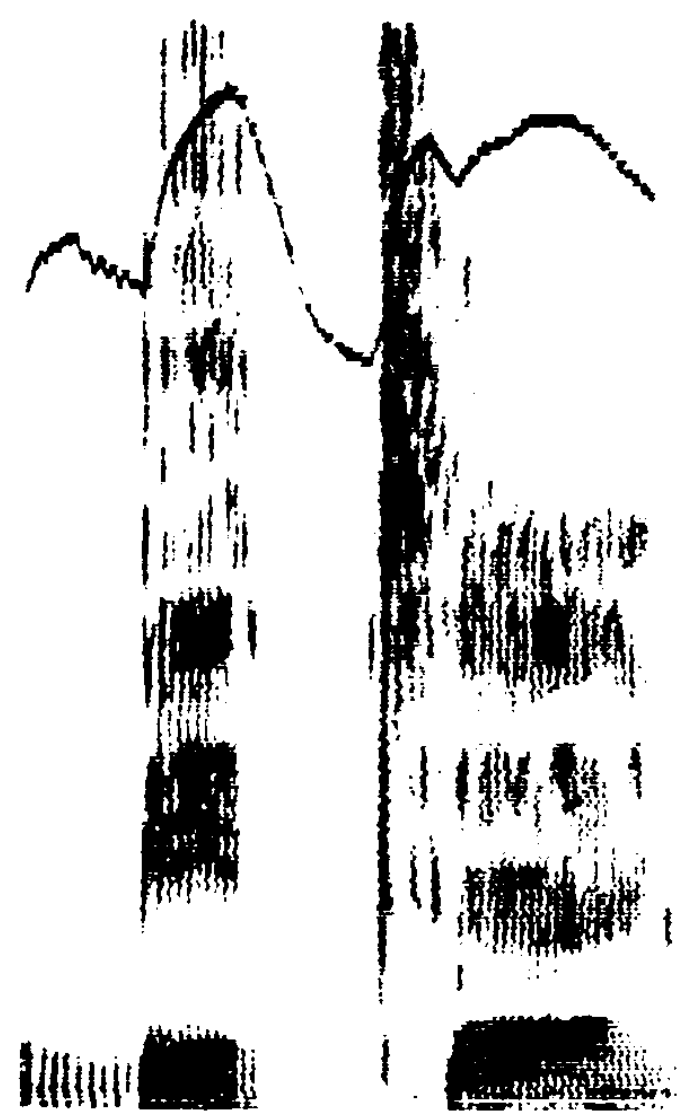

['bite]

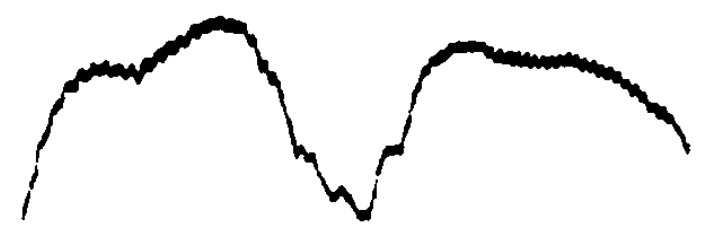

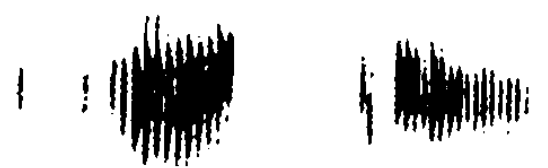

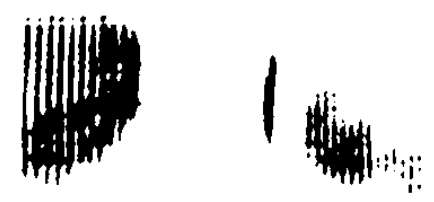

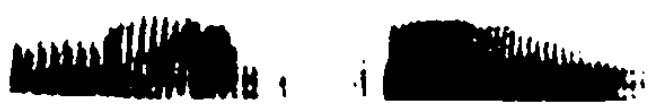

['IEker]
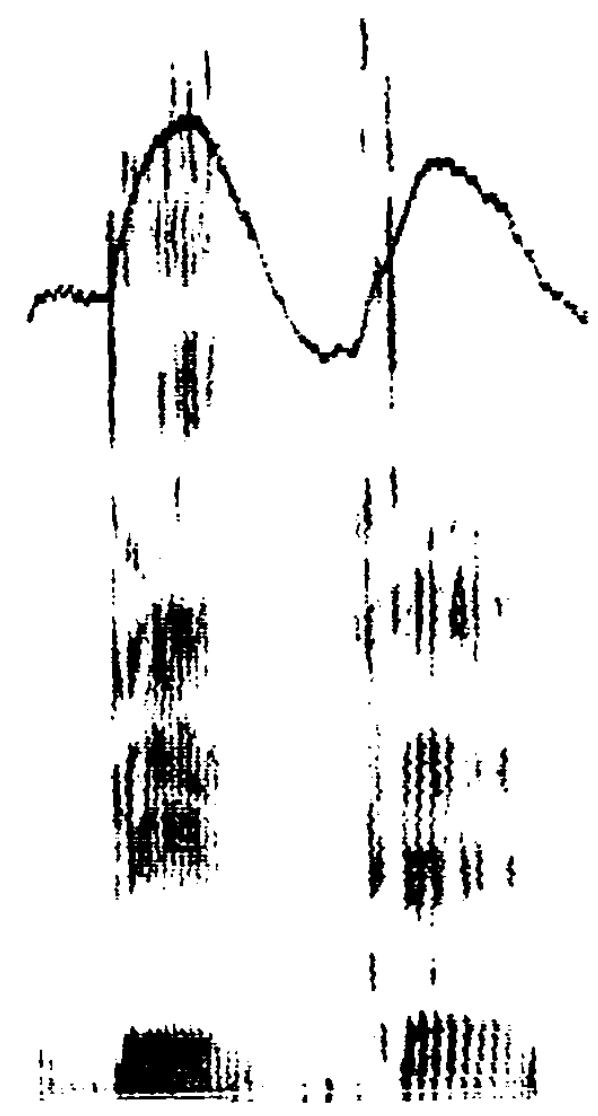

\section{['bito]}

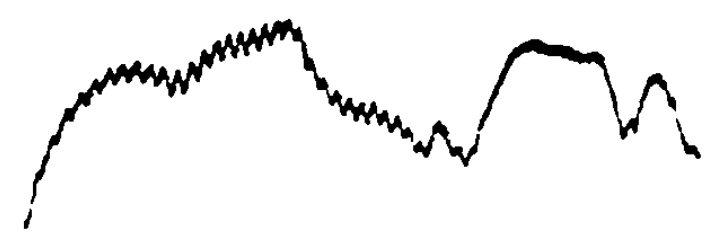

$\left\|^{1} \quad|\| n| m \mid\right.$

I

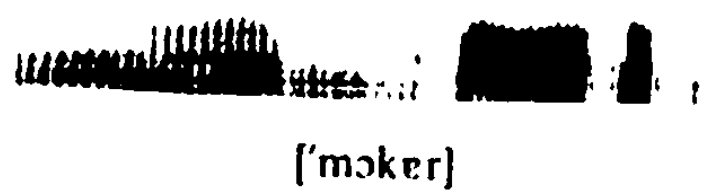


b. $\left[p \int\right]-\left[p t \int\right]-[k s]$ im Anlaut
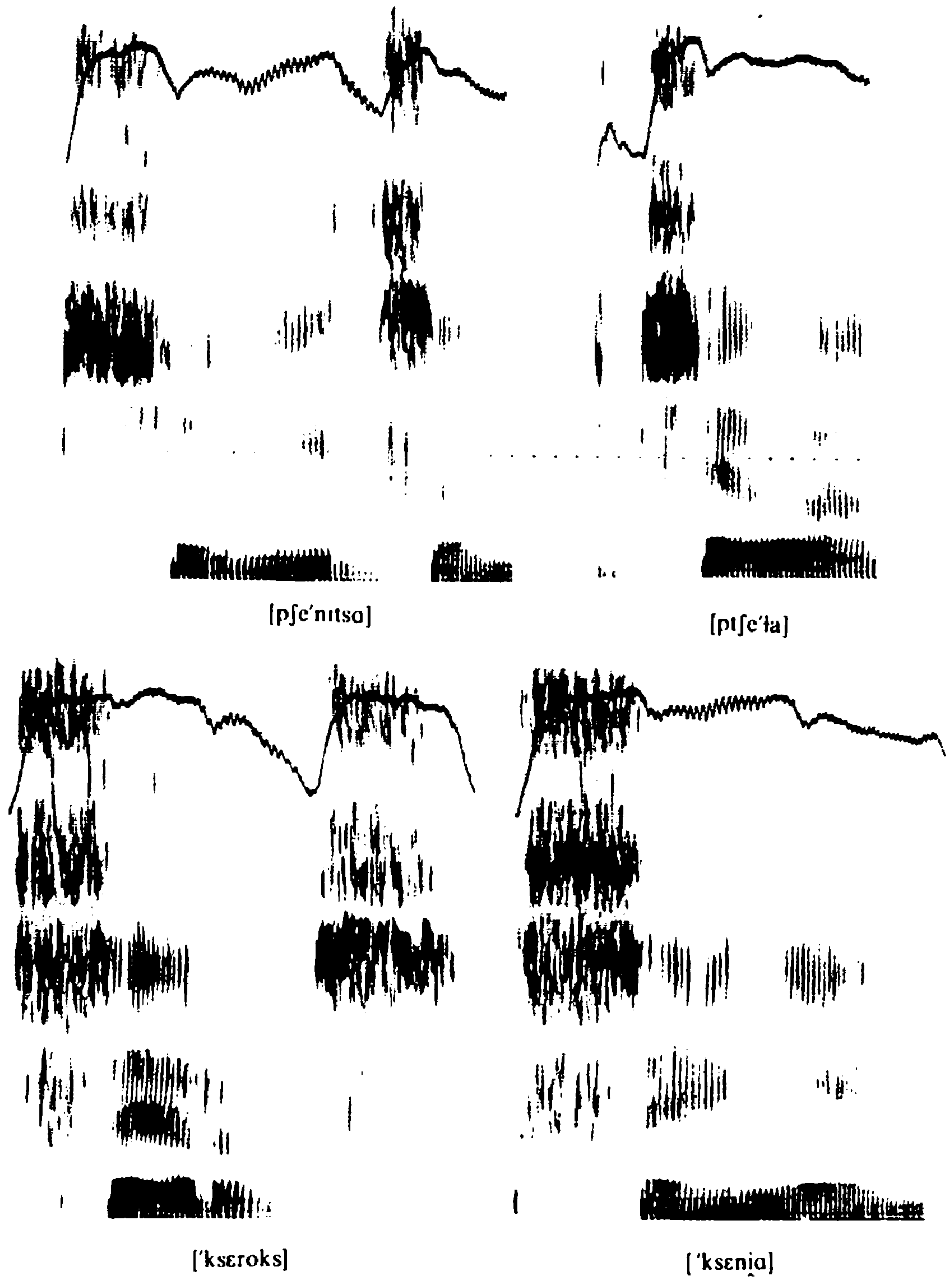
d. [e] an der Morphemgrenze vor ['áe] und [r]
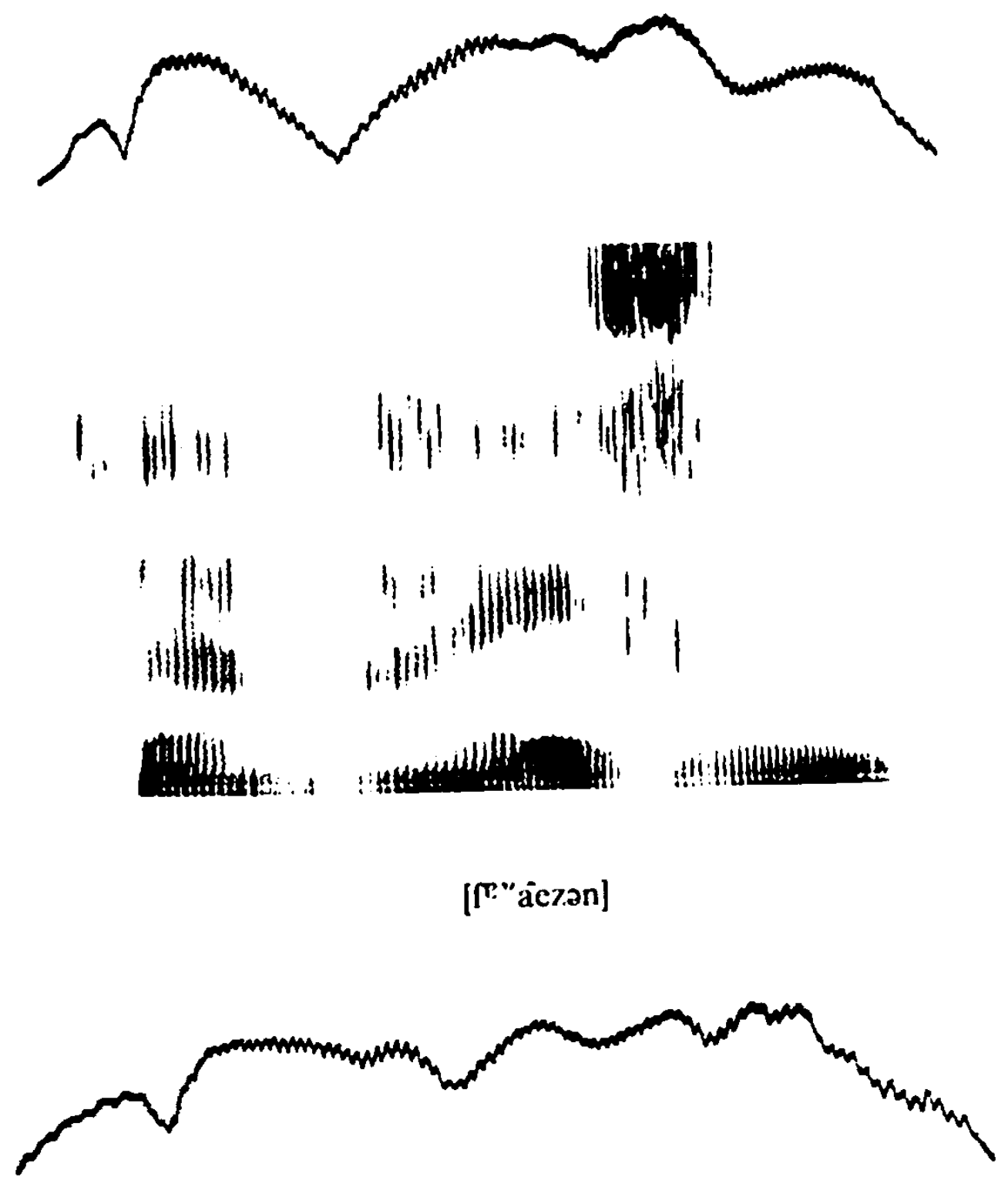

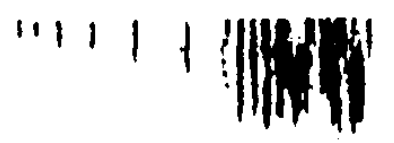

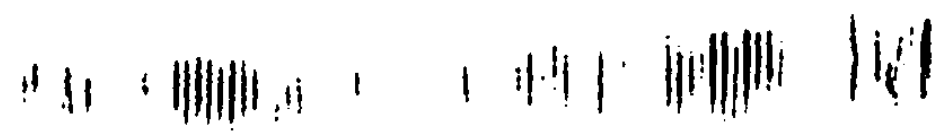

iil $\| 11$

III ii :ilum

$\operatorname{li}_{i j} \|_{1}$

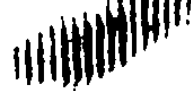

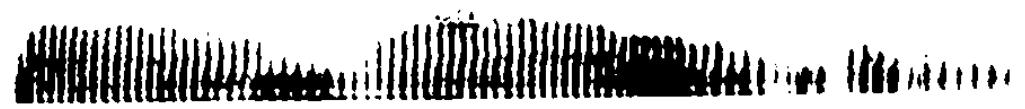

[fe" räezan] 
Sonagramme des deutschen [n], realisiert von einem

Deutschen und einem Bulgaren

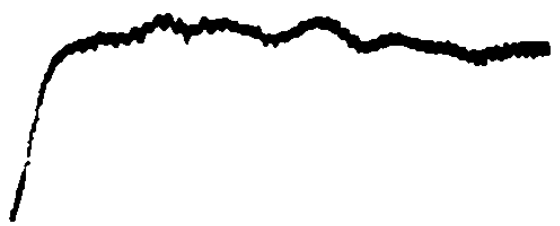

111

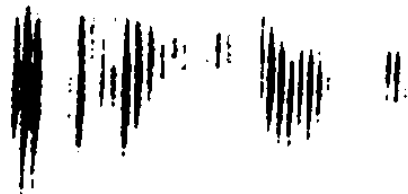

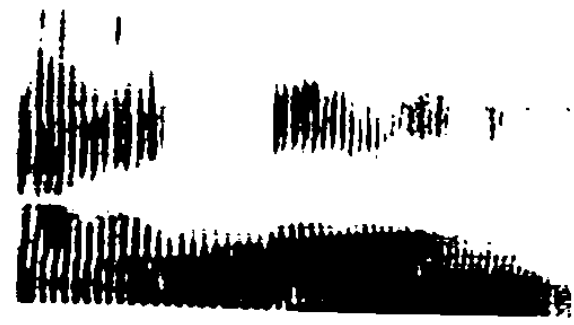

d. ["anal]

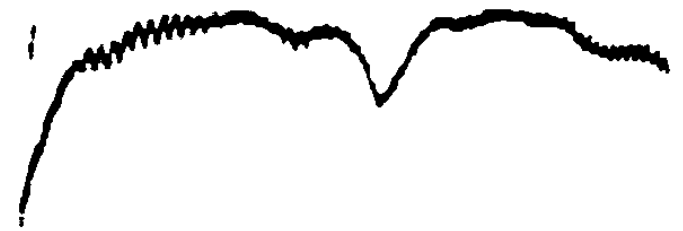

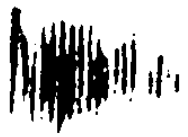

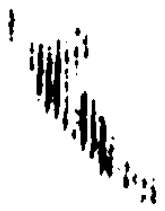

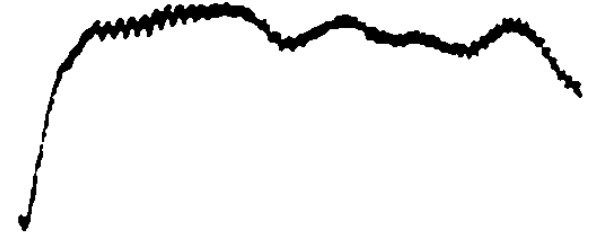

i
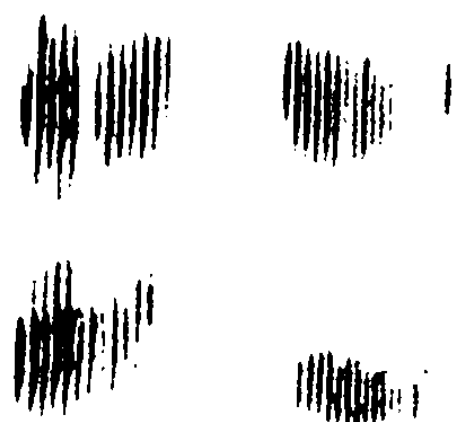

Illy

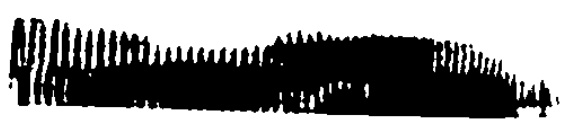

d. ["Enal]

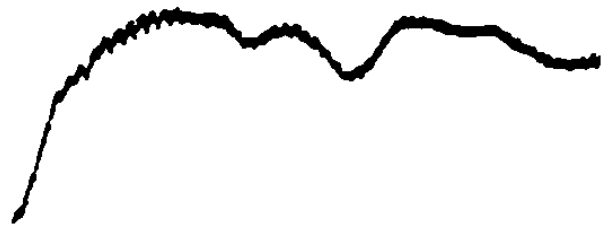

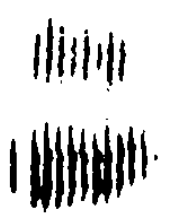

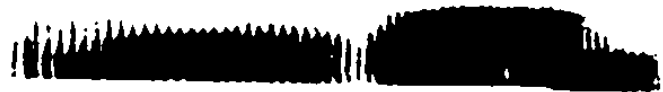

["angal]
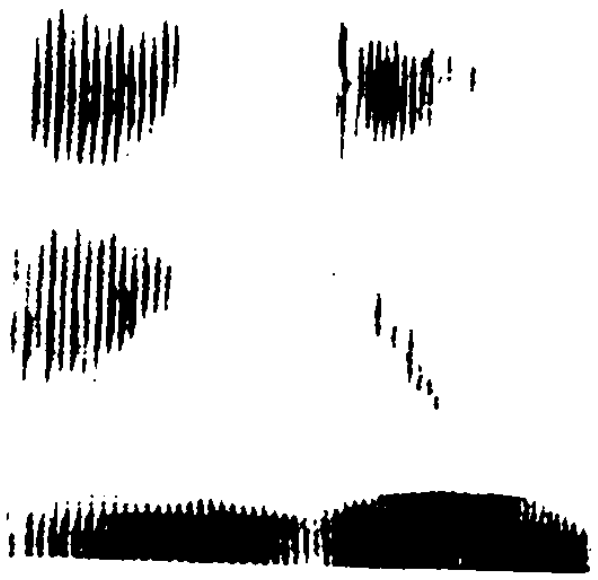

["Engal] 
d. $[\mathbf{n}]-[n]$. gesprochen von einem Deutschen und einem Bulgaren
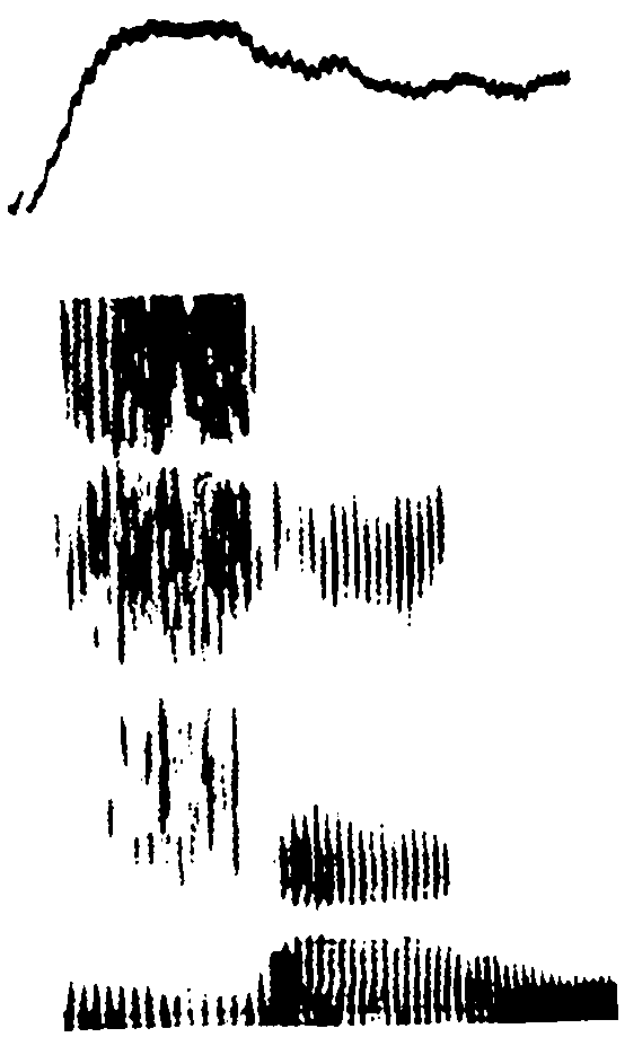

d. [zan]
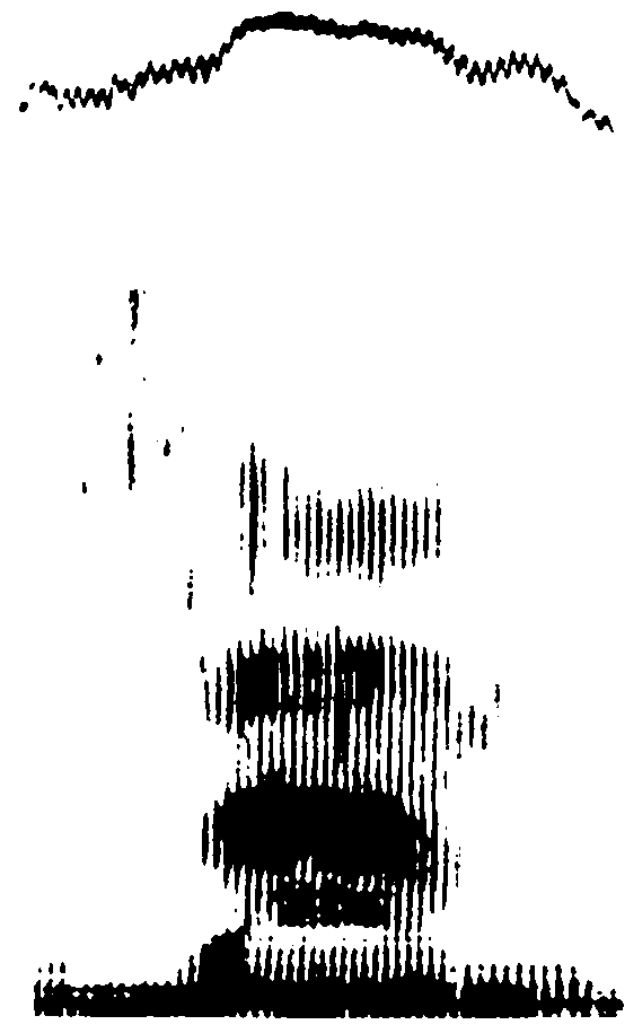

b. [zan]
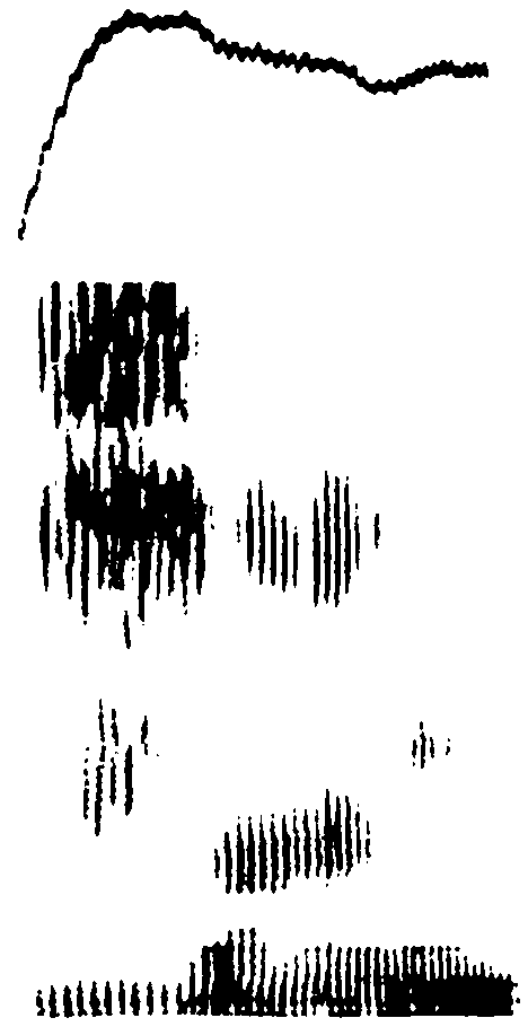

d. [7an]

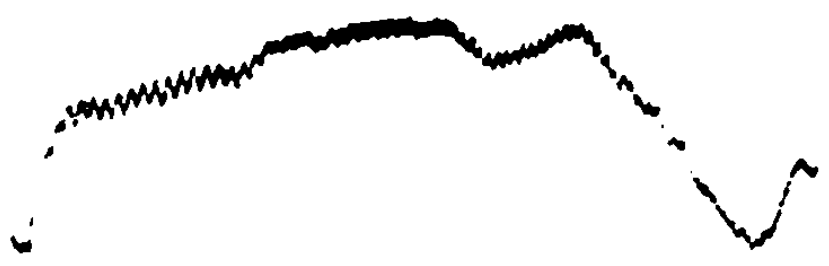

$11+1$

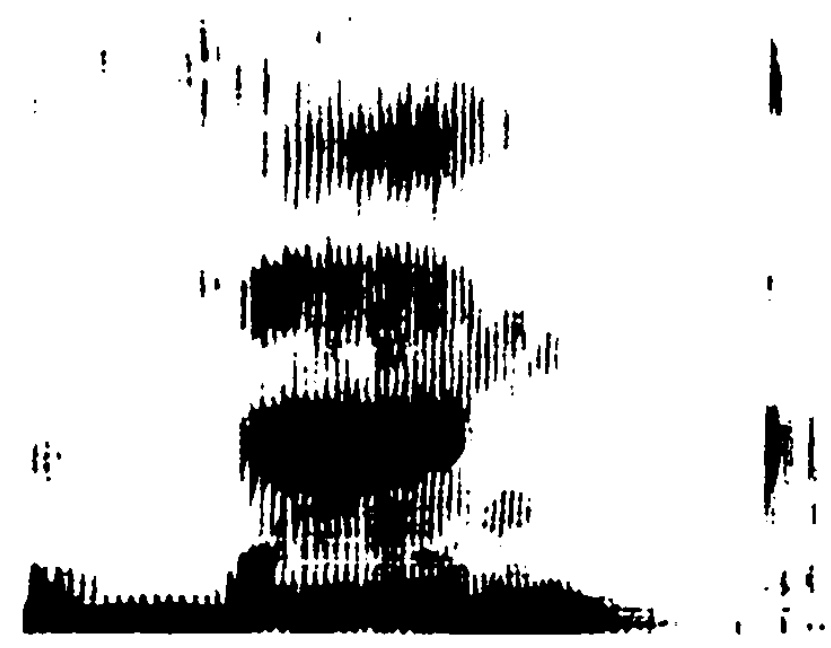

b. [7an] 

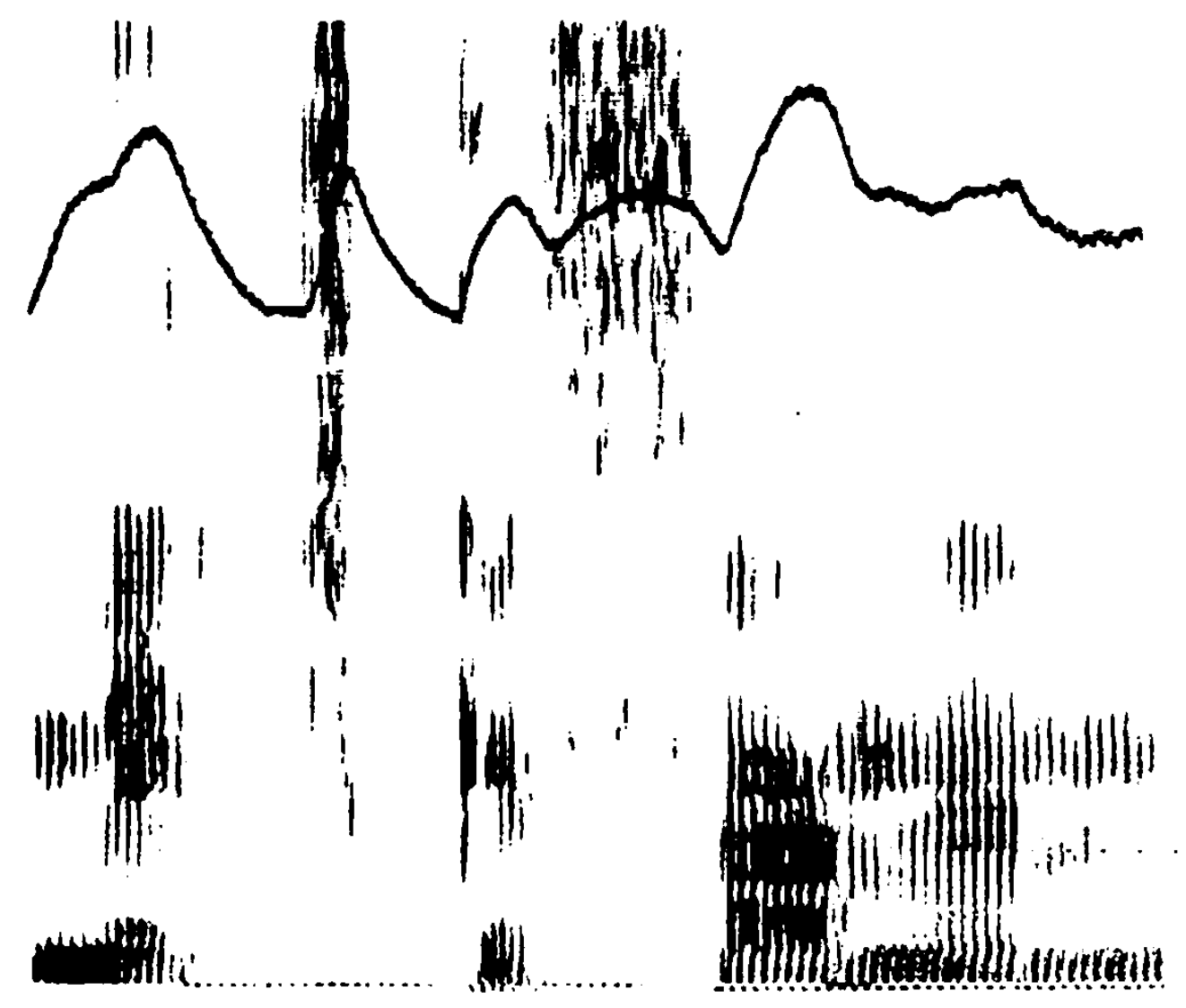

d. ['mitgafanan]
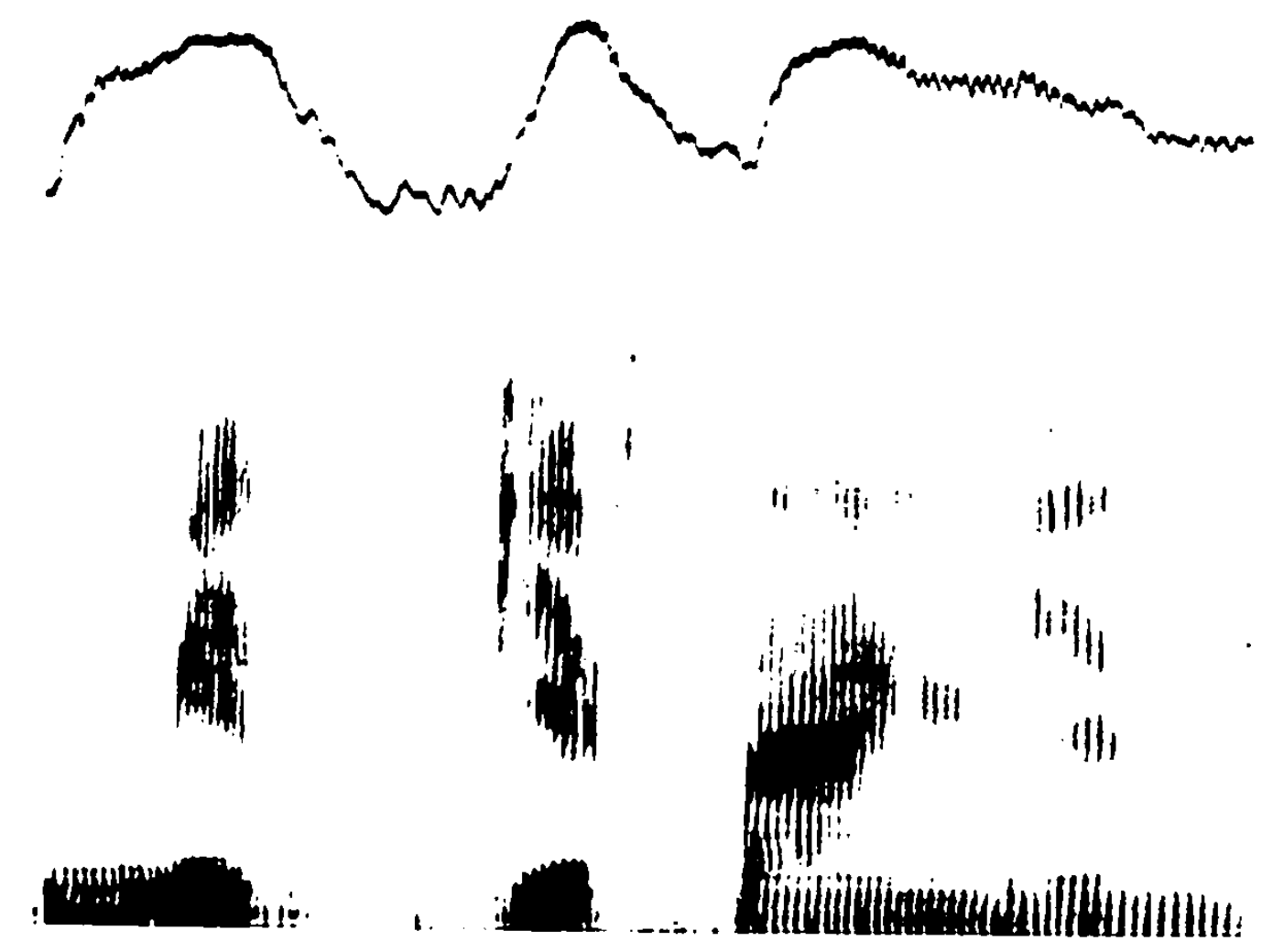

b. ['mitgəfangən] 
d. [1] vor [i]. [c]

b. (II] vor [i]. [c]
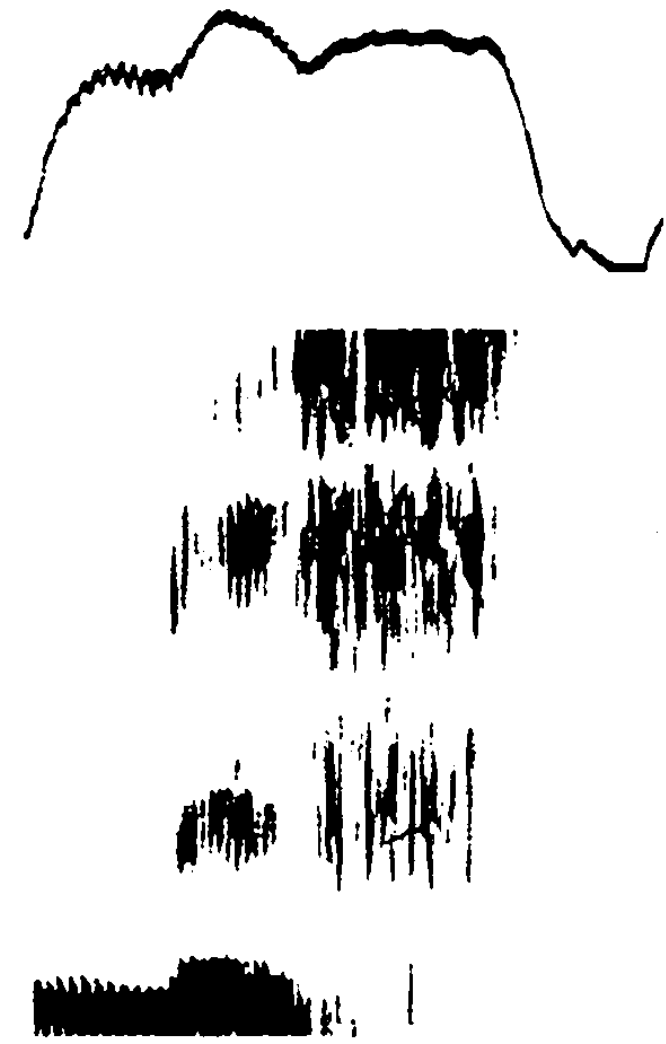

[Itst]
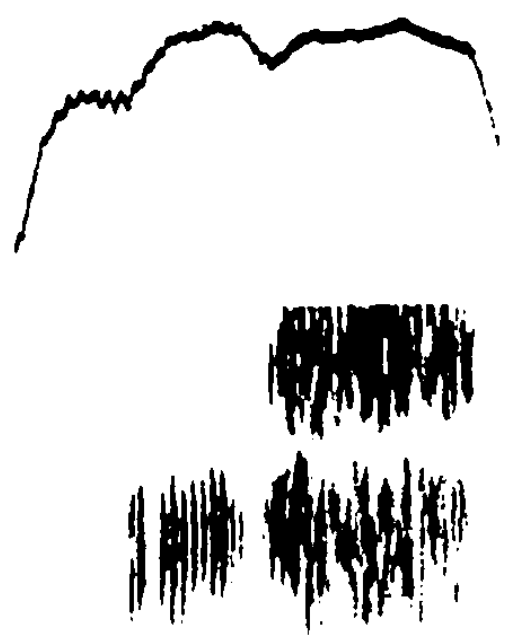

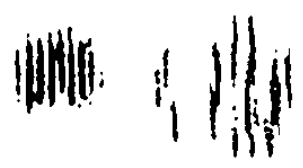

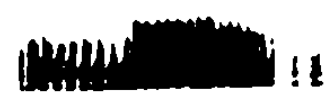

[IIs(t)]

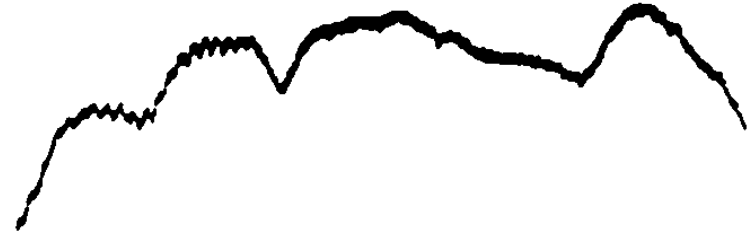

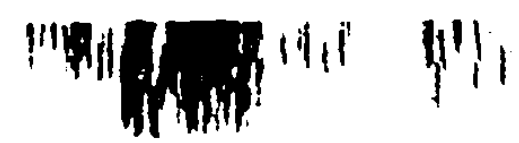

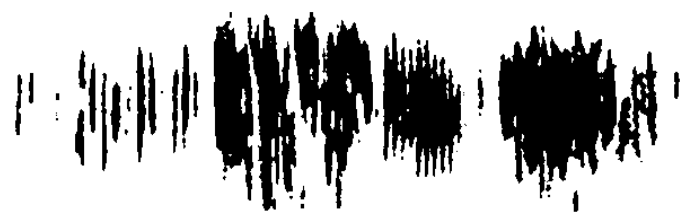

1 mint

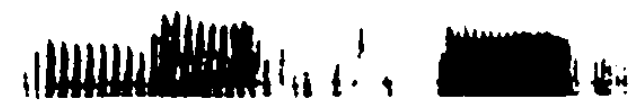

['|ısıç]
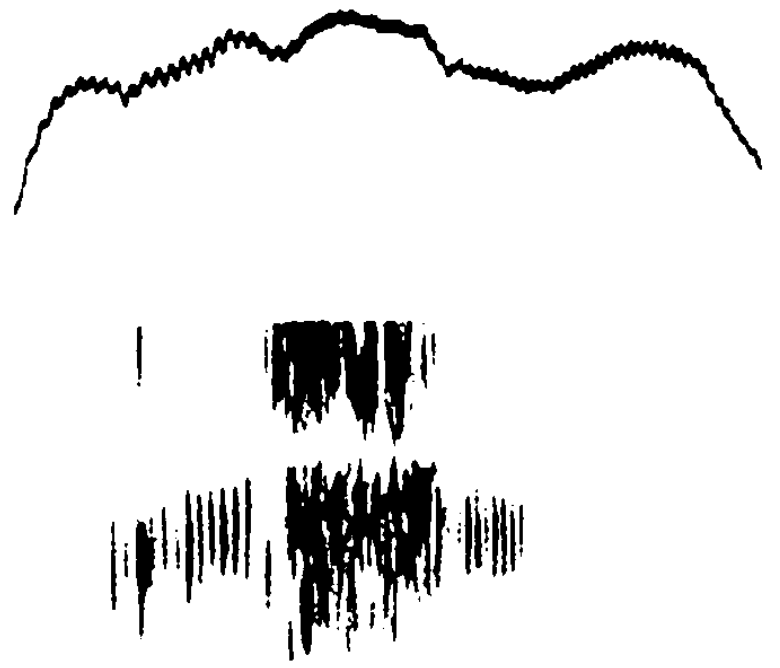

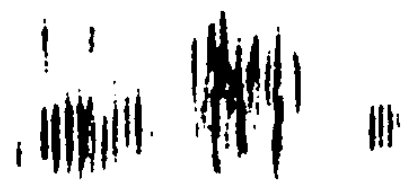

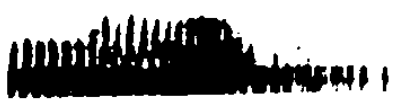

['lesen] 
d. [I] vor [a]. [u]

b. [t] vor $[a] .[u]$
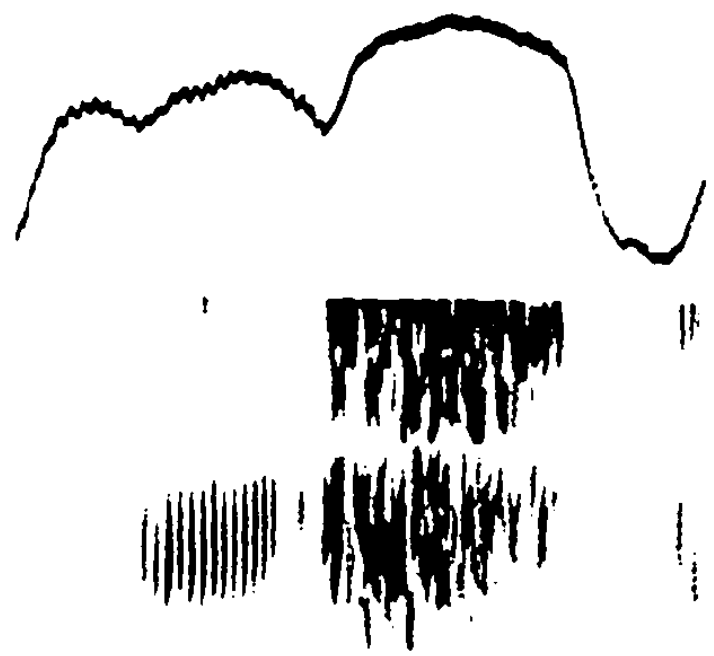

Hillinipi:

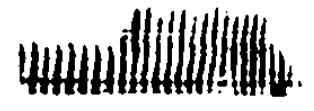

[lasi]
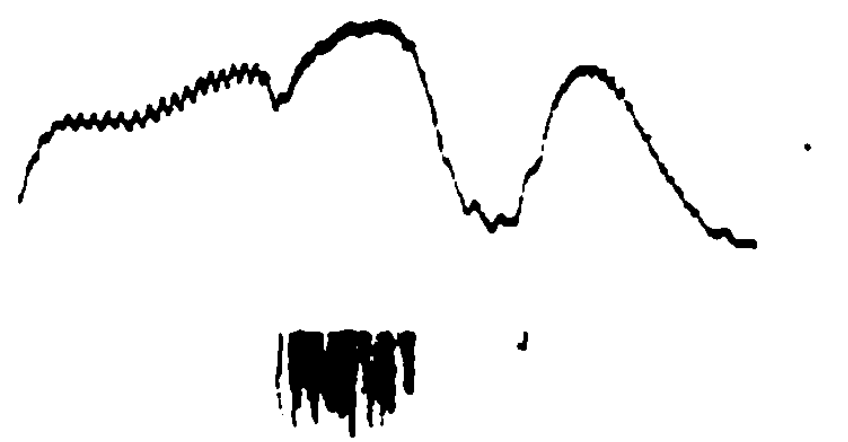

I" || |ी

IIII

it

III.

IIIiililijiliiji...

['lasti(k)]

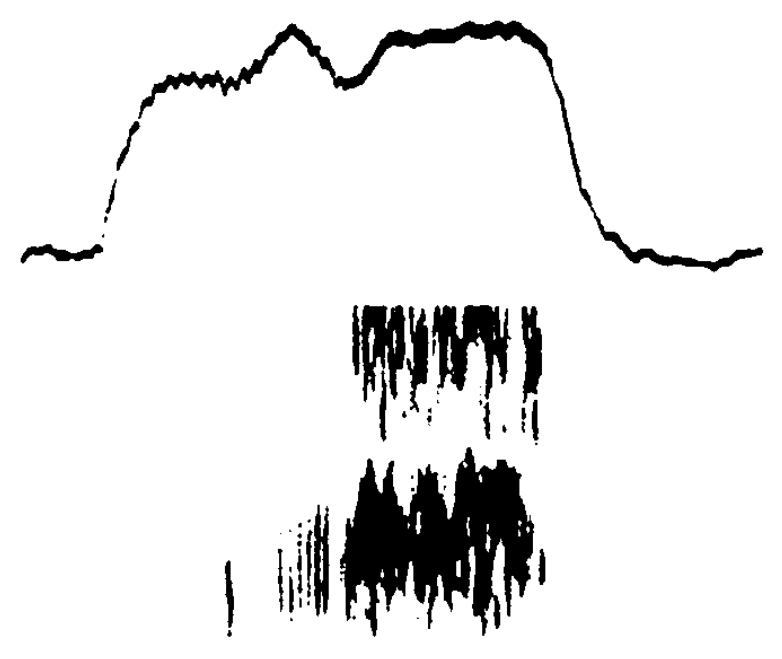

Minnin!)

[lost]

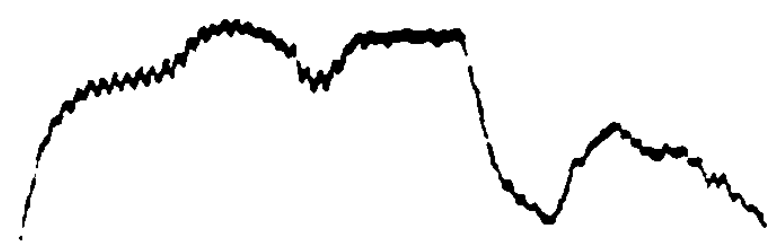

背俳

i 1

1

I

|

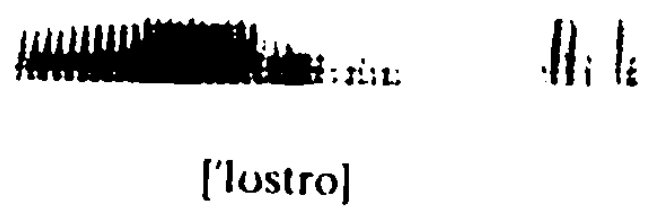


d. [I] vor [a], [u], realisiert von einem Bulgaren

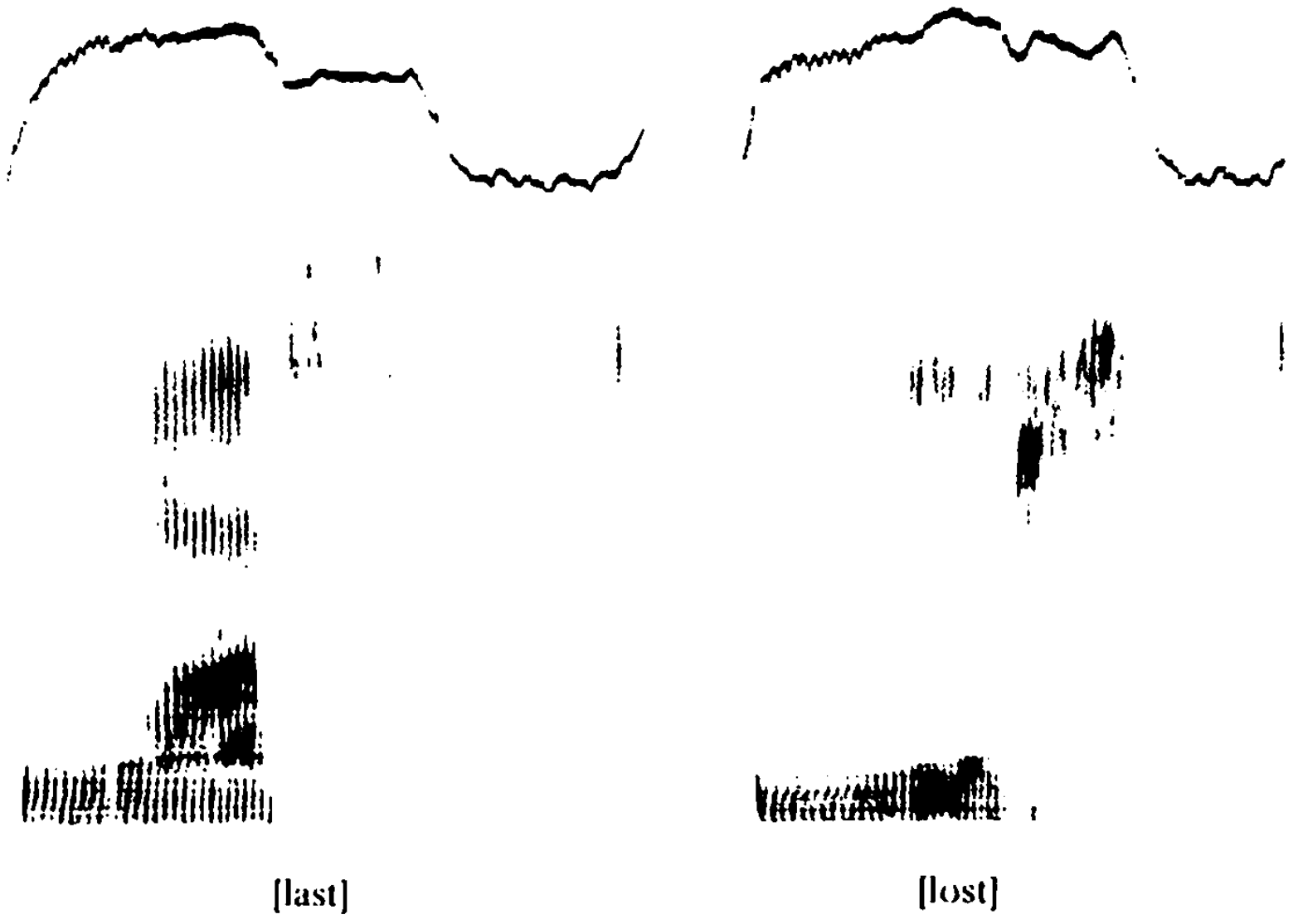


b. [l'] : люляк, пльосна

d. [I] vor [Y], [œ] : Lüftc, plötzlich
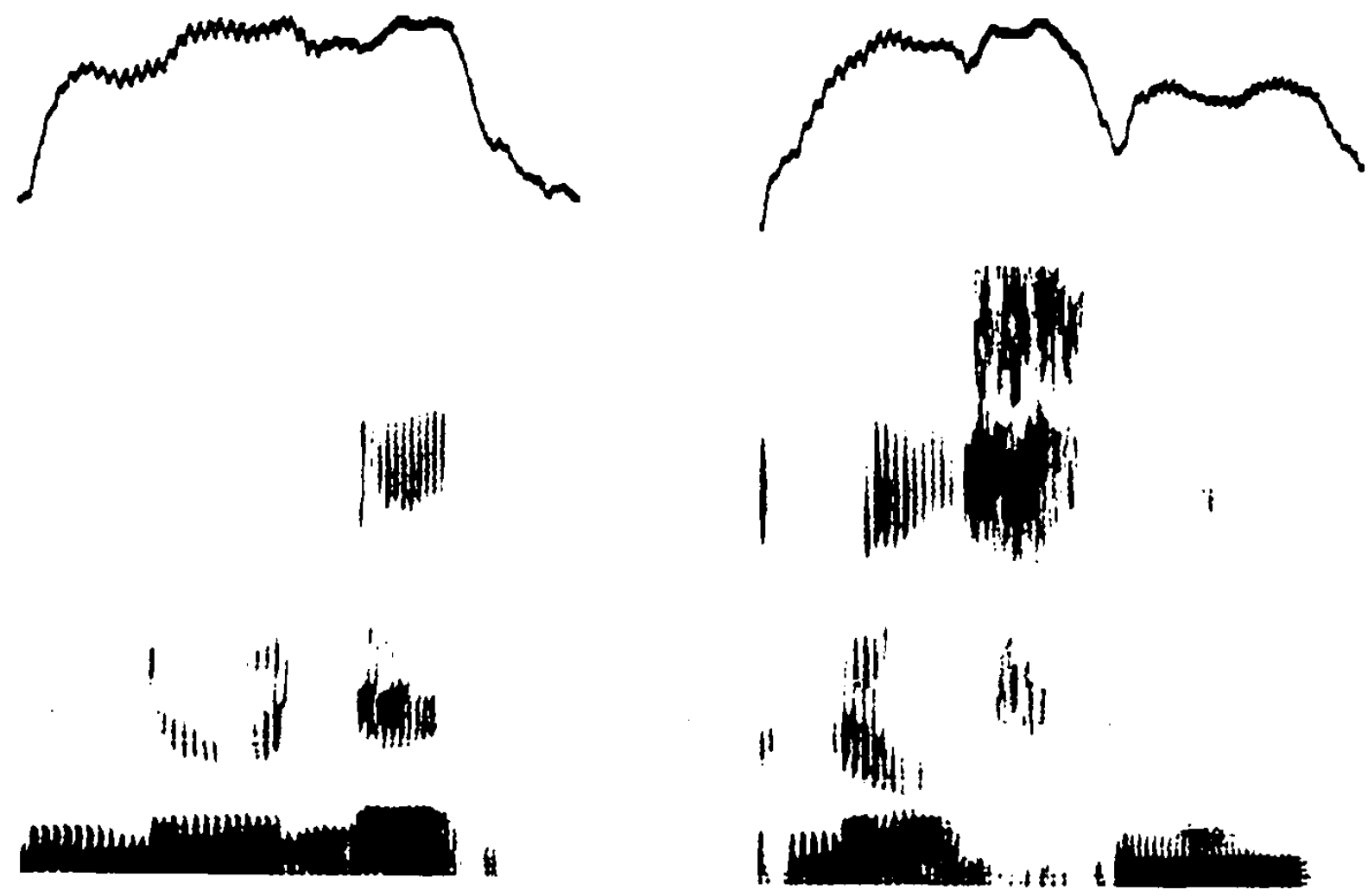

['l'ol'ak]

['pl'oesna]
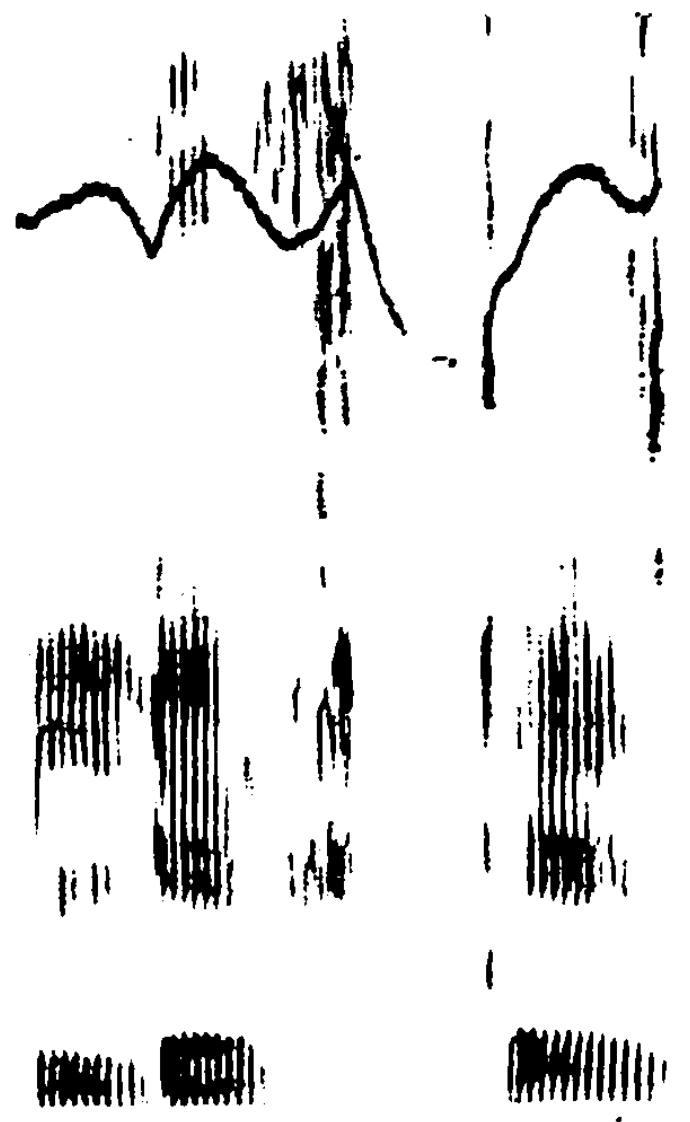

['lyfto]

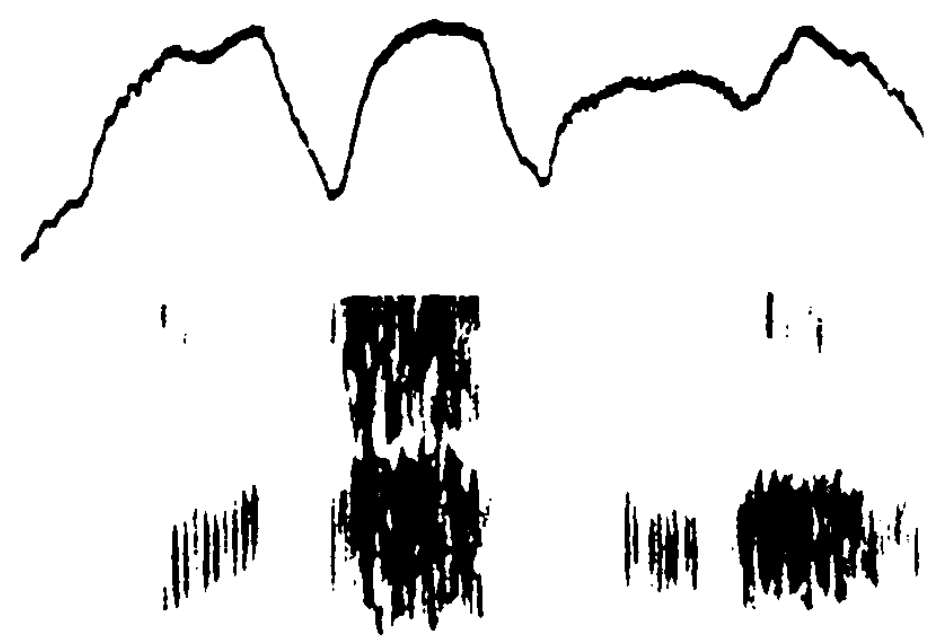

jollingy

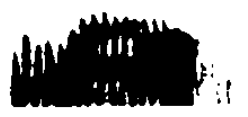

['ploctsliç] 


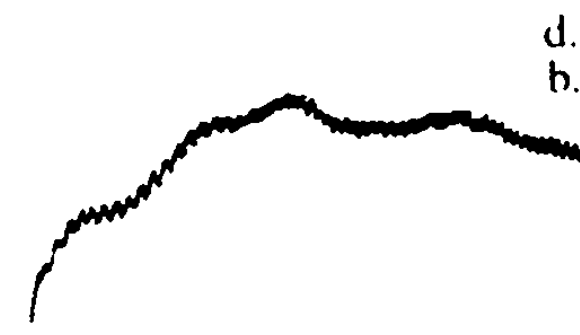

d. uvulares $[r]$ im Anlaut

b. dentales [r] im Anlaut

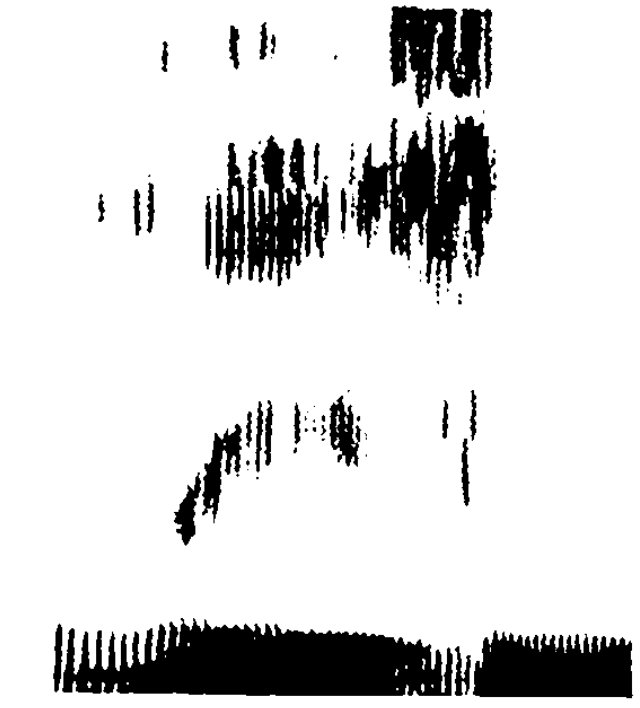

['ri : zan]

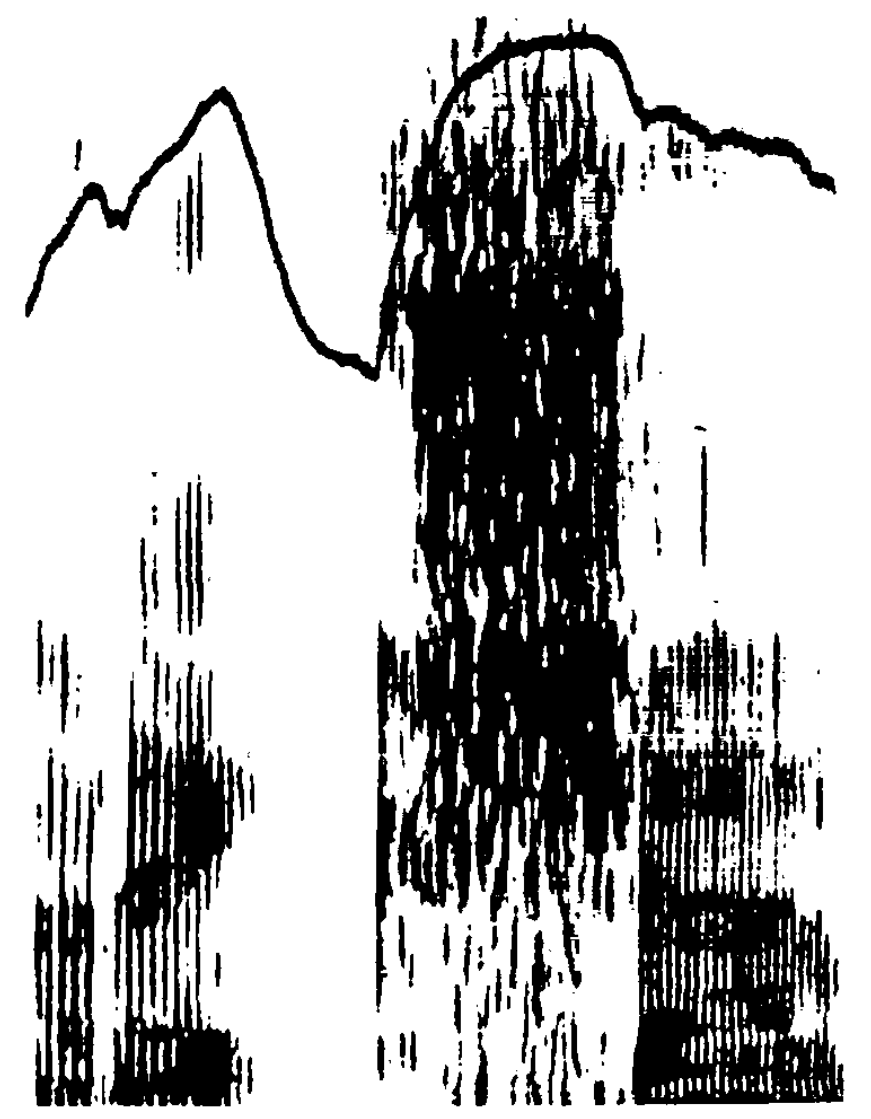

$[r a: t]\left(\int p j^{r} t\right)$
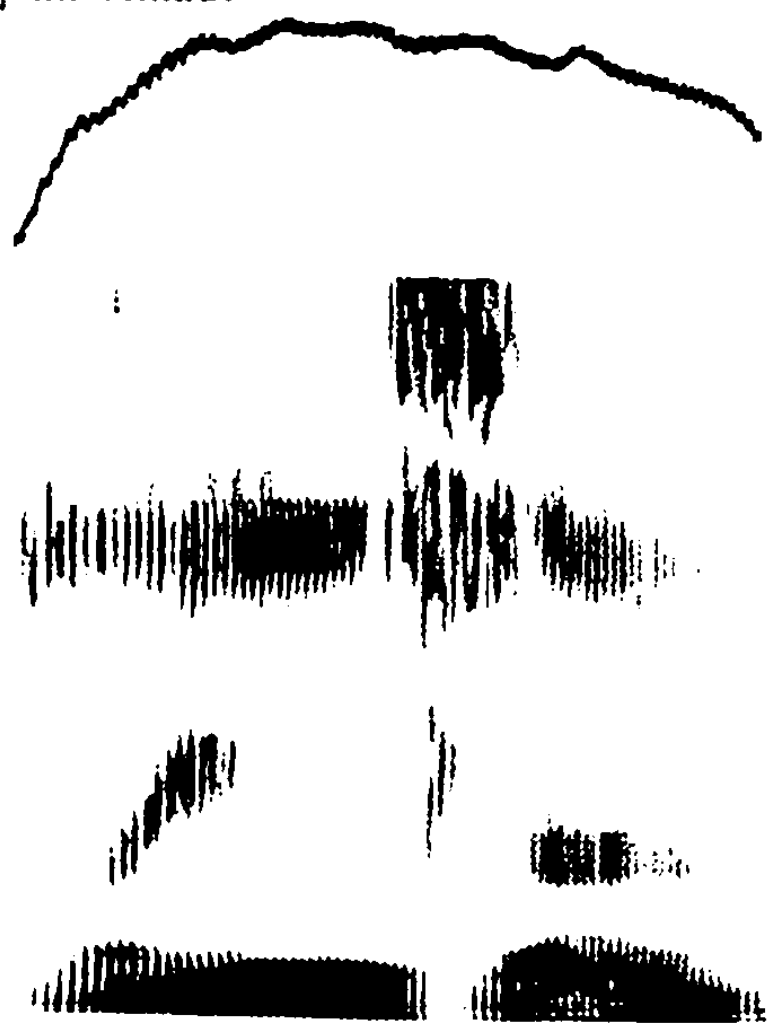

['riza]

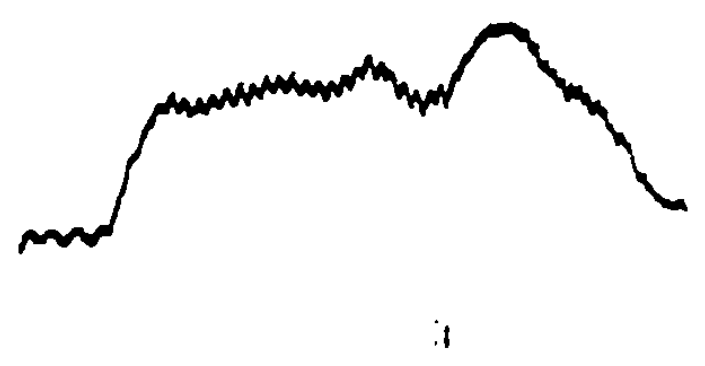

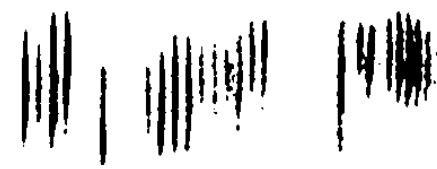

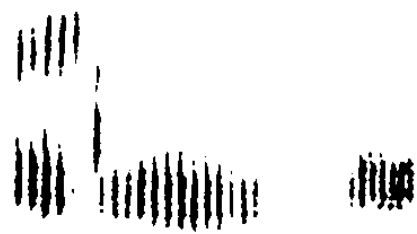

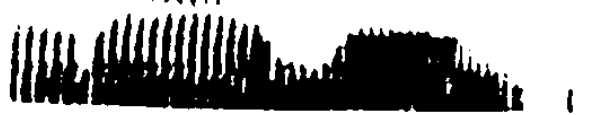

['rada] 


\subsection{VERSUCHSBEISPIELE FÜR DIE INTONOGRAPHISCHE ANALYSE DER AKUSTISCHEN PARAMETER DER DEUTSCHEN UND DER BULGARISCHEN VOKALE}

\section{Deutsche Versuchsheispiele}

Musterbeispiel für den terminalen Rahmenausspruch:

Sag: Bekennen.

bekennen, benennen, bedienen, bewiesen, betupfen, berupfen, bedanken, beranken, getarnt, gewarnt, gegolten, gewollt, Gebelle, Geselle, Getuschel, Gerumpel, Getöse, gewöhne, Gebühr, Geschwür, gekürzt, gewürzt, gebürtig, gewürzig. Babette, Barett, dadurch, dafür, da capo, Damasten, Gagarin, Galaktik, Pakete, Palette, Kabarett, Kalahari, Tabelle, tangieren, Tabular, Tammerfors, tapezieren, talentiert, Papageno, parabolisch, Bikini, binieren, didaktisch, dynamisch, gigantisch, Giraffe, Pikanterie, Pirandello, Tippelei, Tischlerei, titulieren, stimulieren, dekorieren, demontieren, tektonisch, terroristisch, Ketose, Kerosin, pedantisch, pelagisch, Bogota, Boloskop, bombast, Bordell, Botanik, Bonaparte, dokumentieren, dominieren, gotizistisch, goliatisch, Podometer, Polonäse, populär, Popelin, Popularität, Polarität, theokratisch, theoretisch, Totalität, Tonalität, Böttcherei, Börseaner, Köplin, Köslin, typisieren, Tyrranei, Pygmalion, Pyrometer, düpieren, dynamisch, fabulieren, Favoritin, fidel, Finesse, Fassade, fatal, fibril, Ferment, fokussieren, formulieren. figurieren, visieren, vagabundieren, valutieren, Futurist, Fummelei, verbessern, verlieren, verblasen, verlagern, Abitur, Animator, akademisch, alarmieren, egozentrisch, Enormităt, Epigone, Episode, epenthetisch, eremitisch, erkennen, ernennen, Ideal, Illegalität, Operette, Omelette, ökologisch, önologisch, überdecken, überlegen, umgittern, umwittern, Utopie, Urologie, Klage, klare, Klappe, klaffe, Poppe, poche, bette, belle, lege, bitte, bimme, liege, Miene, möge, Göre, möchte, löfle, Rose, rote, lüge, Mühle, Lücke, Küsse, berichtete, entrechtete, rebellieren, referieren, regulieren, resulieren, Rikarda, Rinaldo, robuste, Rosine, Mäcki, Kikeriki, Juni, Juli, Mississippi, Kalahari, Sambesi, quasi, Vati, Haiti, Risiko, Echo, lebendig. Lever, Labor, Lanolin, Libelle, Lisette, Lokomotive, Lollobrigida, Lukkulus, Lumumba, Madonna, Manometer, Medizin, Melitta, Mikado, Milano, modern, Molekül, möblieren. Müllerei, natürlich, Navigator, negativ, Nevada, nitrieren, Novalis, nivelieren, Notar, radieren, raffinieren, rumänisch, Achromatin, Affinität, Alexandriner, fakultativ, Barometrie, paralisieren, Tabulator, Gravitation, alkoholisieren, Astronomie, Faszination, Gastronomie, harmonisieren, Pantheismus. Saxophonist, Transformation, Propaganda, Thema, Medusa, Aorta, Terrakotta, Influenza, jemand, niemals, jemals, Observatorium, Objektivist, Kosmopulit. korrelativ, formalisieren, normalisieren. Somnambulismus, Dissident, distinkt, Instinkt, instruktieren, Disput, intakt, integral, historisch, Dissertation, Disziplin, Fiktion, Firmanent, intellektuell, Examen, Exemplar, eskalieren, Ergonomie, Festival, Fetischismus, generalisieren, heterogen, melodisieren, Sensation, Separatist, Substanz, Vulkan, Pulsator, punktieren, Wulfenit, Ruptur, pulmonal, Puritaner, substituieren, vulkanisieren, multiplizieren, Musikalităt, mumifizieren, Kuriosität, Universum, Gremium, Laboratorium, Forum, Datum. Kaktus, Spiritus, Ameise, Sinus, Fanatismus, immerzu, dazu, Byzantiner, dynamisch, 
Dyskinese, Dyslalie, Gynäkologie, Gymnasium, Hypothese, Hysteriker, Kybernetik, Kymographie, Lymphadenie, Pyramide, systematisch, systematisieren. Symmetrie, symbolisieren, synchronisieren, synthetisch, systematisch, tyrannisieren, Polyglote, Pyrolyse, Önometer, Pönologie, Röntgenologie, ökonomisch, Zökotomie, Zölibat, Zölom, Zönokarp, Obszönität.

\section{Bulgarische Versuchsheispiele}

Musterbeispiel für den terminalen Rahmenausspruch:

Пак: баданосвам.

баданосвам, баламосвам, беглик, безлик, битопис, биволар, бобовиден, боровинка, бъбривка, бъзливка, бюрек, бюфет, вагонетка, варовит, вековсчен, вероломен, видение, винетка, водопой, воловар, вокален, волани, вулкан, вулгарен, въдичар, възвишен, възкликвам, възлизам, вялост, вялостта, гатанка, гаванка, геберясвам, гешефтар, година, горила, губерка, гурелив, гъдулар, гърнета, гювеч, гюрук, датирам, дафинов, дебелана, десетачка, диканя, дилафи, догарям, долавям, дукат, духач, дъбак, дърва, дюкян, дюшек, дяволит, дяволски, единство, езиковед, еталон, ешафот, игумен, изучен, кабаре, кавалер, кебапче, кенарче, кикимора, килимар, кокичка, количка, кукувам, кумувам, къпина, кълчиша, кютюк, кюмюр, лагуна, лазура, легенда, лежешком, лековит, лесовъд, литак, ливада, лопата, лозата, луга, луна, льки, льчи, любезен, люлеян, магарица, маларичен, медовина, мелодичен, митичен, миниран, мислителят, мотив, молив, мутолевя, мухоловка, мътило, мъчило, мюфтия. мющерия. набожен, народен, негоден, неволен, никелирам, нивелирам, необходим, обитавам, онемявам, пакет, палет, патило, парило, пепелища, пелерина, питон, пирон, победител, посетител, пукало, пушкало, пъкии, пълни, редовен, резонен, родина, Розина, рутина, рушител, ръкавица, ръченипа. табан, тамам, тека, теча, титанически, тиранически, топило, точило, тупалка, тухларка, тъпан, тъпея, тънея, тютюнджия, тюрлия, убедителен, увеличителен, укоря, уловя, фотограф, фонолог, факирски, фамилен, федеративен, феминизиран, фигаро, филантроп, фугасен, фуражен, юбиляр, ювелир, ягодар, язовир, ятаган, яхния, бака, бала, бате, Лене, бабо, мамо, куку, шущу, снощи, снопи, лепя, леля, абсурд, ампула, баприло, бакла, балкански, цразнител, драскулка, кандисвам, разграбвам, автомат, антиквар, активист, баклава, баснописеп. вампирясвам, дандания, даскалувам, махмурлия, мармеладен, абонамент, акумулатор, алитерация, благоухание, далечина, мамалигар, магнитофон, нахалността, активността, картотекирам, квантитативен, парламентарен, разгорецявам, безреден, безсилен, германец, ментовка, мерджан, междина, безотговорен, мержелея, меркантилен, междуведомствен, германофил, оставам, болнав, достигам, нормирам, нослето, новдигам, подслаждам, роднина, обвинител, обгорял, плоскодънен, рождество, облагодетелствам, обособявам, побратимявам, простонароден, романизиран, торпедоносец, организиран, огнепоклонник, отдалечавам, постановление, амнистирам, аплодисмент, билкар, близнак, взискателен, игрище, избирам, мислител, аритметичен, диктатура, дип:омат, иглолистен, избирател, истукан, високомерие, височина, диагонал, идеалист, киселина, криминалист, миролюбив. библиограф, 
дезинфектирам, дискриминация, издадина, пипкавостта, пискливостта юни, юли, Тони, моди, макарони, гатанки, съюзници, раци, леяри, двуичник, двувластник, Видин, триптих, Талин, мамин, тепих, субсидирам, сурвакар, суспендирам, рудничар, публикация, пустословя, неустрашим, умопобъркан, уподобявам, усамотен, суматоха, руменина, рудокопач, увеличителен, кукурузяк, рунтавостта, пухкавостта, пукнатина. долу, лулу, кактус, Бакхус, максус, стълкновение, стьрготина, пъстропол, пъстроцветен, първомайски, пълзешката, повърхнина, несъвместимост, льскавина, ръководител, льжовността, кръвопролитен, зъболечение, продължителността, първопричина, объркаността, нетърпеливостта, нихилизъм, ученикът, студентът, пъкъл, мокър, остър, нисък, нисичък, налъм, умисъл, топличък, пелена, ядене, планина, на часа, софра, сьрце, крилще, дете, чети, отиди, донеси, запали, плета(ъ), мерхамла(ъ), махала, хоро, кросно, палто, защо, Перу, банту, конфу.

Beispielsliste für die konfrontative Analyse deutscher Vokale, gesprochen von Bulgaren

Kommst du heute? Alter schützt vor Torheit nicht.

Wer hat es getan? Aufgeschoben ist nicht aufgehoben.

Wie der Anfang, so das Ende. Stille Wasser sind tief.

Im ersten Augenblick. Der gerade Weg ist der beste.

Sie hebt mich in den Himmel. Durch Lehren lernt man.

Schön ist der Frühling.

Höfliche Worte vermögen viel. Röslein auf der Heiden.

Ein König hatte drei Söhne. Das Glück hat Flügel.

Ein Jüngling ist ein junger Mann. Zurück zur Natur.

Lust und Leid und Liebesklagen. Er ist sehr übermütig.

In der Kürze liegt die Würze.

Die Ü-Laute müßte man tüchtig üben.

Wir haben es in Hülle und Fülle. Viele Hirten übel gehütet.

Zwei harte Steine mahlen selten reine.

Morgen, morgen und nicht heute - sagen alle faulen Leute.

Freunde erkennt man in der Not. Ubbermut tut selten gut.

Beredter Mund geht nicht zugrund.

Der erste Trunk macht gesund.

Der zweite Trunk macht fröhlichen Mund.

Der dritte Trunk macht den Menschen zum Hund.

Kleine Sorgen machen viele Worte, große sind stumm.

Hab' ich ist mir lieber als hätt' ich.

Wer die Wahl hat, hat die Qual.

Sie legt die Hände in den Schoß.

Glück und Gias, wie leicht bricht das.

Er sagt es durch die Blume.

Es ist noch nicht aller Tage Abend.

Der erste Gedanke ist immer der beste.

Fliege, niege, weiße Friedenstaube!

Ein jeder fege vor seiner Tür.

In der Not schmeckt jedes Brot. 
Wenn die Sonne scheint, erbleicht der Mond.

Er hat die Flinte ins Korn geworfen.

Er hat sie gut im Zug.

In den Märchen sind alle Königstöchter schön.

Das ist meine wöchentliche Löhnung.

Wohin gehen die Leute?

Er läßt sich öfters öffentlich hören.

Wo Frösche sind, sind auch Störche.

Der Schuster hat die schlechtesten Schuhe.

Das macht er aus lauter Angst.

Woher kommen deine Studenten?

\subsection{BEISPIELE ZUR SPEKTRALANALYSE DER DEUTSCHEN UND DER BULGARISCHEN KONSONANTEN}

Bulgarische Beispiele

Пана, Тана, Кана

пир, тир, кир

праг, трак, крак

плача, клатя, тласък

фас, ваза, 6аза

ефект, лава, браво

йога, Йосиф

яке, юни

Йена, йезуит, Йемен

ихтиол, вехт, зехтин

хитър, Хемус, хей

хубав, хала, хол

хайде, хойкам

Цеца, Цуца, цаца, цоца

Цена, Цона, цум, цял

цвете, цвик, цвръкна

стена, стон, ствол

скверно, скреж, скрънза

чам, чер, чим, чума, чоп

чвор, член, чрез

твар. твой, (из)ткан

тлен, тласък, сплав

сврака, скреж, скромен,

пре3, стрес, страна, здраве

тщеславие

сцена, стена, ствол, страх

оценка, отсенка

вчера. пчелен, пчела

отчел, птица, ктитор

отнел, отсял, отвял

отшелник, отчитам

втори, втривам, втласквам 
счукан, стик, стяг коч, кос, кост, карст здрач, смърч, хленч мра3, Марс, март танц, танк

куче, копче, конче

птичка, Лучка, Мичка

очи, оси, уста, капси

капка, молци, Ломско

танц, гланц, принц

мац, виц, куц, жрец

кос, вис, кост, швепс

Марс, март, Маркс

карст, сфинкс

птица, каца, буца

лице, яйце

каса, бута, буря, бури

буза, боза

вакса, вапцах

капка, капса, капра

капла, фръцла

лист, лесен, лешник

ластик, лустро, лос

лайка, лейка, Лойко

люляк. лято

ало, Оля, Алеко, илария

улица, алхимия, алт

Аляска, Илюшин

плац, плет, плуг, плот

плясък, пльосна, плюска

влак, влек, вливам

влудявам, влог

вляво, влюбен

благ, близо, блуза, блесна

блясък. блюстител

глас, гледам, глист, глупав

длето, длабка

жлеб, жльч

злоба, сльч, зла, зли

клатя, клисав, клепка, клуб

клюка, Клянтев

алабаш, Ели, ало, ела, улей

халба, халва

балдисвам, балтия

Елза, Хелга, елка

алфа, елха, елша

бал. бел, чул, вол

бял, лебед. бял. лен 
риза, роза, Рада

лекар, мокьр, кукер

Ангел, ангария, мангал

чик, щик

uар, стар, чар

Цена, стена

плача, плаща, плаща

кача, каца, каста

плач, пласт, плащ

плачове, плащове

плацове, пластове

плачове, пластове

плача, плаща

плаца, пласта

кача, къша

капса, такса, ксерокс

пшеница, пчела

на рикша, нарича, на Лиска

Bakca. Banca

Baxca, Banua, Baua

Deutsche Beispiele

Panne, Tanne, Kanne

Pilz, Tinte, Kind

Pracht, Tracht, Kraft

Plage, Klage, Atlas

FaB, Vase, Base

äffen, Lava, bravo

Joga, Joseph, Jürgen

Jacke, Juni, Jubel

Jena. Jesuit, Jemen

Ichtiol, Hecht, Heimchen

Ich spreche den Ich-Laut richtig.

mit Haut und Haar

Himmel und Hölle

hoch und heilig

Hinter Heinrichs Hühnerhaus

hängen hundert Hemden 'raus.

Pfanne, Panne, Fahne

Plage, Pracht, Psyche

Zahn, zehn, Zucker

Ziege, Zögern, Züge

Trab, Trieb, TschüB

Tscheche, Chomsky

Opfer, offen, Ibsen

ätzen. Achse. Ozean

atmen, Atlantik, attraktiv

Dampf, Kopf, Strumpf 
Damm, scharf, Schilf

Griff, Treff, Schlaf

Gips, Knicks, links

Fuchs, Mops, Papst

Salz, Holz, Kauz

Tanz, Nerz, Sturz

Sitz, Schutz, Schulz

Putz, Puff, Putsch

Schulz, SchuB, Puls

Matsch, Kitsch, Rutsch

Mann, muß, Muff

Bach, Wachs, Busch

Köpfe, Griffe, Möpse

Hölzer, Walser, Faser

Schwärze, Würfe, Ferse

watschen, watten, waschen

Wasser, Waffen, wallen

List, lässig, Lessing

Last, Lust, Los

allein, alles, Eule

Ulrich, also, Alltag

plagen, Pflug, plötzlich

Ball, Welle, Stille, Stahl

Die linden Lüfte sind erwacht.

Klinge, kleines Frühlingslied!

Eine Last, wohl gefaßt, ist nur eine halbe Last.

Du bist wie eine Blume,

so hold, und schön, und rein.

Angel, Ängel, Bängel

jung, Klang, bange

bang, Bann, Bank

sang, sann, sank

Mitgegangen, mitgefangen, mitgehangen.

Lieben und Singen läßt's sich nicht erzwingen.

Jung gelungert, alt gehungert.

Riesengebirge, Redefreiheit

Regenwurm, Regenschirm

Räderwerk, Radsport

Mohrrübe, Widerrede

vereisen, verreisen

erröten, erörtern

Wart, Wacht, wurden, wuchten

fort, focht, Bord, pocht

weiter, Weite

bitter, Bitte

leiser, leise

Dürres Reis gibt rasches Feuer. 
Guter Rat ist teuer.

Pfennig, Pfingsten, Pforte

stopfen, klopfen, rupfen

Bausch, Rausch, Frosch

Tschibo, stieben

Zehner, Stendal

klatschen, Klaster

glotzen, Kloster

Katze, Kutsche, Küste

Motsch, Most, Mops

platschen, Plattform

klatschen, klapsen

klatschen, Rikscha

Rikscha, Taxe, Tatze 


\section{LITERATUR VERZEICHNIS}

Allgemeine Sprachwissenschaft. Bd. II. Autorenkollektiv unter der Leitung von B. A. Sercbrennikow. Berlin, 1975.

Antoniadis, Z., H. W. Strubc. Untersuchungen zur speziflschen Dauer deutscher Vokalc. - Phonetica, 41, 1984, 72-87.

Barry, W. J. Perzeption und Produktion im subphonemischen Bereich. Tübingen, 1974.

Bergmann, R., P. Pauly. Neuhochdeutsches Arbeitsbuch zum linguistischen Unterricht. 2. Aufl. Göttingen, 1975.

Bethge, W. Das Abhören von Lautmelodic und Silbenmelodic. - ZPSK, 1953, H. 5/6, $320-326$.

Bethge, W. Uber ahgehörte und gemessene Lautmelodic. - ZPSK, 1953, H. 5/6, $339-346$.

Bierwisch, M. Skizze der generativen Phonologie. Berlin, 1967.

Bocl, J. Etude de linteraction source laryngienne - conduit vocal dans la détermination des caractéristiques interinseques des voyelles orales du francais. - Bulletin de l'Institut de Phonetique de Grenoble, I, 1972.

Bojadschiev, T. Zum Bestand der weichen Phoneme in der bulgarischen Literatursprache. - ZPSK, 21, 1976, $722-724$.

Burgschmidt, E., D. Götz. Kontrastive Linguistik Deutsch/Englisch. Theoric und Anwendung. München, 1974.

Die deutsche Sprache. Kleine Enz.yklopädic in zwei Bānden. Bd. 2. Leipzig, 1970.

Dietrich, G. [c] und [x] im Deutschen - ein Phonem oder zwei? - ZPSK, 7, 1953, $28-37$.

Einfuhrung in die Sprechwissenschaft. Autorenkollektiv. Leipzig, 1976.

Endler, D., H. Walter. Wörterbuch Bulgarisch-Deutsch. 3. Aufl. Leipzig. 1984.

Essen, O.v. Über die spezifische Schallwirksamkeit der Laute. - ZPSK, 1953, H. 1/2, $81-88$.

Essen, O.v. Allgemeine und angewandte Phonetik. Berlin, 1962; 5. Aufl. 1979.

Essen, O.v. Grundzüge der hochdeutschen Satzintonation. 3. Aufl. Ratingen/Düsseldorf. 1964.

Fairbanks, G., A. S. House, E. K. Stevens. An experimental study of vowel intensity. - JASA, 21, 1950.

Fant. G. Theory of Speach Production. 's-Gravanhage, 1960.

Fischer-Jorgensen, E. Objektive und subjektive Lautdaucr deutscher Vokale. Arch. vgl. Phon., No 1, 1940.

Fischer-Jorgensen. E. Phonologic. - In: G. Heike. Phonetik und Phonologic, Heidelberg. 1974, 164-178.

Fischer-Jorgensen, E. Phonetische Grundlagen zur Bestimmung phonemischer Elemente. - In: G. Heike. Phonetik und Phonologic. Heidelberg, 1974. 60-98.

Fon a gy. I. Electro-physiological and acoustic correlates of stress perception. - In: J. of Speceh and Hearing Rescarch. 1966. 
Fiuk owski, H. Sprecherzieherisches Elementarbuch. Leipzig, 1978.

Forchhammer, J. Theoric und Technik des Singens und des Sprechens. Leipzig. 1921.

Geratewohl, F. Richtiges Deutschsprechen. Leipzig, 1937.

Gerhardt, D. Phonemtheoric und Phonologic. - Arch. vgl. Phon., 5, 1941.

Gerhardt. D. Phonometric und Phonologie. Berlin, 1963.

GroBe, R. Das phonematische und orthographische System der deutschen Gegenwartssprache. - Germanica Wratislaviensia, I1, Wroclaw, 1967, $119-129$.

Großes Wörterbuch der deutschen Aussprachc. Leipzig, 1982.

Grundzüge einer deutschen Grammatik. Autorenkollektiv. Berlin, 1984.

Häusler, F. Die Begriffsbestimmung der Artikulationsbasis - phonetisch oder phonologisch? - Innbrucker Beiträge zur Kulturwissenschaft. Sonderheft, 15, 1962, 213-221.

Heike, G. Das phonologische System des Deutschen als binäres Distinktionssystem. - Phonetica, 6, 1961, 162-176.

Heike, G. Sprachliche Kommunikation und linguistische Analyse. Heidelberg. 1969. Heike, G. Phonologie. Stuttgart, 1972.

Halle. M. Die Strategie der Phonemik. - In: G. Heike. Phonetik und Phonologic. München, 1974, 179-195.

Horalck k, K. Zum Begriff der phonologischen Korrelationen. - Traveaux linguistique de Prague, 1966, 2, $111-120$.

House, A., G. Fairbanks. The influence of constant environment upon the secondary acoustical characteristics of vowels. - JASA, 25, 1953, 1.

Husson, R. Zur Spektralstruktur menschlicher Vokale aller Stimmstärken. - Phonetica, 10, 1963, 4-21.

Hutterer, Cl. J. Der Vokal der Indifferenzlage in den germanischen Sprachen - Phonem oder Allophon? - Phonetica, 13, 1965, 46-49.

livonnen, A. Experimente zur Erklärung der spektralen Variationen deutscher Phonemrealisation. Helsinki, 1970.

Is ačenk o, A. V. Hat sich die Phonologic überlebt? - In: G. Heike. Phonetik und Phonologic. München, 1974, 196-218.

Jak obson, R., G. Fant. M. Halle. Preliminaries of Speech Analysis. 1952.

Jak obson, R., M. Halle. Grundlagen der Sparche. Berlin, 1960.

Jak obson, R. Bemerkungen zur phonologischen Klassifizierung der Konsonanten. - In: G. Heike. Phonetik und Phonologie. München, 1974, 156-163.

Jcspersen, O. Lehrbuch der Phonetik. Leipzig, 1913.

Jones, D. Die Theorie der Phoneme und ihre Bedeutung für die angewandte Linguistik. - In: G. He ike. Phonetik und Phonologic. München, 1974, 45-46.

Kohler, KI. J. Einführung in die Phonetik des Deutschen. Berlin/West. 1977.

Kohler, Kl. J. Phonetic Explanation in Phonology: The Feature Fortis/Lenis. - Phonetica, 41, 1984, 150-174.

Koschmieder, E. Bemerkungen zur Aussprache des Bulgarischen. - Zschr. f. vergl. Sprachforschung auf dem Gebiet der indogerm. Sprachen. Göttingen, 1951/3-4, $216-224$.

Kozhevnikov, V. A., L. A. Chistovich. Spech: Articulation and Perception, 1965.

Krech. H. Zur Artikulationsbasis der deutschen Hochlautung. - ZPSK, 8, 1974 , $92-107$.

K rech, E. M. Sprechwissenschaftlich-phonetische Untersuchungen zum Gebrauch des Glottisschlageinsatzes in der allgemeinen deutschen Hochlautung. - Bibliotheca Phonetica, 4. Basel-New York, 1968.

Kufner, H. Kontrastive Phonologie Deutsch-Englisch. Stuttgart, 1971.

Laziszius, J. Lehrbuch der Phonetik. Berlin. 1961. 
Lehiste, 1., G. Peters on. Duration of Syllable Nuclei in English. - JASA, 32, 1960. Lehiste, I., G. Peterson. Vowel Amplitude and Phonemic Stress in American English. - JASA, 31, 1959.

Lehiste, I., G. Peterson. Some basic considerations in the analysis of intonation. - JASA, 33, 1961, S. 45f.

Lindner, G. Beurteilung synthetisch erzeugter vokalartiger Klänge durch deutschsprachige Hörer. - ZPSK, 19, 1966, S. 278IT.

Lindner, G. Einführung in die experimentelle Phonetik. Berlin, 1969.

Lindner, G. Veränderungen der Formant-Intensität bei synthetischen Vokalen. - ZPSK, 23, 1970.

Lindner, G. Der Sprechbewegungsablauf. Eine phonetische Studie des Deutschen. Berlin, 1975.

Lindner, G. Urteilsveränderung durch Vokalverkürzung. - ZPSK, 29, 1976.

Lindner, G. Hören und Verstehen. Berlin, 1977.

Lindner, G. Grundlagen und Anwendung der Phonetik. Berlin, 1981.

Ma ack, A. Die spezifísche Lautdauer deutscher Sonanten. - ZPSK, 1949, H. 3/4, $190-232$.

Ma ack, A. Die Beeinflussung der Sonantendauer durch die Nachbarkonsonanten. - ZPSK, 1953, H. 1/2, 104-128.

Ma a ck, A. Höchstlautstärke und Durchschnittslautstärke. - ZPSK, 1953, H. 3/4, $213-230$.

Ma a ck, A. Die Korrelation Akzent - Quantität. - ZPSK, 1954, H. 3/4, 226-238.

Maack, A. Neue Utersuchungen über die Beziehungen des Akzents zum Melodievcrlauf. - ZPSK, 1954, H.5/6, 326-339.

Ma ack. A. Zur dcutschen Wort- und Satzmelodic. - Phonetica, 1, 1957, 230-240.

Ma ngold, M. (Bearbeiter). Duden. Aussprachewörterbuch. Bd. 6. 2. Aufl. Mannheim/ Wien/Zürich, 1974.

Ma ngold, M. Sprachwissenschaft. Berlin, Darmstadt, Wien, 1974.

Martinet. A. Synchronische Sprachwissenschaft. Berlin, 1968.

Ma t e r, E. Rückläufiges Wörterbuch der deutschen Gegenwartssprache. Leipzig. 1967.

Meinhold, G. Deutsche Standardaussprache. Formstufen und Lautschwächungen. Jena, 1973.

Meinhold. G., E. Stock. Phonologie der deutschen Gegenwartssprache. 2. Auf. Leiprig. 1982.

Menzerath, P. Die phonetische Struktur. Eine grundsätzliche Betrachtung. - Acta Physiologica. Amsterdam, 1935.

Me yer. F. A. Englische Lautdauer. Uppsala, 1903.

Me yer, E. A. Zur Vokaldauer im Deutschen. - In: Nordiska Studier. Festschrift für Norren, 1904.

Me yer, E. A. Zur Phonetik der ungarischen Sprache. Uppsala, 1909.

Me yer-Eppler, W. Zum Erzeugungsmcchanismus der Gerāuschlaute. - ZPSK, 7, $1953,196-212$.

Mohr. B. Intrinsic variations of the speech sygnal. - Phonetica, 23, 1971.

Morčinicc, N. Zur phonologischen Wertung der deutschen Affrikaten und Diphthonge. - ZPSK, II, 1958, 49-66.

Morçiniec, N. Distinktive Spracheinhciten im Niederlāndischen und Deutschen. Wroctaw, 1968.

Morčiniec, N. Zum Beschrcibungsmodell phonologischer Kontrastivstudien. - Linguistische Studien, Reihe A, H. 37, Berlin, 1977.

Moulion, W. The sounds of English and German. Chicago/London, 1963.

Moulton. W. Phoncmische Segmenticrungsmerkmalc in der deutschen Hochlautung der Gegenwart. - In: Stegcr. Vorschläge fur eine strukturale Grammatik des Deutschen. Darmstadt. 1970, 429-453. 
Müller, U. Von der statistischen Lautbetrachtung zum Sprechbewegungsablauf. - DaF, 1980/3, 167-171.

Neppert, J., M. Petursson. Elemente einer akustischen Phonetik (Als Manuskript gedruckt). Hamburg, 1984.

Neumann, W. und Autorenkollektiv. Theoreische Probleme der Sprachwissenschaft. Bd. I. Berlin, 1976. Zeichen und Bedeutung. 366-423.

Panconcelli-Calzia, J. Über das Verhalten von Dauer und Höhe im Akzent. Vox, 1917.

Philipp. M. Phonologie des Deutschen. Stuttgart/Berlin/Köln/Mainz, 1974.

Rom portl, M. Zentrum und Peripherie im phonologischen System. - Traveaux linguistique de Prague, 2, 1966, 103-110.

Rossi, M. L'intensite specifique des voyelles. - Phonetica, 24, 1971.

Roussclot. P. J. Principes de phonetique experimentale. Paris, 1924 (1. Aufl. 1897).

Sapir, E. Lautstruktur und Sprache. - In: G. Heike. Phonetik und Phonologie. München, 1974, 14-28.

Scatton, E. Bulgarian Phonology. Michigan, 1983.

Schmidi, L. Lautreduktionen und -assimilationen. Leipzig, 1982.

Schotola, Th. On the use of demilitillables in automatic word recognition. - Speech Communication, 3, 1984, 63-87.

Sicvers, E. Grundzüge der Phonetik zur Einfuhrung in das Studium der Lautlehre der indogermanischen Sprachen. 5. Auf. Leipzig. 1901.

Sime on ova. R. Übungsbuch zur deutschen Aussprache. Sofia, 1973/1982.

Simeonova, R. Korrektive deutsche Phonetik fur Bulgaren. - DaF, H. 1, 1978.

Simeonova, R. Kontrastivităt im Bereich der Aussprache. - Germanistisches Jahrbuch DDR - VRB, 1980/81, 248-258.

Sime on ova, R. Sekundäre akustische Parameter der deutschen und der bulgarischen Vokallaute. - In: E. Stock. Sprechwirkungsforschung, Sprecherzichung, Phonetik und Phonetikunterricht. Halle/Saale, 1982, 366-372.

Simeonova, R. Interferenzcrscheinungen im Bereich des Vokalismus bei Deutsch sprechenden Bulgaren. - In: E. S tock. Sprechwirkungsforschung, Sprecherzichung. Phonetik und Phonetikunterricht. Halle/Saale, 1982, 372-378.

Simeonova. R. Deutsche Standardaussprache und ihre Einbezichung in den Fremdsprachenunterricht. - In: Germanistisches Jahrbuch DDR - VRB 1984, Sofia, $51-68$.

Sime on ova. R. Experimentalphonetische Beobachtungen zu einigen Sandhi-Erscheinungen im Deutschen. - Philologia, 16, 1984, 16-28.

Simeonova, R., H. Kostova-Dobreva. Gesprochenes und geschriebenes Deutsch. Korrektiver Kurs für Germanistikstudenten. Sofia, 1985.

Simeonova, R. Grundzüge einer kontrastiven Phonetik Deutsch/Bulgarisch. Sofia, 1988.

Simič, R. Kontrastive deutsch-serbokroatische Phonologic. Zemun, 1979.

Stötzer, U. (verantw. Bearbeiter). Wörterbuch der deutschen Aussprachc. Leipzig, 1969.

Studiener, M. A. Lange Konsonanten in der bulgarischen Sprache der Gegenwart. - Zschr. f. Slawistik, 1976, 6, 225-229.

Sugarcwa, T. Deutsche Phonetik. Sofia, 1965.

Ternes, E. Probleme der kontrastiven Phonetik. - Forum Phoneticum, 13, Hamburg. 1976.

Ternes, E. Der Einfluß der Silbenstruktur auf die lautliche Interferenz. - Forum Phoneticum, 16, 1978, 93-119.

Tilkov, D. Le phonem indetermine. - Phonetica, 26, 1972, 210-215.

Trubetzkoy, N. S. Grundzüge der Phonologie. 5. Aufl. Göttingen, 1971 (Originalausg. - 1939). 
Tscheschner, W. Methoden und Einrichtungen zur subjektiven und objektiven Analyse von Sprache. - ZPSK, 15, 1962, 227-242.

Ulbrich, H. Instrumentalphonetische r-Untersuchungen im Deutschen. Berlin, 1972. Ungeheuer, G. Das Phonemsystem der deutschen Hochlautung. - In: Th. Siebs. Deutsche Aussprache., 19. Aufl., 1969, $27-42$.

Ven ne ma n n, Th. Zur Silbenstruktur der deutschen Standardsprache. - Linguistische Arbeiten, 126, Tübingen, 1982.

Wängle r, H. Grundriß einer Phonetik des Deutschen. 1. Aufl. Marburg, 1960; 2 . Aufl. 1967.

Wängler, H. Physiologische Phonetik. Marburg, 1972.

Wängler, H. Atlas deutscher Sprachlaute. 3. Auf. Berlin, 1964.

Wendler, J., G. Lindner, H. Ulbrich. Zur auditiven Wahrnehmung der Stimmstärke. - Z. f. Laryng., Rhin., Otol., 48, 1969, 139- 146.

Werner, O. Phonemik des Deutschen. Stuttgart, 1972.

Wolf, O. Sprache und Ohr. Leipzig, 1871.

Wurzel, W. Studien zur deutschen Lautstruktur. - Studia Grammatica, 8, 1970.

Wurzel, W. Die phonologische Komponente. - In: Grundzüge einer deutschen

Grammatik. 2. Auf. Berlin, 1984, 145-150.

Wurzel, W. Hauptklassen von Segmenten. - In: Grundzüge einer deutschen

Grammatik. 2. Aufl. Berlin, 1984, $901-988$.

Zacharias, Chr. Einführung in die Sprecherziehung. Berlin, 1966.

Zacher, O. Deutsche Phonetik. 2. Aufl. Leningrad, 1969.

Zwirner, E. Phonometrischer Beitrag zur Frage der neuhochdeutschen Quantität. - In: Arch. vgl. Phon., 1937.

Ан д рсйчи н. Л. Отг. Ред. Обратсн речник на съвременния български език. С., 1975.

Аронсон, Г. Морфонология болгарского словоизмснения. Москва, 1974.

Бернштейн, С. И. Вопросы обучения пронзнопснию. Москва, 1957.

Бонда рко, Л. В. Фонстичсское описанис языка и фонологическос описанис речи. Mock8a, 1981.

Георгиев, В., Ив. Дуриданов. Езикознание. 2. изд. София, 1965.

Гъл в 6 ов, К. Немска фонетика с оглед на българския език. 2. нзд. Софня, 1956.

Данчс 8, А. Към фонологичната характеристика на „българския“ английски мсжлинен език. - В: Материали от Втората научно-методическа конференция по съпоставитслно сзикознание и ЧЕО. София, 1980, 84-98.

Жинкин, Н. И. Механизмы речи. Москва, 1958.

Зиндер. Л. Р., Т. В. Строев а. Современный немецкий язык. 3. изд. Москва, 1957.

Зиндер, Л. Р., Т. В. Стросва. Исторнческая фонетика немецкого языка. Mосква, 1965.

3 латоустова, Л. В. Фонетическая структура в потоке речи. Казань, 1962, а.

3 лато устова, Л. В. Фонетические особенности словесного ударения в русском и болгарском языках. - В: Ученные записки Казанского гос. университета, 122, 1962a, 5.

Ив анов, В. В. Теория фонологических различительных признаков. - В: Новос в лингвистикс. Вып. 2. Москва, 1962.

Кожевников, В. А., Л. А. Чистович. Язык - артикуляция и восприятие. Ленинграл. 1963.

Котев а, Н. В. За консонанта $九$ пред е и и в българския книжовен изговор. - В: В памет на проф. Ст. Стойков. София, 1974.

Кочев. Ив. Позищионно и фахултативно вариране на фонемите в българския език. - БЕ, 1972, 6, 532-536.

Кочев, Ив. Уникални особености на българската фонол̈огична система. - БЕ, $1981,507-511$. 
Кънчев, Ив. За разстоянията между фонемите и диференциалнитс признаци В консонантните системи на испански и български език. - В: Съпост. езикознание, $2,1978$.

Леков, Ив. Фонологичната стойност на улължените и удвоените съгласни звукове в славянските езици. - В: ГСУ, Ист.-фил. ф-тет, 36, 1939.

Леков. Ив. Опит за фонологична характеристика на българския език. - В: Помагало по българска фонетика. София, 1980, 61-75.

Л сков, Ив. Насоки в развоя на фонологичните системи на славянските езици. София, 1960.

Л еков, Ив. Из стръмния път на фонологията. - БЕ, 1971, 2-3, $148-153$.

Л ско в, Ив. Към двояка - фонетична и фонологична - съпоставка на вокализма и консонантизма на славянските езици. - В: В памет на проф. С. Стойков. София, 1974.

Лилов, М. Фонстичните основи на говорната постановка в българския език. - 6E, 1972, 3, 210-220.

Маринова, М., Ас. Маринов. Статистически изследвания на фонемите в българския книжовен език. - БЕ, 1964, 2-3, 173-179.

Маслов, Ю. С. За някои фонемни редувания в съвременния български език (Опит за фонологичната им интерпретация). - 6Е, 1967, 6, 514-521.

Младенов, Ц. Фонологичен анализ на българската консонантна система (диференциални признаци и съвременни закони). - ИИБЕ, 19, 1970, 63-72.

Москов, М. Езикознание. София, 1981.

Н иколов, Б. Физическа сыщност и функционална стойност на акцентуваността в българския книжовен език. - Език и литература, 1972, 2, 11-22.

Норк. О. Н., К. М. Колосов. Взаимодействие лингвистических и физиологических факторов в образувании артикуляционной базы. - Иностранныс языки в икколе, 1973, I, 9-14.

Пашо в. Ив., Хр. Пврвев. Правоговорен речник на българския език. София, 1975.

П оп ов, К. Относно честотата на някои звукосъчетания в съвременния български език. - В: Помагало по българска фонетика. София, 1980, 167-173.

Радева. В. Рслуване на корслативнитс звучни и беззвучни съгласни фонеми в съвременния български книжовен език. - В: Помагало по българска фонетика. София, 1980, 302-309.

Симеонов, Б. Фонологична характеристика на беззвучните сыгласни в съвременния български книжовен език. - ИИБЕ, 19, 1970, 73-81.

Симеонов, Б. Структурно-фонологична и листрибутивна интерпретация на българските сонантни фонсми. - В: Помагало по българска фонетика. София, 1980, 153-166.

Симсонова. Р. Сравнителна акустична характеристика на гласните звукове в немския и в български език. - ГСУ, Фак. по западни филологии, 70, 1975, 1.

Симсонова, Р. Немският глассн звук [р] и неговите български съответствия. Дисертация, защц. в СУ, 1976.

Симсонов а. Р. За акустичния и фонологичния статус на немския гласен звук [ə] и неговите български съответки. - В: Съпоставително езикознание, $2,4$. 1978.

Си мсонов а, Р. За съдържанието и лефиницията на понятисто ..артикулационна 6аза“" на даден език. - Съпоставително езикознание. $1,1979$.

Симсонова, Р. Българо-нсмска вокална интерференция. - Съпоставително езикознание, 3, 1980.

Скатьн, В. За фонологията на [j]. - 6Е, 1977, 5, 486-487.

Стойков, Ст. Към новобългарския вокализъм. Учлснителен и слухов характер на самогласката [ъ]. - Македонски преглед, 13, 1942а. № 1. 
С тойков, Ст. Български книжовен изговор. Опитно изследване. - В: Сборник на БАН, 37, 19426.

Стойков, Ст. Разпрелялба на гласните в българския книжовен език. - Известия на д-вото на филолозите в България, 1942c, 1, 45-51.

С тойко в, Ст. Характер на съгласните пред гласните е и и в българския книжовен език. - В: Език и литература, 1956, 3, с. 240.

Стойков, Ст. Увод във фонетиката на българския език. София, 1967.

Стойков, Ст. Палаталните съгласни в българския книжовен език. - В: Помагало по българска фонетика. София, 1980, 109-128.

С тойков, Ст. Звук и фонсма. - В: Помагало по българска фонетика. София, $1980,22-45$.

С тойков, Ст. Днешно състояние на фонетичните и фонологичните проучвания на българския литературен език. София, 1975, 299-303.

С тоянов, Ст. Граматика на българския книжовен език. 2. изд. София, 1977.

Т илков, Д. Вокалната фонемна система на българския книжовсн език. - БЕ, 1966, 2, $97-109$.

Тилков, Д. Акустична характеристика на гласните в българския книжовен език. - БЕ, 1968, 2-3, 175- 185.

Тилков, Д. Сонорните съгласни в книжовния български език. - БЕ, 1969, 6. $507-523$.

Тилков, Д. Някои наблюдения върху промяната на интензитета при ударените и нсударсните гласни. - ИИБЕ, 19, 1970, 55-62.

Тилков. Д. Изслелвания върху акустичния състав на съгласните в българския книжовен сзик. - ИИБЕ, 21, 1972, 177-244.

Тилков, Д. Дистрибуция, съчетасмост и фреквснтност на й в съвремснния български език. - В: Славистичен сборник. София, 1973, 165-169.

Тилков. Д. Делимитативната функция на съгласните $\pi, \kappa, 2$ и $x$ в книжовния български сзик. - БЕ, 1973, I-2, 94-95.

Тилков. Д. Сылласната й край гласни в съвремснния български език. - В: Въпрсси на структурата на съвремснния български език. София, 1975, 5- 35 .

Тилков. Д. Количествена характеристика на противопоставянсто по мекостгвърлост в българския език. - БЕ, 1979, 3, $201-203$.

Тилков, Д. Акустичната характеристика и листрибуцията на палаталните съгласни в българския книжовен сзик. - ИИБЕ, 28, 1979, с. 21.

Тилков, Д. Функциониране на фонемата [В] в книжовния български език. - В: ГІомагало по българска фонетика. София, 1980, 148-152.

Тилков, Д. Акустически и перцептивни корелати на признака мекост-твърлост в българския език. - В: Помагало по българска фонетика. София, 1980. $129-133$.

Тилков, Д. Изслслвания върху българския език. София, 1982.

Тилков, Д., Т. Боялжиев. Българска фонетика. София, 1977.

Тилков, Д.. А. Мишсва. Вътрешноприсыщи характеристики на гласните - универсален характер и спсцифични особености. - Сыпоставително сзикознанис, $2,1978,3-11$.

Тилков. Д., А. Мишева. М. Песва. Акустична микроструктура на българските говорни звуковс. Ръкопис. 1977.

Христов. Ф. Нови мстоди за изслелване на фонемите. - Техника, 1962, 6, 215-218.

Христов, Ф. Изеледване върху акустичната структура на сонорните съгласни в българския език. - Техника, 1964, 1, 32-39.

Ч истович, Л. А. Текушес распознавание речи человеком. - В: Машинный перевол и прикладная лингвистика. Москва, 1961.

Я накиев, М. Българско стихознанис. София, 1960. 


\section{INHALTSVERZEICHNIS}

0. EINLEITUNG $\ldots \ldots \ldots \ldots \ldots \ldots \ldots \ldots \ldots \ldots \ldots \ldots \ldots \ldots$

1. KONTRASTIVE BEOBACHTUNGS- UND EXPERIMENTALANALYSE DER ARTIKULATORISCHEN BESONDERHEITEN IM PROZESS DER VOKALBILDUNG DES DEUTSCHEN UND DES BULGA-

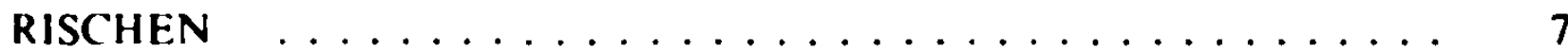

1.1. Segmentierungsmöglichkeiten der Sprachlaute im Redefluß ..... 7

1.2. Vokalartikulation des Deutschen und des Bulgarisehen ........ 10

1.2.1. Relevante Einstellungen und Sprechbewegungsabläufe für die Bildung der Kardinalvokale in den meisten Weltsprachen ....

1.2.2. Relevante Finstellungen und Sprechbewegungsabläufe für dic Vokalartikulation des Deutschen und des Bulgarischen .....

1.2.3. Spannungsgrad der aktiven Sprechorgane und der Wandungen der Mundhöhle

1.2.4. Sprechbewegungen der Lippen und des Unterkiefers ....... 14

1.2.5. Sprechbewegungen der Zunge .............. 16

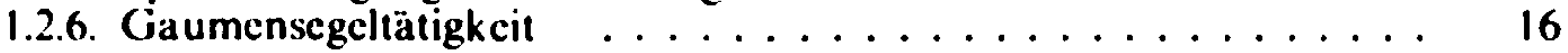

2. KONTRASTIVE ANALYSE DER AKUSTISCHEN STRUKTUR DER DEUTSCHEN UND DER BULGARISCHEN VOKALLAUTE . . . . .

2.1. Primäre akustische Merkmale der Vokallaute des Deutschen und des Bulgarischen

2.1.1. Untersuchungsverfahren

2.1.2. Vergleichende Charakteristik der primären Spektralmerkmale der deutschen und der bulgarischen Vokallaute ...........

2.1.3. Spektrale Besonderheiten der betonten Vokale des Deutschen .

2.1.4. Spektrale Besonderheiten des bulgarischen betonten Vokalismus . .

2.1.5. Vergleichende Charakteristik der primären Spektralmerkmale des deutschen und des bulgarischen betonten Vokalismus .......

2.1.6. Spezifische spektrale Besonderheiten der deutschen betonten Vokale, realisiert von Deutsch sprechenden Bulgaren ........

2.1.7. Vergleichende Charakteristik der primären Spektralparameter des deutschen und des bulgarischen unbetonten Vokalismus ..... 2.1.7.1. Spezifische spektrale Besonderheiten der unbetonten deutschen Vokale, realisiert von Deutsch sprechenden Bulgaren

2.1.7.2. Zusammenfassung der Untersuchungsergebnisse ........

2.1.7.3. Sperifische Besonderheiten des deutschen Vokalismus, realisiert von Deutsch sprechenden Bulgaren (Auditiver Test)

2.2. Sekundäre akustische Merkmale des deutschen und des bulgarischen 
2.2.2. Vergleichendē Charakteristik der sekundären Spektralmerkmale der deutschen und der bulgarischen Vokallaute . . . . . . . .

2.2.3. Grundfrequenz des deutschen und des bulgarischen Vokalismus. Untersuchungsverfahren

2.2.3.1. Grundtonfrequenz der betonten Vokale des Deutschen und des Bulgarischen . . . . . . . . . . . . . . . .

2.2.3.2. Grundfrequenz der unbetonten Vokale des Deutsehen und des Bulgarischen . . . . . . . . . . . . . . .

2.2.3.3. Beeinflussung der Grundfrequenz der Vokallaute des Deutschen und des Bulgarischen durch die konsonantische Lautnachbarschaft . . . . . . . . . . . . . . .

2.2.3.4. Abhängigkeit der Grundfrequenz der Vokale des Deutschen und des Bulgarischen von der Akzentuierung im Wort .

2.2.4. Spezifische Intensität der deutschen und der bulgarischen Vokallaute

2.2.4.1. Dic wichtigsten Untersuchungen zur spezifischen Intensität der Vokale . . . . . . . . . . . . . . . . .

2.2.4.2. Vergleichende Intensitätscharakteristik der deutschen und der bulgarischen Vokallaute . . . . . . . . . . . .

2.2.5. Spezifische Dauer der deutschen und der bulgarischen Vokallautc

2.2.5.1. Die wichtigsten Untersuchungen zur spezifischen Dauer der Vokale

2.2.5.2. Vergleichende Charakteristik der spezifischen Lautdaucr der deutschen und der bulgarischen Vokale . . . . . .

3. VERGLEICHENDE CHARAKTERISTIK DER DEUTSCHEN UND DER BULGARISCHEN VOKALPHONEME .

3.1. Vergleichende phonologische Interpretation des unbetonten Vokalismus des Deutschen und des Bulgarischen

3.2. Distribution der Vokallaute in der deutschen und in der bulgarischen Sprache

4. ZUSAMMENFASSENDE SCHLUSSBEMERKUNGEN

5. DIE KONSONANTENSYSTEME DES DEUTSCHEN UND DES BULGARISCHEN

5.1. Allgemeines zur Konsonantenbildung ............

5.2. Phonetische Klassifizierung der Konsonanten . . . . . . . .

5.3. Phonologische Klassifikation der Konsonanten . . . . . . . .

6. DIE KONSONANTENPHONEME DES DEUTSCHEN UND DES

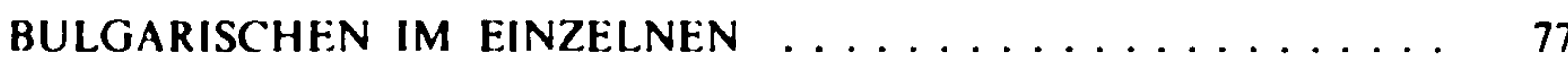

6.1. VerschluBlaute (Explosive) . . . . . . . . . . . . . . . . 77

6.2. Phonetische Charakteristik der deutschen und der bulgarischen

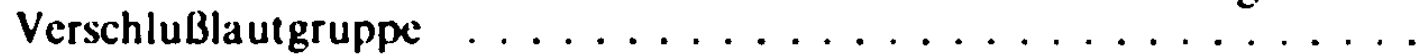

6.3. Paradigmatische und syntagmatische Darstellung der deutschen und der bulgarischen Explosive

6.3.1. Oppositionen der Fortis-Korrelation (für das Deutsche) bzw. der Korrelation stimmlos/stimmhaft (für das Bulgarische) (eindimensional)

6.3.2. Distribution und Kombinatorik der Explosive im Deutschen und im Bulgarischen (Übersicht auf der Grundlage von An- und Auslautstrukturen von konsonantischen Allophonen) ...... 6.3.2.1. Anlautstrukturen 
6.3.2.2. Kombinatorik der Explosive mit Konsonanten als allophonische Auslautstrukturen ...........

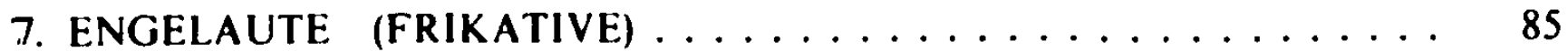

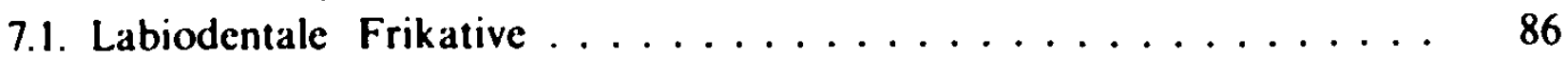

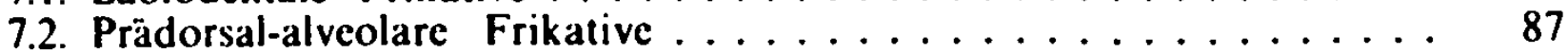

7.3. Prädorsal-palatale Frikative . . . . . . . . . . . . 87

7.3.1. Laryngal-pharyngaler Hauchlaut .............. 90

7.4. Mediodorsal-mediopalatale Frikative ............... 91

7.5. Paradigmatische und syntagmatische Darstellung der Fikative im

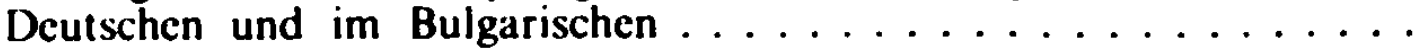

7.5.1. Oppositionen der Fortis-Korrelation (fur das Deutsche) bzw. der Korrelation stimmlos/stimmhaft (für das Bulgarische) (eindimensional)

7.5.2. Distribution und Kombinatorik der Frikative im Deutschen und im Bulgarischen (Übersicht auf der Grundlage von An- und Auslautstrukturen von konsonantischen Allophonen) ...... 7.5.2.1. Anlautstrukturen . . . . . . . . . . . . . . . 7.5.2.2. Auslautstrukturen (vor Pause)

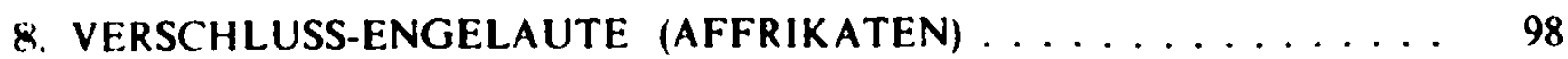

8.1. Physikalische Dauer der deutschen und der bulgarischen Affrikaten . 100

8.1.1. Zusammenfassung der Ergebnisse aus der kontrastiven sonagraphischen Analyse der deutschen und der bulgarischen Afrikaten . . . . . . . . . . . . . . . . .

8.2. Die Affrikaten des Deutschen und des Bulgarischen im einzelnen . .

8.2.1. Deutsches /pI/

8.2.2. Deutsches $/ \mathrm{ts} /$, bulgarisches $/ \mathrm{ts} / . / \mathrm{ts} / ; / \mathrm{dz} /, / \mathrm{si}: / \ldots \ldots \ldots$

8.2.3. Bulgarisches $/ \mathrm{t} / /-/ \mathrm{d} z /$, deutsches $/ \mathrm{t} \mathrm{f} /$.

8.3. Paradigmatische und syntagmatische Darstellung der deutschen und der

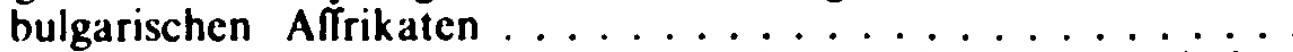

8.3.1. Minimalpaaroppositionen der deutschen und der bulgarisehen Allrikaten . . . . . . . . . . . . . . .

8.3.2. Distribution und Kombinatorik der Afrikaten im Deutsehen und

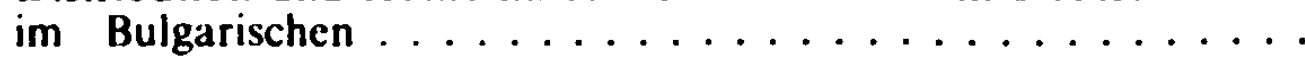

8.3.2.1. Anlautstrukturen $\ldots \ldots \ldots \ldots \ldots \ldots$

8.3.2.2. Auslautstrukturen . . . . . . . . . .

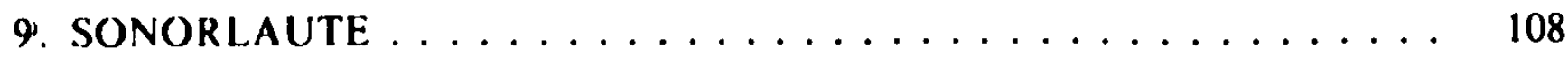

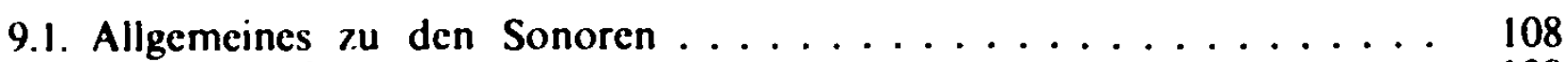

9.1.1. Nasale . . . . . . . . . . . . . . . . 108

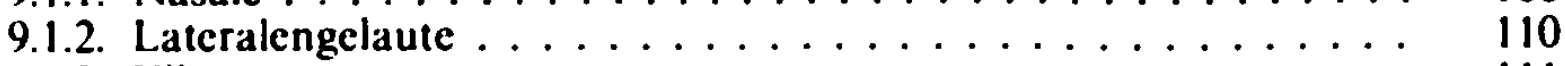

9.1.3. Vibrantes . . . . . . . . . . . . . . . . 111

9.1.3.1. Deutsches $/ \mathrm{r} /$ und seine Allophone . . . . . . . 111

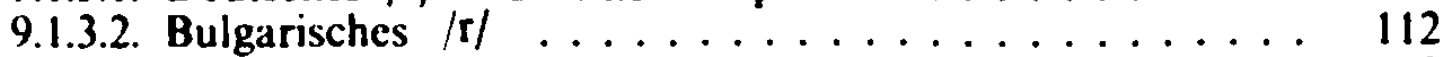

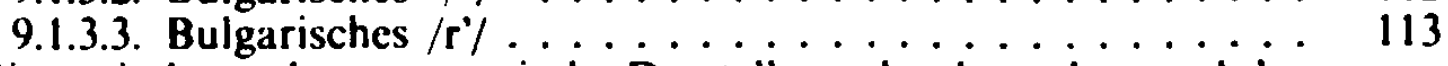

9.2. Paradigmatische und syntagmatische Darstellung der dcutschen und der bulgarischen Sonorlaute

9.2.1. Minimalpaaroppositionen der deutschen und der bulgarischen Sonorlaute

9.2.2. Distribution und Kombinatorik der Sonorlaute im Deutschen und im Bulgarischen (Ühersicht auf der Grundlage von An- und Auslautstrukturen)

9.2.2.2. Auslautstrukturen mit Sonor in Finalstellung . . . . 118 
10. ZUSAMMENFASSENDE SCHLUSSBEMERKUNGEN . . . . . . 119

11. BEILAGE . . . . . . . . . . . . . . . . . . . . . 124

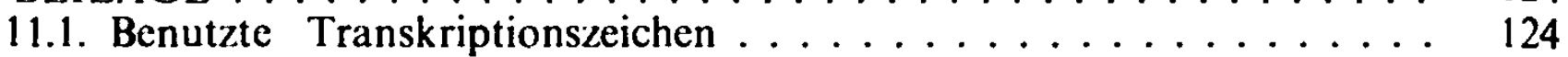

11.2. Abbildungen und Tabellen . . . . . . . . . . . . . 128

11.3. Sonagramme . . . . . . . . . . . . . . . . 156

11.4. Versuchsbeispiele für die intonographische Analyse der akustischen Parameter der deutschen und der bulgarischen Vokale ....... 202

11.5. Beispiele zur Spektralanalyse der deutschen und der bulgarischen Konsonanten ...................... 205

LITERATURVERZEICHNIS . . . . . . . . . . . . . 210

Bayerlsche

Staatsbibliothok

Münctien

Photosatz: Verlag der Bulgarischen Akademie der Wissenschaften

Druck: Druckerei des Verlags der Bulgarischen Akademie der Wissenschaften Bulgarien, 1113 Sofia, Akad. G. Bontschev, Bl. 6 